\title{
2020 ESC Guidelines for the diagnosis and management of atrial fibrillation developed in collaboration with the European Association for Cardio-Thoracic Surgery (EACTS)
}

\section{The Task Force for the diagnosis and management of atrial fibrillation of the European Society of Cardiology (ESC)}

\section{Developed with the special contribution of the European Heart Rhythm Association (EHRA) of the ESC}

Authors/Task Force Members: Gerhard Hindricks* (Chairperson) (Germany), Tatjana Potpara* (Chairperson) (Serbia), Nikolaos Dagres (Germany), Elena Arbelo (Spain), Jeroen J. Bax (Netherlands), Carina Blomström-Lundqvist (Sweden), Giuseppe Boriani (Italy), Manuel Castella ${ }^{1}$ (Spain), Gheorghe-Andrei Dan (Romania), Polychronis E. Dilaveris (Greece), Laurent Fauchier (France), Gerasimos Filippatos (Greece), Jonathan M. Kalman (Australia), Mark La Meir ${ }^{1}$ (Belgium), Deirdre A. Lane (United Kingdom), Jean-Pierre Lebeau (France),

\footnotetext{
* Corresponding authors: The two chairpersons contributed equally to the document.

Gerhard Hindricks, University Clinic of Cardiology, Heart Center Leipzig, Department of Cardiology and Electrophysiology, Leipzig Heart Institute, Strümpellstr. 39, 04289 Leipzig, Germany. Tel: +49 341865 1410, Fax: +49 341865 1460, Email: gerhard.hindricks@helios-gesundheit.de

Tatjana Potpara, School of Medicine, Belgrade University, dr Subotica 8, 11000 Belgrade, Serbia, and Cardiology Clinic, Clinical Centre of Serbia, Visegradska 26, 11000 Belgrade, Serbia. Tel: +3811 1361 6319, Email: tatjana.potpara@med.bg.ac.rs

ESC Committee for Practice Guidelines (CPG) and National Cardiac Societies document reviewers, and Author/Task Force Member affiliations: listed in the Appendix.

${ }^{1}$ Representing the European Association for Cardio-Thoracic Surgery (EACTS)

ESC entities having participated in the development of this document:

Associations: Association for Acute CardioVascular Care (ACVC), Association of Cardiovascular Nursing \& Allied Professions (ACNAP), European Association of Cardiovascular Imaging (EACVI), European Association of Preventive Cardiology (EAPC), European Association of Percutaneous Cardiovascular Interventions (EAPCI), European Heart Rhythm Association (EHRA), Heart Failure Association (HFA)

Councils: Council on Stroke, Council on Valvular Heart Disease.

Working Groups: Cardiac Cellular Electrophysiology, Cardiovascular Pharmacotherapy, Cardiovascular Surgery, e-Cardiology, Thrombosis.

The content of these European Society of Cardiology (ESC) Guidelines has been published for personal and educational use only. No commercial use is authorized. No part of the ESC Guidelines may be translated or reproduced in any form without written permission from the ESC. Permission can be obtained upon submission of a written request to Oxford University Press, the publisher of the European Heart Journal and the party authorized to handle such permissions on behalf of the ESC (journals.permissions@oup.com). Disclaimer The ESC Guidelines represent the views of the ESC and were produced after careful consideration of the scientific and medical knowledge and the evidence available at the time of their publication. The ESC is not responsible in the event of any contradiction, discrepancy and/or ambiguity between the ESC Guidelines and any other official recommendations or guidelines issued by the relevant public health authorities, in particular in relation to good use of healthcare or therapeutic strategies. Health professionals are encouraged to take the ESC Guidelines fully into account when exercising their clinical judgment, as well as in the determination and the implementation of preventive, diagnostic or therapeutic medical strategies; however, the ESC Guidelines do not override, in any way whatsoever, the individual responsibility of health professionals to make appropriate and accurate decisions in consideration of each patient's health condition and in consultation with that patient and, where appropriate and/or necessary, the patient's caregiver. Nor do the ESC Guidelines exempt health professionals from taking into full and careful consideration the relevant official updated recommendations or guidelines issued by the competent public health authorities, in order to manage each patient's case in light of the scientifically accepted data pursuant to their respective ethical and professional obligations. It is also the health professional's responsibility to verify the applicable rules and regulations relating to drugs and medical devices at the time of prescription.
} 


\section{Maddalena Lettino (Italy), Gregory Y. H. Lip (United Kingdom), Fausto J. Pinto} (Portugal), G. Neil Thomas (United Kingdom), Marco Valgimigli (Switzerland), Isabelle C. Van Gelder (Netherlands), Bart P. Van Putte ${ }^{1}$ (Netherlands),

\section{Caroline L. Watkins (United Kingdom)}

Document Reviewers: Paulus Kirchhof (CPG Review Coordinator) (United Kingdom/Germany), Michael Kühne (CPG Review Coordinator) (Switzerland), Victor Aboyans (France), Anders Ahlsson' (Sweden), Pawel Balsam (Poland), Johann Bauersachs (Germany), Stefano Benussi' (Italy), Axel Brandes (Denmark), Frieder Braunschweig (Sweden), A. John Camm (United Kingdom), Davide Capodanno (Italy), Barbara Casadei (United Kingdom), David Conen (Canada), Harry J. G. M. Crijns (Netherlands), Victoria Delgado (Netherlands), Dobromir Dobrev (Germany), Heinz Drexel (Austria), Lars Eckardt (Germany), Donna Fitzsimons (United Kingdom), Thierry Folliguet (France), Chris P. Gale (United Kingdom), Bulent Gorenek (Turkey), Karl Georg Haeusler (Germany), Hein Heidbuchel (Belgium), Bernard Iung (France), Hugo A. Katus (Germany), Dipak Kotecha (United Kingdom), Ulf Landmesser (Germany), Christophe Leclercq (France), Basil S. Lewis (Israel), Julia Mascherbauer (Austria), Jose Luis Merino (Spain), Béla Merkely (Hungary), Lluís Mont (Spain), Christian Mueller (Switzerland), Klaudia V. Nagy (Hungary), Jonas Oldgren (Sweden), Nikola Pavlović (Croatia), Roberto F. E. Pedretti (Italy), Steffen E. Petersen (United Kingdom), Jonathan P. Piccini (United States of America), Bogdan A. Popescu (Romania), Helmut Pürerfellner (Austria), Dimitrios J. Richter (Greece), Marco Roffi (Switzerland), Andrea Rubboli (Italy), Daniel Scherr (Austria), Renate B. Schnabel (Germany), lain A. Simpson (United Kingdom), Evgeny Shlyakhto (Russia), Moritz F. Sinner (Germany), Jan Steffel (Switzerland), Miguel Sousa-Uva (Portugal), Piotr Suwalski' (Poland), Martin Svetlosak (Slovakia), Rhian M. Touyz (United Kingdom)

The disclosure forms of all experts involved in the development of these guidelines are available on the ESC website www.escardio.org/guidelines

SD For the Supplementary Data which include background information and detailed discussion of the data that have provided the basis for the Guidelines see European Heart Journal online.

Click here to access the corresponding chapter in section 4 I - Atrial fibrillation

Keywords

Guidelines $\bullet$ atrial fibrillation $\bullet$ anticoagulation $\bullet$ vitamin $\mathrm{K}$ antagonists $\bullet$ non-vitamin $\mathrm{K}$ antagonist oral anticoagulants - left atrial appendage occlusion • rate control • rhythm control • cardioversion • antiarrhythmic drugs $\bullet$ catheter ablation $\bullet$ pulmonary vein isolation $\bullet$ left atrial ablation $\bullet$ AF surgery $\bullet$ upstream therapy

- $\mathrm{ABC}$ pathway • screening • stroke • recommendations

\section{Table of contents}

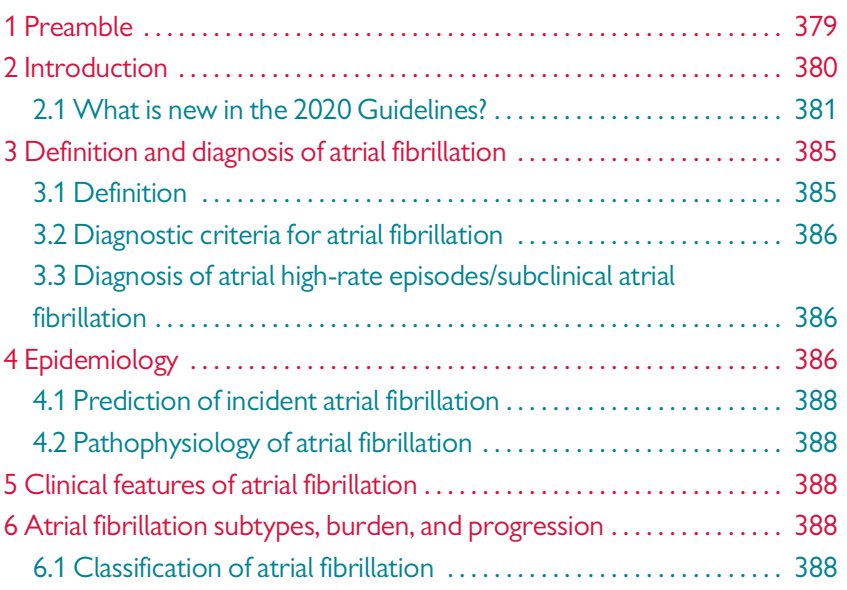

6.2 Definition and assessment of atrial fibrillation burden ........ 391

6.3 Atrial fibrillation progression ........................ 392

6.4 Atrial cardiomyopathy: definition, classification, clinical

implications, and diagnostic assessment .................. 392

7 Screening for atrial fibrillation . . . . . . . . . . . . . . . . . . . 392

7.1 Screening tools ................................ 392

7.2 Screening types and strategies ....................... 394

7.3 Benefits from and risks of screening for atrial fibrillation ...... 394

7.4 Cost-effectiveness of screening for atrial fibrillation .......... 394

7.5 Screening in high-risk populations ..................... 395

7.5.1 Elderly ...................................... 395

8 Diagnostic assessment in atrial fibrillation ................. 395

8.1 Symptoms and quality of life ...................... 395

8.2 Substrate .................................... 395

9 Integrated management of patients with atrial fibrillation ........ 398 
9.1 Definitions and components of integrated management

of atrial fibrillation patients . . . . . . . . . . . . . . . . . . . . . 398

9.2 Multidisciplinary atrial fibrillation teams ................. 398

9.2.1 Role of healthcare systems and budget constraints ...... 398

9.3 Patient involvement and shared decision making ........... 398

9.3.1 Patient values and preferences .................. 398

9.3.2 Patient education ............................ 399

9.4 Healthcare professional education .................... 399

9.5 Adherence to treatment ........................... 399

9.6 Technology tools supporting atrial fibrillation management ... 399

9.7 Advantages of integrated management of atrial

fibrillation patients

9.8 Measures (or approaches) for implementation of

integrated management ............................ 400

9.9 Treatment burden .................................. 400

9.10 Patient-reported outcomes ........................ 400

10 Patient management: the integrated $A B C$ pathway ........... 401

10.1 'A' - Anticoagulation/Avoid stroke .................. 401

10.1.1 Stroke risk assessment ........................ 401

10.1.2 Bleeding risk assessment ......................... 402

10.1.3 Absolute contraindications to oral anticoagulants ...... 404

10.1.4 Stroke prevention therapies ................... 404

10.1.4.1 Vitamin K antagonists ...................... 404

10.1.4.2 Non-vitamin K antagonist oral anticoagulants ...... 405

10.1.4.3 Other antithrombotic drugs ................ 405

10.1.4.4 Combination therapy with oral anticoagulant and

antiplatelet drugs ............................ 406

10.1.4.5 Left atrial appendage occlusion and exclusion ...... 406

10.1.4.5.1 Left atrial appendage occlusion devices ........ 406

10.1.4.5.2 Surgical left atrial appendage

occlusion or exclusion ............................ 406

10.1.4.6 Long-term oral anticoagulation per atrial

fibrillation burden ............................. 407

10.1.4.7 Long-term oral anticoagulation per symptom

control strategy .............................. 407

10.1.5 Management of anticoagulation-related bleeding risk . . . 407

10.1.5.1 Strategies to minimize the risk of bleeding ....... 407

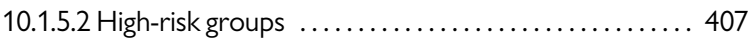

10.1.6 Decision-making to avoid stroke ............... 407

10.2 'B' - Better symptom control ........................ 409

10.2.1 Rate control ................................ 409

10.2.1.1 Target/optimal ventricular rate range ............ 409

10.2.1.2 Drugs .............................. 409

10.2.1.3 Acute rate control ........................ 410

10.2.1.4 Atrioventricular node ablation and pacing ........ 410

10.2.2 Rhythm control ........................... 413

10.2.2.1 Indications for rhythm control ................. 413

10.2.2.2 Cardioversion ................................. 414

10.2.2.2.1 Immediate cardioversion/elective cardioversion .. 414

10.2.2.2.2 Electrical cardioversion ................... 414

10.2.2.2.3 Pharmacological cardioversion

(including 'pill in the pocket') ..................... 414

10.2.2.2.4 Follow-up after cardioversion ............. 414

10.2.2.3 Atrial fibrillation catheter ablation .............. 417

10.2.2.3.1 Indications ........................... 417

10.2.2.3.2 Techniques and technologies ............. 419

10.2.2.3.3 Complications .......................... 419
10.2.2.3.4 AF catheter ablation outcome and impact of modifiable risk factors ........................... 419

10.2.2.3.5 Follow-up after atrial fibrillation ablation ...... 420 10.2.2.3.7 Risk assessment for recurrence of AF post

catheter ablation $\ldots \ldots \ldots \ldots \ldots \ldots \ldots \ldots \ldots \ldots \ldots . \ldots 20$

10.2.2.4 Surgery for atrial fibrillation $\ldots \ldots \ldots \ldots \ldots \ldots \ldots 421$

10.2.2.4.1 Concomitant surgery for atrial fibrillation:

indications, outcome, complications .............. 422

10.2.2.4.2 Stand-alone surgery for atrial fibrillation:

indications, outcome, complications ............... 422

10.2.2.5 Hybrid surgical/catheter ablation procedures ...... 422

10.2.2.6 Peri-procedural stroke risk management in

patients undergoing rhythm control interventions ........ 423

10.2.2.6.1 Management of stroke risk and oral anticoagulant

therapy in atrial fibrillation patients undergoing

cardioversion ................................. 423

10.2.2.6.2 Management of stroke risk and oral

anticoagulant therapy in atrial fibrillation patients

undergoing atrial fibrillation catheter ablation

10.2.2.6.3 Postoperative anticoagulation after surgery

for atrial fibrillation ............................ 424

10.2.2.7 Long-term antiarrhythmic drug therapy for

rhythm control .............................. 424

10.2.2.7.1 Antiarrhythmic drugs $\ldots \ldots \ldots \ldots \ldots \ldots \ldots . \ldots 24$

10.3 ' C - Cardiovascular risk factors and concomitant

diseases: detection and management . ................... 429

10.3.1 Lifestyle interventions ....................... 429

10.3.1.1 Obesity and weight loss ................... 429

10.3.1.2 Alcohol and caffeine use ..................... 430

10.3.1.3 Physical activity ........................... 430

10.3.2 Specific cardiovascular risk factors/comorbidities ...... 430

10.3.2.1 Hypertension ............................ 430

10.3.2.2 Heart failure ........................... 430

10.3.2.3 Coronary artery disease $\ldots \ldots \ldots \ldots \ldots \ldots \ldots \ldots 430$

10.3.2.4 Diabetes mellitus ............................. 430

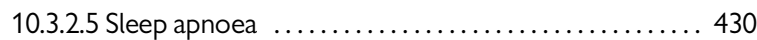

11 The $A B C$ pathway in specific clinical settings/conditions/

patient populations .............................. 431

11.1 Atrial fibrillation with haemodynamic instability .......... 431

11.2 First-diagnosed (new-onset) atrial fibrillation ............. 431

11.3 Acute coronary syndromes, percutaneous coronary

intervention, and chronic coronary syndromes in patients with

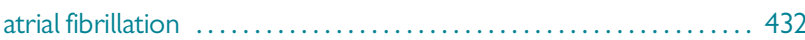

11.4 Acute stroke or intracranial haemorrhage in patients with

atrial fibrillation .................................... 435

11.4.1 Patients with atrial fibrillation and acute ischaemic

stroke or transient ischaemic attack ................... 435

11.4.2 Cryptogenic stroke/embolic stroke with

undetermined source ............................ 435

11.4.3 Post-stroke patients without known atrial fibrillation .... 436

11.4.4 Management of patients with atrial fibrillation

postintracranial haemorrhage $\ldots \ldots \ldots \ldots \ldots \ldots \ldots \ldots \ldots . \ldots 436$

11.5 Active bleeding on anticoagulant therapy: management

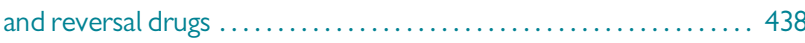

11.6 Atrial fibrillation and heart failure ................... 439

11.7 Atrial fibrillation and valvular heart disease ............. 439

11.8 Atrial fibrillation and chronic kidney disease ............. 440 
11.9 Atrial fibrillation and peripheral artery disease ........... 440

11.10 Atrial fibrillation and endocrine disorders ............... 440

11.11 Atrial fibrillation and gastrointestinal disorders .......... 440

11.12 Atrial fibrillation and haematological disorders ........... 441

11.13 The elderly and frail with atrial fibrillation .............. 441

11.14 Patients with cognitive impairment/dementia ........... 441

11.15 Atrial fibrillation and congenital heart disease ........... 441

11.16 Atrial fibrillation in inherited cardiomyopathies and

primary arrhythmia syndromes ....................... 442

11.17 Atrial fibrillation during pregnancy .................. 442

11.18 Atrial fibrillation in professional athletes .............. 443

11.19 Postoperative atrial fibrillation ...................... 443

11.19.1 Prevention of postoperative AF . ............... 444

11.19.2 Prevention of thrombo-embolic events ............ 444

12 Prevention of atrial fibrillation .......................... 445

12.1 Primary prevention of atrial fibrillation ................. 445

12.2 Secondary prevention of atrial fibrillation ............... 445

13 Sex-related differences in atrial fibrillation ................. 445

14 Implementation of the atrial fibrillation guidelines ............. 446

15 Quality measures and clinical performance indicators

in the management of atrial fibrillation $\ldots \ldots \ldots \ldots \ldots \ldots \ldots \ldots . \ldots 446$

16 Epidemiology, clinical implications, and management of atrial

high-rate episodes/subclinical atrial fibrillation ............... 446

17 Atrial fibrillation and other atrial tachyarrhythmias (atrial flutter

and atrial tachycardias) ............................... 449

18 Key messages ...................................... 449

19 Gaps in evidence .................................. 450

20 'What to do' and 'what not to do' messages from the

Guidelines ............................................. 452

21 Supplementary data ................................ 456

22 Appendix ........................................ 456

23 References ...................................... 457

\section{List of recommendations}

New recommendations ................................ 381

Changes in the recommendations ......................... 383

Recommendations for diagnosis of AF .................. 386

Recommendations for structured characterization of AF ........ 391

Recommendations for screening to detect AF .............. 395

Recommendations for diagnostic evaluation of patients with AF ... 397

Recommendations about integrated AF management ........... 401

Recommendations for the prevention of thrombo-embolic

events in AF ...................................... 408

Recommendations for ventricular rate control in patients with AF .. 412

Recommendations for rhythm control .................... 414

Recommendations for cardioversion ..................... 417

Recommendations for rhythm control/catheter ablation of AF ..... 421

Recommendations for surgical ablation of $A F$............... 422

Recommendations for stroke risk management peri-cardioversion .. 423

Recommendations for stroke risk management peri-catheter ablation .. 424

Recommendations for postoperative anticoagulation after

AF surgery .................................... 424

Recommendations for long-term antiarrhythmic drugs ......... 429

Recommendations for lifestyle interventions and management of risk factors and concomitant diseases in patients with $A F$....... 431
Recommendations for management of AF with haemodynamic

instability ....................................... 431

Recommendations for patients with AF and an ACS, PCl, or CCS .. 434

Recommendations for the search for $A F$ in patients with

cryptogenic stroke ............................... 436

Recommendations for secondary stroke prevention in $\mathrm{AF}$

patients after acute ischaemic stroke ................... 437

Recommendations for stroke prevention in AF patients after intracranial

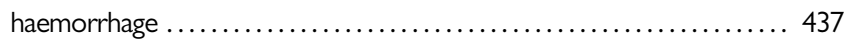

Recommendations for the management of active bleeding on OAC .. 439

Recommendations for patients with valvular heart disease and AF .. 439

Recommendations for the management of $A F$ in patients with

congenital heart disease ............................... 442

Recommendations for the management of AF during pregnancy ... 443

Recommendations for sports activity in patients with AF ........ 443

Recommendations for postoperative AF ................... 445

Recommendations pertaining to sex-related differences in AF ..... 446

Recommendations for quality measures in patients with AF ....... 446

Recommendations for management of patients with AHRE ....... 449

\section{List of tables}

Table 1 Classes of recommendations $\ldots \ldots \ldots \ldots \ldots \ldots \ldots \ldots \ldots \ldots . \ldots \ldots$

Table 2 Levels of evidence ........................... 380

Table 3 Definition of atrial fibrillation ....................... 385

Table 4 Classification of AF ............................. 390

Table 5 Sensitivity and specificity of various AF screening tools

considering the 12-lead ECG as the gold standard ............... 394

Table 6 EHRA symptom scale .............................. 396

Table 7 Stroke risk factors in patients with AF ............... 402

Table $8 \mathrm{CHA}_{2} \mathrm{DS}_{2}$-VASc score ....................... 403

Table 9 Risk factors for bleeding with $\mathrm{OAC}$ and antiplatelet

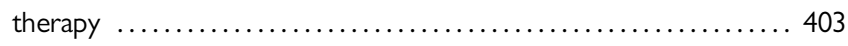

Table 10 Clinical risk factors in the HAS-BLED score ............ 404

Table 11 Dose selection criteria for NOACs ................ 405

Table 12 Antithrombotic therapy after left atrial appendage

occlusion ...................................... 406

Table 13 Drugs for rate control in AF .................... 411

Table 14 Antiarrhythmic drugs used for restoration of sinus rhythm .. 416

Table 15 Goals of follow-up after cardioversion of AF .......... 417

Table 16 Procedure-related complications in catheter ablation and

thoracoscopic ablation of AF ........................... 419

Table 17 Key issues in follow-up after AF catheter ablation ....... 420

Table 18 Principles of antiarrhythmic drug therapy ............. 425

Table 19 Rules to initiate antiarrhythmic drugs for long-term

rhythm control in AF .............................. 425

Table 20 AADs used for long-term maintenance of sinus

rhythm in AF patients ............................. 426

Table 21 Non-antiarrhythmic drugs with antiarrhythmic

properties (upstream therapy) $\ldots \ldots \ldots \ldots \ldots \ldots \ldots \ldots \ldots \ldots . \ldots 28$

Table 22 Summary of quality indicators for the diagnosis

and management of AF

\section{List of figures}

Figure 1 Diagnosis of AHRE/subclinical AF ................. 386 
Figure 2 Epidemiology of AF: prevalence; and lifetime risk and

projected rise in the incidence and prevalence .............. 387

Figure 3 Summary of risk factors for incident AF . ............ 388

Figure 4 Clinical presentation of AF and AF-related outcomes ..... 389

Figure $54 \mathrm{~S}-\mathrm{AF}$ scheme as an example of structured

characterization of AF .............................. 391

Figure 6 Systems used for AF screening . .................. 393

Figure 7 Potential benefits from and risks of screening for AF ...... 394

Figure 8 Diagnostic work-up and follow-up in AF patients ......... 396

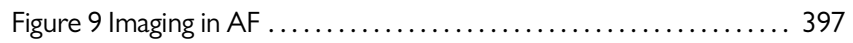

Figure 10 Components of integrated AF management ........... 398

Figure 11 Integrated AF management team (an example) ........ 399

Figure 12 ' $A$ ' - Anticoagulation/Avoid stroke:

The 'AF 3-step' pathway ............................... 408

Figure 13 Outline of rate control therapy $\ldots \ldots \ldots \ldots \ldots \ldots \ldots . \ldots 410$

Figure 14 Choice of rate control drugs .................... 412

Figure 15 Rhythm control strategy ....................... 413

Figure 16 Flowchart for decision making on cardioversion of AF

depending on clinical presentation, AF onset, oral anticoagulation

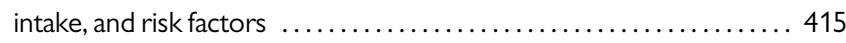

Figure 17 Indications for catheter ablation of symptomatic AF . . ... 418

Figure 18 Risk factors for AF contributing to the development

of an abnormal substrate translating into poorer outcomes with

rhythm control strategies $\ldots \ldots \ldots \ldots \ldots \ldots \ldots \ldots \ldots \ldots \ldots \ldots 420$

Figure 19 Long-term rhythm control therapy ................ 428

Figure 20 Post-procedural management of patients with AF

and $\mathrm{ACS} / \mathrm{PCl}$........................................ 433

Figure 21 (Re-) initiation of anticoagulation post-intracranial bleeding .. 437

Figure 22 Management of active bleeding in patients

receiving anticoagulation ............................... 438

Figure 23 Management of postoperative AF ................. 444

Figure 24 Progression of atrial high-rate episode burden and

stroke rates according to AHRE daily burden and CHA2DS2-VASc

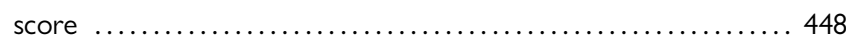

Figure 25 Proposed management of AHRE/subclinical AF ........ 448

Central Illustration. Management of AF..................... 451

\section{List of boxes}

Box 1 About post-procedural management of patients with $\mathrm{AF}$

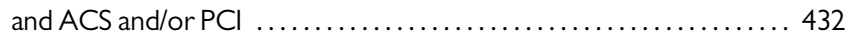

Box 2 About acute ischaemic stroke in patients with AF ........ 435

\section{Abbreviations and acronyms}

Abbreviations and acronyms

4S-AF Stroke risk, Symptom severity, Severity of AF

burden, Substrate severity

AAD Antiarrhythmic drug

$A B C$

Atrial fibrillation Better Care [includes A (avoid stroke), B (better symptom control), and C (cardiovascular risk factors and comorbid conditions management)]

ABC-bleeding Age, Biomarkers (haemoglobin, cTnT hs T, GDF-15), and Clinical history (prior bleeding)

ABC-stroke Age, Biomarkers, Clinical history (stroke risk score)

ACS

Acute coronary syndromes
ACTIVE W

AF

AFFIRM

AFL

AHRE

AMICA

ARCADIA

ARISTOTLE

ARREST-AF

AST

ATRIA

ATTICUS

AVERROES

b.i.d.

BP

bpm

$\mathrm{C}_{2} \mathrm{HEST}$

\section{CABANA}

CAD

CAPTAF

CASTLE-AF

CATCH-ME

CCB

CCS

$\mathrm{CHA}_{2} \mathrm{DS}_{2}-$

VASC

$\mathrm{CHADS}_{2}$

$\mathrm{CHF}$

$\mathrm{Cl}$

CIED

CKD

COP-AF
Atrial Fibrillation Clopidogrel Trial with Irbesartan for Prevention of Vascular Events trial

Atrial fibrillation

Atrial Fibrillation Follow-up Investigation of Rhythm Management

Atrial flutter

Atrial high-rate episode

Atrial Fibrillation Management in Congestive Heart Failure With Ablation

AtRial Cardiopathy and Antithrombotic Drugs In Prevention After Cryptogenic Stroke

Apixaban for Reduction in Stroke and Other Thromboembolic Events in Atrial Fibrillation

Aggressive Risk Factor Reduction Study Implication for AF

Aspartate aminotransferase

Anticoagulation and Risk Factors in Atrial

Fibrillation (score)

Apixaban for treatment of embolic stroke of undetermined source

Apixaban Versus Acetylsalicylic Acid (ASA) to Prevent Stroke in Atrial Fibrillation Patients Who Have Failed or Are Unsuitable for Vitamin K Antagonist Treatment

bis in die (twice a day)

Blood pressure

Beats per minute

CAD/COPD (1 point each), Hypertension (1 point), Elderly ( $\geq 75$ years, 2 points), Systolic heart failure (2 points), and Thyroid disease (hyperthyroidism, 1 point) (score)

Catheter ABlation vs. ANtiarrhythmic Drug

Therapy for Atrial Fibrillation

Coronary artery disease

Catheter Ablation compared with

Pharmacological Therapy for Atrial Fibrillation

Catheter Ablation vs. Standard conventional

Treatment in patients with LEft ventricular dysfunction and Atrial Fibrillation

Characterizing AF by Translating its Causes into Health Modifiers in the Elderly

Calcium channel blocker

Chronic coronary syndrome

Congestive heart failure, Hypertension, Age $\geq 75$ years, Diabetes mellitus, Stroke, Vascular disease, Age 65-74 years, Sex category (female)

CHF history, Hypertension history, Age $\geq 75$ y,

Diabetes mellitus history, Stroke or TIA

symptoms previously

Congestive heart failure

Confidence interval

Cardiac implantable electronic device

Chronic kidney disease

Colchicine For The Prevention Of Perioperative

Atrial Fibrillation In Patients Undergoing

Thoracic Surgery 


\begin{tabular}{|c|c|c|c|}
\hline COPD & Chronic obstructive pulmonary disease & NOAC & Non-vitamin $\mathrm{K}$ antagonist oral anticoagulant \\
\hline CRT & Cardiac resynchronization therapy & o.d. & omni die (once daily) \\
\hline CTI & Cavotricuspid isthmus & OPTIMAS & OPtimal TIMing of Anticoagulation after Stroke \\
\hline cTnT-hs & High-sensitivity troponin T & OSA & Obstructive sleep apnoea \\
\hline EAST & prevention Trial & PAD & Peripheral artery disease \\
\hline ECG & Electrocardiogram & $\mathrm{PCl}$ & Percutaneous coronary intervention \\
\hline EHRA & European Heart Rhythm Association & PCORI & Patient-Centred Outcomes Research Institute \\
\hline \multirow[t]{2}{*}{ ELAN } & Early versus Late initiation of direct oral & PIONEER & OPen-Label, Randomized, Controlled, \\
\hline & $\begin{array}{l}\text { Anticoagulants in post-ischaemic stroke patients } \\
\text { with atrial fibrillatioN }\end{array}$ & $\mathrm{AF}-\mathrm{PCl}$ & $\begin{array}{l}\text { Multicenter Study Explorlng TwO TreatmeNt } \\
\text { StratEgiEs of Rivaroxaban and a Dose-Adjusted }\end{array}$ \\
\hline AF-TIMI 48 & Myocardial Infarction 48 & & Percutaneous Coronary Intervention \\
\hline ENTRUST- & Edoxaban Treatment Versus Vitamin K & PREVAIL & Watchman LAA Closure Device in Patients With \\
\hline \multirow{2}{*}{$\mathrm{AFPCl}$} & Antagonist in Patients With Atrial Fibrillation & & Atrial Fibrillation Versus Long Term Warfarin \\
\hline & Undergoing Percutaneous Coronary Intervention & & Therapy \\
\hline ESC & European Society of Cardiology & PRO & Patient-reported outcome \\
\hline GARFIELD-AF & $\begin{array}{l}\text { Global Anticoagulant Registry in the FIELD - } \\
\text { Atrial Fibrillation }\end{array}$ & PROTECT AF & $\begin{array}{l}\text { Watchman Left Atrial Appendage System for } \\
\text { Embolic Protection in Patients With Atrial }\end{array}$ \\
\hline GDF-15 & Growth differentiation factor-15 & & Fibrillation \\
\hline \multirow[t]{3}{*}{ HAS-BLED } & Hypertension, Abnormal renal/liver function, & $\mathrm{PVI}$ & Pulmonary vein isolation \\
\hline & Stroke, Bleeding history or predisposition, Labile & QoL & Quality of life \\
\hline & INR, Elderly (>65 years), Drugs/alcohol & QRS & QRS interval \\
\hline $\mathrm{ICH}$ & Intracranial haemorrhage & & Fibrillation Undergoing Percutaneous Coronary \\
\hline \multirow[t]{2}{*}{ IMPACT-AF } & Integrated Management Program Advancing & & Intervention \\
\hline & Community Treatment of Atrial Fibrillation & RE-CIRCUIT & Randomized Evaluation of dabigatran etexilate \\
\hline INR & International normalized ratio & & Compared to warfarln in pulmonaRy vein \\
\hline LA & Left atrium/atrial & & ablation: assessment of different peri- \\
\hline LAA & Left atrial appendage & & proCedUral antlcoagulation sTrategies \\
\hline LEGACY & $\begin{array}{l}\text { Long-term Effect of Goal-directed weight } \\
\text { management on an Atrial fibrillation Cohort: a 5- } \\
\text { Year follow-up study }\end{array}$ & REHEARSE-AF & $\begin{array}{l}\text { REmote HEArt Rhythm Sampling using the } \\
\text { AliveCor hear monitor to scrEen for Atrial } \\
\text { Fibrillation }\end{array}$ \\
\hline LGE-CMR & $\begin{array}{l}\text { Late gadolinium contrast-enhanced cardiac } \\
\text { magnetic resonance }\end{array}$ & RE-LY & $\begin{array}{l}\text { Randomized Evaluation of Long Term } \\
\text { Anticoagulant Therapy }\end{array}$ \\
\hline LMWH & Low-molecular-weight heparin & ROCKET AF & Rivaroxaban Once Daily Oral Direct Factor Xa \\
\hline LV & Left ventricular & & Inhibition Compared with Vitamin K Antagonism \\
\hline LVEF & Left ventricular ejection fraction & & for Prevention of Stroke and Embolism Trial in \\
\hline $\mathrm{LVH}$ & Left ventricular hypertrophy & & Atrial Fibrillation \\
\hline mAFA & Mobile AF App & $\mathrm{SAMe}-\mathrm{TT}_{2} \mathrm{R}_{2}$ & Sex (female), Age (<60 years), Medial history, \\
\hline \multirow[t]{3}{*}{ MANTRA-PAF } & Medical Antiarrhythmic Treatment or & & Treatment, Tobacco use, Race (non-Caucasian) \\
\hline & Radiofrequency Ablation in Paroxysmal Atrial & & (score) \\
\hline & Fibrillation & SBP & Systolic blood pressure \\
\hline $\mathrm{MRI}$ & Magnetic resonance imaging & START & Optimal Delay Time to Initiate Anticoagulation \\
\hline
\end{tabular}




$\begin{array}{ll}\text { STEMI } & \text { ST-segment elevation myocardial infarction } \\ \text { TIA } & \text { Transient ischaemic attack } \\ \text { TOE } & \text { Transoesophageal echocardiography } \\ \text { TTR } & \text { Time in therapeutic range } \\ \text { UFH } & \text { Unfractionated heparin } \\ \text { US } & \text { United States of America } \\ \text { VHD } & \text { Valvular heart disease } \\ \text { VKA } & \text { Vitamin K antagonist } \\ \text { WOEST } & \text { What is the Optimal antiplatElet and } \\ & \text { anticoagulant therapy in patients with oral } \\ & \text { anticoagulation and coronary StenTing }\end{array}$

\section{Preamble}

Guidelines summarize and evaluate available evidence with the aim of assisting health professionals in proposing the best management strategies for an individual patient with a given condition. Guidelines and their recommendations should facilitate decision making of health professionals in their daily practice. However, the final decisions concerning an individual patient must be made by the responsible health professional(s) in consultation with the patient and caregiver as appropriate.

A great number of Guidelines have been issued in recent years by the European Society of Cardiology (ESC), as well as by other societies and organizations. Because of their impact on clinical practice, quality criteria for the development of guidelines have been established in order to make all decisions transparent to the user. The recommendations for formulating and issuing ESC Guidelines can be found on the ESC website (https://www.escardio.org/Guidelines/ Clinical-Practice-Guidelines/Guidelines-development/Writing-ESCGuidelines). The ESC Guidelines represent the official position of the ESC on a given topic and are regularly updated.

In addition to the publication of Clinical Practice Guidelines, the ESC carries out the EurObservational Research Programme of international registries of cardiovascular diseases and interventions which are essential to assess, diagnostic/therapeutic processes, use of resources and adherence to Guidelines. These registries aim at providing a better understanding of medical practice in Europe and around the world, based on high-quality data collected during routine clinical practice.

Furthermore, the ESC has developed and embedded, in some of its guidelines, a set of quality indicators (QIs) which are tools to evaluate the level of implementation of the Guidelines and may be used by the ESC, hospitals, healthcare providers and professionals to measure clinical practice as well as used in educational programmes, alongside the key messages from the Guidelines, to improve quality of care and clinical outcomes.

The Members of this Task Force were selected by the ESC, including representation from its relevant ESC sub-specialty groups, in order to represent professionals involved with the medical care of patients with this pathology. Selected experts in the field undertook a comprehensive review of the published evidence for management of a given condition according to ESC Committee for Practice Guidelines (CPG) policy. A critical evaluation of diagnostic and therapeutic procedures was performed, including assessment of the risk-benefit ratio. The level of evidence and the strength of the recommendation of particular management options were weighed and graded according to predefined scales, as outlined below.

The experts of the writing and reviewing panels provided declaration of interest forms for all relationships that might be perceived as real or potential sources of conflicts of interest. Their declarations of interest were reviewed according to the ESC declaration of interest rules and can be found on the ESC website (http://www.escardio.org/ guidelines). This process ensures transparency and prevents potential biases in the development and review processes. Any changes in declarations of interest that arise during the writing period were notified to the ESC and updated. The Task Force received its entire financial support from the ESC without any involvement from the healthcare industry.

\section{Table I Classes of recommendations}

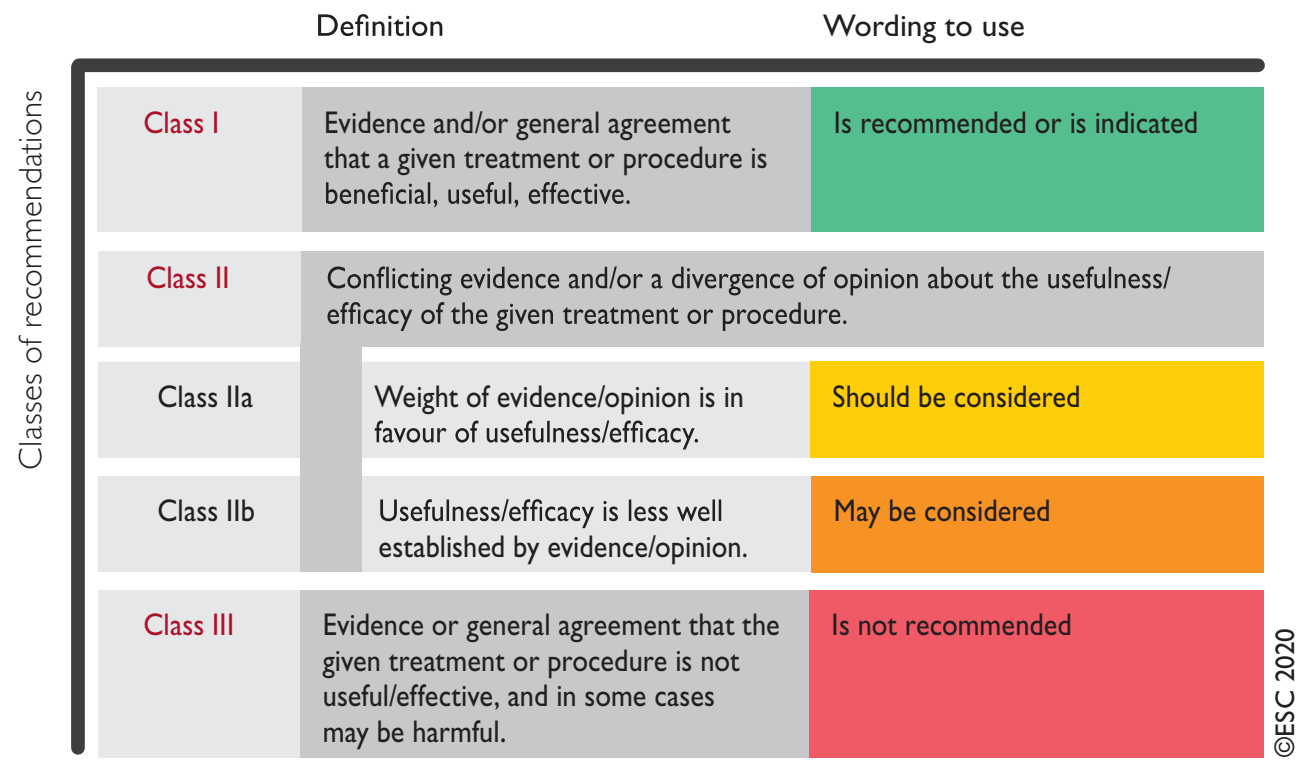


Table 2 Levels of evidence

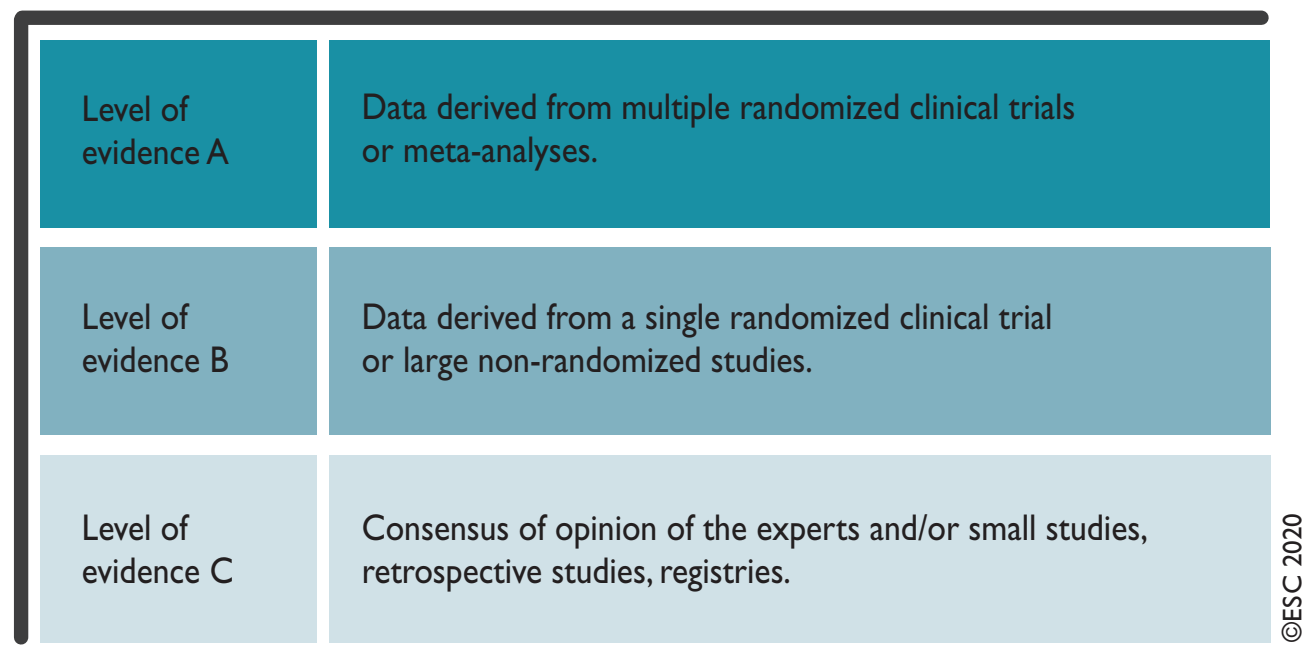

The ESC CPG supervises and coordinates the preparation of new Guidelines. The Committee is also responsible for the endorsement process of these Guidelines. The ESC Guidelines undergo extensive review by the CPG and external experts. After appropriate revisions the Guidelines are approved by all the experts involved in the Task Force. The finalized document is approved by the CPG for publication in the European Heart Journal. The Guidelines were developed after careful consideration of the scientific and medical knowledge and the evidence available at the time of their dating.

The task of developing ESC Guidelines also includes the creation of educational tools and implementation programmes for the recommendations including condensed pocket guideline versions, summary slides, booklets with essential messages, summary cards for non-specialists, and an electronic version for digital applications (smartphones, etc.). These versions are abridged and thus, for more detailed information, the user should always access the full text version of the Guidelines, which is freely available via the ESC website and hosted on the EHJ website. The National Cardiac Societies of the ESC are encouraged to endorse, adopt, translate, and implement all ESC Guidelines. Implementation programmes are needed because it has been shown that the outcome of disease may be favourably influenced by the thorough application of clinical recommendations.

Health professionals are encouraged to take the ESC Guidelines fully into account when exercising their clinical judgment, as well as in the determination and the implementation of preventive, diagnostic or therapeutic medical strategies. However, the ESC Guidelines do not override in any way whatsoever the individual responsibility of health professionals to make appropriate and accurate decisions in consideration of each patient's health condition and in consultation with that patient or the patient's caregiver where appropriate and/or necessary. It is also the health professional's responsibility to verify the rules and regulations applicable in each country to drugs and devices at the time of prescription.

\section{Introduction}

Atrial fibrillation (AF) poses significant burden to patients, physicians, and healthcare systems globally. Substantial research efforts and resources are being directed towards gaining detailed information about the mechanisms underlying AF, its natural course and effective treatments (see also the ESC Textbook of Cardiovascular Medicine: CardioMed) and new evidence is continuously generated and published.

The complexity of AF requires a multifaceted, holistic, and multidisciplinary approach to the management of AF patients, with their active involvement in partnership with clinicians. Streamlining the care of patients with AF in daily clinical practice is a challenging but essential requirement for effective management of AF. In recent years, substantial progress has been made in the detection of $\mathrm{AF}$ and its management, and new evidence is timely integrated in this third edition of the ESC guidelines on AF. The 2016 ESC AF Guidelines introduced the concept of the five domains to facilitate an integrated structured approach to AF care and promote consistent, guideline-adherent management for all patients. The Atrial Fibrillation Better Care (ABC) approach in the 2020 ESC AF Guidelines is a continuum of this approach, with the goal to further improve the structured management of AF patients, promote patient values, and finally improve patient outcomes.

Reflecting the multidisciplinary input into the management of patients with AF and interpretation of new evidence, the Task Force includes cardiologists with varying subspecialty expertise, cardiac surgeons, methodologists, and specialist nurses amongst its members.

Further to adhering to the standards for generating recommendations that are common to all ESC guidelines (see preamble), this Task Force discussed each draft recommendation during web-based conference calls dedicated to specific chapters, followed by consensus modifications and an online vote on each recommendation. Only recommendations that were supported by at least $75 \%$ of the Task Force members were included in the Guidelines. 


\subsection{What is new in the 2020 Guidelines?}

\section{New recommendations}

\section{Recommendations}

\section{Recommendations for diagnosis of AF}

ECG documentation is required to establish the diagnosis of AF.

A standard 12-lead ECG recording or a single-lead ECG tracing of $\geq 30 \mathrm{~s}$ showing heart rhythm with no discernible repeating $P$ waves and irregular RR intervals (when atrioventricular conduction is not impaired) is diagnostic of clinical AF.

\section{Recommendations for structured characterization of AF}

Structured characterization of AF, which includes clinical assessment of stroke risk, symptom status, burden of AF, and evaluation of substrate, should be considered in all AF patients, to streamline the assessment of AF patients at different healthcare levels, inform treatment decision making, and facilitate optimal management of AF patients.

\section{Recommendations for screening to detect AF}

When screening for $A F$ it is recommended that:

- The individuals undergoing screening are informed about the significance and treatment implications of detecting AF.

- A structured referral platform is organized for screen-positive cases for further physician-led clinical evaluation to confirm the diagnosis of AF and provide optimal management of patients with confirmed AF.

- Definite diagnosis of $A F$ in screen-positive cases is established only after the physician reviews the single-lead ECG recording of $\geq 30 \mathrm{~s}$ or 12-lead ECG and confirms that it shows AF.

\section{Recommendations about integrated AF management}

It is recommended to routinely collect PROs to measure treatment success and improve patient care.

\section{Recommendations for the prevention of thrombo-embolic events in AF}

For a formal risk-score-based assessment of bleeding risk, the HAS-BLED score should be considered to help address modifiable bleeding risk factors, and to identify patients at high risk of bleeding (HAS-BLED score $\geq 3$ ) for early and more frequent clinical review and followup.

Stroke and bleeding risk reassessment at periodic intervals is recommended to inform treatment decisions (e.g. initiation of OAC in patients no longer at low risk of stroke) and address potentially modifiable bleeding risk factors

In patients with AF initially at low risk of stroke, first reassessment of stroke risk should be made 4- 6 months after the index evaluation.

Estimated bleeding risk, in the absence of absolute contraindications to OAC, should not in itself guide treatment decisions to use OAC for stroke prevention.

Clinical pattern of AF (i.e. first detected, paroxysmal, persistent, long-standing persistent, permanent) should not condition the indication to thromboprophylaxis.

\section{Recommendations for cardioversion}

Pharmacological cardioversion of AF is indicated only in a haemodynamically stable patient, after consideration of the thrombo-embolic risk.

For patients with sick-sinus syndrome, atrioventricular conduction disturbances or prolonged QTc (>500 ms), pharmacological cardioversion should not be attempted unless risks for proarrhythmia and bradycardia have been considered.

\section{Recommendations for rhythm control/catheter ablation of AF}

\section{General recommendations}

For the decision on AF catheter ablation, it is recommended to take into consideration the procedural risks and the major risk factors for $\mathrm{AF}$ recurrence following the procedure and discuss them with the patient.

Repeated PVI procedures should be considered in patients with AF recurrence provided the patient's symptoms were improved after the initial PVI.

AF catheter ablation after antiarrhythmic drug therapy failure

AF catheter ablation for PVI should be considered for rhythm control after one failed or intolerant to beta-blocker treatment to improve symptoms of $A F$ recurrences in patients with paroxysmal and persistent $A F$.

First-line therapy

AF catheter ablation for PVI should/may be considered as first-line rhythm control therapy to improve symptoms in selected patients with symptomatic:

- Paroxysmal AF episodes, or

- Persistent AF without major risk factors for AF recurrence as an alternative to AAD class I or III, considering patient choice, benefit, and risk. 


\section{Techniques and technologies}

Use of additional ablation lesions beyond PVI (low voltage areas, lines, fragmented activity, ectopic foci, rotors, and others) may be considered but is not well established.

Lifestyle modification and other strategies to improve outcomes of ablation

Strict control of risk factors and avoidance of triggers are recommended as part of rhythm control strategy.

Recommendations for stroke risk management peri-cardioversion

It is recommended that the importance of adherence and persistence to NOAC treatment both before and after cardioversion is

strongly emphasized to patients.

In patients with AF duration of $>24 \mathrm{~h}$ undergoing cardioversion, therapeutic anticoagulation should be continued for at least 4 weeks even after successful cardioversion to sinus rhythm (beyond 4 weeks, the decision about long-term OAC treatment is determined by the presence of stroke risk factors).

In patients with a definite duration of $\mathrm{AF} \leq 24 \mathrm{~h}$ and a very low stroke risk $\left(\mathrm{CHA}_{2} \mathrm{DS}_{2}-\mathrm{VASc}\right.$ of 0 in men or 1 in women) post-cardioversion anticoagulation for 4 weeks may be omitted.

\section{Recommendations for stroke risk management peri-catheter ablation}

In AF patients with stroke risk factors not taking OAC before ablation, it is recommended that pre-procedural management of stroke risk includes initiation of anticoagulation and:

- Preferably, therapeutic OAC for at least 3 weeks before ablation, or

- Alternatively, the use of TOE to exclude LA thrombus before ablation.

For patients undergoing AF catheter ablation who have been therapeutically anticoagulated with warfarin, dabigatran, rivaroxaban, apixaban, or edoxaban, performance of the ablation procedure without OAC interruption is recommended.

\section{Recommendations for long-term AADs}

In AF patients treated with sotalol, close monitoring of QT interval, serum potassium levels, $\mathrm{CrCl}$, and other proarrhythmia risk factors is recommended.

In AF patients treated with flecainide for long-term rhythm control, concomitant use of an atrioventricular nodal-blocking drug (if tolerated) should be considered.

Sotalol may be considered for long-term rhythm control in patients with normal LV function or with ischaemic heart disease if close monitoring of QT interval, serum potassium levels, $\mathrm{CrCl}$, and other proarrhythmia risk factors is provided.

\section{Recommendations for lifestyle interventions and management of risk factors and concomitant diseases in AF}

Identification and management of risk factors and concomitant diseases is recommended as an integral part of treatment in AF patients.

Modification of unhealthy lifestyle and targeted therapy of intercurrent conditions is recommended to reduce AF burden and symptom severity.

Opportunistic screening for $\mathrm{AF}$ is recommended in hypertensive patients.

Opportunistic screening for AF should be considered in patients with OSA.

Recommendations for patients with AF and an ACS, PCI, or CCS

Recommendations for AF patients with ACS

In $\mathrm{AF}$ patients with $\mathrm{ACS}$ undergoing an uncomplicated $\mathrm{PCl}$, early cessation ( $\leq 1$ week) of aspirin and continuation of dual therapy with an $\mathrm{OAC}$ and a $\mathrm{P}_{2} \mathrm{Y}_{12}$ inhibitor (preferably clopidogrel) for up to 12 months is recommended if the risk of stent thrombosis is low or if concerns about bleeding risk prevail over concerns about risk of stent thrombosis, irrespective of the type of stent used.

Recommendations in AF patients with a CCS undergoing $P C I$

After uncomplicated $\mathrm{PCl}$, early cessation ( $\leq 1$ week) of aspirin and continuation of dual therapy with OAC for up to 6 months and clopidogrel is recommended if the risk of stent thrombosis is low or if concerns about bleeding risk prevail over concerns about risk of stent thrombosis, irrespective of the type of stent used.

\section{Recommendations for the management of active bleeding on OAC}

Four-factor prothrombin complex concentrates should be considered in AF patients on VKA who develop a severe bleeding complication.

\section{Recommendations for the management of AF during pregnancy}

\section{Acute management}

In pregnant women with HCM, cardioversion should be considered for persistent AF.

Ibutilide or flecainide i.v. may be considered for termination of AF in stable patients with structurally normal hearts. 


\section{Recommendations for postoperative AF}

Long-term OAC therapy to prevent thrombo-embolic events should be considered in patients at risk for stroke with postoperative AF after non-cardiac surgery, considering the anticipated net clinical benefit of OAC and informed patient preferences.

Beta-blockers should not be used routinely for the prevention of postoperative AF in patients undergoing non-cardiac surgery.

\section{Recommendations pertaining to sex-related differences in AF}

Women with symptomatic paroxysmal or persistent AF should be offered timely access to rhythm control therapies, including AF catheter ablation, when appropriate for medical reasons.

\section{Recommendations for quality measures in AF}

The introduction of tools to measure quality of care and identify opportunities for improved treatment quality and AF patient outcome should be considered by practitioners and institutions.

$\mathrm{AAD}=$ antiarrhythmic drug; $\mathrm{ACS}=$ acute coronary syndrome; $\mathrm{AF}=$ atrial fibrillation; $\mathrm{CCS}=$ chronic coronary syndrome; $\mathrm{CH}_{2} \mathrm{DS} \mathrm{S}_{2}-\mathrm{VASc}=\mathrm{Congestive}$ heart failure, Hypertension, Age $\geq 75$ years, Diabetes mellitus, Stroke, Vascular disease, Age 65-74 years, Sex category (female); $\mathrm{CrCl}=\mathrm{creatinine}$ clearance; ECG = electrocardiogram; HAS-BLED = Hypertension, Abnormal renal/liver function, Stroke, Bleeding history or predisposition, Labile INR, Elderly (>65 years), Drugs/alcohol concomitantly; HCM = hypertrophic cardiomyopathy; i.v. = intravenous; LA = left atrium/atrial; NOAC $=$ non-vitamin $\mathrm{K}$ antagonist oral anticoagulant; OAC $=$ oral anticoagulant; OSA = obstructive sleep apnoea; $\mathrm{PCl}=$ percutaneous coronary intervention; $\mathrm{PRO}=$ patient-reported outcome; $\mathrm{PVI}=$ pulmonary vein isolation; $\mathrm{QTc}=$ corrected $\mathrm{QT}$ interval; $\mathrm{TOE}=$ transoesophageal echocardiography; VKA = vitamin $\mathrm{K}$ antagonist therapy.

${ }^{\mathrm{a} C}$ Class of recommendation.

\section{Changes in the recommendations}

\section{Recommendations about integrated AF management}

2020

To optimize shared decision making about specific AF treatment option(s) in consideration, it is recommended that:

- Physicians inform the patient about advantages/limitations and benefit/risks associated with considered treatment option(s); and

- Discuss the potential burden of the treatment with the patient and include the patient's perception of treatment burden in the treatment decision.

\section{Class $^{\mathrm{a}} \quad 2016$}

Class $^{\mathrm{a}}$

Placing patients in a central role in decision making should be considered in order to tailor management to patient preferences and improve adherence to long-term therapy

\section{Recommendations for the prevention of thrombo-embolic events in AF}

For bleeding risk assessment, a formal structured risk-scorebased bleeding risk assessment is recommended to help identify non-modifiable and address modifiable bleeding risk factors in all AF patients, and to identify patients potentially at high risk of bleeding who should be scheduled for early and more frequent clinical review and follow-up.

In patients on VKAs with low time in INR therapeutic range (e.g. TTR $<70 \%$ ), recommended options are:

- Switching to a NOAC but ensuring good adherence and persistence with therapy; or

- Efforts to improve TTR (e.g. education/counselling and more frequent INR checks).

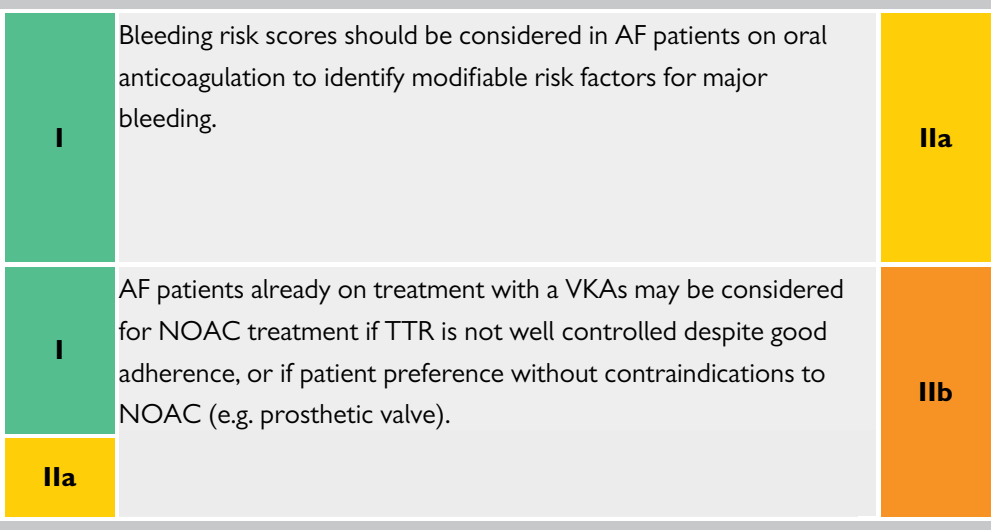

Recommendations for rhythm control/catheter ablation of AF

AF catheter ablation after drug therapy failure

$\mathrm{AF}$ catheter ablation for $\mathrm{PVI}$ is recommended for rhythm control after one failed or intolerant class I or III AAD, to improve symptoms of $\mathrm{AF}$ recurrences in patients with:

- Paroxysmal AF, or

- Persistent AF without major risk factors for AF recurrence, or

- Persistent AF with major risk factors for AF recurrence.
Catheter or surgical ablation should be considered in patients with symptomatic persistent or long-standing persistent AF refractory to $A A D$ therapy to improve symptoms, considering patient choice, benefit and risk, supported by an AF Heart Team.
Ila 


\section{First-line therapy}

AF catheter ablation:

- Is recommended to reverse LV dysfunction in AF patients when tachycardia-induced cardiomyopathy is highly probable, independent of their symptom status.

- Should be considered in selected AF patients with HFrEF to improve survival and reduce HF hospitalization.

\section{Techniques and technologies}

Complete electrical isolation of the pulmonary veins is recommended during all AF catheter-ablation procedures.

If patient has a history of CTI-dependent atrial flutter or if typical atrial flutter is induced at the time of AF ablation, delivery of a CTI lesion may be considered.

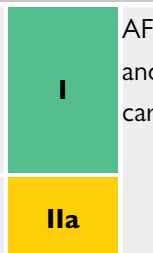

AF ablation should be considered in symptomatic patients with AF and HFrEF to improve symptoms and cardiac function when tachycardiomyopathy is suspected. using radiofrequency ablation or cryothermy balloon catheters.

Ablation of common atrial flutter should be considered to prevent

IIb recurrent flutter as part of an $\mathrm{AF}$ ablation procedure if documented or occurring during the $A F$ ablation Lifestyle modification and other strategies to improve outcomes of ablation Weight loss is recommended in obese patients with AF, particularly those who are being evaluated to undergo AF ablation.

In obese patients with $\mathrm{AF}$, weight loss together with management

I of other risk factors should be considered to reduce AF burden and symptoms.

\section{Recommendations for stroke risk management peri-cardioversion}

In patients with $\mathrm{AF}$ undergoing cardioversion, NOACs are rec-

I

Anticoagulation with heparin or a NOAC should be initiated as soon as possible before every cardioversion of AF or atrial flutter.

\section{Recommendations for stroke risk management peri-catheter ablation}

After AF catheter ablation, it is recommended that:

- Systemic anticoagulation with warfarin or a NOAC is continued for at least 2 months post ablation, and

- Long-term continuation of systemic anticoagulation beyond 2 months post ablation is based on the patient's stroke risk profile and not on the apparent success or failure of the ablation procedure.

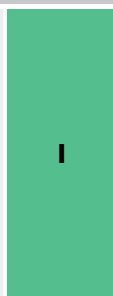

All patients should receive oral anticoagulation for at least 8 weeks after catheter ablation.

\section{Recommendations for long-term antiarrhythmic drugs}

Amiodarone is recommended for long-term rhythm control in all AF patients, including those with HFrEF. However, owing to its extracardiac toxicity, other AADs should be considered first whenever possible. increase with time. For this reason, other AAD should be considered first.

\section{Recommendations for lifestyle interventions and management of risk factors and concomitant diseases in patients with AF}

Attention to good BP control is recommended in AF patients with hypertension to reduce $A F$ recurrences and risk of stroke and bleeding.

Physical activity should be considered to help prevent AF incidence or recurrence, with the exception of excessive endurance exercise, which may promote $\mathrm{AF}$.

Optimal management of OSA may be considered, to reduce AF incidence, $\mathrm{AF}$ progression, $\mathrm{AF}$ recurrences, and symptoms.
BP control in anticoagulated patients with hypertension should be considered to reduce the risk of bleeding

Moderate regular physical activity is recommended to prevent AF,

Ila while athletes should be counselled that long-lasting intense sports participation can promote $\mathrm{AF}$

IIb

OSA treatment should be optimized to reduce $A F$ recurrences and improve AF treatment results.

\section{Recommendations for stroke prevention in AF patients after ICH}

In AF patients at high risk of ischaemic stroke, (re-)initiation of OAC, with preference for NOACs over VKAs in NOAC-eligible patients, should be considered in consultation with a neurologist/ stroke specialist after:

- A trauma-related ICH

- Acute spontaneous ICH (which includes subdural, subarachnoid, or intracerebral haemorrhage), after careful consideration of risks and benefits
After $\mathrm{ICH}$ oral anticoagulation in patients with AF may be reinitiated after 4-8 weeks provided the cause of bleeding or the relevant risk factor has been treated or controlled.

IIa
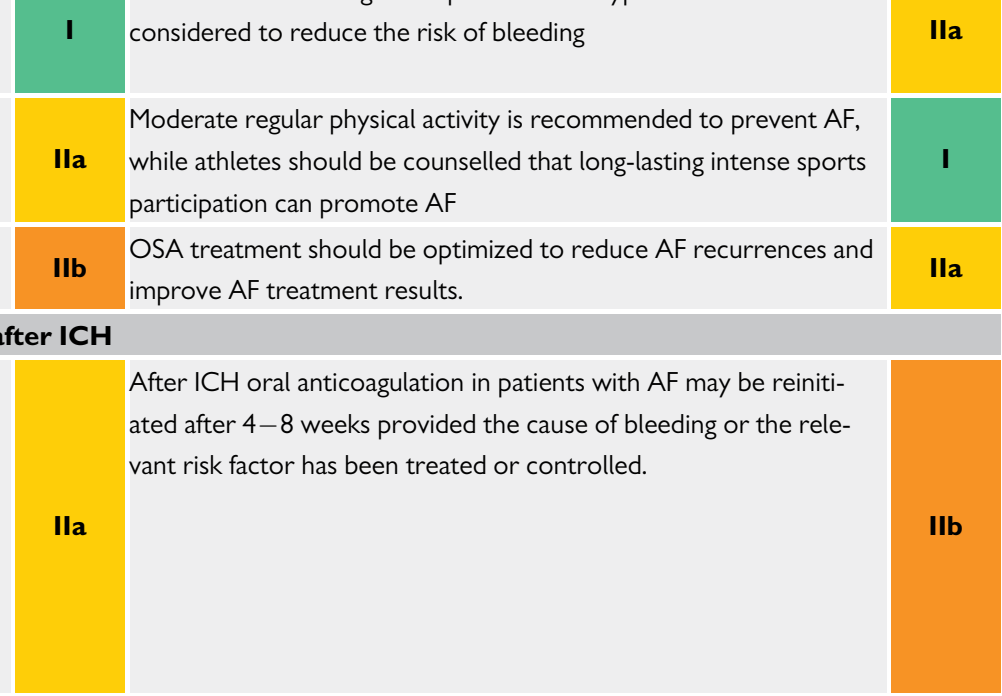


\section{Recommendations for postoperative AF}

Long-term OAC therapy to prevent thrombo-embolic events may be considered in patients at risk for stroke with postoperative AF after cardiac surgery, considering the anticipated net clinical benefit of OAC therapy and informed patient preferences.

$\mathrm{AAD}=$ antiarrhythmic drug; $\mathrm{AF}=$ atrial fibrillation; $\mathrm{BP}=$ blood pressure; $\mathrm{CTI}=$ cavotricuspid isthmus; $\mathrm{HFrEF}=$ heart failure with reduced ejection fraction; $\mathrm{ICH}=$ intracranial haemorrhage; INR = international normalized ratio; LV = left ventricular; LVEF = left ventricular ejection fraction; NOAC = non-vitamin K antagonist oral anticoagulant; OAC = oral anticoagulant or oral anticoagulation; $\mathrm{PVI}=$ pulmonary vein isolation; TTR $=$ time in therapeutic range; VKA $=$ vitamin $\mathrm{K}$ antagonist.

${ }^{\mathrm{a}}$ Class of recommendation.

\section{Definition and diagnosis of atrial fibrillation}

\subsection{Definition}

\section{Table 3 Definition of atrial fibrillation}

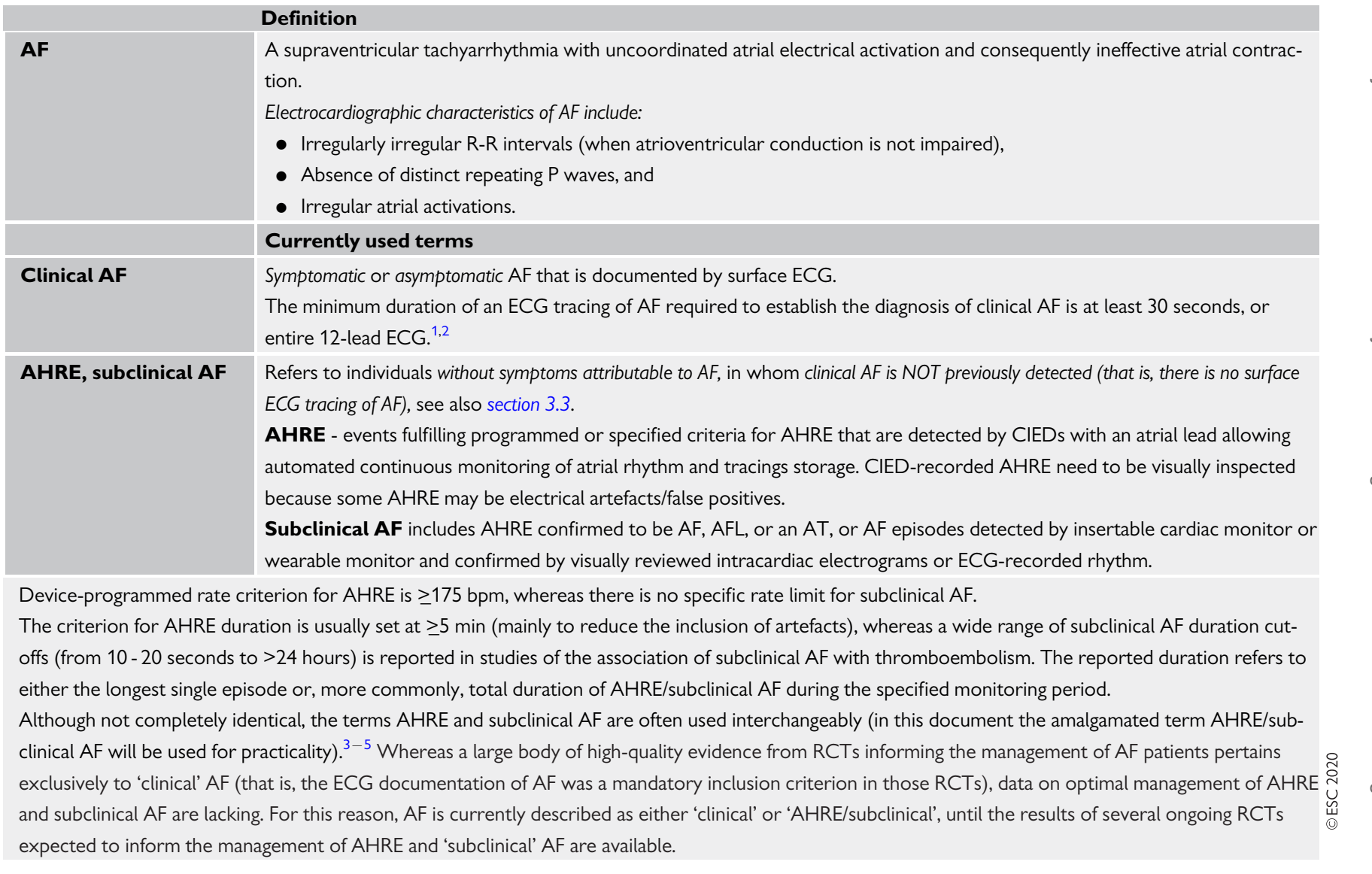

$\mathrm{AHRE}=$ atrial high-rate episode; $\mathrm{AF}=$ atrial fibrillation; $\mathrm{ECG}=$ electrocardiogram; $\mathrm{AFL}=$ atrial flutter; $\mathrm{AT}=$ atrial tachycardia; bpm = beats per minute; $\mathrm{CIED}=$ cardiac implant able electronic device; $\mathrm{ECG}$ = electrocardiogram; $\mathrm{RCT}$ = randomized controlled trial. 


\subsection{Diagnostic criteria for atrial fibrillation}

The diagnosis of AF requires rhythm documentation with an electrocardiogram (ECG) tracing showing AF. By convention, an episode lasting at least $30 \mathrm{~s}$ is diagnostic for clinical $\mathrm{AF}^{6}$

\section{Recommendations for diagnosis of AF}

\begin{tabular}{|c|c|c|}
\hline Recommendations & Class $^{a}$ & Level $^{\mathrm{b}}$ \\
\hline $\begin{array}{l}\text { ECG documentation is required to establish the } \\
\text { diagnosis of AF. } \\
\text { - A standard 12-lead ECG recording or a sin- } \\
\text { gle-lead ECG tracing of } \geq 30 \text { s showing heart } \\
\text { rhythm with no discernible repeating P waves } \\
\text { and irregular RR intervals (when atrioventric- } \\
\text { ular conduction is not impaired) is diagnostic } \\
\text { of clinical AF. }\end{array}$ & $\mathbf{I}$ & B \\
\hline
\end{tabular}

$\mathrm{AF}=$ atrial fibrillation; $\mathrm{ECG}=$ electrocardiogram

${ }^{\mathrm{a} C l a s s}$ of recommendation.

bLevel of evidence.

\subsection{Diagnosis of atrial high-rate episodes/ subclinical atrial fibrillation}

Various implanted devices and wearable monitors allow detection of atrial high-rate episodes (AHRE) /subclinical AF (Figure 1). ${ }^{3}$ Owing to a short monitoring, detection of AHRE/subclinical AF via external ECG is less likely. ${ }^{7}$

When AHRE/subclinical AF is detected by a device/wearable, inspection of the stored electrograms/ECG rhythm strips is recommended to exclude artefacts or other causes of inappropriate detection. $^{8,9}$

\section{Epidemiology}

Worldwide, $A F$ is the most common sustained cardiac arrhythmia in adults $^{10}$ (Figure 2, upper panel). AF is associated with substantial morbidity and mortality, thus portending significant burden to patients, societal health, and health economy (Figure 2, lower panel) (Supplementary section 1).

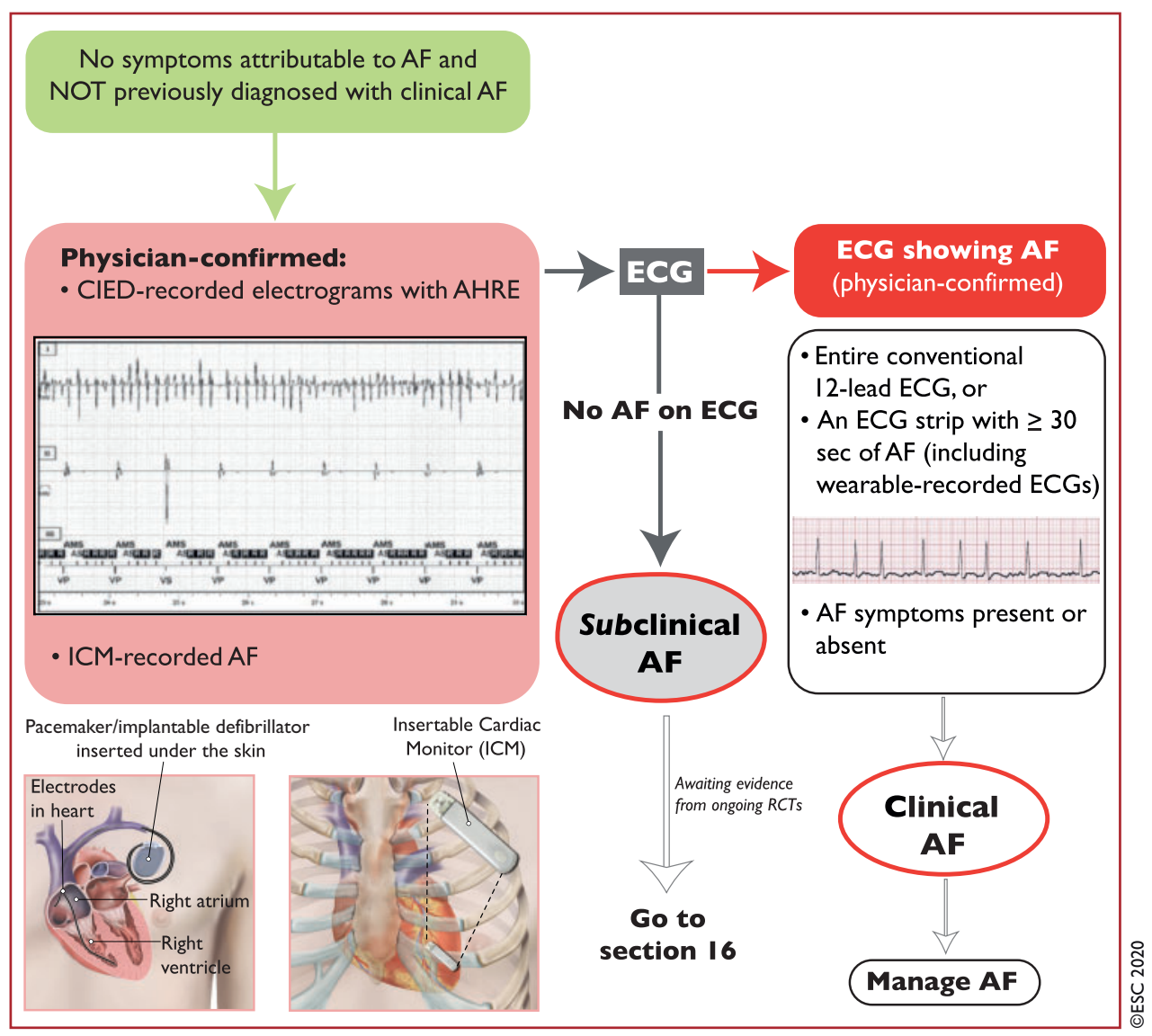

Figure I Diagnosis of AHRE/subclinical AF. CIEDs with an atrial lead can monitor atrial rhythm and store the tracings. ICMs have no intracardiac leads but continuously monitor cardiac electrical activity by recording and analysing a single-lead bipolar surface ECG based on a specific algorithm. Left-bottom image: pacemaker with a right atrial lead, and a ventricular lead in the right ventricular apex. In addition to pacing at either site, these leads can sense activity in the respective cardiac chamber. The device can also detect pre-programmed events, such as AHRE. Right-bottom image: subcutaneous ICM: these devices have no intra-cardiac leads and essentially record a single, bipolar, surface ECG, with inbuilt algorithms for detection of AHRE or AF. AF = atrial fibrillation; $\mathrm{AHRE}=$ atrial high rate episode; $\mathrm{CIED}=$ cardiac implantable electronic device; $\mathrm{ECG}=$ electrocardiogram; ICM = insertable cardiac monitor; $\mathrm{RCT}=$ randomized clinical trial. 


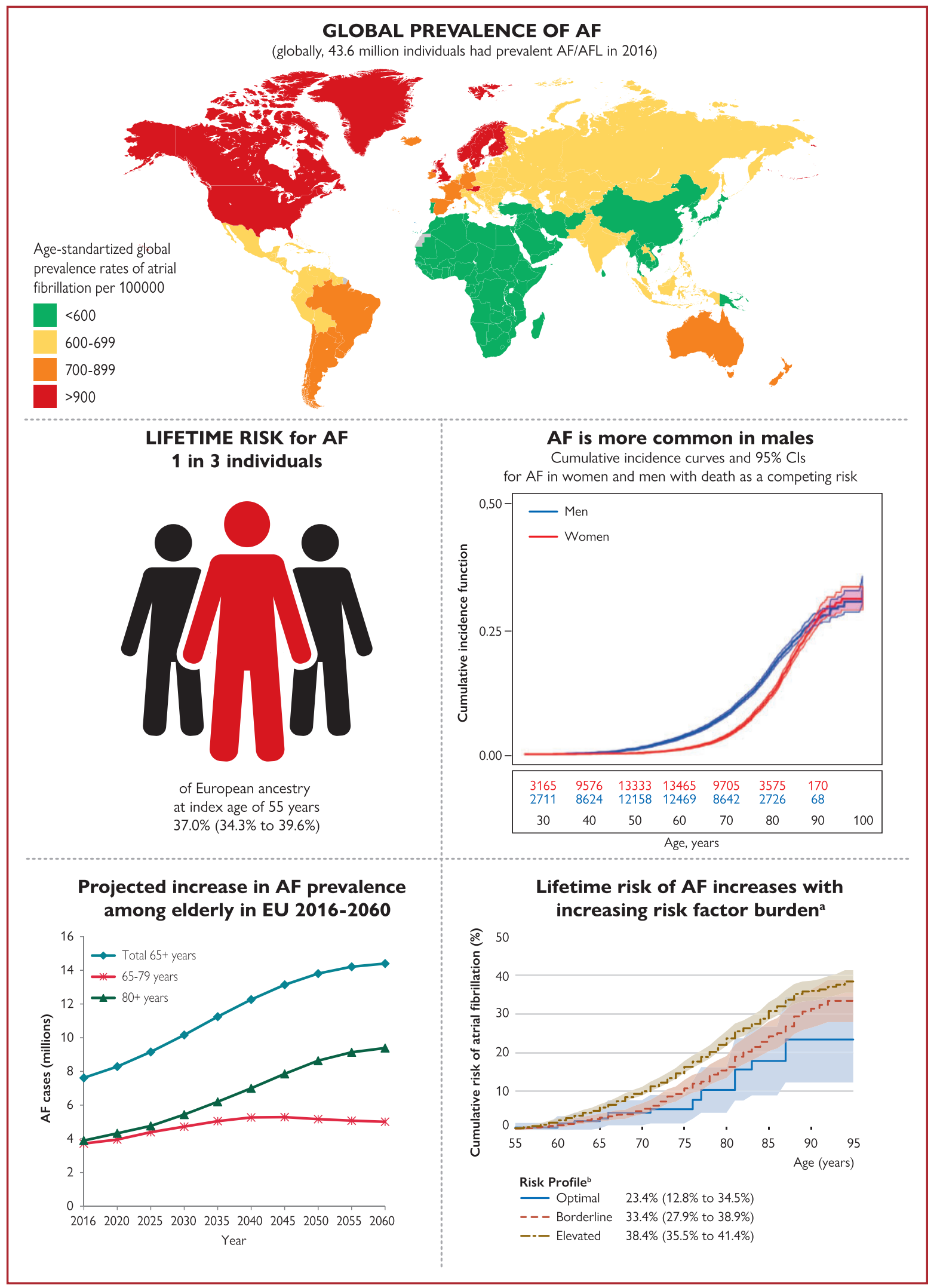

Figure 2 Epidemiology of AF: prevalence (upper panel) ${ }^{10-20}$; and lifetime risk and projected rise in the incidence and prevalence (lower panel). ${ }^{19,21-34}$ $\mathrm{AF}=$ atrial fibrillation; $\mathrm{AFL}=$ atrial flutter; $\mathrm{BP}=$ blood pressure; $\mathrm{Cl}$ = confidence interval; $\mathrm{EU}=$ European Union. ${ }^{\mathrm{a} S m o k i n g}$, alcohol consumption, body

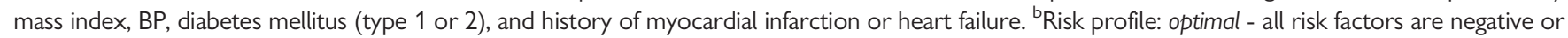
within the normal range; borderline - no elevated risk factors but >1 borderline risk factor; elevated - >1 elevated risk factor. 


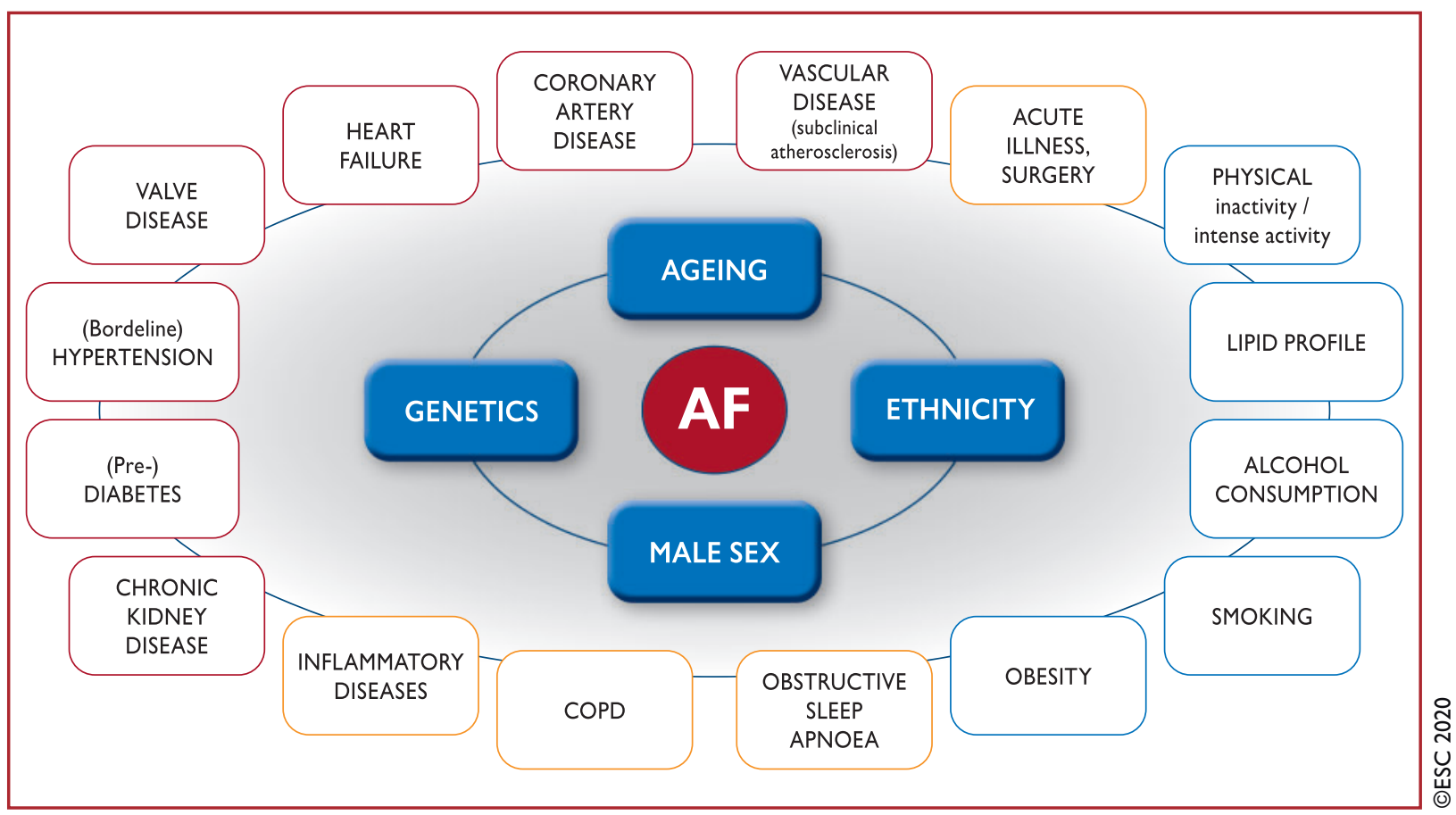

Figure 3 Summary of risk factors for incident $A F^{10,22,33,35-72}$ (Supplementary Table 1 for full list). AF = atrial fibrillation; COPD = chronic obstructive pulmonary disease.

The currently estimated prevalence of AF in adults is between $2 \%$ and $4 \%,{ }^{10}$ and a 2.3 -fold rise ${ }^{11}$ is expected, ${ }^{12,13}$ owing to extended longevity in the general population and intensifying search for undiagnosed AF. ${ }^{15}$ Increasing age is a prominent AF risk factor, but increasing burden of other comorbidities including hypertension, diabetes mellitus, heart failure (HF), coronary artery disease (CAD), chronic kidney disease (CKD), ${ }^{21}$ obesity, and obstructive sleep apnoea (OSA) is also important; ${ }^{22-26}$ modifiable risk factors are potent contributors to AF development and progression ${ }^{27,28}$ (Figure 3). The ageadjusted incidence, prevalence, and lifetime risk of AF are lower in women vs. men and in non-Caucasian vs. Caucasian cohorts. ${ }^{10,14-20}$ A previous lifetime AF risk estimate of 1 in 4 individuals ${ }^{29,30}$ was recently revised to 1 in 3 individuals of European ancestry at index age of 55 years. ${ }^{31,32}$ The AF lifetime risk depends on age, genetic, and (sub)clinical factors. ${ }^{10,33,34}$ The observed impact of clinical risk factor burden/multiple comorbidity on AF risk (Figure 3, lower pane $\left.\right|^{31}$ ) suggests that an early intervention and modifiable risk factor control could reduce incident AF.

\subsection{Prediction of incident atrial fibrillation}

Identifying individuals at higher risk of developing AF in the community could facilitate targeting of preventive interventions and screening programmes for early AF detection, for example in high-risk subgroups such as post-stroke patients. ${ }^{73}$ Various predictive scores for new-onset AF have been proposed (Supplementary Table 2), but none has been widely used in clinical practice.

\subsection{Pathophysiology of atrial fibrillation}

A complex interplay of triggers, perpetuators, and substrate development eventually resulting in AF occurrence is shown in Supplementary Figure 1.

\section{Clinical features of atrial fibrillation}

Clinical presentation of AF and AF-related outcomes are shown in Figure 4 (see also Supplementary section 2 and Supplementary Box 1).

\section{Atrial fibrillation subtypes, burden, and progression}

\subsection{Classification of atrial fibrillation}

Different AF classifications have been proposed but, traditionally, five patterns of AF are distinguished, based on presentation, duration, and spontaneous termination of AF episodes (Table 4). ${ }^{143}$

In patients experiencing both paroxysmal and persistent AF episodes, the more common type should be used for classification. However, clinically determined AF patterns do not correspond well to the AF burden measured by long-term ECG monitoring. ${ }^{144-146}$

Other classifications of $A F$ reflect the presence of symptoms (asymptomatic AF is diagnosed with an opportune 12-lead ECG or rhythm strip in asymptomatic patients) or underlying cause of AF 


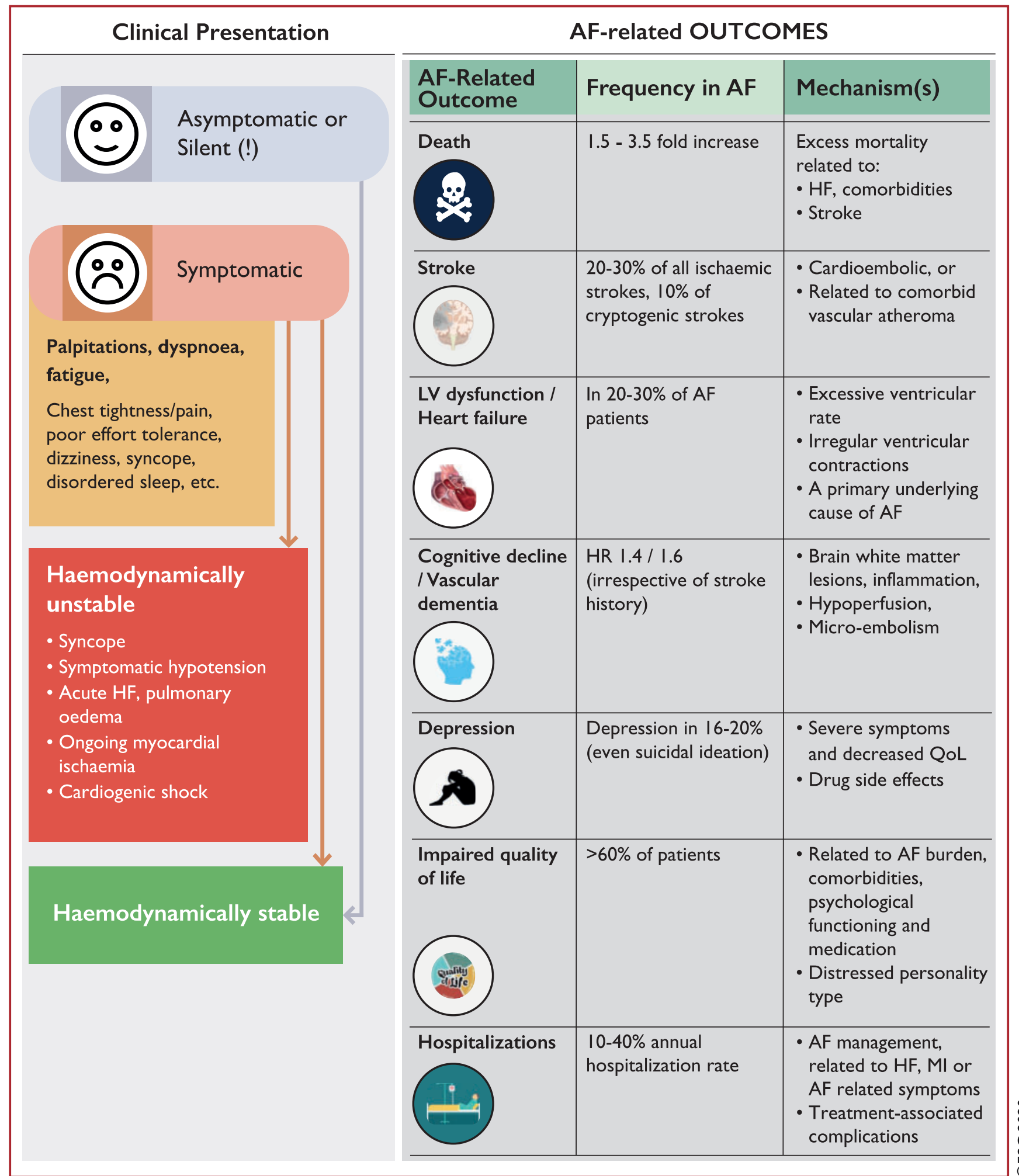

Figure 4 Clinical presentation of AF and AF-related outcomes. ${ }^{10,31,74-140} \mathrm{AF}=$ atrial fibrillation; $\mathrm{HF}=$ heart failure; $\mathrm{HR}=\mathrm{Hazard}$ Ratio; $\mathrm{LV}=$ left ventricle; $\mathrm{MI}=$ myocardial infarction; $\mathrm{QoL}=$ quality of life.

Patients with AF may have various symptoms ${ }^{92,108,109,128,131}$ but $50-87 \%$ are initially asymptomatic, $75,82,88,111,117,120,125,127$ with possibly a less favourable prognosis. ${ }^{79,82,87,88,117,119,127,134,139}$ First-onset AF symptoms are less well studied, ${ }^{92,105,108,109,127}$ may change with treatment ${ }^{119}$ and AF recurrences are commonly asymptomatic. ${ }^{113}$

Stroke/systolic embolism: annual AF-related stroke risk in AF patients depends on comorbidities. ${ }^{78,84,85,91,106,112}$ Cardioembolic strokes associated with AF are usually severe, highly recurrent, often fatal, or with permanent disability. ${ }^{10,83,115}$ In a population-based registry, patients with new-onset AF also had increased rates of systemic embolism. $^{89}$ 


\section{Figure 4 Continued}

Left ventricular (LV) dysfunction and HF: multiple AF-associated mechanisms/myocardial alterations may lead to LV dysfunction and HF, ${ }^{102,138}$ resulting in a high prevalence and incidence of HF among AF patients. Sharing common risk factors, AF and HF often coexist, or may precipitate/exacerbate each other, resulting in significantly greater mortality than either condition alone. ${ }^{140}$

Hospitalization: approximately $30 \%$ of AF patients have at least one, and $10 \%$ have $\geq 2$, hospital admissions annually, ${ }^{99,110,129}$ being twice as likely to be hospitalized as ageand sex-matched non-AF individuals (37.5\% vs. $17.5 \%$, respectively). ${ }^{98}$ In a nationwide cohort, AF was the main cause for admission in $14 \%$ of hospitalized patients but their inhospital mortality was $<1 \% .{ }^{101}$ The most common reasons for hospitalization of AF patients were cardiovascular disorders (49\%), non-cardiovascular causes (43\%) and bleeding $(8 \%) .{ }^{129}$

Quality of life (QoL) and functional status: $>60 \%$ of AF patients have significantly impaired QoL/exercise tolerance, ${ }^{81,88,136}$ but only $17 \%$ have disabling symptoms. ${ }^{88}$ QoL is significantly lower in women, ${ }^{81,107,114,124}$ young individuals, and those with comorbidities. ${ }^{118} \mathrm{AF}$ burden ${ }^{100}$ may also affect QoL, but only psychological functioning consistently predicted symptoms and QoL. ${ }^{136}$ Patients with AF more often developed anxiety disorders, ${ }^{126}$ had a higher burden of depressive symptoms, ${ }^{123}$ and poorer QoL with a Distressed personality type (Type D). ${ }^{103}$ Key symptom and QoL drivers are important to identify optimal AF treatment. It is also important to confirm that symptoms are related to AF or, if absent, to exclude a subconscious adaptation to living with suboptimal physical capacity by asking for breathlessness or fatigue on exertion and recording possible improvements after cardioversion.

Cognitive impairment/dementia: AF may lead to cognitive impairment ranging from mild dysfunction to dementia ${ }^{97,104,141}$ via clinically apparent or silent stroke or insufficiently understood stroke-independent pathways. ${ }^{94,96,97,122}$ Magnetic resonance imaging (MRI) studies have shown that AF is associated with a greater than twofold increase in the odds of having silent cerebral ischaemia. ${ }^{90,121,142}$ A recent expert consensus paper summarized the available data. ${ }^{86}$

Mortality: AF is independently associated with a twofold increased risk of all-cause mortality in women and a 1.5-fold increase in men, ${ }^{77,80,130,137}$ with an overall 3.5-fold mortality risk increase. ${ }^{31}$ Whereas the mechanistic explanation for this association is multifaceted, associated comorbidities play an important role. ${ }^{95}$ In a recent study, the most common causes of death among AF patients were HF (14.5\%), malignancy (23.1\%), and infection/sepsis (17.3\%), whereas stroke-related mortality was only $6.5 \% .^{76}$ These and other recent data indicate that, in addition to anticoagulation and HF treatment, comorbid conditions need to be actively treated in the endeavour to reduce AF-related mortality. ${ }^{77,93,116,133}$

\section{Table 4 Classification of AF}

\begin{tabular}{|c|c|}
\hline AF pattern & Definition \\
\hline First diagnosed & AF not diagnosed before, irrespective of its duration or the presence/severity of AF-related symptoms. \\
\hline Paroxysmal & AF that terminates spontaneously or with intervention within 7 days of onset. \\
\hline Persistent & $\begin{array}{l}\text { AF that is continuously sustained beyond } 7 \text { days, including episodes terminated by cardioversion (drugs or electrical cardioversion) } \\
\text { after } \geq 7 \text { days }\end{array}$ \\
\hline $\begin{array}{l}\text { Long-standing } \\
\text { persistent }\end{array}$ & Continuous AF of $>12$ months' duration when decided to adopt a rhythm control strategy. \\
\hline Permanent & $\begin{array}{l}\text { AF that is accepted by the patient and physician, and no further attempts to restore/maintain sinus rhythm will be undertaken. } \\
\text { Permanent AF represents a therapeutic attitude of the patient and physician rather than an inherent pathophysiological } \\
\text { attribute of AF, and the term should not be used in the context of a rhythm control strategy with antiarrhythmic drug } \\
\text { therapy or AF ablation. Should a rhythm control strategy be adopted, the arrhythmia would be re-classified as 'long-standing persis- } \\
\text { tent AF'. }\end{array}$ \\
\hline \multicolumn{2}{|c|}{ Terminology that should be abandoned } \\
\hline Lone AF & $\begin{array}{l}\text { A historical descriptor. Increasing knowledge about the pathophysiology of AF shows that in every patient a cause is present. Hence, this } \\
\text { term is potentially confusing and should be abandoned. }{ }^{147}\end{array}$ \\
\hline $\begin{array}{l}\text { Valvular/non- } \\
\text { valvular AF }\end{array}$ & $\begin{array}{l}\text { Differentiates patients with moderate/severe mitral stenosis and those with mechanical prosthetic heart valve(s) from other patients } \\
\text { with } A F \text {, but may be confusing }{ }^{148} \text { and should not be used. }\end{array}$ \\
\hline Chronic AF & Has variable definitions and should not be used to describe populations of AF patients. \\
\hline
\end{tabular}

$\mathrm{AF}=$ atrial fibrillation.

(e.g. postoperative AF, see section 11.19). Classifying AF by underlying drivers could inform management, but the evidence in support of the clinical use of such classification is lacking (Supplementary Table 3). Terms that should no longer be used to describe AF are listed in Table 4.

Recommendations for AF management are not based on the temporal AF patterns, except for the restoration of sinus rhythm. ${ }^{143,149,150}$ It is very unlikely that a simple but comprehensive
AF classification will be proposed, given the multiplicity of factors relevant for its management, advances in AF monitoring, multiplicity of risk assessment tools, evolving treatments, and complexity of AF itself. Indeed, a paradigm shift from classification towards a structured characterization of AF, addressing specific domains with treatment and prognostic implications has been recently proposed. ${ }^{151}$ Such a scheme would streamline the assessment of AF patients at any healthcare level, thus facilitating communication among physicians, 


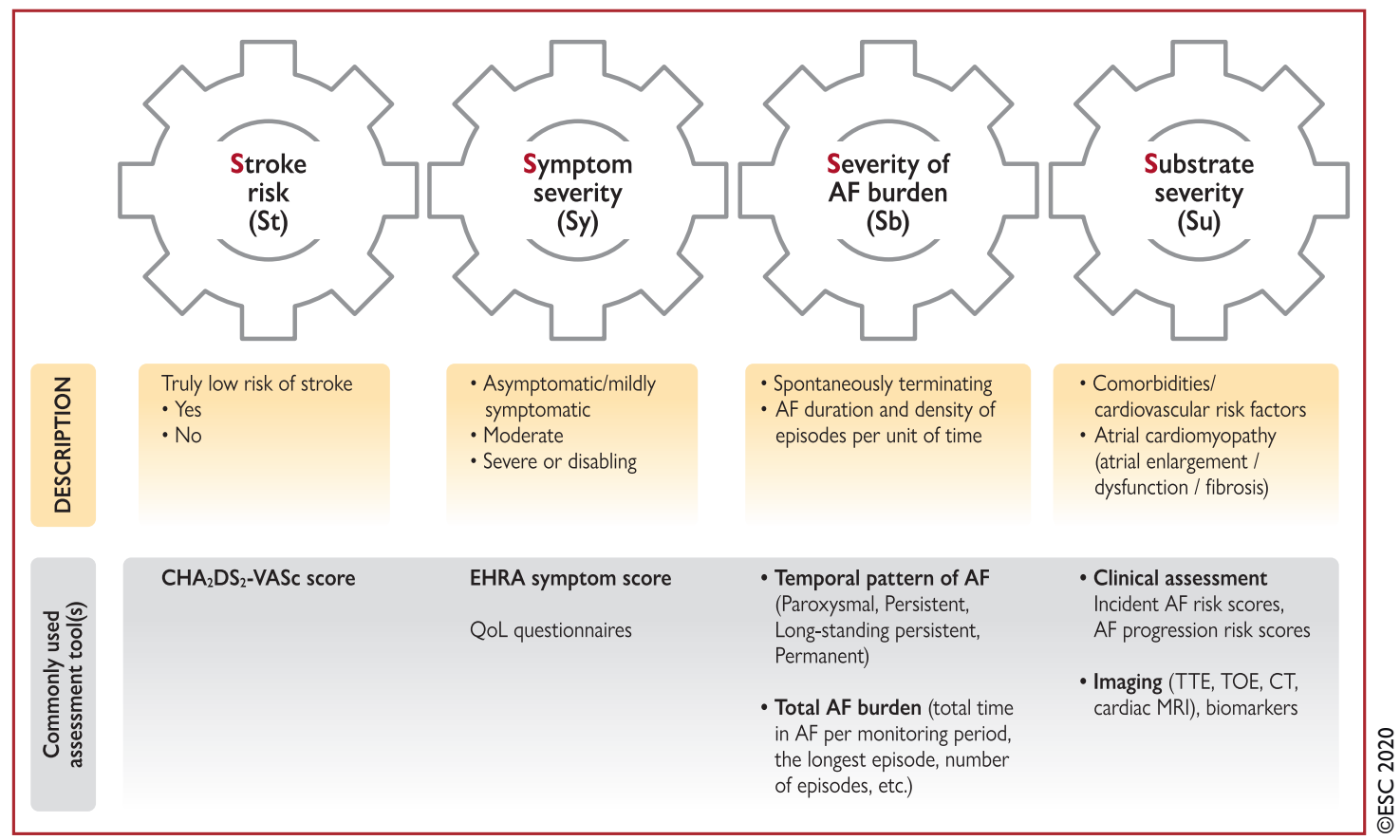

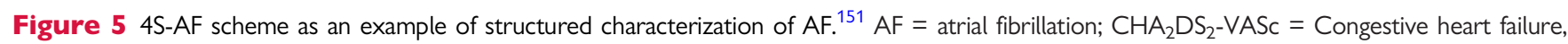
Hypertension, Age $\geq 75$ years, Diabetes mellitus, Stroke, Vascular disease, Age 65 - 74 years, Sex category (female); CT = computed tomography; EHRA = European Heart Rhythm Association; LA = left atrium; MRI = magnetic resonance imaging; QoL = quality of life; TOE = transoesophageal echocardiography; TTE = transthoracic echocardiography.

treatment decision making, and optimal management of AF patients, and should become a standard in clinical practice when reporting an AF case.

The proposed 4S-AF scheme (Stroke risk, Symptom severity, Severity of AF burden, Substrate severity) includes four AF-related domains (Figure 5). ${ }^{151}$ The currently used assessment tools/classifications pertinent to specific domains (e.g. stroke risk scores, symptom scores, clinical factors, imaging modalities, etc.) can be easily fitted in, but the 4S-AF has great potential for future refinements guided by advances in technology, and the most appropriate descriptors of $A F$ domains are yet to be defined. Given the descriptors of AF included in the 4S-AF scheme, the structured characterization of AF patients using 4S-AF could also provide prognostic information, but the clinical utility and prognostic value of the 4S-AF scheme needs extensive validation in different $A F$ cohorts and clinical settings.

\section{Recommendations for structured characterization of AF}

\begin{tabular}{l|l|l|}
\hline Recommendations & Class $^{\mathbf{a}}$ & Level $^{\mathbf{b}}$ \\
\hline $\begin{array}{l}\text { Structured characterization of AF, which } \\
\text { includes clinical assessment of stroke risk, symp- }\end{array}$ & \\
tom status, burden of AF, and evaluation of sub- \\
strate, should be considered in all AF patients, \\
to streamline the assessment of AF patients at \\
different healthcare levels, inform treatment \\
decision-making, and facilitate optimal manage- \\
ment of AF patients.
\end{tabular}

$\mathrm{AF}=$ atrial fibrillation

${ }^{\mathrm{a}}$ Class of recommendation.

bLevel of evidence.

\subsection{Definition and assessment of atrial fibrillation burden}

The term 'burden' refers to various $\mathrm{AF}$ aspects (e.g. epidemiological, economic). ${ }^{144}$ Regarding continuous device-based monitoring, 'AF burden' is currently defined as the overall time spent in AHRE/subclinical AF during a specified monitoring period (e.g. 1 day). Both the time in AF and the monitoring period should be acknowledged when reporting AF burden (most studies reported the maximum time spent in AF over a 24-h period), but optimal measures are yet to be determined. ${ }^{152}$ The term 'AF burden' is different from 'burden of AF', the latter referring to AF consequences.

Clinical AF burden is routinely determined by $A F$ temporal pattern $^{146}$ (Table 4) and intermittent ECG monitoring, ${ }^{153}$ neither corresponding well to the long-term ECG monitoring. The relationship of clinical AF burden with specific outcomes is not well characterized, ${ }^{154}$ but may be associated with higher risk of incident $\mathrm{HF}^{155}$ and all-cause mortality, ${ }^{156}$ while the association with quality of life $(\mathrm{Q} o \mathrm{~L})$ is complex and data about cognitive impairment/dementia are lacking. ${ }^{86}$ Recent randomized controlled trial (RCT) data consistently showed significantly lower residual thrombo-embolic risk among anticoagulated patients with paroxysmal vs. persistent $A F,{ }^{156-159}$ whereas earlier trial-based $^{160}$ and observational data ${ }^{161,162}$ are contradictory. Among non-anticoagulated patients, stroke risk was lower with paroxysmal than non-paroxysmal $A F{ }^{156}$ and a greater total $A F$ burden (but not the longest AF episode) was independently associated with higher thrombo-embolic event rates. ${ }^{163}$ Clinical AF burden may influence the response to rhythm control therapy. ${ }^{164,165}$ The presence of $>6 \mathrm{~h}$ of AF per week (especially when progressing to $>24 \mathrm{~h}$ weekly) was associated with increased mortality, especially in women. ${ }^{166}$ 
Available evidence on the association of AF burden with AFrelated outcomes is insufficient to guide treatment and should not be a major factor in treatment decisions. Comprehensive management of modifiable cardiovascular risk factors/comorbidity reduces AF burden (section 10.3).

\subsection{Atrial fibrillation progression}

Transition from paroxysmal to non-paroxysmal AF (or from subclinical to clinical AF) ${ }^{154,167-169}$ is often characterized by advancing atrial structural remodelling or worsening of atrial cardiomyopathy. ${ }^{170,171}$

Assessment of AF progression depends on duration of rhythm monitoring and underlying substrate. ${ }^{172,173}$ Reported annual rates of paroxysmal AF progression range from $<1 \%$ to $15 \%$ (up to $27-36 \%$ in studies with $\geq 10$-year follow-up). ${ }^{169,174}$ Risk factors for AF progression include age, HF, hypertension, CKD, chronic pulmonary diseases, diabetes mellitus, previous stroke, and left atrial (LA) size, ${ }^{167}$ whereas the added predictive value of biomarkers is presently not well defined. Older age is associated with permanent $A F,{ }^{82,117,154}$ and various triggers may also play a role, with different progression patterns resulting from their interaction with substrate remodelling. ${ }^{171}$ Progression to persistent/permanent AF is associated with adverse cardiovascular events, hospitalizations, and death, ${ }^{166}$ but it is unclear whether AF progression is a determinant of adverse prognosis or rather a marker of an underlying progressive disease/substrate. ${ }^{175,176}$ The true impact of different therapeutic interventions at different disease stages on AF progression and associated outcomes is also less well defined.

\subsection{Atrial cardiomyopathy: definition, classification, clinical implications, and diagnostic assessment}

Important progress in understanding AF mechanisms and thrombogenicity reconsiders the role of atrial cardiomyopathy (i.e. atrial structural, architectural, contractile, or electrophysiological changes with potentially relevant clinical manifestations). ${ }^{170}$

Clinical classification of atrial cardiomyopathy should be based on the atrial structure, morphology, electrical and mechanical function, and the diagnosis could be based on easily accessible parameters (e.g. aetiology, the prothrombotic state, ${ }^{177}$ and abnormal LA volume/ function). ${ }^{178}$ Major clinical issues in AF (i.e. prevention of thromboembolic complications and AF progression) are influenced by atrial remodelling; and, importantly, $A F$ is not only a risk factor for but also a marker of atrial cardiomyopathy, which could explain the lack of temporal relationship between detected $\mathrm{AF}$ and stroke. ${ }^{179}$

The diagnostic algorithm for atrial cardiomyopathy should follow a stepwise approach, identifying risk factors for atrial cardiomyopathy, ${ }^{170}$ atrial electrical and mechanical dysfunction, ${ }^{180}$ and increased thrombotic risk. ${ }^{181}$ More data are needed to define prognostic and treatment implications of different atrial cardiomyopathy morphofunctional forms.

\section{Screening for atrial fibrillation}

Multiple factors (i.e. increasing AF prevalence, previously unknown AF detection in about $10 \%$ of all ischaemic strokes, ${ }^{4,182}$ high prevalence of asymptomatic $A F,{ }^{117}$ potential to prevent $A F$-related strokes with appropriate treatment and increasing availability of AF detection tools) have fuelled international initiatives to implement screening for AF in clinical practice. ${ }^{172}$

Asymptomatic clinical AF has been independently associated with increased risk of stroke and mortality compared with symptomatic AF. ${ }^{82,117,127,183}$ Data derived from studies of incidentally detected asymptomatic AF are the closest possible approximation of the risk of stroke and death in screen-detected AF subjects, because delaying treatment to discern a natural history would be unethical. Observational data suggest that screen-detected AF responds to treatment similarly to AF detected by routine care, ${ }^{183}$ thus favouring AF screening.

Although AF fulfils many of the criteria for disease screening ${ }^{184}$ (Supplementary Figure 2), RCT data to confirm the health benefits from screening for AF and inform the choice of optimal screening programmes and strategies for its implementation are scarce. ${ }^{185,186}$ Advances in wearable technology will likely yield inexpensive and practical options for AF detection and AF burden assessment in the near future.

\subsection{Screening tools}

The systems used for AF screening are shown in Table 5 and Figure $6 .^{173,187}$

Mobile health technologies are rapidly developing for AF detection and other purposes (>100 000 mHealth apps and $\geq 400$ wearable activity monitors are currently available). ${ }^{197}$ Caution is needed in their clinical use, as many are not clinically validated. Several studies evaluated AF detection using smartwatches, ${ }^{198,199}$ thus opening new perspectives for AF detection targeting specific populations at risk. Machine learning and artificial intelligence may be capable of identifying individuals with previous AF episodes from a sinus rhythm ECG recording, ${ }^{200}$ which would be a major technological breakthrough in AF detection. 200

The Apple Heart study ${ }^{201}$ included 419297 self-enrolled smartwatch app users (mean age 40 years) in the United States of America (USA), of whom $0.5 \%$ received an irregular pulse notification $(0.15 \%$ of those aged $<40$ years, $3.2 \%$ among those aged $>65$ years). Subsequent (notification-triggered) 1-week ECG patch monitoring revealed AF in $34 \%$ of monitored participants. The Huawei Heart study $^{202}$ included 187912 individuals (mean age 35 years, 86.7\% male), of whom $0.23 \%$ received a 'suspected AF' notification. Of those effectively followed up, $87.0 \%$ were confirmed as having AF, with the positive predictive value of photoplethysmography signals being 91.6\% [95\% confidence interval $(\mathrm{Cl})$ 91.5 - 91.8]. Of those with identified AF, 95.1\% entered an integrated AF management programme using a mobile AF App (mAFA).

When $A F$ is detected by a screening tool, including mobile or wearable devices, a single-lead ECG tracing of $\geq 30$ s or 12 -lead ECG showing AF analysed by a physician with expertise in ECG rhythm interpretation is necessary to establish a definitive diagnosis of $A F$ (devices capable of ECG recording enable direct analysis of the device-provided tracings). When AF detection is not based on an ECG recording (e.g. with devices using photoplethysmography) or in case of uncertainty in the interpretation of device-provided ECG tracing, a confirmatory ECG diagnosis has to be obtained using additional ECG recording (e.g. 12-lead ECG, Holter monitoring, etc.) 


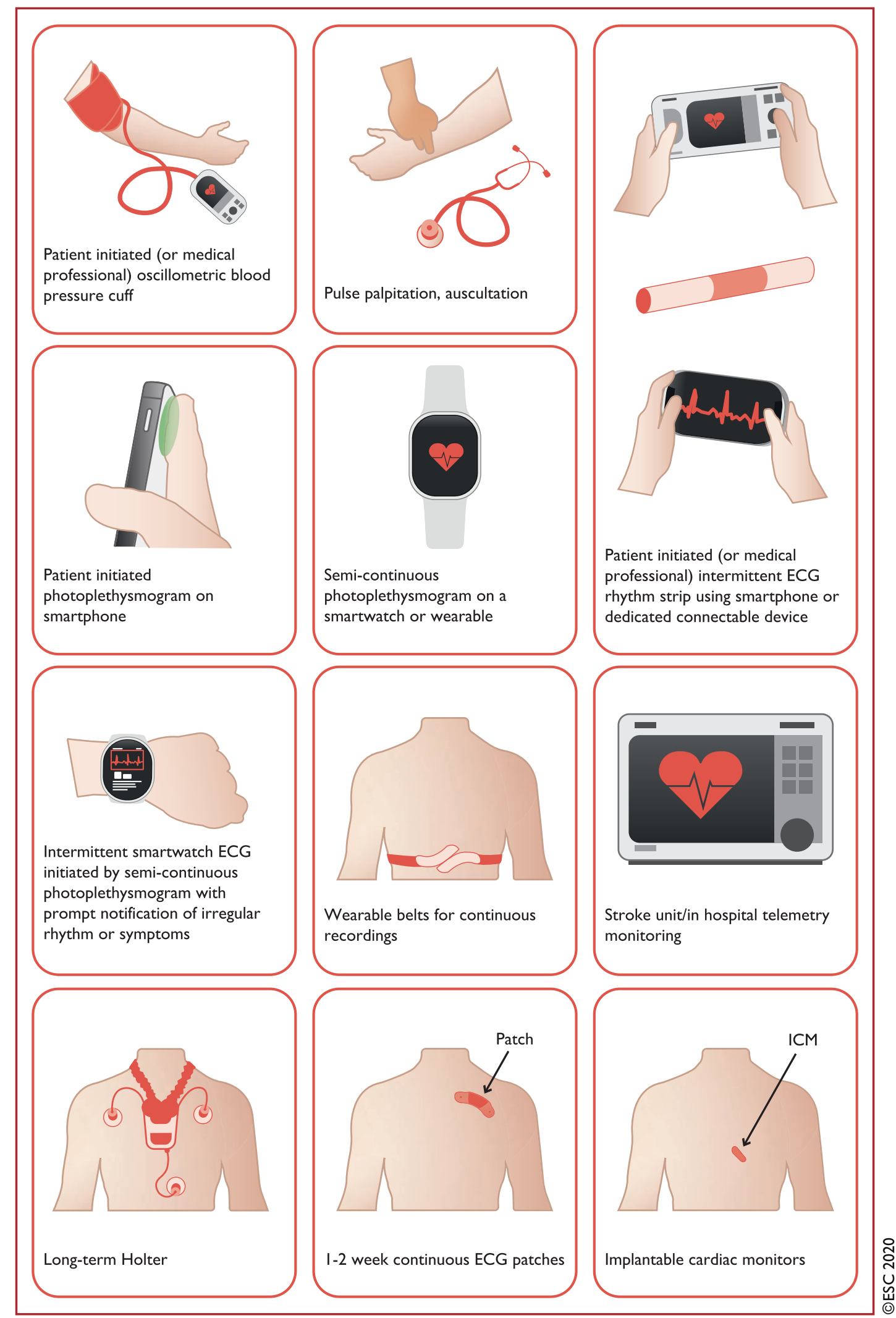

Figure 6 Systems used for AF screening. Pulse palpation, automated BP monitors, single-lead ECG devices, PPG devices, other sensors (using seismocardiography, accelerometers, and gyroscopes, etc.) used in applications for smartphones, wrist bands, and watches. Intermittent smartwatch detection of AF is possible through PPG or ECG recordings. Smartwatches and other 'wearables' can passively measure pulse rate from the wrist using an optical sensor for PPG and alerting the consumer of a pulse irregularity (based on a specific algorithm for AF detection analysing pulse irregularity and variability). ${ }^{172,173,188-196} \mathrm{AF}=$ atrial fibrillation; $\mathrm{BP}=$ blood pressure; $E C G$ = electrocardiogram; PPG = photoplethysmography. 
Table 5 Sensitivity and specificity of various AF screening tools considering the 12-lead ECG as the gold standard ${ }^{173}$

\begin{tabular}{|c|c|c|}
\hline & Sensitivity & Specificity \\
\hline Pulse taking ${ }^{203}$ & $87-97 \%$ & $70-81 \%$ \\
\hline Automated BP monitors ${ }^{204-207}$ & $93-100 \%$ & $86-92 \%$ \\
\hline Single lead ECG ${ }^{208-211}$ & $94-98 \%$ & $76-95 \%$ \\
\hline Smartphone apps ${ }^{188,189,191,195,212,213}$ & $91.5-98.5 \%$ & $91.4-100 \%$ \\
\hline Watches $^{196,198,213,214}$ & $97-99 \%$ & $83-94 \%$ \\
\hline
\end{tabular}

$\mathrm{AF}=$ atrial fibrillation; $\mathrm{BP}=$ blood pressure; $\mathrm{ECG}=$ electrocardiogram .

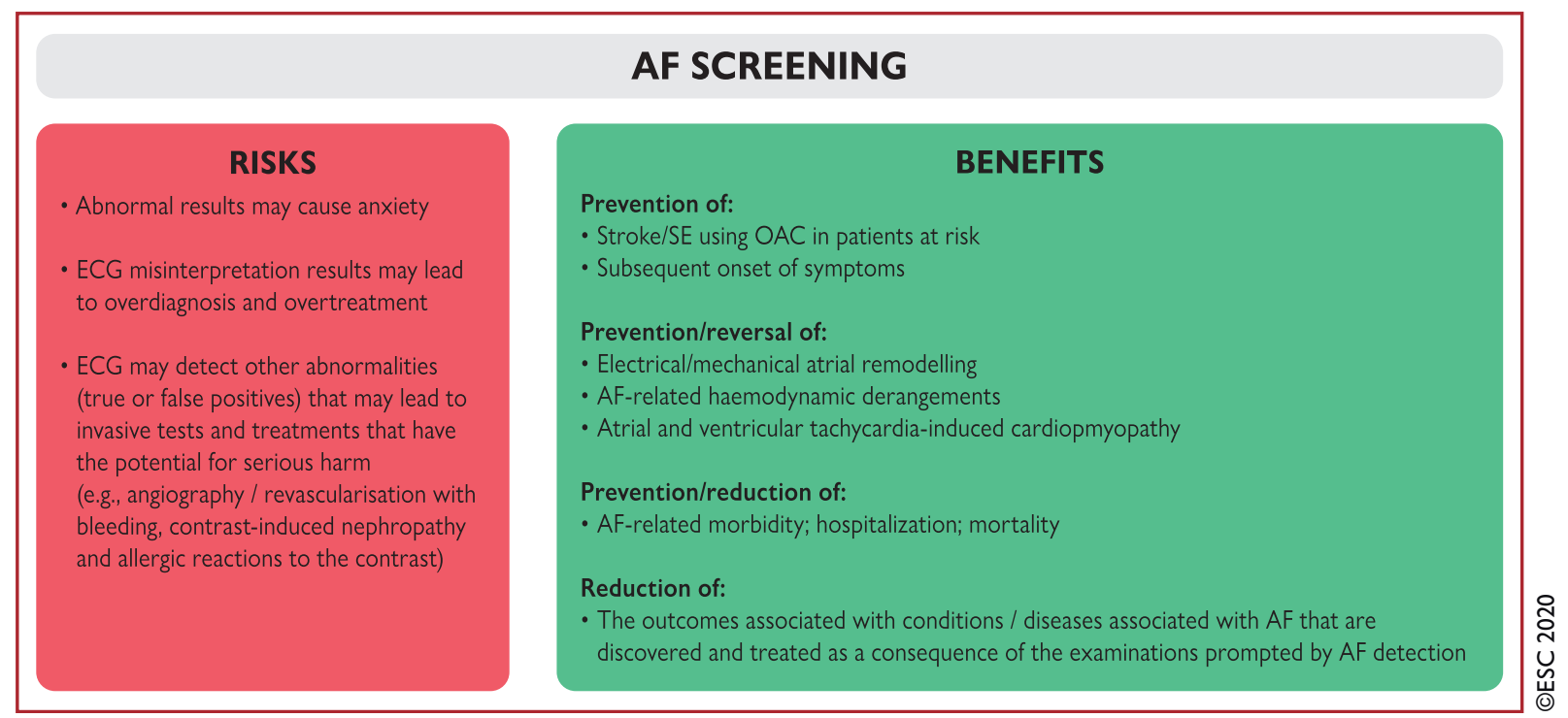

Figure 7 Potential benefits from and risks of screening for $\mathrm{AF} . \mathrm{AF}=$ atrial fibrillation; $\mathrm{ECG}=$ electrocardiogram; $\mathrm{OAC}=$ oral anticoagulant; $\mathrm{SE}=$ systemic embolism.

The data reported in Table 5 should be interpreted with caution, as assessment of sensitivity and specificity in many studies was based on small observational cohorts, with a substantial risk of bias due to signal selection. Moreover, there is a continuous evolution of algorithms and technologies available in commercial devices.

Two recent meta-analyses reported that screening for AF using an ECG would not detect more cases than would screening with pulse palpation. $^{215}$

\subsection{Screening types and strategies}

Commonly used AF screening types and strategies ${ }^{172,173,216}$ include opportunistic or systematic screening of individuals above a certain age (usually $\geq 65$ years) or with other characteristics suggestive of increased stroke risk, using intermittent single-point or repeated 30-s ECG recording over 2 weeks. The appropriate frequency of monitoring using smartphones or watches is undefined. Primary care, pharmacies, or community screening during special events is a good setting for AF screening. ${ }^{172,173}$ Overall, there was no significant difference between systematic vs. opportunistic or general practice vs. community screening in a meta-analysis, but repeated heart rhythm monitoring was associated with significantly better effectiveness compared with single assessment. ${ }^{215}$ Importantly, a structured referral of screen-detected or suspected AF cases for further clinical evaluation should be organized, to provide an appropriate management.

\subsection{Benefits from and risks of screening for atrial fibrillation}

Potential advantages and disadvantages of detecting a previously undiagnosed AF through screening are shown in Figure $7 .^{173}$

Screening can also highlight cases of known suboptimally managed AF. ${ }^{217}$ Intermittent ECG recording increased new AF detection fourfold. ${ }^{217}$ In the REHEARSE-AF (REmote HEArt Rhythm Sampling using the AliveCor heart monitor to scrEen for Atrial Fibrillation) controlled study using a smartphone/tablet-based single-lead ECG system twice weekly over 12 months vs. routine care resulted in a 3.9-fold increase in AF detection in patients aged $\geq 65$ years. ${ }^{218}$ Appropriate patient information and screening programme organization with rapid ECG clarification may reduce anxiety induced by suspicion of abnormality.

\subsection{Cost-effectiveness of screening for atrial fibrillation}

Higher AF-related medical costs justify strategies to identify and treat undiagnosed AF. ${ }^{219}$ Opportunistic AF screening is associated with lower costs than systematic screening. ${ }^{173}$ Appropriate choice of the screening tool and setting is important, ${ }^{220}$ and a favourable cost-effectiveness profile has been estimated for screening programmes based on pulse palpation, hand-held ECG devices, and 
smartphones with pulse photoplethysmography algorithms. ${ }^{172}$ Both systematic and opportunistic screening are more costeffective than routine practice for patients $\geq 65$ years, with opportunistic screening more likely to be cost-effective than systematic population screening. ${ }^{1491}$

\subsection{Screening in high-risk populations}

\subsubsection{Elderly}

The risk of AF (often asymptomatic) and stroke increase with age, ${ }^{82,127,221}$ thus justifying AF screening in the elderly. Opportunistic AF screening seems to be cost-effective in elderly populations ( $\geq 65$ years) ${ }^{222}$ and among 75-76-year-old individuals undergoing a 2week intermittent ECG screening. ${ }^{223}$

Pulse palpation and/or short-term ECG among the elderly $(\geq 65$ years) yielded an AF prevalence of $4.4 \%$, with previously undiagnosed $A F$ in $1.4 \%$, suggesting a number needed to screen of $70 .{ }^{224}$ Repeated hand-held ECG recordings over 2 weeks in an unselected population aged 75 - 76 years increased the detection of asymptomatic AF up to $7.4 \%$ in subjects with $\geq 2$ stroke risk factors. ${ }^{225}$

\section{Recommendations for screening to detect AF}

\section{Recommendation}

Opportunistic screening for AF by pulse taking or ECG rhythm strip is recommended in patients $\geq 65$ years of age. ${ }^{188,211,223,225}$ It is recommended to interrogate pacemakers and implantable cardioverter defibrillators on a regular basis for AHRE. ${ }^{\text {c224,226 }}$

When screening for $A F$ it is recommended that: $^{217,218}$

- The individuals undergoing screening are informed about the significance and treatment implications of detecting AF.

- A structured referral platform is organized for screen-positive cases for further physician-led clinical evaluation to confirm the diagnosis of AF and provide optimal management of patients with confirmed AF.

- Definite diagnosis of AF in screen-positive cases is established only after physician reviews the single-lead ECG recording of $\geq 30$ s or 12 -lead ECG and confirms that it shows AF.

Systematic ECG screening should be considered to detect $A F$ in individuals aged $\geq 75$ years, or those at high risk of stroke. ${ }^{212,224,227}$

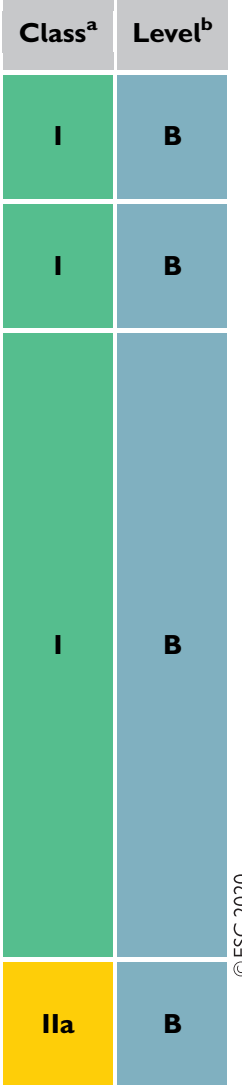

$\mathrm{AF}=$ atrial fibrillation; $\mathrm{AHRE}=$ atrial high-rate episode; $\mathrm{ECG}=$ electrocardiogram.

${ }^{\mathrm{a}}$ Class of recommendation.

bLevel of evidence.

'See sections 3.2 and 3.3 for diagnostic criteria for AF and AHRE, and section 16 for the management of patients with AHRE.

\section{Diagnostic assessment in atrial fibrillation}

Often occurring in patients with cardiovascular risk factors/comorbidities, AF may sometimes be a marker of undiagnosed conditions. Hence, all AF patients will benefit from a comprehensive cardiovascular assessment (Figure 8).

The 'standard package' for diagnostic evaluation of AF patients should include complete medical history and assessment of concomitant conditions, AF pattern, stroke risk, AF-related symptoms, thrombo-embolism, and LV dysfunction. ${ }^{143}$ A 12-lead ECG is recommended in all AF patients, to establish the diagnosis of AF, assess ventricular rate during $A F$, and check for the presence of conduction defects, ischaemia, or signs of structural heart disease. Laboratory tests (thyroid and kidney function, serum electrolytes, full blood count) and transthoracic echocardiography (LV size and function, LA size, valvular disease, and right heart size and systolic function) are needed to guide treatment. Based on the patient's characteristics, specific additional information can be obtained. Most AF patients need regular follow-up (primary care) to ensure continued optimal management.

\subsection{Symptoms and quality of life}

As symptoms related to AF may range from none to disabling, and rhythm control treatment decisions (including catheter ablation) are influenced by symptom severity, symptom status should be characterized using the European Heart Rhythm Association (EHRA) symptom scale 228 (Table 6), and the relation of symptoms (especially if non-specific, such as shortness of breath, fatigue, chest discomfort, etc.) to AF should be elucidated because symptoms may also result from undiagnosed or suboptimally managed concomitant cardiovascular risk factors or pathological conditions. ${ }^{229}$

In selected AF patients, long-term ECG monitoring is recommended to assess the adequacy of rate control or to relate symptoms with AF episodes. Sometimes the association of symptoms with AF can be established only retrospectively, after successful rhythm control intervention. In selected patients, a trial of sinus rhythm using cardioversion and a quantified patient perception of symptoms using a validated assessment tool (Supplementary Table 4) may inform the decision about subsequent AF catheter ablation (section 10.2).

Symptomatic and functional improvement with rhythm control therapies (cardioversion, ${ }^{232-234}$ antiarrhythmic medications, and AF catheter-ablation procedures ${ }^{235-242}$ ) largely depends on sinus rhythm maintenance ${ }^{243}$; however, QoL may improve despite AF recurrences, unless AF burden is high ${ }^{244}$ (e.g. $>2$ h daily ${ }^{100}$ ) owing to optimized management of cardiovascular risk factors or comorbidities $^{245}$ or a treatment expectancy effect. The effect of AF treatment ${ }^{246,247}$ is supported by reports of persistently improved QoL 10 years after paroxysmal AF catheter ablation in patients with a low AF progression rate. $^{248}$

\subsection{Substrate}

The substrate for AF relates to LA dilation and fibrosis with subsequent LA dysfunction and delay in electromechanical conduction. 


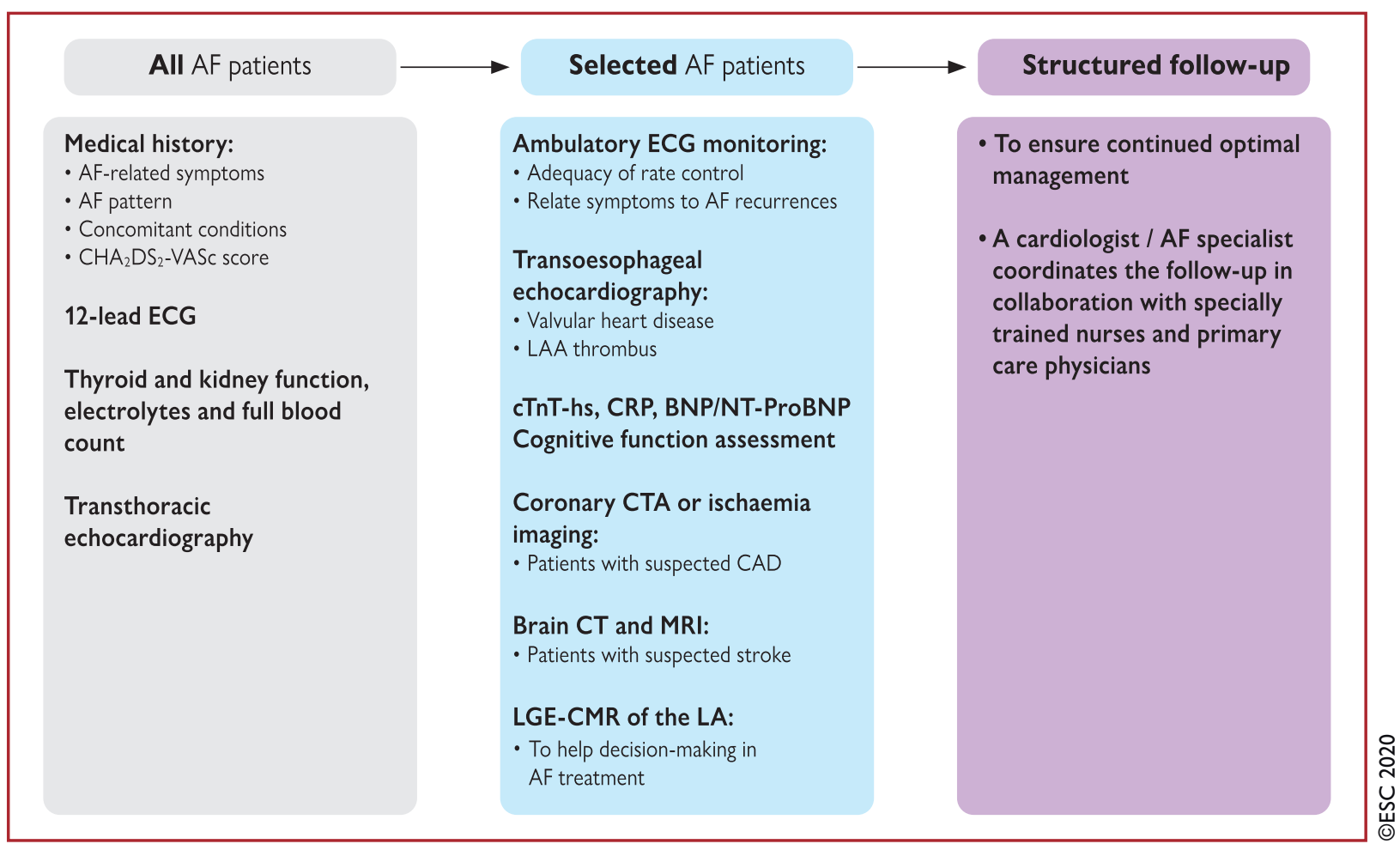

Figure 8 Diagnostic work-up and follow-up in AF patients. $\mathrm{AF}=$ atrial fibrillation; $\mathrm{BNP}=\mathrm{B}$-type natriuretic peptide; $\mathrm{CHA}_{2} \mathrm{DS}_{2}-\mathrm{VASC}_{\mathrm{C}}=\mathrm{Congestive} \mathrm{heart}$ failure, Hypertension, Age $\geq 75$ years, Diabetes mellitus, Stroke, Vascular disease, Age $65-74$ years, Sex category (female); CAD = coronary artery disease; $\mathrm{CRP}=\mathrm{C}$-reactive protein; $\mathrm{CT}=$ computed tomography; $\mathrm{CTA}=$ computed tomography angiography; $\mathrm{cTnT}$-hs = high-sensitivity cardiac troponin T; ECG = electrocardiogram; LAA = left atrial appendage; LGE-CMR = late gadolinium contrast-enhanced cardiac magnetic resonance; $M R I=$ magnetic resonance imaging; NT-ProBNP = N-terminal (NT)-prohormone B-type natriuretic peptide.

\section{Table 6 EHRA symptom scale}

\begin{tabular}{|c|c|c|}
\hline Score & Symptoms & Description \\
\hline 1 & None & AF does not cause any symptoms \\
\hline $2 a$ & Mild & Normal daily activity not affected by symptoms related to AF \\
\hline $2 b$ & Moderate & Normal daily activity not affected by symptoms related to AF, but patient troubled by symptoms \\
\hline 3 & Severe & Normal daily activity affected by symptoms related to AF \\
\hline 4 & Disabling & Normal daily activity discontinued \\
\hline
\end{tabular}

Six symptoms, including palpitations, fatigue, dizziness, dyspnoea, chest pain, and anxiety during AF, are evaluated with regard to how it affects the patient's daily activity, ranging from none to symptom frequency or severity that leads to a discontinuation of daily activities.

To measure treatment effects, QoL and symptom questionnaires should be sensitive to changes in AF burden. The EHRA symptom scale is a physician-assessed tool for quantification of AF-related symptoms that is used to guide symptom-driven AF treatment decisions, ${ }^{228}$ and has been related to adverse outcomes in more symptomatic patients (score 3 - 4) versus those with a score of $1-2.228,230$ However, it does not consider the symptom dimensions such as anxiety, treatment concerns, and medication adverse effects that are captured by general QoL scales, ${ }^{230}$ or the patient-reported symptom-related outcomes. As discrepancies between patient-reported and physician-assessed outcomes are frequently observed, ${ }^{231}$ the AF-related treatment decisions also need to be informed by a quantified patient perception of symptoms, but further research is needed to identify optimal tool(s) for capturing this information.

$\mathrm{AF}=$ atrial fibrillation; EHRA = European Heart Rhythm Association; QoL = quality of life.

Non-invasive, multimodality imaging can provide all needed information (Figure 9). ${ }^{249,250}$

In selected patients, transoesophageal echocardiography (TOE) can be used to evaluate valvular heart disease (VHD) or left atrial appendage (LAA) thrombus; CT coronary angiography can be performed for assessment of CAD; CT/MRI of the brain can be performed when stroke is suspected. Specific predictors of stroke have been suggested: LA dilation, spontaneous LA contrast, reduced LA strain, LAA thrombus, low peak LAA velocity $(<20 \mathrm{~cm} / \mathrm{s})$, and LAA non-chicken wing configuration (on CT). ${ }^{250}$ 


\section{Left atrial remodelling associated with $\mathrm{AF}$}

\section{Anatomy}

Dilatation and change in geometry

Structure
Fibrosis
Function
Altered electrophysiology,
LA reservoir, conduit and booster
pump function

\section{LA/LAA thrombus detection}
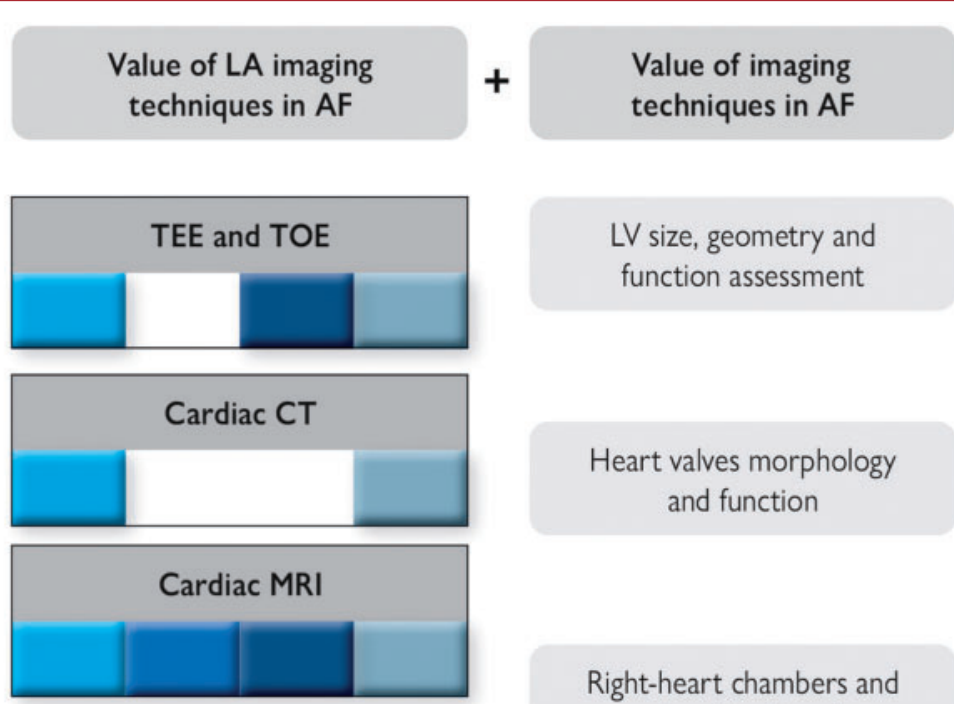

EP mapping

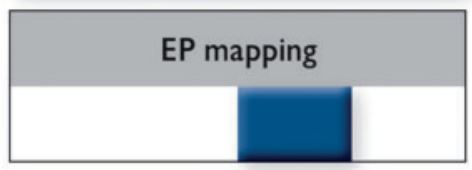

Right-heart chambers and pericardium imaging

Heart valves morphology and function

LV size, geometry and function assessment

\section{Advanced/Investigation imaging: \\ - Echocardiographic TDI and LA strain, etc. \\ - MRI delayed enhancement or T1 imaging \\ - $\mathrm{CT}$ imaging of substrate, etc.}

Figure 9 Imaging in AF. Anatomical imaging provides the LA size, shape, and fibrosis. Most accurate assessment of LA dilation is obtained by CMR or CT. For routine assessment, two-dimensional (2D) or (preferably) three-dimensional (3D) transthoracic echocardiography is used. The 3D echocardiographic normal volume values are $15-42 \mathrm{~mL} / \mathrm{m}^{2}$ for men and $15-39 \mathrm{~mL} / \mathrm{m}^{2}$ for women. ${ }^{250}$ Assessment of LA fibrosis with LGE-CMR has been described but only rarely applied in clinical practice. ${ }^{251}$ Functional imaging includes TDI and strain. TDI measures the velocities of the myocardium in diastole and systole, whereas LA strain reflects active LA contraction. The PA-TDI interval reflects the atrial electromechanical delay (total LA conduction time, the time interval between the P-wave on the ECG and the A' [atrial peak velocity] on TDI) and reflects LA strain. ${ }^{252}$ LA wall infiltration by epicardial fat is a potential early marker of inflammation and can be detected with $\mathrm{CT}$ or cardiac MRI. ${ }^{253}$ Before AF ablation, the pulmonary vein anatomy can be visualized with $\mathrm{CT}$ or $C M R$. AF = atrial fibrillation; $C T$ = computed tomography; $E P=$ electrophysiology; $L A=$ left atrium; $L A A=$ left atrial appendage; $L V=$ left ventricular; LGE-CMR = late gadolinium contrast-enhanced cardiac magnetic resonance; MRI = magnetic resonance imaging; TDI = tissue doppler imaging; TOE = transoesophageal echocardiography; TTE = transthoracic echocardiography.

\section{Recommendations for diagnostic evaluation of patients with AF}

\section{Recommendation}

In patients with $A F$, it is recommended to:

- Evaluate AF-related symptoms (including fatigue, tiredness, exertional shortness of breath, palpitations, and chest pain) and quantify the patient symptom status using the modified EHRA symptom scale before and after initiation of treatment. ${ }^{230,232}$

- Evaluate AF-related symptoms before and after cardioversion of persistent AF to aid rhythm control treatment decisions. ${ }^{230,232}$

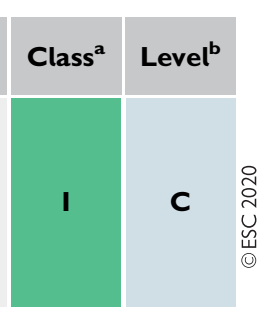

$\mathrm{AF}=$ atrial fibrillation; EHRA = European Heart Rhythm Association.

${ }^{\mathrm{a} C}$ Class of recommendation.

bevel of evidence. 


\section{Integrated management of patients with atrial fibrillation}

\subsection{Definitions and components of integrated management of atrial fibrillation patients}

Integrated management of AF patients requires a coordinated and agreed patient-individualized care pathway to deliver optimized treatment (Figure 10) by an interdisciplinary team (Figure 11). Central to this approach is the patient; treatment options should be discussed, and the management plan agreed in discussion with healthcare professionals. Treatment is subject to change over time with the development of new risk factors, symptoms, disease progression, and the advent of new treatments.

\subsection{Multidisciplinary atrial fibrillation teams}

Integrated AF management requires a coordinated multidisciplinary team (Figure 11) composed according to individual patient needs and local availability of services. Complex patients would benefit from a multidisciplinary team that includes relevant specialists, as well as their primary care physician (for post-discharge care) and their family/carer. Involvement of patient and family/carers is integral to the success of AF management.
9.2.1 Role of healthcare systems and budget constraints Optimized AF treatment requires a well-structured healthcare system and significant financial resources. ${ }^{254}$ Allocation of resources will vary due to differing healthcare system structures and budget constraints in diverse geographies. The significant inequalities in the access to AF management-related resources are documented in the recent ESC Atlas on Cardiovascular Disease. ${ }^{255}$ It is important to consider optimizing use of available resources to reduce stroke, improve symptoms, and treat comorbidities.

\subsection{Patient involvement and shared decision making}

\subsubsection{Patient values and preferences}

Exploring patient's values, goals, and preferences should be the first step of shared decision making. ${ }^{256,257}$ Qualitative research demonstrates recurring discordance between caregivers reporting shared decision making and patients experiencing a paternalistic model, ${ }^{109,258-261}$ and a misperception that many prefer not to be involved in decision making, rather deferring to their physician. ${ }^{259,262-266}$ For shared decision making, ${ }^{261}$ the importance attached by the patient to stroke prevention and rhythm control and the respective risk of death, stroke, and major bleeding, as well as the burden of treatment, should be thoroughly assessed and respected. $257,264,266-268$

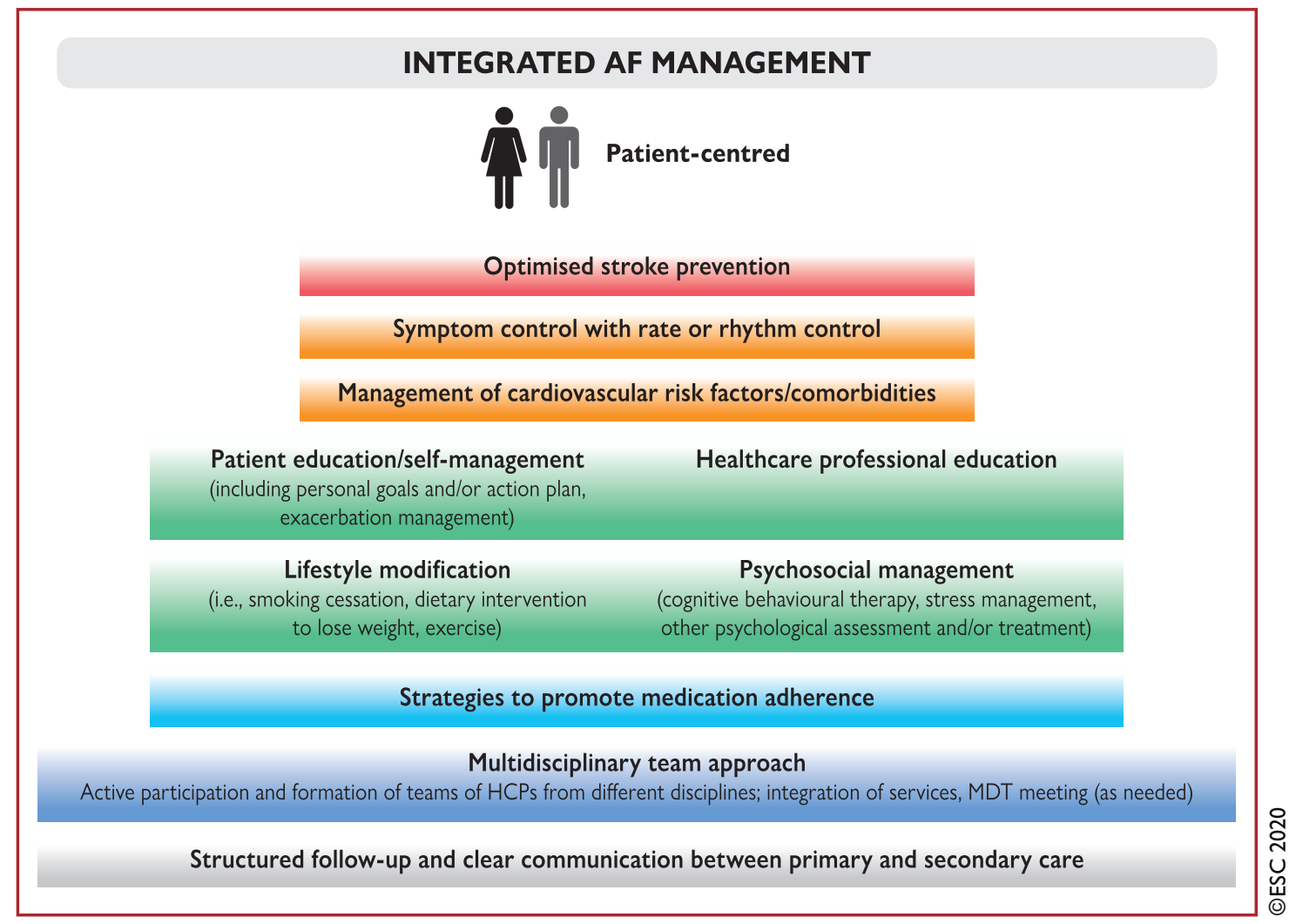

Figure 10 Components of integrated AF management. AF = atrial fibrillation; $\mathrm{HCP}=$ healthcare professional; $\mathrm{MDT}=$ multidisciplinary team . 


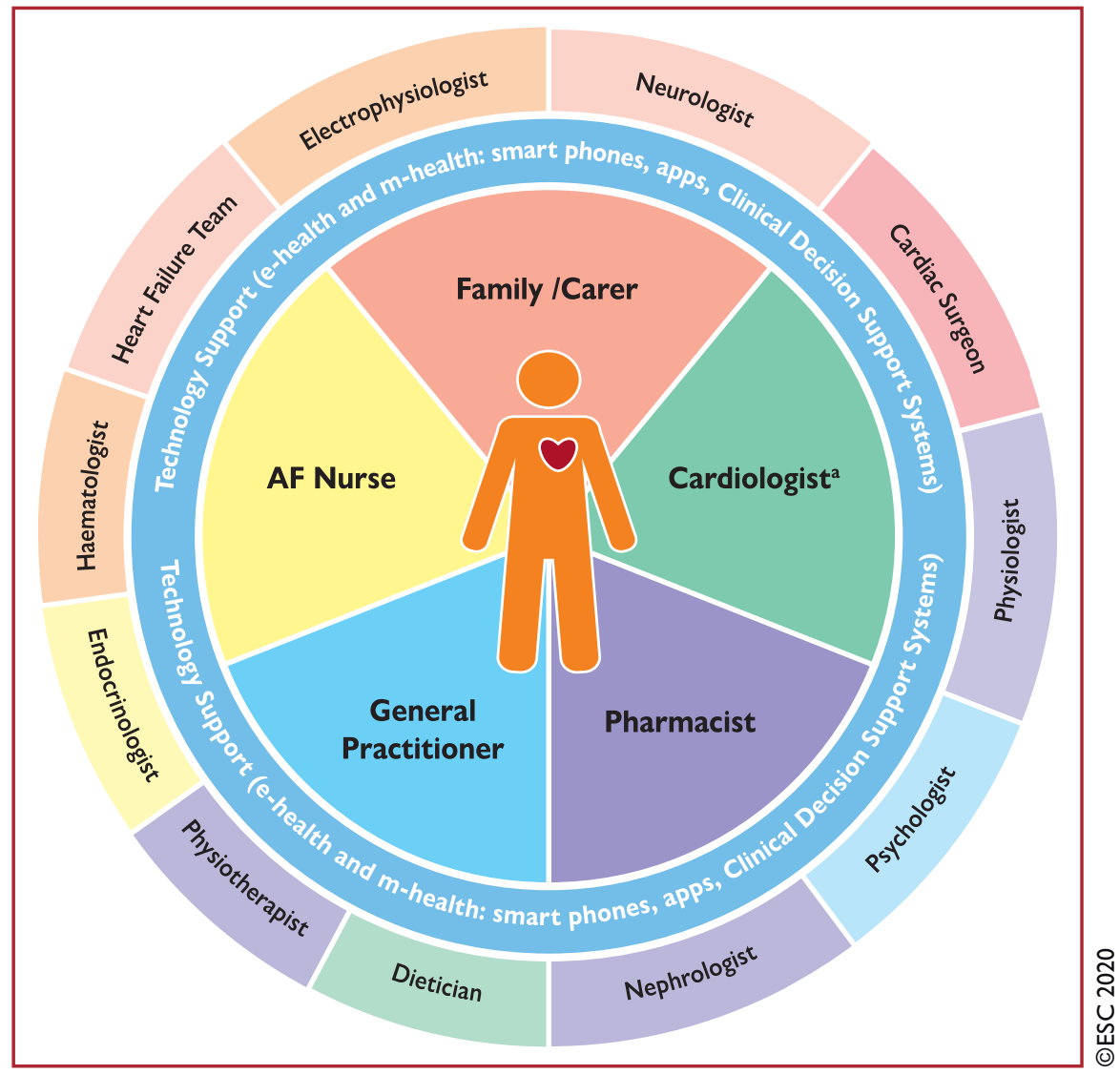

Figure I I Integrated AF management team (an example). The figure gives an example on the potential composition of AF teams showing a variety of different specialists supporting individual patients as needed. AF = atrial fibrillation. ${ }^{a}$ According to local standards, this could be a general cardiologist with special interest in arrhythmias/AF or an electrophysiologist.

\subsubsection{Patient education}

Patient knowledge about AF and its management is often limited $^{257,269-272}$ particularly when first diagnosed, when the majority of treatment decisions are discussed and made.

Information on useful resources to help educate AF patients ${ }^{273}$ can be found in the ESC Textbook of Cardiovascular Medicine, but education alone is often insufficient to produce and maintain medication adherence and lifestyle modifications.

\subsection{Healthcare professional education}

A mixed-methods approach has been used when targeting healthcare professionals including individual needs assessment followed by bespoke education and training, whether by smart technology, online resources, or upskilling face-to-face workshops or a combination. ${ }^{274}$ The mAFA, integrating clinical-decision support and education for healthcare professionals, has been successfully piloted and subsequently tested in an outcome RCT. ${ }^{275}$ Education alone is insufficient to change healthcare-professional behaviour. $^{276}$ In the Integrated Management Program Advancing Community Treatment of Atrial Fibrillation (IMPACT-AF) trial, ${ }^{277}$ a multifaceted educational intervention including healthcareprofessional education and feedback resulted in a significant increase in the proportion of patients treated with oral anticoagulant (OAC) therapy.

\subsection{Adherence to treatment}

Factors affecting adherence to treatment can be grouped into patientrelated (e.g. demographics, comorbidities, cognitive impairment, polypharmacy, treatment side-effects, psychological health, patient understanding of the treatment regimen), physician-related (knowledge, awareness of guidelines, expertise, multidisciplinary team approach), and healthcare system-related (work-setting, access to treatments, cost) factors. ${ }^{278}$

Ensuring patients are appropriately informed about treatment options, how to adhere to treatment, potential consequences of non-adherence, in addition to managing patient's expectations of treatment goals, are crucial to promote adherence. Regular review by any member of the multidisciplinary team is important to identify non-adherence and implement strategies to improve adherence where appropriate.

\subsection{Technology tools supporting atrial fibrillation management}

Clinical decision support systems are intelligent systems that digitize and provide evidence-based guidelines, clinical pathways, and algorithms facilitating personalized, timely, and evidence-based treatment.

The MobiGuide project $^{279}$ and several applications ${ }^{280-283}$ (Supplementary Tables 5 and 6) have been used to enhance patient 
education, improve communication between patients and healthcare professionals, and encourage active patient involvement. The ESC/ CATCH-ME (Characterizing AF by Translating its Causes into Health Modifiers in the Elderly) consortium also has a smartphone/tablet app $^{281}$ for AF patients, but this is yet to be tested prospectively. A Cochrane review ${ }^{284}$ demonstrated that patient decision-support aids reduce decision conflict. ${ }^{285-288}$ Nevertheless, contradictory results $^{277,289,290}$ illustrate the need for more carefully designed studies, including assessment of the intervention's effect on clinical events.

\subsection{Advantages of integrated management of atrial fibrillation patients} Limited evidence exists on the effectiveness of integrated management of AF. Available intervention studies vary widely in number and content of 'integrated care' employed. Six studies—one cluster RCT, ${ }^{291}$ four RCTs, ${ }^{277,292-295}$ and one before-and-after study ${ }^{294}$ of integrated AF management have demonstrated mixed findings (Supplementary Table 7). Two studies ${ }^{292,294}$ and one meta-analysis ${ }^{296}$ report significantly lower rates of cardiovascular hospitalization and death with nurse-led, integrated care, whereas others reported no effect of integrated care on these outcomes. One multifaceted study ${ }^{277}$ demonstrated improved OAC rates in the intervention group at 12 months. The IMPACT-AF study ${ }^{277}$ found no significant difference in the composite efficacy outcome (unplanned emergency department visit or cardiovascular hospitalization) or the primary safety outcome of major bleeding between intervention and usual care.

\subsection{Measures (or approaches) for implementation of integrated management}

Integrated management of AF requires a change in the current approach to patient care, to focus on moving from a multidisciplinary team to interdisciplinary working, including behaviour change for all AF team members and key stakeholders including patients and their family ${ }^{297,298}$ (Supplementary Figure 3).

To understand whether integrated AF management has been implemented into clinical practice and had an impact on important outcomes (mortality, stroke, hospitalization, QoL, symptom reduction, etc.), a specific international standard set of outcome measures should be collected (Supplementary Figure 4). ${ }^{299}$ This would also highlight areas requiring further development.

\subsection{Treatment burden}

Patient-perceived treatment burden ${ }^{300}$ is defined as the workload imposed by healthcare on patients and its effect on patient functioning and well-being apart from specific treatment side-effects. ${ }^{301,302}$ It includes everything patients do for their health (drug management, self-monitoring, visits to the doctor, laboratory tests, lifestyle changes) and healthcare impact on their social relationships, potentially affecting adherence to treatment, ${ }^{303,304}$ QoL, and outcomes (e.g. hospitalization and survival). ${ }^{305,306}$ Patient-perceived treatment burden is influenced by their knowledge about disease. ${ }^{302}$ Patients with similar treatment regimens may have very different treatment burden, ${ }^{307}$ with only a weak agreement between patient's and physicians' treatment burden evaluation, suggesting that the patient's experience is not shared in depth during consultations. ${ }^{302,308,309}$

Treatment burden can be overwhelming for patients with multiple chronic conditions ${ }^{301}$ (e.g. those with three chronic conditions would have to take 6 - 12 medications daily, visit a healthcare giver 1.2 - 5.9 times per month, and spend 49.6-71.0 $\mathrm{h}$ monthly in healthcare-related activities ${ }^{310}$ ). Treatment burden in AF patients is largely unknown. In a single-centre prospective study, AF patient-perceived total treatment burden was higher than in patients with other chronic conditions ( $27.6 \%$ vs. $24.3 \%, P=0.011)$, and 1 in 5 AF patients reported a high treatment burden that could question the sustainability of their treatment. Notably, AF patients attributed the highest proportion of treatment burden to healthcare system-related aspects (e.g. attending appointments etc.) and lifestyle modification requirements. Female sex and younger age were independently significantly associated with a higher treatment burden, whereas non-vitamin $\mathrm{K}$ antagonist oral anticoagulants (NOACs) and rhythm control reduced the odds for high treatment burden by $>50 \%{ }^{311}$

The discussion of treatment burden should be an integral part of shared, informed treatment decision making, and treatment burden can be assessed using a validated questionnaire. ${ }^{312}$

\subsection{Patient-reported outcomes}

There is increasing advocacy for including patient-reported outcomes (PROs) as endpoints in clinical trials ${ }^{313}$ and their routine collection ${ }^{314-316}$ to improve care and assess treatment success from the patient's perspective. Patients' experience of AF and its management is highly subjective; AF management has become increasingly complex, potentially resulting in significant treatment burden and poorer health-related QoL.

Measuring outcomes that are important to patients, in addition to 'hard' clinical endpoints (death, stroke, major bleeding, etc.), can inform AF management. An international consortium of AF patients and healthcare professionals has identified the following PROs as important to measure for AF: health-related QoL, physical and emotional functioning, cognitive function, symptom severity, exercise tolerance, and ability to work (Supplementary Figure 4) ${ }^{299}$; PRO measures can be used to assess these factors and the international standard set of AF outcome measures proposes some tools for assessing PROs. ${ }^{299}$ Health informatics systems could help capture PRO data. Despite increasing support for the role of PRO measures in healthcare management, few studies and registries report collecting PRO data using validated tools. ${ }^{313}$ Implementation of PRO measures in the management of AF patients is addressed in a dedicated expert consensus paper developed in collaboration with patient representatives by the EHRA. ${ }^{317}$ 


\section{Recommendations about integrated AF management}

\begin{tabular}{l} 
Recommendations \\
To optimize shared decision making about specific AF treatment option(s) in consideration, it is recommended that physicians: \\
- Inform the patient about the advantages/limitations and benefit/risks associated with the treatment option(s) being considered; \\
and \\
- Discuss the potential burden of the treatment with the patient and include the patient's perception of treatment burden in the \\
treatment decision. \\
\hline It is recommended to routinely collect PROs to measure treatment success and improve patient care. \\
\hline Integrated management with a structured multidisciplinary approach including healthcare professionals, patients, and their family/ \\
carers, should be used in all AF patients to improve clinical outcomes. ${ }^{277,292-294,296,297}$
\end{tabular}

$\mathrm{AF}=$ atrial fibrillation; $\mathrm{PRO}=$ patient-reported outcome.

${ }^{\mathrm{a}}$ Class of recommendation.

bevel of evidence.

\section{Patient management: the integrated $A B C$ pathway}

The simple Atrial fibrillation Better Care (ABC) holistic pathway ('A' Anticoagulation/Avoid stroke; 'B' Better symptom management; 'C' Cardiovascular and Comorbidity optimization ${ }^{318}$ ) streamlines integrated care of AF patients across all healthcare levels and among different specialties. Compared with usual care, implementation of the $A B C$ pathway has been significantly associated with lower risk of allcause death, composite outcome of stroke/major bleeding/cardiovascular death and first hospitalization, ${ }^{319}$ lower rates of cardiovascular events, ${ }^{320,321}$ and lower health-related costs. ${ }^{322}$ In the prospective, randomized mAFA-II trial, the composite outcome was significantly lowered with $\mathrm{ABC}$ pathway management intervention compared with usual care $[1.9 \%$ vs. $6.0 \%$; hazard ratio $(\mathrm{HR}) \quad 0.39 ; 95 \% \mathrm{Cl}$ $0.22-0.67 ; P<0.001] .^{323}$

\section{1 'A' - Anticoagulation/Avoid stroke}

This section refers to $A F$ in the absence of severe mitral stenosis or prosthetic heart valves (for AF with concomitant VHD see section $11.7) .^{148}$

\subsubsection{Stroke risk assessment}

Overall, AF increases the risk of stroke five-fold, but this risk is not homogeneous, depending on the presence of specific stroke risk factors/modifiers. Main clinical stroke risk factors have been identified from non-anticoagulated arms of the historical RCTs conducted $>20$ years ago, notwithstanding that these trials only randomized $<10 \%$ of patients screened, whereas many common risk factors were not recorded or consistently defined. ${ }^{324}$ These data have been supplemented by evidence from large observational cohorts also studying patients who would not have been included in the RCTs. Subsequently, various imaging, blood, and urine biological markers (biomarkers) have been associated with stroke risk (Table 7). ${ }^{324,325}$ In addition, non-paroxysmal AF is associated with an increase in thrombo-embolism (multivariable adjusted $\mathrm{HR}$ 1.38; $95 \% \mathrm{Cl}$ $1.19-1.61 ; P<0.001)$ compared with paroxysmal AF. ${ }^{156}$ Notably, many of the risk factors for AF-related complications are also risk factors for incident $A F^{33}$
Common stroke risk factors are summarized in the clinical risk-factor-based $\mathrm{CHA}_{2} \mathrm{DS}_{2}$-VASc [Congestive heart failure, Hypertension, Age $\geq 75$ years, Diabetes mellitus, Stroke, Vascular disease, Age 65-74 years, Sex category (female)] score (Table 8). ${ }^{334}$

Stroke risk scores have to balance simplicity and practicality against precision. ${ }^{354-356}$ As any clinical risk-factor-based score, $\mathrm{CHA}_{2} \mathrm{DS}_{2^{-}}$ VASc performs only modestly in predicting high-risk patients who will sustain thrombo-embolic events, but those identified as low-risk $\left[\mathrm{CHA}_{2} \mathrm{DS}_{2}\right.$-VASc 0 (males), or score of 1 (females)] consistently have low ischaemic stroke or mortality rates $(<1 \% /$ year) and do not need any stroke prevention treatment.

Female sex is an age-dependent stroke risk modifier rather than a risk factor per se. ${ }^{357,358}$ Observational studies showed that women with no other risk factors $\left(\mathrm{CHA}_{2} \mathrm{DS}_{2}\right.$-VASc score of 1$)$ have a low stroke risk, similar to men with a $\mathrm{CHA}_{2} \mathrm{DS}_{2}$-VASc score of $0 .{ }^{359}$ The simplified $\mathrm{CHA}_{2} \mathrm{DS}_{2}-\mathrm{VA}$ score could guide the initial decision about OAC in AF patients, but not considering the sex component would underestimate stroke risk in women with $A F .^{360,361}$ In the presence of $>1$ non-sex stroke risk factor, women with AF consistently have significantly higher stroke risk than men. ${ }^{353,362}$

Many clinical stroke risk factors (e.g. renal impairment, OSA, LA dilatation ${ }^{291,326,363-365}$ ) are closely related to the $\mathrm{CHA}_{2} \mathrm{DS}_{2}-\mathrm{VASc}$ components, and their consideration does not improve its predictive value (the relationship of smoking or obesity to stroke risk in AF is also contentious). ${ }^{366}$ Various biomarkers [e.g. troponin, natriuretic peptides, growth differentiation factor (GDF)-15, von Willebrand factor] have shown improved performance of biomarker-based over clinical scores in the assessment of residual stroke risk among anticoagulated AF patients ${ }^{329,367}$; notwithstanding, many of these biomarkers (as well as some clinical risk factors) are predictive of both stroke and bleeding ${ }^{329}$ or non-AF and noncardiovascular conditions, often (non-specifically) reflecting simply a sick heart or patient.

More complex clinical scores [e.g. Global Anticoagulant Registry in the FIELD - Atrial Fibrillation (GARFIELD-AF) $]^{368}$ and those inclusive of biomarkers [e.g. Anticoagulation and Risk Factors in Atrial Fibrillation (ATRIA), ${ }^{369,370}$ Intermountain Risk Score, ${ }^{371}$ ABC-stroke (Age, Biomarkers, Clinical history)] $]^{372}$ improve stroke risk prediction modestly but statistically significantly. The ABC-stroke risk score that considers age, previous stroke/transient ischaemic attack (TIA), highsensitivity troponin T (cTnT-hs) and N-terminal (NT)-prohormone 
Table 7 Stroke risk factors in patients with AF

\begin{tabular}{|c|c|c|c|c|}
\hline $\begin{array}{l}\text { Most commonly studied } \\
\text { clinical risk factors } \\
\text { (a systematic review) }^{324}\end{array}$ & $\begin{array}{l}\text { Positive } \\
\text { studies/All } \\
\text { studies }\end{array}$ & $\begin{array}{l}\text { Other clinical risk } \\
\text { factors }^{325}\end{array}$ & $\begin{array}{l}\text { Imaging } \\
\text { biomarkers }\end{array}$ & $\begin{array}{l}\text { Blood/urine } \\
\text { biomarkers }^{329-332}\end{array}$ \\
\hline Stroke/TIA/systemic embolism & $15 / 16$ & $\begin{array}{l}\text { Impaired renal function/ } \\
\text { CKD }\end{array}$ & Echocardiography & \multirow{8}{*}{$\begin{array}{l}\text { Cardiac troponin T and I } \\
\text { Natriuretic peptides } \\
\text { Cystatin C } \\
\text { Proteinuria } \\
\text { CrCl/eGFR } \\
\text { CRP } \\
\text { IL-6 } \\
\text { GDF-15 } \\
\text { von Willebrand factor } \\
\text { D-dimer }\end{array}$} \\
\hline Hypertension & $11 / 20$ & OSA & LA dilatation & \\
\hline Ageing (per decade) & $9 / 13$ & HCM & Spontaneous contrast or & \\
\hline Structural heart disease & $9 / 13$ & $\begin{array}{l}\text { Amyloidosis in degenerative } \\
\text { cerebral and heart diseases }\end{array}$ & $\begin{array}{l}\text { thrombus in LA } \\
\text { Low LAA velocities }\end{array}$ & \\
\hline Diabetes mellitus & $9 / 14$ & Hyperlipidaemia & Complex aortic plaque & \\
\hline Vascular disease & $6 / 17$ & Smoking & Cerebral imaging & \\
\hline CHF/LV dysfunction & $7 / 18$ & Metabolic syndrome ${ }^{333}$ & Small-vessel disease & \\
\hline Sex category (female) & $8 / 22$ & Malignancy & & \\
\hline
\end{tabular}

$\mathrm{CHF}=$ congestive heart failure; $\mathrm{CKD}=$ chronic kidney disease; $\mathrm{CrCl}=$ creatinine clearance; $\mathrm{CRP}=\mathrm{C}$-reactive protein; eGFR = estimated glomerular filtration rate; GDF-15 = growth differentiation factor-15; IL-6 = interleukin 6; LA = left atrium; LAA = left atrial appendage; LV = left ventricular; OSA = obstructive sleep apnoea; TIA = transient ischaemic attack.

B-type natriuretic peptide has been validated in the cohorts of landmark NOAC trials. ${ }^{373-375}$ A biomarker score-guided treatment strategy to reduce stroke and mortality in AF patients is being evaluated in an ongoing RCT (the ABC-AF Study, NCT03753490).

Whereas the routine use of biomarker-based risk scores currently would not substantially add to initial stroke prevention treatment decisions in patients already qualifying for treatment based on the $\mathrm{CHA}_{2} \mathrm{DS}_{2}$-VASc score (and a limited practicality would be accompanied by increased healthcare costs), ${ }^{355,376,377}$ biomarkers could further refine stroke risk differentiation among patients initially classified as low risk and those with a single non-sex $\mathrm{CHA}_{2} \mathrm{DS}_{2}$-VASc risk factor. $^{378}$

Studies of the $\mathrm{CHA}_{2} \mathrm{DS}_{2}$-VASc score report a broad range of stroke rates depending on study setting (community vs. hospital), methodology (e.g. excluding patients subsequently treated with OAC would bias stroke rates towards lower levels), ethnicity, and prevalence of specific stroke risk factors in the study population (different risk factors carry different weight, and age thresholds for initiating NOACs may even differ for patients with a different single non-sex stroke risk factor, as follows: age 35 years for HF, 50 years for hypertension or diabetes, and 55 years for vascular disease). ${ }^{379,380}$ No RCT has specifically addressed the need for OAC in patients with a single non-sex $\mathrm{CHA}_{2} \mathrm{DS}_{2}$-VASc risk factor (to obtain high event rates and timely complete the study, anticoagulation trials have preferentially included high-risk patients), but an overview of subgroup analyses and observational data suggests that OAC use in such patients confers a positive net clinical benefit when balancing the reduction in stroke against the potential for harm with serious bleeding. ${ }^{339,381}$

For many risk factors (e.g. age), stroke risk is a continuum rather than an artificial low-, moderate-, or high-risk category. Risk factors are dynamic and, given the elderly AF population with multiple (often changing) comorbidities, stroke risk needs to be re-evaluated at each clinical review. Recent studies have shown that patients with a change in their risk profile are more likely to sustain strokes. ${ }^{382,383}$ Many initially low-risk patients (>15\%) would have $\geq 1$ non-sex $\mathrm{CHA}_{2} \mathrm{DS}_{2}$ -
VASc risk factor at 1 year after incident $A F,{ }^{384-386}$ and $90 \%$ of new comorbidities were evident at 4.4 months after AF was diagnosed. ${ }^{387}$

A Patient-Centred Outcomes Research Institute (PCORI)-commissioned systematic review of 61 studies compared diagnostic accuracy and impact on clinical decision making of available clinical and imaging tools and associated risk factors for predicting thromboembolic and bleeding risk in AF patients. ${ }^{388}$ The authors concluded that the $\mathrm{CHADS}_{2}$ (CHF history, Hypertension history, Age $\geq 75 \mathrm{y}$, Diabetes mellitus history, Stroke or TIA symptoms previously), $\mathrm{CHA}_{2} \mathrm{DS}_{2}-\mathrm{VASc}$, and $\mathrm{ABC}$ risk scores have the best evidence for predicting thrombo-embolic risk (moderate strength of evidence for limited prediction ability of each score).

\subsubsection{Bleeding risk assessment}

When initiating antithrombotic therapy, potential risk for bleeding also needs to be assessed. Non-modifiable and partially modifiable bleeding risks (Table 9) are important drivers of bleeding events in synergy with modifiable factors. ${ }^{389}$ Notably, a history of falls is not an independent predictor of bleeding on OAC (a modelling study estimated that a patient would need to fall 295 times per year for the benefits of ischaemic stroke reduction with OAC to be outweighed by the potential for serious bleeding). ${ }^{390}$

Modifiable and non-modifiable bleeding risk factors have been used to formulate various bleeding risk scores, ${ }^{368,391-395}$ generally with a modest predictive ability for bleeding events. ${ }^{396,397}$ Studies comparing specific bleeding risk scores provided conflicting findings. ${ }^{393,394,398}$ Various biomarkers have been proposed as bleeding risk predictors, but many have been studied in anticoagulated trial cohorts (while bleeding risk assessment is needed at all parts of the patient pathway - when initially not using OAC, if taking aspirin, and, subsequently, on $\mathrm{OAC}$ ). Additionally, biomarkers are non-specifically predictive of stroke, death, HF, etc. ${ }^{399,400}$ or even non-cardiovascular conditions (e.g. glaucoma), ${ }^{401}$ and the availability of some biomarkers is limited in routine clinical practice.

The biomarker-based ABC-bleeding risk score [Age, Biomarkers (GDF-15, cTnT-hs, haemoglobin) and Clinical history (prior 


\section{Table 8 CHA $_{2}$ DS $_{2}$-VASc score 334}

\begin{tabular}{|c|c|c|c|}
\hline \multicolumn{2}{|c|}{$\begin{array}{l}\mathrm{CHA}_{2} \mathrm{DS}_{2}-\text { VASc score } \\
\text { Risk factors and definitions }\end{array}$} & \multirow{2}{*}{$\begin{array}{l}\text { Points } \\
\text { awarded } \\
1\end{array}$} & \multirow{2}{*}{$\begin{array}{l}\text { Comment } \\
\text { Recent decompensated HF irrespective of LVEF (thus incorporating HFrEF or HFpEF), or the } \\
\text { presence (even if asymptomatic) of moderate-severe LV systolic impairment on cardiac imag- } \\
\text { ing }^{335} \text {; HCM confers a high stroke risk }\end{array}$} \\
\hline C & $\begin{array}{l}\text { Congestive heart failure } \\
\text { Clinical HF, or objective evi- } \\
\text { dence of moderate to severe } \\
\text { LV dysfunction, or HCM }\end{array}$ & & \\
\hline H & $\begin{array}{l}\text { Hypertension } \\
\text { or on antihypertensive therapy }\end{array}$ & 1 & $\begin{array}{l}\text { History of hypertension may result in vascular changes that predispose to stroke, and a well- } \\
\text { controlled BP today may not be well-controlled over time. }{ }^{324} \text { Uncontrolled BP - the optimal BP } \\
\text { target associated with the lowest risk of ischaemic stroke, death, and other cardiovascular out- } \\
\text { comes is } 120-129 /<80 \mathrm{mmHg} .\end{array}$ \\
\hline A & Age 75 years or older & 2 & $\begin{array}{l}\text { Age is a powerful driver of stroke risk, and most population cohorts show that the risk rises } \\
\text { from age } 65 \text { years upwards. }{ }^{339} \text { Age-related risk is a continuum, but for reasons of simplicity and } \\
\text { practicality, } 1 \text { point is given for age } 65 \text { - } 74 \text { years and } 2 \text { points for age } \geq 75 \text { years. }\end{array}$ \\
\hline D & $\begin{array}{l}\text { Diabetes mellitus } \\
\text { Treatment with oral hypogly- } \\
\text { caemic drugs and/or insulin or } \\
\text { fasting blood glucose } \\
>125 \mathrm{mg} / \mathrm{dL}(7 \mathrm{mmol} / \mathrm{L})\end{array}$ & 1 & 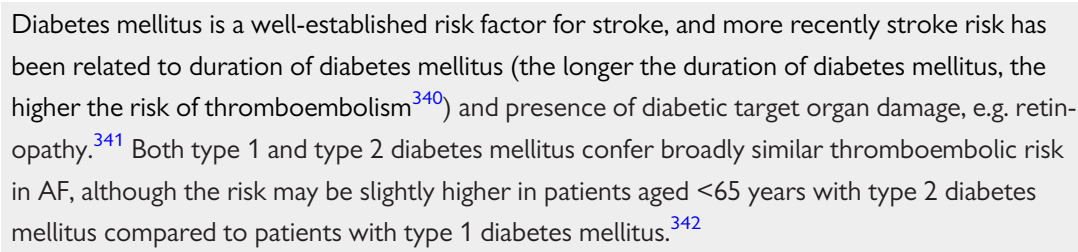 \\
\hline $\mathbf{S}$ & $\begin{array}{l}\text { StrokePrevious stroke, TIA, or } \\
\text { thromboembolism }\end{array}$ & 2 & $\begin{array}{l}\text { Previous stroke, systemic embolism, or TIA confers a particularly high risk of ischaemic stroke, } \\
\text { hence weighted } 2 \text { points. Although excluded from RCTs, AF patients with ICH (including hae- } \\
\text { morrhagic stroke) are at very high risk of subsequent ischaemic stroke, and recent observational } \\
\text { studies suggest that such patients would benefit from oral anticoagulation. }\end{array}$ \\
\hline $\mathbf{V}$ & $\begin{array}{l}\text { Vascular disease } \\
\text { Angiographically significant } \\
\text { CAD, previous myocardial } \\
\text { infarction, PAD, or aortic } \\
\text { plaque }\end{array}$ & 1 & $\begin{array}{l}\text { Vascular disease (PAD or myocardial infarction) confers a } 17-22 \% \text { excess risk, particularly in } \\
\text { Asian patients. }{ }^{346-348} \text { Angiographically significant CAD is also an independent risk factor for } \\
\text { ischaemic stroke among AF patients (adjusted incidence rate ratio } 1.29,95 \% \mathrm{Cl} 1.08-1.53){ }^{349} \\
\text { Complex aortic plaque on the descending aorta, as an indicator of significant vascular disease, is } \\
\text { also a strong predictor of ischaemic stroke. }{ }^{350}\end{array}$ \\
\hline A & Age $65-74$ years & 1 & $\begin{array}{l}\text { See above. Recent data from Asia suggest that the risk of stroke may rise from age } 50-55 \text { years } \\
\text { upwards and that a modified } \mathrm{CHA}_{2} \mathrm{DS}_{2}-\mathrm{VASc} \text { score may be used in Asian patients. }{ }^{351,352}\end{array}$ \\
\hline Sc & Sex category (female) & 1 & A stroke risk modifier rather than a risk factor. ${ }^{353}$ \\
\hline Max & num score & 9 & \\
\hline
\end{tabular}

$\mathrm{AF}=$ atrial fibrillation; $\mathrm{BP}=$ blood pressure; $\mathrm{CAD}=$ coronary artery disease; $\mathrm{CHA}_{2} \mathrm{DS}_{2}-\mathrm{VASc}=$ Congestive heart failure, $\mathrm{Hypertension}$, Age $\geq 75$ years, Diabetes mellitus, Stroke, Vascular disease, Age 65-74 years, Sex category (female); $\mathrm{Cl}=$ confidence interval; $\mathrm{EF}=$ ejection fraction; $\mathrm{HCM}=$ hypertrophic cardiomyopathy; HF = heart failure; $\mathrm{HFpEF}=$ heart failure with preserved ejection fraction; $\mathrm{HFrEF}=$ heart failure with reduced ejection fraction; ICH = intracranial haemorrhage; $L V=$ left ventricular; $L V E F=$ left ventricular ejection fraction; $\mathrm{OAC}=$ oral anticoagulant; $\mathrm{PAD}=$ peripheral artery disease; $\mathrm{RCT}=$ randomized controlled trial; $\mathrm{TIA}=$ transient ischaemic attack.

\section{Table 9 Risk factors for bleeding with OAC and antiplatelet therapy}

\begin{tabular}{|c|c|c|c|}
\hline Non-modifiable & Potentially modifiable & Modifiable & Biomarkers \\
\hline $\begin{array}{l}\text { Age }>65 \text { years } \\
\text { Previous major bleeding } \\
\text { Severe renal impairment (on dialysis or renal } \\
\text { transplant) } \\
\text { Severe hepatic dysfunction (cirrhosis) } \\
\text { Malignancy } \\
\text { Genetic factors (e.g. CYP } 2 \text { C } 9 \text { polymor- } \\
\text { phisms) } \\
\text { Previous stroke, small-vessel disease, etc. } \\
\text { Diabetes mellitus } \\
\text { Cognitive impairment/dementia }\end{array}$ & $\begin{array}{l}\text { Extreme frailty } \pm \text { excessive risk of } \\
\text { falls }^{\mathrm{a}} \\
\text { Anaemia } \\
\text { Reduced platelet count or function } \\
\text { Renal impairment with } \mathrm{CrCl}<60 \\
\mathrm{~mL} / \mathrm{min} \\
\text { VKA management strategy }\end{array}$ & $\begin{array}{l}\text { Hypertension/elevated SBP } \\
\text { Concomitant antiplatelet/NSAID } \\
\text { Excessive alcohol intake } \\
\text { Non-adherence to OAC } \\
\text { Hazardous hobbies/occupations } \\
\text { Bridging therapy with heparin } \\
\text { INR control (target } 2.0-3.0 \text { ), target } \\
\text { TTR }>70 \%^{c} \\
\text { Appropriate choice of OAC and } \\
\text { correct dosing }\end{array}$ & $\begin{array}{l}\text { GDF-15 } \\
\text { Cystatin C/CKD-EPI } \\
\text { cTnT-hs } \\
\text { von Willebrand factor (+ } \\
\text { other coagulation markers) }\end{array}$ \\
\hline
\end{tabular}




\section{Table I0 Clinical risk factors in the HAS-BLED score ${ }^{395}$}

\begin{tabular}{|c|c|c|}
\hline \multicolumn{2}{|c|}{ Risk factors and definitions } & \multirow{2}{*}{$\begin{array}{c}\text { Points awarded } \\
1\end{array}$} \\
\hline $\mathbf{H}$ & $\begin{array}{l}\text { Uncontrolled hypertension } \\
\mathrm{SBP}>160 \mathrm{mmHg}\end{array}$ & \\
\hline A & $\begin{array}{l}\text { Abnormal renal and/or hepatic function } \\
\text { Dialysis, transplant, serum creatinine }>200 \mu \text { mol/L, cirrhosis, bilirubin }>\times 2 \text { upper limit of normal, } \\
\text { AST/ALT/ALP }>3 \times \text { upper limit of normal }\end{array}$ & 1 point for each \\
\hline $\mathbf{S}$ & $\begin{array}{l}\text { Stroke } \\
\text { Previous ischaemic or haemorrhagic }{ }^{a} \text { stroke }\end{array}$ & 1 \\
\hline B & $\begin{array}{l}\text { Bleeding history or predisposition } \\
\text { Previous major haemorrhage or anaemia or severe thrombocytopenia }\end{array}$ & 1 \\
\hline $\mathbf{L}$ & $\begin{array}{l}\text { Labile INR }^{\mathbf{b}} \\
\text { TTR }<60 \% \text { in patient receiving VKA }\end{array}$ & 1 \\
\hline $\mathbf{E}$ & $\begin{array}{l}\text { Elderly } \\
\text { Aged }>65 \text { years or extreme frailty }\end{array}$ & 1 \\
\hline D & $\begin{array}{l}\text { Drugs or excessive alcohol drinking } \\
\text { Concomitant use of antiplatelet or NSAID; and/or excessive }{ }^{c} \text { alcohol per week }\end{array}$ & 1 point for each \\
\hline & & 9 \\
\hline
\end{tabular}

$\mathrm{ALP}=$ alkaline phosphatase; $\mathrm{ALT}=$ alanine aminotransferase; $\mathrm{AST}=$ aspartate aminotransferase; $\mathrm{SBP}=$ systolic blood pressure; INR = international normalized ratio; NSAID = Non-steroidal anti-inflammatory drug; TTR = time in therapeutic range; VKA = vitamin $\mathrm{K}$ antagonist.

a Haemorrhagic stroke would also score 1 point under the 'B' criterion.

${ }^{b}$ Only relevant if patient receiving a VKA.

${ }^{c}$ Alcohol excess or abuse refers to a high intake (e.g. >14 units per week), where the clinician assesses there would be an impact on health or bleeding risk.

bleeding) $]^{375,402}$ reportedly outperformed clinical scores, but in another study there was no long-term advantage of ABC-bleeding over HAS-BLED score (Table 10), whereas HAS-BLED was better in identifying patients at low risk of bleeding (HAS-BLED $0-2) .{ }^{403}$ In the PCORI-commissioned systematic review, ${ }^{388}$ encompassing 38 studies of bleeding risk prediction, the HAS-BLED score had the best evidence for predicting bleeding risk (moderate strength of evidence), consistent with other systematic reviews and meta-analyses comparing bleeding risk prediction approaches. ${ }^{404-406}$

A high bleeding risk score should not lead to withholding OAC, as the net clinical benefit of OAC is even greater amongst such patients. However, the formal assessment of bleeding risk informs management of patients taking OAC, focusing attention on modifiable bleeding risk factors that should be managed and (re)assessed at every patient contact, and identifying high-risk patients with non-modifiable bleeding risk factors who should be reviewed earlier (for instance in 4 weeks rather than 4-6 months) and more frequently. 389,407 Identification of 'high bleeding risk' patients is also needed when determining the antithrombotic strategy in specific AF patient groups, such as those undergoing percutaneous coronary intervention (PCI).

Overall, bleeding risk assessment based solely on modifiable bleeding risk factors is an inferior strategy compared with formal bleeding risk assessment using a bleeding risk score, ${ }^{408-410}$ thus also considering the interaction between modifiable and non-modifiable bleeding risk factors. Bleeding risk is dynamic, and attention to the change in bleeding risk profile is a stronger predictor of major bleeding events compared with simply relying on baseline bleeding risk. In a recent study, there was a 3.5-fold higher risk of major bleeding in the first 3 months amongst patients who had a change in their bleeding risk profile. $^{389}$
In the mAFA-II trial, prospective dynamic monitoring and reassessment using the HAS-BLED score (together with holistic App-based management) was associated with fewer major bleeding events, mitigated modifiable bleeding risk factors, and increased OAC uptake; in contrast, bleeding rates were higher and OAC use overall decreased by $25 \%$ in the 'usual care' arm when comparing baseline with 12 months. ${ }^{411}$

\subsubsection{Absolute contraindications to oral anticoagulants} The few absolute contraindications to OAC include active serious bleeding (where the source should be identified and treated), associated comorbidities (e.g. severe thrombocytopenia $<50$ platelets $/ \mu \mathrm{L}$, severe anaemia under investigation, etc.), or a recent high-risk bleeding event such as intracranial haemorrhage $(\mathrm{ICH})$. Non-drug options may be considered in such cases (section 11.4.3).

\subsubsection{Stroke prevention therapies}

\subsubsection{Vitamin K antagonists}

Compared with control or placebo, vitamin K antagonist (VKA) therapy (mostly warfarin) reduces stroke risk by $64 \%$ and mortality by $26 \%,{ }^{412}$ and is still used in many AF patients worldwide. VKAs are currently the only treatment with established safety in AF patients with rheumatic mitral valve disease and/or an artificial heart valve.

The use of VKAs is limited by the narrow therapeutic interval, necessitating frequent international normalized ratio (INR) monitoring and dose adjustments. ${ }^{413}$ At adequate time in therapeutic range [(TTR) $\left.>70 \%\right]$, VKAs are effective and relatively safe drugs. Quality of VKA management (quantified using the TTR based on the Rosendaal method, or the percentage of INRs in range) correlates with haemorrhagic and thrombo- 
embolic rates. ${ }^{414}$ At high TTR values, the efficacy of VKAs in stroke prevention may be similar to NOACs, whereas the relative safety benefit with NOACs is less affected by TTR, with consistently lower serious bleeding rates (e.g. $\mathrm{ICH}$ ) seen with NOACs compared with warfarin, notwithstanding that the absolute difference is small. ${ }^{415,416}$

Numerous factors (including genetics, concomitant drugs, etc.) influence the intensity of VKA anticoagulant effect; the more common ones have been used to derive and validate the SAMe- $T_{2} R_{2}$ \{Sex [female], Age [ $<60$ years], Medical history of $\geq 2$ comorbidities [hypertension, diabetes mellitus, $C A D / m y o c a r d i a l$ infarction, peripheral artery disease (PAD), HF, previous stroke, pulmonary disease, and hepatic or renal disease], Treatment [interacting drugs, e.g. amiodarone], Tobacco use, Race [non-Caucasian]\} score, ${ }^{417}$ which can help to identify patients who are less likely to achieve a good TTR on VKA therapy (score >2) and would do better with a NOAC. If such patients with SAMe$\Pi_{2} R_{2}>2$ are prescribed a VKA, greater efforts to improve $T T R$, such as more intense regular reviews, education/counselling, and frequent INR monitoring are needed or, more conveniently, the use of a NOAC should be reconsidered. ${ }^{418}$

\subsubsection{Non-vitamin K antagonist oral anticoagulants}

In four pivotal RCTs, apixaban, dabigatran, edoxaban, and rivaroxaban have shown non-inferiority to warfarin in the prevention of stroke/systemic embolism. ${ }^{419-422}$ In a meta-analysis of these RCTs, NOACs were associated with a $19 \%$ significant stroke/systemic embolism risk reduction, a $51 \%$ reduction in haemorrhagic stroke, ${ }^{423}$ and similar ischaemic stroke risk reduction compared with VKAs, but NOACs were associated with a significant $10 \%$ reduction in all-cause mortality (Supplementary Table 8 ). There was a non-significant $14 \%$ reduction in major bleeding risk, significant $52 \%$ reduction in $\mathrm{ICH}$, and $25 \%$ increase in gastrointestinal bleeding with NOACs vs. warfarin. ${ }^{423}$

The major bleeding relative risk reduction with NOACs was significantly greater when INR control was poor (i.e. centre-based TTR $<66 \%$ ). A meta-analysis of the five NOAC trials [RE-LY (Randomized Evaluation of Long Term Anticoagulant Therapy), ROCKET-AF (Rivaroxaban Once Daily Oral Direct Factor Xa Inhibition Compared with Vitamin K Antagonism for Prevention of Stroke and Embolism Trial in Atrial Fibrillation), J-ROCKET AF, ARISTOTLE (Apixaban for Reduction in Stroke and Other Thromboembolic Events in Atrial Fibrillation), and ENGAGE AF TIMI 48 (Effective Anticoagulation with Factor Xa Next Generation in Atrial
Fibrillation-Thrombolysis in Myocardial Infarction 48)] showed that, compared with warfarin, standard-dose NOACs were more effective and safer in Asians than in non-Asians. ${ }^{424}$ In the AVERROES [Apixaban Versus Acetylsalicylic Acid (ASA) to Prevent Stroke in Atrial Fibrillation Patients Who Have Failed or Are Unsuitable for Vitamin K Antagonist Treatment] trial of AF patients who refused or were deemed ineligible for VKA therapy, apixaban $5 \mathrm{mg}$ b.i.d. (twice a day) significantly reduced the risk of stroke/systemic embolism with no significant difference in major bleeding or ICH compared with aspirin. ${ }^{425}$

Post-marketing observational data on the effectiveness and safety of dabigatran, ${ }^{426,427}$ rivaroxaban, ${ }^{428,429}$ apixaban, ${ }^{430}$ and edoxaban ${ }^{431}$ vs. warfarin show general consistency with the respective RCT. Given the compelling evidence about NOACs, AF patients should be informed of this treatment option.

Persistence to NOAC therapy is generally higher than to VKAs, being facilitated by a better pharmacokinetic profile of NOACs ${ }^{432}$ (Supplementary Table 9) and favourable safety and efficacy, especially amongst vulnerable patients including the elderly, those with renal dysfunction or previous stroke, and so on. ${ }^{433}$ Whereas patients with end-stage renal dysfunction were excluded from the pivotal RCTs, reduced dose regimens of rivaroxaban, edoxaban, and apixaban are feasible options for severe CKD [creatinine clearance $(\mathrm{CrCl})$ 15-30 $\mathrm{mL} / \mathrm{min}$ using the Cockcroft-Gault equation]. ${ }^{434,435}$ Considering that inappropriate dose reductions are frequent in clinical practice, ${ }^{436}$ thus increasing the risks of stroke/systemic embolism, hospitalization, and death, but without decreasing bleeding risk, ${ }^{437}$ NOAC therapy should be optimized based on the efficacy and safety profile of each NOAC in different patient subgroups (Table 11).

\subsubsection{Other antithrombotic drugs}

In the ACTIVE W (Atrial Fibrillation Clopidogrel Trial with Irbesartan for Prevention of Vascular Events) trial, dual antiplatelet therapy (DAPT) with aspirin and clopidogrel was less effective than warfarin for prevention of stroke, systemic embolism, myocardial infarction, and vascular death (the annual risk of events was $5.6 \%$ vs. $3.9 \%$, $P=0.0003$ ), with a similar rate of major bleeding. ${ }^{438}$ In the ACTIVE-A trial, patients unsuitable for anticoagulation had a lower rate of thrombo-embolic complications when clopidogrel was added to aspirin compared with aspirin alone, but with a significant increase in major bleeding. ${ }^{439}$ Aspirin monotherapy was ineffective for stroke prevention compared with no antithrombotic treatment and was

\section{Table I I Dose selection criteria for NOACs}

\begin{tabular}{|c|c|c|c|c|}
\hline & Dabigatran & Rivaroxaban & Apixaban & Edoxaban \\
\hline Standard dose & 150 mg b.i.d. & 20 mg o.d. & 5 mg b.i.d. & 60 mg o.d. \\
\hline Lower dose & 110 mg b.i.d. & & & \\
\hline Reduced dose & & 15 mg o.d. & $2.5 \mathrm{mg}$ b.i.d. & 30 mg o.d. \\
\hline $\begin{array}{l}\text { Dose-reduction } \\
\text { criteria }\end{array}$ & $\begin{array}{l}\text { Dabigatran } 110 \text { mg b.i.d. in patients with: } \\
\text { - Age } \geq 80 \text { years } \\
\text { - Concomitant use of verapamil, or } \\
\text { - Increased bleeding risk }\end{array}$ & $\mathrm{CrCl} 15-49 \mathrm{~mL} / \mathrm{min}$ & $\begin{array}{l}\text { At least } 2 \text { of } 3 \text { criteria: } \\
\text { - Age } \geq 80 \text { years, } \\
\text { - } \text { Body weight } \leq 60 \mathrm{~kg} \text {, or } \\
\text { - Serum creatinine } \\
\geq 1.5 \mathrm{mg} / \mathrm{dL}(133 \mu \mathrm{mol} / \mathrm{L})\end{array}$ & $\begin{array}{l}\text { If any of the following: } \\
\text { - } \mathrm{CrCl} 15-50 \mathrm{~mL} / \mathrm{min} \text {, } \\
\text { - Body weight } \leq 60 \mathrm{~kg} \text {, } \\
\text { - Concomitant use of dronedarone, } \\
\text { ciclosporine, erythromycin, or } \\
\text { ketoconazole }\end{array}$ \\
\hline
\end{tabular}


associated with a higher risk of ischaemic stroke in elderly patients. $^{440}$

Overall, antiplatelet monotherapy is ineffective for stroke prevention and is potentially harmful, (especially amongst elderly AF patients), ${ }^{441,442}$ whereas DAPT is associated with a bleeding risk similar to OAC therapy. Hence, antiplatelet therapy should not be used for stroke prevention in AF patients.

\subsubsection{Combination therapy with oral anticoagulant and antiplatelet} drugs

The use of antiplatelet therapy remains common in clinical practice, often in patients without an indication (e.g. PAD, CAD, or cerebrovascular disease) beyond $A F .{ }^{443}$ There is limited evidence to support the combination therapy solely for stroke prevention in AF, with no effect on reductions in stroke, myocardial infarction, or death, but with a substantial increase in the risk of major bleeding and $\mathrm{ICH}^{441,442}$

\subsubsection{Left atrial appendage occlusion and exclusion}

10.1.4.5.1 Left atrial appendage occlusion devices. Only the Watchman device has been compared with VKA therapy in RCTs [the PROTECT AF (WATCHMAN Left Atrial Appendage System for Embolic Protection in Patients With Atrial Fibrillation) and PREVAIL (Watchman LAA Closure Device in Patients With Atrial Fibrillation Versus Long Term Warfarin Therapy)], ${ }^{44-446}$ where LAA occlusion was non-inferior to VKA stroke prevention treatment in AF patients with moderate stroke risk, with a possibility of lower bleeding rates on longer follow-up. ${ }^{447}$ The LAA occlusion may also reduce stroke risk in patients with contraindications to $O A C .{ }^{448,449}$

A large European registry reported a high implantation success rate $(98 \%)$, with an acceptable procedure-related complication rate of $4 \%$ at 30 days. ${ }^{450}$ Nevertheless, the implantation procedure can cause serious complications (higher event rates have been reported in real-world analyses compared with industry-sponsored studies, possibly identifying some reporting bias) and device-related thrombosis may not be a benign finding. ${ }^{451-454}$ Antithrombotic management after LAA occlusion has never been evaluated in a randomized manner and is based on historical studies, at least including aspirin (Table 12). For patients who do not tolerate any antiplatelet therapy, either an epicardial catheter approach (e.g. Lariat system) or thoracoscopic clipping of the LAA may be an option. ${ }^{455,456}$

Notably, the non-inferiority of LAA occlusion to VKA treatment was mostly driven by the prevention of haemorrhagic stroke, with a trend for more ischaemic strokes. The limitations of LAA occlusion as a strategy to reduce the risk of stroke associated with AF also include the consideration that $\mathrm{AF}$ acts as a risk marker of stroke. Withholding OAC after LAA occlusion is likely to result in undertreating the overall risk of stroke related to atrial cardiomyopathy.

10.1.4.5.2 Surgical left atrial appendage occlusion or exclusion. Multiple observational studies indicate the feasibility and safety of surgical LAA occlusion/exclusion, but only limited controlled trial data are available. $^{457-459}$ Residual LAA flow or incomplete LAA occlusion may be associated with an increased risk of stroke. ${ }^{460}$ In most studies, LAA occlusion/exclusion was performed during other open heart surgery, and in more recent years in combination with surgical ablation of $\mathrm{AF}^{459,461}$ or as an isolated thoracoscopic procedure. A large RCT in patients with an associated cardiac surgical procedure is ongoing. ${ }^{462}$

The most common justification for LAA occlusion/exclusion in clinical practice is a perceived high bleeding risk or, less often, contraindications for OAC. ${ }^{450}$ However, LAA occluders have not been randomly tested in such populations. Most patients who some years ago would be considered unsuitable for OAC therapy with VKA now seem to do relatively well on NOAC, ${ }^{433,463,464}$ and LAA occluders have not been compared with NOAC therapy in patients at risk for bleeding, or with surgical LAA occlusion/exclusion. Long-term aspirin is a common strategy in these patients, ${ }^{465}$ and one may question whether a NOAC would not be a better strategy if aspirin is tolerated. There is the need for adequately powered trials to define the best indications of LAA occlusion/exclusion compared with NOAC therapy in patients with relative or absolute contraindications for anticoagulation, in those suffering from an ischaemic stroke on anticoagulant therapy, and for assessment of the appropriate antithrombotic therapy after LAA occlusion.

\section{Table 12 Antithrombotic therapy after left atrial appendage occlusion}

\begin{tabular}{|c|c|c|c|c|}
\hline Device/patient & Aspirin & OAC & Clopidogrel & Comments \\
\hline $\begin{array}{l}\text { Watchman/low } \\
\text { bleeding risk }\end{array}$ & $\begin{array}{l}75-325 \mathrm{mg} / \text { day } \\
\text { indefinitely }\end{array}$ & 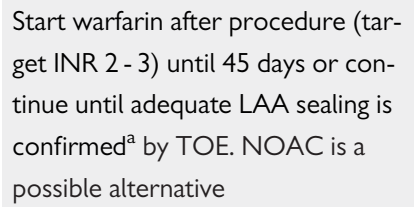 & $\begin{array}{l}\text { Start } 75 \mathrm{mg} / \text { day when } \mathrm{OAC} \\
\text { stopped, continue until } 6 \text { months } \\
\text { after the procedure }\end{array}$ & $\begin{array}{l}\text { Some centres do not withhold } \\
\text { OAC at the time of procedure (no } \\
\text { data to support/deny this } \\
\text { approach) }\end{array}$ \\
\hline $\begin{array}{l}\text { Watchman/high } \\
\text { bleeding risk }\end{array}$ & $\begin{array}{l}75-325 \mathrm{mg} / \text { day } \\
\text { indefinitely }\end{array}$ & None & $\begin{array}{l}75 \mathrm{mg} / \text { day for } 1 \text { - } 6 \text { months while } \\
\text { ensuring adequate LAA sealing }\end{array}$ & $\begin{array}{l}\text { Clopidogrel often given for shorter } \\
\text { time in very high-risk situations }\end{array}$ \\
\hline ACP/Amulet & $\begin{array}{l}75-325 \mathrm{mg} / \text { day } \\
\text { indefinitely }\end{array}$ & None & $\begin{array}{l}75 \mathrm{mg} / \text { day for } 1 \text { - } 6 \text { months while } \\
\text { ensuring adequate LAA sealing }\end{array}$ & $\begin{array}{l}\text { Clopidogrel may replace long-term } \\
\text { aspirin if better tolerated }\end{array}$ \\
\hline
\end{tabular}

$\mathrm{ACP}=$ Amplatzer $^{\mathrm{TM}}$ Cardiac Plug; INR = international normalized ratio; LAA = left atrial appendage; LMWH = low-molecular-weight heparin; NOAC = non-vitamin K antagonist oral anticoagulant; $\mathrm{OAC}=$ oral anticoagulant $; \mathrm{TOE}=$ transoesophageal echocardiography.

Note: Load aspirin or clopidogrel before procedure if untreated. Heparin with activated clotting time $>250$ seconds before or immediately after trans-septal punctures for all patients, followed by LMWH when warfarin needed.

a Less than $5 \mathrm{~mm}$ leak. 
10.1.4.6 Long-term oral anticoagulation per atrial fibrillation burden Although the risk of ischaemic stroke/systemic embolism is higher with non-paroxysmal vs. paroxysmal AF, and AF progression is associated with an excess of adverse outcomes, ${ }^{169,466}$ the clinically determined temporal pattern of AF should not affect the decision regarding long-term $\mathrm{OAC}$, which is driven by the presence of stroke risk factors. ${ }^{156}$ Management of patients with AHRE/subclinical AF is reviewed in section 16. Stroke risk in AHRE patients may be lower than in patients with diagnosed $A F,{ }^{467}$ and strokes often occur without a clear temporal relationship with $A H R E / s u b c l i n i c a l ~ A F,{ }^{179,226}$ underscoring its role as a risk marker rather than a stroke risk factor. ${ }^{4,172}$ Whether AHRE and subclinical AF have the same therapeutic requirements as clinical $A F^{7}$ is presently unclear, and the net clinical benefit of OAC for AHRE/subclinical AF>24 h is currently being studied in several RCTs. ${ }^{4}$

Notably, patients with subclinical AF/AHRE may develop atrial tachyarrhythmias lasting more than $24 h^{468}$ or clinical AF; hence careful monitoring of these patients is recommended, even considering remote monitoring, especially with longer AHRE and higher risk profile. ${ }^{469}$ Given the dynamic nature of AF as well as stroke risk, a recorded duration in one monitoring period would not necessarily be the same in the next.

\subsubsection{Long-term oral anticoagulation per symptom control strategy} Symptom control focuses on patient-centred and symptom-directed approaches to rate or rhythm control. Again, symptom control strategy should not affect the decision regarding long-term OAC, which is driven by the presence of stroke risk factors, and not the estimated success in maintaining sinus rhythm.

\subsubsection{Management of anticoagulation-related bleeding risk}

10.1.5.1 Strategies to minimize the risk of bleeding

Ensuring good quality of VKA treatment (TTR $>70 \%$ ) and selecting the appropriate dose of a NOAC (as per the dose reduction criteria specified on the respective drug label) are important considerations to minimize bleeding risk. As discussed in section 10.1.2, attention to modifiable bleeding risk factors should be made at every patient contact, and formal bleeding risk assessment is needed to help identify high-risk patients who should be followed up or reviewed earlier (e.g. 4 weeks rather than 4 - 6 months). ${ }^{407}$ Concomitant regular administration of antiplatelet drugs or non-steroidal anti-inflammatory drug (NSAID) should be avoided in anticoagulated patients. Bleeding risk is dynamic, and attention to the change in bleeding risk profile is a stronger predictor of major bleeding events, especially in the first 3 months. ${ }^{389}$

\subsubsection{High-risk groups}

Certain high-risk AF populations have been under-represented in RCTs, including the extreme elderly ( $\geq 90$ years), those with cognitive impairment/dementia, recent bleeding or previous $\mathrm{ICH}$, end-stage renal failure, liver impairment, cancer, and so on. Observational data suggest that such patients are at high risk for ischaemic stroke and death, and many would benefit from OAC.

Patients with liver function abnormalities may be at higher risk of bleeding on VKA, possibly less so on NOACs. Observational data in cirrhotic patients suggest that ischaemic stroke reduction may outweigh bleeding risk. ${ }^{470-472}$

In patients with a recent bleeding event, attention should be directed towards addressing the predisposing pathology (e.g. bleeding ulcer or polyp in a patient with gastrointestinal bleeding), and the reintroduction of OAC as soon as feasible, as part of a multidisciplinary team decision. Consideration should be made for drugs such as apixaban or dabigatran $110 \mathrm{mg}$ b.i.d., which are not associated with an excess of gastrointestinal bleeding compared with warfarin. Where OAC is not reintroduced, there is a higher risk of stroke and death compared with restarting OAC, although the risk of rebleeding may be higher. ${ }^{473}$ Similarly, thromboprophylaxis in cancer may require a multidisciplinary team decision balancing stroke reduction against serious bleeding, which may be dependent on cancer type, site(s), staging, anti-cancer therapy and so on.

Thromboprophylaxis in specific high-risk groups is discussed in detail throughout section 11 .

\subsubsection{Decision making to avoid stroke}

In observational population cohorts, both stroke and death are relevant endpoints, as some deaths could be due to fatal strokes (given that endpoints are not adjudicated in population cohorts, and cerebral imaging or post-mortems are not mandated). As OAC significantly reduces stroke (by 64\%) and all-cause mortality (by 26\%) compared with control or placebo, ${ }^{412}$ the endpoints of stroke and/or mortality are relevant in relation to decision making for thromboprophylaxis.

The threshold for initiating OAC for stroke prevention, balancing ischaemic stroke reduction against the risk of $\mathrm{ICH}$ and associated QoL, has been estimated to be $1.7 \%$ /year for warfarin and $0.9 \% / y e a r$ for a NOAC (dabigatran data were used for the modelling analysis). ${ }^{474}$ The threshold for warfarin may be even lower, if good-quality anticoagulation control is achieved, with average TTR $>70 \%$. ${ }^{475}$

Given the limitations of clinical risk scores, the dynamic nature of stroke risk, the greater risk of stroke and death among AF patients with $\geq 1$ non-sex stroke risk factor, and the positive net clinical benefit of OAC among such patients, we recommend a risk-factor-based approach to stroke prevention rather than undue focus on (artificially defined) 'high-risk' patients. As the default is to offer stroke prevention unless the patient is low risk, the $\mathrm{CHA}_{2} \mathrm{DS}_{2^{-}}$ VASc score should be applied in a reductionist manner, to decide on OAC or not. ${ }^{476}$

Thus, the first step in decision making ('A' Anticoagulation/Avoid stroke) is to identify low-risk patients who do not need antithrombotic therapy. Step 2 is to offer stroke prevention (i.e. OAC) to those with $\geq 1$ non-sex stroke risk factors (the strength of evidence differs, with multiple clinical trials for patients with $\geq 2$ stroke risk factors, and subgroups from trials/observational data on patients with 1 nonsex stroke risk factor). Step 3 is the choice of OAC - a NOAC (given their relative effectiveness, safety and convenience, these drugs are generally first choice as OAC for stroke prevention in AF) or VKA (with good TTR at $>70 \%$ ). This 'AF 3-step' patient pathway ${ }^{182,477}$ for stroke risk stratification and treatment decision making is shown in Figure 12. 


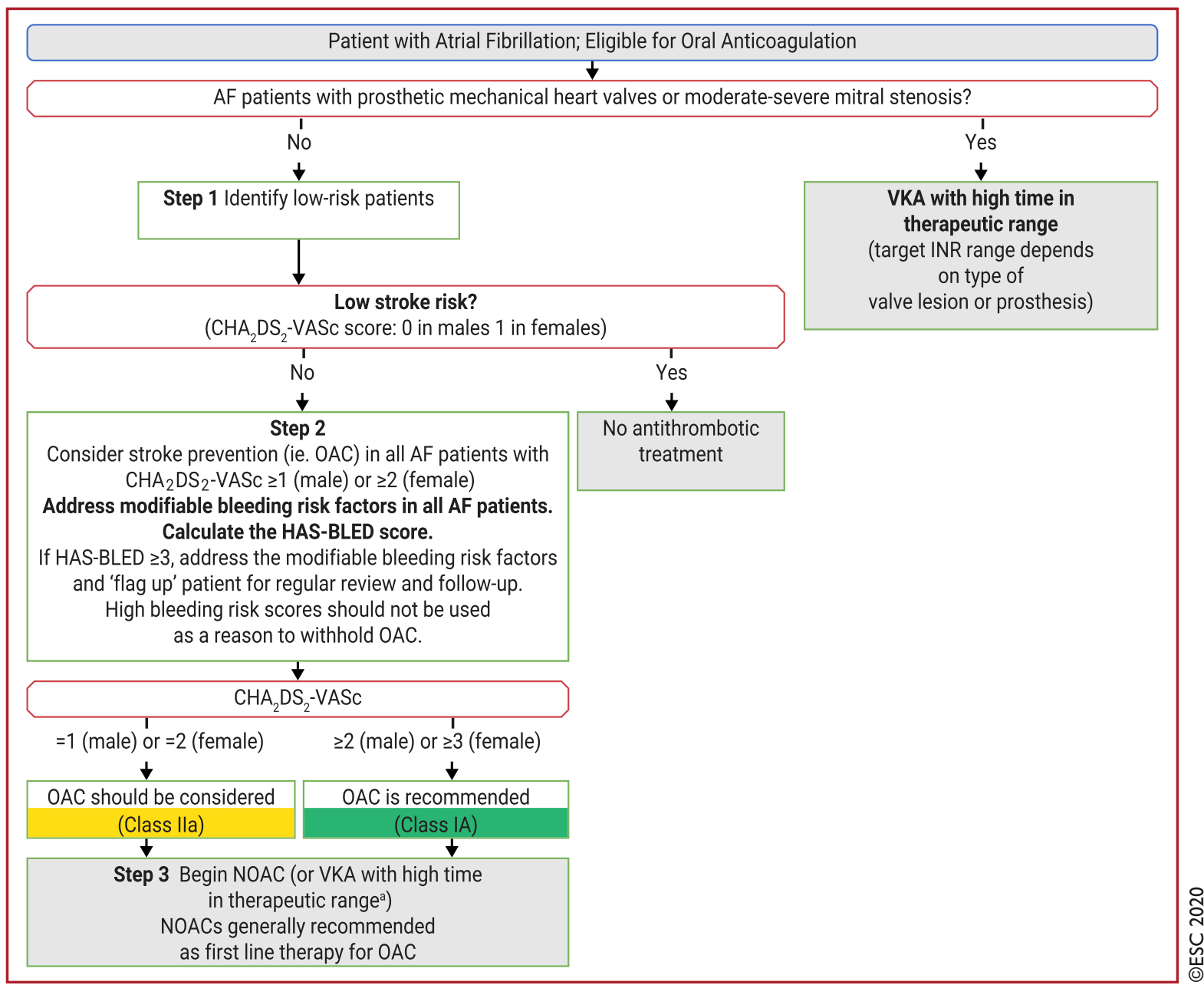

Figure 12 'A' - Anticoagulation/Avoid stroke: The 'AF 3-step' pathway. AF = atrial fibrillation; $\mathrm{CHA}_{2} \mathrm{DS}_{2}$-VASc = Congestive heart failure, Hypertension, Age $\geq 75$ years, Diabetes mellitus, Stroke, Vascular disease, Age 65 - 74 years, Sex category (female); HAS-BLED = Hypertension, Abnormal renal/liver function, Stroke, Bleeding history or predisposition, Labile INR, Elderly (>65 years), Drugs/alcohol concomitantly; INR = international normalized ratio; $\mathrm{NOAC}=$ non-vitamin $\mathrm{K}$ antagonist oral anticoagulant; $\mathrm{OAC}=$ oral anticoagulant; $\mathrm{SAMe}-\mathrm{TT}_{2} \mathrm{R}_{2}=\mathrm{Sex}$ (female), Age (<60 years), Medical history, Treatment (interacting drug(s)), Tobacco use, Race (non-Caucasian) (score); TTR = time in therapeutic range; VKA = vitamin $\mathrm{K}$ antagonist.

${ }^{a}$ If a VKA being considered, calculate SAMe- $\mathrm{TT}_{2} \mathrm{R}_{2}$ score: if score 0-2, may consider VKA treatment (e.g. warfarin) or NOAC; if score $>2$, should arrange regular review/frequent INR checks/ counselling for VKA users to help good anticoagulation control, or reconsider the use of NOAC instead; TTR ideally $>70 \%$.

\section{Recommendations for the prevention of thrombo-embolic events in AF}

\section{Recommendations}

For stroke prevention in AF patients who are eligible for OAC, NOACs are recommended in preference to VKAs (excluding patients with mechanical heart valves or moderate-to-severe mitral stenosis). ${ }^{423,424}$

For stroke risk assessment, a risk-factor-based approach is recommended, using the $\mathrm{CHA}_{2} \mathrm{DS}_{2}-\mathrm{VASc}$ clinical stroke risk score to initially identify patients at 'low stroke risk' $\left(\mathrm{CHA}_{2} \mathrm{DS}_{2}\right.$-VASc score $=0$ in men, or 1 in women) who should not be offered antithrombotic therapy. ${ }^{334,388}$

$\mathrm{OAC}$ is recommended for stroke prevention in AF patients with $\mathrm{CHA}_{2} \mathrm{DS}_{2}-\mathrm{VASc}$ score $\geq 2$ in men or $\geq 3$ in women. ${ }^{412}$

$\mathrm{OAC}$ should be considered for stroke prevention in AF patients with a $\mathrm{CHA}_{2} \mathrm{DS}_{2}-\mathrm{VASc}$ score of 1 in men or 2 in women. Treatment should be individualized based on net clinical benefit and consideration of patient values and preferences. ${ }^{338,378,380}$ For bleeding risk assessment, a formal structured risk-score-based bleeding risk assessment is recommended to help identify nonmodifiable and address modifiable bleeding risk factors in all AF patients, and to identify patients potentially at high risk of bleeding who should be scheduled for early and more frequent clinical review and follow-up. ${ }^{388,395,404,406}$

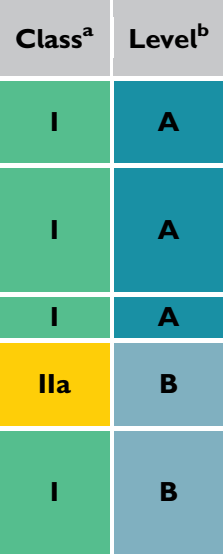

Continued 
For a formal risk-score-based assessment of bleeding risk, the HAS-BLED score should be considered to help address modifiable bleeding risk factors, and to identify patients at high risk of bleeding (HAS-BLED score $\geq 3$ ) for early and more frequent clinical review and follow-up. $388,395,404,406$

Stroke and bleeding risk reassessment at periodic intervals is recommended to inform treatment decisions (e.g. initiation of OAC in patients no longer at low risk of stroke) and address potentially modifiable bleeding risk factors. ${ }^{\text {c389,478,479 }}$

In patients with AF initially at low risk of stroke, first reassessment of stroke risk should be made at $4-6$ months after the index evaluation. $^{385-387}$

If a VKA is used, a target INR of $2.0-3.0$ is recommended, with individual TTR $\geq 70 \%{ }^{414}$

In patients on VKAs with low time in INR therapeutic range (e.g. TTR $<70 \%)$, recommended options are:

- Switching to a NOAC but ensuring good adherence and persistence with therapy ${ }^{415,416}$; or

- Efforts to improve TTR (e.g. education/counselling and more frequent INR checks). ${ }^{480}$

Antiplatelet therapy alone (monotherapy or aspirin in combination with clopidogrel) is not recommended for stroke prevention in $A F .440,441,480,481$

Estimated bleeding risk, in the absence of absolute contraindications to OAC, should not in itself guide treatment decisions to use OAC for stroke prevention.

Clinical pattern of AF (i.e. first detected, paroxysmal, persistent, long-standing persistent, permanent) should not condition the indication to thromboprophylaxis. ${ }^{160}$

\section{Recommendations for occlusion or exclusion of the LAA}

LAA occlusion may be considered for stroke prevention in patients with $\mathrm{AF}$ and contraindications for long-term anticoagulant treatment (e.g. intracranial bleeding without a reversible cause). ${ }^{448,449,481,482}$

Surgical occlusion or exclusion of the LAA may be considered for stroke prevention in patients with AF undergoing cardiac surgery. ${ }^{459,483}$

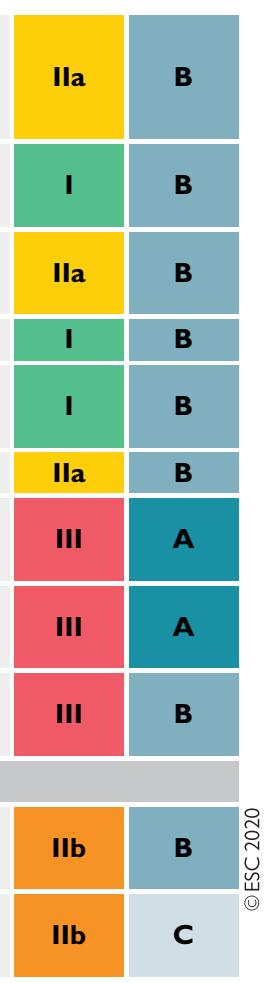

$\mathrm{AF}=$ atrial fibrillation; $\mathrm{BP}=$ blood pressure; $\mathrm{CHA}_{2} \mathrm{DS}_{2}-\mathrm{VASc}=$ Congestive heart failure, Hypertension, Age $\geq 75$ years, Diabetes mellitus, Stroke, Vascular disease, Age $65-74$ years, Sex category (female); HAS-BLED = Hypertension, Abnormal renal/liver function, Stroke, Bleeding history or predisposition, Labile INR, Elderly (>65 years), Drugs/alcohol concomitantly; INR = international normalized ratio; LAA = left atrial appendage; NOAC = non-vitamin K antagonist oral anticoagulant; NSAID = non-steroidal anti-inflammatory drug; $\mathrm{OAC}=$ oral anticoagulant $; \mathrm{TTR}=$ time in therapeutic range; $\mathrm{VKA}=$ vitamin $\mathrm{K}$ antagonist.

${ }^{\mathrm{a} C}$ Class of recommendation.

bLevel of evidence.

'Including uncontrolled BP; labile INRs (in a patient taking VKA); alcohol excess; concomitant use of NSAIDs or aspirin in an anticoagulated patient; bleeding tendency or predisposition (e.g. treat gastric ulcer, optimize renal or liver function, etc.).

\section{2 'B' - Better symptom control}

\subsubsection{Rate control}

Rate control is an integral part of AF management, and is often sufficient to improve AF-related symptoms. Very little robust evidence exists to inform the best type and intensity of rate control treatment. ${ }^{484-486}$

\subsubsection{Target/optimal ventricular rate range}

The optimal heart-rate target in AF patients is unclear. In the RACE (Race Control Efficacy in Permanent Atrial Fibrillation) II RCT of permanent AF patients, there was no difference in a composite of clinical events, New York Heart Association (NYHA) class, or hospitalizations between the strict [target heart rate $<80$ beats per minute (bpm) at rest and $<110 \mathrm{bpm}$ during moderate exercise] and lenient (heart-rate target $<110 \mathrm{bpm}$ ) arm, ${ }^{487,488}$ similar to an analysis from the AFFIRM (Atrial Fibrillation Follow-up Investigation of Rhythm Management) and RACE trials. ${ }^{489}$ Therefore, lenient rate control is an acceptable initial approach, regardless of HF status (with the exception of tachycardia-induced cardiomyopathy), unless symptoms call for stricter rate control (Figure 13).

\subsubsection{Drugs}

Pharmacological rate control can be achieved with beta-blockers, digoxin, diltiazem, and verapamil, or combination therapy (Table 13).
Some antiarrhythmic drugs (AADs) also have rate-limiting properties (e.g. amiodarone, dronedarone, sotalol) but generally they should be used only for rhythm control. The choice of rate control drugs depends on symptoms, comorbidities, and potential side-effects (Table 13).

Beta-blockers are often first-line rate-controlling agents, largely based on better acute rate control. Interestingly, the prognostic benefit of beta-blockers seen in HF with reduced ejection fraction (HFrEF) patients with sinus rhythm has been questioned in patients with AF. ${ }^{491}$

Non-dihydropyridine calcium channel blockers (NDCC) verapamil and diltiazem provide reasonable rate control ${ }^{492}$ and can improve AF-related symptoms ${ }^{486}$ compared with betablockers. In one small trial of patients with preserved LVEF, NDCC preserved exercise capacity and reduced B-type natriuretic peptide. $^{493,494}$

Digoxin and digitoxin are not effective in patients with increased sympathetic drive. Observational studies have associated digoxin use with excess mortality in AF patients. ${ }^{495-497}$ This finding was likely due to selection and prescription biases rather than harm caused by digoxin, ${ }^{498-501}$ particularly as digoxin is commonly prescribed to sicker patients. ${ }^{502}$ Lower doses of digoxin may be associated with 


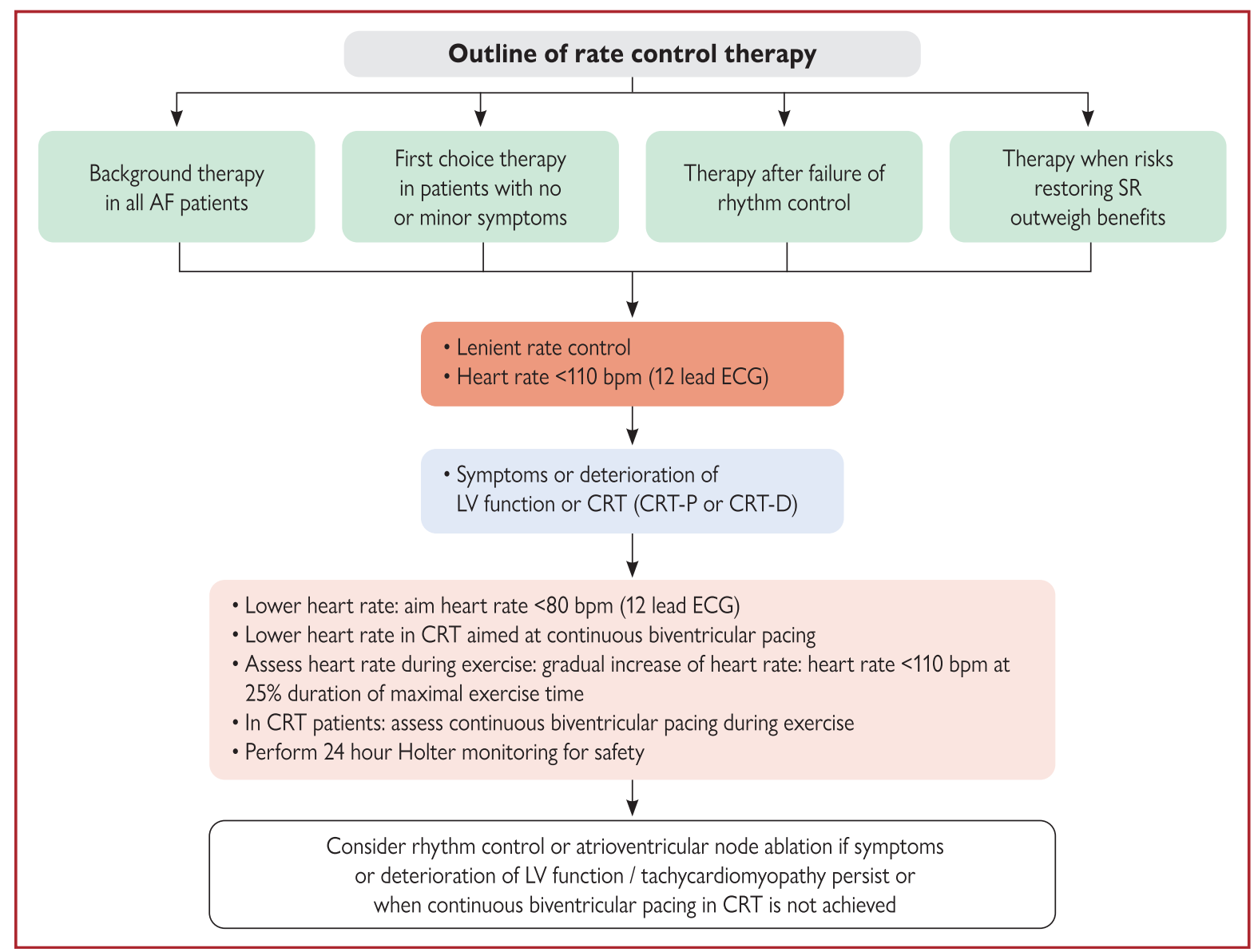

Figure I 3 Outline of rate control therapy. ${ }^{490} \mathrm{AF}=$ atrial fibrillation; $\mathrm{AVN}=$ atrioventricular node; $\mathrm{bpm}=$ beats per minute; $\mathrm{BV}=$ biventricular; $\mathrm{CRT}=$ cardiac resynchronization therapy; CRT-D: cardiac resynchronization therapy defibrillator; CRT-P = cardiac resynchronization therapy pacemaker; ECG = electrocardiogram; $\mathrm{LV}=$ left ventricular; $\mathrm{SR}=$ sinus rhythm.

better prognosis. ${ }^{502}$ An ongoing RCT is addressing digitoxin use in patients with $\mathrm{HFrEF}^{503}$

Amiodarone can be useful as a last resort when heart rate cannot be controlled with combination therapy in patients who do not qualify for non-pharmacological rate control, i.e. atrioventricular node ablation and pacing, notwithstanding the extracardiac adverse effects of the drug ${ }^{504}$ (Table 13).

\subsubsection{Acute rate control}

In acute settings, physicians should always evaluate underlying causes, such as infection or anaemia. Beta-blockers and diltiazem/verapamil are preferred over digoxin because of their rapid onset of action and effectiveness at high sympathetic tone. ${ }^{507-511}$ The choice of drug (Table 13 and Figure 14) and target heart rate will depend on the patient characteristics, symptoms, LVEF value, and haemodynamics, but a lenient initial rate control approach seems acceptable (Figure 13). Combination therapy may be required. In patients with HFrEF, beta-blockers, digitalis, or their combination should be used. $^{512,513}$ In critically ill patients and those with severely impaired LV systolic function, i.v. amiodarone can be used. ${ }^{504,514,515}$ In unstable patients, urgent cardioversion should be considered (section 11.1).
10.2.1.4 Atrioventricular node ablation and pacing

Ablation of the atrioventricular node and pacemaker implantation can control ventricular rate when medication fails. The procedure is relatively simple and has a low complication rate and low long-term mortality risk, ${ }^{516,517}$ especially when the pacemaker is implanted a few weeks before the atrioventricular node ablation and the initial pacing rate after ablation is set at $70-90 \mathrm{bpm} .{ }^{518,519}$ The procedure does not worsen LV function ${ }^{520}$ and may even improve LVEF in selected patients. ${ }^{521-523}$ Most studies have included older patients with limited life expectancy. For younger patients, ablation of the atrioventricular node should only be considered if there is urgent need for rate control and all other pharmacological and nonpharmacological treatment options have been carefully considered. The choice of pacing therapy (right ventricular or biventricular pacing) will depend on patient characteristics. ${ }^{524,525}$ His-bundle pacing after atrioventricular node ablation may evolve as an attractive alternative pacing mode, ${ }^{526}$ as currently tested in ongoing clinical trials (NCT02805465, NCT02700425).

In severely symptomatic patients with permanent AF and at least one hospitalization for HF, atrioventricular node ablation combined with cardiac resynchronization therapy (CRT) may be preferred. In a small RCT, the primary composite outcome (death 
Table I 3 Drugs for rate control in AF $^{a}$

\begin{tabular}{|c|c|c|c|}
\hline & Intravenous administration & Usual oral maintenance dose & Contraindicated \\
\hline \multicolumn{4}{|l|}{ Beta-blockers $^{b}$} \\
\hline Metoprolol tartrate & 2.5 - $5 \mathrm{mg}$ i.v. bolus; up to 4 doses & 25 - 100 mg b.i.d. & \multirow{8}{*}{$\begin{array}{l}\text { In case of asthma use beta-1- } \\
\text { blockers } \\
\text { Contraindicated in acute HF and } \\
\text { history of severe bronchospasm }\end{array}$} \\
\hline Metoprolol XL (succinate) & $\mathrm{N} / \mathrm{A}$ & 50 - 400 mg o.d. & \\
\hline Bisoprolol & $\mathrm{N} / \mathrm{A}$ & $1.25-20 \mathrm{mg}$ o.d. & \\
\hline Atenolol $^{c}$ & N/A & $25-100 \mathrm{mg}$ o.d. & \\
\hline Esmolol & $\begin{array}{l}500 \mu g / \mathrm{kg} \text { i.v. bolus over } 1 \mathrm{~min} \text {; followed by } \\
50-300 \mu \mathrm{g} / \mathrm{kg} / \mathrm{min}\end{array}$ & N/A & \\
\hline Landiolol & $\begin{array}{l}100 \mu \mathrm{g} / \mathrm{kg} \text { i.v. bolus over } 1 \mathrm{~min} \text {, followed by } \\
10-40 \mu \mathrm{g} / \mathrm{kg} / \mathrm{min} \text {; in patients with cardiac } \\
\text { dysfunction: } 1-10 \mu \mathrm{g} / \mathrm{kg} / \mathrm{min}\end{array}$ & N/A & \\
\hline Nebivolol & N/A & $2.5-10 \mathrm{mg}$ o.d. & \\
\hline Carvedilol & $N / A$ & 3.125 - 50 mg b.i.d. & \\
\hline \multicolumn{4}{|c|}{ Non-dihydropyridine calcium channel antagonists } \\
\hline Verapamil & 2.5 - $10 \mathrm{mg}$ i.v. bolus over $5 \mathrm{~min}$ & $\begin{array}{l}40 \mathrm{mg} \text { b.i.d. to } 480 \mathrm{mg} \text { (extended } \\
\text { release) o.d. }\end{array}$ & \multirow{2}{*}{$\begin{array}{l}\text { Contraindicated in HFrEF } \\
\text { Adapt doses in hepatic and renal } \\
\text { impairment }\end{array}$} \\
\hline Diltiazem & $\begin{array}{l}0.25 \mathrm{mg} / \mathrm{kg} \text { i.v. bolus over } 5 \mathrm{~min} \text {, then } 5 \text { - } 15 \\
\mathrm{mg} / \mathrm{h}\end{array}$ & $\begin{array}{l}60 \mathrm{mg} \text { t.i.d. to } 360 \mathrm{mg} \text { (extended } \\
\text { release) o.d. }\end{array}$ & \\
\hline \multicolumn{4}{|l|}{ Digitalis glycosides } \\
\hline Digoxin & $\begin{array}{l}0.5 \mathrm{mg} \text { i.v. bolus ( } 0.75 \text { - } 1.5 \mathrm{mg} \text { over } 24 \\
\text { hours in divided doses) }\end{array}$ & $0.0625-0.25 \mathrm{mg}$ o.d. & $\begin{array}{l}\text { High plasma levels associated with } \\
\text { increased mortality } \\
\text { Check renal function before start- } \\
\text { ing and adapt dose in CKD patients }\end{array}$ \\
\hline Digitoxin & $0.4-0.6 \mathrm{mg}$ & $0.05-0.1 \mathrm{mg}$ o.d. & $\begin{array}{l}\text { High plasma levels associated with } \\
\text { increased mortality }\end{array}$ \\
\hline \multicolumn{4}{|l|}{ Other } \\
\hline Amiodarone & $\begin{array}{l}300 \mathrm{mg} \text { i.v. diluted in } 250 \mathrm{~mL} 5 \% \text { dextrose } \\
\text { over } 30 \text { - } 60 \text { min (preferably via central } \\
\text { venous cannula), followed by } 900-1200 \\
\mathrm{mg} \text { i.v. over } 24 \text { hours diluted in } 500-1000 \\
\mathrm{~mL} \text { via a central venous cannula }\end{array}$ & $\begin{array}{l}200 \mathrm{mg} \text { o.d. after loading } \\
3 \times 200 \mathrm{mg} \text { daily over } 4 \text { weeks, } \\
\text { then } 200 \mathrm{mg} \text { daily }{ }^{536} \text { (reduce other } \\
\text { rate controlling drugs according to } \\
\text { heart rate) }\end{array}$ & $\begin{array}{l}\text { In case of thyroid disease, only if no } \\
\text { other options }\end{array}$ \\
\hline
\end{tabular}

$\mathrm{AF}=$ atrial fibrillation; b.i.d. = bis in die (twice a day); CKD = chronic kidney disease; $\mathrm{HF}=$ heart failure; $\mathrm{HFrEF}=\mathrm{HF}$ with reduced ejection fraction; i.v. = intravenous; min = minutes; $\mathrm{N} / \mathrm{A}=$ not available or not widely available; o.d. = omni die (once daily); t.i.d. = ter in die (three times a day).

${ }^{a}$ All rate control drugs are contraindicated in Wolff-Parkinson-White syndrome, also i.v. amiodarone.

${ }^{b}$ Other beta-blockers are available but not recommended as specific rate control therapy in AF and therefore not mentioned here (e.g. propranolol and labetalol).

'No data on atenolol; should not be used in HFrEF.

'Loading regimen may vary; i.v. dosage should be considered when calculating total load.

or hospitalization for HF, or worsening HF) was significantly less common in the ablation + CRT group vs. the drug arm $(P=0.013)$, and ablation + CRT patients showed a $36 \%$ decrease in symptoms and physical limitations at 1-year follow-up $(P=0.004) .^{527}$ Emerging evidence suggest that His-bundle pacing could be an alternative in these patients. ${ }^{528}$ 


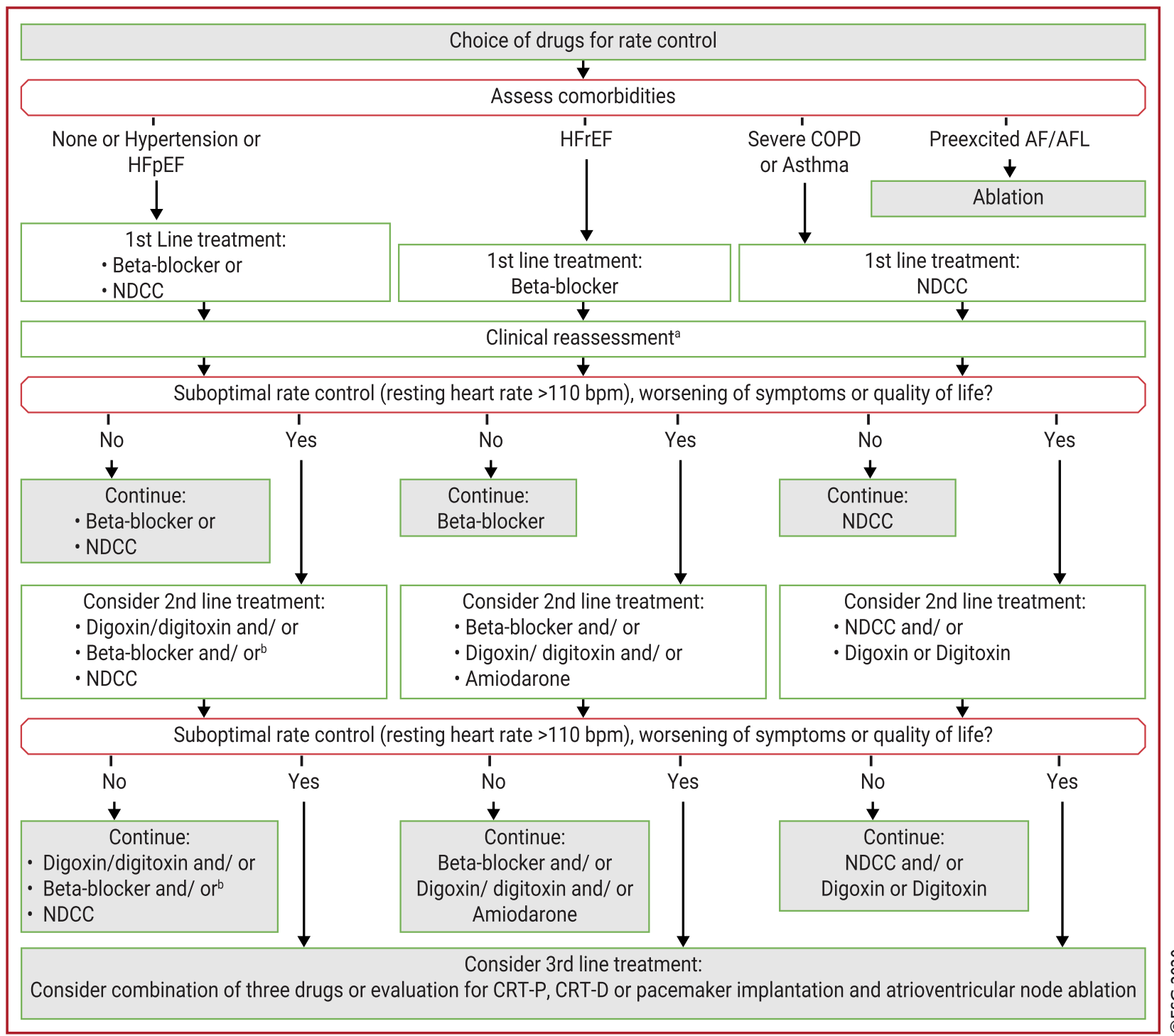

Figure I4 Choice of rate control drugs. ${ }^{490} \mathrm{AF}=$ atrial fibrillation; AFL = atrial flutter; COPD = chronic obstructive pulmonary disease; CRT-D = cardiac resynchronization therapy defibrillator; $\mathrm{CRT}-\mathrm{P}=$ cardiac resynchronization therapy pacemaker; HFpEF = heart failure with preserved ejection fraction; HFrEF =

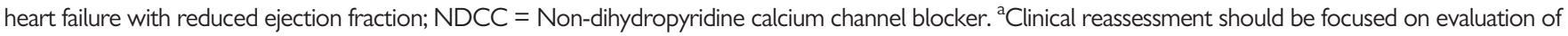
resting heart rate, AF/AFL-related symptoms and quality of life. In case suboptimal rate control (resting heart rate $>110$ bpm), worsening of symptoms or quality of life consider 2 nd line and, if necessary, 3rd line treatment options. ${ }^{b}$ Careful institution of beta-blocker and NDCC, 24-hour Holter to check for bradycardia.

\section{Recommendations for ventricular rate control in patients with $\mathrm{AF}^{\mathrm{a}}$}

\section{Recommendations}

Beta-blockers, diltiazem, or verapamil are recommended as first-choice drugs to control heart rate in AF patients with LVEF $\geq 40 \%$. ${ }^{492,507,511,529}$

Beta-blockers and/or digoxin are recommended to control heart rate in $\mathrm{AF}$ patients with $\mathrm{LVEF}<40 \%$. ${ }^{486,491,502,512,530-532}$

Combination therapy comprising different rate controlling drugs $^{d}$ should be considered if a single drug does not achieve the target heart rate. ${ }^{533,534}$

A resting heart rate of $<110 \mathrm{bpm}$ (i.e. lenient rate control) should be considered as the initial heart rate target for rate control therapy. ${ }^{488}$ Atrioventricular node ablation should be considered to control heart rate in patients unresponsive or intolerant to intensive rate and rhythm control therapy, and not eligible for rhythm control by LA ablation, accepting that these patients will become pacemaker dependent. ${ }^{516,523,535,536}$

In patients with haemodynamic instability or severely depressed LVEF, intravenous amiodarone may be considered for acute control of heart rate. ${ }^{504,514,515}$

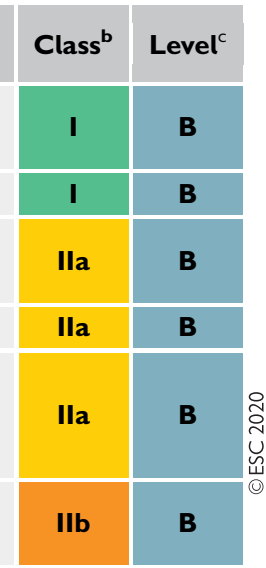

$\mathrm{AF}=$ atrial fibrillation; $\mathrm{bpm}=$ beats per minute; $\mathrm{ECG}=$ electrocardiogram; $\mathrm{LA}=$ left atrial; $\mathrm{LVEF}=$ left ventricular ejection fraction.

${ }^{a}$ See section 11 for ventricular rate control in various concomitant conditions and AF populations

${ }^{\mathrm{b}}$ Class of recommendation.

'Level of evidence.

${ }^{d}$ Combining beta-blocker with verapamil or diltiazem should be performed with careful monitoring of heart rate by $24-\mathrm{h}$ ECG to check for bradycardia. ${ }^{488}$ 


\subsubsection{Rhythm control}

The 'rhythm control strategy' refers to attempts to restore and maintain sinus rhythm, and may engage a combination of treatment approaches, including cardioversion, ${ }^{164,234}$ antiarrhythmic medication, ${ }^{233,537,538}$ and catheter ablation, ${ }^{539-541}$ along with an adequate rate control, anticoagulation therapy (section 10.2.2.6) and comprehensive cardiovascular prophylactic therapy (upstream therapy, including lifestyle and sleep apnoea management) (Figure 15).

\subsubsection{Indications for rhythm control}

Based on the currently available evidence from RCTs, the primary indication for rhythm control is to reduce AF-related symptoms and improve QoL (Figure 15). In case of uncertainty, an attempt to restore sinus rhythm in order to evaluate the response to therapy may be a rational first step. Factors that may favour an attempt at rhythm control should be considered ${ }^{542,543}$ (Figure 15).
As $\mathrm{AF}$ progression is associated with a decrease in $\mathrm{QoL}^{544}$ and, with time, becomes irreversible or less amenable to treatment, ${ }^{176}$ rhythm control may be a relevant choice, although currently there is no substantial evidence that this may result in a different outcome. Reportedly, rates of AF progression were significantly lower with rhythm control than rate control. ${ }^{545}$ Older age, persistent $A F$, and previous stroke/TIA independently predicted AF progression, ${ }^{545}$ which may be considered when deciding the treatment strategy. For many patients, an early intervention to prevent AF progression may be worth considering, ${ }^{546}$ including optimal risk-factor management. $^{245}$ Ongoing trials in patients with newly diagnosed symptomatic AF will assess whether early rhythm control interventions such as AF catheter ablation offer an opportunity to halt the progressive patho-anatomical changes associated with AF. ${ }^{547}$ However, there is evidence that, at least in some patients, a successful rhythm control strategy with AF catheter ablation may not affect atrial substrate

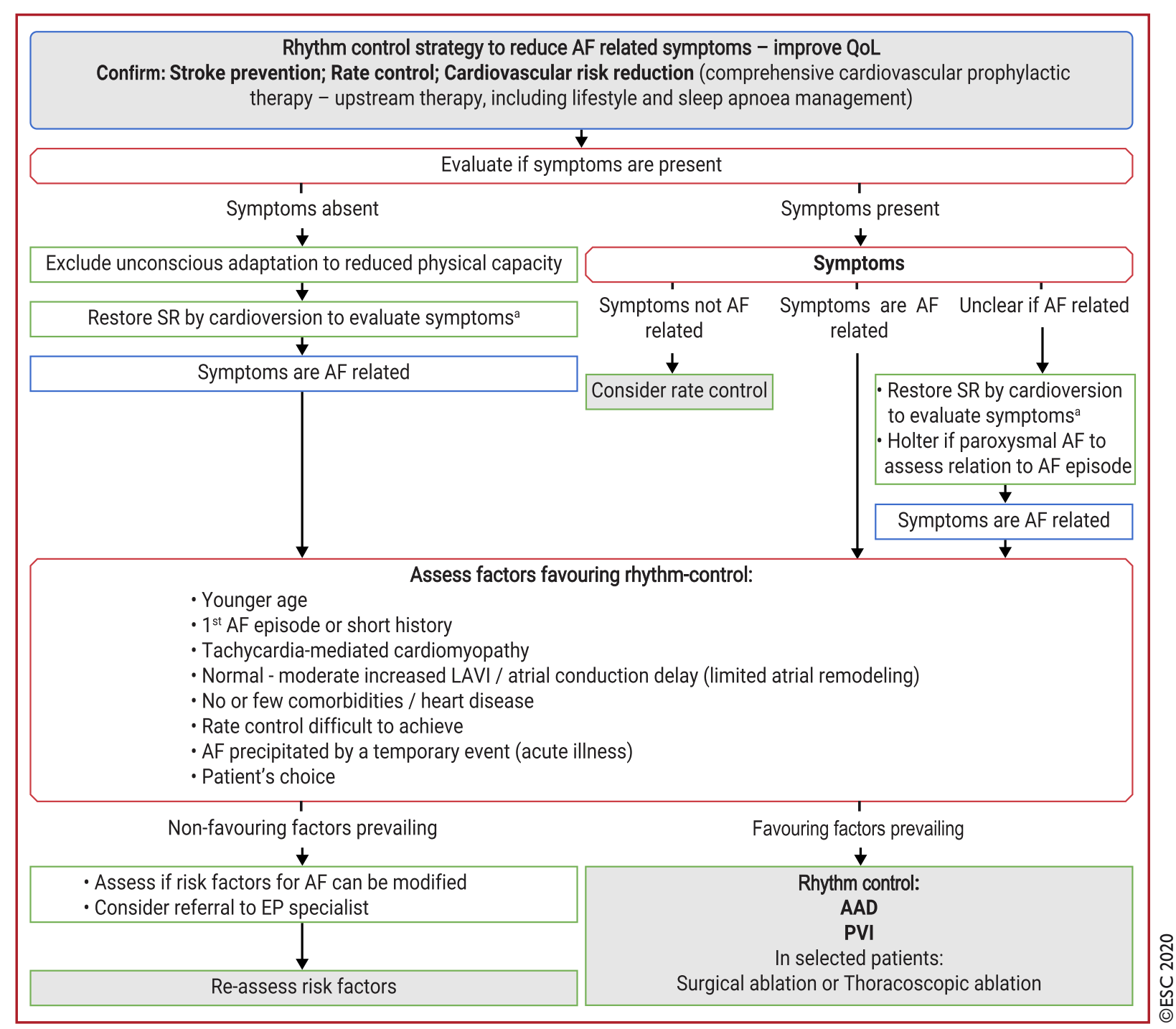

Figure I 5 Rhythm control strategy. $A A D=$ antiarrhythmic drug; $A F=$ atrial fibrillation; $C M P=$ cardiomyopathy; $C V=$ cardioversion; $L A V I=$ left atrial volume index; $\mathrm{PAF}=$ paroxysmal atrial fibrillation; $\mathrm{PVI}=$ pulmonary vein isolation; $\mathrm{QoL}=$ quality of life; $\mathrm{SR}=$ sinus rhythm. ${ }^{\mathrm{a}}$ Consider cardioversion to confirm that the absence of symptoms is not due to unconscious adaptation to reduced physical and/or mental capacity. 
development. ${ }^{548}$ Important evidence regarding the effect of early rhythm control therapy on clinical outcomes are expected in 2020 from the ongoing EAST (Early treatment of Atrial fibrillation for Stoke prevention Trial) trial. ${ }^{549}$

General recommendations regarding active informed patient involvement in shared decision making (section 9) also apply for rhythm control strategies. The same principles should be applied in female and male AF patients when considering rhythm control therapy. ${ }^{550}$

\section{Recommendations for rhythm control}

\begin{tabular}{l|l|l|}
\hline Recommendations & Class $^{\mathbf{a}}$ & Level $^{\mathbf{b}}$ \\
\hline $\begin{array}{l}\text { Rhythm control therapy is recommended for } \\
\text { symptom and QoL improvement in sympto- } \\
\text { matic patients with AF. }\end{array}$. & I & A \\
\hline
\end{tabular}

$\mathrm{AF}=$ atrial fibrillation; $\mathrm{QoL}=$ quality of life.

${ }^{\mathrm{a}}$ Class of recommendation.

bevel of evidence.

\subsubsection{Cardioversion}

10.2.2.2.1 Immediate cardioversion/elective cardioversion. Acute rhythm control can be performed as an emergency cardioversion in a haemodynamically unstable AF patient or in a non-emergency situation. Synchronized direct current electrical cardioversion is the preferred choice in haemodynamically compromised AF patients as it is more effective than pharmacological cardioversion and results in immediate restoration of sinus rhythm. ${ }^{554,555}$ In stable patients, either pharmacological cardioversion or electrical cardioversion can be attempted; pharmacological cardioversion is less effective but does not require sedation. Of note, pre-treatment with AADs can improve the efficacy of elective electrical cardioversion. ${ }^{556}$ A RCT showed maximum fixed-energy electrical cardioversion was more effective than an energy-escalation strategy. ${ }^{557}$

In a RCT, a wait-and-watch approach with rate control medication only and cardioversion when needed within $48 \mathrm{~h}$ of symptom onset was as safe as and non-inferior to immediate cardioversion of paroxysmal AF, which often resolves spontaneously within $24 \mathrm{~h}^{558}$

Elective cardioversion refers to the situation when cardioversion can be planned beyond the nearest hours. Observational data ${ }^{243}$ showed that cardioversion did not result in improved AF-related QoL or halted AF progression, but many of these patients did not receive adjunctive rhythm control therapies. ${ }^{243}$ Other studies reported significant QoL improvement in patients who maintain sinus rhythm after electrical cardioversion and the only variable independently associated with a moderate to large effect size was sinus rhythm at 3 months. ${ }^{232}$

Factors associated with an increased risk for AF recurrence after elective cardioversion include older age, female sex, previous cardioversion, chronic obstructive pulmonary disease (COPD), renal impairment, structural heart disease, larger LA volume index, and HF. ${ }^{164,559,560}$ Treatment of potentially modifiable conditions should be considered before cardioversion to facilitate maintenance of sinus rhythm (Figure 15). ${ }^{245}$ In case of AF recurrence after cardioversion in patients with persistent $A F$, an early re-cardioversion may prolong subsequent duration of sinus rhythm. ${ }^{561}$
Non-emergency cardioversion is contraindicated in the presence of known LA thrombus. Peri-procedural thrombo-embolic risk should be evaluated and peri-procedural and long-term OAC use considered irrespective of cardioversion mode (i.e. pharmacological cardioversion or electrical cardioversion) (section 10.2.2.6). A flowchart for decision making on cardioversion is shown in Figure 16.

10.2.2.2.2 Electrical cardioversion. Electrical cardioversion can be performed safely in sedated patients treated with i.v. midazolam and/or propofol or etomidate. ${ }^{562}$ BP monitoring and oximetry during the procedure should be used routinely. Skin burns may occasionally be observed. Intravenous atropine or isoproterenol, or temporary transcutaneous pacing, should be available in case of post-cardioversion bradycardia. Biphasic defibrillators are standard because of their superior efficacy compared with monophasic defibrillators. ${ }^{563,564}$ Anteriorposterior electrode positions restore sinus rhythm more effectively, ${ }^{554,555}$ while other reports suggest that specific electrical pad positioning is not critically important for successful cardioversion. ${ }^{565}$

10.2.2.2.3 Pharmacological cardioversion (including 'pill in the pocket'). Pharmacological cardioversion to sinus rhythm is an elective procedure indicated in haemodynamically stable patients. Its true efficacy is biased by the spontaneous restoration of sinus rhythm within $48 \mathrm{~h}$ of hospitalization in $76-83 \%$ of patients with recent onset AF (10 - 18\% within first 3 h, $55-66 \%$ within 24 h, and $69 \%$ within 48 h). ${ }^{566-568}$ Therefore, a 'wait-and-watch' strategy (usually for $<24$ h) may be considered in patients with recent-onset AF as a non-inferior alternative to early cardioversion. ${ }^{558}$

The choice of a specific drug is based on the type and severity of associated heart disease (Table 14), and pharmacological cardioversion is more effective in recent onset AF. Flecainide (and other class Ic agents), indicated in patients without significant LV hypertrophy $(\mathrm{LVH}), \mathrm{LV}$ systolic dysfunction, or ischaemic heart disease, results in prompt $(3-5 \mathrm{~h})$ and safe ${ }^{569}$ restoration of sinus rhythm in $>50 \%$ of patients, ${ }^{570-574}$ while i.v. amiodarone, mainly indicated in HF patients, has a limited and delayed effect but can slow heart rate within 12 h. ${ }^{570,575-577}$ Intravenous vernakalant is the most rapidly cardioverting drug, including patients with mild $\mathrm{HF}$ and ischaemic heart disease, and is more effective than amiodarone ${ }^{578-583}$ or flecainide. ${ }^{584}$ Dofetilide is not used in Europe and is rarely used outside Europe. Ibutilide is effective to convert atrial flutter (AFL) to sinus rhythm. ${ }^{585}$

In selected outpatients with rare paroxysmal AF episodes, a selfadministered oral dose of flecainide or propafenone is slightly less effective than in-hospital pharmacological cardioversion but may be preferred (permitting an earlier conversion), provided that the drug safety and efficacy has previously been established in the hospital setting. ${ }^{586}$ An atrioventricular node-blocking drug should be instituted in patients treated with class Ic AADs (especially flecainide) to avoid transformation to AFL with 1:1 conduction. ${ }^{587}$

10.2.2.2.4 Follow-up after cardioversion. The goals of follow-up after cardioversion are shown in Table 15. When assessing the efficacy of a rhythm control strategy, it is important to balance symptoms and AAD side-effects. Patients should be reviewed after cardioversion to detect whether an alternative rhythm control strategy including AF catheter ablation, or a rate control approach is needed instead of current treatment. 


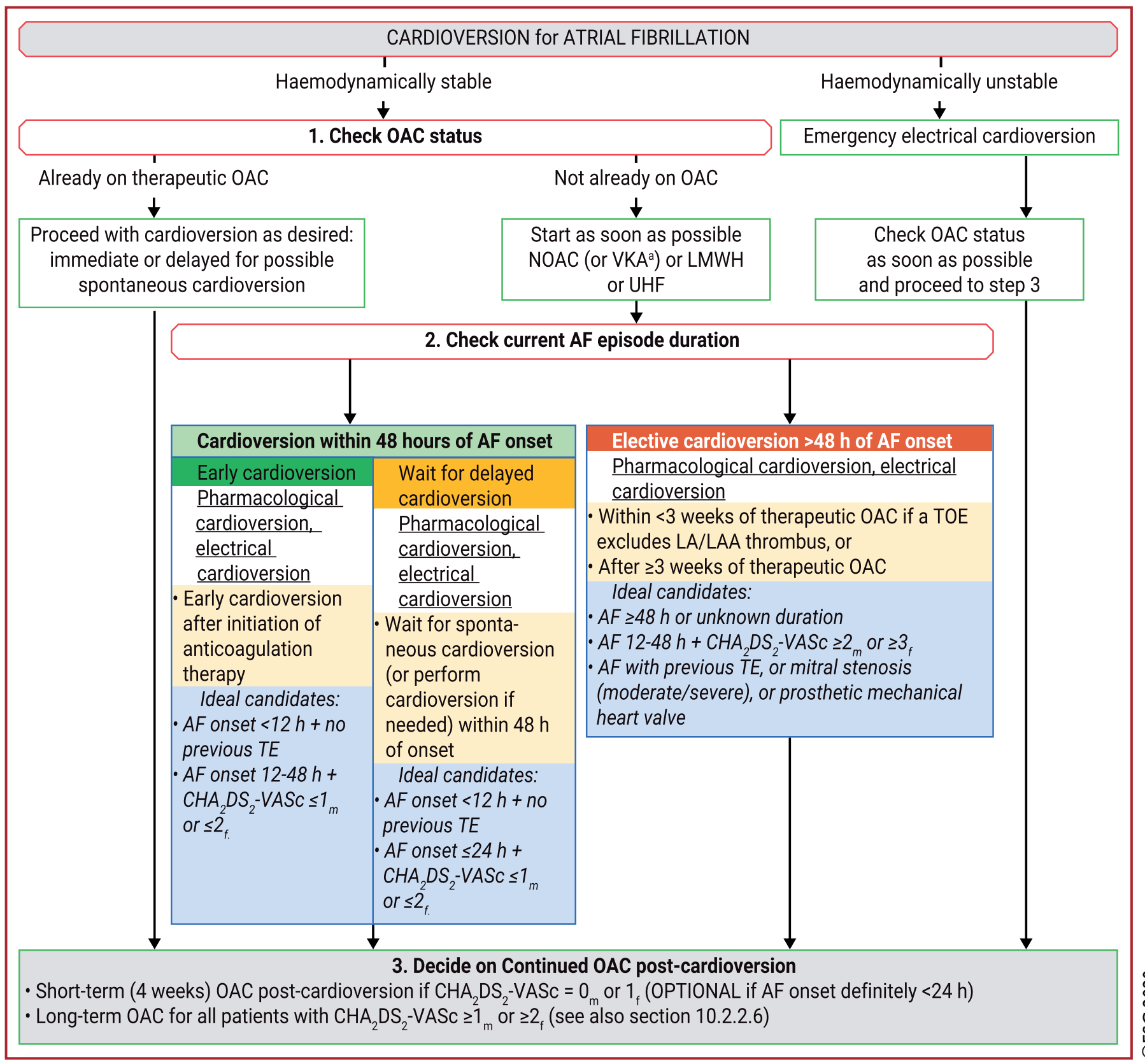

Figure 16 Flowchart for decision making on cardioversion of AF depending on clinical presentation, AF onset, oral anticoagulation intake, and risk factors for stroke. $\mathrm{AF}=$ atrial fibrillation; $\mathrm{CHA}_{2} \mathrm{DS}_{2}-\mathrm{VASc}=$ Congestive heart failure, Hypertension, Age $\geq 75$ years, Diabetes mellitus, Stroke, Vascular disease, Age 65 - 74 years, Sex category (female); cardioversion = cardioversion; $E C V=$ electrical cardioversion; $h=$ hour; $L A=$ left atrium; $L A A=$ left atrial appendage; $\mathrm{LMWH}=$ low-molecular-weight heparin; NOAC = non-vitamin $\mathrm{K}$ antagonist oral anticoagulant; $\mathrm{OAC}=$ oral anticoagulant; TE = thromboembolism; TOE $=$ transoesophageal echocardiography; UFH = unfractionated heparin; VKA = vitamin $\mathrm{K}$ antagonist. 
oror JS

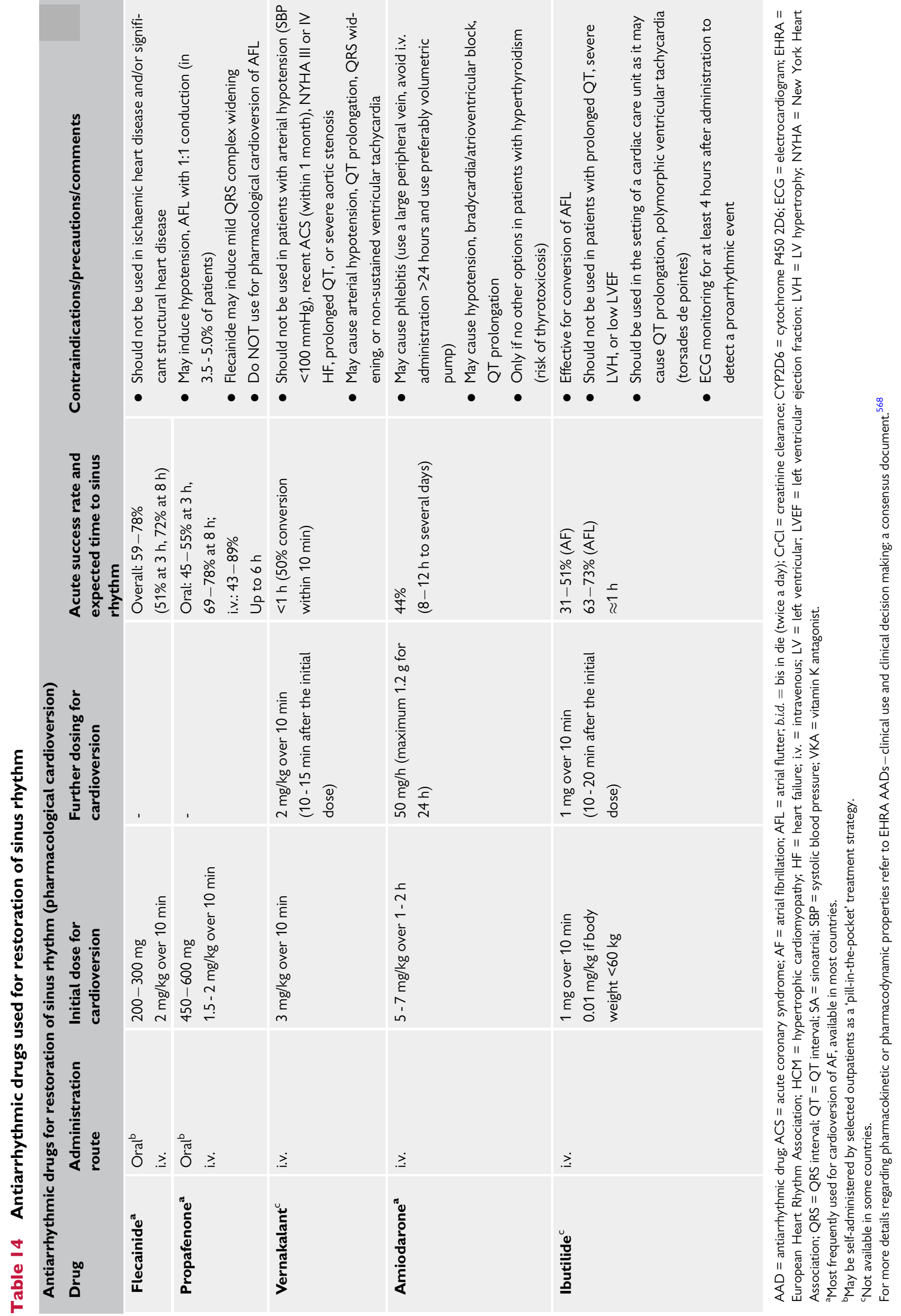




\section{Table I 5 Goals of follow-up after cardioversion of AF}

Early recognition of AF recurrence by ECG recording after cardioversion

Evaluation of the efficacy of rhythm control by symptom assessment

Monitoring of risk for proarrhythmia by regular control of PR, QRS, and QTc intervals in patients on Class I or III AADs

Evaluation of balance between symptoms and side-effects of therapy considering $\mathrm{QoL}$ and symptoms

Evaluation of AF-related morbidities and AAD-related side-effects on concomitant cardiovascular conditions and LV function

Optimization of conditions for maintenance of sinus rhythm including cardiovascular risk management (BP control, HF treatment, increasing cardiorespiratory fitness, and other measures, see section 11).

$A A D=$ antiarrhythmic drug; $A F=$ atrial fibrillation; $B P=$ blood pressure; $E C G=$ electrocardiogram; $H F=$ heart failure; $L V=$ left ventricular; $P R=P R$ interval; $Q \circ L=$ quality of life; QRS = QRS interval; QTc = corrected QT interval.

\section{Recommendations for cardioversion}

\begin{tabular}{|c|c|c|}
\hline Recommendations & Class $^{a}$ & Level $^{\text {b }}$ \\
\hline $\begin{array}{l}\text { For pharmacological cardioversion of recent- } \\
\text { onset AF, i.v. vernakalant (excluding patients } \\
\text { with recent ACS or severe HF) or flecainide or } \\
\text { propafenone (excluding patients with severe } \\
\text { structural heart disease) is } \\
\text { recommended. }\end{array}$ & I & $\mathbf{A}$ \\
\hline $\begin{array}{l}\text { Intravenous amiodarone is recommended for } \\
\text { cardioversion of AF in patients with HF or struc- } \\
\text { tural heart disease, if delayed cardioversion is } \\
\text { consistent with clinical situation. }{ }^{515,591,592}\end{array}$ & I & $\mathbf{A}$ \\
\hline $\begin{array}{l}\text { Cardioversion of AF (either electrical or phar- } \\
\text { macological) is recommended in symptomatic } \\
\text { patients with persistent AF as part of rhythm } \\
\text { control therapy. }\end{array}$ & I & B \\
\hline $\begin{array}{l}\text { Pharmacological cardioversion of AF is } \\
\text { indicated only in a haemodynamically stable } \\
\text { patient, after consideration of the thrombo- } \\
\text { embolic risk. }\end{array}$ & I & B \\
\hline $\begin{array}{l}\text { Pre-treatment with amiodarone, flecainide, ibu- } \\
\text { tilide, or propafenone should be considered to } \\
\text { facilitate the success of electrical } \\
\text { cardioversion. }{ }^{556,596-599}\end{array}$ & Ila & B \\
\hline $\begin{array}{l}\text { In selected patients with infrequent and recent- } \\
\text { onset AF and no significant structural or ischae- } \\
\text { mic heart disease, a single self-administered oral } \\
\text { dose of flecainide or propafenone ('pill in the } \\
\text { pocket' approach) should be considered for } \\
\text { patient-led cardioversion, but only following effi- } \\
\text { cacy and safety assessment. }\end{array}$ & Ila & B \\
\hline $\begin{array}{l}\text { For patients with sick-sinus syndrome, atrioven- } \\
\text { tricular conduction disturbances or prolonged } \\
\text { QTc (>500 ms), pharmacological cardioversion } \\
\text { should not be attempted unless risks for proar- } \\
\text { rhythmia and bradycardia have been considered. }\end{array}$ & III & C \\
\hline
\end{tabular}

$\mathrm{ACS}=$ acute coronary syndrome; $\mathrm{AF}=$ atrial fibrillation; $\mathrm{HF}=$ heart failure; $\mathrm{ms}=$ milliseconds; i.v. = intravenous; QTc = corrected QT interval. Note: For cardioversion in various specific conditions and AF populations see section 11.

${ }^{\mathrm{a}}$ Class of recommendation.

b Level of evidence.
10.2.2.3 Atrial fibrillation catheter ablation

AF catheter ablation is a well-established treatment for the prevention of AF recurrences. ${ }^{1,602-604}$ When performed by appropriately trained operators, AF catheter ablation is a safe and superior alternative to $A A D s$ for maintenance of sinus rhythm and symptom improvement. ${ }^{165,235-242,246,247,605-618}$ It is advised to discuss the efficacy and complication rates of AF catheter ablation and AADs with the patient once rhythm control as long-term management has been selected.

10.2.2.3.1 Indications. In the following section, indications for AF catheter ablation are presented for paroxysmal and persistent $A F$ in patients with and without risk factors for post-ablation AF recurrence. Differentiation of persistent and long-standing persistent AF was omitted because the latter only expresses the duration of persistent AF above an arbitrary and artificial cut-off at 12 months' duration. The significance of such a cut-off as a single measure has never been substantially proven.

A number of risk factors for $A F$ recurrence after $A F$ ablation have been identified, including LA size, AF duration, patient age, renal dysfunction, and substrate visualization by means of MRI. ${ }^{619-625}$ Recent systematic reviews on prediction models for AF recurrence after catheter ablation showed the potential benefits of risk predictions, but a more robust evaluation of such models is desirable. ${ }^{167,626}$ The model variables can be measured before ablation; therefore models could be used pre-procedurally to predict the likelihood of recurrence. ${ }^{627-635}$ However, no single score has been presently identified as consistently superior to others. Thus, at present, for an improved and more balanced indication for ablation in patients with persistent $A F$ and risk factors for recurrence, the most intensely evaluated risk predictors (including duration of AF) should be considered, and adjusted to the individual patient's situation including their preferences. Notably, patients must also be explicitly informed about the importance of treating modifiable risk factors to reduce risk of recurrent $A F .^{621,636-652}$

The indications for $A F$ catheter ablation are summarized in Figure 17. AF catheter ablation is effective in maintaining sinus rhythm in patients with paroxysmal and persistent AF. ${ }^{165,235-242,605-616}$ The main clinical benefit of AF catheter ablation is the reduction of arrhythmia-related symptoms. ${ }^{246,247,603,604,607,617,653,654}$ This has been confirmed in a recent RCT showing that the improvement in QoL was significantly higher in the ablation vs. medical therapy group, 


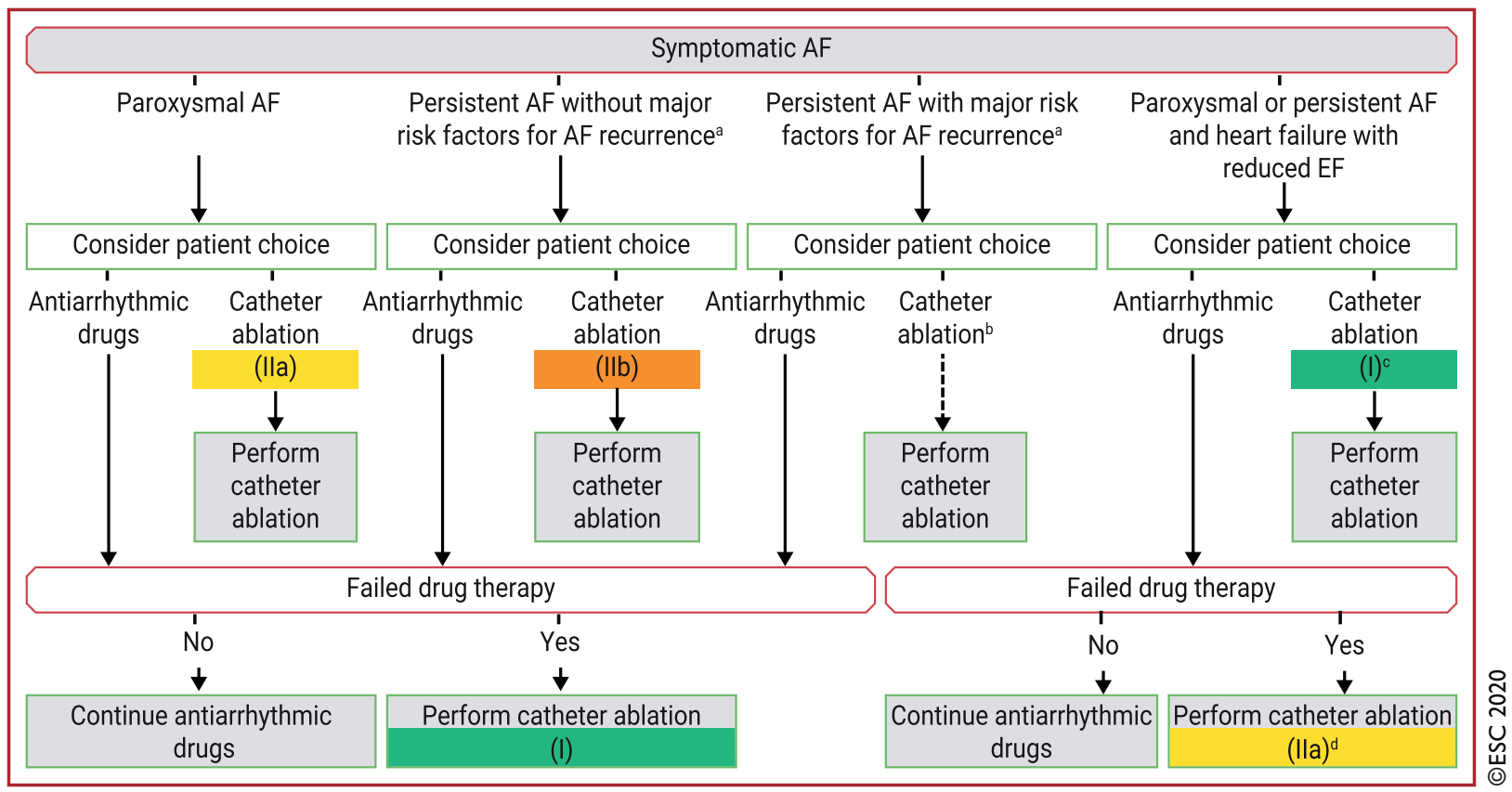

Figure 17 Indications for catheter ablation of symptomatic AF. The arrows from AAD to ablation indicate failed drug therapy. AAD = antiarrhythmic drug; $A F=$ atrial fibrillation; $E F=$ ejection fraction; $L A=$ left atrial. ${ }^{a}$ Significantly enlarged $L A$ volume, advanced age, long $A F$ duration, renal dysfunction, and other cardiovascular risk factors. ${ }^{b}$ In rare individual circumstances, catheter ablation may be carefully considered as first-line therapy. ${ }^{\mathrm{C}}$ Recommended to reverse LV dysfunction when tachycardiomyopathy is highly probable. ${ }^{\mathrm{d}}$ To improve survival and reduce hospitalization.

as was the associated reduction in AF burden. ${ }^{246}$ Symptom improvement has also been confirmed in the recent large CABANA (Catheter ABlation vs. ANtiarrhythmic Drug Therapy for Atrial Fibrillation) $\mathrm{RCT}^{655}$ but the trial showed that the strategy of AF catheter ablation did not significantly reduce the primary composite outcome of death, disabling stroke, serious bleeding, or cardiac arrest compared with medical therapy. ${ }^{617}$ As no RCT has yet demonstrated a significant reduction in all-cause mortality, stroke, or major bleeding with AF catheter ablation in the 'general' AF population, the indications for the procedure have not been broadened beyond symptom relief, ${ }^{617}$ and AF catheter ablation is generally not indicated in asymptomatic patients. Further important evidence regarding the impact of ablation on major cardiovascular events is expected from the EAST trial. ${ }^{656}$

In selected patients with HF and reduced LVEF, two RCTs have shown a reduction in all-cause mortality and hospitalizations with AF catheter ablation, ${ }^{611,657}$ although combined mortality and HF hospitalization was a primary endpoint only in the CASTLE-AF (Catheter Ablation vs. Standard conventional Treatment in patients with LEft ventricular dysfunction and Atrial Fibrillation) trial. ${ }^{657}$ The generalizability of the trial has recently been evaluated in a large HF patient population. ${ }^{658}$ This analysis showed that only a small number of patients met the trial inclusion criteria $(<10 \%)$ and patients who met the CASTLE-AF inclusion criteria had a significant benefit from treatment as demonstrated in the trial. ${ }^{658}$ The smaller AMICA (Atrial Fibrillation Management in Congestive Heart Failure With Ablation) RCT, which included patients with more advanced HFrEF, did not show benefits gained by AF catheter ablation at 1-year follow-up, ${ }^{659}$ whereas a recent CABANA subgroup analysis supported the benefits of $A F$ catheter ablation in patients with $\mathrm{HFrEF}$, showing a significant reduction in the study primary endpoint (death, stroke, bleeding, cardiac arrest) and reduced mortality in the ablation group. ${ }^{617,660}$ Overall, AF catheter ablation in patients with HFrEF results in higher rates of preserved sinus rhythm and greater improvement in LVEF, exercise performance, and QoL compared with AAD and rate control. $^{611,657,661-671}$ Accordingly, ablation should be considered in patients with HFrEF who have been selected for rhythm control treatment to improve QoL and LV function, and to reduce HF hospitalization and, potentially, mortality.

When AF-mediated tachycardia-induced cardiomyopathy (i.e. ventricular dysfunction secondary to rapid and/or asynchronous/irregular myocardial contraction, partially or completely reversed after treatment of the causative arrhythmia) is highly suspected, AF catheter ablation is recommended to restore LV function. ${ }^{672-676}$

Ablation is recommended, in general, as a second-line therapy after failure (or intolerance) of class I or class III AADs. This recommendation is based on the results of multiple RCTs showing superiority of AF catheter ablation vs. AADs regarding freedom from recurrent arrhythmia or improvement in symptoms, exercise capacity, and QoL after medication failure. $^{235-239,246,247,605-607,609,611,613-617}$

Clinical trials considering $A F$ catheter ablation before any $A A D$ suggest that AF catheter ablation is more effective in maintaining sinus rhythm, with comparable complication rates in experienced centres. $^{240-242,614}$ The 5-year follow-up in the MANTRA-PAF (Medical Antiarrhythmic Treatment or Radiofrequency Ablation in Paroxysmal Atrial Fibrillation) trial showed a significantly lower AF burden in the ablation arm that did not, however, translate into improved QoL compared with AAD treatment, ${ }^{615}$ whereas the CAPTAF (Catheter Ablation compared with Pharmacological 
Therapy for Atrial Fibrillation) study showed that, in AF patients mostly naive to class I and III AADs, the greater improvement in QoL in the ablation arm was directly associated with greater reduction in AF burden compared with the AAD arm. ${ }^{246}$ Based on these studies and patient preferences, AF catheter ablation should be considered before a trial of $A A D$ in patients with paroxysmal AF episodes (class Ila), or may be considered in patients with persistent AF without risk factors for recurrence (class Ilb).

10.2.2.3.2 Techniques and technologies. The cornerstone of AF catheter ablation is the complete isolation of pulmonary veins by linear lesions around their antrum, either using point-by-point radiofrequency ablation or single-shot ablation devices. ${ }^{235,237,239,607-609,612,613,654,677-686}$ Unfortunately, persistent pulmonary vein electrical isolation is difficult to achieve (pulmonary vein reconnection rates of $>70 \%$ are reported ${ }^{683,687-697}$, but could be significantly lower with the newer generation of catheters ${ }^{698-700}$ ).

Particularly in persistent and long-standing persistent AF, more extensive ablation has been advocated. This may include linear lesions in the atria, isolation of the LAA or of the superior vena cava, ablation of complex fractionated electrograms, rotors, nonpulmonary foci, or ganglionated plexi, fibrosis-guided voltage and/or MRI-mapping, or ablation of high dominant frequency sites. ${ }^{701-710}$ However, additional benefit vs. pulmonary vein isolation (PVI) alone, justifying its use during the first procedure, is yet to be confirmed. ${ }^{677,680,711-730}$ A RCT-based data suggest improved outcome with targeting extrapulmonary (particularly the LAA) foci and selective ablation of low-voltage areas as adjunct to PVI. ${ }^{708,725}$ In patients with documented cavotricuspid isthmus (CTI)-dependent flutter undergoing AF catheter ablation, right isthmus ablation may be considered. ${ }^{731-734}$ In case of non-CTI-dependent atrial tachycardia, the ablation technique depends on the underlying mechanism and tachycardia focus or circuit. ${ }^{1,614}$

Several RCTs and observational studies have compared point-bypoint radiofrequency and cryoballoon ablation, mostly in the first procedure for paroxysmal AF. ${ }^{612,681,735-755}$ They reported broadly similar arrhythmia-free survival and overall complications with either technique, with slightly shorter procedure duration but longer fluoroscopy time with cryoballoon ablation. ${ }^{612,681,735-755}$ However, some studies showed reduced hospitalization and lower complication rates with cryoballoon ablation. ${ }^{746,756,757}$ The choice of energy source may depend on centre availability, operator preference/experience, and patient preference. Alternative catheter designs and energy sources have been developed in an attempt to simplify the ablation procedure and improve outcomes, ${ }^{613,755,758-761}$ but further evidence is required before changing current recommendations.

10.2.2.3.3 Complications. Prospective, registry-based data show that approximately $4-14 \%$ of patients undergoing AF catheter ablation experience complications, 2 - 3\% of which are potentially life-threatening. ${ }^{602-604,762-765}$ In the recent CABANA trial, mostly including experienced high-volume centres, complications occurred in the lower range of these rates. ${ }^{617}$ Complications occur mostly within the first $24 \mathrm{~h}$ after the procedure, but some may appear 1-2 months after ablation ${ }^{1,602-604}$ (Table 16 and Supplementary Table 10). Periprocedural death is rare $(<0.2 \%)$ and usually related to cardiac tamponade. $^{603,604,766-770}$

10.2.2.3.4 AF catheter ablation outcome and impact of modifiable risk factors. Multiple RCTs have compared AADs with AF catheter ablation using different technologies/energy sources, either as 'first-line' therapy or after AAD failure, showing superiority of AF catheter ablation in arrhythmia-free survival. ${ }^{165,235-242,605-616}$ However, many patients require several procedures and late recurrences are not infrequent. $^{248,639,772-780}$

Key outcomes include QoL, HF, stroke, and mortality. ${ }^{539-541,608,781,782}$ Compared with AADs, AF catheter ablation was associated with significant and sustained improvement in QoL scores in several RCTs and meta-analyses. ${ }^{1,235,239-242,246,247,539-541,783,784}$ To date, there is no RCT sufficiently large to properly evaluate a reduction in stroke by catheter ablation.

Several factors, including AF type and duration, ${ }^{235-237,239,607,609,612,613,654,680,682,785}$ and the presence of comorbidities such as hypertension, ${ }^{621,639-641}$ obesity, ${ }^{638,639,643,646,772,786-791}$ metabolic syndrome, ${ }^{792-794}$ and sleep apnoea ${ }^{643-645,647-652}$ may influence the outcome of catheter

\section{Table 16 Procedure-related complications in catheter ablation and thoracoscopic ablation of $\mathbf{A F}^{\mathbf{7 7 1}}$}

\begin{tabular}{|c|c|c|c|}
\hline \multirow[t]{2}{*}{ Complication severity } & \multirow[t]{2}{*}{ Complication type } & \multicolumn{2}{|l|}{ Complication rate } \\
\hline & & Catheter ablation & Thoracoscopic ablation \\
\hline \multirow[t]{4}{*}{ Life-threatening complications } & Periprocedural death & $<0.1 \%$ & $<0.1 \%$ \\
\hline & Oesophageal perforation/fistula & $<0.5 \%$ & $N / A$ \\
\hline & Periprocedural thromboembolic event & $<1.0 \%$ & $<1.5 \%$ \\
\hline & Cardiac tamponade & $\approx 1 \%$ & $<1.0 \%$ \\
\hline \multirow[t]{5}{*}{ Severe complications } & Pulmonary vein stenosis & $<1.0 \%$ & $\mathrm{~N} / \mathrm{A}$ \\
\hline & Persistent phrenic nerve palsy & $<1.0 \%$ & $N / A$ \\
\hline & Vascular complications & $2-4 \%$ & $\mathrm{~N} / \mathrm{A}$ \\
\hline & Conversion to sternotomy & N/A & $<1.7 \%$ \\
\hline & Pneumothorax & $\mathrm{N} / \mathrm{A}$ & $<6.5 \%$ \\
\hline Moderate or minor complications & Various & $1-2 \%$ & $1-3 \%$ \\
\hline Complications of unknown significance & Asymptomatic cerebral embolism & $5-15 \%$ & N/A \\
\hline
\end{tabular}

NA = not available. 


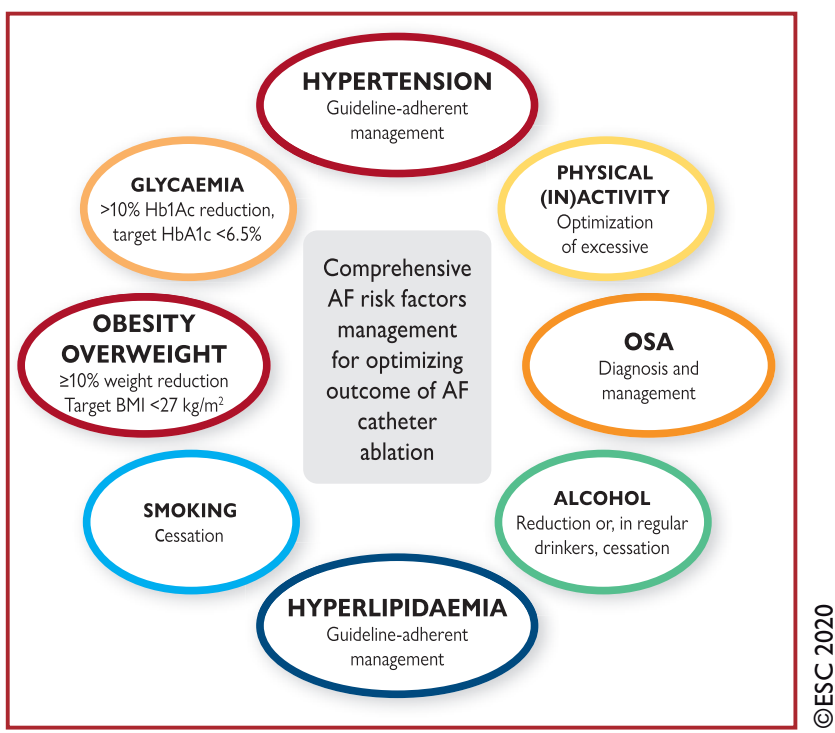

Figure I 8 Risk factors for AF contributing to the development of an abnormal substrate translating into poorer outcomes with rhythm control strategies. $\mathrm{AF}=$ atrial fibrillation; $\mathrm{BMI}=$ body mass index; $\mathrm{CPAP}=$ continuous positive airway pressure; $\mathrm{HbA}_{1 \mathrm{C}}=$ haemoglobin $\mathrm{A} 1 \mathrm{c}$; OSA = obstructive sleep apnoea. Several AF risk factors may contribute to the development of LA substrates and thus affect the outcome of AF catheter ablation, predisposing to a higher recurrence rate. Aggressive control of modifiable risk factors may reduce recurrence rate. ablation (Figure 18 and Supplementary Box 2). Prospective cohort studies suggest that aggressive control of modifiable risk factors may improve arrhythmia-free survival after catheter ablation. ${ }^{636}$

10.2.2.3.5 Follow-up after atrial fibrillation ablation. AF catheter ablation is a complex procedure that may be associated with a range of specific post-procedural complications (section

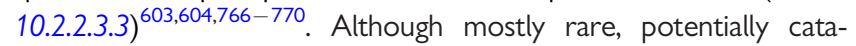
strophic complications may initially present with non-specific symptoms and signs to which managing physicians should be attuned. Key issues in follow-up are shown in Table 17.

10.2.2.3.6 Risk assessment for recurrence of atrial fibrillation post catheter ablation. Recurrence of AF after catheter ablation is driven by the complex interaction of various factors. These include increasing AF duration, age, and LA size, ${ }^{619-624}$ and structural factors such as the abundance of epicardial fat tissue ${ }^{807-810}$ and the presence of atrial substrate as evident from electrical or morphological markers. ${ }^{811}$ A number of risk-prediction scores have been evaluated (for detailed description see Supplementary Table 11 and Supplementary Box 2). Whereas these scores only moderately predict $A F$ recurrence, one of the strongest predictors is early recurrent $A F$, indicating the need for further refinement of these scoring systems. $^{629}$

\section{Table I 7 Key issues in follow-up after AF catheter ablation}

\section{Key issues}

\section{Recognition and management of complications}

- Patients must be fully informed about the clinical signs and symptoms of rare but potentially dangerous ablation-related complications that may occur after hospital discharge (e.g. atrio-oesophageal fistula, pulmonary vein stenosis).

\section{Follow-up monitoring:}

Useful to assess procedural success and correlate symptom status with rhythm. ${ }^{795,796}$ Recurrences beyond the first month post-ablation are generally predictive of late recurrences, ${ }^{797,798}$ but recurrent symptoms may be due to ectopic beats or other non-sustained arrhythmia ${ }^{640,799,800}$; conversely the presence of asymptomatic $\mathrm{AF}$ after ablation is well described. ${ }^{801-803}$

Monitoring may be performed with intermittent ECG, Holter, Patch recordings, external or implanted loop recorder, or smart phone monitor (although the latter has not been validated for such use). Patients should be first reviewed at a minimum of 3 months and annually thereafter. ${ }^{1}$

\section{Management of antiarrhythmic medication and treatment of AF recurrences}

a. Continuing AAD treatment for 6 weeks to 3 months may reduce early AF recurrences, rehospitalizations and cardioversions during this period. ${ }^{797,804}$ Clinical practice regarding routine AAD treatment after ablation varies and there is no convincing evidence that such treatment is routinely needed.

b. Subsequently, AADs may be weaned, ceased, or continued according to symptoms and rhythm status. Recent findings suggest that in AAD-treated patients remaining free of $A F$ at the end of the blanking period, AAD continuation beyond the blanking period reduces arrhythmia recurrences. ${ }^{805}$

\section{Management of anticoagulation therapy}

a. In general, OAC therapy is continued for 2 months following ablation in all patients. ${ }^{1,806}$ Beyond this time, a decision to continue OAC is determined primarily by the presence of $\mathrm{CHA}_{2} \mathrm{DS}_{2}$-VASc stroke risk factors rather than the rhythm status (section 10.2.2.6).

$\mathrm{AAD}=$ antiarrhythmic drug; $\mathrm{AF}=$ atrial fibrillation; $\mathrm{CHA}_{2} \mathrm{DS}_{2}-\mathrm{VASc}=$ Congestive heart failure, Hypertension, Age $\geq 75$ years, Diabetes mellitus, Stroke, Vascular disease, Age $65-74$ years, Sex category (female); $E C G=$ electrocardiogram; $O A C=$ oral anticoagulant. 


\section{Recommendations for rhythm control/catheter ablation of AF}

Recommendations

\section{General recommendations}

For the decision on AF catheter ablation, it is recommended to take into consideration the procedural risks and the major risk factors for AF recurrence following the procedure and discuss them with the patient. ${ }^{235-237,239,607,609,612,613,636,638,652,654,680,682,785,789}$ Repeated PVI procedures should be considered in patients with AF recurrence provided the patient's symptoms were improved after the initial PVI. $812-814$

\section{AF catheter ablation after failure of drug therapy}

AF catheter ablation for $\mathrm{PVI}$ is recommended for rhythm control after one failed or intolerant class I or III AAD, to improve symptoms of AF recurrences in patients with ${ }^{235-238,247,605-609,612,613,615-617,654,677,678,680,682,685,758,779,780,815}$ :

- Paroxysmal AF, or

- Persistent AF without major risk factors for AF recurrence, or

- Persistent AF with major risk factors for AF recurrence.

$\mathrm{AF}$ catheter ablation for $\mathrm{PVI}$ should be considered for rhythm control after one failed or intolerant to beta-blocker treatment to improve symptoms of AF recurrences in patients with paroxysmal and persistent $A F{ }^{246}$

\begin{tabular}{|c|c|}
\hline I & B \\
\hline Ila & B \\
\hline
\end{tabular}

\section{First-line therapy}

AF catheter ablation for PVI should/may be considered as first-line rhythm control therapy to improve symptoms in selected patients with symptomatic:

- Paroxysmal AF episodes, ${ }^{240-242,614,615}$ or

- Persistent AF without major risk factors for AF recurrence. ${ }^{253-255,264,598-601,609,610,633,636,641,724,745,746,832}$

as an alternative to AAD class I or III, considering patient choice, benefit, and risk.

AF catheter ablation:

- Is recommended to reverse LV dysfunction in AF patients when tachycardia-induced cardiomyopathy is highly probable, independent of their symptom status. ${ }^{666,675,676}$

- Should be considered in selected AF patients with HF with reduced LVEF to improve survival and reduce HF hospitalization. ${ }^{612,659,662-666,668-671,817-826}$

AF catheter ablation for PVI should be considered as a strategy to avoid pacemaker implantation in patients with AF-related bradycardia or symptomatic pre-automaticity pause after AF conversion considering the clinical situation. ${ }^{816-818}$

\section{Techniques and technologies}

Complete electrical isolation of the pulmonary veins is recommended during all AF catheter-ablation procedures. $^{235-237,239,606,608-610,613,614,678,679,681,683,684,686,713,731,759,780}$

If patient has history of CTI-dependent AFL or if typical AFL is induced at the time of AF ablation, delivery of a CTI lesion may be considered. ${ }^{731-733,819-821}$

Use of additional ablation lesions beyond PVI (low voltage areas, lines, fragmented activity, ectopic foci, rotors, and others) may be considered but is not well established. ${ }^{677,680,708,711-730}$

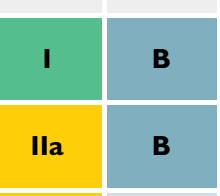

\section{Lifestyle modification and other strategies to improve outcomes of ablation}

Weight loss is recommended in obese patients with AF, particularly those who are being evaluated to undergo AF ablation. $636,638,639,643,646,772,786-791$

Strict control of risk factors and avoidance of triggers are recommended as part of a rhythm control strategy. ${ }^{636,637}$

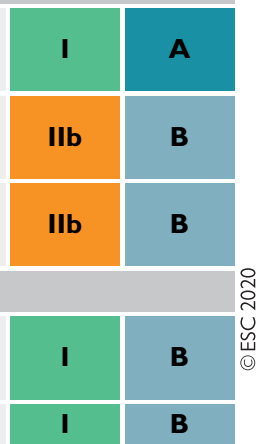

$A A D=$ antiarrhythmic drug; $A F=$ atrial fibrillation; $A F L=$ atrial flutter; $C T I=$ cavotricuspid isthmus; $H F=$ heart failure; $L V=$ left ventricular; $L V E F=$ left ventricular ejection fraction; $\mathrm{PVI}=$ pulmonary vein isolation.

${ }^{a}$ Class of recommendation.

bLevel of evidence.

\subsubsection{Surgery for atrial fibrillation}

With development of the maze procedure for surgical cure from AF, Cox et al. opened up a new window of therapeutic opportunities for AF patients. ${ }^{822}$ The classical cut-and-sew maze procedure underwent several modifications and various device-based surgical ablation procedures have been developed. ${ }^{823,824}$ More than 200 publications documented the application of these techniques and technologies in various clinical scenarios. ${ }^{825}$ Most studies are retrospective and/or observational, but some RCTs and meta-analyses have also been published. ${ }^{771,826-828}$ While the effects of surgical ablation on rhythm outcome (i.e. restoration of sinus rhythm/freedom from AF) have been clearly demonstrated, the effects on endpoints such as QoL, hospitalization, stroke, and mortality are not well established. ${ }^{461,827,829,830}$ The only RCT with longer follow-up has shown a significant reduction in stroke risk at 5 years and a greater likelihood of maintaining sinus rhythm although the trial was underpowered for 
stroke risk assessment. ${ }^{828}$ The largest registry published, from the Polish National Health Service, describes better survival when ablation is performed concomitant to mitral or coronary surgery. ${ }^{831,832}$ Close cooperation between cardiac surgeons and electrophysiologists (heart team) for proper patient selection and postoperative management, especially for handling of arrhythmia recurrences, seems advisable for high-standard quality care.

10.2.2.4.1 Concomitant surgery for atrial fibrillation: indications, outcome, complications. Most trials of concomitant AF ablation have been based mainly on patients undergoing mitral valve repair or replacement. While surgical PVI has been shown to be effective for maintaining sinus rhythm, ${ }^{833}$ the most effective ablation treatment for $\mathrm{AF}$ isolates the pulmonary veins and the LA posterior wall, creates ablation lines that impede electrical impulses around the most important structures (mitral and tricuspid annuli, venae cavae and appendages), and excludes the LAA. Most evidence supports bipolar radiofrequency clamps and cryothermy to perform a maze. ${ }^{834}$ For non-paroxysmal AF, a biatrial lesion pattern is more effective than left-sided only, performed by sternotomy or minimally invasive techniques. ${ }^{826}$

In general, the same preoperative risk factors for AF recurrence after concomitant AF surgery as for AF catheter ablation have been identified. These include LA size, patient age, AF duration, HF/ reduced LVEF, and renal dysfunction. ${ }^{379,636,835-841}$ The significant positive effects of concomitant surgical ablation on freedom from atrial arrhythmias is clearly documented. Most RCTs with 1-year follow-up show no effect on QoL, stroke, and mortality, ${ }^{842-845}$ but some reported reduced event rates. ${ }^{828,830,846}$

Surgical AF ablation concomitant to other cardiac surgery significantly increases the need for pacemaker implantation with biatrial (but not left-sided) lesions, ${ }^{827}$ being reported from $6.8 \%$ to $21.5 \%$, while other complications are not increased. ${ }^{827-830,846,847}$

10.2.2.4.2 Stand-alone surgery for atrial fibrillation: indications, outcome, complications. Thoracoscopic radiofrequency ablation targets the pulmonary veins, LA posterior wall, and LAA closure in AF patients with no structural heart disease. Freedom from $A F$ after the procedure is well documented, but only a few studies have reported improved QoL. ${ }^{844,845,848-850}$ A recent meta-analysis of three RCTs showed a significantly higher freedom from atrial tachyarrhythmia and less need for repeat ablations after thoracoscopic ablation compared with AF catheter ablation for paroxysmal or persistent AF. ${ }^{851}$ The FAST trial randomized patients who were prone to AF catheter-ablation failure (i.e. failed previous ablation or LA dilatation and hypertension) and reported common but substantially lower recurrence after thoracoscopic compared with AF catheter ablation ( $56 \%$ vs. $87 \%$ ) at long-term follow-up (mean 7 years). ${ }^{849}$ Hospitalization was longer and complication rates of surgical ablation were higher compared with catheter ablation $^{771}$ (Table 16). A systematic safety analysis of thoracoscopic ablation showed a 30 -day complication rate of $11.3 \%$, mainly selflimiting, whereas it was significantly lower (3.6\%) in a multicentre registry. ${ }^{456}$ In RCTs, thoracoscopic ablation proved more effective in rhythm control than catheter ablation; however, surgical ablation is more invasive, with higher complication rates and longer hospitalization. ${ }^{461,852}$ Because of this risk-benefit ratio of surgical vs. catheter ablation, it seems reasonable to consider thoracoscopic surgery preferentially in patients with previous failed catheter ablation or with a high risk of catheter-ablation failure. There are no convincing data on the effects on stroke of surgical ablation as a stand-alone procedure or in combination with LAA occlusion or exclusion. Hence, OAC therapy should be continued after the procedure regardless of rhythm outcome in AF patients with stroke risk factors.

\subsubsection{Hybrid surgical/catheter ablation procedures}

Hybrid AF procedures combine a minimally invasive epicardial nonsternotomy ablation not using cardiopulmonary bypass with a percutaneous endocardial approach. They can be performed as a single intervention or sequentially, when the endocardial catheter mapping and, if needed, additional ablations are done within 6 months after the epicardial procedure. ${ }^{853}$ There are no studies comparing these two hybrid strategies.

A systematic review on rhythm outcome and complications with a hybrid procedure or AF catheter ablation in patients with persistent or long-standing persistent AF showed that at 12 months or longer, a hybrid procedure achieved a significantly higher rate of freedom from atrial arrhythmias with and without the use of AAD compared with AF catheter ablation. Although the overall complication rate was low for both strategies, hybrid ablations had more complications $(13.8 \%$ vs. $5.9 \%) .{ }^{854}$ The difference in outcome could be explained by a longlasting isolation of the pulmonary veins after bipolar radiofrequency clamping of the pulmonary veins, epicardial clipping of the LAA, and the add-on possibility of an endocardial touch-up. ${ }^{855,856}$

\section{Recommendations for surgical ablation of AF}

Recommendations
$\begin{aligned} & \text { Concomitant AF ablation should be considered } \\ & \text { in patients undergoing cardiac surgery, balancing }\end{aligned}$
the benefits of freedom from atrial arrhythmias
and the risk factors for recurrence (left atrial
dilatation, years in AF, age, renal dysfunction, and
other cardiovascular risk factors).
Thi,843,857-859
Thoracoscopic-including hybrid surgical abla-
tion-procedures should be considered in
patients who have symptomatic paroxysmal or
persistent AF refractory to AAD therapy and
have failed percutaneous AF ablation, or with
evident risk factors for catheter ablation failure,
to maintain long-term sinus rhythm. The decision
must be supported by an experienced team of
electrophysiologists and surgeons.

$\mathrm{AAD}=$ antiarrhythmic drug; $\mathrm{AF}=$ atrial fibrillation.

${ }^{a}$ Class of recommendation.

bLevel of evidence. 
10.2.2.6 Peri-procedural stroke risk management in patients undergoing rhythm control interventions

10.2.2.6.1 Management of stroke risk and oral anticoagulant therapy in atrial fibrillation patients undergoing cardioversion. Patients undergoing cardioversion of $\mathrm{AF}$ are at increased risk of stroke and thromboembolism, especially in the absence of OAC and if AF has been present for $\geq 12 \mathrm{~h}^{860-862}$ The exact duration of an AF episode before cardioversion may be difficult to ascertain, as many patients develop AF asymptomatically, seeking help only when symptoms or complications occur. If there is uncertainty over the exact onset of $A F$ (i.e. unknown duration of $A F$ ), peri-cardioversion anticoagulation is managed as for AF of $>12 \mathrm{~h}$ to $24 \mathrm{~h}$. Mechanisms of the increased propensity to peri-cardioversion thrombo-embolism include the presence of pre-existing thrombus (especially if not anticoagulated), change in the atrial mechanical function with restoration of sinus rhythm, atrial stunning post-cardioversion, and a transient prothrombotic state. ${ }^{863}$

No RCT has evaluated anticoagulation vs. no anticoagulation in AF patients undergoing cardioversion with a definite duration of $\mathrm{AF}<48$ h. Observational data suggest that the risk of stroke/ thrombo-embolism is very low $(0-0.2 \%)$ in patients with a definite $\mathrm{AF}$ duration of $<12 \mathrm{~h}$ and a very low stroke risk $\left(\mathrm{CHA}_{2} \mathrm{DS}_{2}\right.$-VASc 0 in men, 1 in women), ${ }^{860,864,865}$ in whom the benefit of 4-week anticoagulation after cardioversion is undefined and the prescription of anticoagulants can be optional, based on an individualized approach.

Peri-cardioversion anticoagulation with a VKA results in a significant decrease of stroke and thrombo-embolism, ${ }^{863}$ but achieving the necessary therapeutic anticoagulation (INR 2.0 - 3.0) for a minimum of 3 weeks before cardioversion may be difficult. This 3 -week period is arbitrary, based on the time presumably needed for endothelialization or resolution of pre-existing AF thrombus. To shorten this time, TOE-guided cardioversion was introduced. If there is no atrial thrombus on TOE, cardioversion is performed after administration of heparin, and OAC is continued post-cardioversion. ${ }^{866,867}$

As NOACs act rapidly, cardioversion can be scheduled 3 weeks after NOAC initiation, provided that patients are counselled about the need for compliance to NOAC therapy ${ }^{868-870}$; NOACs have at least comparable efficacy and safety to warfarin in AF patients undergoing cardioversion. ${ }^{871-874}$ A review of the three largest prospective trials ( $n=5203$ patients) showed that the composite primary outcome (stroke/systemic embolism, myocardial infarction, or cardiovascular death) was significantly reduced with NOACs compared with VKA. ${ }^{873}$

Long-term OAC therapy after cardioversion should not be based on successful restoration of sinus rhythm, but on the stroke risk profile (using the $\mathrm{CHA}_{2} \mathrm{DS}_{2}$-VASc score), balanced against bleeding risk (e.g. HAS-BLED score).

For patients in whom a thrombus is identified on TOE, effective anticoagulation for at least 3 weeks before reassessment for cardioversion is recommended. A repeat TOE to ensure thrombus resolution should be considered before cardioversion. ${ }^{875}$ Antithrombotic management for these patients is challenging and decided on an individual basis based on the efficacy (or inefficacy) of previous treatments.

\section{Recommendations for stroke risk management peri- cardioversion} NOACs are recommended with at least similar efficacy and safety to warfarin. ${ }^{868-873}$

For cardioversion of AF/AFL, effective anticoagulation is recommended for a minimum of 3 weeks before cardioversion. ${ }^{866-870}$

TOE is recommended to exclude cardiac thrombus as an alternative to 3-week pre-procedural anticoagulation when early cardioversion is planned. ${ }^{866,868-870,875}$

In patients at risk of stroke, it is recommended that $O A C$ therapy is continued long term after cardioversion according to the long-term anticoagulation recommendations, irrespective of the method of cardioversion, the apparent maintenance of sinus rhythm, or characterization of AF as a 'first-diagnosed episode'. ${ }^{412,872,876}$

When thrombus is identified on TOE, effective anticoagulation is recommended for at least 3 weeks before cardioversion of $A F^{875}$

It is recommended that the importance of adherence and persistence to NOAC treatment both before and after cardioversion is strongly emphasized to patients.

Effective anticoagulation should be initiated as soon as possible before every cardioversion of AF or AFL. ${ }^{866-870}$

Early cardioversion can be performed without TOE in patients with an AF duration of $<48 \mathrm{~h} .^{866}$ In patients with AF duration of $>24 \mathrm{~h}$ undergoing cardioversion, therapeutic anticoagulation should be continued for at least 4 weeks, even after successful cardioversion to sinus rhythm (beyond 4 weeks, the decision about long-term OAC treatment is determined by the presence of stroke risk factors). ${ }^{860,861}$

When thrombus is identified on TOE, a repeat TOE to ensure thrombus resolution should be considered before cardioversion. ${ }^{875}$

In patients with a definite duration of $\mathrm{AF} \leq 24 \mathrm{~h}$ and a very low stroke risk $\left(\mathrm{CHA}_{2} \mathrm{DS}_{2}\right.$-VASc of 0 in men or 1 in women) post-cardioversion anticoagulation for 4 weeks may be omitted. ${ }^{871,876}$

\section{Recommendations}

In patients with $\mathrm{AF}$ undergoing cardioversion,

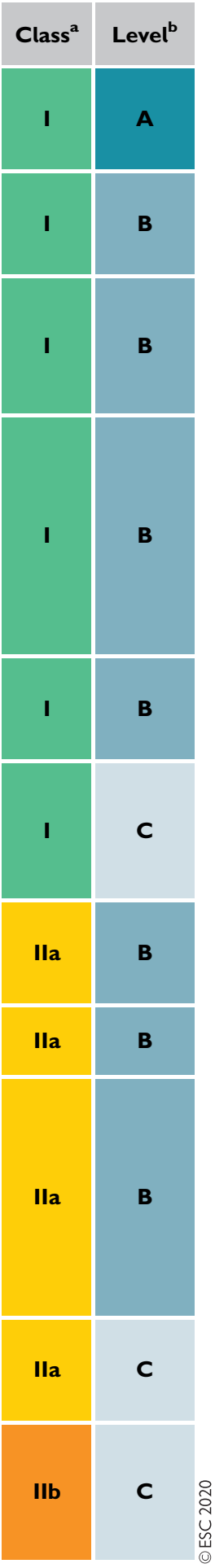

$\mathrm{AF}=$ atrial fibrillation; $\mathrm{AFL}=$ atrial flutter; $\mathrm{CHA}_{2} \mathrm{DS}_{2}-\mathrm{VASc}=$ Congestive heart failure, Hypertension, Age $\geq 75$ years, Diabetes mellitus, Stroke, Vascular disease, Age 65 - 74 years, Sex category (female); NOAC = non-vitamin $\mathrm{K}$ antagonist oral anticoagulant; $\mathrm{OAC}=$ oral anticoagulant; $\mathrm{TOE}=$ transoesophageal echocardiography.

${ }^{\text {a }}$ Class of recommendation.

bLevel of evidence. 
10.2.2.6.2 Management of stroke risk and oral anticoagulant therapy in atrial fibrillation patients undergoing atrial fibrillation catheter ablation. Although there is some variability in the peri-procedural OAC management in patients undergoing AF ablation, more recently operators have moved towards a strategy of performing the ablation under uninterrupted VKA or NOAC treatment, provided the INR is within therapeutic range. In non-anticoagulated patients, initiating therapeutic anticoagulation 3 - 4 weeks before ablation may be considered. ${ }^{1}$

In a meta-analysis of 12 studies, ${ }^{877}$ uninterrupted anticoagulation using NOACs vs. VKAs for AF catheter ablation was associated with low rates of stroke/TIA (NOACs, $0.08 \%$; VKA, 0.16\%) and similar rates of silent cerebral embolic events ( $8.0 \%$ vs $9.6 \%$ ). However, major bleeding was significantly reduced with uninterrupted NOACs (0.9\%) compared with VKAs (2\%).

In the largest RCT comparing peri-procedural NOAC vs. warfarin [the RE-CIRCUIT trial (Randomized Evaluation of dabigatran etexilate Compared to warfarln in pulmonaRy vein ablation: assessment of different peri-proCedUral antlcoagulation sTrategies)], ${ }^{878}$ the incidence of major bleeding events during and up to 8 weeks after ablation was significantly lower with dabigatran vs. warfarin (1.6\% vs. 6.9\%). Other RCTs (VENTURE-AF with rivaroxaban, ${ }^{879}$ AXAFA-AF NET 5 with apixaban, ${ }^{880}$ and ELIMINATE-AF with edoxaban ${ }^{881}$ ) also showed similar event rates under uninterrupted NOACs vs. VKAs. Overall, uninterrupted peri-procedural NOACs were associated with a low incidence of stroke/TIA and a significant reduction in major bleeding compared with uninterrupted VKAs in patients undergoing $\mathrm{AF}$ catheter ablation. In contrast, heparin bridging increases the bleeding risk and should be avoided.

Frequently, the term 'uninterrupted' is used in clinical practice for the description of regimens where one or two NOAC doses are omitted before ablation, whereas in the RCTs comparing uninterrupted NOACs vs. warfarin, NOAC administration before ablation was truly uninterrupted. ${ }^{869,878}$ Hence, there is no reason to recommend omitting one or two NOAC doses before ablation. After the procedure, administration of the first dose the evening after ablation or the next morning (if this corresponds to the timing of the next dose according to the patient's previous $O A C$ regimen) appears to be safe. $^{878,881}$

10.2.2.6.3 Postoperative anticoagulation after surgery for atrial fibrillation. Owing to endothelial damage during ablation, OAC is advisable in all patients after AF surgery, starting as soon as possible (balancing the risk of postoperative bleeding). There are no RCT data regarding interruption of OAC over the long term. Non-randomized studies with longer follow-up have shown better long-term freedom from stroke in patients with persistent sinus rhythm, but not in those with AF despite LAA exclusion. ${ }^{824}$ Therefore, long-term OAC is recommended in all patients at risk of stroke despite a successful maze surgery and appendage closure.

\section{Recommendations for postoperative anticoagulation after AF surgery}

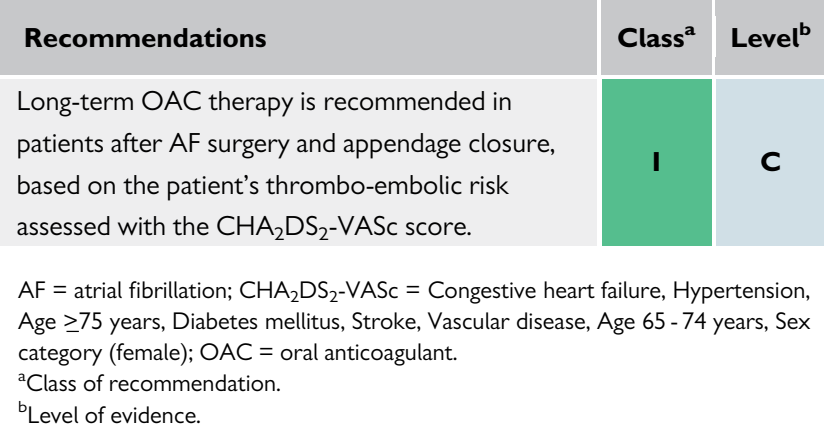

\subsubsection{Long-term antiarrhythmic drug therapy for rhythm control}

10.2.2.7.1 Antiarrhythmic drugs. The aim of AAD therapy is to improve AF-related symptoms. ${ }^{484,882,883}$ Hence, the decision to initiate long-term AAD therapy needs to balance symptom burden, possible adverse drug reactions, and patient preferences. The principles of AAD therapy are shown in Tables 18 and 19.

Compared with no therapy, AAD therapy approximately doubles sinus rhythm maintenance, ${ }^{883}$ but it is difficult to draw firm

\section{Recommendations for stroke risk management peri-catheter ablation}

\section{Recommendations}

In AF patients with stroke risk factors not taking OAC before ablation, it is recommended that pre-procedural management of stroke risk includes initiation of anticoagulation and:

- Preferably, therapeutic OAC for at least 3 weeks before ablation, or

- Alternatively, the use of TOE to exclude LA thrombus before ablation.

For patients undergoing AF catheter ablation who have been therapeutically anticoagulated with warfarin, dabigatran, rivaroxaban, apixaban, or edoxaban, performance of the ablation procedure without OAC interruption is recommended. ${ }^{878,879,881}$

After AF catheter ablation, it is recommended that:

- Systemic anticoagulation with warfarin or a NOAC is continued for at least 2 months post ablation, and

- Long-term continuation of systemic anticoagulation beyond 2 months post ablation is based on the patient's stroke risk profile and not on the apparent success or failure of the ablation procedure.

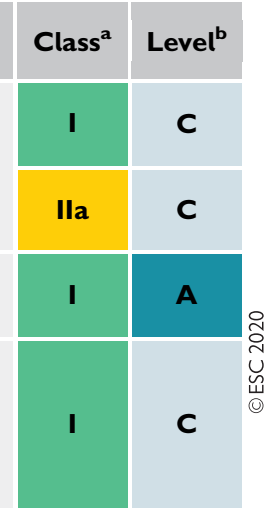

$\mathrm{AF}=$ atrial fibrillation; $\mathrm{LA}=$ left atrial; NOAC = non-vitamin $\mathrm{K}$ antagonist oral anticoagulant; $\mathrm{OAC}=$ oral anticoagulant therapy; TOE=transoesophageal echocardiography

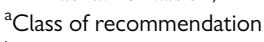

bevel of evidence 
conclusions from existing trials on their comparative efficacy. ${ }^{884}$ In general, AAD therapy is less effective than AF catheter ablation, ${ }^{114,611,615}$ but previously ineffective AADs can be continued after $\mathrm{PVI}$, to reduce recurrent $A F^{805} \mathrm{~A}$ shorter duration of $\mathrm{AAD}$ therapy would likely reduce the risk of side-effects ${ }^{883,885}$ but late recurrences may occur. ${ }^{595}$ Short-term AAD therapy is also used to prevent early AF recurrences after catheter ablation, ${ }^{886}$ although the benefit is still debated ${ }^{797,887}$; this strategy may be reasonable in patients deemed at increased risk of AAD side-effects or in those with a low perceived risk of recurrent AF. Concomitant management of underlying

\section{Table I 8 Principles of antiarrhythmic drug therapy ${ }^{143}$}

Principles
AAD therapy aims to reduce AF-related symptoms
Efficacy of AADs to maintain sinus rhythm is modest
Clinically successful AAD therapy may reduce rather than eliminate AF
recurrences
If one AAD 'fails', a clinically acceptable response may be achieved by
another drug
Drug-induced proarrhythmia or extracardiac side-effects are frequent
Safety rather than efficacy considerations should primarily guide the
choice of AAD
AAD = antiarrhythmic drug; AF = atrial fibrillation.

cardiovascular conditions is pivotal to reduce AF symptom burden and facilitate the maintenance of sinus rhythm. ${ }^{245,636,888,889}$

10.2.2.7.1 Available antiarrhythmic drugs. Several AADs have been shown to reduce AF recurrences (Table 20). ${ }^{890}$ Class la (quinidine and disopyramide) and sotalol have been associated with increased overall mortality. ${ }^{884}$ Again, safety should dictate both the initiation and continuation of AADs.

A flow chart for use of AADs for long-term rhythm control, depending on the underlying disease, is given in Figure 19.

10.2.2.7.2 Non-antiarrhythmic drugs with antiarrhythmic properties (upstream therapy). Either resulting from, or being a marker of structural atrial remodelling, $A F$ is closely related to atrial cardiomyopathy. Drugs that affect the atrial-remodelling process could prevent newonset AF acting as non-conventional AADs (i.e. upstream therapy) (Table 21).

Recently, the RACE 3 study $^{245}$ confirmed the importance of assessing underlying conditions and targeted upstream therapy for intense risk-factor control in AF patients with mild or moderate HF in optimizing rhythm control. The results showed that targeted therapy of underlying conditions improves maintenance of sinus rhythm in patients with persistent AF.

A list of new investigational antiarrhythmic drugs is provided in Supplementary Box 3.

\section{Table 19 Rules to initiate antiarrhythmic drugs for long-term rhythm control in AF}

\begin{tabular}{|c|c|}
\hline Consideration & Criteria \\
\hline Indication for AAD & $\begin{array}{l}\text { - Is the patient symptomatic? } \\
\text { - Are AF symptoms severe enough (EHRA class) to justify AAD use? } \\
\text { - Are there associated conditions predicting poor tolerance of AF episodes? }\end{array}$ \\
\hline When to start AAD & - Usually not for the first episode, but it may enhance efficacy of cardioversion \\
\hline How to choose among AADs & $\begin{array}{l}\text { - Minimize proarrhythmic risk and organ toxicity } \\
\text { Evaluate for: } \\
\text { - basal ECG abnormalities (QRS duration, PR, QTc) and possible interference with AAD } \\
\text { - impact on LV function } \\
\text { - important pharmacokinetic and pharmacodynamic interactions (i.e. antithrombotic drugs) } \\
\text { - Risk factors for proarrhythmia may be dynamic and change over time }\end{array}$ \\
\hline How to minimize proarrhythmic risk & $\begin{array}{l}\text { - Evaluate ECG after the treatment, as indicated in these Guidelines } \\
\text { - Evaluate periodically for organ toxicity (amiodarone) } \\
\text { - Long-term Holter monitoring and exercise test in selected cases } \\
\text { - Avoid AAD combinations }\end{array}$ \\
\hline How to verify efficacy & $\begin{array}{l}\text { - Estimate AF burden under therapy (ask patient for noting episodes) } \\
\text { - If the patient is already on AAD and it was effective but was stopped because of intolerance, } \\
\text { choose preferably from the same class }\end{array}$ \\
\hline Adjuvant interventions and hybrid therapy & $\begin{array}{l}\text { - In patients with atrioventricular conduction abnormalities and/or sinus node dysfunction, } \\
\text { pacemaker implantation should be considered if AAD therapy is deemed necessary } \\
\text { - Short-term AAD therapy could prevent early recurrences after AF ablation }\end{array}$ \\
\hline
\end{tabular}

$\mathrm{AAD}=$ antiarrhythmic drug; $\mathrm{AF}=$ atrial fibrillation; $\mathrm{ECG}=$ electrocardiogram; $\mathrm{EHRA}=$ European Heart Rhythm Association; $\mathrm{LV}=$ left ventricular; $\mathrm{PR}=\mathrm{PR}$ interval; $\mathrm{QRS}=$ QRS interval; QTc = corrected QT interval. 


\section{Table 20 Antiarrhythmic drugs used for long-term maintenance of sinus rhythm in AF patients ${ }^{890}$}

\begin{tabular}{|c|c|c|c|}
\hline Drug & $\begin{array}{l}\text { Administration } \\
\text { route }\end{array}$ & Dose & Contraindications/precautions/comments \\
\hline Amiodarone $\mathrm{e}^{233,506,891-896}$ & Oral & $\begin{array}{l}3 \times 200 \mathrm{mg} \text { daily over } \\
4 \text { weeks, then } 200 \mathrm{mg} \\
\text { daily }^{506}\end{array}$ & 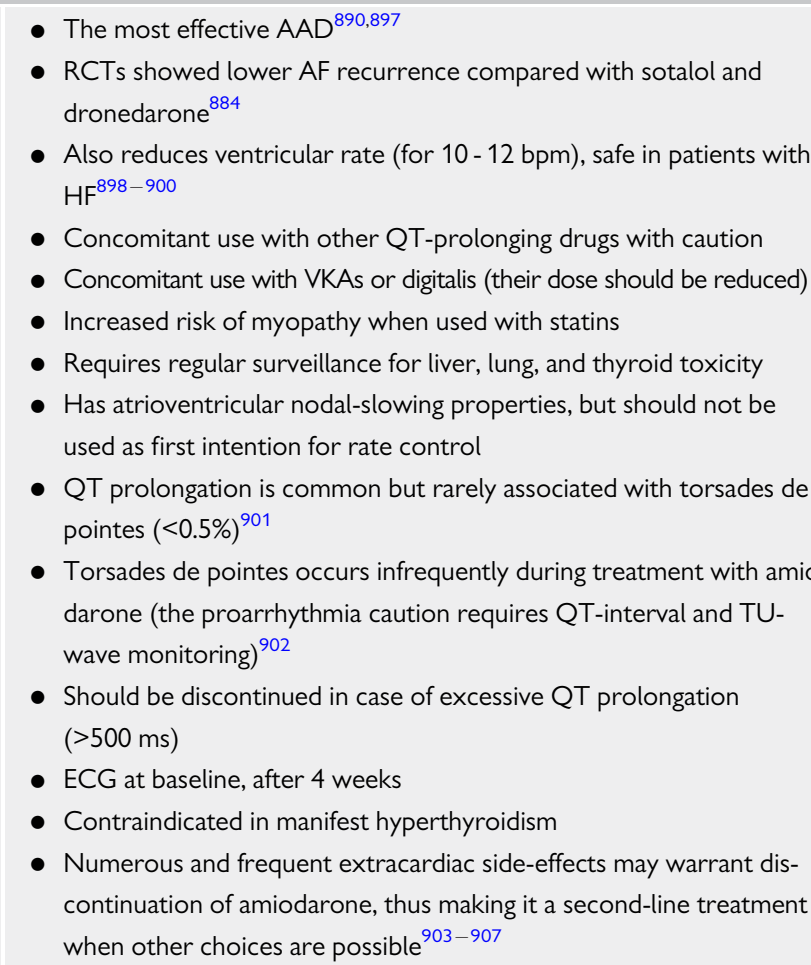 \\
\hline $\begin{array}{l}\text { Flecainide } \\
\text { Flecainide slow release }{ }^{896,908,909}\end{array}$ & Oral & $\begin{array}{l}100 \text { - } 200 \text { mg b.i.d., or } \\
200 \text { mg once daily (fle- } \\
\text { cainide slow release) }\end{array}$ & $\begin{array}{l}\text { - Effective in preventing recurrence of } \mathrm{AF}^{891,908,910} \\
\text { - Should not be used in patients with } \mathrm{CrCl}<35 \mathrm{~mL} / \mathrm{min} / 1.73 \mathrm{~m}^{2} \text { and } \\
\text { - Bignificant liver disease } \\
\text { - Both are contraindicated in patients with ischaemic heart disease or } \\
\text { reduced } \mathrm{LVEF}^{911-913} \\
\text { - Should be discontinued in case of } \mathrm{QRS} \text { widening }>25 \% \text { above base- } \\
\text { line and patients with left bundle-branch block or any other conduc } \\
\text { tion block }>120 \text { ms } \\
\text { - Caution when sinoatrial/atrioventricular conduction disturbances } \\
\text { present }{ }^{\mathrm{a}} \\
\text { - CYP2D6 inhibitors increase concentration } \\
\text { - May increase AFL cycle length, thus promoting } 1: 1 \text { atrioventricular } \\
\text { conduction and increasing ventricular rate. }{ }^{914} \text { This risk can be } \\
\text { reduced by concomitant administration of an atrioventricular nodal } \\
\text { blocking drug such as a beta-blocker or NDCC } \\
\text { - In patients properly screened for propensity to proarrhythmias, bo } \\
\text { flecainide and propafenone are associated with a low proarrhythmi } \\
\text { risk }{ }^{915} \\
\text { - ECG at baseline, after } 1 \text { - } 2 \text { weeks }\end{array}$ \\
\hline $\begin{array}{l}\text { Propafenone } \\
\text { Propafenone slow } \\
\text { release }^{895,896,916-922}\end{array}$ & Oral & $\begin{array}{l}150 \text { - } 300 \text { mg three } \\
\text { times daily, or } \\
225-425 \mathrm{mg} \text { b.i.d. } \\
\text { (propafenone slow } \\
\text { release) }\end{array}$ & $\begin{array}{l}\text { - Should not be used in patients with significant renal or liver disease } \\
\text { ischaemic heart disease, reduced LV systolic function, or asthma } \\
\text { - Should be discontinued in case of QRS widening }>25 \% \text { above base- } \\
\text { line and in patients left bundle-branch block and any other conduc- } \\
\text { tion block }>120 \mathrm{~ms} \\
\text { - Caution when sinoatrial/atrioventricular conduction disturbances } \\
\text { present }^{\text {a }} \\
\text { - Increases concentration of warfarin/acenocoumarin and digoxin } \\
\text { when used in combination } \\
\text { - May increase AFL cycle length, thus promoting 1:1 atrioventricular } \\
\text { conduction and increasing ventricular rate } \\
\text { - ECG at baseline and after } 1 \text { - } 2 \text { weeks }\end{array}$ \\
\hline
\end{tabular}


Table 20 Continued

\begin{tabular}{|c|c|c|c|}
\hline Drug & $\begin{array}{l}\text { Administration } \\
\text { route }\end{array}$ & Dose & Contraindications/precautions/comments \\
\hline Dronedarone $923-927$ & Oral & 400 mg b.i.d. & 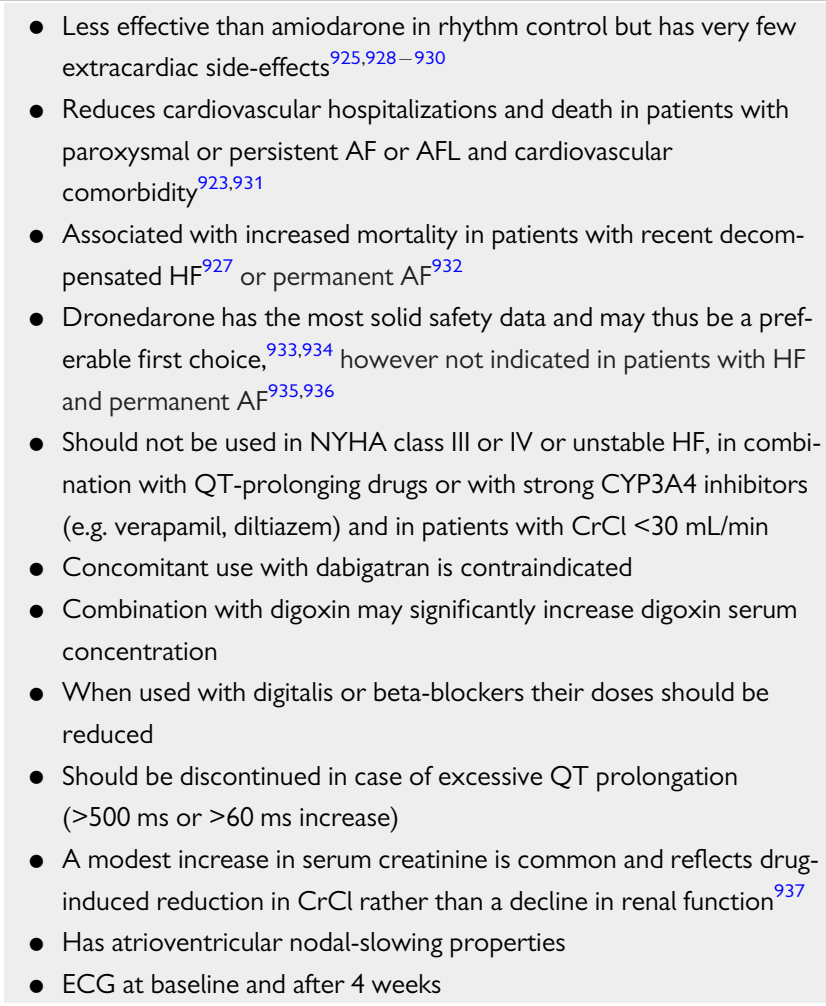 \\
\hline $\begin{array}{l}\text { Sotalol ( } d, 1 \text { racemic } \\
\text { mixture) } 233,891,894,895,920,938-940\end{array}$ & Oral & 80 - 160 mg b.i.d. & $\begin{array}{l}\text { - Only class III effects if dosing }>160 \mathrm{mg} \text { daily } \\
\text { - Considering its safety and efficacy and potential drug alternatives, } \\
\text { sotalol should be used with a caution } \\
\text { - Should not be used in patients with } \mathrm{HFrEF} \text {, significant LVH, pro- } \\
\text { longed QT, asthma, hypokalaemia, or } \mathrm{CrCl}<30 \mathrm{~mL} / \mathrm{min} \\
\text { - Dose-related torsades de pointes may occur in }>2 \% \text { of patients }{ }^{941} \\
\text { - Should be discontinued in case of excessive QT prolongation } \\
\text { (>500 ms or }>60 \text { ms increase) } \\
\text { - The potassium channel-blocking effect increases with increasing dose } \\
\text { and, consequently, the risk of ventricular proarrhythmia (torsades de } \\
\text { pointes) increases } \\
\text { Observational data and a recent meta-analysis revealed a correlation } \\
\text { with an increased all-cause mortality } 890,897,934 \text {, whereas a nationwide } \\
\text { registry analysis and two RCTs found no evidence for increased } \\
\text { safety concerns with sotalol }{ }^{233,933,942,943} \\
\text { ECG at baseline, after } 1 \text { day and after } 1 \text { - } 2 \text { weeks }\end{array}$ \\
\hline Disopyramide $944-946$ & Oral & $\begin{array}{l}100 \text { - } 400 \text { mg two or } \\
\text { t.i.d. (maximum } 800 \text { mg/ } \\
24 \text { h) }\end{array}$ & $\begin{array}{l}\text { - Associated with significantly increased mortality }{ }^{890,947} \text {, and rarely } \\
\text { used for rhythm control in AF. }{ }^{948,949} \text { Should not be used in patients } \\
\text { with a structural heart disease. Rarely used for rhythm control in AF } \\
\text { patients, due to increased mortality and frequent intolerance to side- } \\
\text { effects } \\
\text { - May be useful in 'vagal' AF occurring in athletes or during sleep } \\
\text { - Reduces LV outflow obstruction and symptoms in patients with } \\
\text { HCM }^{950}\end{array}$ \\
\hline
\end{tabular}

$\mathrm{AAD}=$ antiarrhythmic drug; $\mathrm{AF}=$ atrial fibrillation; $\mathrm{AFL}=$ atrial flutter; b.i.d. $=$ bis in die (twice a day); bpm = beats per minute; $\mathrm{CrCl}=\mathrm{creatinine}$ clearance; $\mathrm{CYP2D6}=\mathrm{cyto}-$ chrome P450 2D6; CYP34A = cytochrome 34A; ECG=electrocardiogram; HCM = hypertrophic cardiomyopathy; HF = heart failure; HFrEF = HF with reduced ejection fraction; LV = left ventricular; LVEF = LV ejection fraction; LVH = LV hypertrophy; NDCC = non-dihydropyridinecalcium-channel blocker; NYHA = New York Heart Association; QRS = QRS interval; QT = QT interval; RCT=randomized controlled trial; SBP = systolic blood pressure; t.i.d. = ter in die (three times a day); VKA = vitamin K antagonist. ${ }^{a}$ Caution is needed when using any AAD in patients with conduction-system disease (e.g. sinoatrial or atrioventricular node disease). 


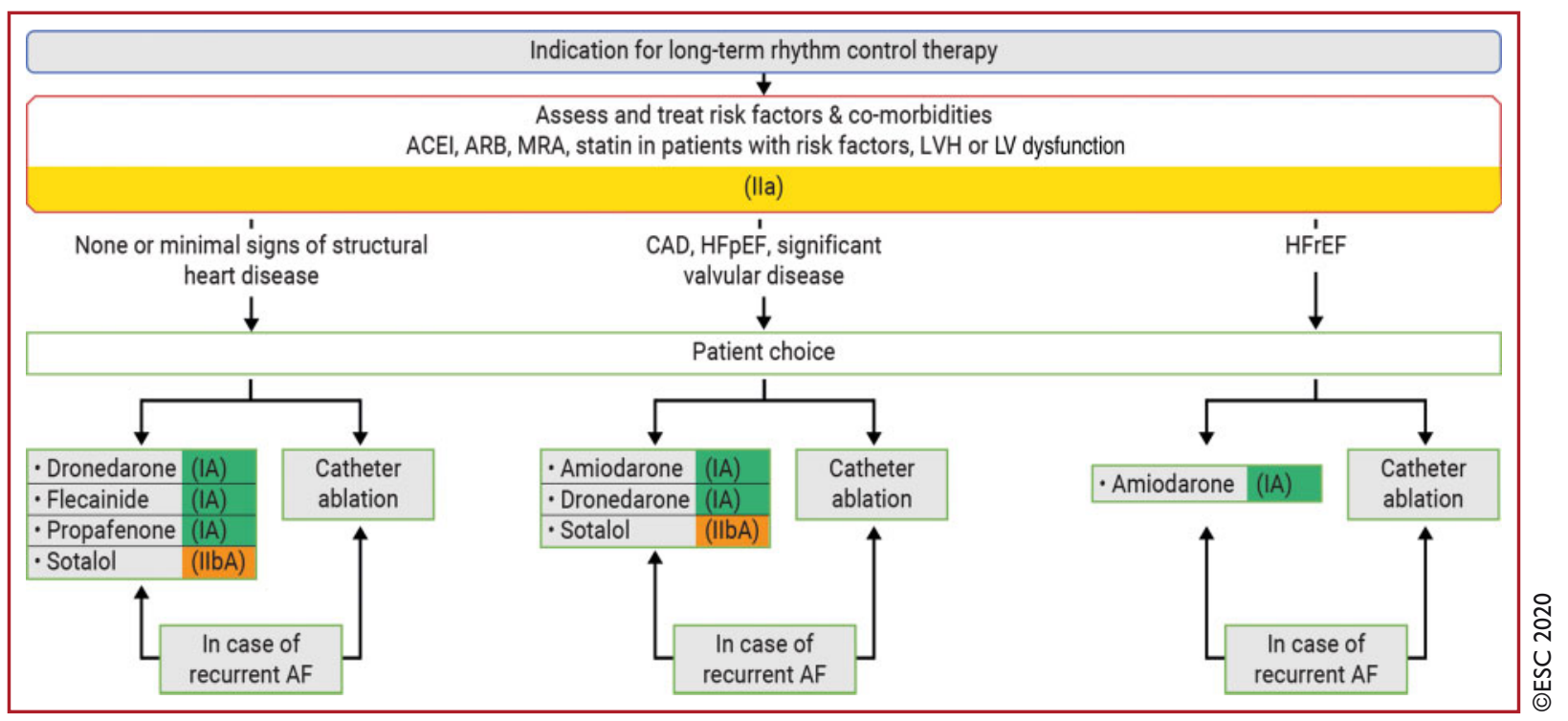

Figure 19 Long-term rhythm control therapy. $A C E i=$ angiotensin converting enzyme inhibitor; $A F=$ atrial fibrillation; $A R B=$ angiotensin receptor blocker; $\mathrm{CAD}=$ coronary artery disease; $\mathrm{HFpEF}=$ heart failure with preserved ejection fraction; $\mathrm{HFrEF}=$ heart failure with reduced ejection fraction; $\mathrm{LV}=$ left ventricular; $\mathrm{LVH}=$ left ventricular hypertrophy; $\mathrm{MRA}=$ mineralocorticoid receptor antagonist.

\section{Table 2 I Non-antiarrhythmic drugs with antiarrhythmic properties (upstream therapy)}

\begin{tabular}{|c|c|}
\hline Drugs & Comment \\
\hline \multirow[t]{3}{*}{ ACEi, ARBs } & $\begin{array}{l}\text { Activated renin-angiotensin-aldosterone system is up-regulated in AF. }{ }^{951,952} \mathrm{ACEi} \text { and } \mathrm{ARB} \text { showed encouraging results in preventing } \\
\text { AF in preclinical studies. }\end{array}$ \\
\hline & $\begin{array}{l}\text { As suggested by retrospective analyses and studies where AF was a prespecified secondary endpoint, ACEi/ARBs could prevent new- } \\
\text { onset } A F \text { in patients with LV dysfunction, LVH, or hypertension. }\end{array}$ \\
\hline & $\begin{array}{l}\text { As initial treatment, } A C E i \text { and ARBs seem to be superior to other antihypertensive regimens, }{ }^{962} \text { but } A R B s \text { did not reduce } A F \text { burden } \\
\text { in patients without structural heart disease. }{ }^{963} \text { Despite several positive small-scale prospective studies and retrospective analyses, } \\
\text { larger RCTs have shown controversial results and failed to confirm the role of ACEi or ARBs in secondary (post-cardioversion) pre- } \\
\text { vention of AF. }{ }^{964} \text { The multifactorial pathways for AF promotion and study design could explain these negative results and should not } \\
\text { discourage the use of ACEi or ARB to AAD in patients with structural heart disease. }\end{array}$ \\
\hline \multirow[t]{2}{*}{ MRAs } & $\begin{array}{l}\text { Aldosterone is implicated in inducibility and perpetuation of AF. }{ }^{965-967} \text { Evidence from RCTs showed that MRAs reduced new-onset } \\
\text { atrial arrhythmias in patients with HFrEF in parallel with improvement of other cardiovascular outcomes. }{ }^{968,969}\end{array}$ \\
\hline & $\begin{array}{l}\text { Recently, the positive impact of MRAs was also shown in patients with } \mathrm{HFpEF}^{970} \text { irrespective of baseline } A F \text { status. Regarding other } \\
\text { renin-angiotensin-aldosterone system inhibitors, the role of MRAs as upstream therapy in rhythm control strategy for patients with } \\
\mathrm{HF} \text { and AF has not been clarified. As AF is a marker of HF severity, the beneficial antiarrhythmic effect could be driven indirectly, } \\
\text { through improvement of HF. A recent meta-analysis showed that MRAs significantly reduced new-onset AF and recurrent AF, but not } \\
\text { postoperative AF. } 971\end{array}$ \\
\hline Beta-blockers & $\begin{array}{l}\text { Several small studies suggested a lower AF recurrence rate with beta-blockers, with a comparable efficacy with sotalol. } \\
\text { However, most evidence pleads against a significant role of beta-blockers in preventing AF. }{ }^{890} \text { The observed beneficial effect could } \\
\text { also result from transformation of clinically manifested AF to silent AF, because of the rate control with beta-blockers. }\end{array}$ \\
\hline Statins & $\begin{array}{l}\text { Statins are attractive candidates for upstream therapy, as the role of inflammation in AF is well established. However, in an adequately } \\
\text { designed RCT, }{ }^{974} \text { statins failed to show a beneficial effect, and their preventive effect was not confirmed in other settings. }{ }^{975,976} \\
\text { Specific patient groups in whom statins could induce reverse remodelling are not identified yet, but findings from the CARAF registry } \\
\text { suggested that AF patients already on beta-blockers could benefit from statin therapy. }{ }^{977} \text { Polyunsaturated fatty acids also failed to } \\
\text { show convincing benefit in preventing AF. }\end{array}$ \\
\hline
\end{tabular}

$\mathrm{AAD}=$ antiarrhythmic drug; $\mathrm{ACE}$ = angiotensin converting enzyme inhibitor; $\mathrm{AF}=$ atrial fibrillation; $\mathrm{ARB}=$ angiotensin receptor blocker; $\mathrm{CARAF}=\mathrm{Canadian}$ Registry of $\mathrm{Atrial}$ Fibrillation; $\mathrm{HF}=$ heart failure; HFrEF = HF with reduced ejection fraction; HFpEF = HF with preserved ejection fraction; LV = left ventricular; LVH = LV hypertrophy; MRA = mineralocorticoid receptor antagonist; RCT = randomized controlled trial. 


\section{Recommendations for long-term antiarrhythmic drugs}

\begin{tabular}{|c|c|c|}
\hline Recommendations & Class $^{a}$ & Level $^{\mathrm{b}}$ \\
\hline $\begin{array}{l}\text { Amiodarone is recommended for long-term rhythm control in all AF patients, including those with HFrEF. However, owing to its } \\
\text { extracardiac toxicity, other AADs should be considered first whenever possible. }{ }^{233,570,884,942,983,985}\end{array}$ & I & $\mathbf{A}$ \\
\hline $\begin{array}{l}\text { Dronedarone is recommended for long-term rhythm control in AF patients with: } \\
\text { - Normal or mildly impaired (but stable) LV function, or } \\
\text { - HFpEF, ischaemic, or VHD. }{ }^{884,923,925,985}\end{array}$ & I & $\mathbf{A}$ \\
\hline $\begin{array}{l}\text { Flecainide or propafenone is recommended for long-term rhythm control in AF patients with normal LV function and without struc- } \\
\text { tural heart disease, including significant LVH and myocardial ischaemia. } 594,884,910,942,983,984\end{array}$ & I & $\mathbf{A}$ \\
\hline $\begin{array}{l}\text { In AF patients treated with sotalol, close monitoring of QT interval, serum potassium levels, } \mathrm{CrCl} \text {, and other proarrhythmia risk fac- } \\
\text { tors is recommended. }{ }^{884,942}\end{array}$ & I & B \\
\hline $\begin{array}{l}\text { In AF patients treated with flecainide for long-term rhythm control, concomitant use of an atrioventricular nodal-blocking drug (if tol- } \\
\text { erated) should be considered. }\end{array}$ & Ila & C \\
\hline $\begin{array}{l}\text { Sotalol may be considered for long-term rhythm control in patients with normal LV function or with ischaemic heart disease if close } \\
\text { monitoring of QT interval, serum potassium levels, } \mathrm{CrCl} \text {, and other proarrhythmia risk factors is provided. }\end{array}$ & IIb & $\mathbf{A}$ \\
\hline $\begin{array}{l}\text { AAD therapy is not recommended in patients with permanent AF under rate control and in patients with advanced conduction dis- } \\
\text { turbances unless antibradycardia pacing is provided. }\end{array}$ & III & C \\
\hline
\end{tabular}

$\mathrm{AAD}=$ antiarrhythmic drug; $\mathrm{AF}=$ atrial fibrillation; $\mathrm{CrCl}=$ Creatinine clearance; $\mathrm{HFpEF}=$ heart failure with preserved ejection fraction; $\mathrm{HFrEF}=$ heart failure with reduced ejection fraction; LV = left ventricular; $L V H=L V$ hypertrophy; VHD = valvular heart disease.

${ }^{\mathrm{a} C}$ Class of recommendation.

bevel of evidence.

10.2.2.7.3 Assessment and long-term monitoring of the risk of proarrhythmia with antiarrhythmic drugs. A variety of clinical, echocardiographic, and ECG criteria have been associated with a higher risk of proarrhythmia. ${ }^{986-989}$ Increasing age, female sex, impaired renal and/ or liver function, and known CAD have been variously identified as associated with higher risk. ${ }^{890,990-992}$ Concomitant AAD use, hypokalaemia, or family history of sudden death have also been implicated. ${ }^{990}$ Proarrhythmic events tend to cluster shortly after drug initiation, especially if a loading dose or a change in usual dosage is prescribed. ${ }^{568}$ For quinidine, the risk is idiosyncratic independent of dosage. Impaired LV function and LVH are echocardiographic markers of increased proarrhythmic risk. Sotalol has a proarrhythmic risk even in the absence of structural heart disease. On the 12-lead ECG, prolonged corrected QT interval (QTc), widened QRS, and prolonged PR interval have all been associated with proarrhythmia. ${ }^{993-995}$ Significant ion-channel mutations have been detected in only a minority of cases of drug-induced torsade. ${ }^{996}$ Periodic ECG analysis for proarrhythmia signs has been used successfully in recent AAD trials ${ }^{594,997}$ Specifically, ECG monitoring was used systematically on days $1-3$ in patients receiving flecainide, propafenone, or sotalol to identify those at risk of proarrhythmia ${ }^{233,594,998}$ The role of routine use of exercise stress testing in patients commencing $1 \mathrm{C}$ drugs who had no evidence of structural heart disease is still debatable ${ }^{915,999}$

\section{3 ' $C$ ' - Cardiovascular risk factors and concomitant diseases: detection and management}

Cardiovascular risk-factor burden and comorbidities, including lifestyle factors and borderline conditions, significantly affect the lifetime risk for AF development (Supplementary Figure 5). The continuum of unhealthy lifestyle, risk factor(s), and cardiovascular disease can contribute to atrial remodelling/cardiomyopathy and development of AF that commonly results from a combined effect of multiple interacting factors (often without specific threshold values).

The 'C' component of the ABC pathway includes identification and management of concomitant diseases, cardiometabolic risk factors, and unhealthy lifestyle factors. Management of risk factors and cardiovascular disease complements stroke prevention and reduces AF burden and symptom severity. In a recent RCT, for example, targeted therapy of underlying conditions significantly improved maintenance of sinus rhythm in patients with persistent $\mathrm{AF}$ and $\mathrm{HF}^{245}$

Whereas strategies on comprehensive risk-factor modification and interventions targeting underlying conditions have shown reduction of AF burden and recurrence, studies addressing isolated management of specific conditions alone (e.g. hypertension) yielded inconsistent findings, ${ }^{1000}$ likely because the condition was not a sole contributor to AF.

\subsubsection{Lifestyle interventions}

\subsubsection{Obesity and weight loss}

Obesity increases the risk for AF progressively according to body mass index. ${ }^{366,1001-1005}$ It may also increase the risk for ischaemic stroke, thrombo-embolism, and death in AF patients, ${ }^{366}$ notwithstanding an obesity paradox in AF patients, especially regarding allcause and cardiovascular death, with an inverse relationship between overweight/obesity and better cardiovascular prognosis in long-term follow-up. ${ }^{1006}$

Intense weight reduction with comprehensive management of concomitant cardiovascular risk factors resulted in fewer AF recurrences and symptoms than general advice in obese patients with $\mathrm{AF}^{636,888,889}$ Achieving a healthy weight may reduce blood pressure (BP), dyslipidaemia, and risk of developing type 2 diabetes mellitus, 
thus improving the cardiovascular risk profile. ${ }^{1007}$ Obesity may increase AF recurrence rates after AF catheter ablation (with OSA as a potential confounder). ${ }^{638,643,789,1008}$ It has also been linked to a higher radiation dose and complication rate during $\mathrm{AF}$ ablation, ${ }^{1009,1010}$ whereas symptom improvement after AF catheter ablation seems comparable in obese and normal-weight patients. ${ }^{1008}$ Given the potential to reduce AF episodes by weight reduction, AF catheter ablation should be offered to obese patients in conjunction with lifestyle modifications for weight reduction (Figure 18).

\subsubsection{Alcohol and caffeine use}

Alcohol excess is a risk factor for incident $A F^{1011-1014}$ and bleeding $^{395}$ in anticoagulated patients (mediated by poor adherence, liver disease, variceal bleeding, and risk of major trauma), and high alcohol intake may be associated with thrombo-embolism or death. ${ }^{1015}$ In a recent $\mathrm{RCT}$, alcohol abstinence reduced arrhythmia recurrence in regular drinkers with $A F^{1016}$

By contrast, it is unlikely that caffeine consumption causes or contributes to AF. ${ }^{47}$ Habitual caffeine consumption might be associated with lower risk of $A F$, but caffeine intake may increase symptoms of palpitations unrelated to AF.

\subsubsection{Physical activity}

Many studies have demonstrated beneficial effects of moderate exercise/physical activity on cardiovascular health. ${ }^{1017-1019}$ Nevertheless, the incidence of AF appears to be increased among elite athletes, and multiple small studies reported a relationship between $A F$ and vigorous physical activity, mainly related to long-term or endurance sport participation. ${ }^{1020-1023}$ A non-linear relationship between physical activity and AF seems likely. Based on these data, patients should be encouraged to undertake moderate-intensity exercise and remain physically active to prevent $A F$ incidence or recurrence, but maybe avoid chronic excessive endurance exercise (such as marathons and long-distance triathlons, etc.), especially if aged $>50$ years. Owing to few randomized patients and outcomes, the effect of exercise-based cardiac rehabilitation on mortality or serious adverse events is uncertain. ${ }^{1024}$

\subsubsection{Specific cardiovascular risk factors/comorbidities}

\subsubsection{Hypertension}

Hypertension is the most common aetiological factor associated with the development of AF, and patients with hypertension have a 1.7-fold higher risk of developing AF compared with normotensives. ${ }^{26,1025}$

Hypertension also adds to the complications of AF, particularly stroke, $\mathrm{HF}$, and bleeding risk. AF patients with a longer hypertension duration or uncontrolled systolic BP (SBP) levels should be categorized as 'high-risk', and strict $\mathrm{BP}$ control in addition to $\mathrm{OAC}$ is important to reduce the risk of ischaemic stroke and $\mathrm{ICH}$.

Given the importance of hypertension as a precipitating factor for $\mathrm{AF}$, which should be regarded as a manifestation of hypertension target-organ damage, treatment of hypertension consistent with current $\mathrm{BP}$ guidelines ${ }^{1026}$ is mandatory in AF patients, aiming to achieve $\mathrm{BP} \leq 130 / 80 \mathrm{mmHg}$ to reduce adverse outcomes. ${ }^{338,1027,1028} \mathrm{~A}$ recent randomized trial in patients with paroxysmal $A F$ and hypertension reported fewer recurrences in patients undergoing renal denervation in addition to $\mathrm{PVI}$ compared with patients undergoing PVI only. ${ }^{1029}$ Sotalol should not be used in the presence of hypertensive LVH or renal impairment, owing to the risk of proarrhythmia. There is some evidence of angiotensin converting enzyme or angiotensin receptor blocker use to improve outcomes in AF or reduce progression of the arrhythmia $^{26,1025}$ Other lifestyle changes, including obesity management, alcohol reduction, and attention to OSA, may also help in patients with AF and hypertension.

\subsubsection{Heart failure}

The interactions between $\mathrm{AF}$ and $\mathrm{HF}$ and the optimal management of patients with both $\mathrm{AF}$ and $\mathrm{HF}$ are discussed in section 11.6.

\subsubsection{Coronary artery disease}

The interactions between $A F$ and $C A D$ and the optimal management of patients with both AF and CAD are discussed in section 11.3.

\subsubsection{Diabetes mellitus}

In addition to shared risk factors (e.g. hypertension and obesity), ${ }^{1004,1030}$ diabetes is an independent risk factor for AF, especially in young patients. ${ }^{1031}$ Silent AF episodes are favoured by concurrent autonomic dysfunction, ${ }^{1032}$ thus suggesting an opportunity for routine screening for $A F$ in diabetes mellitus patients. The prevalence of $A F$ is at least two-fold higher in patients with diabetes compared with people without diabetes, ${ }^{1033}$ and AF incidence rises with increasing severity of microvascular complications (retinopathy, renal disease). ${ }^{1034}$ Both type 1 and type 2 diabetes mellitus are the risk factors for stroke. ${ }^{342,1035}$

Intensive glycaemic control does not affect the rate of new-onset $A F,{ }^{1036}$ but metformin and pioglitazone could be associated with lower long-term risk of AF in patients with diabetes, ${ }^{1037}$ while this was not confirmed for rosiglitazone. ${ }^{1038}$ Currently there is no evidence that glucagon-like peptide-1 agonists, sodium glucose cotransporter-2 inhibitors, and dipeptidyl peptidase-4 inhibitors affect the development of AF. ${ }^{1039}$

Previous meta-analyses showed no significant interaction between diabetes mellitus and NOAC effects in AF patients, ${ }^{423,1040}$ but vascular mortality was lower in patients with diabetes treated with NOACs than in those on warfarin. ${ }^{1040}$ Bleeding risk reduction with NOACs was similar in diabetic and non-diabetic patients except for apixaban, where a lower reduction in haemorrhagic complications was reported in the AF patients with diabetes compared with AF patients without diabetes. ${ }^{1041}$ Regarding potential side-effects of OAC, there is no evidence that bleeding risk is increased in patients with diabetes and retinopathy. ${ }^{341}$

Optimal glycaemic control in 12 months before AF catheter ablation was associated with significant reduction in recurrent $A F$ after ablation. $^{1042}$

\subsubsection{Sleep apnoea}

The most common form of sleep-disordered breathing, OSA, is highly prevalent in patients with $\mathrm{AF}, \mathrm{HF}$, and hypertension, and is associated with increased risk of mortality or major cardiovascular events. $^{1043}$ In a prospective analysis, approximately $50 \%$ of AF patients had OSA compared with $32 \%$ of controls. ${ }^{1044}$ The mechanisms facilitating AF include intermittent nocturnal hypoxemia/hypercapnia, intrathoracic pressure shifts, sympathovagal imbalance, oxidative stress, inflammation, and neurohumoral activation. ${ }^{1045}$ OSA has been shown to reduce success rates of AADs, electrical cardioversion, and catheter ablation in AF. ${ }^{1045}$ 
Continuous positive airway pressure (CPAP) is the therapy of choice for OSA, and may ameliorate OSA effects on AF recurrences. ${ }^{1046,1047}$ Observational studies and meta-analyses showed that appropriate CPAP treatment of OSA may improve rhythm control in AF patients. ${ }^{648,649,1047-1051}$

It seems reasonable to test for OSA before the initiation of rhythm control therapy in symptomatic AF patients, with the aim to reduce symptomatic AF recurrences (Figure 18). In the ARREST-AF (Aggressive Risk Factor Reduction Study - Implication for AF) and LEGACY (Long-term Effect of Goal-directed weight management on an Atrial fibrillation Cohort: a 5-Year follow-up study) studies, an aggressive risk-factor reduction programme focusing on weight management, hyperlipidaemia, OSA, hypertension, diabetes, smoking cessation, and alcohol-intake reduction significantly reduced AF burden after PVI. 636,1052 However, it remains unclear how and when to test for OSA and implement OSA management in the standard work-up of AF patients.

\section{Recommendations for lifestyle interventions and man- agement of risk factors and concomitant diseases in patients with AF}

\section{Recommendations}

Identification and management of risk factors and concomitant diseases is recommended as an integral part of treatment in AF patients. ${ }^{888}$

Modification of unhealthy lifestyle and targeted therapy of intercurrent conditions is recommended to reduce AF burden and symptom severity. ${ }^{245,636,887,889,1016,1052}$

Opportunistic screening for $\mathrm{AF}$ is recommended in hypertensive patients. ${ }^{26,172,222}$

Attention to good BP control is recommended in AF patients with hypertension to reduce AF recurrences and risk of stroke and bleeding. ${ }^{26,1035}$

In obese patients with AF, weight loss together with management of other risk factors should be considered to reduce $A F$ incidence, $A F$ progression, AF recurrences, and symptoms. ${ }^{898,899,1011}$

Advice and management to avoid alcohol excess should be considered for AF prevention and in AF patients considered for OAC therapy. ${ }^{324,1012,1014,1016}$

Physical activity should be considered to help prevent AF incidence or recurrence, with the exception of excessive endurance exercise, which may promote AF. ${ }^{1027-1033,1063}$

Opportunistic screening for AF should be considered in patients with OSA. ${ }^{172}$

Optimal management of OSA may be considered, to reduce $A F$ incidence, $A F$ progression, AF recurrences, and symptoms. ${ }^{650,651,1047-1051}$

$\mathrm{AF}=$ atrial fibrillation; $\mathrm{BP}=$ blood pressure; $\mathrm{OAC}=$ oral anticoagulant; $\mathrm{OSA}=$ obstructive sleep apnoea.

${ }^{a}$ Class of recommendation.

bLevel of evidence.

\section{The ABC pathway in specific clinical settings/conditions/patient populations}

In this section, the management of $A F$ in patient populations with specific conditions is described. The principles of the $A B C$ pathway apply in these settings as well. Additionally, specific considerations are given for each of these special conditions and populations.

\subsection{Atrial fibrillation with haemodynamic instability}

Acute haemodynamic instability (i.e. syncope, acute pulmonary oedema, ongoing myocardial ischaemia, symptomatic hypotension, or cardiogenic shock) in AF patients presenting with a rapid ventricular rate requires prompt intervention. In severely compromised patients, emergency electrical cardioversion should be attempted without delay, and anticoagulation should be started as soon as possible.

In critically ill patients and those with severely impaired LV systolic function, AF is often precipitated/exacerbated by increased sympathetic tone, inotropes, and vasopressors, and rhythm control is often unsuccessful. It is important to identify and correct precipitating factors and secondary causes and optimize background treatment. Owing to their rate-controlling effect during exertion and increased sympathetic tone, rather than only at rest, beta-blockers are preferred over digitalis glycosides for ventricular rate control in AF. ${ }^{490}$ Beta-blockers and NDCC antagonists may exert a negative inotropic effect (the latter are contraindicated in HFrEF). Digoxin is often unsuccessful due to the increased sympathetic tone in these patients.

As conventional therapy is often ineffective or not well-tolerated, ${ }^{490}$ electrical cardioversion should always be considered, even as initial therapy, whereas intravenous amiodarone may be instituted for rate control (or potential cardioversion to sinus rhythm), with or without electrical cardioversion. ${ }^{504,514,515}$ Intravenous administration of amiodarone may lead to a further decrease in BP.

\section{Recommendations for management of AF with haemo-} dynamic instability

\begin{tabular}{|c|c|c|}
\hline Recommendations & Class $^{a}$ & Level $^{b}$ \\
\hline $\begin{array}{l}\text { Emergency electrical cardioversion is recom- } \\
\text { mended in AF patients with acute or worsening } \\
\text { haemodynamic instability. }{ }^{1053,1054}\end{array}$ & I & B \\
\hline $\begin{array}{l}\text { In AF patients with haemodynamic instability, } \\
\text { amiodarone may be considered for acute con- } \\
\text { trol of heart rate. } \\
503,511,512\end{array}$ & Illb & B \\
\hline
\end{tabular}

$\mathrm{AF}=$ atrial fibrillation

${ }^{\mathrm{a} C l a s s}$ of recommendation.

bevel of evidence.

\subsection{First-diagnosed (new-onset) atrial fibrillation}

First-diagnosed or new-onset $A F$ is a working diagnosis in a patient without a history of AF, until the pattern of AF can be defined more 
precisely. Although the clinical profile and outcome of patients with first-diagnosed $A F$ in $A F$ registries were less favourable than those with paroxysmal $A F$, rather resembling permanent $A F,{ }^{1055,1056} \mathrm{OAC}$ prescription rates were the lowest in patients with first-diagnosed AF. ${ }^{1057}$ In patients with first-diagnosed AF, the $A B C$ pathway should resemble all steps outlined in the Central Illustration.

\subsection{Acute coronary syndromes, percutaneous coronary intervention, and chronic coronary syndromes in patients with atrial fibrillation}

The incidence of $A F$ in acute coronary syndromes (ACS) ranges from $2-23 \%$, ${ }^{1058}$ the risk of new-onset $A F$ is increased by $60-77 \%{ }^{1059}$ in myocardial infarction patients, and AF per se may be associated with an increased risk of ST-segment elevation myocardial infarction (STEMI) or non-STEMI ACS. ${ }^{381,1060-1063}$ Overall, $10-15 \%$ of AF patients undergo $\mathrm{PCl}$ for $\mathrm{CAD} .^{1064}$ In observational studies, patients with $A F$ and $A C S$ were less likely to receive appropriate antithrombotic therapy ${ }^{1065}$ and more likely to experience adverse outcomes $^{1066}$ than ACS patients without AF.

Peri-procedural management of patients with an ACS or undergoing $\mathrm{PCl}$ is detailed in the respective ESC Guidelines on myocardial revascularization ${ }^{1067}$ and chronic coronary syndromes (CCS). ${ }^{1068}$

\section{Post-procedural management of atrial fibrillation patients with acute coronary syndrome and/or percutaneous coronary intervention}

In AF patients having an ACS or undergoing $\mathrm{PCl}$, concomitant risks of ischaemic stroke/systemic embolism, coronary ischaemic events, and antithrombotic treatment-related bleeding need to be carefully balanced when considering the use and duration of combined antithrombotic therapy. ${ }^{1069}$ Overall, dual antithrombotic therapy including OAC (preferably NOAC) and a P2Y 12 inhibitor (preferably clopidogrel) is associated with significantly less major bleeding (and ICH) than triple therapy. However, available evidence suggests that at least a short course of triple therapy (e.g. $\leq 1$ week) would be desirable in some AF patients after a recent $\mathrm{ACS}$ or undergoing $\mathrm{PCl}$, especially in those at increased risk of ischaemic events ${ }^{1070,1071}$ (Figure 20).

\section{Box 1 About post-procedural manage- ment of patients with AF and ACS and/ or $\mathrm{PCl}$}

Shorter courses of triple therapy (OAC + DAPT) may be safe in post-ACS/PCI patients requiring OAC. ${ }^{1076}$ Observational data ${ }^{1077}$ and the WOEST trial with warfarin (a safety RCT, underpowered for ischaemic outcomes) ${ }^{1078}$ suggested better safety and similar efficacy with dual (OAC + clopidogrel) vs. triple therapy.

$R C T$ s of NOACs in AF patients after a recent ACS/PCI

Four RCTs compared dual therapy with a $\mathrm{P}_{2} \mathrm{Y}_{12}$ inhibitor (mostly clopidogrel) plus a NOAC - dabigatran $110 \mathrm{mg}$ or $150 \mathrm{mg}$ b.i.d. (REDUAL PCl), ${ }^{1079}$ rivaroxaban $15 \mathrm{mg}$ o.d. (PIONEER AF-PCI), ${ }^{1080}$ apixaban $5 \mathrm{mg}$ b.i.d. (AUGUSTUS), ${ }^{1081}$ or edoxaban $60 \mathrm{mg}$ o.d. $\left(\right.$ ENTRUST-AF PCl) ${ }^{1082}$-vs. triple therapy with a VKA in AF patients with a recent ACS or undergoing $\mathrm{PCl}$. The two-by-two factorial AUGUSTUS trial design enabled the comparison of aspirin vs. placebo (see Supplementary Table 12 for detailed information about these studies). All four trials had a primary safety endpoint (i.e. bleeding) and were underpowered to assess ischaemic outcomes.

Despite some heterogeneity among these trials, all have consistently:

- Included a proportion of patients with an ACS/PCI (37-52\%); nevertheless, the highest risk patients (e.g. previous stent thrombosis or a complex $\mathrm{PCl}$ with stent-in-stent placement) were largely under-represented;

- Used triple therapy during $\mathrm{PCl}$ and until randomization (1-14 days post $\mathrm{PCl}$ );

- Most commonly used the P2Y 12 inhibitor clopidogrel (overall, $>90 \%$; and

- Reported a significant reduction of major/clinically significant bleeding, comparable rates of ischaemic stroke, similar or non-significantly higher rates of myocardial infarction and stent thrombosis, and a neutral effect on trial-defined major adverse cardiovascular events and all-cause mortality with dual (NOAC $\left.+\mathrm{P}_{2} \mathrm{Y}_{12}\right)$ vs. triple $\left(\mathrm{VKA}+\mathrm{P}_{2} \mathrm{Y}_{12}+\right.$ aspirin) therapy.

In AUGUSTUS, ${ }^{1081}$ both placebo (vs. aspirin) and apixaban (vs. VKA) regimens were associated with significant reduction in bleeding, and apixaban (vs. VKA) was associated with significantly lower rates of stroke, death, or hospitalization.

Meta-analyses of RCTs

- Bleeding outcomes: Meta-analyses ${ }^{1070,1071,1083,1084}$ consistently showed a significant reduction in major bleeding with dual vs. triple and NOAC- vs. VKA-based therapies (NOAC-based treatments were also associated with a significant reduction in $\mathrm{ICH}$ ).

- Ischaemic events: Stroke rates were similar across all treatment arms, but the rates of myocardial infarction and stent thrombosis were numerically higher with dual vs. triple therapy. In two meta-analyses ${ }^{1070,1071}$ stent thrombosis was statistically significantly increased on dual (i.e. no aspirin) vs. triple therapy. Also, the risk of myocardial infarction or stent thrombosis was slightly higher with dabigatran $110 \mathrm{mg}$ but not dabigatran $150 \mathrm{mg}$.

- The trial-defined major adverse cardiovascular events and mortality rates were similar in all treatment arms, suggesting that the benefit from major bleeding and $\mathrm{ICH}$ reduction is counterbalanced by a higher risk for coronary (mainly stent-related) ischaemic events with dual therapy.

$\mathrm{ACS}=$ acute coronary syndromes; $\mathrm{AF}=$ atrial fibrillation; b.i.d. $=$ bis in die (twice a day); DAPT = dual antiplatelet therapy; ENTRUST-AF PCI = Edoxaban Treatment Versus Vitamin K Antagonist in Patients With Atrial Fibrillation Undergoing Percutaneous Coronary Intervention; $\mathrm{ICH}=$ intracranial haemorrhage; $\mathrm{NOAC}=$ non-vitamin $\mathrm{K}$ antagonist oral anticoagulant; $\mathrm{OAC}=$ oral anticoagulant; o.d. = omni die (once daily); $\mathrm{PCl}=$ percutaneous coronary intervention; PIONEER AF-PCI = (OPen-Label, Randomized, Controlled, Multicenter Study Explorlng TwO TreatmeNt StratEgiEs of Rivaroxaban and a Dose-Adjusted Oral Vitamin K Antagonist Treatment Strategy in Subjects with Atrial Fibrillation who Undergo Percutaneous Coronary Intervention; RCT = randomized controlled trial; RE-DUAL PCI = Randomized Evaluation of Dual Antithrombotic Therapy with Dabigatran vs. Triple Therapy with Warfarin in Patients with Nonvalvular Atrial Fibrillation Undergoing Percutaneous Coronary Intervention; VKA = vitamin $\mathrm{K}$ antagonist; WOEST $=$ What is the Optimal antiplatElet and anticoagulant therapy in patients with oral anticoagulation and coronary StenTing. 


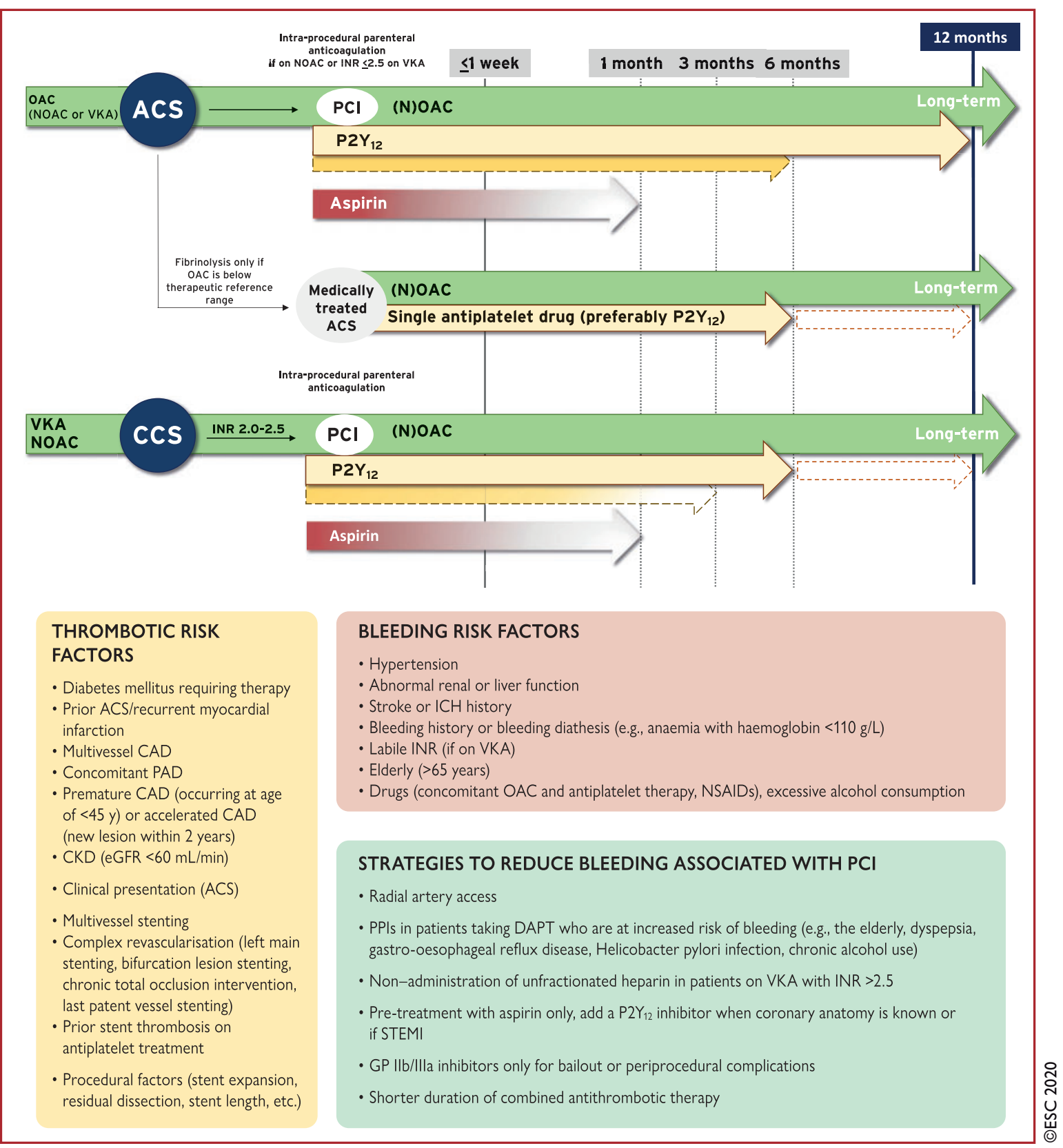

Figure 20 Post-procedural management of patients with AF and ACS/PCI (full-outlined arrows represent a default strategy; graded/dashed arrows show treatment modifications depending on individual patient's ischaemic and bleeding risks).

Pretreatment with a P2Y 12 inhibitor is recommended in STEMI patients or when coronary anatomy is known; it should be withheld in non-STEMI ACS until the time of coronary angiography in case of an early invasive strategy within 24 hours. Observational studies indicate that PCl on uninterrupted VKAs is generally safe compared with OAC interruption and heparin-bridging therapy, ${ }^{1073}$ particularly with radial artery access; in contrast, studies on NOACs are conflicting, predominantly discouraging a PCl on fully uninterrupted NOAC therapy. ${ }^{1074,1075}$ If urgent $\mathrm{PCl}$ is needed, administration of a parenteral anticoagulant (UFH, LMWH, or bivalirudin) is suggested, with temporary withdrawal of NOAC at least for the initial post-procedural period (e.g. 24 h) depending on the patient's thrombotic and bleeding risk profile. Where thrombolysis is being considered in a patient with STEMI, the initial step should be to assess the anticoagulation status (e.g. INR in a patient taking VKA; with a NOAC, assessing, for example, activated partial thromboplastin time on dabigatran or anti-factor Xa activity on factor Xa inhibitors). Thrombolytic therapy may be associated with an increased risk of bleeding in systemically anticoagulated patients, especially if parenteral heparin and antiplatelet drugs are coadministered. A balance between the potential benefit (e.g. large anterior myocardial infarction) and harm (e.g. ICH) is needed, as well as the reassessment of urgent transfer to a PCl centre. If the supposedly anticoagulated patient does not have evidence of a therapeutic anticoagulation effect (e.g. INR $<2.0$ on warfarin; or no NOAC anticoagulant effect detected), systemic thrombolysis may be considered if no access to primary $\mathrm{PCl}$ is possible.

ACS = acute coronary syndromes; $A S A=$ acetylsalicylic acid; $C A D=$ coronary artery disease; $C C S=$ chronic coronary syndromes; $C K D=$ chronic kidney disease; DAPT = dual antithrombotic therapy; eGFR = estimated glomerular filtration rate; ICH = intracranial haemorrhage; INR = international normalized ratio; $\mathrm{LMWH}=$ low-molecular-weight heparin; $\mathrm{MI}=$ myocardial infarction; $\mathrm{NOAC}=$ non-vitamin $\mathrm{K}$ antagonist oral anticoagulant; NSAID = non-steroidal anti-inflammatory drug; $\mathrm{OAC}=$ oral anticoagulant; $\mathrm{PAD}=$ peripheral artery disease; $\mathrm{PCl}=$ percutaneous coronary intervention; $\mathrm{PPI}=$ proton-pump inhibitor; STEMI = ST-segment elevation myocardial infarction; UFH = unfractionated heparin; VKA = vitamin K antagonist. 
Whichever initial treatment plan was chosen, dual therapy with OAC and an antiplatelet drug (preferably clopidogrel) is recommended for the first 12 months after $\mathrm{PCl}$ for $\mathrm{ACS}$, or 6 months after $\mathrm{PCl}$ in patients with CCS. ${ }^{1067}$ Thereafter, OAC monotherapy is to be continued (irrespective of the stent type) provided that there were no recurrent ischaemic events in the interim. In 1-year event-free (i.e. 'stable') AF patients with $\mathrm{CAD}$ and no $\mathrm{PCl}, \mathrm{OAC}$ monotherapy is also recommended. ${ }^{1072}$

Use of prasugrel or ticagrelor has been associated with a greater risk of major bleeding compared with clopidogrel ${ }^{1085-1089}$ and should be avoided in ACS patients with AF. In the RE-DUAL PCl (Randomized Evaluation of Dual Antithrombotic Therapy with Dabigatran vs. Triple Therapy with Warfarin in Patients with Nonvalvular Atrial Fibrillation Undergoing Percutaneous Coronary Intervention) trial, $12 \%$ of patients received ticagrelor with dabigatran, but experience with ticagrelor or prasugrel was minimal in PIONEER-AF (OPen-Label, Randomized, Controlled, Multicenter Study Explorlng TwO TreatmeNt StratEgiEs of Rivaroxaban and a Dose-Adjusted Oral Vitamin K Antagonist Treatment Strategy in Subjects with Atrial Fibrillation who Undergo
Percutaneous Coronary Intervention), AUGUSTUS, and ENTRUSTAF PCl (Edoxaban Treatment Versus Vitamin K Antagonist in Patients With Atrial Fibrillation Undergoing Percutaneous Coronary Intervention). In patients at potential risk of gastrointestinal bleeding, concomitant use of proton-pump inhibitors is reasonable. ${ }^{1084}$

In AF patients treated with surgical coronary revascularization, OAC should be resumed as soon as bleeding is controlled, possibly in combination with clopidogrel, and triple therapy should be avoided.

Poor ventricular rate control during AF may exacerbate symptoms of myocardial ischaemia and precipitate or worsen HF. Appropriate treatment may include a beta-blocker or rate-limiting calcium antagonist. In haemodynamic instability, acute cardioversion may be indicated. Vernakalant, flecainide, and propafenone should not be used for rhythm control in patients with known CAD (section 10.2.2.2).

In all AF patients with an ACS/CCS, optimized management of risk factors is needed, and cardiovascular prevention strategies such as good BP control, ${ }^{338}$ lipid management, and other cardiovascular prevention interventions ${ }^{1007}$ should be implemented as needed, once the acute presentation is stabilized.

\section{Recommendations for patients with AF and an ACS, PCI, or CCS $^{1068}$}

\section{General recommendations for patients with AF and an indication for concomitant antiplatelet therapy}

In AF patients eligible for NOACs, it is recommended to use a NOAC ${ }^{\mathrm{c}}$ in preference to a VKA in combination with antiplatelet therapy. ${ }^{1079,1081}$

In patients at high bleeding risk (HAS-BLED $\geq 3$ ), rivaroxaban $15 \mathrm{mg}$ o.d. should be considered in preference to rivaroxaban 20 mg o.d. for the duration of concomitant single or DAPT, to mitigate bleeding risk. ${ }^{1080}$

In patients at high bleeding risk (HAS-BLED $\geq 3$ ), dabigatran $110 \mathrm{mg}$ b.i.d. should be considered in preference to dabigatran $150 \mathrm{mg}$ b.i.d. for the duration of concomitant single or DAPT, to mitigate bleeding risk. ${ }^{1079}$

In AF patients with an indication for a VKA in combination with antiplatelet therapy, the VKA dosing should be carefully regulated with a target INR of $2.0-2.5$ and TTR $>70 \%{ }^{1094,1095,1104,1105}$

\section{Recommendations for AF patients with ACS}

In AF patients with ACS undergoing an uncomplicated $\mathrm{PCl}$, early cessation ( $\leq 1$ week) of aspirin and continuation of dual therapy with an OAC and a P2Y 12 inhibitor (preferably clopidogrel) for up to 12 months is recommended if the risk of stent thrombosis ${ }^{\mathrm{d}}$ is low or if concerns about bleeding risk ${ }^{\mathrm{e}}$ prevail over concerns about risk of stent thrombosis, ${ }^{d}$ irrespective of the type of stent used. ${ }^{1090,1092-1095}$ Triple therapy with aspirin, clopidogrel, and an OAC for longer than 1 week after an ACS should be considered when risk of stent thrombosis ${ }^{d}$ outweighs the bleeding risk, ${ }^{e}$ with the total duration ( $\leq 1$ month) decided according to assessment of these risks, and the treatment plan should be clearly specified at hospital discharge.

\section{Recommendations in AF patients with a CCS undergoing $\mathbf{P C I}$}

After uncomplicated $\mathrm{PCl}$, early cessation ( $\leq 1$ week) of aspirin and continuation of dual therapy with OAC for up to 6 months and clopidogrel is recommended if the risk of stent thrombosis ${ }^{d}$ is low or if concerns about bleeding risk ${ }^{e}$ prevail over concerns about risk of stent thrombosis, ${ }^{d}$ irrespective of the type of stent used. ${ }^{1076,1078-1081}$

Triple therapy with aspirin, clopidogrel, and an $O A C^{f}$ for longer than 1 week should be considered when risk of stent thrombosis ${ }^{d}$ outweighs the bleeding risk, ${ }^{e}$ with the total duration ( $\leq 1$ month) decided according to assessment of these risks, and the treatment plan should be clearly specified at hospital discharge.

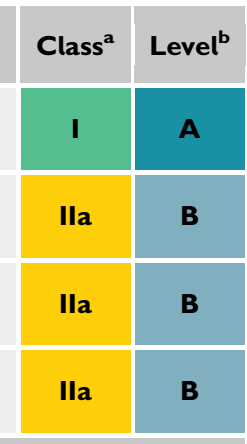

ACS = acute coronary syndrome; AF = atrial fibrillation; b.i.d. = bis in die (twice a day); CCS = chronic coronary syndrome; CKD = chronic kidney disease; DAPT = Dual antiplatelet therapy; HAS-BLED = Hypertension, Abnormal renal/liver function, Stroke, Bleeding history or predisposition, Labile INR, Elderly ( $>65$ years), Drugs/alcohol concomitantly; INR = international normalized ratio; NOAC = non-vitamin $\mathrm{K}$ antagonist oral anticoagulant; o.d. = omni die (once daily); OAC = oral anticoagulant; PCl=percutaneous coronary intervention; TTR = time in therapeutic range; VKA = vitamin $\mathrm{K}$ antagonist.

${ }^{\mathrm{a}}$ Class of recommendation.

bevel of evidence.

'See summary of product characteristics for reduced doses or contraindications for each NOAC in patients with CKD, body weight $<60$ kg, age $>75-80$ years, and/or drug interactions.

${ }^{d}$ Risk of stent thrombosis encompasses: (i) risk of thrombosis occurring, and (ii) risk of death should stent thrombosis occur, both of which relate to anatomical, procedural, and clinical characteristics. Risk factors for CCS patients include: stenting of left main stem or last remaining patent artery; suboptimal stent deployment; stent length > 60 mm; diabetes mellitus; CKD; bifurcation with two stents implanted; treatment of chronic total occlusion; and previous stent thrombosis on adequate antithrombotic therapy.

eBleeding risk in AF patients may be assessed using the HAS-BLED score (section 10.1.2), which draws attention to modifiable bleeding risk factors; those at high risk (score $\geq 3$ ) can have more frequent or early review and follow-up. Bleeding risk is highly dynamic and does not remain static, and relying on modifiable bleeding risk factors alone is an inferior strategy to evaluate bleeding risk. ${ }^{389}$

'When dabigatran is used in triple therapy, dabigatran $110 \mathrm{mg}$ b.i.d may be used instead of $150 \mathrm{mg}$ b.i.d, but the evidence is insufficient. 


\subsection{Acute stroke or intracranial haemorrhage in patients with atrial fibrillation}

\subsubsection{Patients with atrial fibrillation and acute ischaemic stroke or transient ischaemic attack}

Management of acute stroke in AF patients is beyond the scope of this document. In AF patients presenting with acute ischaemic stroke while taking OAC, acute therapy depends on the treatment regimen and intensity of anticoagulation. Patients on VKA with an INR $<1.7$ are eligible for thrombolysis according to the neurological indication (if presenting with a clinically relevant neurological deficit within the appropriate time window and $\mathrm{ICH}$ is excluded with cerebral imaging). In patients taking NOACs, measurement of activated partial thromboplastin time or thrombin time (for dabigatran), or antifactor Xa levels (for factor Xa inhibitors) will provide information on whether the patient is systemically anticoagulated. Whenever possible, the time when the last NOAC dose was taken should be elucidated (generally, thrombolysis is considered to be safe in patients with last NOAC intake being $\geq 48 \mathrm{~h}$, assuming normal renal function). ${ }^{1090}$

If the patient is systemically anticoagulated, thrombolysis should not be performed due to the risk of haemorrhage, and endovascular treatment should be considered. In patients taking dabigatran, systemic thrombolysis may be performed after reversal of the dabigatran action by idarucizumab. ${ }^{1091}$

Secondary prevention of stroke/systemic embolism in patients after acute AF-related ischaemic stroke or TIA includes early prevention of recurrent ischaemic stroke in the 2 weeks after the index event and long-term prevention thereafter.

Whereas infarct size/stroke severity is used clinically to guide timing of OAC initiation, ${ }^{1090}$ the usefulness of such an approach in estimating the net benefit of early treatment may be limited. Robust data to inform optimal timing for (re)initiation of OAC after acute stroke are lacking. From the cardiological perspective, OAC should be (re)initiated as soon as considered possible from the neurological perspective (in most cases within the first 2 weeks). A multidisciplinary approach with involvement of stroke specialists, cardiologists, and patients is considered appropriate.

In AF patients who presented with acute ischaemic stroke despite taking OAC, optimization of OAC therapy is of key importance - if on VKA, optimize TTR (ideally $>70 \%$ ) or switch to a NOAC; if on NOAC, ensure appropriate dosing and good adherence to treatment. Inappropriate NOAC under-dosing using lower or reduced doses of specific NOACs has been associated with increased risk of stroke/systemic embolism, hospitalization, and deaths without appreciable reduction in major bleeding. ${ }^{1107}$

\subsubsection{Cryptogenic stroke/embolic stroke with undetermined source}

Currently available evidence including two recently completed $\mathrm{RCTs}^{1108,1109}$ does not support routine OAC use in patients with acute ischaemic stroke of uncertain aetiology (cryptogenic stroke) or acute embolic stroke of undetermined source in patients without documented AF (Supplementary Box 4). Of note, subgroup

\section{Box 2 About acute ischaemic stroke in patients with AF}

AF-related ischaemic strokes are often fatal or disabling ${ }^{106}$, with increased risk of early recurrence within $48 \mathrm{~h}{ }^{1092}$ to 2 weeks, ${ }^{1092-1095}$ or haemorrhagic transformation, ${ }^{1096}$ especially in the first days after large cardio-embolic lesions and acute recanalization therapy. ${ }^{1097,1098}$ Notably, ICH is generally associated with higher mortality and morbidity than recurrent ischaemic stroke.

Timing of OAC (re)initiation after acute ischaemic stroke

- Early anticoagulation after acute ischaemic stroke might cause parenchymal haemorrhage, with potentially serious clinical consequences ${ }^{1097,}$. $^{1099}$ Using UFH, LMWH, heparinoids, or VKAs $<48 \mathrm{~h}$ after acute ischaemic stroke was associated with an increased risk of symptomatic ICH, without significant reduction in recurrent ischaemic stroke. ${ }^{1095}$

- Reportedly, the 90-day risk of recurrent ischaemic stroke outweighs the risk of symptomatic ICH in AF patients receiving a NOAC 4-14 days after the acute event ${ }^{1100-1102}$ (ischaemic stroke recurrence rates after mild/moderate ischaemic stroke significantly increased with a later NOAC administration, ${ }^{1101}$ e.g. $>14$ days). ${ }^{1100}$ In a small RCT, rivaroxaban use within 5 days after mild ischaemic stroke in AF patients was associated with similar event rates compared with VKA. ${ }^{1103}$

As high-quality RCT-derived evidence to inform optimal timing of anticoagulation after acute ischaemic stroke is lacking, OAC use in the early post-stroke period is currently based on expert consensus. ${ }^{505}$ Several ongoing RCTs [ELAN (NCT03148457), OPTIMAS (EudraCT, 2018-003859-3), TIMING (NCT02961348), and START (NCT03021928)] are investigating early (<1 week) vs. late NOAC initiation in patients with AF-related ischaemic stroke (first results are not expected before 2021).

Long-term secondary stroke prevention

- There is no evidence that the addition of aspirin to OAC or supratherapeutic INRs would improve outcomes in secondary stroke prevention.

- Compared with VKAs, NOACs were associated with better efficacy in secondary stroke prevention and better safety regarding $\mathrm{ICH}$ in a meta-analysis of landmark NOAC AF trial. ${ }^{1104}$

- Good adherence to OAC treatment is essential for effective secondary stroke prevention.

There is some evidence to support that strokes can induce AF through neurogenic mechanisms ${ }^{1105,1106}$ The first study showed that damage to the insula increases the odds of AF detection after ischaemic stroke and is more prevalent in patients with AF diagnosed after stroke than among those without AF. ${ }^{1105}$ The second study explained the reason why AFDAS detected soon after ischaemic stroke is associated with a low risk of ischaemic stroke recurrence. ${ }^{1106}$

$\mathrm{AF}=$ atrial fibrillation; ELAN = Early versus Late Initiation of Direct Oral Anticoagulants in Post-ischaemic Stroke Patients With AF; ICH = intracranial haemorrhage; INR = international normalized ratio; LMWH = low-molecular-weight heparin; NOAC = non-vitamin $\mathrm{K}$ antagonist oral anticoagulant; OAC = oral anticoagulant; OPTIMAS = OPtimal TIMing of Anticoagulation after Stroke; RCT = randomized controlled trial; START = Optimal Delay Time to Initiate Anticoagulation After Ischemic Stroke in AF; TIMING = TIMING of Oral Anticoagulant Therapy in Acute Ischemic Stroke With AF; UFH = unfractionated heparin; VKA = vitamin $\mathrm{K}$ antagonist. 
analyses of those two RCTs suggested that certain subgroups (i.e. age $\geq 75$ years, impaired renal function, ${ }^{1109}$ or enlarged $L A^{1110}$ ) could benefit from OAC, but more data are needed to inform optimal use of NOACs among patients with a cryptogenic stroke. Two ongoing trials will study the use of apixaban in this setting [ATTICUS (Apixaban for treatment of embolic stroke of undetermined source) ${ }^{1111}$ and ARCADIA [(AtRial Cardiopathy and Antithrombotic Drugs In Prevention After Cryptogenic Stroke) (NCT03192215)].

Efforts to improve detection of $A F$ are needed in such patients (see also section 8). Clinical risk scores $\left\{\right.$ e.g. $\mathrm{C}_{2}$ HEST [CAD/COPD (1 point each), Hypertension (1 point), Elderly ( $\geq 75$ years, 2 points), Systolic heart failure ( 2 points), and Thyroid disease (hyperthyroidism, 1 point) (score)]\} have been proposed for identification of 'highrisk' patients for $A F$ diagnosis ${ }^{1112}$ and facilitation of prolonged monitoring.

\section{Recommendations for the search for AF in patients with cryptogenic stroke}

\begin{tabular}{l|l|l|}
\hline Recommendations & Class $^{\mathbf{a}}$ & Level $^{\mathbf{b}}$ \\
\hline $\begin{array}{l}\text { In patients with acute ischaemic stroke or TIA } \\
\text { and without previously known AF, monitoring } \\
\text { for AF is recommended using a short-term ECG } \\
\text { recording for at least the first } 24 \mathrm{~h} \text {, followed by } \\
\text { continuous ECG monitoring for at least } 72 \mathrm{~h} \\
\text { whenever possible. }\end{array}$ I113-1116 & B \\
\hline $\begin{array}{l}\text { In selected } \text { stroke patients without previously }_{\text {known AF, additional ECG monitoring using }} \\
\text { long-term non-invasive ECG monitors or insert- } \\
\text { able cardiac monitors should be considered, to } \\
\text { detect AF. }{ }^{1112}\end{array}$ & Ila & B \\
\hline
\end{tabular}

$\mathrm{AF}=$ atrial fibrillation; $\mathrm{C}_{2} \mathrm{HEST}=\mathrm{CAD} / \mathrm{COPD}$ (1 point each), Hypertension (1 point), Elderly ( $\geq 75$ years, 2 points), Systolic heart failure ( 2 points), and Thyroid disease (hyperthyroidism, 1 point) (score); ECG=electrocardiogram; LA = left atrial; $\mathrm{TIA}=$ transient ischaemic attack.

${ }^{a}$ Class of recommendation.

${ }^{\mathrm{b}}$ Level of evidence.

${ }^{\mathrm{N}}$ Not all stroke patients would benefit from prolonged ECG monitoring; those deemed at risk of developing AF (e.g. elderly, with cardiovascular risk factors or comorbidities, indices of LA remodelling, high $\mathrm{C}_{2} \mathrm{HEST}$ score, etc.) or those with cryptogenic stroke and stroke characteristics suggestive of an embolic stroke should be scheduled for prolonged ECG monitoring.

\subsubsection{Post-stroke patients without known atrial fibrillation}

Detection of previously unknown AF after stroke has important implications for secondary prevention. Several RCTs have established the effectiveness of ECG monitoring for post-stroke AF detection, with numbers needed to screen of $8-14$.

Looking harder and longer and using more sophisticated monitoring may generally improve AF detection. In a meta-analysis ${ }^{1118}$ of 50 post-stroke studies, the proportion of patients with poststroke AF was $7.7 \%$ in the emergency room using admission ECG; $5.1 \%$ in the wards using serial ECG, continuous inpatient ECG monitoring/cardiac telemetry, and in-hospital Holter monitoring; $10.7 \%$ in the first ambulatory period using ambulatory Holter; and, after discharge, $16.9 \%$ using mobile cardiac outpatient telemetry and external or implantable loop recording. The overall poststroke AF detection after all phases of cardiac monitoring reached $23.7 \%{ }^{1118}$

In patients with ischaemic stroke/TIA, monitoring for AF is recommended by short-term ECG recording followed by continuous ECG monitoring for at least $72 \mathrm{~h}$, also considering a tiered longer ECG monitoring approach ${ }^{1113}$ and insertion of an intracardiac monitor in case of cryptogenic stroke. ${ }^{1114,1119}$ Post-stroke ECG monitoring is likely cost-effective ${ }^{1120,1121}$; however, RCTs have not been powered to assess the effect of prolonged ECG monitoring and subsequent prescription of OAC on stroke or mortality in patients with detected AF.

\subsubsection{Management of patients with atrial fibrillation post-intracranial haemorrhage}

As ICH is the most feared, often lethal, complication of anticoagulant and antiplatelet therapy, there is a considerable reluctance to (re)initiate OAC in AF patients who survived an $\mathrm{ICH}$, despite their high estimated risk of AF-related ischaemic stroke.

Patients with a history of recent ICH were excluded from RCTs of stroke prevention in AF, but available observational data suggest than many AF patients would benefit from (re)institution of OAC, depending on the cause(s) of $\mathrm{ICH}$ and findings on brain CT and MRI (Supplementary Box 5).

Treatment decision to (re)start $\mathrm{OAC}$ in $\mathrm{AF}$ patients after an $\mathrm{ICH}$ requires multidisciplinary-team input from cardiologists, stroke specialists, neurosurgeons, patients, and their family/carers. After acute spontaneous ICH (which includes epidural, subdural, subarachnoid, or intracerebral haemorrhage), OAC may be considered after careful assessment of risks and benefits, and cerebral imaging may help. The risk of recurrent $\mathrm{ICH}$ may be increased in the presence of specific risk factors, shown in Figure 21. Of note, the risk of OAC-related ICH is increased especially in Asian patients. ${ }^{1122}$

Compared with VKAs, the use of NOACs in patients without previous $\mathrm{ICH}$ is associated with an approximately $50 \%$ lower risk of $\mathrm{ICH},{ }^{423}$ whereas the size and outcome of $\mathrm{OAC}$-related $\mathrm{ICH}$ is similar with NOACs and VKAs. ${ }^{1124}$ Hence, NOACs should be preferred in NOAC-eligible ICH survivors with AF although there is no RCT to prove this.

The optimal timing of anticoagulation after $\mathrm{ICH}$ is unknown, but should be delayed beyond the acute phase, probably for at least 4 weeks; in AF patients at very high risk of recurrent ICH, LAA occlusion may be considered. Ongoing RCTs of NOACs and LAA occlusion may inform decision making in the future. 


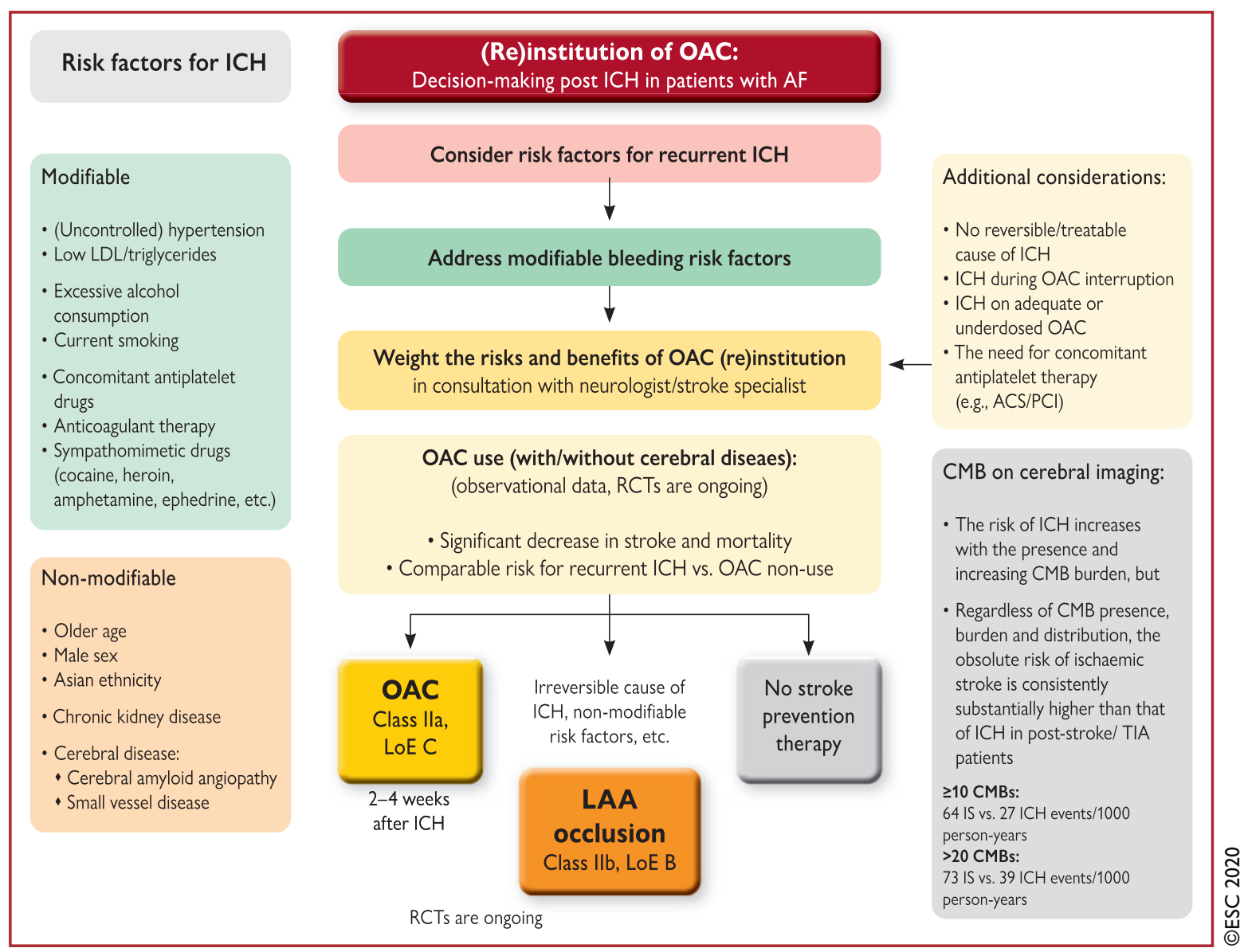

Figure 2 I (Re-) initiation of anticoagulation post-intracranial bleeding.

A pooled analysis of individual patient data from cohort studies ( $n=20322$ patients; 38 cohorts; >35 225 patient-years) showed that although cerebral microbleeds can inform regarding the risk for $\mathrm{ICH}$ in patients with recent ischaemic stroke/TIA treated with antithrombotic therapy, the absolute risk of ischaemic stroke is substantially higher than that of $\mathrm{ICH}$, regardless of the presence, burden, or location of cerebral microbleeds. ${ }^{505,1123}$

IS = ischaemic stroke;ACS = acute coronary syndrome; $C M B=$ cerebral microbleeds; ICH = intracranial haemorrhage; LAA = left atrial appendage; LDL = low-density lipoprotein; $\mathrm{LoE}=$ level of evidence; $\mathrm{NOAC}=$ non-vitamin $\mathrm{K}$ antagonist oral anticoagulant; $\mathrm{OAC}=$ oral anticoagulant; $\mathrm{PCl}=$ percutaneous coronary intervention; $\mathrm{RCT}=$ randomized controlled trial; $\mathrm{TIA}=$ transient ischaemic attack.

\section{Recommendations for secondary stroke prevention in AF patients after acute ischaemic stroke}

In AF patients with an ischaemic stroke or TIA, long-term secondary prevention of stroke using OAC is recommended if there is no strict contraindication to OAC use, with a preference for NOACs over VKAs in NOAC-eligible patients. ${ }^{1125-1130}$

In AF patients presenting with acute ischaemic stroke, very early anticoagulation ( $<48 \mathrm{~h}$ ) using UFH, LMWH, or VKAs is not recommended. ${ }^{1095}$

\section{Recommendations for stroke prevention in AF patients after intracranial haemorrhage}

In AF patients at high risk of ischaemic stroke, (re-)initiation of OAC, with preference for NOACs over VKAs in NOAC-eligible patients, should be considered in consultation with a neurologist/stroke specialist after:

- A trauma-related $\mathrm{ICH}$

- Acute spontaneous ICH (which includes subdural, subarachnoid, or intracerebral haemorrhage), after careful consideration of risks and benefits. ${ }^{c}$

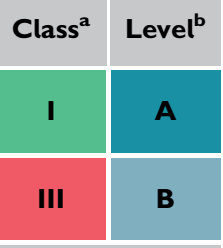

$\mathrm{AF}=$ atrial fibrillation; $\mathrm{ICH}=$ intracranial haemorrhage; $\mathrm{LMWH}=$ low-molecular-weight heparin; $\mathrm{NOAC}=$ non-vitamin $\mathrm{K}$ antagonist oral anticoagulant; $\mathrm{OAC}=$ oral anticoagulant; TIA = transient ischaemic attack; UFH = unfractionated heparin; VKA = vitamin $\mathrm{K}$ antagonist.

${ }^{\mathrm{a}}$ Class of recommendation.

bLevel of evidence.

${ }^{\mathrm{c}} \mathrm{A}$ more favourable net benefit is likely with deep $\mathrm{ICH}$ or without neuroimaging evidence of cerebral amyloid angiopathy or microbleeds. 


\subsection{Active bleeding on anticoagulant therapy: management and reversal drugs}

Management of patients with active bleeding while on $O A C$ is shown in Figure 22. General assessment should include detection of the bleeding site, assessment of bleeding severity, and evaluation of the time-point of last OAC intake. Concomitant antithrombotic drugs and other factors influencing bleeding risk (alcohol abuse, renal function) should be explored. Laboratory tests, such as INR, are useful in case of VKA therapy. More specific coagulation tests for NOACs include diluted thrombin time, ecarin clotting time, or ecarin chromogenic assay for dabigatran, and chromogenic anti-factor $\mathrm{Xa}$ assay for rivaroxaban, apixaban, and edoxaban. ${ }^{1131}$ However, these tests or measurement of NOAC plasma levels are not always readily available in practice and are often unnecessary for bleeding management. ${ }^{1132}$ An overview of reversal drugs for NOACs is given in Supplementary Table 13 and Supplementary Figure 6.

Notably, the time of last drug ingestion combined with assessment of renal function, haemoglobin, haematocrit, and platelet count enable appropriate clinical decision making in most of the cases.

Minor bleeding events should be treated with supportive measures such as mechanical compression or minor surgery to achieve haemostasis. Withdrawal of VKAs is not associated with a prompt reduction of anticoagulant effect, while NOACs have a short plasma half-life and haemostasis can be expected within 12-24 h after an omitted dose.
Treatment of moderate bleeding events may require blood transfusions and fluid replacement. If the last intake of NOACs was less than 2 - $4 \mathrm{~h}$ before bleeding assessment, charcoal administration and/ or gastric lavage will reduce further exposure. Specific diagnostic and treatment interventions to identify and manage the cause of bleeding (e.g. gastroscopy) should be performed promptly. Dialysis is effective in reducing dabigatran concentration and has been associated with reduction in the duration and/or severity of associated bleeding. ${ }^{1133}$

Severe or life-threatening bleeding requires immediate reversal of the antithrombotic effect of OACs. For VKAs, administration of fresh frozen plasma restores coagulation more rapidly than vitamin $\mathrm{K}$, but prothrombin complex concentrates achieve even faster blood coagulation $^{1134}$ and are first-line therapy for VKA reversal. ${ }^{1135}$ Specific reversal drugs are available for NOACs: idarucizumab (for dabigatran) and andexanet alfa (for factor $\mathrm{Xa}$ inhibitors) effectively reverse the anticoagulation action of NOACs and restore physiological haemostasis. ${ }^{1136,1137}$ However, their use is often associated with subsequent non-reinitiation of $O A C$ and increased rates of thrombotic events. These drugs can be effectively applied in case of severe lifethreatening bleeding or urgent surgery, but their use is only very rarely necessary in daily clinical practice. Ciraparantag is an investigational synthetic drug that binds and inhibits direct factor Xa inhibitors, dabigatran, and heparin. The use of four-factor prothrombin complex concentrates may be considered as an alternative treatment for reversing the anticoagulant effect of rivaroxaban, apixaban, and edoxaban, although scientific evidence is very limited in this context and is frequently from healthy volunteers. ${ }^{1138-1140}$

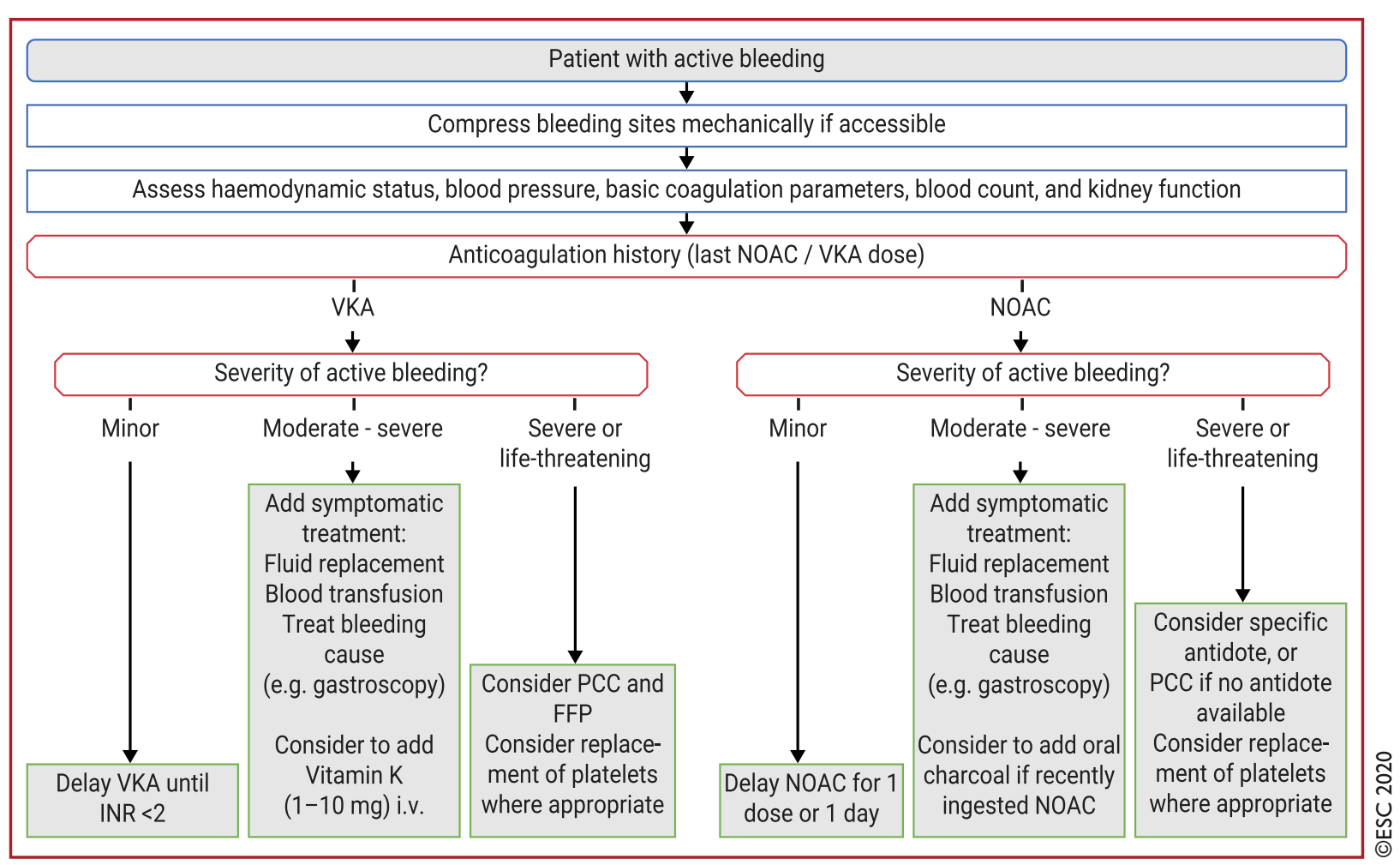

Figure 22 Management of active bleeding in patients receiving anticoagulation (institutions should have an agreed procedure in place). ${ }^{143} \mathrm{FFP}=$ fresh frozen plasma; INR = international normalized ratio; i.v. = intravenous; NOAC = non-vitamin $\mathrm{K}$ antagonist oral anticoagulant; $\mathrm{OAC}=$ oral anticoagulation therapy; $\mathrm{PCC}=$ prothrombin complex concentrates; $\mathrm{VKA}=$ vitamin $\mathrm{K}$ antagonist. 


\section{Recommendations for the management of active bleed- ing on OAC}

\begin{tabular}{|c|c|c|}
\hline & Class $^{a}$ & Level $^{b}$ \\
\hline $\begin{array}{l}\text { In an AF patient with severe active bleeding, it is } \\
\text { recommended to: } \\
\text { - Interrupt OAC until the cause of bleeding is } \\
\text { identified and active bleeding is resolved; and } \\
\text { - Promptly perform specific diagnostic and } \\
\text { treatment interventions to identify and man- } \\
\text { age the cause(s) and source(s) of bleeding. }\end{array}$ & I & C \\
\hline $\begin{array}{l}\text { Four-factor prothrombin complex concentrates } \\
\text { should be considered in AF patients on VKA } \\
\text { who develop a severe bleeding complication. }\end{array}$ & Ila & C \\
\hline
\end{tabular}

$\mathrm{AF}=$ atrial fibrillation; $\mathrm{OAC}=$ oral anticoagulant; $\mathrm{VKA}=\mathrm{vitamin} \mathrm{K}$ antagonist. ${ }^{\mathrm{a}}$ Class of recommendation.

bevel of evidence.

\subsection{Atrial fibrillation and heart failure}

Both $\mathrm{AF}$ and $\mathrm{HF}$ facilitate the occurrence and aggravate the prognosis of each other, and often coexist (see also sections 4.2 and 5.3); HF is also a thrombo-embolic risk factor in AF. The efficacy and safety of NOACs do not seem to differ in AF patients with and without $\mathrm{HF}^{1141,1142}$

The management of patients with $\mathrm{AF}$ and $\mathrm{HF}$ is often challenging (section 10.2). The optimal heart-rate target in AF patients with $\mathrm{HF}$ remains unclear, but a rate of $<100-110 \mathrm{bpm}$ is usually recommended. ${ }^{1143-1145}$ Pharmacological rate control strategies are different for patients with heart failure with preserved ejection fraction (HFpEF) and HFrEF. Beta-blockers, diltiazem, verapamil, and digoxin are all viable options in HFpEF, while beta-blockers and digoxin can be used in those with HFrEF. Amiodarone may be considered for rate control in both forms of HF, but only in the acute setting. Atrioventricular-node ablation and pacing can control ventricular rate when medication fails (section 10.2.1.). However, in an observation study, rhythm control strategies showed a lower 1-year all-cause death over rate control in older patients ( $\geq 65$ years) with HFpEF. ${ }^{1146}$

Haemodynamic instability or worsening of HF may require emergency or immediate electrical cardioversion of AF, whereas pharmacological cardioversion using i.v. amiodarone may be attempted if a delayed cardioversion is consistent with the clinical situation (section 10.2.2.2.2). AF catheter ablation has been shown to improve symptoms, exercise capacity, QoL, and LVEF in AF patients with $\mathrm{HF}^{661}$ whereas the recent CASTLE-AF RCT showed a reduction in allcause mortality and hospitalization for worsening HF after AF catheter ablation in patients with $\mathrm{HFrEF}^{657}$ (section 10.2.2.3).

All patients with $\mathrm{HF}$ and $\mathrm{AF}$ should receive guideline-adherent $\mathrm{HF}$ therapy. ${ }^{1145}$ The benefit of beta-blocker therapy in reducing mortality in AF patients with HFrEF has been questioned by some meta-analyses, ${ }^{491}$ although this is not a universal finding, especially with some real-world studies supporting an improved prognosis. $^{1147,1148}$

\subsection{Atrial fibrillation and valvular heart disease}

VHD is independently associated with $A F^{1149}$ and more than onethird of patients with AF have some form of $\mathrm{VHD}{ }^{512}$

Among patients with severe $\mathrm{VHD}$, including those undergoing surgical and transcatheter aortic or mitral valve intervention, $A F$ is associated with less favourable clinical outcomes. ${ }^{1150-1155}$ Compared to AF patients without VHD, the risk of thrombo-embolism and stroke is increased among AF patients with VHD other than mitral stenosis and mechanical heart prostheses, mostly owing to older age and more frequent comorbidities. ${ }^{1156,1157}$ While patients with moderateto-severe mitral stenosis and mechanical prosthetic heart valves require anticoagulation with VKAs, ${ }^{1158}$ there is no evidence that the presence of other VHDs including aortic stenosis/regurgitation, mitral regurgitation, bioprostheses, or valve repair should modify the choice of OAC. ${ }^{1156,1159}$ In a meta-analysis of the four pivotal RCTs comparing NOACs with VKAs, the effects of NOACs vs. VKAs in terms of stroke/systemic embolism and bleeding risk in patients with VHD other than mitral stenosis and mechanical prosthetic heart valves were consistent with those in the main RCTs. ${ }^{1160}$ In an observational study, NOACs were associated with better outcomes, with reduced rates of ischaemic stroke and major bleeding compared to warfarin in AF patients with mitral stenosis. ${ }^{1161}$

Recently, a functional categorization of VHD in relation to OAC use was introduced, categorizing patients with moderate-severe or rheumatic mitral stenosis as type 1 and all other VHD as type 2. ${ }^{148,1157,1162}$ There are gaps in evidence on NOAC use in AF patients with rheumatic mitral valve disease, and during the first 3 months after surgical or transcatheter implantation of a bioprosthesis, and observational data regarding NOACs use after transcatheter aortic valve implantation are conflicting. ${ }^{1163}$ An RCT in non-AF patients comparing rivaroxaban $10 \mathrm{mg}$ daily with aspirin after transcatheter aortic valve implantation was stopped early due to higher risks of death or thrombo-embolic complications and bleeding in the rivaroxaban arm. ${ }^{1164}$

\section{Recommendations for patients with valvular heart dis- ease and AF}

\begin{tabular}{l|l|l} 
Recommendations & Class $^{\mathbf{a}}$ & Le \\
$\begin{array}{l}\text { NOACs are contraindicated in patients with a } \\
\text { prosthetic mechanical valve. }\end{array}{ }^{165}$ & III \\
\hline $\begin{array}{l}\text { Use of NOACs is not recommended in patients } \\
\text { with AF and moderate-to-severe mitral stenosis. }\end{array}$ & \\
$\mathrm{AF}=$ atrial fibrillation; NOAC = non-vitamin K antagonist oral anticoagulant. \\
a'Class of recommendation. \\
bLevel of evidence.
\end{tabular}




\subsection{Atrial fibrillation and chronic kidney disease}

Independently of AF, CKD is a prothrombotic and prohaemorrhagic condition (Supplementary Figure 7), ${ }^{166,1167,1168}$ and AF may accelerate CKD progression. Coexisting in 15-20\% of CKD patients, ${ }^{1169}$ $\mathrm{AF}$ is associated with increased mortality, ${ }^{1170}$ whereas CKD may be present in $40-50 \%$ of $A F$ patients. ${ }^{1171}$ In AF patients, renal function can deteriorate over time, ${ }^{1172}$ and worsening $\mathrm{CrCl}$ is a better independent predictor of ischaemic stroke/systemic embolism and bleeding than renal impairment per se. ${ }^{1172}$ In RCTs of OAC for stroke prevention in AF, renal function was usually estimated using the Cockcroft-Gault formula for $\mathrm{CrCl}$, and a $\mathrm{CrCl}$ cut-off of $<50 \mathrm{~mL}$ min was used to adapt NOAC dosage.

In patients with mild-to-moderate CKD ( $\mathrm{CrCl} 30-49 \mathrm{~mL} / \mathrm{min})$, the safety and efficacy of NOACs vs. warfarin was consistent with patients without CKD in landmark NOAC trials ${ }^{1173-1176}$, hence the same considerations for stroke risk assessment and choice of OAC may apply.

In patients with $\mathrm{CrCl} 15-29 \mathrm{~mL} / \mathrm{min}, \mathrm{RCT}$-derived data on the effect of VKA or NOACs are lacking. These patients were essentially excluded from the major RCTs. The evidence for the benefits of $\mathrm{OAC}$ in patients with end-stage kidney disease with $\mathrm{CrCl} \leq 15 \mathrm{~mL}$ min or on dialysis is even more limited, and to some extent controversial. There are no RCTs, whereas observational data question the benefit of OAC in this patient population. Data from observational studies suggest possible bleeding risk reduction in patients with endstage kidney disease taking a NOAC compared with VKA, ${ }^{435,1177}$ but there is no solid evidence for a reduction in embolic events with either NOACs or VKAs, as recently shown in a systematic review. ${ }^{1178}$ Notably, NOACs have not been approved in Europe for patients with $\mathrm{CrCl} \leq 15 \mathrm{~mL} / \mathrm{min}$ or on dialysis.

Several RCTs are currently assessing OAC use and comparing NOACs with VKAs in patients with end-stage renal disease (NCT02933697, NCT03987711). The RENAL-AF trial, investigating apixaban vs. warfarin in AF patients on haemodialysis, was terminated early with inconclusive data on relative stroke and bleeding rates. ${ }^{1179}$

There are no RCT data on OAC use in patients with AF after kidney transplantation. The prescription and dosing of NOACs should be guided by the estimated glomerular filtration rate of the transplanted kidney and taking into account potential interactions with concomitant medication.

Particular attention must be given to the dosing of NOACs in patients with CKD (Supplementary Table 9).

\subsection{Atrial fibrillation and peripheral artery disease}

Patients with AF often have atherosclerotic vascular disease. With the inclusion of asymptomatic ankle-brachial index $\leq 0.90$ in the definition PAD, the prevalence of vascular disease increased significantly. ${ }^{1180}$ In a systematic review and meta-analysis, the presence of PAD was significantly associated with a 1.3 - to 2.5 -fold increased risk of stroke. ${ }^{347}$ Complex aortic plaque in the descending aorta, as identified on TOE, is also a significant vascular stroke risk factor (section 10.1.1).

In patients with asymptomatic PAD, the risk of cardiovascular events progressively increases with increasing vascular disease burden. ${ }^{470}$ Therefore, PAD patients should be opportunistically screened for AF. Patients with AF and PAD should be prescribed OAC, unless contraindicated. Those with stable vascular disease (arbitrarily defined as no new vascular event in the past 12 months) should be managed with OAC alone (section 11.3), as concomitant use of antiplatelet therapy has not been shown to reduce stroke or other cardiovascular events, but may increase serious bleeds, including $\mathrm{ICH}$.

The principles of rate and rhythm control outlined in section 10.2 also apply for AF patients with PAD. Special considerations include possibly limited exercise capacity in these patients, owing to intermittent claudication. Beta-blockers may exacerbate PAD symptoms in some patients, in whom NDCC blockers may be more appropriate for rate control.

\subsection{Atrial fibrillation and endocrine disorders}

Electrolyte disturbances and altered glucose and/or hormone levels in endocrine disorders such as thyroid disorders, acromegaly, pheochromocytoma, diseases of adrenal cortex, parathyroid disease, or pancreas dysfunction including diabetes mellitus may contribute to development of AF. Data on management of AF in these settings are limited. ${ }^{3}$ Diabetes is discussed in section 10.3.2.4. Stroke prevention should follow the same principles as in other AF patients, with risk stratification using the $\mathrm{CHA}_{2} \mathrm{DS}_{2}$-VASc score. ${ }^{3,1181}$ In AF patients with hyperthyroidism, spontaneous conversion of AF often occurs once a euthyroid state is achieved. ${ }^{1182}$ Withdrawal of amiodarone is mandatory in hyperthyroidism. AF catheter ablation should be performed under stable electrolytic and metabolic conditions and should not be carried out during active hyperthyroidism.

\subsection{Atrial fibrillation and gastrointestinal disorders}

While gastrointestinal lesions can lead to bleeding events in anticoagulated AF patients, some gastrointestinal conditions such as active inflammatory bowel disease increase the risk of AF and stroke. ${ }^{1183}$ Gastrointestinal bleeding is a well-known complication of OAC. Overall, NOAC use is associated with an increased risk of gastrointestinal bleeding, ${ }^{184,1185}$ but in patients treated with apixaban or dabigatran $110 \mathrm{mg}$ the risk is similar to warfarin. ${ }^{419,421}$ Bleeding lesions can be identified in more than $50 \%$ of cases of major gastrointestinal bleeding. ${ }^{1186}$ After correction of the bleeding source, OAC should be restarted, as this strategy has been associated with decreased risks of thrombo-embolism and death. ${ }^{1187}$

Patients treated with dabigatran may experience dyspepsia (about $11 \%$ in the RE-LY trial, and $2 \%$ discontinued the drug because of gastrointestinal symptoms ${ }^{419}$ ). After-meal ingestion of dabigatran and/or the addition of proton-pump inhibitors improves symptoms. ${ }^{1188}$

Management of AF patients with liver disease is challenging, owing to increased bleeding risk (associated with decreased hepatic synthetic function in advanced liver disease, thrombocytopenia, and gastrointestinal variceal lesions), as well as increased ischaemic risk $^{1189,1190}$ ). Patients with hepatic dysfunction were generally excluded from the RCTs, ${ }^{1191}$ especially those with abnormal clotting tests, as such patients may be at higher risk of bleeding on VKA, possibly less so on NOACs. Despite the paucity of data, observational 
studies did not raise concerns regarding the use of NOACs in advanced hepatic disease. ${ }^{1192}$ In a recent study, AF patients with liver fibrosis had no increase in bleeding on NOACs compared with VKAs. ${ }^{470}$ Other reassuring data for NOACs come from a large nationwide cohort. ${ }^{472} \mathrm{~A}$ number of patients may be started on a NOAC while having unrecognized significant liver damage and, in cirrhotic patients, ischaemic stroke reduction may outweigh bleeding risk. ${ }^{471}$ NOACs are contraindicated in patients within ChildTurcotte-Pugh $C$ hepatic dysfunction, and rivaroxaban is not recommended for patients in the Child-Turcotte-Pugh B or C category. ${ }^{1193}$

\subsection{Atrial fibrillation and haematological disorders}

Anaemia is an independent predictor of OAC-related major bleeding. ${ }^{393,402}$ In a population-based AF cohort, anaemia was associated with major bleeding and lower TTR, whereas OAC use in AF patients with moderate or severe anaemia was associated with more major bleeding but no reduction in thrombo-embolic risk. ${ }^{1194}$ Thrombocytopenia is also associated with increased bleeding risk. Before and during anticoagulation treatment, both anaemia and thrombocytopenia should be investigated and corrected, if possible. Decision making on OAC use in patients with platelet counts $<100 /$ $\mu \mathrm{L}$ requires a multidisciplinary approach including haematologists, balancing thrombotic and bleeding risks and addressing modifiable bleeding risk factors. Some chemotherapeutic drugs may increase the risk of incident AF (e.g. ibrutinib, melphalan, anthracyclines) ${ }^{1195-1197}$ or impair platelet function, thus increasing the risk of bleeding (e.g. ibrutinib). ${ }^{1198,1199}$

\subsection{The elderly and frail with atrial fibrillation}

The prevalence of AF increases progressively with age ${ }^{67,1200-1206}$, and age is an independent risk factor for adverse outcomes in AF. ${ }^{372,1200,1207,1208}$ Older people are less likely to receive OAC ${ }^{1209-1216}$ despite sufficient evidence supporting the use of OAC in this population. Frailty, comorbidities, and increased risk of falls $^{1217-1219}$ do not outweigh the benefits of OAC given the small absolute risk of bleeding in anticoagulated elderly patients. ${ }^{339,390,391,1220-1223}$ Evidence from RCTs, ${ }^{441,1224}$ meta-analyses $^{423,1225}$ and large registries $339,433,1209,1226$ support the use of OAC in this age group. Antiplatelets are neither more effective nor safer than warfarin and may even be harmful, ${ }^{433}$ whereas NOACs appear to have a better overall risk-benefit profile compared with warfarin. ${ }^{423,433,441,1035,1225,1227-1236}$ Prescribing a reduced dose of OAC is less effective in preventing AF adverse outcomes. ${ }^{1107,1211,1237,1238}$

Rate control is traditionally the preferred strategy, but evidence informing the choice between rate and rhythm control in the elderly is insufficient. ${ }^{1239-1242}$ Limited evidence on other AF treatments supports the use of all rate and rhythm control options, including cardioversion, pacemaker implantation, and AF catheter ablation without any age discrimination. AF catheter ablation may be an effective and safe option in selected older individuals with success rates comparable to younger patients ${ }^{1243-1255}$ and acceptable complication rates. ${ }^{1243,1245-1247,1249-1260}$ Nevertheless, age was a predictor of complications in AF catheter ablation in some studies ${ }^{1261-1263}$ and longer follow-up studies suggested an age-related increase in multivariable-adjusted risk for AF/AFL recurrence, death, and major adverse cardiac events. ${ }^{1257}$

\subsection{Patients with cognitive impairment/dementia}

Evidence regarding effective prevention of cognitive impairment in $A F$ is derived mainly from observational studies, suggesting that $O A C$ could play a protective role in AF patients with stroke risk factors, not only for stroke prevention but also for prevention of cognitive decline. $^{1264}$ The quality of anticoagulation with VKAs (i.e. TTR) seems to play an additional role: low TTR and supratherapeutic INR values were associated with higher risk of dementia. ${ }^{1265,1266}$ Limited evidence suggests that NOACs may be superior to VKA for preventing cognitive impairment in some, ${ }^{1267,1268}$ but not all, studies. ${ }^{1269}$ Recent observational data indicate a protective effect of OAC even in lowrisk $\mathrm{AF}$ patients who do not need OAC for stroke prevention. ${ }^{1270} \mathrm{~A}$ number of RCTs with cognitive function as an endpoint are ongoing and will provide more insights into the role of anticoagulation (NOACs and VKAs) for prevention of cognitive impairment in $A F^{86}$

Conversely, cognitive impairment can influence treatment adherence, ${ }^{1271,1272}$ thus affecting outcomes in AF patients. After AF catheter ablation, silent brain lesions are detected by MRI, but this has not led to cognitive impairment in the AXAFA-AFNET 5 trial, although underpowered. ${ }^{880}$

\subsection{Atrial fibrillation and congenital heart disease}

Survival of patients with congenital heart disease has increased over time, but robust data on the management of AF are missing and available evidence is derived mainly from observational studies and/or extrapolation from large clinical trials.

In patients with AF (or AFL or intra-atrial re-entrant tachycardia) and congenital heart disease, $O A C$ treatment is recommended for all patients with intracardiac repair, cyanotic congenital heart disease, Fontan palliation, or systemic right ventricle. ${ }^{1273}$ Patients with $A F$ and other congenital heart diseases should follow the general risk stratification for OAC use in AF. Notably, NOACs are contraindicated in patients with mechanical heart valves, ${ }^{1165}$ whereas they seem safe in those with a valvular bioprosthesis. ${ }^{1274,1275}$

Rate control drugs such as beta-blockers, verapamil, diltiazem, and digitalis can be used with caution due to the risk of bradycardia and hypotension. Rhythm control strategies (i.e. amiodarone) may be effective. In Fontan patients, sodium-channel blockers suppress half of the atrial arrhythmias, but caution is needed for proarrhythmia. When cardioversion is planned, both 3 weeks of anticoagulation and TOE may be considered as thrombi are common in patients with congenital heart disease and atrial tachyarrhythmias. ${ }^{1276,1277}$

In patients with atrial septal defect, closure may be considered before the fourth decade of life to decrease the risk of AF or AFL. ${ }^{1278}$ Patients with stroke who underwent closure of the patent foramen ovale may have an increased risk of $A F^{1279}$ but in patients with patent foramen ovale and AF, closure is not recommended for stroke prevention; and OAC use should be decided using the conventional stroke risk assessment tool. In patients with a history of AF, AF surgery or AF catheter ablation should be considered at the time of 
closure of the septal defect. ${ }^{1280-1282}$ AF catheter ablation of late atrial arrhythmias is likely to be effective after surgical atrial septal defect closure. ${ }^{1283}$

\section{Recommendations for the management of AF in patients with congenital heart disease}

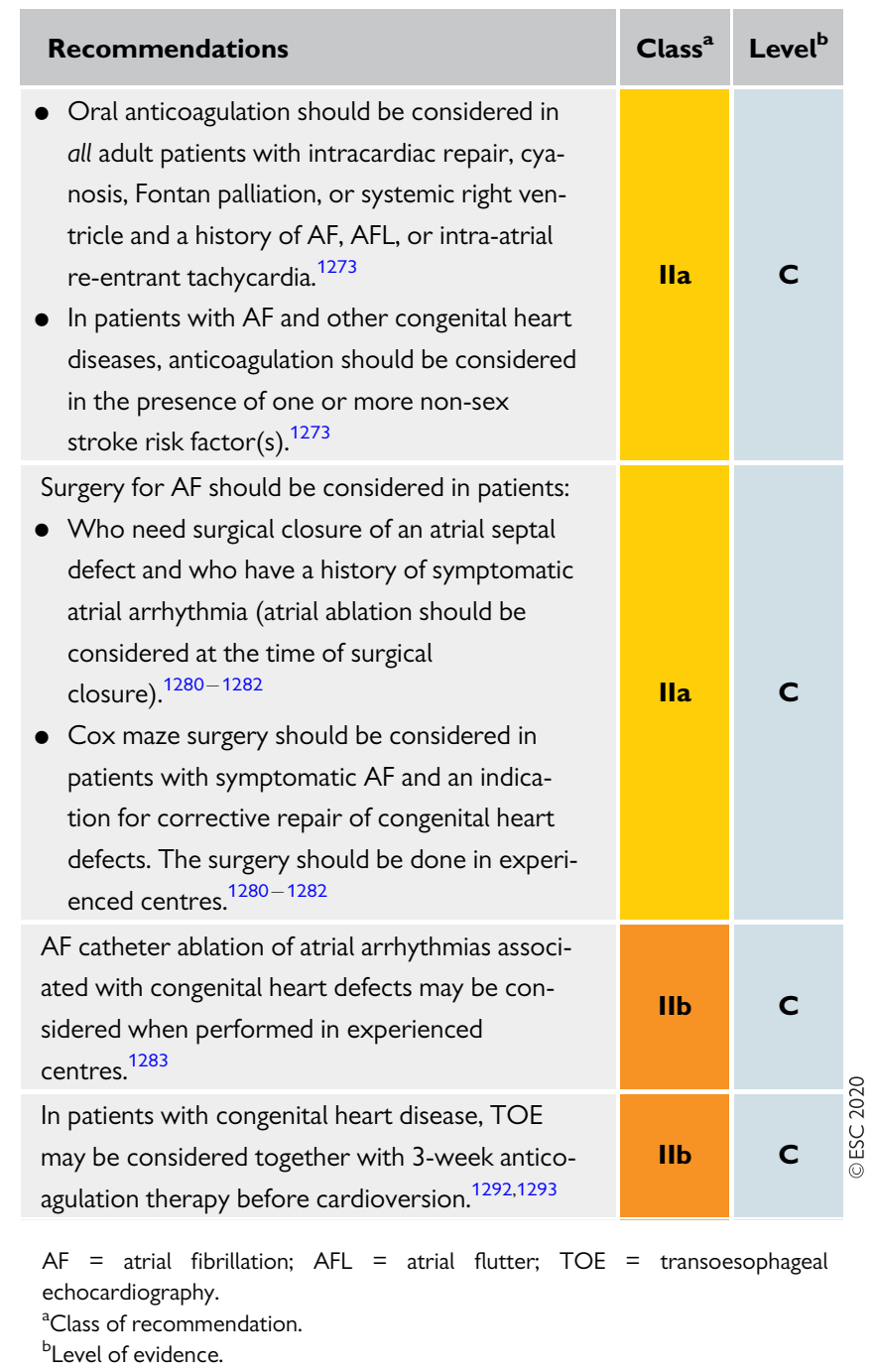

\subsection{Atrial fibrillation in inherited cardiomyopathies and primary arrhythmia syndromes}

A higher incidence and prevalence of AF have been described in patients with inherited cardiomyopathies and primary arrhythmia syndromes. ${ }^{1284-1318}$ Sometimes AF is the presenting or only clinically overt feature, ${ }^{1319-1323}$ is often associated with adverse clinical outcomes, ${ }^{1292,1299,1301,1307,1308,1310,1324-1329}$ and has important implications:

- The use of AADs may be challenging. In congenital long QT syndrome, many drugs are contraindicated owing to increased risk of QT prolongation and torsade de pointes (http://www.credible meds.org/); in Brugada syndrome, class I drugs are contraindicated (http://www.brugadadrugs.org/). Owing to its long-term adverse effects, chronic use of amiodarone is problematic in these typically young individuals.

- In patients with an implantable cardioverter defibrillator, AF is a common cause of inappropriate shocks. ${ }^{1307,1311,1330-1333}$ Programming a single high-rate ventricular fibrillation zone $\geq 210$ - 220 bpm with long detection time is safe, ${ }^{1295,1296,1334}$ and is recommended in patients without documented slow monomorphic ventricular tachycardia. Implantation of an atrial lead may be considered in case of significant bradycardia under betablocker treatment.

Supplementary Table 14 summarizes the main clinical features of AF in patients with inherited cardiac diseases.

Patients with Wolff-Parkinson-White syndrome and AF are at risk of fast ventricular rates resulting from rapid conduction of atrial electrical activity to the ventricles via the accessory pathway, and at increased risk of ventricular fibrillation and sudden death. ${ }^{1335,1336}$ Electrical cardioversion should be readily available for haemodynamically compromised patients with pre-excited $\mathrm{AF}$, and atrioventricular node-modulating drugs (e.g. verapamil, beta-blockers, digoxin) should be avoided. ${ }^{1337,1338}$ Pharmacological cardioversion can be attempted using ibutilide, ${ }^{1339}$ whereas AADs class la (procainamide) and Ic (propafenone, flecainide) should be used with caution owing to their effect on the atrioventricular node. ${ }^{1340-1343}$ Amiodarone may not be safe in pre-excited $\mathrm{AF}$ as it may enhance pathway conduction. ${ }^{1343}$

\subsection{Atrial fibrillation during pregnancy} $\mathrm{AF}$ is one of the most frequent arrhythmias during pregnancy, ${ }^{1344}$

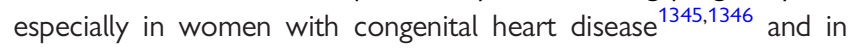
older gravidae, ${ }^{1344,1347,1348}$ and is associated with increased risk of death. ${ }^{1344}$ Rapid atrioventricular conduction may have serious haemodynamic consequences for mother and foetus.

Pregnancy is associated with a hypercoagulable state and increased thrombo-embolic risk. Given the lack of specific data, the same rules for stroke risk assessment should be used as in non-pregnant women. ${ }^{1349}$ Detailed practical recommendations on oral and parenteral anticoagulation regimens depending on the pregnancy trimester, such as low- and high-dose VKA use during the second and third trimesters, timing of low-molecular-weight heparin (LMWH) to unfractionated heparin (UFH) relative delivery, and control of therapeutic effects are given in the recent ESC Pregnancy Guidelines. ${ }^{1349}$ Immediate anticoagulation is required in clinically significant mitral stenosis, using LMWH at therapeutic doses in the first and last trimesters, and VKA with the usual INR targets or LMWH for the second trimester. Use of NOACs is prohibited during pregnancy. Vaginal delivery should be advised for most women but is contraindicated while the mother is on VKAs because of the risk of foetal intracranial bleeding. ${ }^{1349}$

Intravenous beta-blockers are recommended for acute rate control. Beta-1 selective blockers (e.g. metoprolol and bisoprolol) are generally safe and are recommended as the first choice. ${ }^{1349}$ If beta-blockers fail, digoxin and verapamil should be considered for rate control.

Rhythm control should be considered the preferred strategy during pregnancy. Electrical cardioversion is recommended if there is haemodynamic instability or considerable risk for mother or foetus. It can be performed safely without compromising foetal blood flow ${ }^{1350}$ and the consequent risk for foetal arrhythmias or preterm 
labour is low. ${ }^{1351,1352}$ The fetal heart rate should routinely be controlled after cardioversion. ${ }^{1353}$ Cardioversion should generally be preceded by anticoagulation (section 10.2.2.6). ${ }^{1349}$ In haemodynamically stable patients without structural heart disease, i.v. ibutilide or flecainide may be considered for termination of AF but experience is limited. ${ }^{1354,1355}$ Flecainide, propafenone, or sotalol should be considered to prevent AF if atrioventricular nodal-blocking drugs fail. AF catheter ablation has no role during pregnancy.

\section{Recommendations for the management of AF during pregnancy}

\begin{tabular}{|c|c|c|}
\hline Recommendations & Class $^{a}$ & Level $^{\mathrm{b}}$ \\
\hline \multicolumn{3}{|l|}{ Acute management } \\
\hline $\begin{array}{l}\text { Immediate electrical cardioversion }{ }^{c} \text { is recom- } \\
\text { mended in case of haemodynamic instability or } \\
\text { pre-excited AF. }{ }^{1350,1351,1354}\end{array}$ & $\mathbf{I}$ & C \\
\hline $\begin{array}{l}\text { In pregnant women with } \mathrm{HCM} \text {, cardioversion }{ }^{\mathrm{c}} \\
\text { should be considered for persistent } \mathrm{AF} .\end{array}$ & Illa & C \\
\hline $\begin{array}{l}\text { Ibutilide or flecainide i.v. may be considered for } \\
\text { termination of AF in stable patients with struc- } \\
\text { turally normal hearts. }\end{array}$ & Illb & C \\
\hline \multicolumn{3}{|l|}{ Long-term management (oral administration of drugs) } \\
\hline $\begin{array}{l}\text { Therapeutic anticoagulation with heparin or } \\
\text { VKA according to the stage of pregnancy is rec- } \\
\text { ommended for patients with AF. }{ }^{1349}\end{array}$ & $\mathbf{I}$ & C \\
\hline $\begin{array}{l}\text { Beta-selective blockers are recommended for } \\
\text { rate control in } \mathrm{AF} .^{\text {d }}\end{array}$ & $\mathbf{I}$ & C \\
\hline $\begin{array}{l}\text { Flecainide, }{ }^{e} \text { propafenone, }{ }^{e} \text { or sotalol } f^{f} \text { should be } \\
\text { considered to prevent AF if atrioventricular } \\
\text { nodal-blocking drugs }{ }^{f} \text { fail. }\end{array}$ & Ila & C \\
\hline $\begin{array}{l}\text { Digoxin }{ }^{g} \text { or verapamilg should be considered for } \\
\text { rate control if beta-blockers fail. }\end{array}$ & Ila & C \\
\hline
\end{tabular}

$\mathrm{AF}=$ atrial fibrillation; $\mathrm{ECG}=$ electrocardiogram; US FDA = United States Food and Drug Administration; i.v. = intravenous; $L V=$ left ventricular; $\mathrm{HCM}=$ hypertrophic cardiomyopathy; $\mathrm{QTc}=$ corrected $\mathrm{QT}$ interval; $\mathrm{VKA}=$ vitamin $\mathrm{K}$ antagonist.

${ }^{\mathrm{a} C}$ Class of recommendation.

bLevel of evidence.

${ }^{c}$ Cardioversion of AF should generally be preceded by anticoagulation.

${ }^{d}$ Atenolol has been associated with higher rates of foetal growth retardation and is not recommended. ${ }^{1356}$

eFlecainide and propafenone should be combined with atrioventricular nodalblocking drugs, but structural heart disease, reduced LV function, and bundle branch block should be excluded.

${ }^{f}$ Class III drugs should not be used in prolonged QTc.

${ }^{\mathrm{g}}$ Atrioventricular nodal-blocking drugs should not be used in patients with preexcitation on resting ECG or pre-excited AF.

Note that the former $A$ to $X$ categories of drugs - the classification system for counselling of pregnant women requiring drug therapy-was replaced by the Pregnancy and Lactation Labelling Rule, which provides a descriptive risk summary and detailed information on animal and clinical data, by the US FDA in June 2015.

\subsection{Atrial fibrillation in professional athletes}

Moderate physical activity improves cardiovascular health and prevents $A F$, whereas intense sports activity increases the risk of
AF. ${ }^{35,1357}$ Athletes have an approximate five-fold increased lifetime risk of AF compared with sedentary individuals despite a lower prevalence of conventional AF risk factors. ${ }^{35,1020}$ Risk factors for AF in athletes include male sex, middle age, endurance sports, tall stature, and total lifetime exercise dose exceeding $1500-2000$ hours. ${ }^{1020,1358-1361}$ Endurance sports such as running, cycling, and cross-country skiing ${ }^{35,1362}$ carry the highest risk.

In the absence of RCTs, recommendations for AF management in athletes are based largely on evidence in non-athletes, observational data, and expert consensus. ${ }^{143}$ The need for anticoagulation is determined by clinical risk factors. Sports with direct bodily contact or prone to trauma should be avoided in patients on OAC. As athletes have a high prevalence of sinus bradycardia and sinus pauses, medical therapy is frequently contraindicated or poorly tolerated. ${ }^{1021,1363}$ Digoxin and verapamil are often ineffective for rate control during exertional AF, whereas beta-blockers may not be well tolerated or are sometimes prohibited. Pill-in-the-pocket therapy has been used, but sports activity should be avoided after ingestion of flecainide or propafenone until AF ceases and two half-lives of the drug have elapsed. ${ }^{586}$ AF catheter ablation is often preferred by athletes and was similarly efficacious in both the athletic and non-athletic populations in small studies. ${ }^{1364,1365}$

\section{Recommendations for sports activity in patients with AF}

\begin{tabular}{|c|c|c|}
\hline Recommendation & Class $^{a}$ & Level $^{\mathbf{b}}$ \\
\hline $\begin{array}{l}\text { It is recommended to counsel professional ath- } \\
\text { letes that long-lasting intense sports participation } \\
\text { may promote AF, while moderate physical activ- } \\
\text { ity is recommended to prevent } \\
\text { AF. }^{35,38,1020,1360,1366-1368}\end{array}$ & I & B \\
\hline
\end{tabular}

$\mathrm{AF}=$ atrial fibrillation

${ }^{\text {a }}$ Class of recommendation.

'Level of evidence.

\subsection{Postoperative atrial fibrillation}

Perioperative AF describes the onset of the arrhythmia during an ongoing intervention. This is most relevant in patients undergoing cardiac surgery. While multiple strategies to reduce the incidence of perioperative AF with pretreatment or acute drug treatment have been described, there is lack of evidence from large RCTs. Amiodarone is the most frequently used drug for prevention of perioperative AF. $^{1369}$

Postoperative AF, defined as new-onset $A F$ in the immediate postoperative period, is a clinically relevant problem, ${ }^{1370,1371}$ occurring in $20-50 \%$ of patients after cardiac surgery, ${ }^{1372,1373} 10$ - 30\% after noncardiac thoracic surgery, ${ }^{1374}$ and in 5 - 10\% after vascular or large colorectal surgery, ${ }^{1375}$ with peak incidence between postoperative day 2 and $4 .{ }^{1376}$ Intra- and postoperative changes affecting AF triggers and pre-existing atrial substrate may increase atrial vulnerability to AF. Many episodes of postoperative AF are self-terminating and some are asymptomatic, but postoperative AF has been associated with a four- to five-fold risk of recurrent $A F$ in the next 5 years. ${ }^{1377,1378}$ It has also been shown to be a risk factor for stroke, 


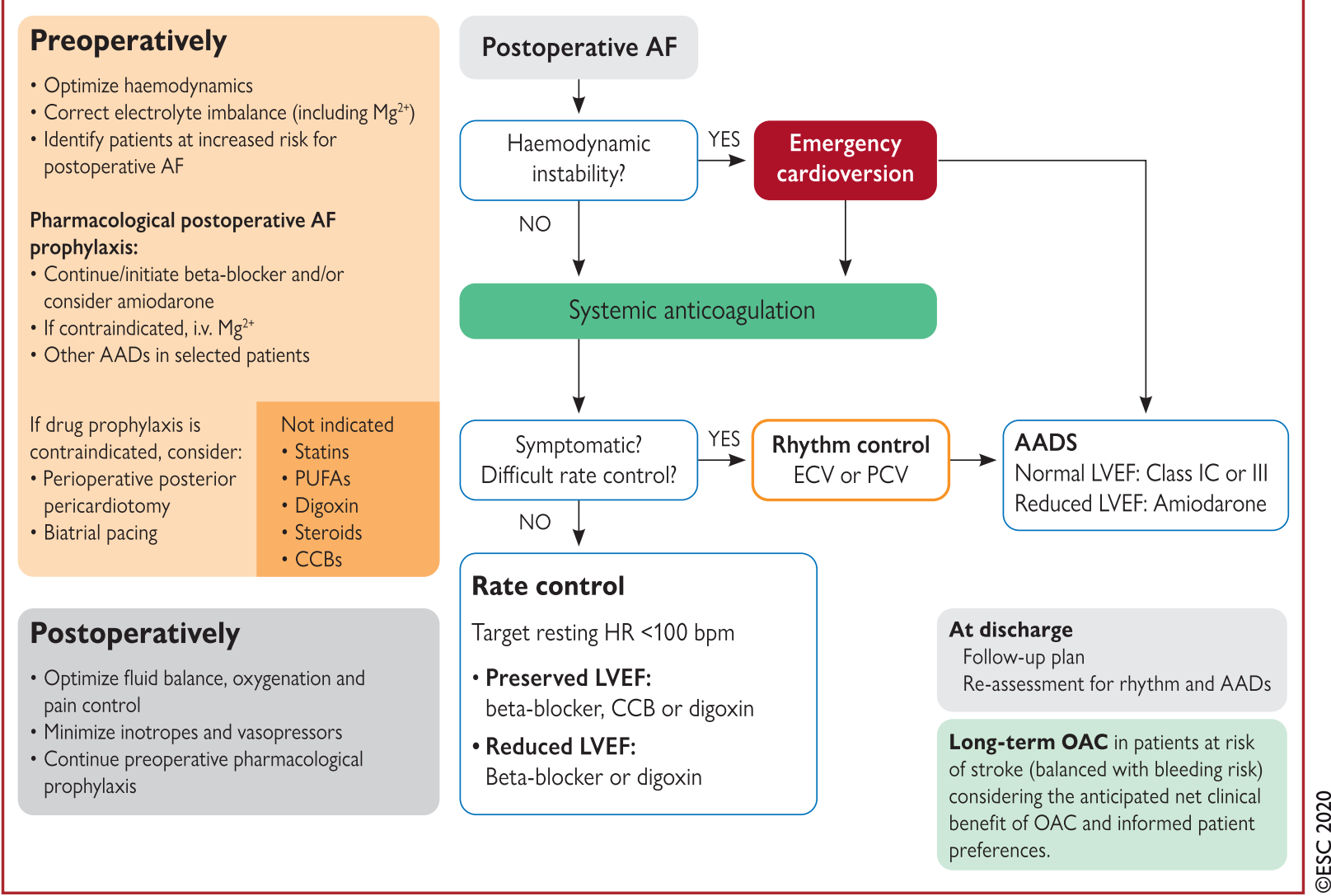

Figure 23 Management of postoperative AF. AAD = antiarrhythmic drug; bpm = beats per minute; $C C B=$ calcium channel blocker; $E C V=$ electrical cardioversion; LVEF = left ventricular ejection fraction; $\mathrm{Mg}^{2+}$ magnesium; $\mathrm{OAC}=$ oral anticoagulation; $\mathrm{PCV}=$ pharmacological cardioversion; PUFA=polyunsaturated fatty acid.

myocardial infarction, and death compared with non-postoperative AF patients. ${ }^{1379,1380}$

Other adverse consequences of postoperative AF include haemodynamic instability, prolonged hospital stay, infections, renal complications, bleeding, increased in-hospital death, and greater healthcare costs. $^{1371,1381,1382}$ Management of postoperative AF is shown in Figure 23.

\subsubsection{Prevention of postoperative AF}

Preoperative beta-blocker (propranolol, carvedilol plus $\mathrm{N}$-acetyl cysteine) use in cardiac and non-cardiac surgery is associated with a reduced incidence of postoperative $A F,{ }^{1383-1386}$ but not major adverse events such as death, stroke, or acute kidney injury. ${ }^{1387}$ Notably, in non-cardiac surgery, perioperative metoprolol was associated with increased risk of death in a large RCT. ${ }^{1388}$ In a metaanalysis, amiodarone (oral or i.v.), and beta-blockers were equally effective in reducing postoperative $A F,{ }^{1389}$ but their combination was better than beta-blockers alone. ${ }^{1390}$ Lower cumulative doses of amiodarone $(<3000 \mathrm{mg}$ ) could be effective, with fewer adverse events. ${ }^{1391-1393}$ Data for other interventions such as statins ${ }^{974,}, 1394$ magnesium, ${ }^{1395}$ sotalol, ${ }^{1385}$ colchicine, ${ }^{1396}$ posterior pericardiotomy, ${ }^{1397,1398}$ (bi)atrial pacing, ${ }^{1385}$ and corticosteroids ${ }^{1399}$ are not robust. Two large RCTs showed no significant effect of i.v. steroids on the incidence of postoperative AF after cardiac surgery, ${ }^{1400,1401}$ and colchicine is currently being investigated in the prevention of postoperative AF [COP-AF (Colchicine For The Prevention Of Perioperative Atrial Fibrillation In Patients Undergoing Thoracic Surgery): NCT03310125].

\subsubsection{Prevention of thrombo-embolic events}

In a large meta-analysis, patients with postoperative AF had a $62 \%$ higher odds of early and $37 \%$ higher risk of long-term stroke compared with those without postoperative AF ( $\geq 1$-year stroke rates were $2.4 \%$ vs. $0.4 \%$, respectively), as well as $44 \%$ higher odds of early and $37 \%$ higher risk of long-term mortality; long-term stroke risk was substantially higher with non-cardiac than cardiac postoperative AF (HR 2.00; 95\% Cl 1.70-2.35 for non-cardiac vs. HR 1.20; 95\% Cl 1.07-1.34 for cardiac postoperative AF; $P$ for subgroup difference $<0.0001)^{1379}$

Nevertheless, the evidence on OAC effects in patients with postoperative AF is not very robust. ${ }^{1382,1402-1407}$ Observational data ${ }^{1408}$ suggest that although coronary artery bypass graft-related postoperative AF might not be equivalent to non-surgery AF regarding the long-term risk of adverse outcomes, OAC use during follow-up was associated with a significantly lower risk of thrombo-embolic events in both postoperative AF and non-surgery AF compared with no OAC. ${ }^{1408}$ Reportedly, postoperative AF occurring after non-cardiac surgery was associated with a similar long-term thrombo-embolic risk to non-surgery AF, and OAC therapy was associated with comparably lower risk of thrombo-embolic events and all-cause death in 
both groups. ${ }^{1409}$ Ongoing RCTs in cardiac [PACES (Anticoagulation for New-Onset Post-Operative Atrial Fibrillation After CABG); NCT04045665] and non-cardiac (ASPIRE-AF; NCT03968393) surgery will inform optimal long-term OAC use among patients developing postoperative AF.

In haemodynamically unstable patients with postoperative AF, emergency electrical cardioversion (or i.v. administration of amiodarone $^{1385}$ or vernakalant, ${ }^{583}$ if consistent with the clinical situation) is indicated. In a recent RCT of postoperative AF patients after cardiac surgery, neither rate nor rhythm control showed net clinical advantage over each other. ${ }^{1373}$ Hence, rate or rhythm control treatment decisions should be based on symptoms, and non-emergency cardioversion should follow the principles of peri-cardioversion anticoagulation outlined in section 10.2.

\section{Recommendations for postoperative AF}

\begin{tabular}{|c|c|c|}
\hline Recommendations & Class $^{a}$ & Level $^{\mathbf{b}}$ \\
\hline $\begin{array}{l}\text { Perioperative amiodarone or beta blocker ther- } \\
\text { apy is recommended for the prevention of post- } \\
\text { operative AF after cardiac surgery. }{ }^{1390,1492}\end{array}$ & $\mathbf{I}$ & $\mathbf{A}$ \\
\hline $\begin{array}{l}\text { Long-term OAC therapy to prevent thrombo- } \\
\text { embolic events should be considered in patients } \\
\text { at risk for stroke with postoperative AF after } \\
\text { non-cardiac surgery, considering the anticipated } \\
\text { net clinical benefit of OAC therapy and informed } \\
\text { patient preferences. }{ }^{1404,1405,1408,1409}\end{array}$ & Ila & B \\
\hline $\begin{array}{l}\text { Long-term OAC therapy to prevent thrombo- } \\
\text { embolic events may be considered in patients at } \\
\text { risk for stroke with postoperative AF after car- } \\
\text { diac surgery, considering the anticipated net clin- } \\
\text { ical benefit of OAC therapy and informed } \\
\text { patient preferences. }{ }^{1404,1405,1408,1409}\end{array}$ & Ilb & B \\
\hline $\begin{array}{l}\text { Beta-blockers should not be used routinely for } \\
\text { the prevention of postoperative } A F \text { in patients } \\
\text { undergoing non-cardiac surgery. }{ }^{1410}\end{array}$ & III & B \\
\hline
\end{tabular}

$\mathrm{AF}=$ atrial fibrillation; $\mathrm{OAC}=$ oral anticoagulant.

${ }^{\mathrm{a}}$ Class of recommendation.

bLevel of evidence.

\section{Prevention of atrial fibrillation}

\subsection{Primary prevention of atrial fibrillation}

Primary prevention of AF refers to the implementation of preventive measures in patients at risk but without previous documentation of AF. This strategy relies on the identification and management of risk factors and comorbidities predisposing to AF, before the development of atrial remodelling and fibrosis ${ }^{964,1411}$ Upstream therapy refers to the use of non-AADs that modify the atrial substrate or target-specific mechanisms of AF to prevent the occurrence or recurrence of the arrhythmia. The key targets of upstream therapy are structural changes in the atria (e.g. fibrosis, hypertrophy, inflammation, oxidative stress), but effects on atrial ion channels, gap junctions, and calcium handling are also evident. ${ }^{964}$
Adequate management of hypertension and $\mathrm{HF}$ may prevent $\mathrm{AF}$ by reducing atrial stretch, but inhibition of the renin-angiotensinaldosterone system may exert an additional protective role by suppressing electrical and structural cardiac remodelling. ${ }^{964,1411,1412}$ Large RCTs and meta-analyses have yielded equivocal results, either in favour ${ }^{1413-1416}$ or against ${ }^{1417-1421}$ statin use for primary prevention of AF. Controversial results have also been reported for the effects of fish oils on primary prevention of AF. ${ }^{1422}$

For primary prevention of postoperative AF after cardiac and noncardiac surgery, see section 11.19 .

\subsection{Secondary prevention of atrial fibrillation}

For secondary AF prevention see section 11.3 and Supplementary section 12.

\section{Sex-related differences in atrial fibrillation}

Female patients are generally under-represented in RCTs, including AF trials. Sex-related differences in the epidemiology, pathophysiology, clinical presentation, and prognosis of AF that are consistently reported ${ }^{19,107,124,1423,1424}$ may influence the effectiveness of AF treatment, and hence should be considered in a personalized, individual patient-centred approach to AF management in clinical practice. ${ }^{1425}$ Understanding the underlying pathophysiological mechanisms and biology may help to improve personalized treatments. Adequate representation of women in future AF trials is recommended, as well as the identification and resolution of sex-specific barriers to implementation of guideline-recommended treatments for AF.

Women presenting with $A F$ are older, have a higher prevalence of hypertension, $\mathrm{VHD}$, and $\mathrm{HFpEF}$, and a lower prevalence of CAD compared with men. Women with AF are more often symptomatic than men with $A F$, with greater symptom severity. ${ }^{1423,1426}$

Female sex is a stroke risk modifier that increases the risk of AFassociated stroke in the presence of other stroke risk factors. ${ }^{353}$ Women with AF have a greater stroke severity and permanent disability than men with AF. ${ }^{1427}$ Anticoagulation with warfarin may be less well controlled in women, and they have a greater residual stroke risk even with well-controlled VKAs. ${ }^{1428}$ The efficacy and safety of NOACs in landmark RCTs were consistent in both sexes, but women were largely under-represented. ${ }^{423}$

In women with AF, the use of AADs for rhythm control is associated with significantly higher rates of life-threatening adverse events (e.g. acquired long QT syndrome with class la or III AADs) ${ }^{1429,1430}$ or sinus-node disease/bradyarrhythmia requiring pacemaker implantation ${ }^{19}$ compared with male patients. Women with AF are less likely to undergo electrical cardioversion, ${ }^{1426}$ and are referred for AF catheter ablation later than men, possibly reflecting AF occurrence later in life among women. ${ }^{107,1431,1432}$ The result of PVI may be less favourable in women, ${ }^{1431,1432}$ with higher rates of procedure-related complications. ${ }^{1431}$ Women are more likely to undergo atrioventricular nodal ablation for AF than men. ${ }^{124}$ Sex-specific data on cardiovascular risk management in women with AF are lacking. Principles outlined in section 11.3 apply to women with AF. 


\section{Recommendations pertaining to sex-related differences in AF}

\section{Recommendation}

It is recommended that women and men with $\mathrm{AF}$ are equally offered diagnostic assessment and therapies to prevent stroke and other AFrelated complications. ${ }^{423,1433}$

Women with symptomatic paroxysmal or persistent AF should be offered timely access to rhythm control therapies, including AF catheter ablation, when appropriate for medical reasons. ${ }^{1448,1451}$

$\mathrm{AF}=$ atrial fibrillation

${ }^{\mathrm{a}}$ Class of recommendation.

bevel of evidence.

\section{Implementation of the atrial fibrillation guidelines}

Guideline-adherent care (i.e. the implementation of guidelinerecommended management to individual AF patients) aims to improve patient outcomes and reduce healthcare costs, ${ }^{1238,1434,1435}$ but adherence to guidelines is modest worldwide. $^{124,1436-1439,1440,1441}$ Reportedly, the adoption of NOACs as first-line therapy has been associated with increasing guideline-adherent stroke prevention. ${ }^{1442,1443}$

Guideline non-adherence is multifactorial, ${ }^{1215,1444,1445}$ including physician/healthcare professional- and healthcare system-related factors. ${ }^{1446}$ Integrated AF management may facilitate adherence to guidelines. Various educational interventions ${ }^{280,284,290,1447,1448}$ based on guideline-provided recommendations ${ }^{284}$ and tailored to close specific knowledge gaps among healthcare professionals and/or AF patients ${ }^{1446}$ may facilitate the implementation of guideline-based AF management to improve patient outcomes. ${ }^{277,1449-1452}$ Further research is needed to identify the cost-effective intervention type(s) that would more effectively improve patient clinical outcomes, medication adherence, and QoL.

\section{Quality measures and clinical performance indicators in the management of atrial fibrillation}

Measurable service quality has been identified as a cornerstone for optimal AF management and is a mandatory step towards valuebased healthcare. Quality and performance indicator sets should provide practitioners and institutions with the tools to measure the quality of care (e.g. adherence to guideline class I recommendations upon discharge/end of visit, complications after procedures, access/waiting list times) and identify opportunities for improvement. They should capture important aspects of care quality, including structure, process, outcome measures, and patient-centrednes, while the reporting burden for hospitals, practices, and practitioners should be kept to a minimum. $^{658,1453-1455}$

A collaborative effort involving the ESC, EHRA, Asia Pacific Heart Rhythm Society, Heart Rhythm Society, and Latin American Heart Rhythm Society was put in place to develop quality indicators for the diagnosis and management of AF; a summary form of these quality indicators is provided in Table 22, with the full set published separately. $^{317}$ The ESC quality indicators are intended for quality improvement and performance measurement through meaningul surveillance, as well as for integration within registries that specifically aim to identify areas for improvement in clinical practice and are not intended for ranking healthcare professionals/providers or payment incentives.

\section{Recommendations for quality measures in patients with} AF

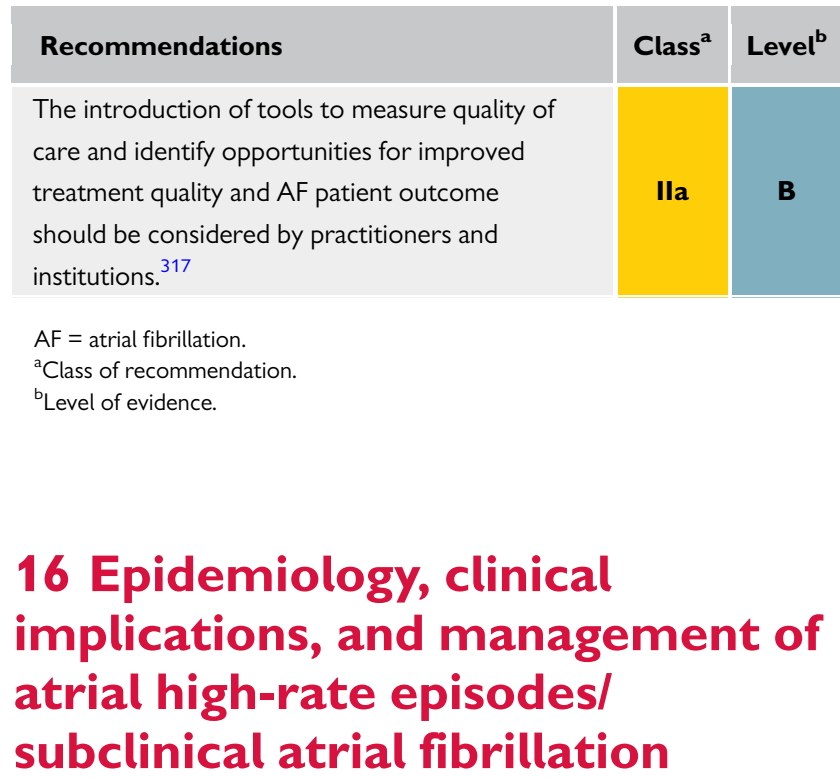

The incidence of AHRE/subclinical AF in patients with a pacemaker/ implanted device is $30-70 \%$, but it may be lower in the general population. ${ }^{1458}$ Very short episodes ( $\leq 10-20$ s/day) are considered clinically irrelevant, as they are not significantly associated with longer episodes or an increased risk of stroke or systemic embolism. ${ }^{1459}$ However, longer episodes of AHRE/subclinical AF (minimum of 5 - 6 $\mathrm{min}$ ) are associated with an increased risk of clinical AF, 467,469 ischaemic stroke, ${ }^{168,467}$ major adverse cardiovascular events, ${ }^{1460}$ and cardiovascular death. ${ }^{1461}$

Overall, the absolute risk of stroke associated with AHRE/subclinical AF may be lower than with clinical AF. ${ }^{160,168,226,467}$ The temporal dissociation from acute stroke suggests that AHRE/subclinical AF may represent a marker rather than a risk factor for stroke $e^{4,7,1462}$ (Supplementary Box 6).

Whereas current data were obtained mostly from pacemakers/ implantable cardioverter defibrillators or post-stroke patients, $A H R E / s u b c l i n i c a l ~ A F$ is increasingly reported in a variety of patients undergoing cardiac monitoring. Clinical AF will reportedly develop in 1 in 5 - 6 of patients within 2.5 years after diagnosing AHRE/subclinical AF. ${ }^{168}$ Notwithstanding that more high-quality evidence is needed to inform optimal management of these patients, more intense 


\section{Table 22 Summary of quality indicators for the diagnosis and management of AF}

\section{Domain: Patient assessment (at baseline and follow-up)}

Main quality indicator: $\mathrm{CHA}_{2} \mathrm{DS}_{2}$-VASc cardioembolic risk assessment.

Main quality indicator: bleeding risk assessment using a validated method such as the HAS-BLED score.

Numerator: Number of AF patients who have their respective score documented at the time of diagnosis and at every follow-up appointment.

Denominator: Number of AF patients.

\section{Domain: Anticoagulation}

Main quality indicator: inappropriate prescription of anticoagulation to patients with a $\mathrm{CHA}_{2} \mathrm{DS}_{2}-\mathrm{VASc}$ score of 0 for men and 1 for women.

Numerator: number of $\mathrm{AF}$ patients with $\mathrm{CHA}_{2} \mathrm{DS}_{2}$-VASc score of 0 for men and 1 for women, who are inappropriately prescribed anticoagulation.

Denominator: number of AF patients with $\mathrm{CHA}_{2} \mathrm{DS}_{2}$-VASc score of 0 for men and 1 for women who do not have other indication for anticoagulation.

Main quality indicator: proportion of patients with a $\mathrm{CHA}_{2} \mathrm{DS}_{2}-\mathrm{VASc}$ score of $\geq 1$ for men and $\geq 2$ for women who are prescribed anticoagulation.

Numerator: Number of $A F$ patients with $\mathrm{CHA}_{2} \mathrm{DS}_{2}$-VASc score of $\geq 1$ for men and $\geq 2$ for women who are prescribed anticoagulation.

Denominator: Number of AF patients with $\mathrm{CHA}_{2} \mathrm{DS}_{2}-\mathrm{VASc}$ score of $\geq 1$ for men and $\geq 2$ for women who are eligible for anticoagulation with no contraindication or refusal.

\section{Domain: rate control}

Main quality indicator: inappropriate prescription of $A A D s^{a}$ to patients with permanent AF (i.e. where no attempt to restore sinus rhythm is planned).

Numerator: Number of patients with permanent AF who are prescribed one or more AADs ${ }^{\mathrm{a}}$ for rhythm control.

Denominator: Number of patients with permanent AF.

\section{Domain: rhythm control}

Main quality indicator: inappropriate prescription of class IC AADs to patients with structural heart disease.

Numerator: number of AF patients with structural heart disease who are inappropriately prescribed class IC AADs.

Denominator: number of AF patients with structural heart disease.

Main quality indicator: proportion of patients with symptomatic paroxysmal or persistent $A F$ who are offered $A F$ catheter ablation after failure of/intolerance to one class I or class III AAD.

Numerator: Number of patients with paroxysmal or persistent AF who are offered catheter ablation after the failure of, or intolerance to, at least one class I or class III AAD.

Denominator: Number of patients with paroxysmal or persistent AF with no contraindications (or refusal) to catheter ablation who remain symptomatic on, or intolerant to at least one class I or class III AAD.

\section{Domain: risk factor management}

Main quality indicator: Proportion of patients who have their modifiable risk factors identified.

Numerator: number of AF patients who have their modifiable risk factors (e.g. BP, obesity, OSA, alcohol excess, lack of exercise, poor glycaemic control and smoking) identified

Denominator: number of AF patients.

\section{Domain: outcomes}

Main quality indicator: ischaemic stroke or TIA.

Main quality indicator: life-threatening or major bleeding events. ${ }^{\text {b }}$

Numerator: number of AF patients who have a documented ischaemic or bleeding event

Denominator: number of $A F$ patients or number of patients prescribed an $\mathrm{OAC}$, respectively.

$\mathrm{AAD}=$ antiarrhythmic drug; $\mathrm{AF}=$ atrial fibrillation; $\mathrm{BP}=$ blood pressure; $\mathrm{CHA}_{2} \mathrm{DS}_{2}-\mathrm{VASc}=$ Congestive heart failure, Hypertension, Age $\geq 75$ years, Diabetes mellitus, Stroke, Vascular disease, Age 65-74 years, Sex category (female); HAS-BLED = Hypertension, Abnormal renal/liver function, Stroke, Bleeding history or predisposition, Labile INR, Elderly (>65 years), Drugs/alcohol concomitantly; OAC = oral anticoagulant; OSA = obstructive sleep apnoea; TIA = transient ischaemic attack.

${ }^{a}$ Flecainide, propafenone, amiodarone, dronedarone, sotalol and disopyramide.

'Using the definitions of the International Society of Thrombosis and Haemostasis. ${ }^{1456,1457}$

follow-up and monitoring to detect clinical AF early is prudent (preferably with the support of remote monitoring). Notably, the AHRE/ subclinical AF burden is not static but may change on daily basis, ${ }^{469}$ hence should be regularly reassessed - the greater the AHRE/subclinical AF burden at diagnosis, the higher the risk of subsequent progression to longer episodes ${ }^{469}$ (Figure 24).
Whereas available evidence is insufficient to justify routine $O A C$ use in patients with AHRE/subclinical AF, modifiable stroke risk factors should be identified and managed in each patient.

The use of OAC may be considered in selected patients with longer durations of AHRE/subclinical AF ( $\geq 24 \mathrm{~h}$ ) and an estimated high individual risk of stroke, ${ }^{4,1462}$ accounting for the anticipated net 


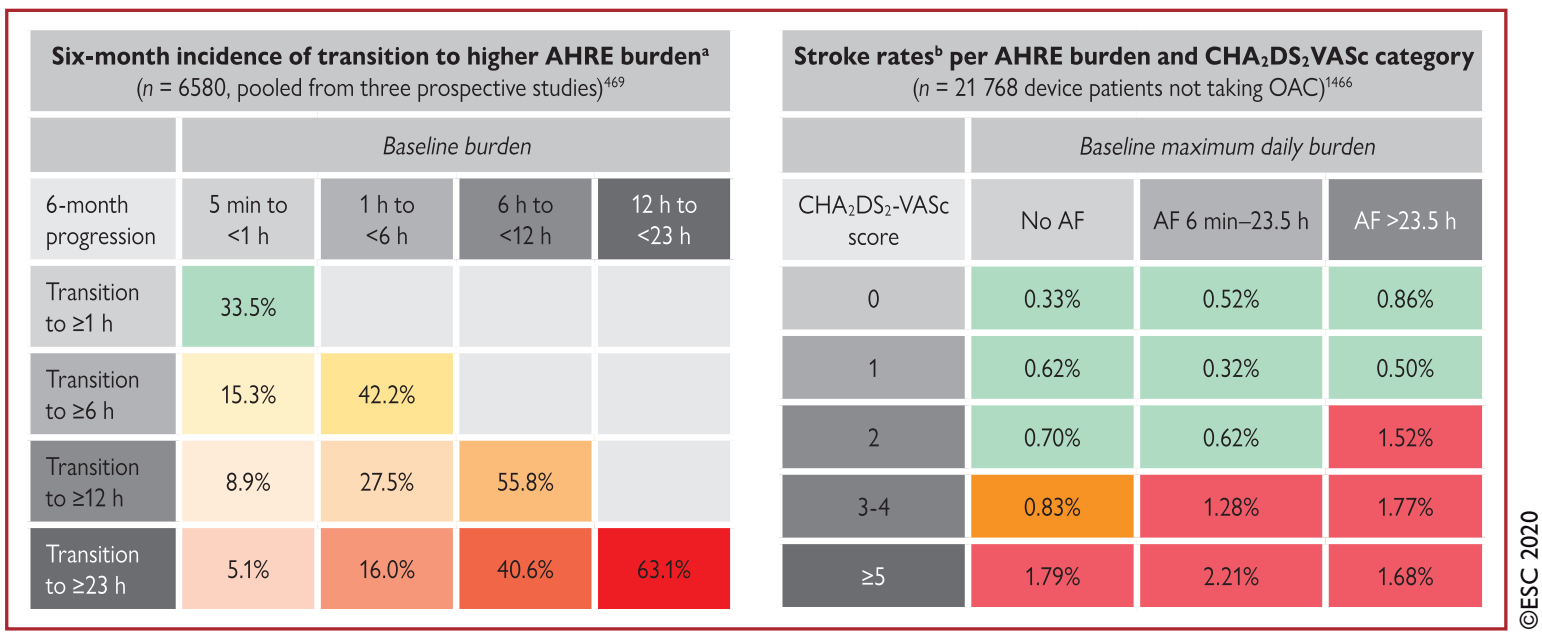

Figure 24 Progression of atrial high-rate episode burden (left panel) and stroke rates according to AHRE daily burden and $\mathrm{CHA}_{2} \mathrm{DS} \mathrm{S}_{2}-\mathrm{VASc} \mathrm{score}$ (right panel). $\mathrm{AHRE}=$ atrial high-rate episodes; $\mathrm{CHA}_{2} \mathrm{DS}_{2}$-VASc = Congestive heart failure, Hypertension, Age $\geq 75$ years, Diabetes mellitus, Stroke, Vascular disease, Age 65 - 74 years, Sex category (female); OAC = oral anticoagulant. ${ }^{\text {T}}$ The higher the burden at diagnosis, the greater the incidence of progression in the next 6 months and thereafter. ${ }^{\mathrm{b}}$ Stroke rates above the threshold for $\mathrm{OAC}$ are shown in red.

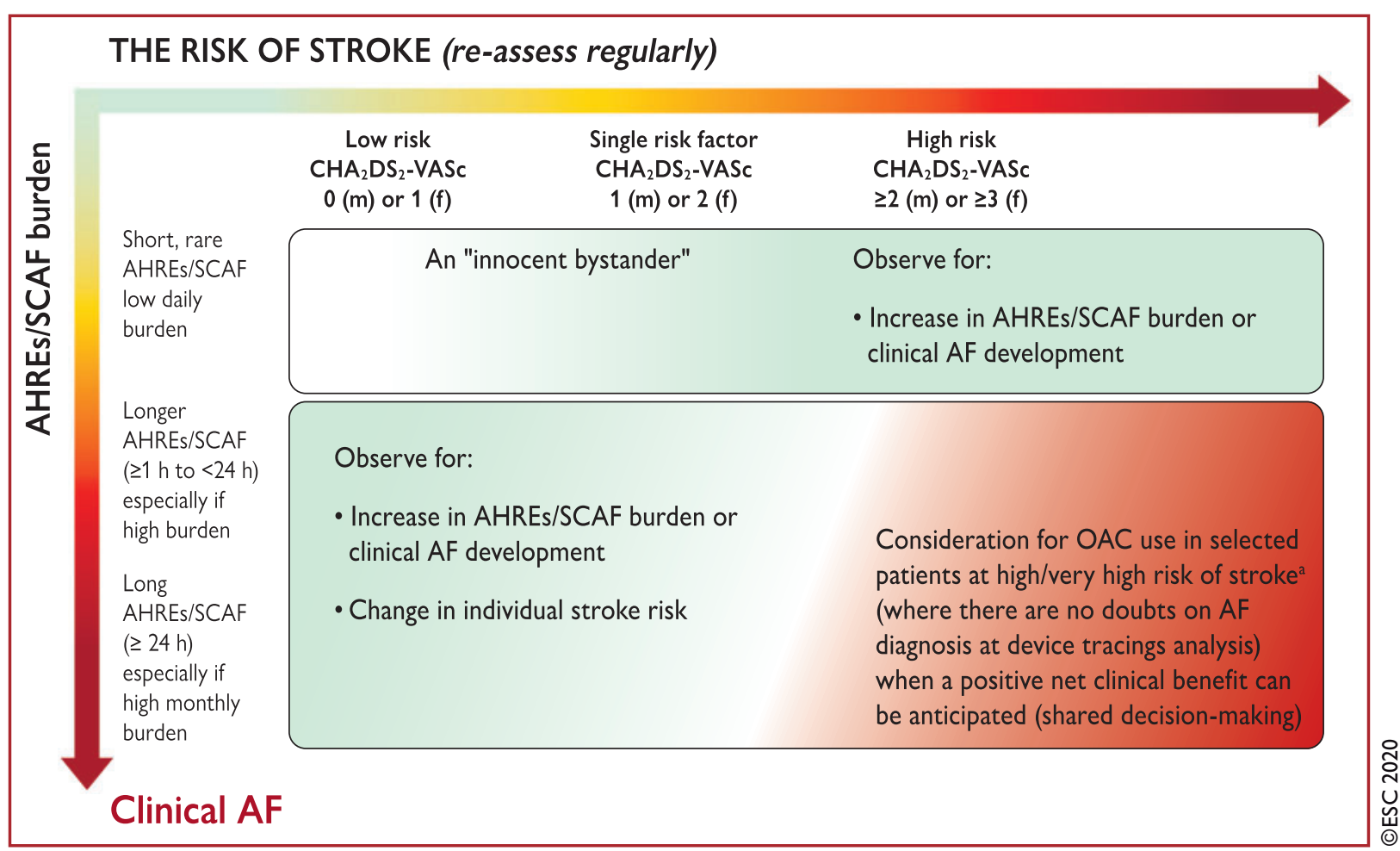

Figure 25 Proposed management of AHRE/subclinical AF. AF = atrial fibrillation; AHRE = atrial high-rate episode; CKD = chronic kidney disease; $\mathrm{CHA}_{2} \mathrm{DS}_{2}-\mathrm{VASc}=$ Congestive heart failure, Hypertension, Age $\geq 75$ years, Diabetes mellitus, Stroke, Vascular disease, Age $65-74$ years, Sex category (female); $\mathrm{f}=$ female; $\mathrm{LA}=$ left atrium; $\mathrm{LoE}=$ level of evidence; $\mathrm{m}=$ male; $\mathrm{OAC}=$ oral anticoagulant; $\mathrm{SCAF}=$ subclinical atrial fibrillation. ${ }^{\mathrm{a}} \mathrm{Highly}$ selected patients (e.g. with previous stroke and/or age $\geq 75$ years, or $\geq 3 \mathrm{CHA}_{2} \mathrm{DS}_{2}-\mathrm{VASc}$ risk factors, and additional non-CHA $\mathrm{DS}_{2}$-VASc stroke factors such as $C K D$, elevated blood biomarkers, spontaneous echo contrast in dilated LA, etc); selected patients (e.g. with previous stroke and/or age $\geq 75$ years, or $\geq 3$ $\mathrm{CHA}_{2} \mathrm{DS}_{2}$-VASc risk factors, etc).

clinical benefit and informed patient's preferences (Figures 24 and 25). In the recent trials, OAC was initiated in $76.4 \%$ and $56.3 \%$ of patients with $\geq 2$ clinical stroke risk factors and insertable cardiac monitordetected physician-confirmed $A F \geq 6$ min, but follow-up bleeding rates were not reported. ${ }^{1463,1464}$ In a large retrospective cohort study using remote monitoring data about daily AF burden, there was large practice variation in OAC initiation. Across increasing AF burden strata (from $>6$ min to $>24 \mathrm{~h}$ ) the risk of stroke in untreated 
patients increased numerically, and the strongest association of OAC with reduction in stroke was observed among patients with devicedetected AF episodes of $>24$ h. $^{5}$

\section{Recommendations for management of patients with AHRE}

\begin{tabular}{|c|c|c|}
\hline Recommendations & Class $^{a}$ & Level $^{\mathbf{b}}$ \\
\hline $\begin{array}{l}\text { In patients with AHRE/subclinical AF detected by } \\
\text { CIED or insertable cardiac monitor, it is recom- } \\
\text { mended to conduct: } \\
\text { - Complete cardiovascular evaluation with ECG } \\
\text { recording, clinical risk factors/comorbidity } \\
\text { evaluation, and thrombo-embolic risk assess- } \\
\text { ment using the } \mathrm{CHA}_{2} \mathrm{DS}_{2} \text {-VASc score. }{ }^{469} \\
\text { - Continued patient follow-up and monitoring } \\
\text { (preferably with the support of remote moni- } \\
\text { toring) to detect progression to clinical AF, } \\
\text { monitor the AHRE/subclinical AF burden } \\
\text { (especially transition to } \geq 24 \mathrm{~h} \text { ), and detect } \\
\text { changes in underlying clinical conditions. }{ }^{469}\end{array}$ & I & B \\
\hline
\end{tabular}

$\mathrm{AF}=$ atrial fibrillation; $\mathrm{AHRE}=$ atrial high-rate episode; $\mathrm{CIED}=$ cardiac implantable electronic device; ECG = electrocardiogram.

${ }^{a}$ Class of recommendation.

'Level of evidence.

\section{Atrial fibrillation and other atrial tachyarrhythmias (atrial flutter and atrial tachycardias)}

Although AFL may exist as a solitary atrial arrhythmia, a significant proportion of patients will subsequently develop AF. ${ }^{1466-1470}$ Typical AFL may occur in those taking class IC AADs or amiodarone. ${ }^{1467,1468,1471}$ The $A B C$ pathway for integrated AF management largely applies to patients with AFL. It is recommended that strokeprevention strategies in patients with solitary AFL, including periprocedural management of stroke risk, follow the same principles as in patients with $\mathrm{AF} .^{1472}$

Rate control should be the first step in symptom management. However, cardioversion to sinus rhythm may be more effective, especially electrical cardioversion or (where feasible) high-rate stimulation. ${ }^{1473,1474}$ Of note, the class III AADs dofetilide and ibutilide i.v. are very effective in interrupting AFL, whereas the class Ic drugs flecainide and propafenone ${ }^{1475-1478}$ should not be used in the absence of atrioventricular-blocking drugs as they may slow the atrial rate, thus facilitating $1: 1$ atrioventricular conduction with a rapid ventricular rate. ${ }^{1479,1480} \mathrm{AF}$ catheter ablation of the CTI is the most effective rhythm control treatment for CTI-dependent AFL. ${ }^{732,1481,1482}$ When typical AFL develops in AF patients during treatment with class Ic drugs or amiodarone, $\mathrm{CTI}$ ablation should be considered to ensure that AADs can be continued for AF rhythm control. ${ }^{732,1481}$

Atypical AFL (i.e. macro re-entrant atrial tachycardia) most commonly occurs in diseased or scarred atrial myocardium. Clinical management of atypical AFL/macro re-entrant atrial tachycardia broadly follows the principles of typical AFL management, but the use of AADs is often limited by significant structural heart disease, and ablation is more complex. ${ }^{1336}$

Notably, the intervention to treat atrial tachycardias (AFL/macro re-entrant atrial tachycardia) occurring early after AF catheter ablation (or surgery) should be delayed, and initial rate control or the use of AADs should be considered instead, as some of these tachyarrhythmias are transient and cease after maturation of the lesions deployed by the index procedure. ${ }^{1483-1485}$ For additional details about AFL, see Supplementary Box 7 and the 2019 ESC Guidelines on supraventricular tachycardias. ${ }^{1336}$

\section{Key messages}

(1) The diagnosis of AF needs to be confirmed by a conventional 12lead ECG tracing or rhythm strip showing AF for $\geq 30 \mathrm{~s}$.

(2) Structured characterization of $A F$, including stroke risk, symptom severity, severity of AF burden, and AF substrate, helps improve personalized treatment of AF patients.

(3) Novel tools and technologies for screening and detection of AF such as (micro-)implants and wearables substantially add to the diagnostic opportunities in patients at risk for AF. However, appropriate management pathways based on such tools are still incompletely defined.

(4) Integrated holistic management of AF patients is essential to improving their outcomes.

(5) Patient values need to be considered in treatment decision making and incorporated into the AF management pathways; the structured assessment of PRO measures is an important element to document and measure treatment success.

(6) The $A B C$ pathway streamlines integrated care of $A F$ patients across healthcare levels and among different specialties.

(7) Structured, clinical, risk-score-based assessment of individual thrombo-embolic risk, using the $\mathrm{CHA}_{2} \mathrm{DS}_{2}$-VASc score, should be performed as the first step in optimal thrombo-embolic risk management in AF patients.

(8) Patients with $\mathrm{AF}$ and risk factors for stroke need to be treated with OAC for stroke prevention. In NOAC-eligible patients, NOACs are preferred over VKAs.

(9) A formal structured risk-score-based bleeding risk assessment using, for example, the HAS-BLED score, helps to identify non-modifiable and address modifiable bleeding risk factors in AF patients.

(10) An elevated bleeding risk should not automatically lead to withholding $O A C$ in patients with $A F$ and stroke risk. Instead, modifiable bleeding risk factors should be addressed, and high-risk patients scheduled for a more frequent clinical review and follow-up.

(11) Rate control is an integral part of AF management and is often sufficient to improve AF-related symptoms.

(12) The primary indication for rhythm control using cardioversion, AADs, and/or catheter ablation is reduction in AF-related symptoms and improvement of QoL.

(13) The decision to initiate long-term AAD therapy needs to balance symptom burden, possible adverse drug reactions, particularly drug-induced proarrhythmia or extracardiac side-effects, and patient preferences. 
(14) Catheter ablation is a well-established treatment for prevention of $\mathrm{AF}$ recurrences. When performed by appropriately trained operators, catheter ablation is a safe and superior alternative to AADs for maintenance of sinus rhythm and symptom improvement.

(15) Major risk factors for AF recurrence should be assessed and considered in the decision making for interventional therapy.

(16) In patients with AF and normal LVEF, catheter ablation has not been shown to reduce total mortality or stroke. In patients with $\mathrm{AF}$ and tachycardia-induced cardiomyopathy, catheter ablation reverses LV dysfunction in most cases.

(17) Weight loss, strict control of risk factors, and avoidance of triggers for $\mathrm{AF}$ are important strategies to improve outcome of rhythm control.

(18) Identification and management of risk factors and concomitant diseases is an integral part of the treatment of AF patients.

(19) In AF patients with ACS undergoing uncomplicated $\mathrm{PCl}$, an early discontinuation of aspirin and switch to dual antithrombotic therapy with OAC and a P2Y 12 inhibitor should be considered.

(20) Patients with AHRE should be regularly monitored for progression to clinical $A F$ and changes in the individual thrombo-embolic risk (i.e. change in $\mathrm{CHA}_{2} \mathrm{DS}_{2}-\mathrm{VASc}$ score). In patients with longer AHRE (especially $>24 \mathrm{~h}$ ) and a high $\mathrm{CHA}_{2} \mathrm{DS}_{2}-\mathrm{VASc}$ score, it is reasonable to consider the use of $\mathrm{OAC}$ when a positive net clinical benefit from OAC is anticipated in a shared, informed, treatment decision-making process.

\section{Gaps in evidence}

Whereas some progress has been made since publication of the 2016 ESC AF Guidelines, major gaps identified in those guidelines persist in 2020, calling for more intense research. In 2019, the EHRA published a white paper that covers major gaps in the field of AF in detail. ${ }^{1486}$ The following bullet-list gives the most important knowledge gaps:

\section{$\square$ Major health modifiers causing atrial fibrillation}

Mechanisms of AF are not yet fully understood. Improvement in understanding of these mechanisms in individual patients, e.g. patients with cardiac structural remodelling or HF, would allow better selection of treatments including the best rate and rhythm control strategies and $\mathrm{OAC}$.

It is uncertain how educational interventions translate into actual behavioural change (patients and physicians) that leads to improvements in clinical management and outcomes, especially in the multimorbid AF patient.

$\square$ Implementation of digital technologies for screening, diagnosis, and risk stratification in the atrial fibrillation patient

New techniques for digital ECG analysis (e.g. machine learning and artificial intelligence) and new technologies (e.g. wearables and injectables) have opened up potentially significant opportunities for the detection and diagnosis of AF. These innovations may help to personalize therapy and risk stratification. Studies are needed to evaluate such opportunities and to define for which groups of patients this is worthwhile.

\section{$\square$ Type of atrial fibrillation}

There is a gap in knowledge regarding classification of AF. Recent data suggest that paroxysmal AF is not one entity. According to the pattern, type of therapy and outcome may differ. ${ }^{1487}$ More studies are needed. $\square$ How much atrial fibrillation constitutes a mandate for therapy?

The threshold of AF burden at which to initiate OAC therapy needs to be defined more clearly. This knowledge gap has resulted in substantial variation in physician attitudes and practice patterns. ${ }^{5}$

We are still waiting for the results of two ongoing RCTs in subclinical AF patients who are detected with cardiac implantable electronic device (CIED) [(Apixaban for the Reduction of Thrombo-Embolism in Patients With Device-Detected Sub-Clinical Atrial Fibrillation) (NCT 01938248) and NOAH (Non-vitamin K Antagonist Oral Anticoagulants in Patients With Atrial High Rate Episodes) (NCT 02618577)].

\section{$\square$ Role of biomarkers in atrial fibrillation management}

Although some studies have demonstrated an effective role of biomarkers (including natriuretic peptides and troponin) in AF risk assessment, there is uncertainty over the exact time point of biomarker assessment, optimal cut-offs, and the effect on management decision making based on changes in biomarker levels over time, especially with increasing age and incident comorbidities.

\section{$\square$ Stroke risk in specific populations}

Some studies have tested the effect of biomarkers in predicting risk of AF-related complications, including stroke, in specific populations. However, it is unknown if biomarkers and biomarker-based scores practically help physicians in refining stroke risk, especially in prospective non-anticoagulated cohorts, particularly given the dynamic nature of stroke risk and how many current biomarkers are nonspecific for AF or AF-related outcomes.

There is uncertainty of actual stroke risk in AHRE, compared with actual stroke risk in overt AF, in properly matched cohorts in similar settings, and the effect of appropriate management pathways.

The effect of sex in AF patients has been more investigated. Men with $\mathrm{AF}$ are less likely to have hypertension or VHD vs. women. ${ }^{1488}$ Women often present with atypical symptoms related to AF. Further comparative studies are needed in different settings and ethnic groups on the effect of different stroke risk factors and female sex on stroke and bleeding risks.

\section{$\square$ Anticoagulant therapy in specific patients}

There is a gap in knowledge regarding optimal NOAC dosing in specific groups, including those with mild-to-moderate CKD, with very low/high body mass index, and patients receiving medications with a high risk of metabolic interaction. ${ }^{1489}$

In patients with $\mathrm{CrCl} \leq 25 \mathrm{~mL} / \mathrm{min}, \mathrm{RCT}$-derived data on the effect of VKA or NOACs is still lacking, due to the exclusion of these patients from the major RCTs. However, two RCTs (NCT02933697, NCT03987711) are currently assessing OAC use and comparing NOACs with VKAs in patients with end-stage renal disease.

\section{$\square$ Anticoagulation in patients with heart valve diseases}

There are gaps in evidence on NOAC use in AF patients with rheumatic mitral valve disease and during the first 3 months after surgical or transcatheter implantation of a bioprosthesis; observational data regarding the use of NOACs after transcatheter aortic valve implantation are conflicting. ${ }^{1163}$

\section{$\square$ Anticoagulation in atrial fibrillation patients after a bleeding or stroke event}

As high-quality RCT-derived evidence to inform optimal timing of anticoagulation after acute ischaemic stroke is lacking, OAC use in the early post-stroke period is currently based on expert consensus. Several ongoing RCTs [ELAN (NCT03148457), OPTIMAS 


\section{To ABC}

\section{Confirm AF}

A 12-lead ECG or a rhythm strip showing AF pattern for $\geq 30 \mathrm{~s}$

\section{Characterise AF (the 4S-AF scheme)}

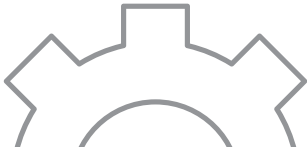

Stroke risk (St)

(e.g., $\mathrm{CHA}_{2} \mathrm{DS}_{2}$-VASc score)
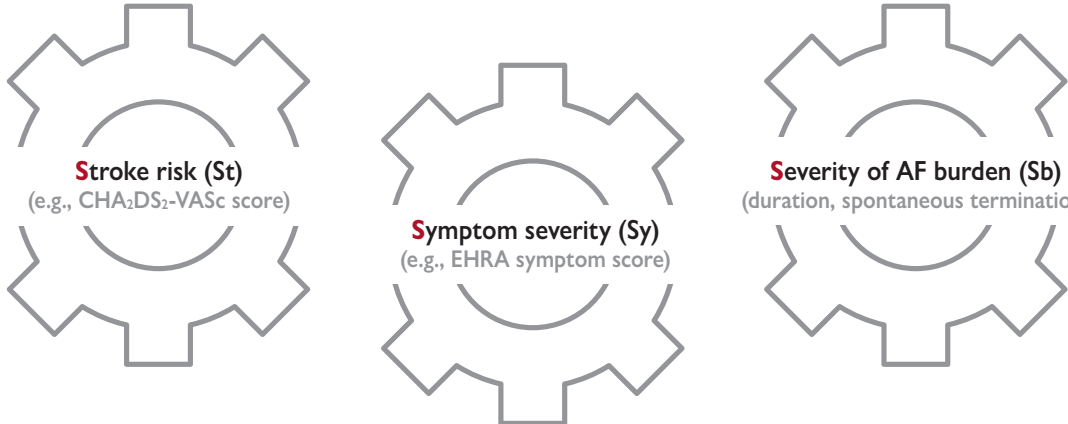

Severity of AF burden (Sb) (duration, spontaneous termination)
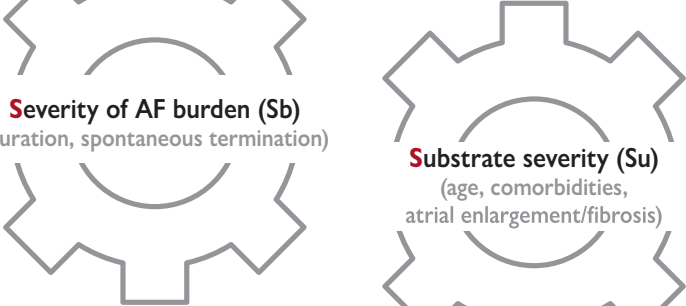

Substrate severity (Su)

(age, comorbidities,

atrial enlargement/fibrosis)

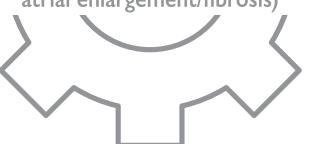

\section{Treat AF: The ABC pathway}
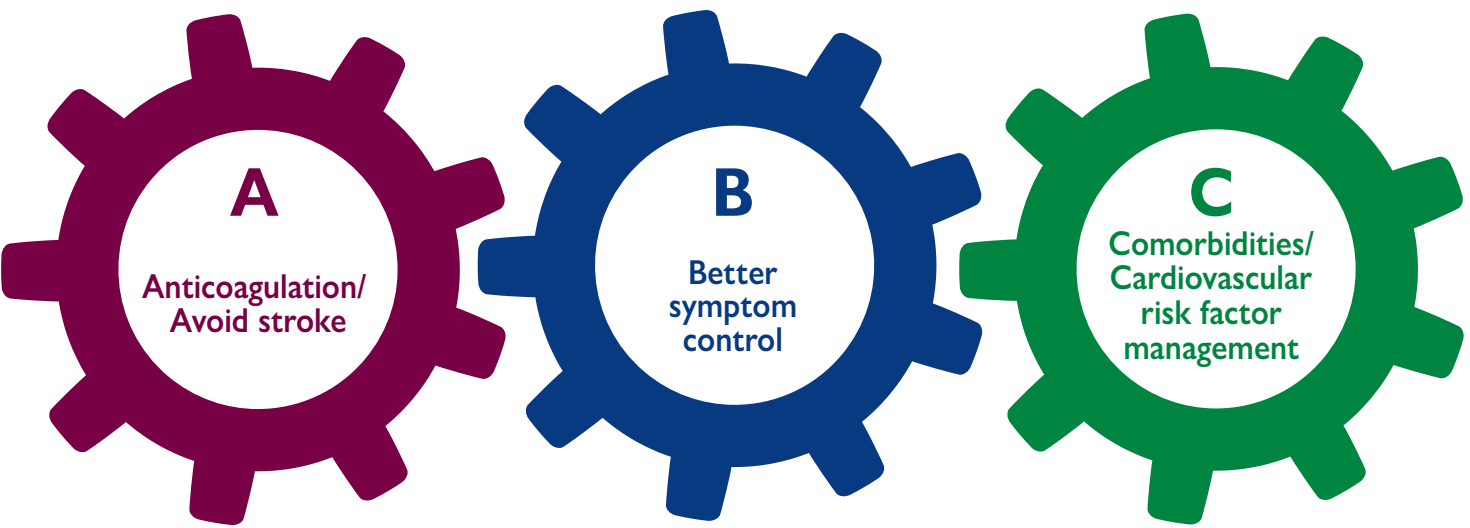

1. Identify low-risk patients $\mathrm{CHA}_{2} \mathrm{DS}_{2}$-VASc $0(\mathrm{~m}), 1(\mathrm{f})$

2. Offer stroke prevention if $\mathrm{CHA}_{2} \mathrm{DS}_{2} \mathrm{VASc} \geq 1(\mathrm{~m}), 2(\mathrm{f})$

Assess bleeding risk, address modifiable bleeding risk factors

3. Choose OAC (NOAC or VKA with well-managed TTR)

\section{Assess symptoms, QoL and patient's preferences \\ Optimize rate control}

Consider a rhythm control strategy (CV, AADs, ablation)
Comorbidities and cardiovascular risk factors

Lifestyle changes (obesity reduction, regular exercise, reduction of alcohol use, etc.)

Central Illustration Management of AF. AAD = antiarrhythmic drug; AF = atrial fibrillation; ECG = electrocardiogram; EHRA = European Heart Rhythm Association; $\mathrm{CHA}_{2} \mathrm{DS}_{2}$-VASc = Congestive HF, Hypertension, Age $\geq 75$ years, diabetes mellitus, Stroke, Vascular disease, Age $65-74$ years, Sex category (female); $\mathrm{CV}=$ cardioversion; $\mathrm{NOAC}=$ non-vitamin $\mathrm{K}$ antagonist oral anticoagulant; $\mathrm{OAC}=$ oral anticoagulant; TTR = time in therapeutic range; $\mathrm{VKA}=$ vitamin $\mathrm{K}$ antagonist. 
(EudraCT, 2018-003859-3), TIMING (NCT02961348), and START (NCT03021928)] will try to assess the differences between the two approaches, including early ( $<1$ week) vs. late NOAC initiation in patients with AF-related ischaemic stroke.

\section{$\square$ Left atrial appendage occlusion for stroke prevention}

More studies have been conducted in this field. There is clearer evidence of the safety and possible complications of the LAA closure procedure. ${ }^{450-454}$ However, there are still knowledge gaps to be addressed: (i) antithrombotic management after LAA occlusion has not been evaluated in a randomized manner; and (ii) the efficacy and safety of LAA closure vs. OAC therapy needs to be assessed in randomized trials.

LAA occluders have not been compared with NOAC therapy in patients at risk for bleeding, or with surgical LAA occlusion/ exclusion.

\section{$\square$ Surgical exclusion of Left atrial appendage}

Only limited RCT data are available ${ }^{457-459}$ on surgical exclusion of the LAA. Although a large RCT in patients with an associated cardiac surgical procedure is ongoing, ${ }^{462}$ adequately powered RCTs are needed.

There is the need for adequately powered trials to define the best indications for LAA occlusion/exclusion compared with NOAC therapy in patients with relative or absolute contraindications for anticoagulation, in those with an ischaemic stroke on anticoagulant therapy, and for assessment of the appropriate antithrombotic therapy after LAA occlusion.

\section{$\square$ Atrial fibrillation catheter ablation technique}

The best approach to safely and expeditiously achieve permanent $\mathrm{PVI}$ in a single procedure is still one of the knowledge gaps in relation to emerging technologies for catheter ablation of AF. Moreover, it remains unknown if ablating additional targets will improve the outcomes of AF catheter ablation. ${ }^{1490}$

\section{$\square$ Outcome of atrial fibrillation catheter ablation}

The following issues need to be addressed in further studies:

- The value of early AF ablation to prevent AF progression.

- The optimal outcome measure (AF $30 \mathrm{~s}, \mathrm{AF}$ burden, etc.) for AFrelated outcome.
- How much reduction in AF burden is needed to achieve an effect on hard endpoints, including survival, stroke, and comorbidity.

- The main mechanism of PVI translating into freedom of AF.

- The potential effect of cardiac structure and function on the likelihood of success of AF ablation.

Despite the publication of CABANA and CASTLE-AF, more data are needed on the effect of AF catheter ablation on clinical outcomes, including death, stroke, serious bleeding, AF recurrence, QoL, and cardiac arrest.

The relationship between the degree of atrial dilation/fibrosis and successful ablation of AF needs to be addressed. Additionally, the impact of specific components of structural heart disease, including LA structure/function, LV structure, etc., on the success of AF catheter ablation and the likelihood of recurrence requires further investigation.

\section{$\square$ Who may benefit less from atrial fibrillation catheter} ablation

There are gaps in knowledge about subgroups of patients who may benefit less from AF catheter ablation, including (i) persistent and long-standing persistent AF; (ii) patients with enlarged atrial size and/ or atrial fibrosis; (iii) patients with atypical AFL; and (iv) patients with risk factors for AF recurrence, including obesity or sleep apnoea.

\section{$\square$ Thoracoscopic 'stand-alone' atrial fibrillation surgery}

There are no convincing data on the effects on stroke of surgical ablation as a stand-alone procedure or in combination with LAA occlusion or exclusion on various outcomes including QoL, stroke, and death.

\section{$\square$ Personalized therapy}

The arrhythmia phenotype may differ among patients. Improved assessment of the pathophysiological process involved in the individual patient by using clinical characteristics, blood biomarkers, and non-invasive substrate determination (echo/MRI/CT) may improve personalized therapy (e.g. selection of rhythm control, yes or no; treatment of risk factors and comorbidities; type of antiarrhythmic drug; atrial ablation; and which type/techniques used for AF).

\section{0 'What to do' and 'what not to do' messages from the Guidelines}

\section{Recommendations}

Recommendations for diagnosis of AF

ECG documentation is required to establish the diagnosis of AF.

- A standard 12-lead ECG recording or a single-lead ECG tracing of $\geq 30$ s showing heart rhythm with no discernible repeating $P$ waves and irregular RR intervals (when atrioventricular conduction is not impaired) is diagnostic of clinical AF.

\section{Recommendations for screening of AF}

Opportunistic screening for AF by pulse taking or ECG rhythm strip is recommended in patients $\geq 65$ years of age. It is recommended to interrogate pacemakers and implantable cardioverter defibrillators on a regular basis for AHRE. When screening for $A F$ it is recommended that:

- The individuals undergoing screening are informed about the significance and treatment implications of detecting AF.

- A structured referral platform is organized for screen-positive cases for further physician-led clinical evaluation to confirm the diagnosis of AF and provide optimal management of patients with confirmed AF.

- Definite diagnosis of AF in screen-positive cases is established only after the physician reviews the single-lead ECG recording of $\geq 30$ s or 12 -lead ECG and confirms that it shows AF. 


\section{Recommendations for diagnostic evaluation of patients with AF}

In patients with $A F$, it is recommended to:

- Evaluate AF-related symptoms (including fatigue, tiredness, exertional shortness of breath, palpitations, and chest pain) and quantify the patient symptom status using the modified EHRA symptom scale before and after initiation of treatment.

- Evaluate AF-related symptoms before and after cardioversion of persistent AF to aid rhythm control treatment decisions.

In patients with AHRE/subclinical AF detected by CIED or insertable cardiac monitor, it is recommended to conduct:

- Complete cardiovascular evaluation with ECG recording, clinical risk factors/comorbidity evaluation, and thrombo-embolic risk assessment using the $\mathrm{CHA}_{2} \mathrm{DS}_{2}$-VASc score.

- Continued patient follow-up and monitoring (preferably with the support of remote monitoring) to detect progression to clinical AF, monitor the AHRE/subclinical AF burden (especially transition to $\geq 24 \mathrm{~h}$ ), and detect changes in underlying clinical conditions.

\section{Recommendations about integrated AF management}

To optimize shared decision making about specific AF treatment option(s) in consideration, it is recommended that physicians:

- Inform the patient about the advantages/limitations and benefit/risks associated with the treatment option(s) being considered; and

- Discuss the potential burden of the treatment with the patient and include the patient's perception of treatment burden in the treatment decision.

It is recommended to routinely collect PROs to measure treatment success and improve patient care.

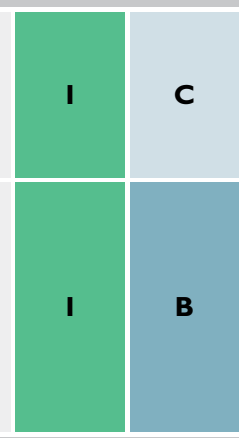

Recommendations for the prevention of thrombo-embolic events in AF

For stroke prevention in AF patients who are eligible for OAC, NOACs are recommended in preference to VKAs (excluding patients with mechanical heart valves or moderate-to-severe mitral stenosis).

For stroke risk assessment, a risk-factor-based approach is recommended, using the $\mathrm{CHA}_{2} \mathrm{DS}_{2}$-VASc clinical stroke risk score to initially identify patients at 'low stroke risk' $\left(\mathrm{CHA}_{2} \mathrm{DS}_{2}\right.$-VASc score $=0$ in men, or 1 in women) who should not be offered antithrombotic therapy.

$\mathrm{OAC}$ is recommended for stroke prevention in $\mathrm{AF}$ patients with $\mathrm{CHA}_{2} \mathrm{DS}_{2}-\mathrm{VASc}$ score $\geq 2$ in men or $\geq 3$ in women.

For bleeding risk assessment, a formal structured risk-score-based bleeding risk assessment is recommended to help identify nonmodifiable and address modifiable bleeding risk factors in all AF patients, and to identify patients potentially at high risk of bleeding who should be scheduled for early and more frequent clinical review and follow-up.

Stroke and bleeding risk reassessment at periodic intervals is recommended to inform treatment decisions (e.g. initiation of OAC in patients no longer at low risk of stroke) and address potentially modifiable bleeding risk factors.

If a VKA is used, a target INR of $2.0-3.0$ is recommended, with individual TTR $\geq 70 \%$.

In patients on VKAs with low time in INR therapeutic range (e.g. TTR<70\%), switching to a NOAC but ensuring good adherence and persistence with therapy is recommended.

Antiplatelet therapy alone (monotherapy or aspirin in combination with clopidogrel) is not recommended for stroke prevention in AF.

Estimated bleeding risk, in the absence of absolute contraindications to OAC, should not in itself guide treatment decisions to use OAC for stroke prevention.

Clinical pattern of AF (i.e. first detected, paroxysmal, persistent, long-standing persistent, permanent) should not condition the indication to thromboprophylaxis.

\section{Recommendations for stroke risk management peri-cardioversion}

In patients with AF undergoing cardioversion, NOACs are recommended with at least similar efficacy and safety as warfarin. For cardioversion of AF/AFL, effective anticoagulation is recommended for a minimum of 3 weeks before cardioversion.

TOE is recommended to exclude cardiac thrombus as an alternative to 3-week pre-procedural anticoagulation when early cardioversion is planned.

In patients at risk of stroke, it is recommended that OAC therapy is continued long term after cardioversion according to the long-term anticoagulation recommendations, irrespective of the method of cardioversion, the apparent maintenance of sinus rhythm, or characterization of AF as a 'first-diagnosed episode'.

When thrombus is identified on TOE, effective anticoagulation is recommended for at least 3 weeks before cardioversion of AF. It is recommended that the importance of adherence and persistence to NOAC treatment both before and after cardioversion is strongly emphasized to patients.

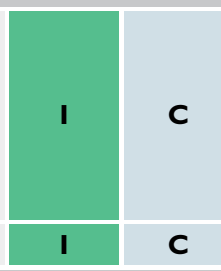

Recommendations for stroke risk management peri-catheter ablation

In AF patients with stroke risk factors not taking OAC before ablation, it is recommended that pre-procedural management of stroke risk includes initiation of anticoagulation and, preferably, therapeutic OAC for at least 3 weeks before ablation.
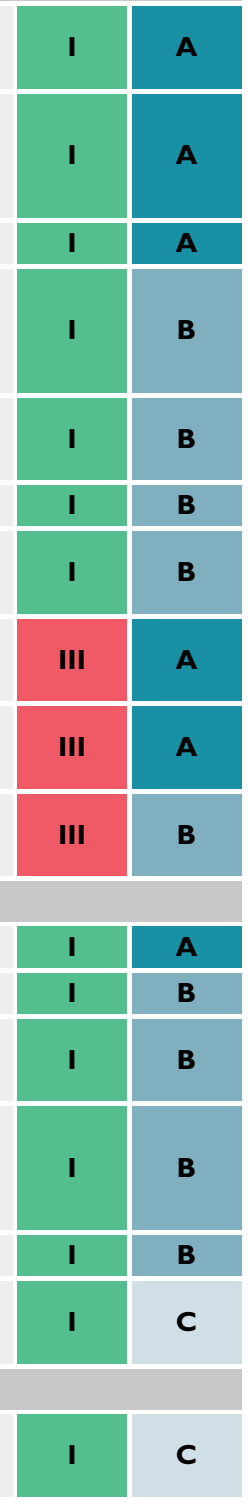
For patients undergoing AF catheter ablation who have been therapeutically anticoagulated with warfarin, dabigatran, rivaroxaban, apixaban, or edoxaban, performance of the ablation procedure without OAC interruption is recommended.

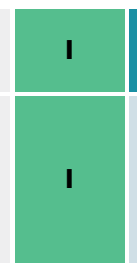

After AF catheter ablation, it is recommended that:

- Systemic anticoagulation with warfarin or a NOAC is continued for at least 2 months post ablation, and

- Long-term continuation of systemic anticoagulation beyond 2 months post ablation is based on the patient's stroke risk profile and not on the apparent success or failure of the ablation procedure.

\section{Recommendations for postoperative anticoagulation after AF surgery}

Long-term $\mathrm{OAC}$ is recommended in patients after AF surgery and appendage closure, based on the patient's thrombo-embolic risk assessed with the $\mathrm{CHA}_{2} \mathrm{DS}_{2}$-VASc score.

\section{Recommendations for patients with AF and an ACS, PCI, or CCS}

In AF patients eligible for NOACs, it is recommended to use a NOAC in preference to a VKA in combination with antiplatelet therapy.

In $\mathrm{AF}$ patients with $\mathrm{ACS}$ undergoing an uncomplicated $\mathrm{PCl}$, early cessation ( $\leq 1$ week) of aspirin and continuation of dual therapy with an $\mathrm{OAC}$ and a $\mathrm{P}_{2} \mathrm{Y}_{12}$ inhibitor (preferably clopidogrel) for up to 12 months is recommended if the risk of stent thrombosis is low or if concerns about bleeding risk prevail over concerns about risk of stent thrombosis, irrespective of the type of stent used.

After uncomplicated $\mathrm{PCl}$, early cessation ( $\leq 1$ week) of aspirin and continuation of dual therapy with OAC for up to 6 months and clopidogrel is recommended if the risk of stent thrombosis is low or if concerns about bleeding risk prevail over concerns about risk of stent thrombosis, irrespective of the type of stent used.

I

\section{Recommendations for secondary stroke prevention in AF patients after acute ischaemic stroke}

In AF patients with an ischaemic stroke or TIA, long-term secondary prevention of stroke using OAC is recommended if there is no strict contraindication to OAC use, with a preference for NOACs over VKAs in NOAC-eligible patients.

In $\mathrm{AF}$ patients presenting with acute ischaemic stroke, very early anticoagulation ( $<48 \mathrm{~h})$ using UFH, LMWH, or VKAs is not recommended.

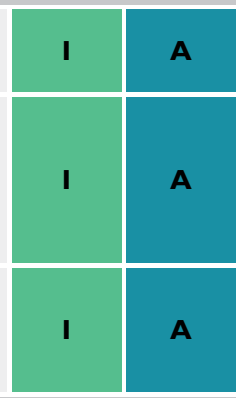

\section{Recommendations for patients with valvular heart disease and AF}

NOACs are contraindicated in patients with a prosthetic mechanical valve.

Use of NOACs is not recommended in patients with AF and moderate-to-severe mitral stenosis.

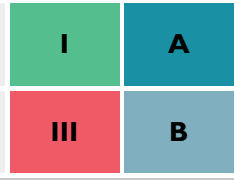

Recommendations for the management of AF during pregnancy

Therapeutic anticoagulation with heparin or VKA according to the stage of pregnancy is recommended for patients with AF.

\begin{tabular}{|l|l|}
\hline III & B \\
\hline III & C \\
\hline
\end{tabular}

Recommendations for the management of active bleeding on OAC

In an AF patient with severe active bleeding, it is recommended to:

- Interrupt OAC until the cause of bleeding is identified and active bleeding is resolved; and

- Promptly perform specific diagnostic and treatment interventions to identify and manage the cause(s) and source(s) of bleeding.

\section{Recommendations for ventricular rate control in patients with AF}

Beta-blockers, diltiazem, or verapamil are recommended as first-choice drugs to control heart rate in AF patients with $\mathrm{LVEF} \geq 40 \%$.

Beta-blockers and/or digoxin are recommended to control heart rate in $\mathrm{AF}$ patients with $\mathrm{LVEF}<40 \%$.

Recommendations for the management of AF during pregnancy

Beta-selective blockers are recommended for rate control in AF.

Recommendations for rhythm control

Rhythm control therapy is recommended for symptom and QoL improvement in symptomatic patients with AF.

I A

Recommendations for cardioversion

For pharmacological cardioversion of new-onset AF, i.v. vernakalant (excluding patients with recent ACS or severe HF) or flecainide or propafenone (excluding patients with severe structural heart disease) is recommended.

Intravenous amiodarone is recommended for cardioversion of AF in patients with HF or structural heart disease, if delayed cardioversion is consistent with clinical situation.

Cardioversion of AF (either electrical or pharmacological) is recommended in symptomatic patients with persistent or longstanding persistent $\mathrm{AF}$ as part of rhythm control therapy.

Pharmacological cardioversion of $\mathrm{AF}$ is indicated only in a haemodynamically stable patient, after consideration of the thromboembolic risk.

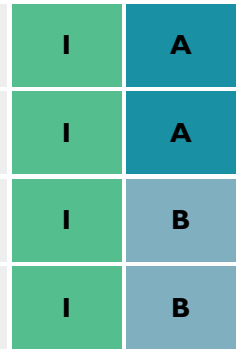

Continued 
Emergency electrical cardioversion is recommended in AF patients with acute or worsening haemodynamic instability. For patients with sick-sinus syndrome, atrioventricular conduction disturbances or prolonged QTc ( $>500 \mathrm{~ms})$, pharmacological cardioversion should not be attempted unless risks for proarrhythmia and bradycardia have been considered.

\begin{tabular}{|c|c|}
\hline I & B \\
\hline III & C \\
\hline
\end{tabular}

Recommendations for the management of AF during pregnancy

Immediate electrical cardioversion is recommended in case of haemodynamic instability or pre-excited AF.

I C

Recommendations for rhythm control/catheter ablation of AF

For the decision on AF catheter ablation, it is recommended to take into consideration the procedural risks and the major risk factors for $A F$ recurrence following the procedure and discuss them with the patient.

\section{AF catheter ablation after failure of drug therapy}

AF catheter ablation for PVI is recommended for rhythm control after one failed or intolerant class I or III AAD, to improve symptoms of $A F$ recurrences in patients with:

- Paroxysmal AF, or

- Persistent AF without major risk factors for AF recurrence, or

- Persistent AF with major risk factors for AF recurrence.

\section{First-line therapy}

AF catheter ablation is recommended to reverse LV dysfunction in AF patients when tachycardia-induced cardiomyopathy is highly probable, independent of their symptom status.

\section{Techniques and technologies}

Complete electrical isolation of the pulmonary veins is recommended during all AF catheter-ablation procedures.

\section{Lifestyle modification and other strategies to improve outcomes of ablation}

Weight loss is recommended in obese patients with AF, particularly those who are being evaluated to undergo AF ablation. Strict control of risk factors and avoidance of triggers are recommended as part of a rhythm control strategy.

\section{Recommendations for long-term antiarrhythmic drugs}

Flecainide or propafenone are recommended for long-term rhythm control in AF patients with normal LV function and without structural heart disease, including significant LVH and myocardial ischaemia.

Dronedarone is recommended for long-term rhythm control in AF patients with:

- Normal or mildly impaired (but stable) LV function, or

- HFpEF, ischaemic, or VHD.

Amiodarone is recommended for long-term rhythm control in all AF patients, including those with HFrEF. However, owing to its extracardiac toxicity, other AADs should be considered first whenever possible.

In AF patients treated with sotalol, close monitoring of QT interval, serum potassium levels, $\mathrm{CrCl}$, and other proarrhythmia risk factors is recommended.

AAD therapy is not recommended in patients with permanent AF under rate control and in patients with advanced conduction disturbances unless antibradycardia pacing is provided.

\begin{tabular}{l|l|l|l}
\hline & B
\end{tabular}

Recommendations for lifestyle interventions and management of risk factors and concomitant diseases in patients with AF

Identification and management of risk factors and concomitant diseases is recommended as an integral part of treatment in AF patients.

Modification of unhealthy lifestyle and targeted therapy of intercurrent conditions is recommended to reduce AF burden and symptom severity.

Opportunistic screening for $\mathrm{AF}$ is recommended in hypertensive patients.

Attention to good $\mathrm{BP}$ control is recommended in $\mathrm{AF}$ patients with hypertension to reduce $\mathrm{AF}$ recurrences and risk of stroke and bleeding.

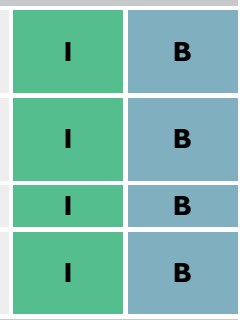

Recommendations for sports activity in patients with AF

It is recommended to counsel professional athletes that long-lasting intense sports participation may promote AF, while moderate physical activity is recommended to prevent AF.

\section{Recommendations for postoperative AF}

Perioperative amiodarone or beta blocker therapy is recommended for the prevention of postoperative AF after cardiac surgery. Beta-blockers should not be used routinely for the prevention of postoperative AF in patients undergoing non-cardiac surgery.

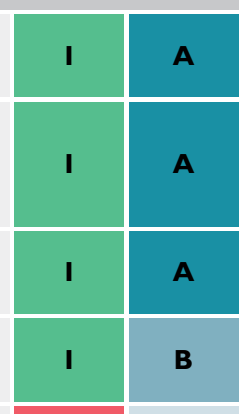

III

\begin{tabular}{|c|c|}
\hline I & A \\
\hline III & B \\
\hline
\end{tabular}




\section{Recommendations pertaining to sex-related differences in AF}

It is recommended that women and men with AF are equally offered diagnostic assessment and therapies to prevent stroke and other AF-related complications.

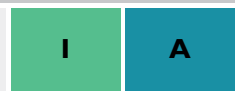

$\mathrm{AAD}=$ antiarrhythmic drug; $\mathrm{ACS}=$ acute coronary syndrome; $\mathrm{AF}=$ atrial fibrillation; $\mathrm{AFL}=$ atrial flutter; $\mathrm{AHRE}=$ atrial high-rate episodes; $\mathrm{BP}=$ blood pressure; $\mathrm{CCS}=$ chronic coronary syndrome; $\mathrm{CHA}_{2} \mathrm{DS}_{2}-\mathrm{VASc}=$ Congestive heart failure, Hypertension, Age $\geq 75$ years, Diabetes mellitus, Stroke, Vascular disease, Age $65-74$ years, Sex category (female); $\mathrm{ClED}=$ cardiac implantable electronic device; $\mathrm{CrCl}=$ creatinine clearance; $\mathrm{ECG}=$ electrocardiogram; $\mathrm{HF}=$ heart failure; $\mathrm{HFpEF}=$ heart failure with preserved ejection fraction; $\mathrm{HFrEF}=$ heart failure with reduced ejection fraction; i.v. = intravenous; INR = international normalized ratio; $L M W H=$ low-molecular-weight heparin; $L V=$ left ventricular; $\mathrm{LVEF}=$ left ventricular ejection fraction; $\mathrm{LVH}=$ left ventricular hypertrophy; $\mathrm{NOAC}=$ non-vitamin $\mathrm{K}$ antagonist oral anticoagulant; $\mathrm{OAC}=$ oral anticoagulant; $\mathrm{PCl}$ = percutaneous coronary intervention; $\mathrm{PRO}=$ patient-reported outcome; $\mathrm{PVI}=$ pulmonary vein isolation; $\mathrm{QoL}=$ quality of life; TIA = transient ischaemic attack; TOE = transoesophageal echocardiography; TTR = time in therapeutic range; UFH = unfractionated heparin; VHD = Valvular heart disease; VKA = vitamin K antagonist.

\section{Supplementary data}

Supplementary Data with additional Supplementary Figures, Tables, and text complementing the full text are available on the European Heart Journal website and via the ESC website at www.escardio.org/ guidelines.

\section{Appendix}

Author/Task Force Member Affiliations: Nikolaos Dagres, Department of Electrophysiology, Heart Center Leipzig at the University of Leipzig, Leipzig, Germany; Elena Arbelo, Arrhythmia Department, Cardiovascular Institute, Hospital Clinic de Barcelona, Barcelona, Catalonia, Spain; Jeroen J. Bax, Cardiology, Leiden University Medical Center, Leiden, Netherlands; Carina Blomström-Lundqvist, Department of Medical Science and Cardiology, Medicine, Uppsala, Sweden; Giuseppe Boriani, Cardiology Division, Department of Biomedical, Metabolic and Neural Sciences, University of Modena and Reggio Emilia, Policlinico di Modena, Modena, Italy; Manuel Castella, ${ }^{1}$ Cardiovascular Surgery, Hospital Clínic, University of Barcelona, Barcelona, Spain; Gheorghe-Andrei Dan, Cardiology Department, Internal Medicine Clinic, 'Carol Davila' University of Medicine, Colentina University Hospital, Bucharest, Romania; Polychronis E. Dilaveris, $1^{\text {st }}$ University Department of Cardiology, National \& Kapodistrian University of Athens School of Medicine, Athens, Attica, Greece; Laurent Fauchier, Department of Cardiology, Centre Hospitalier Universitaire Trousseau and University of Tours, Tours, France; Gerasimos Filippatos, Department of Cardiology, Attikon University Hospital, National and Kapodistrian University of Athens, Athens, Greece; Jonathan M. Kalman, Department of Cardiology, Royal Melbourne Hospital and University of Melbourne, Melbourne, Australia; Mark La Meir, ${ }^{1}$ Cardiac surgery, UZ Brussel, Brussels, Belgium; Deirdre A. Lane, Liverpool Centre for Cardiovascular Science, University of Liverpool and Liverpool Heart \& Chest Hospital, Liverpool, United Kingdom, and Department of Clinical Medicine, Aalborg University, Aalborg, Denmark; Jean-Pierre Lebeau, Department of General Practice, University of Tours, Tours, France; Maddalena Lettino, Cardiovascular, San Gerardo Hospital, ASST-Monza, Monza, Italy; Gregory Y. H. Lip, Liverpool Centre for Cardiovascular Science, University of Liverpool and Liverpool Heart \& Chest Hospital, Liverpool, United Kingdom, and Department of Clinical Medicine, Aalborg University, Aalborg, Denmark; Fausto J. Pinto, Cardiology, CCUL, Faculdade de
Medicina, Universidade de Lisboa, Lisbon, Portugal; G. Neil Thomas, Institute of Applied Health Research, University of Birmingham, Birmingham, United Kingdom; Marco Valgimigli, Cardiocentro Ticino, Lugano, Switzerland; Isabelle C. Van Gelder, Department of Cardiology, University of Groningen, University Medical Center Groningen, Groningen, Netherlands; Bart P. Van Putte, ${ }^{1}$ Cardiothoracic Surgery, St Antonius Hospital, Nieuwegein, Netherlands; Caroline L. Watkins, Faculty of Health and Wellbeing, University of Central Lancashire, Preston, United Kingdom.

ESC Committee for Practice Guidelines (CPG): Stephan Windecker (Chairperson) (Switzerland), Victor Aboyans (France), Colin Baigent (United Kingdom), Jean-Philippe Collet (France), Veronica Dean (France), Victoria Delgado (Netherlands), Donna Fitzsimons (United Kingdom), Chris P. Gale (United Kingdom), Diederick E. Grobbee (Netherlands), Sigrun Halvorsen (Norway), Gerhard Hindricks (Germany), Bernard lung (France), Peter Jüni (Canada), Hugo A. Katus (Germany), Ulf Landmesser (Germany), Christophe Leclercq (France), Maddalena Lettino (Italy), Basil S. Lewis (Israel), Béla Merkely (Hungary), Christian Mueller (Switzerland), Steffen E. Petersen (United Kingdom), Anna Sonia Petronio (Italy), Dimitrios J. Richter (Greece), Marco Roffi (Switzerland), Evgeny Shlyakhto (Russian Federation), lain A. Simpson (United Kingdom), Miguel Sousa-Uva (Portugal), Rhian M. Touyz (United Kingdom).

ESC National Cardiac Societies actively involved in the review process of the 2020 ESC Guidelines for the diagnosis and management of atrial fibrillation.

Algeria: Algerian Society of Cardiology, Tahar Delassi; Armenia: Armenian Cardiologists Association, Hamayak S. Sisakian; Austria: Austrian Society of Cardiology, Daniel Scherr; Belarus: Belorussian Scientific Society of Cardiologists, Alexandr Chasnoits; Belgium: Belgian Society of Cardiology, Michel De Pauw; Bosnia and Herzegovina: Association of Cardiologists of Bosnia and Herzegovina, Elnur Smajic; Bulgaria: Bulgarian Society of Cardiology, Tchavdar Shalganov; Cyprus: Cyprus Society of Cardiology, Panayiotis Avraamides; Czech Republic: Czech Society of Cardiology, Josef Kautzner; Denmark: Danish Society of Cardiology, Christian Gerdes; Egypt: Egyptian Society of Cardiology, Ahmad Abd Alaziz; Estonia: Estonian Society of Cardiology, Priit Kampus; Finland: Finnish Cardiac Society, Pekka Raatikainen; France: French Society of Cardiology, Serge Boveda; 
Georgia: Georgian Society of Cardiology, Giorgi Papiashvili; Germany: German Cardiac Society, Lars Eckardt; Greece: Hellenic Society of Cardiology, Vassilios P. Vassilikos; Hungary: Hungarian Society of Cardiology, Zoltán Csanádi; Iceland: Icelandic Society of Cardiology, David O. Arnar; Ireland: Irish Cardiac Society, Joseph Galvin; Israel: Israel Heart Society, Alon Barsheshet; Italy: Italian Federation of Cardiology, Pasquale Caldarola; Kazakhstan: Association of Cardiologists of Kazakhstan, Amina Rakisheva; Kosovo (Republic of): Kosovo Society of Cardiology, Ibadete Bytyçi; Kyrgyzstan: Kyrgyz Society of Cardiology, Alina Kerimkulova; Latvia: Latvian Society of Cardiology, Oskars Kalejs; Lebanon: Lebanese Society of Cardiology, Mario Njeim; Lithuania: Lithuanian Society of Cardiology, Aras Puodziukynas; Luxembourg: Luxembourg Society of Cardiology, Laurent Groben; Malta: Maltese Cardiac Society, Mark A. Sammut; Moldova (Republic of): Moldavian Society of Cardiology, Aurel Grosu; Montenegro: Montenegro Society of Cardiology, Aneta Boskovic; Morocco: Moroccan Society of Cardiology, Abdelhamid Moustaghfir; Netherlands: Netherlands Society of Cardiology, Natasja de Groot; North Macedonia: North Macedonian Society of Cardiology, Lidija Poposka; Norway: Norwegian Society of Cardiology, Ole-Gunnar Anfinsen; Poland: Polish Cardiac Society, Przemyslaw P. Mitkowski; Portugal: Portuguese Society of Cardiology, Diogo Magalhães Cavaco; Romania: Romanian Society of Cardiology, Calin Siliste; Russian Federation: Russian Society of Cardiology, Evgeny N. Mikhaylov; San Marino: San Marino Society of Cardiology, Luca Bertelli; Serbia: Cardiology Society of Serbia, Dejan Kojic; Slovakia: Slovak Society of Cardiology, Robert Hatala; Slovenia: Slovenian Society of Cardiology, Zlatko Fras; Spain: Spanish Society of Cardiology, Fernando Arribas; Sweden: Swedish Society of Cardiology, Tord Juhlin; Switzerland: Swiss Society of Cardiology, Christian Sticherling; Tunisia: Tunisian Society of Cardiology and Cardio-Vascular Surgery, Leila Abid; Turkey: Turkish Society of Cardiology, Ilyas Atar; Ukraine: Ukrainian Association of Cardiology, Oleg Sychov; United Kingdom of Great Britain and Northern Ireland: British Cardiovascular Society, Matthew G. D. Bates; Uzbekistan: Association of Cardiologists of Uzbekistan, Nodir U. Zakirov.

\section{References}

1. Calkins H, Hindricks G, Cappato R, Kim YH, Saad EB, Aguinaga L, Akar JG, Badhwar V, Brugada J, Camm J, Chen PS, Chen SA, Chung MK, Nielsen JC, Curtis $A B$, Davies DW, Day JD, d'Avila A, de Groot N, Di Biase L, Duytschaever M, Edgerton JR, Ellenbogen KA, Ellinor PT, Ernst S, Fenelon G, Gerstenfeld EP, Haines DE, Haissaguerre M, Helm RH, Hylek E, Jackman WM, Jalife J, Kalman JM, Kautzner J, Kottkamp H, Kuck KH, Kumagai K, Lee R, Lewalter T, Lindsay BD, Macle L, Mansour M, Marchlinski FE, Michaud GF, Nakagawa H, Natale A, Nattel S, Okumura K, Packer D, Pokushalov E, Reynolds MR, Sanders P, Scanavacca M, Schilling R, Tondo C, Tsao HM, Verma A, Wilber DJ, Yamane T. 2017 HRS/ EHRA/ECAS/APHRS/SOLAECE expert consensus statement on catheter and surgical ablation of atrial fibrillation: executive summary. Europace 2018;20:157-208.

2. Charitos El, Stierle U, Ziegler PD, Baldewig M, Robinson DR, Sievers HH, Hanke T. A comprehensive evaluation of rhythm monitoring strategies for the detection of atrial fibrillation recurrence: insights from 647 continuously monitored patients and implications for monitoring after therapeutic interventions. Circulation 2012;126:806-814.

3. Gorenek B, Boriani G, Dan GA, Fauchier L, Fenelon G, Huang H, Kudaiberdieva G, Lip GYH, Mahajan R, Potpara T, Ramirez JD, Vos MA, Marin F, ESC Scientific Document Group. European Heart Rhythm Association (EHRA) position paper on arrhythmia management and device therapies in endocrine disorders, endorsed by Asia Pacific Heart Rhythm Society (APHRS) and Latin American Heart Rhythm Society (LAHRS). Europace 2018;20:895-896.
4. Freedman B, Boriani G, Glotzer TV, Healey JS, Kirchhof P, Potpara TS. Management of atrial high-rate episodes detected by cardiac implanted electronic devices. Nat Rev Cardiol 2017;14:701-714.

5. Perino AC, Fan J, Askari M, Heidenreich PA, Keung E, Raitt MH, Piccini JP, Ziegler PD, Turakhia MP. Practice variation in anticoagulation prescription and outcomes after device-detected atrial fibrillation. Circulation 2019;139:2502-2512.

6. Steinberg JS, O'Connell H, Li S, Ziegler PD. Thirty-second gold standard definition of atrial fibrillation and its relationship with subsequent arrhythmia patterns: analysis of a large prospective device database. Circ Arrhythm Electrophysiol 2018;11:e006274.

7. Camm AJ, Simantirakis E, Goette A, Lip GY, Vardas P, Calvert M, Chlouverakis G, Diener HC, Kirchhof P. Atrial high-rate episodes and stroke prevention. Europace 2017;19:169-179.

8. Pollak WM, Simmons JD, Interian A, Jr., Atapattu SA, Castellanos A, Myerburg RJ, Mitrani RD. Clinical utility of intraatrial pacemaker stored electrograms to diagnose atrial fibrillation and flutter. Pacing Clin Electrophysiol 2001;24:424-429.

9. Kaufman ES, Israel CW, Nair GM, Armaganijan L, Divakaramenon S, Mairesse GH, Brandes A, Crystal E, Costantini O, Sandhu RK, Parkash R, Connolly SJ, Hohnloser SH, Healey JS; ASSERT Steering Committee and Investigators. Positive predictive value of device-detected atrial high-rate episodes at different rates and durations: an analysis from ASSERT. Heart Rhythm 2012;9:1241-1246.

10. Benjamin EJ, Muntner P, Alonso A, Bittencourt MS, Callaway CW, Carson AP, Chamberlain AM, Chang AR, Cheng S, Das SR, Delling FN, Djousse L, Elkind MSV, Ferguson JF, Fornage M, Jordan LC, Khan SS, Kissela BM, Knutson KL, Kwan TW, Lackland DT, Lewis TT, Lichtman JH, Longenecker CT, Loop MS, Lutsey PL, Martin SS, Matsushita K, Moran AE, Mussolino ME, O'Flaherty M, Pandey A, Perak AM, Rosamond WD, Roth GA, Sampson UKA, Satou GM, Schroeder EB, Shah SH, Spartano NL, Stokes A, Tirschwell DL, Tsao CW, Turakhia MP, VanWagner LB, Wilkins JT, Wong SS, Virani SS, American Heart Association Council on Epidemiology and Prevention Statistics Committee and Stroke Statistics Subcommittee. Heart disease and stroke statistics - 2019 update: a report from the American Heart Association. Circulation 2019;139:e56-e528.

11. Chugh SS, Havmoeller R, Narayanan K, Singh D, Rienstra M, Benjamin EJ, Gillum RF, Kim YH, McAnulty JH Jr, Zheng Z], Forouzanfar MH, Naghavi M, Mensah GA, Ezzati M, Murray CJ. Worldwide epidemiology of atrial fibrillation: a Global Burden of Disease 2010 Study. Circulation 2014;129:837-847.

12. Colilla S, Crow A, Petkun W, Singer DE, Simon T, Liu X. Estimates of current and future incidence and prevalence of atrial fibrillation in the US adult population. Am J Cardiol 2013;112:1142-1147.

13. Krijthe BP, Kunst A, Benjamin EJ, Lip GY, Franco OH, Hofman A, Witteman JC, Stricker $\mathrm{BH}$, Heeringa J. Projections on the number of individuals with atrial fibrillation in the European Union, from 2000 to 2060. Eur Heart J 2013;34:2746-2751.

14. Dewland TA, Olgin JE, Vittinghoff E, Marcus GM. Incident atrial fibrillation among Asians, Hispanics, blacks, and whites. Circulation 2013;128:2470-2477.

15. Staerk L, Sherer JA, Ko D, Benjamin EJ, Helm RH. Atrial fibrillation: epidemiology, pathophysiology, and clinical outcomes. Circ Res 2017;120:1501-1517.

16. Alonso A, Agarwal SK, Soliman EZ, Ambrose M, Chamberlain AM, Prineas RJ, Folsom AR. Incidence of atrial fibrillation in whites and African Americans: the Atherosclerosis Risk in Communities (ARIC) study. Am Heart J 2009;158:111-117

17. Chao TF, Liu C], Tuan TC, Chen TJ, Hsieh MH, Lip GYH, Chen SA. Lifetime risks, projected numbers, and adverse outcomes in Asian patients with atrial fibrillation: a report from the Taiwan Nationwide AF Cohort Study. Chest 2018;153:453-466

18. Guo Y, Tian Y, Wang H, Si Q, Wang Y, Lip GYH. Prevalence, incidence, and lifetime risk of atrial fibrillation in China: new insights into the global burden of atrial fibrillation. Chest 2015;147:109-119.

19. Di Carlo A, Bellino L, Consoli D, Mori F, Zaninelli A, Baldereschi M, Baldereschi M, Cattarinussi A, D’Alfonso MG, Gradia C, Sgherzi B, Pracucci G, Piccardi B, Polizzi B, Inzitari D, National Research Program: Progetto FAl. La Fibrillazione Atriale in Italia. Prevalence of atrial fibrillation in the Italian elderly population and projections from 2020 to 2060 for Italy and the European Union: the FAI Project. Europace 2019;21:1468-1475.

20. Mou L, Norby FL, Chen LY, O'Neal WT, Lewis TT, Loehr LR, Soliman EZ, Alonso A. Lifetime risk of atrial fibrillation by race and socioeconomic status: ARIC study (Atherosclerosis Risk in Communities). Circ Arrhythm Electrophysiol 2018;11:e006350.

21. Boriani G, Savelieva I, Dan GA, Deharo JC, Ferro C, Israel CW, Lane DA, La Manna G, Morton J, Mitjans AM, Vos MA, Turakhia MP, Lip GY. Chronic kidney disease in patients with cardiac rhythm disturbances or implantable electrical devices: clinical significance and implications for decision making - a position paper of the European Heart Rhythm Association endorsed by the Heart Rhythm Society and the Asia Pacific Heart Rhythm Society. Europace 2015;17:1169-1196. 
22. Aune D, Feng T, Schlesinger S, Janszky I, Norat T, Riboli E. Diabetes mellitus, blood glucose and the risk of atrial fibrillation: a systematic review and metaanalysis of cohort studies. J Diabetes Complications 2018;32:501-511.

23. Cadby G, McArdle N, Briffa T, Hillman DR, Simpson L, Knuiman M, Hung J. Severity of OSA is an independent predictor of incident atrial fibrillation hospitalization in a large sleep-clinic cohort. Chest 2015;148:945 -952.

24. Hobbelt AH, Siland JE, Geelhoed B, Van Der Harst P, Hillege HL, Van Gelder IC, Rienstra M. Clinical, biomarker, and genetic predictors of specific types of atrial fibrillation in a community-based cohort: data of the PREVEND study. Europace 2017; 19:226-232.

25. Nalliah CJ, Sanders P, Kalman JM. The impact of diet and lifestyle on atrial fibrillation. Curr Cardiol Rep 2018;20:137.

26. Lip GYH, Coca A, Kahan T, Boriani G, Manolis AS, Olsen MH, Oto A, Potpara TS, Steffel J, Marin F, de Oliveira Figueiredo MJ, de Simone G, Tzou WS, Chiang CE, Williams B, Reviewers, Dan GA, Gorenek B, Fauchier L, Savelieva I, Hatala R, van Gelder I, Brguljan-Hitij J, Erdine S, Lovic D, Kim YH, Salinas-Arce J, Field M. Hypertension and cardiac arrhythmias: a consensus document from the European Heart Rhythm Association (EHRA) and ESC Council on Hypertension, endorsed by the Heart Rhythm Society (HRS), Asia-Pacific Heart Rhythm Society (APHRS) and Sociedad Latinoamericana de Estimulacion Cardiaca y Electrofisiologia (SOLEACE). Europace 2017;19:891-911.

27. Gallagher C, Hendriks JML, Elliott AD, Wong CX, Rangnekar G, Middeldorp ME, Mahajan R, Lau DH, Sanders P. Alcohol and incident atrial fibrillation - a systematic review and meta-analysis. Int J Cardiol 2017;246:46-52.

28. Ricci C, Gervasi F, Gaeta M, Smuts CM, Schutte AE, Leitzmann MF. Physical activity volume in relation to risk of atrial fibrillation. A non-linear meta-regression analysis. Eur J Prev Cardiol 2018;25:857-866.

29. Heeringa J, van der Kuip DA, Hofman A, Kors JA, van Herpen G, Stricker BH, Stijnen T, Lip GY, Witteman JC. Prevalence, incidence and lifetime risk of atrial fibrillation: the Rotterdam Study. Eur Heart J 2006;27:949-953.

30. Lloyd-Jones DM, Wang TJ, Leip EP, Larson MG, Levy D, Vasan RS, D'Agostino RB, Massaro JM, Beiser A, Wolf PA, Benjamin EJ. Lifetime risk for development of atrial fibrillation: the Framingham Heart Study. Circulation 2004:110:10421046.

31. Magnussen C, Niiranen TJ, Ojeda FM, Gianfagna F, Blankenberg S, Njolstad I, Vartiainen E, Sans S, Pasterkamp G, Hughes M, Costanzo S, Donati MB, Jousilahti P, Linneberg A, Palosaari T, de Gaetano G, Bobak M, den Ruijter HM, Mathiesen E, Jorgensen T, Soderberg S, Kuulasmaa K, Zeller T, lacoviello L, Salomaa V, Schnabel RB, BiomarCaRE Consortium. Sex differences and similarities in atrial fibrillation epidemiology, risk factors, and mortality in community cohorts: results from the BiomarCaRE Consortium (Biomarker for Cardiovascular Risk Assessment in Europe). Circulation 2017;136:1588-1597.

32. Staerk L, Wang B, Preis SR, Larson MG, Lubitz SA, Ellinor PT, McManus DD, Ko $D$, Weng $L C$, Lunetta KL, Frost L, Benjamin E), Trinquart L. Lifetime risk of atrial fibrillation according to optimal, borderline, or elevated levels of risk factors: cohort study based on longitudinal data from the Framingham Heart Study. BMJ 2018;361:k1453.

33. Allan V, Honarbakhsh S, Casas JP, Wallace J, Hunter R, Schilling R, Perel P, Morley K, Banerjee A, Hemingway $\mathrm{H}$. Are cardiovascular risk factors also associated with the incidence of atrial fibrillation? A systematic review and field synopsis of 23 factors in 32 population-based cohorts of 20 million participants. Thromb Haemost 2017;117:837-850.

34. Feghaly J, Zakka P, London B, MacRae CA, Refaat MM. Genetics of atrial fibrillation. J Am Heart Assoc 2018;7:e009884.

35. Abdulla J, Nielsen JR. Is the risk of atrial fibrillation higher in athletes than in the general population? A systematic review and meta-analysis. Europace 2009;11:1156-1159.

36. Alonso A, Jensen PN, Lopez FL, Chen LY, Psaty BM, Folsom AR, Heckbert SR. Association of sick sinus syndrome with incident cardiovascular disease and mortality: the Atherosclerosis Risk in Communities Study and Cardiovascular Health Study. PLoS One 2014;9:e109662.

37. Alonso A, Lopez FL, Matsushita K, Loehr LR, Agarwal SK, Chen LY, Soliman EZ, Astor BC, Coresh J. Chronic kidney disease is associated with the incidence of atrial fibrillation: the Atherosclerosis Risk in Communities (ARIC) study. Circulation 2011;123:2946-2953.

38. Andersen K, Farahmand B, Ahlbom A, Held C, Ljunghall S, Michaelsson K, Sundstrom J. Risk of arrhythmias in 52755 long-distance cross-country skiers: a cohort study. Eur Heart J 2013;34:3624-3631.

39. Asad Z, Abbas M, Javed I, Korantzopoulos P, Stavrakis S. Obesity is associated with incident atrial fibrillation independent of gender: a meta-analysis. J Cardiovasc Electrophysiol 2018;29:725-732.

40. Aune D, Sen A, Schlesinger S, Norat T, Janszky I, Romundstad P, Tonstad S, Riboli E, Vatten LJ. Body mass index, abdominal fatness, fat mass and the risk of atrial fibrillation: a systematic review and dose-response meta-analysis of prospective studies. Eur J Epidemiol 2017;32:181-192.
41. Bansal N, Zelnick LR, Alonso A, Benjamin EJ, de Boer IH, Deo R, Katz R, Kestenbaum B, Mathew J, Robinson-Cohen C, Sarnak MJ, Shlipak MG, Sotoodehnia N, Young B, Heckbert SR. eGFR and albuminuria in relation to risk of incident atrial fibrillation: a meta-analysis of the Jackson Heart Study, the Multi-Ethnic Study of Atherosclerosis, and the Cardiovascular Health Study. Clin J Am Soc Nephrol 2017;12:1386-1398.

42. Baumgartner C, da Costa BR, Collet TH, Feller M, Floriani C, Bauer DC, Cappola AR, Heckbert SR, Ceresini G, Gussekloo J, den Elzen WPJ, Peeters RP, Luben R, Volzke H, Dorr M, Walsh JP, Bremner A, lacoviello M, Macfarlane P, Heeringa J, Stott DJ, Westendorp RGJ, Khaw KT, Magnani JW, Aujesky D, Rodondi N, Thyroid Studies Collaboration. Thyroid function within the normal range, subclinical hypothyroidism, and the risk of atrial fibrillation. Circulation 2017;136:2100-2116.

43. Benjamin EJ, Levy D, Vaziri SM, D’Agostino RB, Belanger AJ, Wolf PA. Independent risk factors for atrial fibrillation in a population-based cohort. The Framingham Heart Study. JAMA 1994;271:840-844.

44. Bunch TJ, May HT, Bair TL, Anderson JL, Crandall BG, Cutler MJ, Jacobs V, Mallender C, Muhlestein JB, Osborn JS, Weiss JP, Day JD. Long-term natural history of adult Wolff-Parkinson-White syndrome patients treated with and without catheter ablation. Circ Arrhythm Electrophysiol 2015;8:1465-1471.

45. Chang SH, Kuo CF, Chou IJ, See LC, Yu KH, Luo SF, Huang LH, Zhang W, Doherty M, Wen MS, Kuo CT, Yeh YH. Association of a family history of atrial fibrillation with incidence and outcomes of atrial fibrillation: a population-based family cohort study. JAMA Cardiol 2017;2:863-870.

46. Chen LY, Leening MJ, Norby FL, Roetker NS, Hofman A, Franco OH, Pan W, Polak JF, Witteman JC, Kronmal RA, Folsom AR, Nazarian S, Stricker BH, Heckbert SR, Alonso A. Carotid intima-media thickness and arterial stiffness and the risk of atrial fibrillation: the Atherosclerosis Risk in Communities (ARIC) study, Multi-Ethnic Study of Atherosclerosis (MESA), and the Rotterdam Study. J Am Heart Assoc 2016;5.

47. Cheng M, Hu Z, Lu X, Huang J, Gu D. Caffeine intake and atrial fibrillation incidence: dose response meta-analysis of prospective cohort studies. Can J Cardiol 2014;30:448-454.

48. Cheng S, Keyes MJ, Larson MG, McCabe EL, Newton-Cheh C, Levy D, Benjamin EJ, Vasan RS, Wang TJ. Long-term outcomes in individuals with prolonged PR interval or first-degree atrioventricular block. JAMA 2009;301:2571-2577.

49. Conen D, Chiuve SE, Everett BM, Zhang SM, Buring JE, Albert CM. Caffeine consumption and incident atrial fibrillation in women. Am J Clin Nutr 2010;92:509-514.

50. Desai R, Patel U, Singh S, Bhuva R, Fong HK, Nunna P, Zalavadia D, Dave H, Savani S, Doshi R. The burden and impact of arrhythmia in chronic obstructive pulmonary disease: insights from the National Inpatient Sample. Int J Cardiol 2019:281:49-55.

51. Eaker ED, Sullivan LM, Kelly-Hayes M, D’Agostino RB Sr, Benjamin EJ. Anger and hostility predict the development of atrial fibrillation in men in the Framingham Offspring Study. Circulation 2004;109:1267-1271.

52. Fox CS, Parise H, D'Agostino RB Sr, Lloyd-Jones DM, Vasan RS, Wang TJ, Levy $D$, Wolf PA, Benjamin EJ. Parental atrial fibrillation as a risk factor for atrial fibrillation in offspring. JAMA 2004:291:2851-2855.

53. Furberg CD, Psaty BM, Manolio TA, Gardin JM, Smith VE, Rautaharju PM. Prevalence of atrial fibrillation in elderly subjects (the Cardiovascular Health Study). Am J Cardiol 1994;74:236-241.

54. Giacomantonio NB, Bredin SS, Foulds HJ, Warburton DE. A systematic review of the health benefits of exercise rehabilitation in persons living with atrial fibrillation. Can J Cardiol 2013;29:483-491.

55. Kirchhof P, Lip GY, Van Gelder IC, Bax J, Hylek E, Kaab S, Schotten U, Wegscheider K, Boriani G, Brandes A, Ezekowitz M, Diener H, Haegeli L, Heidbuchel H, Lane D, Mont L, Willems S, Dorian P, Aunes-Jansson M, Blomstrom-Lundqvist C, Borentain M, Breitenstein S, Brueckmann M, Cater N, Clemens A, Dobrev D, Dubner S, Edvardsson NG, Friberg L, Goette A, Gulizia M, Hatala R, Horwood J, Szumowski L, Kappenberger L, Kautzner J, Leute A, Lobban T, Meyer R, Millerhagen J, Morgan J, Muenzel F, Nabauer M, Baertels C, Oeff M, Paar D, Polifka J, Ravens U, Rosin L, Stegink W, Steinbeck G, Vardas P, Vincent A, Walter M, Breithardt G, Camm AJ. Comprehensive risk reduction in patients with atrial fibrillation: emerging diagnostic and therapeutic options - a report from the 3rd Atrial Fibrillation Competence NETwork/European Heart Rhythm Association consensus conference. Europace 2012;14:8-27.

56. Ko D, Benson MD, Ngo D, Yang Q, Larson MG, Wang TJ, Trinquart L, McManus DD, Lubitz SA, Ellinor PT, Vasan RS, Gerszten RE, Benjamin EJ, Lin H. Proteomics profiling and risk of new-onset atrial fibrillation: Framingham Heart Study. J Am Heart Assoc 2019;8:e010976.

57. Kwok CS, Anderson SG, Myint PK, Mamas MA, Loke YK. Physical activity and incidence of atrial fibrillation: a systematic review and meta-analysis. Int J Cardiol 2014;177:467-476.

58. Lip GYH, Collet JP, de Caterina R, Fauchier L, Lane DA, Larsen TB, Marin F, Morais J, Narasimhan C, Olshansky B, Pierard L, Potpara T, Sarrafzadegan N, 
Sliwa K, Varela G, Vilahur G, Weiss T, Boriani G, Rocca B. Antithrombotic therapy in atrial fibrillation associated with valvular heart disease: executive summary of a joint consensus document from the European Heart Rhythm Association (EHRA) and European Society of Cardiology Working Group on Thrombosis, Endorsed by the ESC Working Group on Valvular Heart Disease, Cardiac Arrhythmia Society of Southern Africa (CASSA), Heart Rhythm Society (HRS), Asia Pacific Heart Rhythm Society (APHRS), South African Heart (SA Heart) Association and Sociedad Latinoamericana de Estimulacion Cardiaca y Electrofisiologia (SOLEACE). Thromb Haemost 2017;117:2215-2236.

59. Loomba RS, Buelow MW, Aggarwal S, Arora RR, Kovach J, Ginde S. Arrhythmias in adults with congenital heart disease: what are risk factors for specific arrhythmias?Pacing Clin Electrophysiol 2017;40:353-361.

60. Lubitz SA, Yin X, Fontes JD, Magnani JW, Rienstra M, Pai M, Villalon ML, Vasan RS, Pencina MJ, Levy D, Larson MG, Ellinor PT, Benjamin EJ. Association between familial atrial fibrillation and risk of new-onset atrial fibrillation. JAMA 2010;304:2263-2269.

61. May AM, Blackwell T, Stone PH, Stone KL, Cawthon PM, Sauer WH, Varosy PD, Redline S, Mehra R, Sleep MrOS (Outcomes of Sleep Disorders in Older Men) Study Group. Central sleep-disordered breathing predicts incident atrial fibrillation in older men. Am J Respir Crit Care Med 2016;193:783-791.

62. Michniewicz E, Mlodawska E, Lopatowska P, Tomaszuk-Kazberuk A, Malyszko J. Patients with atrial fibrillation and coronary artery disease - double trouble. Adv Med Sci 2018;63:30-35.

63. Monrad M, Sajadieh A, Christensen JS, Ketzel M, Raaschou-Nielsen O, Tjonneland A, Overvad K, Loft S, Sorensen M. Long-term exposure to trafficrelated air pollution and risk of incident atrial fibrillation: a cohort study. Environ Health Perspect 2017;125:422-427.

64. O'Neal WT, Efird JT, Qureshi WT, Yeboah J, Alonso A, Heckbert SR, Nazarian S, Soliman EZ. Coronary artery calcium progression and atrial fibrillation: the Multi-Ethnic Study of Atherosclerosis. Circ Cardiovasc Imaging 2015;8:pii: e003786.

65. Qureshi WT, Alirhayim Z, Blaha MJ, Juraschek SP, Keteyian SJ, Brawner CA, AlMallah $\mathrm{MH}$. Cardiorespiratory fitness and risk of incident atrial fibrillation: results from the Henry Ford Exercise Testing (FIT) Project. Circulation 2015;131:1827-1834.

66. Santhanakrishnan R, Wang N, Larson MG, Magnani JW, McManus DD, Lubitz SA, Ellinor PT, Cheng S, Vasan RS, Lee DS, Wang TJ, Levy D, Benjamin EJ, Ho JE. Atrial fibrillation begets heart failure and vice versa: temporal associations and differences in preserved versus reduced ejection fraction. Circulation 2016;133:484-492.

67. Schnabel RB, Yin X, Gona P, Larson MG, Beiser AS, McManus DD, NewtonCheh C, Lubitz SA, Magnani JW, Ellinor PT, Seshadri S, Wolf PA, Vasan RS, Benjamin EJ, Levy D. 50-Year trends in atrial fibrillation prevalence, incidence, risk factors, and mortality in the Framingham Heart Study: a cohort study. Lancet 2015;386:154-162.

68. Shen J, Johnson VM, Sullivan LM, Jacques PF, Magnani JW, Lubitz SA, Pandey S, Levy D, Vasan RS, Quatromoni PA, Junyent M, Ordovas JM, Benjamin EJ. Dietary factors and incident atrial fibrillation: the Framingham Heart Study. Am J Clin Nutr 2011;93:261-266.

69. Svensson T, Kitlinski M, Engstrom G, Melander O. Psychological stress and risk of incident atrial fibrillation in men and women with known atrial fibrillation genetic risk scores. Sci Rep 2017;7:42613.

70. Tung P, Levitzky YS, Wang R, Weng J, Quan SF, Gottlieb DJ, Rueschman M, Punjabi NM, Mehra R, Bertisch S, Benjamin EJ, Redline S. Obstructive and central sleep apnea and the risk of incident atrial fibrillation in a community cohort of men and women. J Am Heart Assoc 2017;6:pii.

71. Walkey AJ, Greiner MA, Heckbert SR, Jensen PN, Piccini JP, Sinner MF, Curtis LH, Benjamin EJ. Atrial fibrillation among Medicare beneficiaries hospitalized with sepsis: incidence and risk factors. Am Heart J 2013;165:949-955.e3.

72. Zoller B, Ohlsson H, Sundquist J, Sundquist K. High familial risk of atrial fibrillation/atrial flutter in multiplex families: a nationwide family study in Sweden. J Am Heart Assoc 2012;2:e003384.

73. Lip GYH, Skjoth F, Nielsen PB, Larsen TB. Evaluation of the C2HEST risk score as a possible opportunistic screening tool for incident atrial fibrillation in a healthy population (from a nationwide Danish cohort study). Am J Cardiol 2020;125:48-54.

74. Yiin GSC, Li L, Bejot Y, Rothwell PM. Time trends in atrial fibrillation-associated stroke and premorbid anticoagulation. Stroke 2018:STROKEAHA118022249.

75. Akao M, Chun YH, Wada H, Esato M, Hashimoto T, Abe M, Hasegawa K, Tsuji $\mathrm{H}$, Furuke K; Fushimi AFRegistry Investigators. Current status of clinical background of patients with atrial fibrillation in a community-based survey: the Fushimi AF Registry. J Cardiol 2013;61:260-266.

76. An $Y$, Ogawa H, Yamashita $Y$, Ishii M, Iguchi M, Masunaga N, Esato M, Tsuji H, Wada H, Hasegawa K, Abe M, Lip GYH, Akao M. Causes of death in Japanese patients with atrial fibrillation: the Fushimi Atrial Fibrillation Registry. Eur Heart J Qual Care Clin Outcomes 2019;5:35-42.
77. Andersson T, Magnuson A, Bryngelsson IL, Frobert O, Henriksson KM, Edvardsson N, Poci D. All-cause mortality in 272,186 patients hospitalized with incident atrial fibrillation 1995-2008: a Swedish nationwide long-term case-control study. Eur Heart J 2013;34:1061-1067.

78. Andrew NE, Thrift AG, Cadilhac DA. The prevalence, impact and economic implications of atrial fibrillation in stroke: what progress has been made? Neuroepidemiology 2013;40:227-239.

79. Bakhai A, Darius H, De Caterina R, Smart A, Le Heuzey JY, Schilling RJ, Zamorano JL, Shah M, Bramlage P, Kirchhof P. Characteristics and outcomes of atrial fibrillation patients with or without specific symptoms: results from the PREFER in AF registry. Eur Heart J Qual Care Clin Outcomes 2016;2:299-305.

80. Benjamin EJ, Wolf PA, D’Agostino RB, Silbershatz H, Kannel WB, Levy D. Impact of atrial fibrillation on the risk of death: the Framingham Heart Study. Circulation 1998;98:946-952.

81. Blum S, Muff C, Aeschbacher S, Ammann P, Erne P, Moschovitis G, Di Valentino M, Shah D, Schlapfer J, Fischer A, Merkel T, Kuhne M, Sticherling C, Osswald S, Conen D. Prospective assessment of sex-related differences in symptom status and health perception among patients with atrial fibrillation. J Am Heart Assoc 2017;6:e005401.

82. Boriani G, Laroche C, Diemberger I, Fantecchi E, Popescu MI, Rasmussen LH, Sinagra G, Petrescu L, Tavazzi L, Maggioni AP, Lip GY. Asymptomatic atrial fibrillation: clinical correlates, management, and outcomes in the EORP-AF Pilot General Registry. Am J Med 2015;128:509-518.e2.

83. Ceornodolea AD, Bal R, Severens JL. Epidemiology and management of atrial fibrillation and stroke: review of data from four European countries. Stroke Res Treat 2017;2017:8593207.

84. Chao TF, Lip GY, Liu CJ, Tuan TC, Chen SJ, Wang KL, Lin YJ, Chang SL, Lo LW, Hu YF, Chen TJ, Chiang CE, Chen SA. Validation of a modified CHA2DS2-VASc score for stroke risk stratification in Asian patients with atrial fibrillation: a nationwide cohort study. Stroke 2016;47:2462-2469.

85. Chao T-F, Liu C-J, Wang K-L, Lin Y-J, Chang S-L, Lo L-W, Hu Y-F, Tuan T-C, Chen T-J, Lip GY. Should atrial fibrillation patients with 1 additional risk factor of the CHA2DS2-VASc score (beyond sex) receive oral anticoagulation?] Am Coll Cardiol 2015;65:635-642.

86. Dagres N, Chao T-F, Fenelon G, Aguinaga L, Benhayon D, Benjamin EJ, Bunch TJ, Chen LY, Chen S-A, Darrieux F, de Paola A, Fauchier L, Goette A, Kalman J, Kalra L, Kim Y-H, Lane DA, Lip GYH, Lubitz SA, Márquez MF, Potpara T, Pozzer DL, Ruskin JN, Savelieva I, Teo WS, Tse H-F, Verma A, Zhang S, Chung MK, Bautista-Vargas W-F, Chiang C-E, Cuesta A, Dan G-A, Frankel DS, Guo Y, Hatala R, Lee YS, Murakawa Y, Pellegrini CN, Pinho C, Milan DJ, Morin DP, Nadalin E, Ntaios G, Prabhu MA, Proietti M, Rivard L, Valentino M, Shantsila A. European Heart Rhythm Association (EHRA)/Heart Rhythm Society (HRS)/Asia Pacific Heart Rhythm Society (APHRS)/Latin American Heart Rhythm Society (LAHRS) expert consensus on arrhythmias and cognitive function: what is the best practice?EP Europace 2018;20:1399-1421.

87. Esato M, Chun YH, An Y, Ogawa H, Wada H, Hasegawa K, Tsuji H, Abe M, Lip GYH, Akao M. Clinical impact of asymptomatic presentation status in patients with paroxysmal and sustained atrial fibrillation: the Fushimi AF Registry. Chest 2017;152:1266-1275.

88. Freeman JV, Simon DN, Go AS, Spertus J, Fonarow GC, Gersh BJ, Hylek EM, Kowey PR, Mahaffey KW, Thomas LE, Chang P, Peterson ED, Piccini JP: Outcomes Registry for Better Informed Treatment of Atrial Fibrillation Investigators. Association between atrial fibrillation symptoms, quality of life, and patient outcomes: results from the Outcomes Registry for Better Informed Treatment of Atrial Fibrillation (ORBIT-AF). Circ Cardiovasc Qual Outcomes 2015;8:393-402.

89. Frost L, Engholm G, Johnsen S, Moller H, Henneberg EW, Husted S. Incident thromboembolism in the aorta and the renal, mesenteric, pelvic, and extremity arteries after discharge from the hospital with a diagnosis of atrial fibrillation. Arch Intern Med 2001;161:272-276.

90. Gaita F, Corsinovi L, Anselmino M, Raimondo C, Pianelli M, Toso E, Bergamasco L, Boffano C, Valentini MC, Cesarani F, Scaglione M. Prevalence of silent cerebral ischemia in paroxysmal and persistent atrial fibrillation and correlation with cognitive function. J Am Coll Cardiol 2013;62:1990-1997.

91. Garcia-Fernandez A, Roldan V, Rivera-Caravaca JM, Lip GYH, Marin F. Applicability of the modified CHA2DS2-VASc score for stroke risk stratification in Caucasian atrial fibrillation patients. Eur J Intern Med 2017;38:e21-e22.

92. Gleason KT, Nazarian S, Dennison Himmelfarb CR. Atrial fibrillation symptoms and sex, race, and psychological distress: a literature review. J Cardiovasc Nurs 2018;33:137-143.

93. Gomez-Outes A, Lagunar-Ruiz J, Terleira-Fernandez Al, Calvo-Rojas G, SuarezGea ML, Vargas-Castrillon E. Causes of death in anticoagulated patients with atrial fibrillation. J Am Coll Cardiol 2016;68:2508-2521.

94. Graves KG, May HT, Jacobs V, Bair TL, Stevens SM, Woller SC, Crandall BG, Cutler MJ, Day JD, Mallender C, Osborn JS, Peter Weiss J, Jared Bunch T. Atrial fibrillation incrementally increases dementia risk across all CHADS2 and 
CHA2DS2VASc strata in patients receiving long-term warfarin. Am Heart J 2017;188:93-98.

95. John RM, Michaud GF, Stevenson WG. Atrial fibrillation hospitalization, mortality, and therapy. Eur Heart J 2018;39:3958-3960.

96. Kalantarian S, Ruskin JN. Atrial fibrillation and cognitive decline: phenomenon or epiphenomenon?Cardiol Clin 2016;34:279-285.

97. Kalantarian S, Stern TA, Mansour M, Ruskin JN. Cognitive impairment associated with atrial fibrillation: a meta-analysis. Ann Intern Med 2013;158:338-46.

98. Kim MH, Johnston SS, Chu BC, Dalal MR, Schulman KL. Estimation of total incremental health care costs in patients with atrial fibrillation in the United States. Circ Cardiovasc Qual Outcomes 2011;4:313-320.

99. Kirchhof P, Schmalowsky J, Pittrow D, Rosin L, Kirch W, Wegscheider K, Meinertz T, ATRIUM Study Group. Management of patients with atrial fibrillation by primary-care physicians in Germany: 1-year results of the ATRIUM registry. Clin Cardiol 2014:37:277-284.

100. Kochhauser S, Joza J, Essebag V, Proietti R, Koehler J, Tsang B, Wulffhart Z, Pantano A, Khaykin Y, Ziegler PD, Verma A. The impact of duration of atrial fibrillation recurrences on measures of health-related quality of life and symptoms. Pacing Clin Electrophysiol 2016;39:166-72.

101. Konig S, Ueberham L, Schuler E, Wiedemann M, Reithmann C, Seyfarth M, Sause A, Tebbenjohanns J, Schade A, Shin DI, Staudt A, Zacharzowsky U, Andrie R, Wetzel U, Neuser H, Wunderlich C, Kuhlen R, Tijssen JGP, Hindricks G, Bollmann A. In-hospital mortality of patients with atrial arrhythmias: insights from the German-wide Helios hospital network of 161502 patients and 34025 arrhythmia-related procedures. Eur Heart J 2018;39:3947-3957.

102. Kotecha D, Lam CS, Van Veldhuisen DJ, Van Gelder IC, Voors AA, Rienstra M. Heart failure with preserved ejection fraction and atrial fibrillation: vicious twins. J Am Coll Cardiol 2016;68:2217-2228.

103. Kupper N, van den Broek K, Haagh E, van der Voort P, Widdershoven J, Denollet J. Type D personality affects health-related quality of life in patients with lone atrial fibrillation by increasing symptoms related to sympathetic activation. J Psychosom Res 2018;115:44-52.

104. Kwok CS, Loke YK, Hale R, Potter JF, Myint PK. Atrial fibrillation and incidence of dementia: a systematic review and meta-analysis. Neurology 2011;76:914-922.

105. Levy S, Maarek M, Coumel P, Guize L, Lekieffre J, Medvedowsky JL, Sebaoun A. Characterization of different subsets of atrial fibrillation in general practice in France: the ALFA study. The College of French Cardiologists. Circulation 1999;99:3028-3035.

106. Lin HJ, Wolf PA, Kelly-Hayes M, Beiser AS, Kase CS, Benjamin EJ, D'Agostino RB. Stroke severity in atrial fibrillation. The Framingham Study. Stroke 1996;27:1760-1764

107. Lip GY, Laroche C, Boriani G, Cimaglia P, Dan GA, Santini M, Kalarus Z, Rasmussen LH, Popescu MI, Tica O, Hellum CF, Mortensen B, Tavazzi L, Maggioni AP. Sex-related differences in presentation, treatment, and outcome of patients with atrial fibrillation in Europe: a report from the Euro Observational Research Programme Pilot Survey on Atrial Fibrillation. Europace 2015;17:24-31.

108. McCabe PJ, Rhudy LM, DeVon HA. Patients' experiences from symptom onset to initial treatment for atrial fibrillation. J Clin Nurs 2015;24:786-796.

109. McCabe PJ, Schumacher K, Barnason SA. Living with atrial fibrillation: a qualitative study. J Cardiovasc Nurs 2011;26:336-344.

110. Meyre P, Blum S, Berger S, Aeschbacher S, Schoepfer H, Briel M, Osswald S, Conen D. Risk of hospital admissions in patients with atrial fibrillation: a systematic review and meta-analysis. Can J Cardiol 2019;35:1332-1343.

111. Nieuwlaat R, Capucci A, Camm AJ, Olsson SB, Andresen D, Davies DW, Cobbe S, Breithardt G, Le Heuzey JY, Prins MH, Levy S, Crijns HJ; European Heart Survey Investigators. Atrial fibrillation management: a prospective survey in ESC member countries: the Euro Heart Survey on Atrial Fibrillation. Eur Heart J 2005; 26:2422-2434.

112. Overvad TF, Nielsen PB, Lip GY. Treatment thresholds for stroke prevention in atrial fibrillation: observations on the CHA2DS2-VASc score. Eur Heart J Cardiovasc Pharmacother 2017;3:37-41.

113. Page RL, Wilkinson WE, Clair WK, McCarthy EA, Pritchett EL. Asymptomatic arrhythmias in patients with symptomatic paroxysmal atrial fibrillation and paroxysmal supraventricular tachycardia. Circulation 1994;89:224-227.

114. Piccini JP, Fauchier L. Rhythm control in atrial fibrillation. Lancet 2016;388:829-840.

115. Pistoia F, Sacco S, Tiseo C, Degan D, Ornello R, Carolei A. The epidemiology of atrial fibrillation and stroke. Cardiol Clin 2016;34:255-268.

116. Pokorney SD, Piccini JP, Stevens SR, Patel MR, Pieper KS, Halperin JL, Breithardt G, Singer DE, Hankey GJ, Hacke W, Becker RC, Berkowitz SD, Nessel CC, Mahaffey KW, Fox KA, Califf RM; ROCKET AF Steering Committee Investigators. Cause of death and predictors of all-cause mortality in anticoagulated patients with nonvalvular atrial fibrillation: data from ROCKET AF. J Am Heart Assoc 2016;5:e002197.
117. Potpara TS, Polovina MM, Marinkovic JM, Lip GY. A comparison of clinical characteristics and long-term prognosis in asymptomatic and symptomatic patients with first-diagnosed atrial fibrillation: the Belgrade Atrial Fibrillation Study. Int J Cardiol 2013;168:4744-4749.

118. Randolph TC, Simon DN, Thomas L, Allen LA, Fonarow GC, Gersh BJ, Kowey PR, Reiffel JA, Naccarelli GV, Chan PS, Spertus JA, Peterson ED, Piccini JP; ORBIT AF Investigators. Patient factors associated with quality of life in atrial fibrillation. Am Heart J 2016;182:135-143.

119. Rienstra M, Lubitz SA, Mahida S, Magnani JW, Fontes JD, Sinner MF, Van Gelder IC, Ellinor PT, Benjamin EJ. Symptoms and functional status of patients with atrial fibrillation: state-of-the-art and future research opportunities. Circulation 2012;125:2933-2943.

120. Rienstra M, Vermond RA, Crijns HJ, Tijssen JG, Van Gelder IC; RACE Investigators. Asymptomatic persistent atrial fibrillation and outcome: results of the RACE study. Heart Rhythm 2014;11:939-945.

121. Rivard L, Khairy P. Mechanisms, clinical significance, and prevention of cognitive impairment in patients with atrial fibrillation. Can J Cardiol 2017;33:1556-1564.

122. Santangeli P, Di Biase L, Bai R, Mohanty S, Pump A, Cereceda Brantes M, Horton R, Burkhardt JD, Lakkireddy D, Reddy YM, Casella M, Dello Russo A, Tondo C, Natale A. Atrial fibrillation and the risk of incident dementia: a metaanalysis. Heart Rhythm 2012;9:1761-1768.

123. Schnabel RB, Michal M, Wilde S, Wiltink J, Wild PS, Sinning CR, Lubos E, Ojeda FM, Zeller T, Munzel T, Blankenberg S, Beutel ME. Depression in atrial fibrillation in the general population. PLoS One 2013;8:e79109.

124. Schnabel RB, Pecen L, Ojeda FM, Lucerna M, Rzayeva N, Blankenberg S, Darius $H$, Kotecha D, Caterina R, Kirchhof P. Gender differences in clinical presentation and 1-year outcomes in atrial fibrillation. Heart 2017;103:1024-1030.

125. Senoo K, Suzuki S, Sagara K, Otsuka T, Matsuno S, Funada R, Uejima T, Oikawa Y, Yajima J, Koike A, Nagashima K, Kirigaya H, Sawada H, Aizawa T, Yamashita $\mathrm{T}$. Distribution of first-detected atrial fibrillation patients without structural heart diseases in symptom classifications. Circ J 2012;76:1020-1023.

126. Serpytis R, Navickaite A, Serpytiene E, Barysiene J, Marinskis G, Jatuzis D, Petrulioniene Z, Laucevicius A, Serpytis P. Impact of atrial fibrillation on cognitive function, psychological distress, quality of life, and impulsiveness. Am J Med 2018;131:703.e1-703e5.

127. Siontis KC, Gersh BJ, Killian JM, Noseworthy PA, McCabe P, Weston SA, Roger VL, Chamberlain AM. Typical, atypical, and asymptomatic presentations of newonset atrial fibrillation in the community: characteristics and prognostic implications. Heart Rhythm 2016;13:1418-1424.

128. Steg PG, Alam S, Chiang CE, Gamra H, Goethals M, Inoue H, Krapf L, Lewalter T, Merioua I, Murin J, Naditch-Brule L, Ponikowski P, Rosenqvist M, SilvaCardoso J, Zharinov O, Brette S, Neill JO; RealiseAF investigators. Symptoms, functional status and quality of life in patients with controlled and uncontrolled atrial fibrillation: data from the RealiseAF cross-sectional international registry. Heart 2012;98:195-201.

129. Steinberg BA, Kim S, Fonarow GC, Thomas L, Ansell J, Kowey PR, Mahaffey KW, Gersh BJ, Hylek E, Naccarelli G, Go AS, Reiffel J, Chang P, Peterson ED, Piccini JP. Drivers of hospitalization for patients with atrial fibrillation: results from the Outcomes Registry for Better Informed Treatment of Atrial Fibrillation (ORBIT-AF). Am Heart J 2014;167:735-742.e2.

130. Stewart S, Hart CL, Hole DJ, McMurray JJ. A population-based study of the long-term risks associated with atrial fibrillation: 20-year follow-up of the Renfrew/Paisley study. Am J Med 2002;113:359-364.

131. Streur M, Ratcliffe SJ, Ball J, Stewart S, Riegel B. Symptom clusters in adults with chronic atrial fibrillation. J Cardiovasc Nurs 2017;32:296-303.

132. Thrall G, Lip GY, Carroll D, Lane D. Depression, anxiety, and quality of life in patients with atrial fibrillation. Chest 2007;132:1259-1264.

133. Ugowe FE, Jackson LRn. Atrial fibrillation and mortality risk: seeing the big picture. Eur Heart J Qual Care Clin Outcomes 2019;5:6-7.

134. Vermond RA, Crijns HJ, Tijssen JG, Alings AM, Van den Berg MP, Hillege HL, Van Veldhuisen DJ, Van Gelder IC, Rienstra M; RACE II investigators. Symptom severity is associated with cardiovascular outcome in patients with permanent atrial fibrillation in the RACE II study. Europace 2014;16:1417-1425.

135. Walters TE, Wick K, Tan G, Mearns M, Joseph SA, Morton JB, Sanders P, Bryant C, Kistler PM, Kalman JM. Psychological distress and suicidal ideation in patients with atrial fibrillation: prevalence and response to management strategy. J Am Heart Assoc 2018;7:e005502.

136. Walters TE, Wick K, Tan G, Mearns M, Joseph SA, Morton JB, Sanders P, Bryant C, Kistler PM, Kalman JM. Symptom severity and quality of life in patients with atrial fibrillation: psychological function outweighs clinical predictors. Int J Cardiol 2019;279:84-89.

137. Wang TJ, Massaro JM, Levy D, Vasan RS, Wolf PA, D'Agostino RB, Larson MG, Kannel WB, Benjamin EJ. A risk score for predicting stroke or death in individuals with new-onset atrial fibrillation in the community: the Framingham Heart Study. JAMA 2003;290:1049-1056. 
138. Wijesurendra RS, Casadei $\quad$ B. Atrial fibrillation: effects beyond the atrium?Cardiovasc Res 2015;105:238-247.

139. Xiong Q, Proietti M, Senoo K, Lip GY. Asymptomatic versus symptomatic atrial fibrillation: a systematic review of age/gender differences and cardiovascular outcomes. Int J Cardiol 2015;191:172-177.

140. Ziff OJ, Carter PR, McGowan J, Uppal H, Chandran S, Russell S, Bainey KR, Potluri R. The interplay between atrial fibrillation and heart failure on long-term mortality and length of stay: insights from the United Kingdom ACALM registry. Int J Cardiol 2018;252:117-121.

141. Sepehri Shamloo A, Dagres N, Mussigbrodt A, Stauber A, Kircher S, Richter S, Dinov B, Bertagnolli L, Husser-Bollmann D, Bollmann A, Hindricks G, Arya A. Atrial fibrillation and cognitive impairment: new insights and future directions. Heart Lung Circ 2020;29:69-85.

142. Conen D, Rodondi N, Muller A, Beer JH, Ammann P, Moschovitis G, Auricchio A, Hayoz D, Kobza R, Shah D, Novak J, Schlapfer J, Di Valentino M, Aeschbacher S, Blum S, Meyre P, Sticherling C, Bonati LH, Ehret G, Moutzouri E, Fischer U, Monsch AU, Stippich C, Wuerfel J, Sinnecker T, Coslovsky M, Schwenkglenks M, Kuhne M, Osswald S, Swiss AFSI. Relationships of overt and silent brain lesions with cognitive function in patients with atrial fibrillation. J Am Coll Cardiol 2019;73:989-999.

143. Kirchhof P, Benussi S, Kotecha D, Ahlsson A, Atar D, Casadei B, Castella M, Diener HC, Heidbuchel H, Hendriks J, Hindricks G, Manolis AS, Oldgren J, Popescu BA, Schotten U, Van Putte B, Vardas P, ESC Scientific Document Group. 2016 ESC Guidelines for the management of atrial fibrillation developed in collaboration with EACTS. Eur Heart J 2016;37:2893-2962.

144. Boriani G, Diemberger I, Ziacchi M, Valzania C, Gardini B, Cimaglia P, Martignani C, Biffi M. AF burden is important - fact or fiction?Int J Clin Pract 2014;68:444-452.

145. Boriani G, Pettorelli D. Atrial fibrillation burden and atrial fibrillation type: clinical significance and impact on the risk of stroke and decision making for longterm anticoagulation. Vascul Pharmacol 2016;83:26-35.

146. Charitos El, Purerfellner H, Glotzer TV, Ziegler PD. Clinical classifications of atrial fibrillation poorly reflect its temporal persistence: insights from 1,195 patients continuously monitored with implantable devices. J Am Coll Cardiol 2014;63:2840-2848.

147. Wyse DG, Van Gelder IC, Ellinor PT, Go AS, Kalman JM, Narayan SM, Nattel S, Schotten U, Rienstra M. Lone atrial fibrillation: does it exist?] Am Coll Cardiol 2014:63:1715-1723.

148. Lip GYH, Collet JP, Caterina R, Fauchier L, Lane DA, Larsen TB, Marin F, Morais J, Narasimhan C, Olshansky B, Pierard L, Potpara T, Sarrafzadegan N, Sliwa K, Varela G, Vilahur G, Weiss T, Boriani G, Rocca B, ESC Scientific Document Group. Antithrombotic therapy in atrial fibrillation associated with valvular heart disease: a joint consensus document from the European Heart Rhythm Association (EHRA) and European Society of Cardiology Working Group on Thrombosis, endorsed by the ESC Working Group on Valvular Heart Disease, Cardiac Arrhythmia Society of Southern Africa (CASSA), Heart Rhythm Society (HRS), Asia Pacific Heart Rhythm Society (APHRS), South African Heart (SA Heart) Association and Sociedad Latinoamericana de Estimulacion Cardiaca y Electrofisiologia (SOLEACE). Europace 2017;19:1757-1758.

149. January CT, Wann LS, Calkins H, Chen LY, Cigarroa JE, Cleveland JC, Jr., Ellinor PT, Ezekowitz MD, Field ME, Furie KL, Heidenreich PA, Murray KT, Shea JB, Tracy CM, Yancy CW. 2019 AHA/ACC/HRS focused update of the 2014 AHA/ ACC/HRS Guideline for the management of patients with atrial fibrillation. Circulation 2019;;140:e125-e151.

150. NHFA CSANZ Atrial Fibrillation Guideline Working Group, Brieger D, Amerena J, Attia J, Bajorek B, Chan KH, Connell C, Freedman B, Ferguson C, Hall T, Haqqani H, Hendriks J, Hespe C, Hung J, Kalman JM, Sanders P, Worthington J, Yan TD, Zwar N. National Heart Foundation of Australia and the Cardiac Society of Australia and New Zealand: Australian Clinical Guidelines for the diagnosis and management of atrial fibrillation 2018. Heart Lung Circ 2018;27:1209-1266.

151. Potpara TS, Lip GYH, Blomstrom-Lundqvist C, Boriani G, Van Gelder IC, Heidbuchel H, Hindricks G, Camm AJ. The 4S-AF scheme (Stroke Risk; Symptoms; Severity of Burden; Substrate): A novel approach to in-depth characterization (rather than Classification) of atrial fibrillation. Thromb Haemost 2020; doi: 10.1055/s-0040-1716408.

152. Chen LY, Chung MK, Allen LA, Ezekowitz M, Furie KL, McCabe P, Noseworthy PA, Perez MV, Turakhia MP,American Heart Association Council on Clinical Cardiology, Council on Cardiovascular and Stroke Nursing, Council on Quality of Care and Outcomes Research, and Stroke Council. Atrial fibrillation burden: moving beyond atrial fibrillation as a binary entity: a scientific statement from the American Heart Association. Circulation 2018;137:e623-e644.

153. Ziegler PD, Koehler JL, Mehra R. Comparison of continuous versus intermittent monitoring of atrial arrhythmias. Heart Rhythm 2006;3:1445-1452.
154. Boriani G, Proietti M, Laroche C, Fauchier L, Marin F, Nabauer M, Potpara T, Dan GA, Kalarus Z, Diemberger I, Tavazzi L, Maggioni AP, Lip GYH; EORP-AF Long-Term General Registry Investigators Steering Committee (National Coordinators). Contemporary stroke prevention strategies in 11096 European patients with atrial fibrillation: a report from the EURObservational Research Programme on Atrial Fibrillation (EORP-AF) long-term general registry. Europace 2018;20:747-757.

155. Pandey A, Kim S, Moore C, Thomas L, Gersh B, Allen LA, Kowey PR, Mahaffey KW, Hylek E, Peterson ED, Piccini JP, Fonarow GC; ORBIT-AF Investigators and Patients. Predictors and prognostic implications of incident heart failure in patients with prevalent atrial fibrillation. JACC Heart Fail 2017;5:44-52.

156. Ganesan AN, Chew DP, Hartshorne T, Selvanayagam JB, Aylward PE, Sanders $P$, McGavigan AD. The impact of atrial fibrillation type on the risk of thromboembolism, mortality, and bleeding: a systematic review and meta-analysis. Eur Heart J 2016;37:1591-1602.

157. Al-Khatib SM, Thomas L, Wallentin L, Lopes RD, Gersh B, Garcia D, Ezekowitz J, Alings M, Yang H, Alexander JH, Flaker G, Hanna M, Granger CB. Outcomes of apixaban vs. warfarin by type and duration of atrial fibrillation: results from the ARISTOTLE trial. Eur Heart J 2013;34:2464-2471.

158. Link MS, Giugliano RP, Ruff CT, Scirica BM, Huikuri H, Oto A, Crompton AE, Murphy SA, Lanz H, Mercuri MF, Antman EM, Braunwald E; ENGAGE AF-TIMI 48 Investigators. Stroke and mortality risk in patients with various patterns of atrial fibrillation: results from the ENGAGE AF-TIMI 48 Trial (Effective Anticoagulation With Factor $\mathrm{Xa}$ Next Generation in Atrial FibrillationThrombolysis in Myocardial Infarction 48). Circ Arrhythm Electrophysio 2017;10:e004267.

159. Steinberg BA, Hellkamp AS, Lokhnygina Y, Patel MR, Breithardt G, Hankey G], Becker RC, Singer DE, Halperin JL, Hacke W, Nessel CC, Berkowitz SD, Mahaffey KW, Fox KA, Califf RM, Piccini JP; ROCKET-AF Steering Committee and Investigators. Higher risk of death and stroke in patients with persistent vs. paroxysmal atrial fibrillation: results from the ROCKET-AF Trial. Eur Heart 2015;36:288-296

160. Hart RG, Pearce LA, Rothbart RM, McAnulty JH, Asinger RW, Halperin JL. Stroke with intermittent atrial fibrillation: incidence and predictors during aspirin therapy. Stroke Prevention in Atrial Fibrillation Investigators. J Am Coll Cardiol 2000;35:183-187.

161. Takabayashi K, Hamatani Y, Yamashita Y, Takagi D, Unoki T, Ishii M, Iguchi M, Masunaga N, Ogawa H, Esato M, Chun YH, Tsuji H, Wada H, Hasegawa K, Abe M, Lip GY, Akao M. Incidence of stroke or systemic embolism in paroxysmal versus sustained atrial fibrillation: the Fushimi Atrial Fibrillation Registry. Stroke 2015;46:3354-3361.

162. Nieuwlaat R, Dinh T, Olsson SB, Camm AJ, Capucci A, Tieleman RG, Lip GY, Crijns HJ; Euro Heart Survey Investigators. Should we abandon the common practice of withholding oral anticoagulation in paroxysmal atrial fibrillation? Eur Heart J 2008;29:915-922.

163. Go AS, Reynolds K, Yang J, Gupta N, Lenane J, Sung SH, Harrison TN, Liu TI, Solomon MD. Association of burden of atrial fibrillation with risk of ischemic stroke in adults with paroxysmal atrial fibrillation: the KP-RHYTHM Study. JAMA Cardiol 2018;3:601-608.

164. Ecker V, Knoery C, Rushworth G, Rudd I, Ortner A, Begley D, Leslie SJ. A review of factors associated with maintenance of sinus rhythm after elective electrical cardioversion for atrial fibrillation. Clin Cardiol 2018;41:862-870.

165. Nyong J, Amit G, Adler AJ, Owolabi OO, Perel P, Prieto-Merino D, Lambiase P, Casas JP, Morillo CA. Efficacy and safety of ablation for people with nonparoxysmal atrial fibrillation. Cochrane Database Syst Rev 2016;11:CD012088.

166. Piccini JP, Passman R, Turakhia M, Connolly AT, Nabutovsky Y, Varma N. Atrial fibrillation burden, progression, and the risk of death: a case-crossover analysis in patients with cardiac implantable electronic devices. Europace 2019;21:404-413.

167. Deng H, Bai Y, Shantsila A, Fauchier L, Potpara TS, Lip GYH. Clinical scores for outcomes of rhythm control or arrhythmia progression in patients with atrial fibrillation: a systematic review. Clin Res Cardiol 2017;106:813-823.

168. Healey JS, Connolly SJ, Gold MR, Israel CW, Van Gelder IC, Capucci A, Lau CP, Fain E, Yang S, Bailleul C, Morillo CA, Carlson M, Themeles E, Kaufman ES, Hohnloser SH; ASSERT Investigators. Subclinical atrial fibrillation and the risk of stroke. N Engl J Med 2012;366:120-129.

169. Potpara TS, Stankovic GR, Beleslin BD, Polovina MM, Marinkovic JM, Ostojic MC, Lip GYH. A 12-year follow-up study of patients with newly diagnosed lone atrial fibrillation: implications of arrhythmia progression on prognosis: the Belgrade Atrial Fibrillation Study. Chest 2012;141:339-347.

170. Goette A, Kalman JM, Aguinaga L, Akar J, Cabrera JA, Chen SA, Chugh SS, Corradi D, D'Avila A, Dobrev D, Fenelon G, Gonzalez M, Hatem SN, Helm R, Hindricks G, Ho SY, Hoit B, Jalife J, Kim YH, Lip GY, Ma CS, Marcus GM, Murray K, Nogami A, Sanders P, Uribe W, Van Wagoner DR, Nattel S. EHRA/ HRS/APHRS/SOLAECE expert consensus on atrial cardiomyopathies: definition, characterization, and clinical implication. Europace 2016;18:1455-1490. 
171. Nattel S, Guasch E, Savelieva I, Cosio FG, Valverde I, Halperin JL, Conroy JM, Al-Khatib SM, Hess PL, Kirchhof P, De Bono J, Lip GY, Banerjee A, Ruskin J, Blendea D, Camm AJ. Early management of atrial fibrillation to prevent cardiovascular complications. Eur Heart J 2014;35:1448-1456.

172. Freedman B, Camm J, Calkins H, Healey JS, Rosenqvist M, Wang J, Albert CM, Anderson CS, Antoniou S, Benjamin EJ, Boriani G, Brachmann J, Brandes A, Chao TF, Conen D, Engdahl J, Fauchier L, Fitzmaurice DA, Friberg L, Gersh BJ, Gladstone DJ, Glotzer TV, Gwynne K, Hankey GJ, Harbison J, Hillis GS, Hills MT, Kamel H, Kirchhof P, Kowey PR, Krieger D, Lee VWY, Levin LA, Lip GYH, Lobban T, Lowres N, Mairesse GH, Martinez C, Neubeck L, Orchard J, Piccini JP, Poppe K, Potpara TS, Puererfellner H, Rienstra M, Sandhu RK, Schnabel RB, Siu CW, Steinhubl S, Svendsen JH, Svennberg E, Themistoclakis S, Tieleman RG, Turakhia MP, Tveit A, Uittenbogaart SB, Van Gelder IC, Verma A, Wachter $R$, Yan BP, SCREEN CollaboratorsAF-. Screening for atrial fibrillation: a report of the AF-SCREEN International Collaboration. Circulation 2017; 135:1851-1867.

173. Mairesse GH, Moran P, Van Gelder IC, Elsner C, Rosenqvist M, Mant J, Banerjee A, Gorenek B, Brachmann J, Varma N, Glotz de Lima G, Kalman J, Claes N, Lobban T, Lane D, Lip GYH, Boriani G, ESC Scientific Document Group. Screening for atrial fibrillation: a European Heart Rhythm Association (EHRA) consensus document endorsed by the Heart Rhythm Society (HRS), Asia Pacific Heart Rhythm Society (APHRS), and Sociedad Latinoamericana de Estimulacion Cardiaca y Electrofisiologia (SOLAECE). Europace 2017;19:1589-1623.

174. Padfield G], Steinberg C, Swampillai J, Qian H, Connolly SJ, Dorian P, Green MS, Humphries KH, Klein G], Sheldon R, Talajic M, Kerr CR. Progression of paroxysmal to persistent atrial fibrillation: 10-year follow-up in the Canadian Registry of Atrial Fibrillation. Heart Rhythm 2017;14:801-807.

175. Vidal-Perez R, Otero-Ravina F, Lado-Lopez M, Turrado-Turrado V, RodriguezMoldes E, Gomez-Vazquez JL, de Frutos-de Marcos C, de Blas-Abad P, BesadaGesto R, Gonzalez-Juanatey JR; BARBANZA Investigators. The change in the atrial fibrillation type as a prognosis marker in a community study: long-term data from AFBAR (Atrial Fibrillation in the BARbanza) study. Int J Cardiol 2013;168:2146-2152.

176. de Vos CB, Pisters R, Nieuwlaat R, Prins MH, Tieleman RG, Coelen RJ, van den Heijkant AC, Allessie MA, Crijns HJ. Progression from paroxysmal to persistent atrial fibrillation clinical correlates and prognosis. I Am Coll Cardiol 2010;55:725-731.

177. Hobbelt AH, Spronk HM, Crijns H, Ten Cate H, Rienstra M, Van Gelder IC. Prethrombotic state in young very low-risk patients with atrial fibrillation. J Am Coll Cardiol 2017;69:1990-1992.

178. Habibi M, Samiei S, Ambale Venkatesh B, Opdahl A, Helle-Valle TM, Zareian M, Almeida AL, Choi EY, Wu C, Alonso A, Heckbert SR, Bluemke DA, Lima JA. Cardiac magnetic resonance-measured left atrial volume and function and incident atrial fibrillation: results from MESA (Multi-Ethnic Study of Atherosclerosis). Circ Cardiovasc Imaging 2016;9:e004299.

179. Brambatti M, Connolly SJ, Gold MR, Morillo CA, Capucci A, Muto C, Lau CP, Van Gelder IC, Hohnloser SH, Carlson M, Fain E, Nakamya J, Mairesse GH, Halytska M, Deng WQ, Israel CW, Healey JS; ASSERT Investigators. Temporal relationship between subclinical atrial fibrillation and embolic events. Circulation 2014;129:2094-2099.

180. Guichard JB, Nattel S. Atrial Cardiomyopathy: A useful notion in cardiac disease management or a passing fad?] Am Coll Cardiol 2017;70:756-765.

181. Hirsh BJ, Copeland-Halperin RS, Halperin JL. Fibrotic atrial cardiomyopathy, atrial fibrillation, and thromboembolism: mechanistic links and clinical inferences. J Am Coll Cardiol 2015;65:2239-2251.

182. Freedman B, Potpara TS, Lip GY. Stroke prevention in atrial fibrillation. Lancet 2016;388:806-817.

183. Martinez C, Katholing A, Freedman SB. Adverse prognosis of incidentally detected ambulatory atrial fibrillation. A cohort study. Thromb Haemost 2014;112:276-286.

184. Wilson JM, Jungner YG. [Principles and practice of mass screening for disease]. Bol Oficina Sanit Panam 1968;65:281-393.

185. Welton NJ, McAleenan A, Thom HH, Davies P, Hollingworth W, Higgins JP, Okoli G, Sterne JA, Feder G, Eaton D, Hingorani A, Fawsitt C, Lobban T, Bryden P, Richards A, Sofat R. Screening strategies for atrial fibrillation: a systematic review and cost-effectiveness analysis. Health Technol Assess 2017;21:1-236.

186. Steinhubl SR, Waalen J, Edwards AM, Ariniello LM, Mehta RR, Ebner GS, Carter C, Baca-Motes K, Felicione E, Sarich T, Topol EJ. Effect of a home-based wearable continuous ECG monitoring patch on detection of undiagnosed atrial fibrillation: the mSToPS randomized clinical trial. JAMA 2018;320:146-155.

187. Schnabel RB, Haeusler KG, Healey JS, Freedman B, Boriani G, Brachmann J, Brandes A, Bustamante A, Casadei B, Crijns H, Doehner W, Engstrom G, Fauchier L, Friberg L, Gladstone DJ, Glotzer TV, Goto S, Hankey G], Harbison JA, Hobbs FDR, Johnson LSB, Kamel H, Kirchhof P, Korompoki E, Krieger DW, Lip GYH, Lochen ML, Mairesse GH, Montaner J, Neubeck L, Ntaios G, Piccini
JP, Potpara TS, Quinn TJ, Reiffel JA, Ribeiro ALP, Rienstra M, Rosenqvist M, Sakis T, Sinner MF, Svendsen JH, Van Gelder IC, Wachter R, Wijeratne T, Yan B. Searching for atrial fibrillation poststroke: a white paper of the AF-SCREEN International Collaboration. Circulation 2019;140:1834-1850.

188. Yan BP, Lai WHS, Chan CKY, Chan SC, Chan LH, Lam KM, Lau HW, Ng CM, Tai LY, Yip KW, To OTL, Freedman B, Poh YC, Poh MZ. Contact-free screening of atrial fibrillation by a smartphone using facial pulsatile photoplethysmographic signals. J Am Heart Assoc 2018;7.

189. Orchard J, Lowres N, Freedman SB, Ladak L, Lee W, Zwar N, Peiris D, Kamaladasa Y, Li J, Neubeck L. Screening for atrial fibrillation during influenza vaccinations by primary care nurses using a smartphone electrocardiograph (iECG): a feasibility study. Eur J Prev Cardiol 2016;23:13-20.

190. Lampert R. Screening for atrial fibrillation using smartphone-based technology and layperson volunteers: high-tech meets community participatory research for the best of both worlds. Heart Rhythm 2018;15:1312-1313.

191. Lahdenoja O, Hurnanen T, Iftikhar Z, Nieminen S, Knuutila T, Saraste A, Kiviniemi T, Vasankari T, Airaksinen J, Pankaala M, Koivisto T. Atrial fibrillation detection via accelerometer and gyroscope of a smartphone. IEEE J Biomed Health Inform 2018;22:108-118.

192. Freedman B. Screening for atrial fibrillation using a smartphone: is there an app for that?] Am Heart Assoc 2016;5.

193. Chan NY, Choy CC. Screening for atrial fibrillation in 13122 Hong Kong citizens with smartphone electrocardiogram. Heart 2017;103:24-31.

194. Chan PH, Wong CK, Poh YC, Pun L, Leung WW, Wong YF, Wong MM, Poh MZ, Chu DW, Siu CW. Diagnostic performance of a smartphone-based photoplethysmographic application for atrial fibrillation screening in a primary care setting. J Am Heart Assoc 2016;5.

195. Brasier N, Raichle CJ, Dorr M, Becke A, Nohturfft V, Weber S, Bulacher F, Salomon L, Noah T, Birkemeyer R, Eckstein J. Detection of atrial fibrillation with a smartphone camera: first prospective, international, two-centre, clinical validation study (DETECT AF PRO). Europace 2019;21:41-47.

196. Tison GH, Sanchez JM, Ballinger B, Singh A, Olgin JE, Pletcher MJ, Vittinghoff E, Lee ES, Fan SM, Gladstone RA, Mikell C, Sohoni N, Hsieh J, Marcus GM. Passive detection of atrial fibrillation using a commercially available smartwatch. JAMA Cardiol 2018;3:409-416.

197. Li KHC, White FA, Tipoe T, Liu T, Wong MC, Jesuthasan A, Baranchuk A, Tse G, Yan BP. The current state of mobile phone apps for monitoring heart rate, heart rate variability, and atrial fibrillation: narrative review. JMIR Mhealth Uhealth 2019;7:e11606.

198. Bumgarner JM, Lambert CT, Hussein AA, Cantillon DJ, Baranowski B, Wolski K, Lindsay BD, Wazni OM, Tarakji KG. Smartwatch algorithm for automated detection of atrial fibrillation. J Am Coll Cardiol 2018;71:2381-2388.

199. Wasserlauf J, You C, Patel R, Valys A, Albert D, Passman R. Smartwatch performance for the detection and quantification of atrial fibrillation. Circ Arrhythm Electrophysiol 2019;12:e006834.

200. Attia ZI, Noseworthy PA, Lopez-Jimenez F, Asirvatham SJ, Deshmukh AJ, Gersh BJ, Carter RE, Yao X, Rabinstein AA, Erickson BJ, Kapa S, Friedman PA. Anartificial intelligence-enabled ECG algorithm for the identification of patients with atrial fibrillation during sinus rhythm: a retrospective analysis of outcome prediction. Lancet 2019;394:861-867.

201. Turakhia MP, Desai M, Hedlin H, Rajmane A, Talati N, Ferris T, Desai S, Nag D, Patel M, Kowey P, Rumsfeld JS, Russo AM, Hills MT, Granger CB, Mahaffey $\mathrm{KW}$, Perez MV. Rationale and design of a large-scale, app-based study to identify cardiac arrhythmias using a smartwatch: the Apple Heart Study. Am Heart J 2019;207:66-75.

202. Guo Y, Wang H, Zhang H, Liu T, Liang Z, Xia Y, Yan L, Xing Y, Shi H, Li S, Liu Y, Liu F, Feng M, Chen Y, Lip GYH; MAFA II Investigators. Mobile photoplethysmographic technology to detect atrial fibrillation. J Am Coll Cardiol 2019;74:2365-2375.

203. Harris K, Edwards D, Mant J. How can we best detect atrial fibrillation?] R Coll Physicians Edinb 2012;42 Suppl 18:5-22.

204. Wiesel J, Wiesel D, Suri R, Messineo FC. The use of a modified sphygmomanometer to detect atrial fibrillation in outpatients. Pacing Clin Electrophysiol 2004;27:639-643.

205. Wiesel J, Fitzig L, Herschman Y, Messineo FC. Detection of atrial fibrillation using a modified microlife blood pressure monitor. Am J Hypertens 2009;22:848-852.

206. Stergiou GS, Karpettas N, Protogerou A, Nasothimiou EG, Kyriakidis M. Diagnostic accuracy of a home blood pressure monitor to detect atrial fibrillation. J Hum Hypertens 2009;23:654-658.

207. Willits I, Keltie K, Craig J, Sims A. WatchBP Home A for opportunistically detecting atrial fibrillation during diagnosis and monitoring of hypertension: aNICE Medical Technology Guidance. Appl Health Econ Health Policy 2014;12:255-265.

208. Desteghe L, Raymaekers Z, Lutin M, Vijgen J, Dilling-Boer D, Koopman P, Schurmans J, Vanduynhoven P, Dendale P, Heidbuchel H. Performance of 
handheld electrocardiogram devices to detect atrial fibrillation in a cardiology and geriatric ward setting. Europace 2017;19:29-39.

209. Kaasenbrood F, Hollander M, Rutten FH, Gerhards LJ, Hoes AW, Tieleman RG. Yield of screening for atrial fibrillation in primary care with a hand-held, singlelead electrocardiogram device during influenza vaccination. Europace 2016:18:1514-1520.

210. Wiesel J, Abraham S, Messineo FC. Screening for asymptomatic atrial fibrillation while monitoring the blood pressure at home: trial of regular versus irregular pulse for prevention of stroke (TRIPPS 2.0). Am J Cardiol 2013;111:1598-1601.

211. Jacobs MS, Kaasenbrood F, Postma MJ, van Hulst M, Tieleman RG. Cost-effectiveness of screening for atrial fibrillation in primary care with a handheld, single-lead electrocardiogram device in the Netherlands. Europace 2018;20:12-18.

212. Lowres N, Neubeck L, Salkeld G, Krass I, McLachlan AJ, Redfern J, Bennett AA, Briffa T, Bauman A, Martinez C, Wallenhorst C, Lau JK, Brieger DB, Sy RW, Freedman SB. Feasibility and cost-effectiveness of stroke prevention through community screening for atrial fibrillation using iPhone ECG in pharmacies. The SEARCH-AF study. Thromb Haemost 2014;111:1167-1176.

213. William AD, Kanbour M, Callahan T, Bhargava M, Varma N, Rickard J, Saliba W, Wolski K, Hussein A, Lindsay BD, Wazni OM, Tarakji KG. Assessing the accuracy of an automated atrial fibrillation detection algorithm using smartphone technology: the iREAD Study. Heart Rhythm 2018; 15:1561-1565

214. Nemati S, Ghassemi MM, Ambai V, Isakadze N, Levantsevych $O$, Shah A, Clifford GD. Monitoring and detecting atrial fibrillation using wearable technology. Conf Proc IEEE Eng Med Biol Soc 2016;2016:3394-3397.

215. Petryszyn P, Niewinski P, Staniak A, Piotrowski P, Well A, Well M, Jeskowiak I, Lip G, Ponikowski P. Effectiveness of screening for atrial fibrillation and its determinants. A meta-analysis. PLoS One 2019;14:e0213198.

216. Orchard J, Lowres N, Neubeck L, Freedman B. Atrial fibrillation: is there enough evidence to recommend opportunistic or systematic screening?!nt J Epidemiol 2018:47:1361.

217. Svennberg E, Engdahl J, Al-Khalili F, Friberg L, Frykman V, Rosenqvist M. Mass screening for untreated atrial fibrillation: the STROKESTOP Study. Circulation 2015;131:2176-2184.

218. Halcox JPJ, Wareham K, Cardew A, Gilmore M, Barry JP, Phillips C, Gravenor MB. Assessment of remote heart rhythm sampling using the AliveCor heart monitor to screen for atrial fibrillation: the REHEARSE-AF Study. Circulation 2017;136:1784-1794.

219. Turakhia MP, Shafrin J, Bognar K, Goldman DP, Mendys PM, Abdulsattar Y, Wiederkehr D, Trocio J. Economic burden of undiagnosed nonvalvular atrial fibrillation in the United States. Am J Cardiol 2015;116:733-739.

220. Fay MR, Fitzmaurice DA, Freedman B. Screening of older patients for atrial fibrillation in general practice: current evidence and its implications for future practice. Eur J Gen Pract 2017;23:246-253.

221. Boriani G, Valzania C, Biffi M, Diemberger I, Ziacchi M, Martignani C. Asymptomatic lone atrial fibrillation - how can we detect the arrhythmia?Curr Pharm Des 2015;21:659-666.

222. Hobbs FD, Fitzmaurice DA, Mant J, Murray E, Jowett S, Bryan S, Raftery J, Davies M, Lip G. A randomised controlled trial and cost-effectiveness study of systematic screening (targeted and total population screening) versus routine practice for the detection of atrial fibrillation in people aged 65 and over. The SAFE study. Health Technol Assess 2005;9:iii-iv, ix-x, 1-74.

223. Aronsson M, Svennberg E, Rosenqvist M, Engdahl J, Al-Khalili F, Friberg L, FrykmanKull V, Levin LA. Cost-effectiveness of mass screening for untreated atrial fibrillation using intermittent ECG recording. Europace 2015;17:1023-1029.

224. Lowres N, Neubeck L, Redfern J, Freedman SB. Screening to identify unknown atrial fibrillation. A systematic review. Thromb Haemost 2013;110:213-222.

225. Engdahl J, Andersson L, Mirskaya M, Rosenqvist M. Stepwise screening of atrial fibrillation in a 75 -year-old population: implications for stroke prevention. Circulation 2013;127:930-937.

226. Boriani G, Glotzer TV, Santini M, West TM, De Melis M, Sepsi M, Gasparini M, Lewalter T, Camm JA, Singer DE. Device-detected atrial fibrillation and risk for stroke: an analysis of $>10,000$ patients from the SOS AF project (Stroke preventiOn Strategies based on Atrial Fibrillation information from implanted devices). Eur Heart J 2014;35:508-516.

227. Lowres N, Krass I, Neubeck L, Redfern J, McLachlan AJ, Bennett AA, Freedman SB. Atrial fibrillation screening in pharmacies using an iPhone ECG: a qualitative review of implementation. Int J Clin Pharm 2015;37:1111-1120.

228. Wynn GJ, Todd DM, Webber M, Bonnett L, McShane J, Kirchhof P, Gupta D. The European Heart Rhythm Association symptom classification for atrial fibrillation: validation and improvement through a simple modification. Europace 2014:16:965-972.

229. De With RR, Rienstra M, Smit MD, Weijs B, Zwartkruis VW, Hobbelt AH, Alings M, Tijssen JGP, Brugemann J, Geelhoed B, Hillege HL, Tukkie R, Hemels
ME, Tieleman RG, Ranchor AV, Van Veldhuisen DJ, Crijns H, Van Gelder IC. Targeted therapy of underlying conditions improves quality of life in patients with persistent atrial fibrillation: results of the RACE 3 study. Europace 2019;21:563-571.

230. Schnabel RB, Pecen L, Rzayeva N, Lucerna M, Purmah Y, Ojeda FM, De Caterina R, Kirchhof P. Symptom burden of atrial fibrillation and its relation to interventions and outcome in Europe. J Am Heart Assoc 2018;7.

231. Björkenheim A, Brandes A, Magnuson A, Chemnitz A, Svedberg L, Edvardsson $\mathrm{N}$, Poçi D. Assessment of atrial fibrillation - specific symptoms before and 2 years after atrial fibrillation ablation: do patients and physicians differ in their perception of symptom relief?]ACC: Clinical Electrophysiology 2017;3:1168-1176.

232. Sandhu RK, Smigorowsky M, Lockwood E, Savu A, Kaul P, McAlister FA. Impact of electrical cardioversion on quality of life for the treatment of atrial fibrillation. Can J Cardiol 2017;33:450-455.

233. Singh BN, Singh SN, Reda DJ, Tang XC, Lopez B, Harris CL, Fletcher RD, Sharma SC, Atwood JE, Jacobson AK, Lewis HD, Jr., Raisch DW, Ezekowitz MD; Sotalol Amiodarone Atrial Fibrillation Efficacy Trial Investigators. Amiodarone versus sotalol for atrial fibrillation. $N$ Engl J Med 2005;352:1861-1872.

234. Gilbert KA, Hogarth AJ, MacDonald W, Lewis NT, Tan LB, Tayebjee MH. Restoration of sinus rhythm results in early and late improvements in the functional reserve of the heart following direct current cardioversion of persistent AF: FRESH-AF. Int J Cardiol 2015;199:121-125.

235. Jais $P$, Cauchemez B, Macle L, Daoud E, Khairy P, Subbiah R, Hocini M, Extramiana F, Sacher F, Bordachar P, Klein G, Weerasooriya R, Clementy J, Haissaguerre M. Catheter ablation versus antiarrhythmic drugs for atrial fibrillation: the A4 study. Circulation 2008;118:2498-2505.

236. Oral H, Pappone C, Chugh A, Good E, Bogun F, Pelosi F, Jr., Bates ER, Lehmann MH, Vicedomini G, Augello G, Agricola E, Sala S, Santinelli V, Morady F. Circumferential pulmonary-vein ablation for chronic atrial fibrillation. N Engl J Med 2006;354:934-941.

237. Mont L, Bisbal F, Hernandez-Madrid A, Perez-Castellano N, Vinolas X, Arenal A, Arribas F, Fernandez-Lozano I, Bodegas A, Cobos A, Matia R, PerezVillacastin J, Guerra JM, Avila P, Lopez-Gil M, Castro V, Arana Jl, Brugada J: SARA investigators. Catheter ablation vs. antiarrhythmic drug treatment of persistent atrial fibrillation: a multicentre, randomized, controlled trial (SARA study). Eur Heart J 2014;35:501-507.

238. Forleo GB, Mantica M, De Luca L, Leo R, Santini L, Panigada S, De Sanctis V, Pappalardo A, Laurenzi F, Avella A, Casella M, Dello Russo A, Romeo F, Pelargonio G, Tondo C. Catheter ablation of atrial fibrillation in patients with diabetes mellitus type 2: results from a randomized study comparing pulmonary vein isolation versus antiarrhythmic drug therapy. I Cardiovasc Electrophysiol 2009;20:22-28.

239. Wilber DJ, Pappone C, Neuzil P, De Paola A, Marchlinski F, Natale A, Macle L, Daoud EG, Calkins H, Hall B, Reddy V, Augello G, Reynolds MR, Vinekar C, Liu CY, Berry SM, Berry DA; ThermoCool AF Trial Investigators. Comparison of antiarrhythmic drug therapy and radiofrequency catheter ablation in patients with paroxysmal atrial fibrillation: a randomized controlled trial. JAMA 2010;303:333-340.

240. Wazni OM, Marrouche NF, Martin DO, Verma A, Bhargava M, Saliba W, Bash D, Schweikert R, Brachmann J, Gunther J, Gutleben K, Pisano E, Potenza D, Fanelli R, Raviele A, Themistoclakis S, Rossillo A, Bonso A, Natale A. Radiofrequency ablation vs antiarrhythmic drugs as first-line treatment of symptomatic atrial fibrillation: a randomized trial. JAMA 2005;293:2634-2640.

241. Morillo CA, Verma A, Connolly SJ, Kuck KH, Nair GM, Champagne J, Sterns LD, Beresh H, Healey JS, Natale A; RAAFT Investigators. Radiofrequency ablation vs antiarrhythmic drugs as first-line treatment of paroxysmal atrial fibrillation (RAAFT-2): a randomized trial. JAMA 2014;311:692-700.

242. Cosedis Nielsen J, Johannessen A, Raatikainen P, Hindricks G, Walfridsson H, Kongstad O, Pehrson S, Englund A, Hartikainen J, Mortensen LS, Hansen PS. Radiofrequency ablation as initial therapy in paroxysmal atrial fibrillation. $N$ Engl Med 2012;367:1587-1595.

243. Pokorney SD, Kim S, Thomas L, Fonarow GC, Kowey PR, Gersh BJ, Mahaffey KW, Peterson ED, Piccini JP; Outcomes Registry for Better Informed Treatment of Atrial Fibrillation Investigators. Cardioversion and subsequent quality of life and natural history of atrial fibrillation. Am Heart J 2017;185:59-66.

244. Mantovan R, Macle L, De Martino G, Chen J, Morillo CA, Novak P, Calzolari V, Khaykin Y, Guerra PG, Nair G, Torrecilla EG, Verma A. Relationship of quality of life with procedural success of atrial fibrillation (AF) ablation and postablation AF burden: substudy of the STAR AF randomized trial. Can J Cardiol 2013:29:1211-1217.

245. Rienstra M, Hobbelt AH, Alings M, Tijssen JGP, Smit MD, Brugemann J, Geelhoed B, Tieleman RG, Hillege HL, Tukkie R, Van Veldhuisen DJ, Crijns H, Van Gelder IC; RACE Investigators. Targeted therapy of underlying conditions improves sinus rhythm maintenance in patients with persistent atrial fibrillation: results of the RACE 3 trial. Eur Heart J 2018;39:2987-2996. 
246. Blomstrom-Lundqvist C, Gizurarson S, Schwieler J, Jensen SM, Bergeldt L, Kenneback G, Rubulis A, Malmborg H, Raatikainen P, Lonnerholm S, Hoglund $\mathrm{N}$, Mortsell D. Effect of catheter ablation vs antiarrhythmic medication on quality of life in patients with atrial fibrillation: the CAPTAF randomized clinical trial. JAMA 2019;321:1059-1068.

247. Mark DB, Anstrom KJ, Sheng S, Piccini JP, Baloch KN, Monahan KH, Daniels MR, Bahnson TD, Poole JE, Rosenberg Y, Lee KL, Packer DL; CABANA Investigators. Effect of catheter ablation vs medical therapy on quality of life among patients with atrial fibrillation: the CABANA randomized clinical trial. JAMA 2019.

248. Gaita F, Scaglione M, Battaglia A, Matta M, Gallo C, Galata M, Caponi D, D Donna P, Anselmino M. Very long-term outcome following transcatheter ablation of atrial fibrillation. Are results maintained after 10 years of followup?Europace 2018:20:443-450.

249. Donal E, Lip GY, Galderisi M, Goette A, Shah D, Marwan M, Lederlin M, Mondillo S, Edvardsen T, Sitges M, Grapsa J, Garbi M, Senior R, Gimelli A, Potpara TS, Van Gelder IC, Gorenek B, Mabo P, Lancellotti P, Kuck KH, Popescu BA, Hindricks G, Habib G, Cardim NM, Cosyns B, Delgado V, Haugaa KH, Muraru D, Nieman K, Boriani G, Cohen A. EACVI/EHRA Expert Consensus Document on the role of multi-modality imaging for the evaluation of patients with atrial fibrillation. Eur Heart J Cardiovasc Imaging 2016;17:355-383.

250. Delgado V, Di Biase L, Leung M, Romero J, Tops LF, Casadei B, Marrouche N, Bax JJ. Structure and function of the left atrium and left atrial appendage: $A F$ and stroke implications. J Am Coll Cardiol 2017;70:3157-3172.

251. Oakes RS, Badger TJ, Kholmovski EG, Akoum N, Burgon NS, Fish EN, Blauer JJ, Rao SN, DiBella EV, Segerson NM, Daccarett M, Windfelder J, McGann CJ, Parker D, MacLeod RS, Marrouche NF. Detection and quantification of left atrial structural remodeling with delayed-enhancement magnetic resonance imaging in patients with atrial fibrillation. Circulation 2009;119:1758-1767.

252. Cameli M, Lisi M, Righini FM, Massoni A, Natali BM, Focardi M, Tacchini D, Geyer A, Curci V, Di Tommaso C, Lisi G, Maccherini M, Chiavarelli M, Massetti $M$, Tanganelli $P$, Mondillo $S$. Usefulness of atrial deformation analysis to predict left atrial fibrosis and endocardial thickness in patients undergoing mitral valve operations for severe mitral regurgitation secondary to mitral valve prolapse. Am J Cardiol 2013;111:595-601.

253. Nakamori S, Nezafat M, Ngo LH, Manning WJ, Nezafat R. Left atrial epicardial fat volume is associated with atrial fibrillation: a prospective cardiovascular magnetic resonance 3D Dixon Study. J Am Heart Assoc 2018;7.

254. Murphy A, Banerjee A, Breithardt G, Camm A), Commerford P, Freedman B, Gonzalez-Hermosillo JA, Halperin JL, Lau CP, Perel P, Xavier D, Wood D, Jouven $X$, Morillo CA. The World Heart Federation roadmap for nonvalvular atrial fibrillation. Glob Heart 2017;12:273-284.

255. Timmis A, Townsend N, Gale CP, Torbica A, Lettino M, Petersen SE, Mossialos EA, Maggioni AP, Kazakiewicz D, May HT, De Smedt D, Flather M, Zuhlke L, Beltrame JF, Huculeci R, Tavazzi L, Hindricks G, Bax J, Casadei B, Achenbach S, Wright L, Vardas P. European Society of Cardiology: Cardiovascular Disease Statistics 2019. Eur Heart J 2020;41:12-85.

256. Charles C, Whelan T, Gafni A. What do we mean by partnership in making decisions about treatment?BMJ 1999;319:780-782.

257. Lane DA, Aguinaga L, Blomstrom-Lundqvist C, Boriani G, Dan GA, Hills MT, Hylek EM, LaHaye SA, Lip GY, Lobban T, Mandrola J, McCabe PJ, Pedersen SS, Pisters R, Stewart S, Wood K, Potpara TS, Gorenek B, Conti JB, Keegan R, Power S, Hendriks J, Ritter P, Calkins H, Violi F, Hurwitz J. Cardiac tachyarrhythmias and patient values and preferences for their management: the European Heart Rhythm Association (EHRA) consensus document endorsed by the Heart Rhythm Society (HRS), Asia Pacific Heart Rhythm Society (APHRS), and Sociedad Latinoamericana de Estimulacion Cardiaca y Electrofisiologia (SOLEACE). Europace 2015;17:1747-1769.

258. Bergtun S, Oterhals K, Fridlund B. Patients' experiences 1-6 months after atrial fibrillation ablation: an holistic perspective. J Adv Nurs 2019;75:150 - 160

259. Borg Xuereb C, Shaw RL, Lane DA. Patients' and physicians' experiences of atrial fibrillation consultations and anticoagulation decision-making: a multiperspective IPA design. Psychol Health 2016;31:436-455.

260. Loewen PS, Ji AT, Kapanen A, McClean A. Patient values and preferences for antithrombotic therapy in atrial fibrillation. A narrative systematic review. Thromb Haemost 2017;117:1007-1022.

261. Seaburg L, Hess EP, Coylewright M, Ting HH, McLeod CJ, Montori VM. Shared decision making in atrial fibrillation: where we are and where we should be going. Circulation 2014;129:704-710.

262. Bajorek BV, Ogle SJ, Duguid MJ, Shenfield GM, Krass I. Management of warfarin in atrial fibrillation: views of health professionals, older patients and their carers. Med J Aust 2007;186:175-180.

263. Hess EP, Knoedler MA, Shah ND, Kline JA, Breslin M, Branda ME, Pencille LJ, Asplin BR, Nestler DM, Sadosty AT, Stiell IG, Ting HH, Montori VM. The chest pain choice decision aid: a randomized trial. Circ Cardiovasc Qual Outcomes 2012;5:251-259.
264. Lane DA, Meyerhoff J, Rohner U, Lip GYH. Atrial fibrillation patient preferences for oral anticoagulation and stroke knowledge: results of a conjoint analysis. Clin Cardiol 2018;41:855-861.

265. Lindberg T, Sanmartin Berglund J, Elmstahl S, Bohman DM. Older individuals' need for knowledge and follow-up about their chronic atrial fibrillation, lifelong medical treatment and medical controls. Scand J Caring Sci 2017;31:1022-1030.

266. Palacio AM, Kirolos I, Tamariz L. Patient values and preferences when choosing anticoagulants. Patient Prefer Adherence 2015;9:133-138.

267. Lane DA, Lip GY. Patient's values and preferences for stroke prevention in atrial fibrillation: balancing stroke and bleeding risk with oral anticoagulation. Thromb Haemost 2014:111:381-383.

268. MacLean S, Mulla S, Akl EA, Jankowski M, Vandvik PO, Ebrahim S, McLeod S, Bhatnagar N, Guyatt GH. Patient values and preferences in decision making for antithrombotic therapy: a systematic review: Antithrombotic Therapy and Prevention of Thrombosis, 9th ed: American College of Chest Physicians Evidence-Based Clinical Practice Guidelines. Chest 2012;141:e1S-e23S.

269. Desteghe L, Engelhard L, Raymaekers Z, Kluts K, Vijgen J, Dilling-Boer D, Koopman P, Schurmans J, Dendale P, Heidbuchel H. Knowledge gaps in patients with atrial fibrillation revealed by a new validated knowledge questionnaire. Int J Cardiol 2016;223:906-914.

270. Frankel DS, Parker SE, Rosenfeld LE, Gorelick PB. HRS/NSA 2014 Survey of atrial fibrillation and stroke: gaps in knowledge and perspective, opportunities for improvement.J Stroke Cerebrovasc Dis 2015;24:1691-700.

271. Lane DA, Ponsford J, Shelley A, Sirpal A, Lip GY. Patient knowledge and perceptions of atrial fibrillation and anticoagulant therapy: effects of an educational intervention programme. The West Birmingham Atrial Fibrillation Project. Int J Cardiol 2006;110:354-358.

272. McCabe PJ, Schad S, Hampton A, Holland DE. Knowledge and self-management behaviors of patients with recently detected atrial fibrillation. Heart Lung 2008:37:79-90.

273. Ihara M, Washida K. Linking atrial fibrillation with Alzheimer's disease: epidemiological, pathological, and mechanistic evidence. I Alzheimers Dis 2018;62:61-72.

274. Lip GYH, Lane DA, Sarwar S. Streamlining primary and secondary care management pathways for stroke prevention in atrial fibrillation. Eur Heart J 2017:38:2980-2982.

275. Guo Y, Lane DA, Wang L, Chen Y, Lip GYH; mAF-App II Trial investigators. Mobile Health (mHealth) technology for improved screening, patient involvement and optimising integrated care in atrial fibrillation: the mAFA (mAF-App) II randomised trial. Int J Clin Pract 2019:e13352.

276. Franchi C, Antoniazzi S, Ardoino I, Proietti M, Marcucci M, Santalucia P, Monzani V, Mannucci PM, Nobili A, Collaborators S-A. Simulation-based education for physicians to increase oral anticoagulants in hospitalized elderly patients with atrial fibrillation. Am J Med 2019;132:e634-e647.

277. Vinereanu D, Lopes RD, Bahit MC, Xavier D, Jiang J, Al-Khalidi HR, He W, Xian Y, Ciobanu AO, Kamath DY, Fox KA, Rao MP, Pokorney SD, Berwanger O, Tajer C, de Barros ESPGM, Roettig ML, Huo Y, Granger CB; IMPACT-AF Investigators. A multifaceted intervention to improve treatment with oral anticoagulants in atrial fibrillation (IMPACT-AF): an international, clusterrandomised trial. Lancet 2017;390:1737-1746

278. Raparelli V, Proietti M, Cangemi R, Lip GY, Lane DA, Basili S. Adherence to oral anticoagulant therapy in patients with atrial fibrillation. Focus on nonvitamin $\mathrm{K}$ antagonist oral anticoagulants. Thromb Haemost 2017;117:209-218.

279. Parimbelli E, Sacchi L, Budasu R, Napolitano C, Peleg M, Quaglini S. The role of nurses in e-health: the MobiGuide project experience. Stud Health Technol Inform 2016;225:153-157.

280. Guo Y, Chen Y, Lane DA, Liu L, Wang Y, Lip GYH. Mobile health technology for atrial fibrillation management integrating decision support, education, and patient involvement: mAF App trial. Am J Med 2017;130:1388-1396.e6.

281. Kotecha D, Chua WWL, Fabritz L, Hendriks J, Casadei B, Schotten U, Vardas P, Heidbuchel H, Dean V, Kirchhof P, European Society of Cardiology (ESC) Atrial Fibrillation Guidelines Taskforce, the CATCH ME consortium, and the European Heart Rhythm Association (EHRA). European Society of Cardiology smartphone and tablet applications for patients with atrial fibrillation and their health care providers. Europace 2018;20:225-233.

282. Lee J-A, Evangelista LS, Moore AA, Juth V, Guo Y, Gago-Masague S, Lem CG, Nguyen M, Khatibi P, Baje M, Amin AN. Feasibility study of a mobile health intervention for older adults on oral anticoagulation therapy. Gerontol Geriatr Med 2016;2. doi:10.1177/2333721416672970. Published 2016 Oct 7.

283. Stephan LS, Dytz Almeida E, Guimaraes RB, Ley AG, Mathias RG, Assis MV, Leiria TL. Processes and recommendations for creating $\mathrm{mHealth}$ apps for lowincome populations. JMIR Mhealth Uhealth 2017;5:e41.

284. Clarkesmith DE, Pattison HM, Khaing PH, Lane DA. Educational and behavioural interventions for anticoagulant therapy in patients with atrial fibrillation. Cochrane Database Syst Rev 2017;4:CD008600. 
285. Man-Son-Hing M, Laupacis A, O'Connor AM, Biggs J, Drake E, Yetisir E, Hart RG. A patient decision aid regarding antithrombotic therapy for stroke prevention in atrial fibrillation: a randomized controlled trial. JAMA 1999;282:737-743.

286. McAlister FA, Man-Son-Hing M, Straus SE, Ghali WA, Anderson D, Majumdar SR, Gibson P, Cox JL, Fradette M; Decision Aid in Atrial Fibrillation Investigators. Impact of a patient decision aid on care among patients with nonvalvular atrial fibrillation: a cluster randomized trial. CMAJ 2005;173:496-501.

287. Thomson RG, Eccles MP, Steen IN, Greenaway J, Stobbart L, Murtagh MJ, May CR. A patient decision aid to support shared decision-making on antithrombotic treatment of patients with atrial fibrillation: randomised controlled trial. Qual Saf Health Care 2007;16:216-223.

288. Eckman MH, Costea A, Attari M, Munjal J, Wise RE, Knochelmann C, Flaherty ML, Baker P, Ireton R, Harnett BM, Leonard AC, Steen D, Rose A, Kues J. Shared decision-making tool for thromboprophylaxis in atrial fibrillation - a feasibility study. Am Heart J 2018;199:13-21.

289. Eckman MH, Lip GY, Wise RE, Speer B, Sullivan M, Walker N, Kissela B, Flaherty ML, Kleindorfer D, Baker P, Ireton R, Hoskins D, Harnett BM, Aguilar C, Leonard AC, Arduser L, Steen D, Costea A, Kues J. Impact of an atrial fibrillation decision support tool on thromboprophylaxis for atrial fibrillation. Am Heart J 2016;176:17-27.

290. Karlsson LO, Nilsson S, Bang M, Nilsson L, Charitakis E, Janzon M. A clinical decision support tool for improving adherence to guidelines on anticoagulant therapy in patients with atrial fibrillation at risk of stroke: a cluster-randomized trial in a Swedish primary care setting (the CDS-AF study). PLoS Med 2018;15:e1002528.

291. Vinereanu D, Lopes RD, Mulder H, Gersh BJ, Hanna M, de Barros ESPGM, Atar $\mathrm{D}$, Wallentin L, Granger CB, Alexander JH; ARISTOTLE Investigators. Echocardiographic risk factors for stroke and outcomes in patients with atrial fibrillation anticoagulated with apixaban or warfarin. Stroke 2017; 48:3266-3273.

292. Hendriks JM, de Wit R, Crijns HJ, Vrijhoef HJ, Prins MH, Pisters R, Pison LA, Blaauw Y, Tieleman RG. Nurse-led care vs. usual care for patients with atrial fibrillation: results of a randomized trial of integrated chronic care vs. routine clinical care in ambulatory patients with atrial fibrillation. Eur Heart J 2012;33:2692-2699.

293. Stewart S, Ball J, Horowitz JD, Marwick TH, Mahadevan G, Wong C, Abhayaratna WP, Chan YK, Esterman A, Thompson DR, Scuffham PA, Carrington MJ. Standard versus atrial fibrillation-specific management strategy (SAFETY) to reduce recurrent admission and prolong survival: pragmatic, multicentre, randomised controlled trial. Lancet 2015;385:775-784.

294. Carter L, Gardner M, Magee K, Fearon A, Morgulis I, Doucette S, Sapp JL, Gray C, Abdelwahab A, Parkash R. An integrated management approach to atrial fibrillation. J Am Heart Assoc 2016;5.

295. Wijtvliet E, Tieleman RG, van Gelder IC, Pluymaekers NAHA, Rienstra M, Folkeringa RJ, Bronzwaer P, Elvan A, Elders J, Tukkie R, Luermans JGLM, Van Asselt ADIT, Van Kuijk SMJ, Tijssen JG, Crijns HJGM; RACE Investigators. Nurse-led vs. usual-care for atrial fibrillation. Eur Heart J 2020;41:634-641.

296. Gallagher C, Elliott AD, Wong CX, Rangnekar G, Middeldorp ME, Mahajan R, Lau DH, Sanders P, Hendriks JML. Integrated care in atrial fibrillation: a systematic review and meta-analysis. Heart 2017;103:1947-1953.

297. Michie S, van Stralen MM, West R. The behaviour change wheel: a new method for characterising and designing behaviour change interventions. Implement Sci 2011;6:42.

298. Lip GYH, Lane DA, Potpara TS. Innovative strategies to improve adherence to non-vitamin $\mathrm{K}$ antagonist oral anticoagulants for stroke prevention in atrial fibrillation. Eur Heart J 2018;39:1404-1406.

299. Seligman WH, Das-Gupta Z, Jobi-Odeneye AO, Arbelo E, Banerjee A, Bollmann A, Caffrey-Armstrong B, Cehic DA, Corbalan R, Collins M, Dandamudi G, Dorairaj P, Fay M, Van Gelder IC, Goto S, Granger CB, Gyorgy B, Healey JS, Hendriks JM, Hills MT, Hobbs FDR, Huisman MV, Koplan KE, Lane DA, Lewis WR, Lobban T, Steinberg BA, McLeod CJ, Moseley S, Timmis A, Yutao G, Camm AJ. Development of an international standard set of outcome measures for patients with atrial fibrillation: a report of the International Consortium for Health Outcomes Measurement (ICHOM) atrial fibrillation working group. Eur Heart J 2020;41:1132-1140.

300. Dobler CC, Harb N, Maguire CA, Armour CL, Coleman C, Murad MH. Treatment burden should be included in clinical practice guidelines. BM] 2018;363:k4065.

301. Eton DT, Ramalho de Oliveira D, Egginton JS, Ridgeway JL, Odell L, May CR, Montori VM. Building a measurement framework of burden of treatment in complex patients with chronic conditions: a qualitative study. Patient Relat Outcome Meas 2012;3:39-49.

302. Tran VT, Montori VM, Eton DT, Baruch D, Falissard B, Ravaud P. Development and description of measurement properties of an instrument to assess treatment burden among patients with multiple chronic conditions. BMC Med 2012;10:68.

303. Vijan S, Hayward RA, Ronis DL, Hofer TP. Brief report: the burden of diabetes therapy: implications for the design of effective patient-centered treatment regimens. J Gen Intern Med 2005;20:479-482.

304. Vermeire E, Hearnshaw H, Van Royen P, Denekens J. Patient adherence to treatment: three decades of research. A comprehensive review. J Clin Pharm Ther 2001;26:331-342.

305. Ho PM, Rumsfeld JS, Masoudi FA, McClure DL, Plomondon ME, Steiner JF Magid DJ. Effect of medication nonadherence on hospitalization and mortality among patients with diabetes mellitus. Arch Intern Med 2006;166:1836-1841.

306. Rasmussen JN, Chong A, Alter DA. Relationship between adherence to evidence-based pharmacotherapy and long-term mortality after acute myocardial infarction. JAMA 2007;297:177-186.

307. May C, Montori VM, Mair FS. We need minimally disruptive medicine. BM 2009;339:b2803.

308. Wilcox AR, Dragnev MC, Darcey CJ, Siegel CA. A new tool to measure the burden of Crohn's disease and its treatment: do patient and physician perceptions match?Inflamm Bowel Dis 2010;16:645 - 650.

309. Bohlen K, Scoville E, Shippee ND, May CR, Montori VM. Overwhelmed patients: a videographic analysis of how patients with type 2 diabetes and clinicians articulate and address treatment burden during clinical encounters. Diabetes Care 2012;35:47-49.

310. Buffel du Vaure C, Ravaud P, Baron G, Barnes C, Gilberg S, Boutron I. Potential workload in applying clinical practice guidelines for patients with chronic conditions and multimorbidity: a systematic analysis. BMJ Open 2016;6:e010119

311. Potpara TS, Mihajlovic M, Zec N, Marinkovic M, Kovacevic V, Simic J, Kocijancic A, Vajagic L, Jotic A, Mujovic N, Stankovic G. Self-reported treatment burden in patients with atrial fibrillation: quantification, major determinants and implications for integrated holistic management of the arrhythmia. Europace 2020 doi:10.1093/europace/euaa210.

312. Tran VT, Harrington M, Montori VM, Barnes C, Wicks P, Ravaud P. Adaptation and validation of the Treatment Burden Questionnaire (TBQ) in English using an internet platform. BMC Med 2014;12:109.

313. Steinberg BA, Dorian P, Anstrom KJ, Hess R, Mark DB, Noseworthy PA Spertus JA, Piccini JP. Patient-reported outcomes in atrial fibrillation research: results of a Clinicaltrials.gov analysis. JACC Clin Electrophysiol 2019;5:599-605.

314. Calvert M, Kyte D, Price G, Valderas JM, Hjollund NH. Maximising the impact of patient reported outcome assessment for patients and society. BMJ 2019;364:k5267.

315. Rotenstein LS, Huckman RS, Wagle NW. Making patients and doctors happier - the potential of patient-reported outcomes. $N$ Engl J Med 2017;377:1309-1312.

316. Van Der Wees PJ, Nijhuis-Van Der Sanden MW, Ayanian JZ, Black N, Westert GP, Schneider EC. Integrating the use of patient-reported outcomes for both clinical practice and performance measurement: views of experts from 3 countries. Milbank Q 2014;92:754-775.

317. Arbelo E, Aktaa S, Bollmann A, D'Avila A, Drossart I, Dwight J, Hills MT, Hindricks G, Kusumoto FM, Lane DA, Lau DH, Lettino M, Lip GYH, Lobban T, Pak H-N, Potpara T, Saenz LC, Van Gelder IC, Varosy P, Gale CP, Dagres N. Quality indicators for the care and outcomes of adults with atrial fibrillation. Task Force for the development of quality indicators in Atrial Fibrillation of the European Heart Rhythm Association (EHRA) and of the European Society of Cardiology (ESC): Developed in collaboration with the Heart Rhythm Society (HRS), the Asia Pacific Heart Rhythm Society (APHRS) and the Latin-American Heart Rhythm Society (LAHRS). Europace 2020;doi:10.1093/europace/euaa253.

318. Lip GYH. The ABC pathway: an integrated approach to improve AF management. Nat Rev Cardiol 2017;14:627-628.

319. Proietti M, Romiti GF, Olshansky B, Lane DA, Lip GYH. Improved outcomes by integrated care of anticoagulated patients with atrial fibrillation using the simple ABC (Atrial Fibrillation Better Care) Pathway. Am J Med 2018;131:1359-1366.e6

320. Yoon M, Yang PS, Jang E, Yu HT, Kim TH, Uhm JS, Kim JY, Sung JH, Pak HN Lee MH, Joung B, Lip GYH. Improved population-based clinical outcomes of patients with atrial fibrillation by compliance with the simple ABC (Atria Fibrillation Better Care) pathway for integrated care management: a nationwide cohort study. Thromb Haemost 2019;19:1695-1703.

321. Pastori D, Pignatelli P, Menichelli D, Violi F, Lip GYH. Integrated care management of patients with atrial fibrillation and risk of cardiovascular events: the ABC (Atrial fibrillation Better Care) pathway in the ATHERO-AF study cohort. Mayo Clin Proc 2019;94:1261-1267

322. Pastori D, Farcomeni A, Pignatelli P, Violi F, Lip GY. ABC (Atrial fibrillation Better Care) pathway and healthcare costs in atrial fibrillation: the ATHEROAF study. Am J Med 2019;132:856-861. 
323. Guo $Y$, Lane DA, Wang L, Zhang $H$, Wang $H$, Zhang W, Wen J, Xing Y, Wu F, Xia Y, Liu T, Wu F, Liang Z, Liu F, Zhao Y, Li R, Li X, Zhang L, Guo J, Burnside G, Chen Y, Lip GYH; mAF-App II Trial Investigators. Mobile health technology to improve care for patients with atrial fibrillation. I Am Coll Cardiol 2020;75:1523-1534

324. Pisters R, Lane DA, Marin F, Camm AJ, Lip GY. Stroke and thromboembolism in atrial fibrillation. Circ J 2012;76:2289-2304.

325. Szymanski FM, Lip GY, Filipiak KJ, Platek AE, Hrynkiewicz-Szymanska A, Opolski G. Stroke risk factors beyond the CHA(2)DS(2)-VASc score: can we improve our identification of 'high stroke risk' patients with atrial fibrillation?Am J Cardiol 2015;116:1781-1788.

326. Atrial Fibrillation Investigators. Echocardiographic predictors of stroke in patients with atrial fibrillation: a prospective study of 1066 patients from 3 clinical trials. Arch Intern Med 1998;158:1316-1320.

327. Ntaios G, Lip GY, Lambrou D, Papavasileiou V, Manios E, Milionis H, Spengos $\mathrm{K}$, Makaritsis K, Vemmos K. Leukoaraiosis and stroke recurrence risk in patients with and without atrial fibrillation. Neurology 2015;84:1213-1219.

328. Esteve-Pastor MA, Roldan V, Rivera-Caravaca JM, Ramirez-Macias I, Lip GYH, Marin F. The use of biomarkers in clinical management guidelines: a critical appraisal. Thromb Haemost 2019;119:1901-1919.

329. Hijazi Z, Oldgren J, Siegbahn A, Wallentin L. Application of biomarkers for risk stratification in patients with atrial fibrillation. Clin Chem 2017;63:152-164.

330. Yaghi S, Kamel H. Stratifying stroke risk in atrial fibrillation: beyond clinical risk scores. Stroke 2017;48:2665-2670.

331. loannou A, Papageorgiou N, Falconer D, Rehal O, Sewart E, Zacharia E, Toutouzas K, Vlachopoulos C, Siasos G, Tsioufis C, Tousoulis D. Biomarkers associated with stroke risk in atrial fibrillation. Curr Med Chem 2019;26:803-823.

332. Sepehri Shamloo A, Bollmann A, Dagres N, Hindricks G, Arya A. Natriuretic peptides: biomarkers for atrial fibrillation management. Clin Res Cardiol 2020;109:957-966.

333. Decker JJ, Norby FL, Rooney MR, Soliman EZ, Lutsey PL, Pankow JS, Alonso A, Chen LY. Metabolic syndrome and risk of ischemic stroke in atrial fibrillation: ARIC Study. Stroke 2019;50:3045-3050.

334. Lip GY, Nieuwlaat R, Pisters R, Lane DA, Crijns HJ. Refining clinical risk stratification for predicting stroke and thromboembolism in atrial fibrillation using a novel risk factor-based approach: the Euro Heart Survey on atrial fibrillation. Chest 2010;137:263-272.

335. Banerjee A, Taillandier S, Olesen JB, Lane DA, Lallemand B, Lip GY, Fauchier L. Ejection fraction and outcomes in patients with atrial fibrillation and heart failure: the Loire Valley Atrial Fibrillation Project. Eur J Heart Fail 2012;14:295-301.

336. Jung $H$, Sung JH, Yang PS, Jang E, Yu HT, Kim TH, Pak HN, Lee MH, Joung B, Lip GYH. Stroke risk stratification for atrial fibrillation patients with hypertrophic cardiomyopathy. J Am Coll Cardiol 2018;72:2409-2411.

337. Jung $H$, Yang PS, Jang E, Yu HT, Kim TH, Uhm JS, Kim JY, Pak HN, Lee MH, Joung B, Lip GYH. Effectiveness and safety of non-vitamin $\mathrm{K}$ antagonist oral anticoagulants in patients with atrial fibrillation with hypertrophic cardiomyopathy: a nationwide cohort study. Chest 2019;155:354-363.

338. Kim D, Yang PS, Kim TH, Jang E, Shin H, Kim HY, Yu HT, Uhm JS, Kim JY, Pak $\mathrm{HN}$, Lee MH, Joung B, Lip GYH. Ideal blood pressure in patients with atrial fibrillation. J Am Coll Cardiol 2018;72:1233-1245.

339. Lip GY, Clementy N, Pericart L, Banerjee A, Fauchier L. Stroke and major bleeding risk in elderly patients aged $>/=75$ years with atrial fibrillation: the Loire Valley Atrial Fibrillation Project. Stroke 2015;46:143-50.

340. Overvad TF, Skjoth F, Lip GY, Lane DA, Albertsen IE, Rasmussen LH, Larsen TB. Duration of diabetes mellitus and risk of thromboembolism and bleeding in atrial fibrillation: nationwide cohort study. Stroke 2015;46:2168-74.

341. Lip GYH, Clementy N, Pierre B, Boyer M, Fauchier L. The impact of associated diabetic retinopathy on stroke and severe bleeding risk in diabetic patients with atrial fibrillation: the Loire Valley Atrial Fibrillation Project. Chest 2015;147:1103-1110.

342. Fangel MV, Nielsen PB, Larsen TB, Christensen B, Overvad TF, Lip GYH, Goldhaber SZ, Jensen MB. Type 1 versus type 2 diabetes and thromboembolic risk in patients with atrial fibrillation: a Danish nationwide cohort study. Int J Cardiol 2018;268:137-142.

343. Chao TF, Liu CJ, Liao JN, Wang KL, Lin YJ, Chang SL, Lo LW, Hu YF, Tuan TC, Chung FP, Chen TJ, Lip GY, Chen SA. Use of oral anticoagulants for stroke prevention in patients with atrial fibrillation who have a history of intracranial hemorrhage. Circulation 2016;133:1540-1547.

344. Bronnum Nielsen P, Larsen TB, Gorst-Rasmussen A, Skjoth F, Rasmussen LH, Lip GYH. Intracranial hemorrhage and subsequent ischemic stroke in patients with atrial fibrillation: a nationwide cohort study. Chest 2015;147:1651-1658.

345. Nielsen PB, Larsen TB, Skjoth F, Gorst-Rasmussen A, Rasmussen LH, Lip GY. Restarting anticoagulant treatment after intracranial hemorrhage in patients with atrial fibrillation and the impact on recurrent stroke, mortality, and bleeding: a nationwide cohort study. Circulation 2015;132:517-525.

346. Lin LY, Lee CH, Yu CC, Tsai CT, Lai LP, Hwang JJ, Chen PC, Lin JL. Risk factors and incidence of ischemic stroke in Taiwanese with nonvalvular atrial fibrillation - a nation-wide database analysis. Atherosclerosis 2011;217:292-295.

347. Anandasundaram B, Lane DA, Apostolakis S, Lip GY. The impact of atherosclerotic vascular disease in predicting a stroke, thromboembolism and mortality in atrial fibrillation patients: a systematic review. J Thromb Haemost 2013;11:975-987.

348. Friberg L, Rosenqvist M, Lip GY. Evaluation of risk stratification schemes for ischaemic stroke and bleeding in 182678 patients with atrial fibrillation: the Swedish Atrial Fibrillation cohort study. Eur Heart J 2012;33:1500-1510.

349. Steensig K, Olesen KKW, Thim T, Nielsen JC, Jensen SE, Jensen LO, Kristensen $\mathrm{SD}$, Botker HE, Lip GYH, Maeng M. Should the presence or extent of coronary artery disease be quantified in the CHA2DS2-VASc score in atrial fibrillation? A report from the Western Denmark Heart Registry. Thromb Haemost 2018;118:2162-2170.

350. Zabalgoitia M, Halperin JL, Pearce LA, Blackshear JL, Asinger RW, Hart RG. Transesophageal echocardiographic correlates of clinical risk of thromboembolism in nonvalvular atrial fibrillation. Stroke Prevention in Atrial Fibrillation III Investigators. J Am Coll Cardiol 1998;31:1622-1626.

351. Kim TH, Yang PS, Yu HT, Jang E, Uhm JS, Kim JY, Pak HN, Lee MH, Joung B, Lip $\mathrm{GYH}$. Age threshold for ischemic stroke risk in atrial fibrillation. Stroke 2018;49:1872-1879.

352. Chao TF, Wang KL, Liu CJ, Lin YJ, Chang SL, Lo LW, Hu YF, Tuan TC, Chung FP, Liao JN, Chen TJ, Chiang CE, Lip GY, Chen SA. Age threshold for increased stroke risk among patients with atrial fibrillation: a nationwide cohort study from Taiwan. J Am Coll Cardiol 2015;66:1339-1347.

353. Nielsen PB, Skjoth F, Overvad TF, Larsen TB, Lip GYH. Female sex is a risk modifier rather than a risk factor for stroke in atrial fibrillation: should we use a CHA2DS2-VA score rather than CHA2DS2-VASc?Circulation 2018;137:832-840.

354. Killu AM, Granger CB, Gersh BJ. Risk stratification for stroke in atrial fibrillation: a critique. Eur Heart J 2019;40:1294-1302.

355. Rivera-Caravaca JM, Roldan V, Esteve-Pastor MA, Valdes M, Vicente V, Lip GYH, Marin F. Long-term stroke risk prediction in patients with atrial fibrillation: comparison of the ABC-Stroke and CHA2DS2-VASc scores. J Am Heart Assoc 2017;6: pii: JAHA.117.006490. doi: 10.1161/JAHA.117.006490.

356. Alkhouli M, Friedman PA. Ischemic stroke risk in patients with nonvalvular atrial fibrillation: JACC review topic of the week. I Am Coll Cardiol 2019;74:3050-3065.

357. Wu VC, Wu M, Aboyans V, Chang SH, Chen SW, Chen MC, Wang CL, Hsieh IC, Chu PH, Lin YS. Female sex as a risk factor for ischaemic stroke varies with age in patients with atrial fibrillation. Heart 2020;106:534-540.

358. Tomasdottir M, Friberg L, Hijazi Z, Lindback J, Oldgren J. Risk of ischemic stroke and utility of CHA2 DS2 -VASc score in women and men with atrial fibrillation. Clin Cardiol 2019;42:1003-1009.

359. Friberg L, Benson L, Rosenqvist M, Lip GY. Assessment of female sex as a risk factor in atrial fibrillation in Sweden: nationwide retrospective cohort study. BMJ 2012;344:e3522.

360. Overvad TF, Potpara TS, Nielsen PB. Stroke risk stratification: CHA2DS2-VA or CHA2DS2-VASc?Heart Lung Circ 2019;28:e14-e15.

361. Nielsen PB, Overvad TF. Female sex as a risk modifier for stroke risk in atrial fibrillation: using CHA2DS2-VASc versus CHA2DS2-VA for stroke risk stratification in atrial fibrillation: a note of caution. Thromb Haemost 2020. doi: 10.1055/s-0040-1710014. Epub ahead of print.

362. Marzona I, Proietti M, Farcomeni A, Romiti GF, Romanazzi I, Raparelli V, Basili S, Lip GYH, Nobili A, Roncaglioni MC. Sex differences in stroke and major adverse clinical events in patients with atrial fibrillation: a systematic review and meta-analysis of 993,600 patients. Int J Cardiol 2018;269:182-191.

363. Friberg L, Benson L, Lip GY. Balancing stroke and bleeding risks in patients with atrial fibrillation and renal failure: the Swedish Atrial Fibrillation Cohort study. Eur Heart J 2015;36:297-306.

364. Poli M, Philip P, Taillard J, Debruxelles S, Renou P, Orgogozo JM, Rouanet F, Sibon I. Atrial fibrillation is a major cause of stroke in apneic patients: a prospective study. Sleep Med 2017;30:251-254.

365. Bassand JP, Accetta G, Al Mahmeed W, Corbalan R, Eikelboom J, Fitzmaurice DA, Fox KAA, Gao H, Goldhaber SZ, Goto S, Haas S, Kayani G, Pieper K, Turpie AGG, van Eickels M, Verheugt FWA, Kakkar AK; GARFIELD-AF Investigators. Risk factors for death, stroke, and bleeding in 28,628 patients from the GARFIELD-AF registry: rationale for comprehensive management of atrial fibrillation. PLoS One 2018;13:e0191592.

366. Overvad TF, Rasmussen LH, Skjoth F, Overvad K, Lip GY, Larsen TB. Body mass index and adverse events in patients with incident atrial fibrillation. Am J Med 2013;126:640.e9-17. 
367. Lip GY, Lane D, Van Walraven C, Hart RG. Additive role of plasma von Willebrand factor levels to clinical factors for risk stratification of patients with atrial fibrillation. Stroke 2006;37:2294-2300.

368. Fox KAA, Lucas JE, Pieper KS, Bassand JP, Camm AJ, Fitzmaurice DA, Goldhaber SZ, Goto S, Haas S, Hacke W, Kayani G, Oto A, Mantovani LG, Misselwitz F, Piccini JP, Turpie AGG, Verheugt FWA, Kakkar AK; GARFIELDAF Investigators. Improved risk stratification of patients with atrial fibrillation: an integrated GARFIELD-AF tool for the prediction of mortality, stroke and bleed in patients with and without anticoagulation. BMJ Open 2017;7:e017157.

369. Zhu W, Fu L, Ding Y, Huang L, Xu Z, Hu J, Hong K. Meta-analysis of ATRIA versus CHA2DS2-VASc for predicting stroke and thromboembolism in patients with atrial fibrillation. Int J Cardiol 2017;227:436-442.

370. Singer DE, Chang Y, Borowsky LH, Fang MC, Pomernacki NK, Udaltsova N, Reynolds K, Go AS. A new risk scheme to predict ischemic stroke and other thromboembolism in atrial fibrillation: the ATRIA study stroke risk score. J Am Heart Assoc 2013;2:e000250.

371. Graves KG, May HT, Knowlton KU, Muhlestein JB, Jacobs V, Lappe DL, Anderson JL, Horne BD, Bunch TJ. Improving CHA2DS2-VASc stratification of non-fatal stroke and mortality risk using the Intermountain Mortality Risk Score among patients with atrial fibrillation. Open Heart 2018;5:e000907.

372. Hijazi Z, Lindback J, Alexander JH, Hanna M, Held C, Hylek EM, Lopes RD, Oldgren J, Siegbahn A, Stewart RA, White HD, Granger CB, Wallentin L; ARISTOTLE and STABILITY Investigators. The ABC (age, biomarkers, clinical history) stroke risk score: a biomarker-based risk score for predicting stroke in atrial fibrillation. Eur Heart J 2016;37:1582-90.

373. Hijazi Z, Lindahl B, Oldgren J, Andersson U, Lindback J, Granger CB, Alexander JH, Gersh BJ, Hanna M, Harjola VP, Hylek EM, Lopes RD, Siegbahn A, Wallentin $L$. Repeated measurements of cardiac biomarkers in atrial fibrillation and validation of the ABC stroke score over time. J Am Heart Assoc 2017;6.

374. Oldgren J, Hijazi Z, Lindback J, Alexander JH, Connolly SJ, Eikelboom JW, Ezekowitz MD, Granger CB, Hylek EM, Lopes RD, Siegbahn A, Yusuf S, Wallentin L; RE-LY and ARISTOTLE Investigators. Performance and validation of a novel biomarker-based stroke risk score for atrial fibrillation. Circulation 2016;134:1697-1707.

375. Berg DD, Ruff CT, Jarolim P, Giugliano RP, Nordio F, Lanz HJ, Mercuri MF, Antman EM, Braunwald E, Morrow DA. Performance of the ABC scores for assessing the risk of stroke or systemic embolism and bleeding in patients with atrial fibrillation in ENGAGE AF-TIMI 48. Circulation 2019;139:760-771.

376. Rivera-Caravaca JM, Marin F, Vilchez JA, Galvez J, Esteve-Pastor MA, Vicente V, Lip GYH, Roldan V. Refining stroke and bleeding prediction in atrial fibrillation by adding consecutive biomarkers to clinical risk scores. Stroke 2019;50:1372-1379.

377. Esteve-Pastor MA, Rivera-Caravaca JM, Roldan V, Vicente V, Valdes M, Marin F, Lip GY. Long-term bleeding risk prediction in 'real world'patients with atrial fibrillation: comparison of the HAS-BLED and ABC-Bleeding risk scores. Thromb Haemost 2017;117:1848-1858.

378. Shin SY, Han SJ, Kim JS, Im SI, Shim J, Ahn J, Lee EM, Park YM, Kim JH, Lip GYH, Lim HE. Identification of markers associated with development of stroke in 'clinically low-risk' atrial fibrillation patients. J Am Heart Assoc 2019;8:e012697.

379. Chao TF, Lip GYH, Lin YJ, Chang SL, Lo LW, Hu YF, Tuan TC, Liao JN, Chung FP, Chen TJ, Chen SA. Age threshold for the use of non-vitamin $\mathrm{K}$ antagonist oral anticoagulants for stroke prevention in patients with atrial fibrillation: insights into the optimal assessment of age and incident comorbidities. Eur Heart J 2019;40:1504-1514.

380. Nielsen PB, Larsen TB, Skjoth F, Overvad TF, Lip GY. Stroke and thromboembolic event rates in atrial fibrillation according to different guideline treatment thresholds: a nationwide cohort study. Sci Rep 2016;6:27410.

381. Fauchier L, Clementy N, Bisson A, Ivanes F, Angoulvant D, Babuty D, Lip GY. Should atrial fibrillation patients with only 1 nongender-related CHA2DS2VASc risk factor be anticoagulated? Stroke 2016;47:1831-1836.

382. Chao TF, Lip GYH, Liu CJ, Lin YJ, Chang SL, Lo LW, Hu YF, Tuan TC, Liao JN, Chung FP, Chen TJ, Chen SA. Relationship of aging and incident comorbidities to stroke risk in patients with atrial fibrillation. I Am Coll Cardiol 2018; 71:122-132.

383. Yoon M, Yang PS, Jang E, Yu HT, Kim TH, Uhm JS, Kim JY, Pak HN, Lee MH, Lip GYH, Joung B. Dynamic changes of CHA2DS2-VASc score and the risk of ischaemic stroke in Asian patients with atrial fibrillation: a nationwide cohort study. Thromb Haemost 2018;118:1296-1304.

384. Chao TF, Chiang CE, Chen TJ, Lip GYH, Chen SA. Reassessment of risk for stroke during follow-up of patients with atrial fibrillation. Ann Intern Med 2019;170:663-664

385. Potpara TS, Polovina MM, Licina MM, Marinkovic JM, Prostran MS, Lip GY. Reliable identification of 'truly low' thromboembolic risk in patients initially diagnosed with 'lone' atrial fibrillation: the Belgrade Atrial Fibrillation Study. Circ Arrhythm Electrophysiol 2012;5:319-326.
386. Weijs B, Dudink E, de Vos CB, Limantoro I, Tieleman RG, Pisters R, Cheriex EC, Luermans J, Crijns H. Idiopathic atrial fibrillation patients rapidly outgrow their low thromboembolic risk: a 10-year follow-up study. Neth Heart J 2019;27:487-497.

387. Chao TF, Liao JN, Tuan TC, Lin YJ, Chang SL, Lo LW, Hu YF, Chung FP, Chen TJ, Lip GYH, Chen SA. Incident co-morbidities in patients with atrial fibrillation initially with a CHA2DS2-VASc score of 0 (males) or 1 (females): implications for reassessment of stroke risk in initially 'low-risk' patients. Thromb Haemost 2019;119:1162-1170

388. Borre ED, Goode A, Raitz G, Shah B, Lowenstern A, Chatterjee R, Sharan L, Allen LaPointe NM, Yapa R, Davis JK, Lallinger K, Schmidt R, Kosinski A, AlKhatib SM, Sanders GD. Predicting thromboembolic and bleeding event risk in patients with non-valvular atrial fibrillation: a systematic review. Thromb Haemost 2018;118:2171-2187.

389. Chao TF, Lip GYH, Lin YJ, Chang SL, Lo LW, Hu YF, Tuan TC, Liao JN, Chung FP, Chen TJ, Chen SA. Incident risk factors and major bleeding in patients with atrial fibrillation treated with oral anticoagulants: a comparison of baseline, follow-up and Delta HAS-BLED scores with an approach focused on modifiable bleeding risk factors. Thromb Haemost 2018;118:768-777.

390. Man-Son-Hing M, Nichol G, Lau A, Laupacis A. Choosing antithrombotic therapy for elderly patients with atrial fibrillation who are at risk for falls. Arch Intern Med 1999;159:677-685.

391. Gage BF, Yan Y, Milligan PE, Waterman AD, Culverhouse R, Rich MW, Radford MJ. Clinical classification schemes for predicting hemorrhage: results from the National Registry of Atrial Fibrillation (NRAF). Am Heart J 2006;151:713-719.

392. Fang MC, Go AS, Chang Y, Borowsky LH, Pomernacki NK, Udaltsova N, Singer DE. A new risk scheme to predict warfarin-associated hemorrhage: the ATRIA (Anticoagulation and Risk Factors in Atrial Fibrillation) study. J Am Coll Cardiol 2011;58:395-401.

393. O'Brien EC, Simon DN, Thomas LE, Hylek EM, Gersh BJ, Ansell JE, Kowey PR, Mahaffey KW, Chang P, Fonarow GC, Pencina MJ, Piccini JP, Peterson ED. The ORBIT bleeding score: a simple bedside score to assess bleeding risk in atrial fibrillation. Eur Heart J 2015;36:3258-3264.

394. Rohla M, Weiss TW, Pecen L, Patti G, Siller-Matula JM, Schnabel RB, Schilling R, Kotecha D, Lucerna M, Huber K, De Caterina R, Kirchhof P. Risk factors for thromboembolic and bleeding events in anticoagulated patients with atrial fibrillation: the prospective, multicentre observational PREvention of thromboembolic events - European Registry in Atrial Fibrillation (PREFER in AF). BM Open 2019;9:e022478.

395. Pisters R, Lane DA, Nieuwlaat R, de Vos CB, Crijns HJ, Lip GY. A novel userfriendly score (HAS-BLED) to assess 1-year risk of major bleeding in patients with atrial fibrillation: the Euro Heart Survey. Chest 2010;138:1093-1100.

396. Mori N, Sotomi Y, Hirata A, Hirayama A, Sakata Y, Higuchi Y. External validation of the ORBIT bleeding score and the HAS-BLED score in nonvalvular atrial fibrillation patients using direct oral anticoagulants (Asian data from the DIRECT registry). Am J Cardiol 2019;124:1044-1048.

397. Yao X, Gersh BJ, Sangaralingham LR, Kent DM, Shah ND, Abraham NS, Noseworthy PA. Comparison of the CHA2DS2-VASc, CHADS2, HAS-BLED, ORBIT, and ATRIA risk scores in predicting non-vitamin $\mathrm{K}$ antagonist oral anticoagulants-associated bleeding in patients with atrial fibrillation. Am J Cardiol 2017; 120:1549-1556.

398. Rutherford OW, Jonasson C, Ghanima W, Holst R, Halvorsen S. New score for assessing bleeding risk in patients with atrial fibrillation treated with NOACs. Open Heart 2018;5:e000931.

399. Thomas MR, Lip GY. Novel risk markers and risk assessments for cardiovascular disease. Circ Res 2017;120:133-149.

400. Khan AA, Lip GYH. The prothrombotic state in atrial fibrillation: pathophysiological and management implications. Cardiovasc Res 2019;115:31-45.

401. Ban N, Siegfried CJ, Lin JB, Shui YB, Sein J, Pita-Thomas W, Sene A, Santeford A, Gordon M, Lamb R, Dong Z, Kelly SC, Cavalli V, Yoshino J, Apte RS. GDF15 is elevated in mice following retinal ganglion cell death and in glaucoma patients. JCI Insight 2017;2:pii: 91455. doi: 10.1172/jci.insight.91455.

402. Hijazi Z, Oldgren J, Lindback J, Alexander JH, Connolly SJ, Eikelboom JW Ezekowitz MD, Held C, Hylek EM, Lopes RD, Siegbahn A, Yusuf S, Granger CB, Wallentin L; ARISTOTLE and RE-LY Investigators. The novel biomarker-based $A B C$ (age, biomarkers, clinical history)-bleeding risk score for patients with atrial fibrillation: a derivation and validation study. Lancet 2016;387:2302-2311.

403. Esteve-Pastor MA, Rivera-Caravaca JM, Roldan V, Vicente V, Valdes M, Marin F, Lip GYH. Long-term bleeding risk prediction in 'real world' patients with atrial fibrillation: comparison of the HAS-BLED and ABC-Bleeding risk scores. The Murcia Atrial Fibrillation Project. Thromb Haemost 2017;117:1848-1858.

404. Caldeira D, Costa J, Fernandes RM, Pinto FJ, Ferreira JJ. Performance of the HAS-BLED high bleeding-risk category, compared to ATRIA and HEMORR2HAGES in patients with atrial fibrillation: a systematic review and meta-analysis. J Interv Card Electrophysiol 2014;40:277-284. 
405. Zhu W, He W, Guo L, Wang X, Hong K. The HAS-BLED score for predicting major bleeding risk in anticoagulated patients with atrial fibrillation: a systematic review and meta-analysis. Clin Cardiol 2015;38:555-561.

406. Chang G, Xie Q, Ma L, Hu K, Zhang Z, Mu G, Cui Y. Accuracy of HAS-BLED and other bleeding risk assessment tools in predicting major bleeding events in atrial fibrillation: a network meta-analysis. J Thromb Haemost 2020;18:791-801.

407. Lip GY, Lane DA. Bleeding risk assessment in atrial fibrillation: observations on the use and misuse of bleeding risk scores. I Thromb Haemost 2016:14:1711-1714

408. Chao TF, Lip GYH, Lin YJ, Chang SL, Lo LW, Hu YF, Tuan TC, Liao JN, Chung FP, Chen TJ, Chen SA. Major bleeding and intracranial hemorrhage risk prediction in patients with atrial fibrillation: attention to modifiable bleeding risk factors or use of a bleeding risk stratification score? A nationwide cohort study. Int J Cardiol 2018;254:157-161.

409. Guo Y, Zhu H, Chen Y, Lip GYH. Comparing bleeding risk assessment focused on modifiable risk factors only versus validated bleeding risk scores in atrial fibrillation. Am J Med 2018;131:185-192.

410. Esteve-Pastor MA, Rivera-Caravaca JM, Shantsila A, Roldan V, Lip GYH, Marin F. Assessing bleeding risk in atrial fibrillation patients: comparing a bleeding risk score based only on modifiable bleeding risk factors against the HAS-BLED score. The AMADEUS trial. Thromb Haemost 2017;117:2261-2266.

411. Guo Y, Lane DA, Chen Y, Lip GYH; mAF-App II Trial investigators. Regular bleeding risk assessment associated with reduction in bleeding outcomes: the mAFA-II randomized trial. Am J Med 2020:pii: S0002-9343(20)30274-6.

412. Hart RG, Pearce LA, Aguilar MI. Meta-analysis: antithrombotic therapy to prevent stroke in patients who have nonvalvular atrial fibrillation. Ann Intern Med 2007; 146:857-867.

413. De Caterina R, Husted S, Wallentin L, Andreotti F, Arnesen H, Bachmann F, Baigent C, Huber K, Jespersen J, Kristensen SD, Lip GY, Morais J, Rasmussen LH, Siegbahn A, Verheugt FW, Weitz Jl. Vitamin K antagonists in heart disease: current status and perspectives (Section III). Position paper of the ESC working group on thrombosis - Task Force on anticoagulants in heart disease. Thromb Haemost 2013;110:1087-1107.

414. Wan Y, Heneghan C, Perera R, Roberts N, Hollowell J, Glasziou P, Bankhead C, Xu $Y$. Anticoagulation control and prediction of adverse events in patients with atrial fibrillation: a systematic review. Circ Cardiovasc Qual Outcomes 2008;1:84-91.

415. Sjalander S, Sjogren V, Renlund H, Norrving B, Sjalander A. Dabigatran, rivaroxaban and apixaban vs. high TTR warfarin in atrial fibrillation. Thromb Res 2018;167:113-118.

416. Amin A, Deitelzweig S, Jing Y, Makenbaeva D, Wiederkehr D, Lin J, Graham J. Estimation of the impact of warfarin's time-in-therapeutic range on stroke and major bleeding rates and its influence on the medical cost avoidance associated with novel oral anticoagulant use-learnings from ARISTOTLE, ROCKET-AF, and RE-LY trials. J Thromb Thrombolysis 2014;38:150-159.

417. Apostolakis S, Sullivan RM, Olshansky B, Lip GYH. Factors affecting quality of anticoagulation control among patients with atrial fibrillation on warfarin: the SAMe-TT(2)R(2) score. Chest 2013;144:1555-1563.

418. Proietti M, Lip GY. Simple decision-making between a vitamin $\mathrm{K}$ antagonist and a non-vitamin $\mathrm{K}$ antagonist oral anticoagulant: using the SAMe-TT2R2 score. Eur Heart J Cardiovasc Pharmacother 2015;1:150-152.

419. Connolly SJ, Ezekowitz MD, Yusuf S, Eikelboom J, Oldgren J, Parekh A, Pogue J, Reilly PA, Themeles E, Varrone J, Wang S, Alings M, Xavier D, Zhu J, Diaz R, Lewis BS, Darius H, Diener HC, Joyner CD, Wallentin L; RE-LY Steering Committee Investigators. Dabigatran versus warfarin in patients with atrial fibrillation. N Engl J Med 2009;361:1139-1151.

420. Patel MR, Mahaffey KW, Garg J, Pan G, Singer DE, Hacke W, Breithardt G, Halperin JL, Hankey GJ, Piccini JP, Becker RC, Nessel CC, Paolini JF, Berkowitz SD, Fox KA, Califf RM; ROCKET AF Investigators. Rivaroxaban versus warfarin in nonvalvular atrial fibrillation. N EnglJ Med 2011;365:883-891.

421. Granger CB, Alexander JH, McMurray J], Lopes RD, Hylek EM, Hanna M, AlKhalidi HR, Ansell J, Atar D, Avezum A, Bahit MC, Diaz R, Easton JD, Ezekowitz JA, Flaker G, Garcia D, Geraldes M, Gersh BJ, Golitsyn S, Goto S, Hermosillo AG, Hohnloser SH, Horowitz J, Mohan P, Jansky P, Lewis BS, Lopez-Sendon JL, Pais P, Parkhomenko A, Verheugt FW, Zhu J, Wallentin L; ARISTOTLE Committees and Investigators. Apixaban versus warfarin in patients with atrial fibrillation. N Engl J Med 2011;365:981-992.

422. Giugliano RP, Ruff CT, Braunwald E, Murphy SA, Wiviott SD, Halperin IL, Waldo AL, Ezekowitz MD, Weitz JI, Spinar J, Ruzyllo W, Ruda M, Koretsune Y, Betcher J, Shi M, Grip LT, Patel SP, Patel I, Hanyok J], Mercuri M, Antman EM; ENGAGE AF-TIMI Investigators. Edoxaban versus warfarin in patients with atrial fibrillation. N Engl J Med 2013;369:2093-2104.

423. Ruff CT, Giugliano RP, Braunwald E, Hoffman EB, Deenadayalu N, Ezekowitz MD, Camm AJ, Weitz Jl, Lewis BS, Parkhomenko A, Yamashita T, Antman EM. Comparison of the efficacy and safety of new oral anticoagulants with warfarin in patients with atrial fibrillation: a meta-analysis of randomised trials. Lancet 2014:383:955-962.
424. Wang KL, Lip GY, Lin SJ, Chiang CE. Non-vitamin K antagonist oral anticoagulants for stroke prevention in Asian patients with nonvalvular atrial fibrillation: meta-analysis. Stroke 2015;46:2555-2561.

425. Connolly S), Eikelboom J, Joyner C, Diener HC, Hart R, Golitsyn S, Flaker G, Avezum A, Hohnloser SH, Diaz R, Talajic M, Zhu J, Pais P, Budaj A, Parkhomenko A, Jansky P, Commerford P, Tan RS, Sim KH, Lewis BS, Van Mieghem W, Lip GY, Kim JH, Lanas-Zanetti F, Gonzalez-Hermosillo A, Dans AL, Munawar M, O'Donnell M, Lawrence J, Lewis G, Afzal R, Yusuf S; AVERROES Steering Committee Investigators. Apixaban in patients with atrial fibrillation. N Engl J Med 2011;364:806-817.

426. Carmo J, Moscoso Costa F, Ferreira J, Mendes M. Dabigatran in real-world atrial fibrillation. Meta-analysis of observational comparison studies with vitamin $\mathrm{K}$ antagonists. Thromb Haemost 2016;116:754-763.

427. Huisman MV, Rothman KJ, Paquette M, Teutsch C, Diener HC, Dubner SJ, Halperin JL, Ma CS, Zint K, Elsaesser A, Lu S, Bartels DB, Lip GYH; GLORIAAF Investigators. Two-year follow-up of patients treated with dabigatran for stroke prevention in atrial fibrillation: Global Registry on Long-Term Antithrombotic Treatment in Patients with Atrial Fibrillation (GLORIA-AF) registry. Am Heart J 2018;198:55-63.

428. Camm AJ, Amarenco P, Haas S, Hess S, Kirchhof P, Kuhls S, van Eickels M, Turpie AG; XANTUS Investigators. XANTUS: a real-world, prospective, observational study of patients treated with rivaroxaban for stroke prevention in atrial fibrillation. Eur Heart J 2016;37:1145-1153.

429. Martinez CAA, Lanas F, Radaideh G, Kharabsheh SM, Lambelet M, Viaud MAL, Ziadeh NS, Turpie AGG; XANTUS Investigators. XANTUS-EL: a real-world, prospective, observational study of patients treated with rivaroxaban for stroke prevention in atrial fibrillation in Eastern Europe, Middle East, Africa and Latin America. Egypt Heart J 2018;70:307-313.

430. Li XS, Deitelzweig S, Keshishian A, Hamilton M, Horblyuk R, Gupta K, Luo X, Mardekian J, Friend K, Nadkarni A, Pan X, Lip GYH. Effectiveness and safety of apixaban versus warfarin in non-valvular atrial fibrillation patients in 'real-world' clinical practice. A propensity-matched analysis of 76,940 patients. Thromb Haemost 2017;117:1072-1082.

431. Lee SR, Choi EK, Han KD, Jung JH, Oh S, Lip GYH. Edoxaban in Asian patients with atrial fibrillation: effectiveness and safety. I Am Coll Cardiol 2018;72:838-853.

432. Ingrasciotta Y, Crisafulli S, Pizzimenti V, Marciano I, Mancuso A, Ando G, Corrao S, Capranzano P, Trifiro G. Pharmacokinetics of new oral anticoagulants: implications for use in routine care. Expert Opin Drug Metab Toxicol 2018;14:1057-1069.

433. Chao TF, Liu CJ, Lin YJ, Chang SL, Lo LW, Hu YF, Tuan TC, Liao JN, Chung FP, Chen TJ, Lip GYH, Chen SA. Oral anticoagulation in very elderly patients with atrial fibrillation: a nationwide cohort study. Circulation 2018;138:37-47.

434. Stanton BE, Barasch NS, Tellor KB. Comparison of the safety and effectiveness of apixaban versus warfarin in patients with severe renal impairment. Pharmacotherapy 2017;37:412-419.

435. Siontis KC, Zhang X, Eckard A, Bhave N, Schaubel DE, He K, Tilea A, Stack AG, Balkrishnan R, Yao X, Noseworthy PA, Shah ND, Saran R, Nallamothu BK. Outcomes associated with apixaban use in patients with end-stage kidney disease and atrial fibrillation in the United States. Circulation 2018;138:1519-1529.

436. Steinberg BA, Shrader P, Thomas L, Ansell J, Fonarow GC, Gersh B], Kowey PR, Mahaffey KW, Naccarelli G, Reiffel J, Singer DE, Peterson ED, Piccini JP; ORBIT-AF Investigators and Patients. Off-label dosing of non-vitamin $\mathrm{K}$ antagonist oral anticoagulants and adverse outcomes: the ORBIT-AF II registry. J Am Coll Cardiol 2016;68:2597-2604.

437. Yao X, Shah ND, Sangaralingham LR, Gersh BJ, Noseworthy PA. Non-vitamin K antagonist oral anticoagulant dosing in patients with atrial fibrillation and renal dysfunction. J Am Coll Cardiol 2017;69:2779-2790.

438. ACTIVE Writing Group of the ACTIVE Investigators, Connolly S, Pogue J, Hart R, Pfeffer M, Hohnloser S, Chrolavicius S, Pfeffer M, Hohnloser S, Yusuf S. Clopidogrel plus aspirin versus oral anticoagulation for atrial fibrillation in the Atrial fibrillation Clopidogrel Trial with Irbesartan for prevention of Vascular Events (ACTIVE W): a randomised controlled trial. Lancet 2006;367:1903-1912.

439. ACTIVE Investigators, Connolly SJ, Pogue J, Hart RG, Hohnloser SH, Pfeffer M, Chrolavicius S, Yusuf S. Effect of clopidogrel added to aspirin in patients with atrial fibrillation. N Engl J Med 2009;360:2066-2078.

440. Sjalander S, Sjalander A, Svensson PJ, Friberg L. Atrial fibrillation patients do not benefit from acetylsalicylic acid. Europace 2014;16:631-638.

441. Mant J, Hobbs FD, Fletcher K, Roalfe A, Fitzmaurice D, Lip GY, Murray E; BAFTA investigators, Midland Research Practices Network (MidReC). Warfarin versus aspirin for stroke prevention in an elderly community population with atrial fibrillation (the Birmingham Atrial Fibrillation Treatment of the Aged Study, BAFTA): a randomised controlled trial. Lancet 2007;370:493 - 503.

442. Lip GY. The role of aspirin for stroke prevention in atrial fibrillation. Nat Rev Cardiol 2011;8:602-606. 
443. Verheugt FWA, Gao H, Al Mahmeed W, Ambrosio G, Angchaisuksiri P, Atar D, Bassand JP, Camm AJ, Cools F, Eikelboom J, Kayani G, Lim TW, Misselwitz F, Pieper KS, van Eickels M, Kakkar AK; GARFIELD-AF Investigators. Characteristics of patients with atrial fibrillation prescribed antiplatelet monotherapy compared with those on anticoagulants: insights from the GARFIELDAF registry. Eur Heart J 2018;39:464-473.

444. Holmes DR, Reddy VY, Turi ZG, Doshi SK, Sievert H, Buchbinder M, Mullin CM, Sick P; PROTECT AF Investigators. Percutaneous closure of the left atrial appendage versus warfarin therapy for prevention of stroke in patients with atrial fibrillation: a randomised non-inferiority trial. Lancet 2009;374:534-542.

445. Reddy VY, Doshi SK, Sievert H, Buchbinder M, Neuzil P, Huber K, Halperin JL, Holmes D; PROTECT AF Investigators. Percutaneous left atrial appendage closure for stroke prophylaxis in patients with atrial fibrillation: 2.3-year follow-up of the PROTECT AF (Watchman Left Atrial Appendage System for Embolic Protection in Patients with Atrial Fibrillation) trial. Circulation 2013;127:720-729.

446. Holmes DR Jr, Kar S, Price MJ, Whisenant B, Sievert H, Doshi SK, Huber K, Reddy VY. Prospective randomized evaluation of the Watchman left atrial appendage closure device in patients with atrial fibrillation versus long-term warfarin therapy: the PREVAIL trial. J Am Coll Cardiol 2014;64:1-12.

447. Holmes DR, Jr., Doshi SK, Kar S, Price MJ, Sanchez JM, Sievert H, Valderrabano $M$, Reddy VY. Left atrial appendage closure as an alternative to warfarin for stroke prevention in atrial fibrillation: a patient-level meta-analysis. J Am Coll Cardiol 2015;65:2614-2623.

448. Reddy VY, Mobius-Winkler S, Miller MA, Neuzil P, Schuler G, Wiebe J, Sick P, Sievert $\mathrm{H}$. Left atrial appendage closure with the Watchman device in patients with a contraindication for oral anticoagulation: the ASAP study (ASA Plavix Feasibility Study With Watchman Left Atrial Appendage Closure Technology). J Am Coll Cardiol 2013;61:2551-2556.

449. Boersma LV, Schmidt B, Betts TR, Sievert H, Tamburino C, Teiger E, Pokushalov E, Kische S, Schmitz T, Stein KM, Bergmann MW, on behalf of the EWOLUTION investigators. Implant success and safety of left atrial appendage closure with the WATCHMAN device: peri-procedural outcomes from the EWOLUTION registry. Eur Heart J 2016;37:2465-2474.

450. Boersma LV, Ince H, Kische S, Pokushalov E, Schmitz T, Schmidt B, Gori T, Meincke F, Protopopov AV, Betts T, Foley D, Sievert H, Mazzone P, De Potter $T$, Vireca E, Stein K, Bergmann MW, for the EWOLUTION Investigators. Efficacy and safety of left atrial appendage closure with WATCHMAN in patients with or without contraindication to oral anticoagulation: 1-year followup outcome data of the EWOLUTION trial. Heart Rhythm 2017; 14:1302-1308.

451. Badheka AO, Chothani A, Mehta K, Patel NJ, Deshmukh A, Hoosien M, Shah N, Singh V, Grover P, Savani GT, Panaich SS, Rathod A, Patel N, Arora S, Bhalara V, Coffey JO, O'Neill W, Makkar R, Grines CL, Schreiber T, Di Biase L, Natale A, Viles-Gonzalez JF. Utilization and adverse outcomes of percutaneous left atrial appendage closure for stroke prevention in atrial fibrillation in the United States: influence of hospital volume. Circ Arrhythm Electrophysiol 2015;8:42-48.

452. Pison L, Potpara TS, Chen J, Larsen TB, Bongiorni MG, Blomstrom-Lundqvist C; Scientific Initiative Committee EHRA. Left atrial appendage closure-indications, techniques, and outcomes: results of the European Heart Rhythm Association Survey. Europace 2015;17:642-646.

453. Price MJ, Gibson DN, Yakubov SJ, Schultz JC, Di Biase L, Natale A, Burkhardt JD, Pershad A, Byrne TJ, Gidney B, Aragon JR, Goldstein J, Moulton K, Patel T, Knight B, Lin AC, Valderrabano M. Early safety and efficacy of percutaneous left atrial appendage suture ligation: results from the US transcatheter LAA ligation consortium. J Am Coll Cardiol 2014;64:565-572.

454. Fauchier L, Cinaud A, Brigadeau F, Lepillier A, Pierre B, Abbey S, Fatemi M, Franceschi F, Guedeney P, Jacon P, Paziaud O, Venier S, Deharo JC, Gras D, Klug D, Mansourati J, Montalescot G, Piot O, Defaye P. Device-related thrombosis after percutaneous left atrial appendage occlusion for atrial fibrillation. J Am Coll Cardiol 2018;71:1528-1536.

455. Lakkireddy D, Afzal MR, Lee RJ, Nagaraj H, Tschopp D, Gidney B, Ellis C, Altman E, Lee B, Kar S, Bhadwar N, Sanchez M, Gadiyaram V, Evonich R, Rasekh A, Cheng J, Cuoco F, Chandhok S, Gunda S, Reddy M, Atkins D, Bommana S, Cuculich P, Gibson D, Nath J, Ferrell R, Matthew E, Wilber D. Short and long-term outcomes of percutaneous left atrial appendage suture ligation: results from a US multicenter evaluation. Heart Rhythm 2016;13:1030-1036.

456. van Laar C, Verberkmoes NJ, van Es HW, Lewalter T, Dunnington G, Stark S, Longoria J, Hofman FH, Pierce CM, Kotecha D, van Putte BP. Thoracoscopic left atrial appendage clipping: a multicenter cohort analysis. JACC Clin Electrophysiol 2018;4:893-901.

457. Healey JS, Crystal E, Lamy A, Teoh K, Semelhago L, Hohnloser SH, Cybulsky I, Abouzahr L, Sawchuck C, Carroll S, Morillo C, Kleine P, Chu V, Lonn E, Connolly SJ. Left Atrial Appendage Occlusion Study (LAAOS): results of a randomized controlled pilot study of left atrial appendage occlusion during coronary bypass surgery in patients at risk for stroke. Am Heart J 2005; 150:288-293.

458. Whitlock RP, Vincent J, Blackall MH, Hirsh J, Fremes S, Novick R, Devereaux PJ, Teoh K, Lamy A, Connolly SJ, Yusuf S, Carrier M, Healey JS. Left Atrial Appendage Occlusion Study II (LAAOS II). Can J Cardiol 2013;29:1443-1447.

459. Tsai YC, Phan K, Munkholm-Larsen S, Tian DH, La Meir M, Yan TD. Surgical left atrial appendage occlusion during cardiac surgery for patients with atrial fibrillation: a meta-analysis. Eur J Cardiothorac Surg 2015;47:847-854.

460. Aryana A, Singh SK, Singh SM, O'Neill PG, Bowers MR, Allen SL, Lewandowski SL, Vierra EC, d'Avila A. Association between incomplete surgical ligation of left atrial appendage and stroke and systemic embolization. Heart Rhythm 2015;12:1431-1437.

461. Gillinov AM, Gelijns AC, Parides MK, DeRose JJ Jr, Moskowitz AJ, Voisine P, Ailawadi G, Bouchard D, Smith PK, Mack MJ, Acker MA, Mullen JC, Rose EA, Chang HL, Puskas JD, Couderc JP, Gardner TJ, Varghese R, Horvath KA, Bolling SF, Michler RE, Geller NL, Ascheim DD, Miller MA, Bagiella E, Moquete EG, Williams P, Taddei-Peters WC, O'Gara PT, Blackstone EH, Argenziano M: CTSN Investigators. Surgical ablation of atrial fibrillation during mitral-valve surgery. N Engl J Med 2015;372:1399-1409.

462. Whitlock R, Healey J, Vincent J, Brady K, Teoh K, Royse A, Shah P, Guo Y, Alings M, Folkeringa RJ, Paparella D, Colli A, Meyer SR, Legare JF, Lamontagne F, Reents W, Boning A, Connolly S. Rationale and design of the Left Atrial Appendage Occlusion Study (LAAOS) III. Ann Cardiothorac Surg 2014;3:45-54.

463. Nielsen PB, Skjoth F, Sogaard M, Kjaeldgaard JN, Lip GY, Larsen TB. Effectiveness and safety of reduced dose non-vitamin $K$ antagonist oral anticoagulants and warfarin in patients with atrial fibrillation: propensity weighted nationwide cohort study. BMJ 2017;356:j510.

464. Larsen TB, Skjoth F, Nielsen PB, Kjaeldgaard JN, Lip GY. Comparative effectiveness and safety of non-vitamin $K$ antagonist oral anticoagulants and warfarin in patients with atrial fibrillation: propensity weighted nationwide cohort study. BMJ 2016;353:i3189.

465. Tilz RR, Potpara T, Chen J, Dobreanu D, Larsen TB, Haugaa KH, Dagres N. Left atrial appendage occluder implantation in Europe: indications and anticoagulation post-implantation. Results of the European Heart Rhythm Association Survey. Europace 2017;19:1737-1742.

466. Ogawa H, An Y, lkeda S, Aono Y, Doi K, Ishii M, Iguchi M, Masunaga N, Esato M, Tsuji H, Wada H, Hasegawa K, Abe M, Lip GYH, Akao M; Fushimi AF Registry Investigators. Progression from paroxysmal to sustained atrial fibrillation is associated with increased adverse events. Stroke 2018;49:2301-2308.

467. Mahajan R, Perera T, Elliott AD, Twomey DJ, Kumar S, Munwar DA, Khokhar KB, Thiyagarajah A, Middeldorp ME, Nalliah CJ, Hendriks JML, Kalman JM, Lau $\mathrm{DH}$, Sanders P. Subclinical device-detected atrial fibrillation and stroke risk: a systematic review and meta-analysis. Eur Heart J 2018;39:1407-1415.

468. Van Gelder IC, Healey JS, Crijns H, Wang J, Hohnloser SH, Gold MR, Capucci A, Lau CP, Morillo CA, Hobbelt AH, Rienstra M, Connolly SJ. Duration of device-detected subclinical atrial fibrillation and occurrence of stroke in ASSERT. Eur Heart J 2017;38:1339-1344.

469. Boriani G, Glotzer TV, Ziegler PD, De Melis M, Mangoni di SSL, Sepsi M, Landolina M, Lunati M, Lewalter T, Camm AJ. Detection of new atrial fibrillation in patients with cardiac implanted electronic devices and factors associated with transition to higher device-detected atrial fibrillation burden. Heart Rhythm 2018;15:376-383.

470. Pastori D, Lip GYH, Farcomeni A, Del Sole F, Sciacqua A, Perticone F, Marcucci R, Grifoni E, Pignatelli P, Violi F, ATHERO-AF study group. Incidence of bleeding in patients with atrial fibrillation and advanced liver fibrosis on treatment with vitamin $\mathrm{K}$ or non-vitamin $\mathrm{K}$ antagonist oral anticoagulants. Int J Cardiol 2018;264:58-63.

471. Kuo L, Chao TF, Liu CJ, Lin YJ, Chang SL, Lo LW, Hu YF, Tuan TC, Liao JN, Chung FP, Chen TJ, Lip GYH, Chen SA. Liver cirrhosis in patients with atrial fibrillation: would oral anticoagulation have a net clinical benefit for stroke prevention?] Am Heart Assoc 2017;6

472. Lee SR, Lee HJ, Choi EK, Han KD, Jung JH, Cha MJ, On S, Lip GYH. Direct oral anticoagulants in patients with atrial fibrillation and liver disease. I Am Coll Cardiol 2019;73:3295-3308.

473. Staerk L, Lip GY, Olesen JB, Fosbol EL, Pallisgaard JL, Bonde AN, Gundlund A Lindhardt TB, Hansen ML, Torp-Pedersen C, Gislason GH. Stroke and recurrent haemorrhage associated with antithrombotic treatment after gastrointestinal bleeding in patients with atrial fibrillation: nationwide cohort study. BMJ 2015;351:h5876.

474. Eckman MH, Singer DE, Rosand J, Greenberg SM. Moving the tipping point: the decision to anticoagulate patients with atrial fibrillation. Circ Cardiovasc Qual Outcomes 2011;4:14-21.

475. Proietti M, Lip GY. Major outcomes in atrial fibrillation patients with one risk factor: impact of time in therapeutic range observations from the SPORTIF trials. Am J Med 2016;129:1110-1116. 
476. Lip GY, Nielsen PB. Should patients with atrial fibrillation and 1 stroke risk factor (CHA2DS2-VASc Score 1 in Men, 2 in Women) be anticoagulated? Yes: even 1 stroke risk factor confers a real risk of stroke. Circulation 2016;133:1498-1503; discussion 1503.

477. Lip GY, Lane DA. Stroke prevention in atrial fibrillation: a systematic review. JAMA 2015;313:1950-1962.

478. Hijazi Z, Hohnloser SH, Andersson U, Alexander JH, Hanna M, Keltai M, Parkhomenko A, Lopez-Sendon JL, Lopes RD, Siegbahn A, Granger CB, Wallentin L. Efficacy and safety of apixaban compared with warfarin in patients with atrial fibrillation in relation to renal function over time: insights from the ARISTOTLE randomized clinical trial. JAMA Cardiol 2016;1:451-460.

479. Bohm M, Ezekowitz MD, Connolly SJ, Eikelboom JW, Hohnloser SH, Reilly PA, Schumacher H, Brueckmann M, Schirmer SH, Kratz MT, Yusuf S, Diener HC, Hijazi Z, Wallentin L. Changes in renal function in patients with atrial fibrillation: an analysis from the RE-LY trial. J Am Coll Cardiol 2015;65:2481-2493.

480. Clarkesmith DE, Pattison HM, Lip GY, Lane DA. Educational intervention improves anticoagulation control in atrial fibrillation patients: the TREAT randomised trial. PLoS One 2013;8:e74037.

481. Teiger E, Thambo JB, Defaye P, Hermida JS, Abbey S, Klug D, Juliard JM, Pasquie JL, Rioufol G, Lepillier A, Elbaz M, Horvilleur J, Brenot P, Pierre B, Le Corvoisier P. Percutaneous left atrial appendage closure is a reasonable option for patients with atrial fibrillation at high risk for cerebrovascular events. Circ Cardiovasc Interv 2018;11:e005841.

482. Saw J, Fahmy P, Azzalini L, Marquis JF, Hibbert B, Morillo C, Carrizo A, Ibrahim R. Early Canadian multicenter experience with WATCHMAN for percutaneous left atrial appendage closure. J Cardiovasc Electrophysiol 2017;28:396-401.

483. Martin Gutierrez E, Castano M, Gualis J, Martinez-Comendador JM, Maiorano P, Castillo L, Laguna G. Beneficial effect of left atrial appendage closure during cardiac surgery: a meta-analysis of 280585 patients. Eur J Cardiothorac Surg 2020;57:252-262.

484. Al-Khatib SM, Allen LaPointe NM, Chatterjee R, Crowley MJ, Dupre ME, Kong DF, Lopes RD, Povsic TJ, Raju SS, Shah B, Kosinski AS, McBroom AJ, Sanders GD. Rate- and rhythm-control therapies in patients with atrial fibrillation: a systematic review. Ann Intern Med 2014;160:760-773.

485. Tamariz LJ, Bass EB. Pharmacological rate control of atrial fibrillation. Cardiol Clin 2004:22:35-45.

486. Nikolaidou T, Channer KS. Chronic atrial fibrillation: a systematic review of medical heart rate control management. Postgrad Med J 2009;85:303-312.

487. Groenveld HF, Crijns HJ, Van den Berg MP, Van Sonderen E, Alings AM, Tijssen JG, Hillege HL, Tuininga YS, Van Veldhuisen DJ, Ranchor AV, Van Gelder IC; RACE II Investigators. The effect of rate control on quality of life in patients with permanent atrial fibrillation: data from the RACE II (Rate Control Efficacy in Permanent Atrial Fibrillation II) study. J Am Coll Cardiol 2011;58:1795-1803.

488. Van Gelder IC, Groenveld HF, Crijns HJ, Tuininga YS, Tijssen JG, Alings AM, Hillege HL, Bergsma-Kadijk JA, Cornel JH, Kamp O, Tukkie R, Bosker HA, Van Veldhuisen DJ, Van den Berg MP; RACE II Investigators. Lenient versus strict rate control in patients with atrial fibrillation. $N$ Engl I Med 2010;362:1363-1373.

489. Van Gelder IC, Wyse DG, Chandler ML, Cooper HA, Olshansky B, Hagens VE, Crijns HJ; RACE and AFFIRM Investigators. Does intensity of rate-control influence outcome in atrial fibrillation? An analysis of pooled data from the RACE and AFFIRM studies. Europace 2006;8:935-942.

490. Van Gelder IC, Rienstra M, Crijns HJ, Olshansky B. Rate control in atrial fibrillation. Lancet 2016;388:818-828.

491. Kotecha D, Holmes J, Krum H, Altman DG, Manzano L, Cleland JG, Lip GY, Coats AJ, Andersson B, Kirchhof P, von Lueder TG, Wedel H, Rosano G, Shibata MC, Rigby A, Flather MD. Efficacy of beta blockers in patients with heart failure plus atrial fibrillation: an individual-patient data meta-analysis. Lancet 2014;384:2235-2243.

492. Ulimoen SR, Enger S, Carlson J, Platonov PG, Pripp AH, Abdelnoor M, Arnesen $H$, Gjesdal K, Tveit A. Comparison of four single-drug regimens on ventricular rate and arrhythmia-related symptoms in patients with permanent atrial fibrillation. Am J Cardiol 2013;111:225-230.

493. Ulimoen SR, Enger S, Pripp AH, Abdelnoor M, Arnesen H, Gjesdal K, Tveit A. Calcium channel blockers improve exercise capacity and reduce $\mathrm{N}$-terminal Pro-B-type natriuretic peptide levels compared with beta-blockers in patients with permanent atrial fibrillation. Eur Heart J 2014;35:517-524.

494. Figulla HR, Gietzen F, Zeymer U, Raiber M, Hegselmann J, Soballa R, Hilgers R. Diltiazem improves cardiac function and exercise capacity in patients with idiopathic dilated cardiomyopathy. Results of the Diltiazem in Dilated Cardiomyopathy trial. Circulation 1996;94:346-352.

495. Hallberg P, Lindback J, Lindahl B, Stenestrand U, Melhus H, group R-H. Digoxin and mortality in atrial fibrillation: a prospective cohort study. Eur J Clin Pharmacol 2007;63:959-971.

496. Turakhia MP, Santangeli P, Winkelmayer WC, Xu X, Ullal AJ, Than CT, Schmitt
S, Holmes TH, Frayne SM, Phibbs CS, Yang F, Hoang DD, Ho PM, Heidenreich PA. Increased mortality associated with digoxin in contemporary patients with atrial fibrillation: findings from the TREAT-AF study. I Am Coll Cardiol 2014;64:660-668.

497. Whitbeck MG, Charnigo RJ, Khairy P, Ziada K, Bailey AL, Zegarra MM, Shah J, Morales G, Macaulay T, Sorrell VL, Campbell CL, Gurley J, Anaya P, Nasr H, Bai R, Di Biase L, Booth DC, Jondeau G, Natale A, Roy D, Smyth S, Moliterno DJ, Elayi CS. Increased mortality among patients taking digoxin - analysis from the AFFIRM study. Eur Heart J 2013;34:1481-1488.

498. Andrey JL, Romero S, Garcia-Egido A, Escobar MA, Corzo R, GarciaDominguez G, Lechuga V, Gomez F. Mortality and morbidity of heart failure treated with digoxin. A propensity-matched study. Int J Clin Pract 2011; 65:1250-1258.

499. Flory JH, Ky B, Haynes K, S MB, Munson J, Rowan, C, Strom BL, Hennessy S. Observational cohort study of the safety of digoxin use in women with heart failure. BMJ Open 2012;2:e000888.

500. Gheorghiade M, Fonarow GC, van Veldhuisen DJ, Cleland JG, Butler J, Epstein AE, Patel K, Aban IB, Aronow WS, Anker SD, Ahmed A. Lack of evidence of increased mortality among patients with atrial fibrillation taking digoxin: findings from post hoc propensity-matched analysis of the AFFIRM trial. Eur Heart J 2013;34:1489-1497.

501. Aguirre Davila L, Weber K, Bavendiek U, Bauersachs J, Wittes J, Yusuf S, Koch A. Digoxin-mortality: randomized vs. observational comparison in the DIG trial. Eur Heart J 2019;40:3336-3341.

502. Ziff OJ, Lane DA, Samra M, Griffith M, Kirchhof P, Lip GY, Steeds RP, Townend J, Kotecha D. Safety and efficacy of digoxin: systematic review and meta-analysis of observational and controlled trial data. BM/ 2015;351:h4451.

503. Bavendiek U, Berliner D, Davila LA, Schwab J, Maier L, Philipp SA, Rieth A, Westenfeld R, Piorkowski C, Weber K, Hanselmann A, Oldhafer M, Schallhorn S, von der Leyen H, Schroder C, Veltmann C, Stork S, Bohm M, Koch A, Bauersachs J; DIGIT-HF Investigators and Committees. Rationale and design of the DIGIT-HF trial (DIGitoxin to Improve ouTcomes in patients with advanced chronic Heart Failure): a randomized, double-blind, placebo-controlled study. Eur J Heart Fail 2019;21:676-684.

504. Clemo HF, Wood MA, Gilligan DM, Ellenbogen KA. Intravenous amiodarone for acute heart rate control in the critically ill patient with atrial tachyarrhythmias. Am J Cardiol 1998;81:594-598.

505. Klijn CJ, Paciaroni M, Berge E, Korompoki E, Korv J, Lal A, Putaala J, Werring DJ. Antithrombotic treatment for secondary prevention of stroke and other thromboembolic events in patients with stroke or transient ischemic attack and non-valvular atrial fibrillation: a European Stroke Organisation guideline. Eur Stroke J 2019;4:198-223.

506. Gosselink AT, Crijns HJ, Van Gelder IC, Hillige H, Wiesfeld AC, Lie KI. Lowdose amiodarone for maintenance of sinus rhythm after cardioversion of atrial fibrillation or flutter. JAMA 1992;267:3289-3293.

507. Scheuermeyer FX, Grafstein E, Stenstrom R, Christenson J, Heslop C, Heilbron B, McGrath L, Innes G. Safety and efficiency of calcium channel blockers versus beta-blockers for rate control in patients with atrial fibrillation and no acute underlying medical illness. Acad Emerg Med 2013;20:222-230.

508. Schreck DM, Rivera AR, Tricarico VJ. Emergency management of atrial fibrillation and flutter: intravenous diltiazem versus intravenous digoxin. Ann Emerg Med 1997;29:135-140.

509. Segal JB, McNamara RL, Miller MR, Kim N, Goodman SN, Powe NR, Robinson $\mathrm{K}, \mathrm{Yu} \mathrm{D}$, Bass EB. The evidence regarding the drugs used for ventricular rate control. J Fam Pract 2000;49:47-59.

510. Siu CW, Lau CP, Lee WL, Lam KF, Tse HF. Intravenous diltiazem is superior to intravenous amiodarone or digoxin for achieving ventricular rate control in patients with acute uncomplicated atrial fibrillation. Crit Care Med 2009;37:2174-2179; quiz 2180.

511. Tisdale JE, Padhi ID, Goldberg AD, Silverman NA, Webb CR, Higgins RS, Paone G, Frank DM, Borzak S. A randomized, double-blind comparison of intravenous diltiazem and digoxin for atrial fibrillation after coronary artery bypass surgery. Am Heart J 1998;135:739-747.

512. Darby AE, Dimarco JP. Management of atrial fibrillation in patients with structural heart disease. Circulation 2012;125:945-957.

513. Kotecha D, Piccini JP. Atrial fibrillation in heart failure: what should we do?Eur Heart J 2015;36:3250-3257.

514. Delle Karth G, Geppert A, Neunteufl T, Priglinger U, Haumer M, Gschwandtner M, Siostrzonek P, Heinz G. Amiodarone versus diltiazem for rate control in critically ill patients with atrial tachyarrhythmias. Crit Care Med 2001;29:1149-1153.

515. Hou ZY, Chang MS, Chen CY, Tu MS, Lin SL, Chiang HT, Woosley RL. Acute treatment of recent-onset atrial fibrillation and flutter with a tailored dosing regimen of intravenous amiodarone. A randomized, digoxin-controlled study. Eur Heart J 1995;16:521-528. 
516. Lim KT, Davis MJ, Powell A, Arnolda L, Moulden K, Bulsara M, Weerasooriya R. Ablate and pace strategy for atrial fibrillation: long-term outcome of AIRCRAFT trial. Europace 2007;9:498-505.

517. Queiroga A, Marshall HJ, Clune M, Gammage MD. Ablate and pace revisited: long term survival and predictors of permanent atrial fibrillation. Heart 2003;89:1035-1038

518. Geelen P, Brugada J, Andries E, Brugada P. Ventricular fibrillation and sudden death after radiofrequency catheter ablation of the atrioventricular junction. Pacing Clin Electrophysiol 1997;20:343-348.

519. Wang RX, Lee HC, Hodge DO, Cha YM, Friedman PA, Rea RF, Munger TM, Jahangir A, Srivathsan K, Shen WK. Effect of pacing method on risk of sudden death after atrioventricular node ablation and pacemaker implantation in patients with atrial fibrillation. Heart Rhythm 2013;10:696-701.

520. Chatterjee NA, Upadhyay GA, Ellenbogen KA, McAlister FA, Choudhry NK, Singh JP. Atrioventricular nodal ablation in atrial fibrillation: a meta-analysis and systematic review. Circ Arrhythm Electrophysiol 2012;5:68-76.

521. Bradley DJ, Shen WK. Overview of management of atrial fibrillation in symptomatic elderly patients: pharmacologic therapy versus AV node ablation. Clin Pharmacol Ther 2007;81:284-287.

522. Ozcan C, Jahangir A, Friedman PA, Patel PJ, Munger TM, Rea RF, Lloyd MA, Packer DL, Hodge DO, Gersh BJ, Hammill SC, Shen WK. Long-term survival after ablation of the atrioventricular node and implantation of a permanent pacemaker in patients with atrial fibrillation. $N$ Engl J Med 2001;344:1043-1051.

523. Wood MA, Brown-Mahoney C, Kay GN, Ellenbogen KA. Clinical outcomes after ablation and pacing therapy for atrial fibrillation: a meta-analysis. Circulation 2000;101:1138-1144.

524. Brignole M, Auricchio A, Baron-Esquivias G, Bordachar P, Boriani G, Breithardt OA, Cleland J, Deharo JC, Delgado V, Elliott PM, Gorenek B, Israel CW, Leclercq C, Linde C, Mont L, Padeletti L, Sutton R, Vardas PE, ESC Committee for Practice Guidelines, Zamorano JL, Achenbach S, Baumgartner H, Bax JJ, Bueno H, Dean V, Deaton C, Erol C, Fagard R, Ferrari R, Hasdai D, Hoes AW, Kirchhof P, Knuuti J, Kolh P, Lancellotti P, Linhart A, Nihoyannopoulos P, Piepoli MF, Ponikowski P, Sirnes PA, Tamargo JL, Tendera M, Torbicki A, Wijns W, Windecker S, Document R, Kirchhof P, Blomstrom-Lundqvist C, Badano LP, Aliyev F, Bansch D, Baumgartner H, Bsata W, Buser P, Charron P, Daubert JC, Dobreanu D, Faerestrand S, Hasdai D, Hoes AW, Le Heuzey JY, Mavrakis H, McDonagh T, Merino JL, Nawar MM, Nielsen JC, Pieske B, Poposka L, Ruschitzka F, Tendera M, Van Gelder IC, Wilson CM. 2013 ESC Guidelines on cardiac pacing and cardiac resynchronization therapy: the Task Force on cardiac pacing and resynchronization therapy of the European Society of Cardiology (ESC). Developed in collaboration with the European Heart Rhythm Association (EHRA). Eur Heart J 2013;34:2281-2329.

525. Chatterjee NA, Upadhyay GA, Ellenbogen KA, Hayes DL, Singh JP. Atrioventricular nodal ablation in atrial fibrillation: a meta-analysis of biventricular vs. right ventricular pacing mode. Eur I Heart Fail 2012;14:661-667.

526. Huang W, Su L, Wu S. Pacing treatment of atrial fibrillation patients with heart failure: His bundle pacing combined with atrioventricular node ablation. Card Electrophysiol Clin 2018;10:519-535.

527. Brignole M, Pokushalov E, Pentimalli F, Palmisano P, Chieffo E, Occhetta E, Quartieri F, Calo L, Ungar A, Mont L; APAF-CRT Investigators. A randomized controlled trial of atrioventricular junction ablation and cardiac resynchronization therapy in patients with permanent atrial fibrillation and narrow QRS. Eur Heart J 2018;39:3999-4008.

528. Huang W, Su L, Wu S, Xu L, Xiao F, Zhou X, Ellenbogen KA. Benefits of permanent His bundle pacing combined with atrioventricular node ablation in atrial fibrillation patients with heart failure with both preserved and reduced left ventricular ejection fraction. J Am Heart Assoc 2017;6.

529. Farshi R, Kistner D, Sarma JS, Longmate JA, Singh BN. Ventricular rate control in chronic atrial fibrillation during daily activity and programmed exercise: a crossover open-label study of five drug regimens. I Am Coll Cardiol 1999;33:304-310.

530. Khand AU, Rankin AC, Martin W, Taylor J, Gemmell I, Cleland JG. Carvedilol alone or in combination with digoxin for the management of atrial fibrillation in patients with heart failure?] Am Coll Cardiol 2003;42:1944-1951.

531. Lewis RV, Irvine N, McDevitt DG. Relationships between heart rate, exercise tolerance and cardiac output in atrial fibrillation: the effects of treatment with digoxin, verapamil and diltiazem. Eur Heart J 1988;9:777-781.

532. Mulder BA, Van Veldhuisen DJ, Crijns HJ, Tijssen JG, Hillege HL, Alings M, Rienstra M, Van den Berg MP, Van Gelder IC. Digoxin in patients with permanent atrial fibrillation: data from the RACE II study. Heart Rhythm 2014;11:1543-1550.

533. Roth A, Harrison E, Mitani G, Cohen J, Rahimtoola SH, Elkayam U. Efficacy and safety of medium- and high-dose diltiazem alone and in combination with digoxin for control of heart rate at rest and during exercise in patients with chronic atrial fibrillation. Circulation 1986;73:316-324

534. David D, Segni ED, Klein HO, Kaplinsky E. Inefficacy of digitalis in the control of heart rate in patients with chronic atrial fibrillation: beneficial effect of an added beta adrenergic blocking agent. Am J Cardiol 1979;44:1378-1382.

535. Weerasooriya R, Davis M, Powell A, Szili-Torok T, Shah C, Whalley D, Kanagaratnam L, Heddle W, Leitch J, Perks A, Ferguson L, Bulsara M. The Australian Intervention Randomized Control of Rate in Atrial Fibrillation Trial (AIRCRAFT). J Am Coll Cardiol 2003;41:1697-1702.

536. Vijayaraman $P$, Subzposh FA, Naperkowski A. Atrioventricular node ablation and His bundle pacing. Europace 2017;19:iv10-iv16.

537. Shiga T, Yoshioka K, Watanabe E, Omori H, Yagi M, Okumura Y, Matsumoto N, Kusano K, Oshiro C, lkeda T, Takahashi N, Komatsu T, Suzuki A, Suzuki T, Sato Y, Yamashita T; AF-QOL study investigators. Paroxysmal atrial fibrillation recurrences and quality of life in symptomatic patients: a crossover study of flecainide and pilsicainide. J Arrhythm 2017;33:310-317.

538. Capucci A, Piangerelli L, Ricciotti J, Gabrielli D, Guerra F. Flecainide-metoprolol combination reduces atrial fibrillation clinical recurrences and improves tolerability at 1-year follow-up in persistent symptomatic atrial fibrillation. Europace 2016;18:1698-1704.

539. Shi LZ, Heng R, Liu SM, Leng FY. Effect of catheter ablation versus antiarrhythmic drugs on atrial fibrillation: a meta-analysis of randomized controlled trials. Exp Ther Med 2015;10:816-822.

540. Siontis KC, loannidis JPA, Katritsis GD, Noseworthy PA, Packer DL, Hummel JD, Jais P, Krittayaphong R, Mont L, Morillo CA, Nielsen JC, Oral H, Pappone C, Santinelli V, Weerasooriya R, Wilber DJ, Gersh BJ, Josephson ME, Katritsis DG. Radiofrequency ablation versus antiarrhythmic drug therapy for atrial fibrillation: meta-analysis of quality of life, morbidity, and mortality. JACC Clin Electrophysiol 2016;2:170-180.

541. Kim YG, Shim J, Choi Jl, Kim YH. Radiofrequency catheter ablation improves the quality of life measured with a short form-36 questionnaire in atrial fibrillation patients: a systematic review and meta-analysis. PLoS One 2016;11:e0163755.

542. Bayes de Luna A, Platonov P, Cosio FG, Cygankiewicz I, Pastore C, Baranowski R, Bayes-Genis A, Guindo J, Vinolas X, Garcia-Niebla J, Barbosa R, Stern S, Spodick D. Interatrial blocks. A separate entity from left atrial enlargement: a consensus report. J Electrocardiol 2012;45:445-451.

543. Jadidi A, Muller-Edenborn B, Chen J, Keyl C, Weber R, Allgeier J, MorenoWeidmann Z, Trenk D, Neumann Fj, Lehrmann H, Arentz T. The duration of the amplified sinus-p-wave identifies presence of left atrial low voltage substrate and predicts outcome after pulmonary vein isolation in patients with persistent atrial fibrillation. JACC Clin Electrophysiol 2018;4:531-543.

544. Dudink E, Erkuner O, Berg J, Nieuwlaat R, de Vos CB, Weijs B, Capucci A, Camm AJ, Breithardt G, Le Heuzey JY, Luermans J, Crijns $H$. The influence of progression of atrial fibrillation on quality of life: a report from the Euro Heart Survey. Europace 2018;20:929-934.

545. Zhang YY, Qiu C, Davis PJ, Jhaveri M, Prystowsky EN, Kowey P, Weintraub WS. Predictors of progression of recently diagnosed atrial fibrillation in REgistry on Cardiac Rhythm DisORDers Assessing the Control of Atrial Fibrillation (RecordAF) - United States cohort. Am J Cardiol 2013;112:79-84.

546. Bunch TJ, May HT, Bair TL, Johnson DL, Weiss JP, Crandall BG, Osborn JS, Anderson JL, Muhlestein JB, Lappe DL, Day JD. Increasing time between first diagnosis of atrial fibrillation and catheter ablation adversely affects long-term outcomes. Heart Rhythm 2013;10:1257-1262.

547. Andrade JG, Champagne J, Deyell MW, Essebag V, Lauck S, Morillo C, Sapp J, Skanes A, Theoret-Patrick P, Wells GA, Verma A; EARLY-AF Study Investigators. A randomized clinical trial of early invasive intervention for atrial fibrillation (EARLY-AF) - methods and rationale. Am Heart J 2018;206:94-104

548. Teh AW, Kistler PM, Lee G, Medi C, Heck PM, Spence SJ, Morton JB, Sanders $\mathrm{P}$, Kalman JM. Long-term effects of catheter ablation for lone atrial fibrillation: progressive atrial electroanatomic substrate remodeling despite successful ablation. Heart Rhythm 2012;9:473-480.

549. Aliot E, Brandes A, Eckardt L, Elvan A, Gulizia M, Heidbuchel H, Kautzner J, Mont L, Morgan J, Ng A, Szumowski L, Themistoclakis S, Van Gelder IC Willems S, Kirchhof P. The EAST study: redefining the role of rhythmcontrol therapy in atrial fibrillation: EAST, the Early treatment of Atrial fibrillation for Stroke prevention Trial. Eur Heart J 2015;36:255-256.

550. Michelena HI, Powell BD, Brady PA, Friedman PA, Ezekowitz MD. Gender in atrial fibrillation: ten years later. Gend Med 2010;7:206-217.

551. Sethi NJ, Feinberg J, Nielsen EE, Safi S, Gluud C, Jakobsen JC. The effects of rhythm control strategies versus rate control strategies for atrial fibrillation and atrial flutter: a systematic review with meta-analysis and trial sequential analysis. PLoS One 2017;12:e0186856.

552. Ha AC, Breithardt G, Camm AJ, Crijns HJ, Fitzmaurice GM, Kowey PR, Le Heuzey JY, Naditch-Brule L, Prystowsky EN, Schwartz PJ, Torp-Pedersen C, 
Weintraub WS, Dorian P. Health-related quality of life in patients with atrial fibrillation treated with rhythm control versus rate control: insights from a prospective international registry (Registry on Cardiac Rhythm Disorders Assessing the Control of Atrial Fibrillation: RECORD-AF). Circ Cardiovasc Qual Outcomes 2014;7:896-904

553. Bulkova V, Fiala M, Havranek S, Simek J, Sknouril L, Januska J, Spinar J, Wichterle D. Improvement in quality of life after catheter ablation for paroxysmal versus long-standing persistent atrial fibrillation: a prospective study with 3year follow-up. J Am Heart Assoc 2014;3.

554. Kirchhof P, Monnig G, Wasmer K, Heinecke A, Breithardt G, Eckardt L, Bocker $D$. A trial of self-adhesive patch electrodes and hand-held paddle electrodes for external cardioversion of atrial fibrillation (MOBIPAPA). Eur Heart $J$ 2005;26:1292-1297.

555. Kirchhof P, Eckardt L, Loh P, Weber K, Fischer RJ, Seidl KH, Bocker D, Breithardt G, Haverkamp W, Borggrefe M. Anterior-posterior versus anteriorlateral electrode positions for external cardioversion of atrial fibrillation: a randomised trial. Lancet 2002;360:1275-1279.

556. Um KJ, Mclntyre WF, Healey JS, Mendoza PA, Koziarz A, Amit G, Chu VA, Whitlock RP, Belley-Cote EP. Pre- and post-treatment with amiodarone for elective electrical cardioversion of atrial fibrillation: a systematic review and meta-analysis. Europace 2019;21:856-863.

557. Schmidt AS, Lauridsen KG, Torp P, Bach LF, Rickers H, Lofgren B. Maximumfixed energy shocks for cardioverting atrial fibrillation. Eur Heart J 2020;41:626-631.

558. Pluymaekers N, Dudink E, Luermans J, Meeder JG, Lenderink T, Widdershoven J, Bucx JJJ, Rienstra M, Kamp O, Van Opstal JM, Alings M, Oomen A, Kirchhof C], Van Dijk VF, Ramanna H, Liem A, Dekker LR, Essers BAB, Tijssen JGP, Van Gelder IC, Crijns H; RACE ACWAS Investigators. Early or delayed cardioversion in recent-onset atrial fibrillation. N Engl J Med 2019;380:1499-1508.

559. Baranchuk A, Yeung C. Advanced interatrial block predicts atrial fibrillation recurrence across different populations: learning Bayes syndrome. Int J Cardiol 2018;272:221-222.

560. Toufan M, Kazemi B, Molazadeh N. The significance of the left atrial volume index in prediction of atrial fibrillation recurrence after electrical cardioversion. J Cardiovasc Thorac Res 2017;9:54-59.

561. Voskoboinik A, Kalman E, Plunkett G, Knott J, Moskovitch J, Sanders P, Kistler PM, Kalman JM. A comparison of early versus delayed elective electrical cardioversion for recurrent episodes of persistent atrial fibrillation: a multi-center study. Int J Cardiol 2019;284:33-37.

562. Furniss SS, Sneyd JR. Safe sedation in modern cardiological practice. Heart 2015;101:1526-1530.

563. Mittal S, Ayati S, Stein KM, Schwartzman D, Cavlovich D, Tchou PJ, Markowitz SM, Slotwiner DJ, Scheiner MA, Lerman BB. Transthoracic cardioversion of atrial fibrillation: comparison of rectilinear biphasic versus damped sine wave monophasic shocks. Circulation 2000;101:1282-1287.

564. Inacio JF, da Rosa Mdos S, Shah J, Rosario J, Vissoci JR, Manica AL, Rodrigues CG. Monophasic and biphasic shock for transthoracic conversion of atrial fibrillation: systematic review and network meta-analysis. Resuscitation 2016;100:66-75.

565. Kirkland S, Stiell I, AlShawabkeh T, Campbell S, Dickinson G, Rowe BH. The efficacy of pad placement for electrical cardioversion of atrial fibrillation/flutter: a systematic review. Acad Emerg Med 2014;21:717-726.

566. Boriani G, Diemberger I, Biffi M, Martignani C, Branzi A. Pharmacological cardioversion of atrial fibrillation: current management and treatment options. Drugs 2004;64:2741-2762.

567. Danias PG, Caulfield TA, Weigner MJ, Silverman DI, Manning WJ. Likelihood of spontaneous conversion of atrial fibrillation to sinus rhythm. J Am Coll Cardiol 1998;31:588-592.

568. Dan GA, Martinez-Rubio A, Agewall S, Boriani G, Borggrefe M, Gaita F, van Gelder I, Gorenek B, Kaski JC, Kjeldsen K, Lip GYH, Merkely B, Okumura K, Piccini JP, Potpara T, Poulsen BK, Saba M, Savelieva I, Tamargo JL, Wolpert C, ESC Scientific Document Group. Antiarrhythmic drugs-clinical use and clinical decision making: a consensus document from the European Heart Rhythm Association (EHRA) and European Society of Cardiology (ESC) Working Group on Cardiovascular Pharmacology, endorsed by the Heart Rhythm Society (HRS), Asia-Pacific Heart Rhythm Society (APHRS) and International Society of Cardiovascular Pharmacotherapy (ISCP). Europace 2018;20:731-732.

569. Markey GC, Salter N, Ryan J. Intravenous flecainide for emergency department management of acute atrial fibrillation. J Emerg Med 2018;54:320-327.

570. Chevalier P, Durand-Dubief A, Burri H, Cucherat M, Kirkorian G, Touboul P. Amiodarone versus placebo and class lc drugs for cardioversion of recentonset atrial fibrillation: a meta-analysis. J Am Coll Cardiol 2003;41:255-262.

571. Capucci A, Lenzi T, Boriani G, Trisolino G, Binetti N, Cavazza M, Fontana G, Magnani B. Effectiveness of loading oral flecainide for converting recent-onset atrial fibrillation to sinus rhythm in patients without organic heart disease or with only systemic hypertension. Am J Cardiol 1992;70:69-72.

572. Donovan KD, Dobb GJ, Coombs LJ, Lee KY, Weekes JN, Murdock CJ, Clarke GM. Efficacy of flecainide for the reversion of acute onset atrial fibrillation. Am J Cardiol 1992;70:50A-54A; discussion 54A-55A.

573. Reisinger J, Gatterer E, Lang W, Vanicek T, Eisserer G, Bachleitner T, Niemeth C, Aicher F, Grander W, Heinze G, Kuhn P, Siostrzonek P. Flecainide versus ibutilide for immediate cardioversion of atrial fibrillation of recent onset. Eur Heart J 2004;25:1318-1324.

574. Khan IA. Oral loading single dose flecainide for pharmacological cardioversion of recent-onset atrial fibrillation. Int J Cardiol 2003;87:121-128.

575. Galve E, Rius T, Ballester R, Artaza MA, Arnau JM, Garcia-Dorado D, SolerSoler J. Intravenous amiodarone in treatment of recent-onset atrial fibrillation: results of a randomized, controlled study. I Am Coll Cardiol 1996;27:1079-1082.

576. Vardas PE, Kochiadakis GE, Igoumenidis NE, Tsatsakis AM, Simantirakis EN, Chlouverakis $\mathrm{Gl}$. Amiodarone as a first-choice drug for restoring sinus rhythm in patients with atrial fibrillation: a randomized, controlled study. Chest 2000;117:1538-1545.

577. Letelier LM, Udol K, Ena J, Weaver B, Guyatt GH. Effectiveness of amiodarone for conversion of atrial fibrillation to sinus rhythm: a meta-analysis. Arch Intern Med 2003;163:777-785.

578. Bash LD, Buono JL, Davies GM, Martin A, Fahrbach K, Phatak H, Avetisyan R, Mwamburi M. Systematic review and meta-analysis of the efficacy of cardioversion by vernakalant and comparators in patients with atrial fibrillation. Cardiovasc Drugs Ther 2012;26:167-179.

579. Camm AJ, Capucci A, Hohnloser SH, Torp-Pedersen C, Van Gelder IC, Mangal $B$, Beatch G; AVRO Investigators. A randomized active-controlled study comparing the efficacy and safety of vernakalant to amiodarone in recent-onset atrial fibrillation. J Am Coll Cardiol 2011;57:313-321.

580. Akel T, Lafferty J. Efficacy and safety of intravenous vernakalant for the rapid conversion of recent-onset atrial fibrillation: a meta-analysis. Ann Noninvasive Electrocardiol 2018;23:e12508.

581. Beatch GN, Mangal B. Safety and efficacy of vernakalant for the conversion of atrial fibrillation to sinus rhythm; a phase $3 \mathrm{~b}$ randomized controlled trial. BMC Cardiovasc Disord 2016;16:113.

582. Roy D, Pratt CM, Torp-Pedersen C, Wyse DG, Toft E, Juul-Moller S, Nielsen T, Rasmussen SL, Stiell IG, Coutu B, Ip JH, Pritchett EL, Camm AJ; Atrial Arrhythmia Conversion Trial Investigators. Vernakalant hydrochloride for rapid conversion of atrial fibrillation: a phase 3, randomized, placebo-controlled trial. Circulation 2008;117:1518-1525.

583. Kowey PR, Dorian P, Mitchell LB, Pratt CM, Roy D, Schwartz PJ, Sadowski J, Sobczyk D, Bochenek A, Toft E; Atrial Arrhythmia Conversion Trial Investigators. Vernakalant hydrochloride for the rapid conversion of atrial fibrillation after cardiac surgery: a randomized, double-blind, placebo-controlled trial. Circ Arrhythm Electrophysiol 2009;2:652-659.

584. Pohjantahti-Maaroos H, Hyppola H, Lekkala M, Sinisalo E, Heikkola A, Hartikainen J. Intravenous vernakalant in comparison with intravenous flecainide in the cardioversion of recent-onset atrial fibrillation. Eur Heart J Acute Cardiovasc Care 2019;8:114-120.

585. Vos MA, Golitsyn SR, Stangl K, Ruda MY, Van Wijk LV, Harry JD, Perry KT, Touboul P, Steinbeck G, Wellens HJ. Superiority of ibutilide (a new class III agent) over DL-sotalol in converting atrial flutter and atrial fibrillation. The Ibutilide/Sotalol Comparator Study Group. Heart 1998;79:568-575.

586. Alboni P, Botto GL, Baldi N, Luzi M, Russo V, Gianfranchi L, Marchi P, Calzolari M, Solano A, Baroffio R, Gaggioli G. Outpatient treatment of recent-onset atrial fibrillation with the 'pill-in-the-pocket' approach. N Engl J Med 2004;351:2384-2391.

587. Brembilla-Perrot B, Houriez P, Beurrier D, Claudon O, Terrier de la Chaise A, Louis $P$. Predictors of atrial flutter with 1:1 conduction in patients treated with class I antiarrhythmic drugs for atrial tachyarrhythmias. Int I Cardiol 2001;80:7-15.

588. Zhang N, Guo JH, Zhang H, Li XB, Zhang P, Xn Y. Comparison of intravenous ibutilide vs. propafenone for rapid termination of recent onset atrial fibrillation. Int J Clin Pract 2005;59:1395-1400.

589. Conde D, Costabel JP, Caro M, Ferro A, Lambardi F, Corrales Barboza A, Lavalle Cobo A, Trivi M. Flecainide versus vernakalant for conversion of recentonset atrial fibrillation. Int J Cardiol 2013;168:2423-2425.

590. Martinez-Marcos FJ, Garcia-Garmendia JL, Ortega-Carpio A, Fernandez-Gomez JM, Santos JM, Camacho C. Comparison of intravenous flecainide, propafenone, and amiodarone for conversion of acute atrial fibrillation to sinus rhythm. Am J Cardiol 2000;86:950-953.

591. Deedwania PC, Singh BN, Ellenbogen K, Fisher S, Fletcher R, Singh SN Spontaneous conversion and maintenance of sinus rhythm by amiodarone in patients with heart failure and atrial fibrillation: observations from the veterans 
affairs congestive heart failure survival trial of antiarrhythmic therapy (CHFSTAT). The Department of Veterans Affairs CHF-STAT Investigators. Circulation 1998;98:2574-2579.

592. Hofmann R, Steinwender C, Kammler J, Kypta A, Wimmer G, Leisch F. Intravenous amiodarone bolus for treatment of atrial fibrillation in patients with advanced congestive heart failure or cardiogenic shock. Wien Klin Wochenschr 2004;116:744-749.

593. Crijns HJ, Weijs B, Fairley AM, Lewalter T, Maggioni AP, Martin A, Ponikowski P, Rosenqvist M, Sanders P, Scanavacca M, Bash LD, Chazelle F, Bernhardt A, Gitt AK, Lip GY, Le Heuzey JY. Contemporary real life cardioversion of atrial fibrillation: results from the multinational RHYTHM-AF study. Int I Cardiol 2014;172:588-594

594. Kirchhof $P$, Andresen D, Bosch R, Borggrefe M, Meinertz T, Parade U, Ravens U, Samol A, Steinbeck G, Treszl A, Wegscheider K, Breithardt G. Short-term versus long-term antiarrhythmic drug treatment after cardioversion of atrial fibrillation (Flec-SL): a prospective, randomised, open-label, blinded endpoint assessment trial. Lancet 2012;380:238-246.

595. Van Gelder IC, Tuinenburg AE, Schoonderwoerd BS, Tieleman RG, Crijns HJ. Pharmacologic versus direct-current electrical cardioversion of atrial flutter and fibrillation. Am J Cardiol 1999:84:147R-151R.

596. Climent VE, Marin F, Mainar L, Gomez-Aldaravi R, Martinez JG, Chorro FJ, Roman P, Sogorb F. Effects of pretreatment with intravenous flecainide on efficacy of external cardioversion of persistent atrial fibrillation. Pacing Clin Electrophysiol 2004;27:368-372.

597. Mussigbrodt A, John S, Kosiuk J, Richter S, Hindricks G, Bollmann A. Vernakalant-facilitated electrical cardioversion: comparison of intravenous vernakalant and amiodarone for drug-enhanced electrical cardioversion of atrial fibrillation after failed electrical cardioversion. Europace 2016;18:51-56.

598. Singh SN, Tang XC, Reda D, Singh BN. Systematic electrocardioversion for atrial fibrillation and role of antiarrhythmic drugs: a substudy of the SAFE-T trial. Heart Rhythm 2009;6:152-155.

599. Oral H, Souza J], Michaud GF, Knight BP, Goyal R, Strickberger SA, Morady F. Facilitating transthoracic cardioversion of atrial fibrillation with ibutilide pretreatment. N Engl J Med 1999;340:1849-1854.

600. Khan IA. Single oral loading dose of propafenone for pharmacological cardioversion of recent-onset atrial fibrillation. I Am Coll Cardiol 2001;37:542-547.

601. Alboni P, Botto GL, Boriani G, Russo G, Pacchioni F, lori M, Pasanisi G, Mancini M, Mariconti B, Capucci A. Intravenous administration of flecainide or propafenone in patients with recent-onset atrial fibrillation does not predict adverse effects during 'pill-in-the-pocket' treatment. Heart 2010;96:546-549.

602. Arbelo E, Brugada J, Hindricks G, Maggioni A, Tavazzi L, Vardas P, Anselme F, Inama G, Jais P, Kalarus Z, Kautzner J, Lewalter T, Mairesse G, Perez-Villacastin J, Riahi S, Taborsky M, Theodorakis G, Trines S; Atrial Fibrillation Ablation Pilot Study Investigators. ESC-EURObservational Research Programme: the Atrial Fibrillation Ablation Pilot Study, conducted by the European Heart Rhythm Association. Europace 2012;14:1094-1103.

603. Arbelo E, Brugada J, Hindricks G, Maggioni AP, Tavazzi L, Vardas P, Laroche C, Anselme $F$, Inama G, Jais P, Kalarus Z, Kautzner J, Lewalter T, Mairesse GH, Perez-Villacastin J, Riahi S, Taborsky M, Theodorakis G, Trines SA; Atrial Fibrillation Ablation Pilot Study Investigators. The atrial fibrillation ablation pilot study: a European Survey on Methodology and results of catheter ablation for atrial fibrillation conducted by the European Heart Rhythm Association. Eur Heart J 2014;35:1466-1478.

604. Arbelo E, Brugada J, Blomstrom-Lundqvist C, Laroche C, Kautzner J, Pokushalov E, Raatikainen P, Efremidis M, Hindricks G, Barrera A, Maggioni A, Tavazzi L, Dagres N, on the behalf of the ESC EHRA Atrial Fibrillation Ablation Long-term Registry Investigators. Contemporary management of patients undergoing atrial fibrillation ablation: in-hospital and 1-year follow-up findings from the ESC-EHRA atrial fibrillation ablation long-term registry. Eur Heart J 2017;38:1303-1316.

605. Krittayaphong R, Raungrattanaamporn O, Bhuripanyo K, Sriratanasathavorn C, Pooranawattanakul S, Punlee K, Kangkagate C. A randomized clinical trial of the efficacy of radiofrequency catheter ablation and amiodarone in the treatment of symptomatic atrial fibrillation. J Med Assoc Thai 2003;86 Suppl 1:S8-16.

606. Stabile G, Bertaglia E, Senatore G, De Simone A, Zoppo F, Donnici G, Turco P, Pascotto P, Fazzari M, Vitale DF. Catheter ablation treatment in patients with drug-refractory atrial fibrillation: a prospective, multi-centre, randomized, controlled study (Catheter Ablation For The Cure Of Atrial Fibrillation Study). Eur Heart / 2006;27:216-221.

607. Pappone C, Augello G, Sala S, Gugliotta F, Vicedomini G, Gulletta S, Paglino G, Mazzone P, Sora N, Greiss I, Santagostino A, LiVolsi L, Pappone N, Radinovic A, Manguso F, Santinelli V. A randomized trial of circumferential pulmonary vein ablation versus antiarrhythmic drug therapy in paroxysmal atrial fibrillation: the APAF Study. I Am Coll Cardiol 2006;48:2340-2347.
608. Calkins H, Reynolds MR, Spector P, Sondhi M, Xu Y, Martin A, Williams CJ, Sledge I. Treatment of atrial fibrillation with antiarrhythmic drugs or radiofrequency ablation: two systematic literature reviews and meta-analyses. Circ Arrhythm Electrophysiol 2009:2:349-361.

609. Packer DL, Kowal RC, Wheelan KR, Irwin JM, Champagne J, Guerra PG, Dubuc M, Reddy V, Nelson L, Holcomb RG, Lehmann JW, Ruskin JN; STOP AF Cryoablation Investigators. Cryoballoon ablation of pulmonary veins for paroxysmal atrial fibrillation: first results of the North American Arctic Front (STOP AF) pivotal trial. J Am Coll Cardiol 2013;61:1713-1723.

610. Ganesan AN, Shipp NJ, Brooks AG, Kuklik P, Lau DH, Lim HS, Sullivan T, Roberts-Thomson KC, Sanders P. Long-term outcomes of catheter ablation of atrial fibrillation: a systematic review and meta-analysis. J Am Heart Assoc 2013;2:e004549.

611. Di Biase L, Mohanty P, Mohanty S, Santangeli P, Trivedi C, Lakkireddy D, Reddy M, Jais P, Themistoclakis S, Dello Russo A, Casella M, Pelargonio G, Narducci ML, Schweikert R, Neuzil P, Sanchez J, Horton R, Beheiry S, Hongo R, Hao S, Rossillo A, Forleo G, Tondo C, Burkhardt JD, Haissaguerre M, Natale A. Ablation versus amiodarone for treatment of persistent atrial fibrillation in patients with congestive heart failure and an implanted device: results from the AATAC multicenter randomized trial. Circulation 2016;133:1637-1644.

612. Kuck KH, Brugada J, Furnkranz A, Metzner A, Ouyang F, Chun KR, Elvan A, Arentz T, Bestehorn K, Pocock SJ, Albenque JP, Tondo C; FIRE AND ICE Investigators. Cryoballoon or radiofrequency ablation for paroxysmal atrial fibrillation. N Engl J Med 2016;374:2235-2245.

613. Sohara H, Ohe T, Okumura K, Naito S, Hirao K, Shoda M, Kobayashi Y, Yamauchi Y, Yamaguchi Y, Kuwahara T, Hirayama H, YeongHwa C, Kusano K, Kaitani K, Banba K, Fujii S, Kumagai K, Yoshida H, Matsushita M, Satake S, Aonuma K. HotBalloon ablation of the pulmonary veins for paroxysmal AF: a multicenter randomized trial in Japan. J Am Coll Cardiol 2016;68:2747-2757.

614. Hakalahti A, Biancari F, Nielsen JC, Raatikainen MJ. Radiofrequency ablation vs. antiarrhythmic drug therapy as first line treatment of symptomatic atrial fibrillation: systematic review and meta-analysis. Europace 2015;17:370-378.

615. Nielsen JC, Johannessen A, Raatikainen P, Hindricks G, Walfridsson H, Pehrson SM, Englund A, Hartikainen J, Mortensen LS, Hansen PS; MANTRA-PAF Investigators. Long-term efficacy of catheter ablation as first-line therapy for paroxysmal atrial fibrillation: 5-year outcome in a randomised clinical trial. Heart 2017;103:368-376.

616. Chen C, Zhou X, Zhu M, Chen S, Chen J, Cai H, Dai J, Xu X, Mao W. Catheter ablation versus medical therapy for patients with persistent atrial fibrillation: a systematic review and meta-analysis of evidence from randomized controlled trials. J Interv Card Electrophysiol 2018;52:9-18.

617. Packer DL, Mark DB, Robb RA, Monahan KH, Bahnson TD, Poole JE, Noseworthy PA, Rosenberg YD, Jeffries N, Mitchell LB, Flaker GC, Pokushalov E, Romanov A, Bunch TJ, Noelker G, Ardashev A, Revishvili A, Wilber DJ, Cappato R, Kuck KH, Hindricks G, Davies DW, Kowey PR, Naccarelli GV, Reiffel JA, Piccini JP, Silverstein AP, Al-Khalidi HR, Lee KL; CABANA Investigators. Effect of catheter ablation vs antiarrhythmic drug therapy on mortality, stroke, bleeding, and cardiac arrest among patients with atrial fibrillation: the CABANA randomized clinical trial. JAMA 2019;321:1261-1274.

618. Noseworthy PA, Gersh BJ, Kent DM, Piccini JP, Packer DL, Shah ND, Yao X. Atrial fibrillation ablation in practice: assessing CABANA generalizability. Eur Heart J 2019:40:1257-1264.

619. Teh AW, Kistler PM, Lee G, Medi C, Heck PM, Spence SJ, Sparks PB, Morton JB, Kalman JM. Electroanatomic remodeling of the left atrium in paroxysmal and persistent atrial fibrillation patients without structural heart disease. J Cardiovasc Electrophysiol 2012;23:232-238.

620. D'Ascenzo F, Corleto A, Biondi-Zoccai G, Anselmino M, Ferraris F, di Biase L, Natale A, Hunter RJ, Schilling RJ, Miyazaki S, Tada H, Aonuma K, Yenn-jiang L, Tao H, Ma C, Packer D, Hammill S, Gaita F. Which are the most reliable predictors of recurrence of atrial fibrillation after transcatheter ablation?: a meta-analysis. Int J Cardiol 2013;167:1984-1989.

621. Berruezo A, Tamborero D, Mont L, Benito B, Tolosana JM, Sitges M, Vidal B, Arriagada G, Mendez F, Matiello M, Molina I, Brugada J. Pre-procedural predictors of atrial fibrillation recurrence after circumferential pulmonary vein ablation. Eur Heart J 2007; 28:836-841.

622. Nedios S, Kosiuk J, Koutalas E, Kornej J, Sommer P, Arya A, Richter S, Rolf S, Husser D, Hindricks G, Bollmann A. Comparison of left atrial dimensions in CT and echocardiography as predictors of long-term success after catheter ablation of atrial fibrillation. J Interv Card Electrophysiol 2015;43:237-244.

623. Njoku A, Kannabhiran M, Arora R, Reddy P, Gopinathannair R, Lakkireddy D, Dominic $P$. Left atrial volume predicts atrial fibrillation recurrence after radiofrequency ablation: a meta-analysis. Europace 2018;20:33-42.

624. Costa FM, Ferreira AM, Oliveira S, Santos PG, Durazzo A, Carmo P, Santos KR, Cavaco D, Parreira L, Morgado F, Adragao P. Left atrial volume is more important than the type of atrial fibrillation in predicting the long-term success of catheter ablation. Int / Cardiol 2015;184:56-61. 
625. Marrouche NF, Wilber D, Hindricks G, Jais P, Akoum N, Marchlinski F, Kholmovski E, Burgon N, Hu N, Mont L, Deneke T, Duytschaever M, Neumann T, Mansour M, Mahnkopf C, Herweg B, Daoud E, Wissner E, Bansmann P, Brachmann J. Association of atrial tissue fibrosis identified by delayed enhancement MRI and atrial fibrillation catheter ablation: the DECAAF study. JAMA 2014;311:498-506.

626. Kosich F, Schumacher K, Potpara T, Lip GY, Hindricks G, Kornej J. Clinical scores used for the prediction of negative events in patients undergoing catheter ablation for atrial fibrillation. Clin Cardiol 2019;42:320 - 329.

627. Kornej J, Hindricks G, Shoemaker MB, Husser D, Arya A, Sommer P, Rolf S, Saavedra P, Kanagasundram A, Patrick Whalen S, Montgomery J, Ellis CR, Darbar D, Bollmann A. The APPLE score: a novel and simple score for the prediction of rhythm outcomes after catheter ablation of atrial fibrillation. Clin Res Cardiol 2015;104:871-876.

628. Kornej J, Hindricks G, Arya A, Sommer P, Husser D, Bollmann A. The APPLE score - a novel score for the prediction of rhythm outcomes after repeat catheter ablation of atrial fibrillation. PLoS One 2017;12:e0169933.

629. Kornej J, Schumacher K, Dinov B, Kosich F, Sommer P, Arya A, Husser D, Bollmann A, Lip GYH, Hindricks G. Prediction of electro-anatomical substrate and arrhythmia recurrences using APPLE, DR-FLASH and MB-LATER scores in patients with atrial fibrillation undergoing catheter ablation. Sci Rep 2018;8:12686.

630. Kosiuk J, Dinov B, Kornej J, Acou WJ, Schonbauer R, Fiedler L, Buchta P, Myrda K, Gasior M, Polonski L, Kircher S, Arya A, Sommer P, Bollmann A, Hindricks $\mathrm{G}$, Rolf S. Prospective, multicenter validation of a clinical risk score for left atrial arrhythmogenic substrate based on voltage analysis: DR-FLASH score. Heart Rhythm 2015;12:2207-2212.

631. Mujovic N, Marinkovic M, Markovic N, Shantsila A, Lip GY, Potpara TS. Prediction of very late arrhythmia recurrence after radiofrequency catheter ablation of atrial fibrillation: the MB-LATER clinical score. Sci Rep 2017;7:40828.

632. Mesquita J, Ferreira AM, Cavaco D, Moscoso Costa F, Carmo P, Marques H, Morgado F, Mendes M, Adragao P. Development and validation of a risk score for predicting atrial fibrillation recurrence after a first catheter ablation procedure - ATLAS score. Europace 2018;20:f428-f435.

633. Winkle RA, Jarman JW, Mead RH, Engel G, Kong MH, Fleming W, Patrawala RA. Predicting atrial fibrillation ablation outcome: the CAAP-AF score. Heart Rhythm 2016;13:2119-2125.

634. Canpolat U, Aytemir K, Yorgun H, Sahiner L, Kaya EB, Oto A. A proposal for a new scoring system in the prediction of catheter ablation outcomes: promising results from the Turkish Cryoablation Registry. Int / Cardiol 2013;169:201-206.

635. Wojcik M, Berkowitsch A, Greiss H, Zaltsberg S, Pajitnev D, Deubner N, Hamm CW, Pitschner HF, Kuniss M, Neumann T. Repeated catheter ablation of atrial fibrillation: how to predict outcome?Circ J 2013;77:2271-2279.

636. Pathak RK, Middeldorp ME, Lau DH, Mehta AB, Mahajan R, Twomey D, Alasady M, Hanley L, Antic NA, McEvoy RD, Kalman JM, Abhayaratna WP, Sanders P. Aggressive risk factor reduction study for atrial fibrillation and implications for the outcome of ablation: the ARREST-AF cohort study. J Am Coll Cardiol 2014;64:2222-2231.

637. Trines SA, Stabile G, Arbelo E, Dagres N, Brugada J, Kautzner J, Pokushalov E, Maggioni AP, Laroche C, Anselmino M, Beinart R, Traykov V, BlomstromLundqvist $C$. Influence of risk factors in the ESC-EHRA EORP atrial fibrillation ablation long-term registry. Pacing Clin Electrophysiol 2019;42:1365-1373.

638. Wong CX, Sullivan T, Sun MT, Mahajan R, Pathak RK, Middeldorp M, Twomey D, Ganesan AN, Rangnekar G, Roberts-Thomson KC, Lau DH, Sanders P. Obesity and the risk of incident, post-operative, and post-ablation atrial fibrillation: a meta-analysis of 626,603 individuals in 51 studies. JACC Clin Electrophysiol 2015;1:139-152.

639. Wokhlu A, Hodge DO, Monahan KH, Asirvatham SJ, Friedman PA, Munger TM, Cha YM, Shen WK, Brady PA, Bluhm CM, Haroldson JM, Hammill SC, Packer DL. Long-term outcome of atrial fibrillation ablation: impact and predictors of very late recurrence. J Cardiovasc Electrophysiol 2010;21:1071-1078.

640. Arya A, Hindricks G, Sommer P, Huo Y, Bollmann A, Gaspar T, Bode K, Husser D, Kottkamp H, Piorkowski C. Long-term results and the predictors of outcome of catheter ablation of atrial fibrillation using steerable sheath catheter navigation after single procedure in 674 patients. Europace 2010;12:173-180.

641. Santoro F, Di Biase L, Trivedi C, Burkhardt JD, Paoletti Perini A, Sanchez J, Horton R, Mohanty P, Mohanty S, Bai R, Santangeli P, Lakkireddy D, Reddy M, Elayi CS, Hongo R, Beheiry S, Hao S, Schweikert RA, Viles-Gonzalez J, Fassini G, Casella M, Dello Russo A, Tondo C, Natale A. Impact of uncontrolled hypertension on atrial fibrillation ablation outcome. JACC Clin Electrophysiol 2015; 1:164-173.

642. Letsas KP, Weber R, Burkle G, Mihas CC, Minners J, Kalusche D, Arentz T. Preablative predictors of atrial fibrillation recurrence following pulmonary vein isolation: the potential role of inflammation. Europace 2009;11:158-163.

643. Jongnarangsin K, Chugh A, Good E, Mukerji S, Dey S, Crawford T, Sarrazin JF, Kuhne M, Chalfoun N, Wells D, Boonyapisit W, Pelosi F, Jr., Bogun F,
Morady F, Oral H. Body mass index, obstructive sleep apnea, and outcomes of catheter ablation of atrial fibrillation. J Cardiovasc Electrophysiol 2008; 19:668-672.

644. Patel D, Mohanty P, Di Biase L, Shaheen M, Lewis WR, Quan K, Cummings JE, Wang P, Al-Ahmad A, Venkatraman P, Nashawati E, Lakkireddy D, Schweikert R, Horton R, Sanchez J, Gallinghouse J, Hao S, Beheiry S, Cardinal DS, Zagrodzky J, Canby R, Bailey S, Burkhardt JD, Natale A. Safety and efficacy of pulmonary vein antral isolation in patients with obstructive sleep apnea: the impact of continuous positive airway pressure. Circ Arrhythm Electrophysiol 2010;3:445-451.

645. Matiello M, Nadal M, Tamborero D, Berruezo A, Montserrat J, Embid C, Rios J, Villacastin J, Brugada J, Mont L. Low efficacy of atrial fibrillation ablation in severe obstructive sleep apnoea patients. Europace 2010;12:1084-1089.

646. Chilukuri K, Dalal D, Gadrey S, Marine JE, Macpherson E, Henrikson CA, Cheng A, Nazarian S, Sinha S, Spragg D, Berger R, Calkins H. A prospective study evaluating the role of obesity and obstructive sleep apnea for outcomes after catheter ablation of atrial fibrillation. I Cardiovasc Electrophysiol 2010;21:521- 525 .

647. Ng CY, Liu T, Shehata M, Stevens S, Chugh SS, Wang X. Meta-analysis of obstructive sleep apnea as predictor of atrial fibrillation recurrence after catheter ablation. Am J Cardiol 2011;108:47-51.

648. Naruse $Y$, Tada $H$, Satoh $M$, Yanagihara $M$, Tsuneoka $H$, Hirata $Y$, Ito $Y$, Kuroki K, Machino T, Yamasaki H, Igarashi M, Sekiguchi $Y$, Sato A, Aonuma K. Concomitant obstructive sleep apnea increases the recurrence of atrial fibrillation following radiofrequency catheter ablation of atrial fibrillation: clinical impact of continuous positive airway pressure therapy. Heart Rhythm 2013;10:331-337.

649. Li L, Wang ZW, Li J, Ge X, Guo LZ, Wang Y, Guo WH, Jiang CX, Ma CS. Efficacy of catheter ablation of atrial fibrillation in patients with obstructive sleep apnoea with and without continuous positive airway pressure treatment: a meta-analysis of observational studies. Europace 2014;16:1309-1314.

650. Kawakami H, Nagai T, Fujii A, Uetani T, Nishimura K, Inoue K, Suzuki J, Oka Y, Okura T, Higaki J, Ogimoto A, Ikeda S. Apnea-hypopnea index as a predictor of atrial fibrillation recurrence following initial pulmonary vein isolation: usefulness of type-3 portable monitor for sleep-disordered breathing. J Interv Card Electrophysiol 2016;47:237-244.

651. Congrete S, Bintvihok M, Thongprayoon C, Bathini T, Boonpheng B, Sharma K, Chokesuwattanaskul R, Srivali N, Tanawuttiwat T, Cheungpasitporn W. Effect of obstructive sleep apnea and its treatment of atrial fibrillation recurrence after radiofrequency catheter ablation: a meta-analysis. J Evid Based Med 2018;11:145-151.

652. Deng F, Raza A, Guo J. Treating obstructive sleep apnea with continuous positive airway pressure reduces risk of recurrent atrial fibrillation after catheter ablation: a meta-analysis. Sleep Med 2018;46:5-11.

653. Wokhlu A, Monahan KH, Hodge DO, Asirvatham SJ, Friedman PA, Munger TM, Bradley DJ, Bluhm CM, Haroldson JM, Packer DL. Long-term quality of life after ablation of atrial fibrillation the impact of recurrence, symptom relief, and placebo effect. J Am Coll Cardiol 2010;55:2308-2316.

654. Reddy VY, Dukkipati SR, Neuzil P, Natale A, Albenque JP, Kautzner J, Shah D, Michaud G, Wharton M, Harari D, Mahapatra S, Lambert H, Mansour M. Randomized, controlled trial of the safety and effectiveness of a contact forcesensing irrigated catheter for ablation of paroxysmal atrial fibrillation: results of the TactiCath Contact Force Ablation Catheter Study for Atrial Fibrillation (TOCCASTAR) Study. Circulation 2015;132:907-915.

655. Mark DB, Anstrom KJ, Sheng S, Piccini JP, Baloch KN, Monahan KH, Daniels MR, Bahnson TD, Poole JE, Rosenberg Y, Lee KL, Packer DL. Effect of catheter ablation vs medical therapy on quality of life among patients with atrial fibrillation: the CABANA randomized clinical trial. JAMA 2019;321:1275-1285.

656. Kirchhof P, Breithardt G, Camm AJ, Crijns HJ, Kuck KH, Vardas P, Wegscheider K. Improving outcomes in patients with atrial fibrillation: rationale and design of the Early treatment of Atrial fibrillation for Stroke prevention Trial. Am Heart J 2013;166:442-448.

657. Marrouche NF, Brachmann J, Andresen D, Siebels J, Boersma L, Jordaens L, Merkely B, Pokushalov E, Sanders P, Proff J, Schunkert H, Christ H, Vogt J, Bansch D; CASTLE-AF Investigators. Catheter ablation for atrial fibrillation with heart failure. N Engl J Med 2018;378:417-427.

658. Noseworthy PA, Van Houten HK, Gersh BJ, Packer DL, Friedman PA, Shah ND, Dunlay SM, Siontis KC, Piccini JP, Yao X. Generalizability of the CASTLEAF trial: catheter ablation for patients with atrial fibrillation and heart failure in routine practice. Heart Rhythm 2020;17:1057-1065.

659. Kuck KH, Merkely B, Zahn R, Arentz T, Seidl K, Schluter M, Tilz RR, Piorkowski C, Geller L, Kleemann T, Hindricks G. Catheter ablation versus best medical therapy in patients with persistent atrial fibrillation and congestive heart failure: the randomized AMICA trial. Circ Arrhythm Electrophysiol 2019;12:e07731.

660. Packer DL, Monahan KH, Al-KhalidiHR, Silverstein AP, Poole JP, Bahnson TD, Mark DB, Lee KL. Ablation of Atrial Fibrillation in Heart Failure Patients: Additional outcomes of the CABANATrial. Heart Rhythm 2019;16(suppl):S35. 
661. Khan MN, Jais P, Cummings J, Di Biase L, Sanders P, Martin DO, Kautzner J, Hao S, Themistoclakis S, Fanelli R, Potenza D, Massaro R, Wazni O, Schweikert R, Saliba W, Wang P, Al-Ahmad A, Beheiry S, Santarelli P, Starling RC, Dello Russo A, Pelargonio G, Brachmann J, Schibgilla V, Bonso A, Casella M, Raviele $A$, Haissaguerre $M$, Natale A; PABA-CHF Investigators. Pulmonary-vein isolation for atrial fibrillation in patients with heart failure. $N$ Engl J Med 2008;359:1778-1785.

662. MacDonald MR, Connelly DT, Hawkins NM, Steedman T, Payne J, Shaw M, Denvir M, Bhagra S, Small S, Martin W, McMurray JJ, Petrie MC. Radiofrequency ablation for persistent atrial fibrillation in patients with advanced heart failure and severe left ventricular systolic dysfunction: a randomised controlled trial. Heart 2011;97:740-747.

663. Jones DG, Haldar SK, Hussain W, Sharma R, Francis DP, Rahman-Haley SL, McDonagh TA, Underwood SR, Markides $V$, Wong $T$. A randomized trial to assess catheter ablation versus rate control in the management of persistent atrial fibrillation in heart failure. J Am Coll Cardiol 2013;61:1894-1903.

664. Hunter RJ, Berriman TJ, Diab I, Kamdar R, Richmond L, Baker V, Goromonzi F, Sawhney V, Duncan E, Page SP, Ullah W, Unsworth B, Mayet J, Dhinoja M, Earley MJ, Sporton S, Schilling RJ. A randomized controlled trial of catheter ablation versus medical treatment of atrial fibrillation in heart failure (the CAMTAF trial). Circ Arrhythm Electrophysiol 2014;7:31-38.

665. Al Halabi S, Qintar M, Hussein A, Alraies MC, Jones DG, Wong T, MacDonald MR, Petrie MC, Cantillon D, Tarakji KG, Kanj M, Bhargava M, Varma N, Baranowski B, Wilkoff BL, Wazni O, Callahan T, Saliba W, Chung MK. Catheter ablation for atrial fibrillation in heart failure patients: a meta-analysis of randomized controlled trials. JACC Clin Electrophysiol 2015;1:200-209.

666. Prabhu S, Taylor AJ, Costello BT, Kaye DM, McLellan AJA, Voskoboinik A, Sugumar H, Lockwood SM, Stokes MB, Pathik B, Nalliah CJ, Wong GR, Azzopardi SM, Gutman SJ, Lee G, Layland J, Mariani JA, Ling LH, Kalman JM, Kistler PM. Catheter ablation versus medical rate control in atrial fibrillation and systolic dysfunction: the CAMERA-MRI study. I Am Coll Cardiol 2017;70:1949-1961.

667. Elgendy AY, Mahmoud AN, Khan MS, Sheikh MR, Mojadidi MK, Omer M, Elgendy IY, Bavry AA, Ellenbogen KA, Miles WM, McKillop M. Meta-analysis comparing catheter-guided ablation versus conventional medical therapy for patients with atrial fibrillation and heart failure with reduced ejection fraction. Am J Cardiol 2018;122:806-813

668. Briceno DF, Markman TM, Lupercio F, Romero J, Liang JJ, Villablanca PA, Birati EY, Garcia FC, Di Biase L, Natale A, Marchlinski FE, Santangeli P. Catheter ablation versus conventional treatment of atrial fibrillation in patients with heart failure with reduced ejection fraction: a systematic review and meta-analysis of randomized controlled trials. J Interv Card Electrophysiol 2018;53:19-29.

669. Ma Y, Bai F, Qin F, Li Y, Tu T, Sun C, Zhou S, Liu Q. Catheter ablation for treatment of patients with atrial fibrillation and heart failure: a meta-analysis of randomized controlled trials. BMC Cardiovasc Disord 2018;18:165.

670. Kheiri B, Osman M, Abdalla A, Haykal T, Ahmed S, Bachuwa G, Hassan M, Bhatt DL. Catheter ablation of atrial fibrillation with heart failure: an updated meta-analysis of randomized trials. Int J Cardiol 2018;269:170-173.

671. Khan SU, Rahman H, Talluri S, Kaluski E. The clinical benefits and mortality reduction associated with catheter ablation in subjects with atrial fibrillation: a systematic review and meta-analysis. JACC Clin Electrophysiol 2018;4:626-635.

672. Martin CA, Lambiase PD. Pathophysiology, diagnosis and treatment of tachycardiomyopathy. Heart 2017;103:1543-1552.

673. Raymond-Paquin A, Nattel S, Wakili R, Tadros R. Mechanisms and clinical significance of arrhythmia-induced cardiomyopathy. Can J Cardiol 2018;34:1449-1460.

674. Brembilla-Perrot B, Ferreira JP, Manenti V, Sellal JM, Olivier A, Villemin T, Beurrier D, De Chillou C, Louis P, Brembilla A, Juilliere Y, Girerd N. Predictors and prognostic significance of tachycardiomyopathy: insights from a cohort of 1269 patients undergoing atrial flutter ablation. Eur I Heart Fail 2016;18:394-401.

675. Dagres N, Varounis C, Gaspar T, Piorkowski C, Eitel C, lliodromitis EK, Lekakis JP, Flevari P, Simeonidou E, Rallidis LS, Tsougos E, Hindricks G, Sommer P, Anastasiou-Nana M. Catheter ablation for atrial fibrillation in patients with left ventricular systolic dysfunction. A systematic review and meta-analysis. J Card Fail 2011;17:964-970.

676. Prabhu S, Costello BT, Taylor AJ, Gutman SJ, Voskoboinik A, McLellan AJA, Peck KY, Sugumar H, lles L, Pathik B, Nalliah C], Wong GR, Azzopardi SM, Lee G, Mariani J, Kaye DM, Ling LH, Kalman JM, Kistler PM. Regression of diffuse ventricular fibrosis following restoration of sinus rhythm with catheter ablation in patients with atrial fibrillation and systolic dysfunction: a substudy of the CAMERA MRI trial. JACC Clin Electrophysiol 2018;4:999-1007.

677. Tamborero D, Mont L, Berruezo A, Matiello M, Benito B, Sitges M, Vidal B, de Caralt TM, Perea RJ, Vatasescu R, Brugada J. Left atrial posterior wall isolation does not improve the outcome of circumferential pulmonary vein ablation for atrial fibrillation: a prospective randomized study. Circ Arrhythm Electrophysiol 2009;2:35-40.

678. Natale A, Reddy VY, Monir G, Wilber DJ, Lindsay BD, McElderry HT, Kantipudi C, Mansour MC, Melby DP, Packer DL, Nakagawa H, Zhang B, Stagg RB, Boo LM, Marchlinski FE. Paroxysmal AF catheter ablation with a contact force sensing catheter: results of the prospective, multicenter SMART-AF trial. J Am Coll Cardiol 2014;64:647-656.

679. McLellan AJ, Ling LH, Azzopardi S, Lee GA, Lee G, Kumar S, Wong MC Walters TE, Lee JM, Looi KL, Halloran K, Stiles MK, Lever NA, Fynn SP, Heck PM, Sanders P, Morton JB, Kalman JM, Kistler PM. A minimal or maximal ablation strategy to achieve pulmonary vein isolation for paroxysmal atrial fibrillation: a prospective multi-centre randomized controlled trial (the Minimax study). Eur Heart J 2015;36:1812-1821.

680. Verma A, Jiang CY, Betts TR, Chen J, Deisenhofer I, Mantovan R, Macle L, Morillo CA, Haverkamp W, Weerasooriya R, Albenque JP, Nardi S, Menardi E, Novak P, Sanders P; STAR AF II Investigators. Approaches to catheter ablation for persistent atrial fibrillation. N Engl J Med 2015;372:1812-1822.

681. Luik A, Radzewitz A, Kieser M, Walter M, Bramlage P, Hormann P, Schmidt K Horn N, Brinkmeier-Theofanopoulou M, Kunzmann K, Riexinger T, Schymik G, Merkel M, Schmitt C. Cryoballoon versus open irrigated radiofrequency ablation in patients with paroxysmal atrial fibrillation: the prospective, randomized, controlled, noninferiority FreezeAF study. Circulation 2015;132:1311-1319.

682. Dukkipati SR, Cuoco F, Kutinsky I, Aryana A, Bahnson TD, Lakkireddy D, Woollett I, Issa ZF, Natale A, Reddy VY; HeartLight Study Investigators. Pulmonary vein isolation using the visually guided laser balloon: a prospective, multicenter, and randomized comparison to standard radiofrequency ablation. J Am Coll Cardiol 2015;66:1350-1360.

683. Kuck KH, Hoffmann BA, Ernst S, Wegscheider K, Treszl A, Metzner A, Eckardt L, Lewalter T, Breithardt G, Willems S; Gap-AF-AFNET 1 Investigators. Impact of complete versus incomplete circumferential lines around the pulmonary veins during catheter ablation of paroxysmal atrial fibrillation: results from the Gap-Atrial Fibrillation-German Atrial Fibrillation Competence Network 1 trial. Circ Arrhythm Electrophysiol 2016;9:e003337.

684. Nery PB, Belliveau D, Nair GM, Bernick J, Redpath C], Szczotka A, Sadek MM, Green MS, Wells G, Birnie DH. Relationship between pulmonary vein reconnection and atrial fibrillation recurrence: a systematic review and meta-analysis. JACC Clin Electrophysiol 2016;2:474-483.

685. Bassiouny M, Saliba W, Hussein A, Rickard J, Diab M, Aman W, Dresing T, Tt Callahan, Bhargava M, Martin DO, Shao M, Baranowski B, Tarakji K, Tchou PJ, Hakim A, Kanj M, Lindsay B, Wazni O. Randomized study of persistent atrial fibrillation ablation: ablate in sinus rhythm versus ablate complex-fractionated atrial electrograms in atrial fibrillation. Circ Arrhythm Electrophysio 2016;9:e003596.

686. Hindricks G, Sepehri Shamloo A, Lenarczyk R, Kalarus Z, Arya A, Kircher S, Darma A, Dagres N. Catheter ablation of atrial fibrillation: current status, techniques, outcomes and challenges. Kardiol Pol 2018;76:1680-1686.

687. Nanthakumar K, Plumb VJ, Epstein AE, Veenhuyzen GD, Link D, Kay GN Resumption of electrical conduction in previously isolated pulmonary veins: rationale for a different strategy? Circulation 2004;109:1226-1229.

688. Verma A, Kilicaslan F, Pisano E, Marrouche NF, Fanelli R, Brachmann J, Geunther J, Potenza D, Martin DO, Cummings J, Burkhardt JD, Saliba W Schweikert RA, Natale A. Response of atrial fibrillation to pulmonary vein antrum isolation is directly related to resumption and delay of pulmonary vein conduction. Circulation 2005;112:627-635.

689. Ouyang F, Antz M, Ernst S, Hachiya H, Mavrakis H, Deger FT, Schaumann A Chun J, Falk P, Hennig D, Liu X, Bansch D, Kuck KH. Recovered pulmonary vein conduction as a dominant factor for recurrent atrial tachyarrhythmias after complete circular isolation of the pulmonary veins: lessons from double Lasso technique. Circulation 2005;111:127-135.

690. Cheema A, Dong J, Dalal D, Marine JE, Henrikson CA, Spragg D, Cheng A, Nazarian S, Bilchick K, Sinha S, Scherr D, Almasry I, Halperin H, Berger R, Calkins $\mathrm{H}$. Incidence and time course of early recovery of pulmonary vein conduction after catheter ablation of atrial fibrillation. J Cardiovasc Electrophysiol 2007; 18:387-391.

691. Pratola C, Baldo E, Notarstefano P, Toselli T, Ferrari R. Radiofrequency ablation of atrial fibrillation: is the persistence of all intraprocedural targets necessary for long-term maintenance of sinus rhythm?Circulation 2008;117:136-143.

692. Rajappan K, Kistler PM, Earley MJ, Thomas G, Izquierdo M, Sporton SC, Schilling RJ. Acute and chronic pulmonary vein reconnection after atrial fibrillation ablation: a prospective characterization of anatomical sites. Pacing Clin Electrophysiol 2008;31:1598-1605.

693. Bansch D, Bittkau J, Schneider R, Schneider C, Wendig I, Akin I, Nienaber CA. Circumferential pulmonary vein isolation: wait or stop early after initial successful pulmonary vein isolation?Europace 2013;15:183-188. 
694. Nakamura K, Naito S, Kaseno K, Tsukada N, Sasaki T, Hayano M, Nishiuchi S, Fuke E, Miki Y, Sakamoto T, Nakamura K, Kumagai K, Kataoka A, Takaoka H, Kobayashi Y, Funabashi N, Oshima S. Optimal observation time after completion of circumferential pulmonary vein isolation for atrial fibrillation to prevent chronic pulmonary vein reconnections. Int J Cardiol 2013;168:5300-5310.

695. Neuzil P, Reddy VY, Kautzner J, Petru J, Wichterle D, Shah D, Lambert H, Yulzari A, Wissner E, Kuck $\mathrm{KH}$. Electrical reconnection after pulmonary vein isolation is contingent on contact force during initial treatment: results from the EFFICAS I study. Circ Arrhythm Electrophysiol 2013;6:327-333.

696. Jiang RH, Po SS, Tung R, Liu Q, Sheng X, Zhang ZW, Sun YX, Yu L, Zhang P, Fu $\mathrm{GS}$, Jiang $\mathrm{CY}$. Incidence of pulmonary vein conduction recovery in patients without clinical recurrence after ablation of paroxysmal atrial fibrillation: mechanistic implications. Heart Rhythm 2014;11:969-976.

697. Kim TH, Park J, Uhm JS, Joung B, Lee MH, Pak HN. Pulmonary vein reconnection predicts good clinical outcome after second catheter ablation for atrial fibrillation. Europace 2017;19:961-967.

698. Bordignon S, Furnkranz A, Perrotta L, Dugo D, Konstantinou A, Nowak B, Schulte-Hahn B, Schmidt B, Chun KR. High rate of durable pulmonary vein isolation after second-generation cryoballoon ablation: analysis of repeat procedures. Europace 2015;17:725-731.

699. Ullah W, McLean A, Tayebjee MH, Gupta D, Ginks MR, Haywood GA, O'Neill M, Lambiase PD, Earley MJ, Schilling RJ, Group UKMT. Randomized trial comparing pulmonary vein isolation using the SmartTouch catheter with or without real-time contact force data. Heart Rhythm 2016;13:1761-1767.

700. Phlips T, Taghji P, El Haddad M, Wolf M, Knecht S, Vandekerckhove Y, Tavernier R, Duytschaever M. Improving procedural and one-year outcome after contact force-guided pulmonary vein isolation: the role of interlesion distance, ablation index, and contact force variability in the 'CLOSE'-protocol. Europace 2018;20:f419-f427.

701. Shah D, Haissaguerre M, Jais P, Hocini M. Nonpulmonary vein foci: do they exist?Pacing Clin Electrophysiol 2003;26:1631-1635.

702. Nademanee K, McKenzie J, Kosar E, Schwab M, Sunsaneewitayakul B, Vasavakul T, Khunnawat C, Ngarmukos T. A new approach for catheter ablation of atrial fibrillation: mapping of the electrophysiologic substrate. I Am Coll Cardiol 2004;43:2044-2053.

703. Haissaguerre M, Sanders P, Hocini M, Takahashi Y, Rotter M, Sacher F, Rostock T, Hsu LF, Bordachar P, Reuter S, Roudaut R, Clementy J, Jais P. Catheter ablation of long-lasting persistent atrial fibrillation: critical structures for termination. J Cardiovasc Electrophysiol 2005;16:1125-1137.

704. Haissaguerre M, Hocini M, Sanders P, Sacher F, Rotter M, Takahashi Y, Rostock T, Hsu LF, Bordachar P, Reuter S, Roudaut R, Clementy J, Jais P. Catheter ablation of long-lasting persistent atrial fibrillation: clinical outcome and mechanisms of subsequent arrhythmias. J Cardiovasc Electrophysiol 2005;16:1138-1147.

705. Jaïs P, O'Neill MD, Takahashi Y, Jönsson A, Hocini M, Sacher F, Sanders P, Kodali S, Rostock T, Rotter M, Clémenty J, Haïssaguerre M. Stepwise catheter ablation of chronic atrial fibrillation:importance of discrete anatomic sites for termination. J Cardiovasc Electrophysiol 2006;17:S28-S36.

706. Atienza F, Almendral J, Jalife J, Zlochiver S, Ploutz-Snyder R, Torrecilla EG, Arenal A, Kalifa J, Fernandez-Aviles F, Berenfeld O. Real-time dominant frequency mapping and ablation of dominant frequency sites in atrial fibrillation with left-to-right frequency gradients predicts long-term maintenance of sinus rhythm. Heart Rhythm 2009;6:33-40.

707. Stavrakis S, Nakagawa H, Po SS, Scherlag BJ, Lazzara R, Jackman WM. The role of the autonomic ganglia in atrial fibrillation. JACC Clin Electrophysiol 2015;1:1-13.

708. Di Biase L, Burkhardt JD, Mohanty P, Mohanty S, Sanchez JE, Trivedi C, Gunes M, Gokoglan Y, Gianni C, Horton RP, Themistoclakis S, Gallinghouse G], Bailey S, Zagrodzky JD, Hongo RH, Beheiry S, Santangeli P, Casella M, Dello Russo A, Al-Ahmad A, Hranitzky P, Lakkireddy D, Tondo C, Natale A. Left atrial appendage isolation in patients with longstanding persistent af undergoing catheter ablation: BELIEF trial. J Am Coll Cardiol 2016;68:1929-1940.

709. Gianni C, Mohanty S, Di Biase L, Metz T, Trivedi C, Gokoglan Y, Gunes MF, Bai R, Al-Ahmad A, Burkhardt JD, Gallinghouse GJ, Horton RP, Hranitzky PM, Sanchez JE, Halbfass P, Muller P, Schade A, Deneke T, Tomassoni GF, Natale A. Acute and early outcomes of focal impulse and rotor modulation (FIRM)-guided rotors-only ablation in patients with nonparoxysmal atrial fibrillation. Heart Rhythm 2016;13:830-835.

710. Santangeli P, Zado ES, Hutchinson MD, Riley MP, Lin D, Frankel DS, Supple GE, Garcia FC, Dixit S, Callans DJ, Marchlinski FE. Prevalence and distribution of focal triggers in persistent and long-standing persistent atrial fibrillation. Heart Rhythm 2016;13:374-382.

711. Katritsis DG, Pokushalov E, Romanov A, Giazitzoglou E, Siontis GC, Po SS, Camm AJ, loannidis JP. Autonomic denervation added to pulmonary vein isolation for paroxysmal atrial fibrillation: a randomized clinical trial. J Am Coll Cardiol 2013;62:2318-2325.
712. Arbelo E, Guiu E, Ramos P, Bisbal F, Borras R, Andreu D, Tolosana JM, Berruezo A, Brugada J, Mont L. Benefit of left atrial roof linear ablation in paroxysmal atrial fibrillation: a prospective, randomized study. J Am Heart Assoc 2014;3:e000877.

713. Da Costa A, Levallois M, Romeyer-Bouchard C, Bisch L, Gate-Martinet A, Isaaz $K$. Remote-controlled magnetic pulmonary vein isolation combined with superior vena cava isolation for paroxysmal atrial fibrillation: a prospective randomized study. Arch Cardiovasc Dis 2015;108:163-171.

714. Wong KC, Paisey JR, Sopher M, Balasubramaniam R, Jones M, Qureshi N, Hayes CR, Ginks MR, Rajappan K, Bashir Y, Betts TR. No benefit of complex fractionated atrial electrogram ablation in addition to circumferential pulmonary vein ablation and linear ablation: Benefit of Complex Ablation Study. Circ Arrhythm Electrophysiol 2015;8:1316-1324.

715. Vogler J, Willems S, Sultan A, Schreiber D, Luker J, Servatius H, Schaffer B, Moser J, Hoffmann BA, Steven D. Pulmonary vein isolation versus defragmentation: the CHASE-AF clinical trial. J Am Coll Cardiol 2015;66:2743-2752.

716. Faustino M, Pizzi C, Agricola T, Xhyheri B, Costa GM, Flacco ME, Capasso L, Cicolini G, Di Girolamo E, Leonzio L, Manzoli L. Stepwise ablation approach versus pulmonary vein isolation in patients with paroxysmal atrial fibrillation: randomized controlled trial. Heart Rhythm 2015;12:1907-1915.

717. Scott PA, Silberbauer J, Murgatroyd FD. The impact of adjunctive complex fractionated atrial electrogram ablation and linear lesions on outcomes in persistent atrial fibrillation: a meta-analysis. Europace 2016;18:359-367.

718. Driessen AHG, Berger WR, Krul SPJ, van den Berg NWE, Neefs J, Piersma FR, Chan Pin Yin D, de Jong J, van Boven WP, de Groot JR. Ganglion plexus ablation in advanced atrial fibrillation: the AFACT study. I Am Coll Cardiol 2016;68:1155-1165.

719. Qin M, Liu X, Wu SH, Zhang XD. Atrial substrate modification in atrial fibrillation: targeting GP or CFAE? Evidence from meta-analysis of clinical trials. PLoS One 2016;11:e0164989.

720. Hu X, Jiang J, Ma Y, Tang A. Is there still a role for additional linear ablation in addition to pulmonary vein isolation in patients with paroxysmal atrial fibrillation? An updated meta-analysis of randomized controlled trials. Int J Cardiol 2016;209:266-274.

721. Wynn GJ, Panikker S, Morgan M, Hall M, Waktare J, Markides V, Hussain W, Salukhe T, Modi S, Jarman J, Jones DG, Snowdon R, Todd D, Wong T, Gupta $D$. Biatrial linear ablation in sustained nonpermanent AF: results of the substrate modification with ablation and antiarrhythmic drugs in nonpermanent atrial fibrillation (SMAN-PAF) trial. Heart Rhythm 2016;13:399-406.

722. Zhang Z, Letsas KP, Zhang N, Efremidis M, Xu G, Li G, Liu T. Linear ablation following pulmonary vein isolation in patients with atrial fibrillation: a meta-analysis. Pacing Clin Electrophysiol 2016;39:623-630.

723. Fink T, Schluter M, Heeger CH, Lemes C, Maurer T, Reissmann B, Riedl J, Rottner L, Santoro F, Schmidt B, Wohlmuth P, Mathew S, Sohns C, Ouyang F, Metzner A, Kuck KH. Stand-alone pulmonary vein isolation versus pulmonary vein isolation with additional substrate modification as index ablation procedures in patients with persistent and long-standing persistent atrial fibrillation: the randomized Alster-Lost-AF trial (Ablation at St. Georg Hospital for longstanding persistent atrial fibrillation). Circ Arrhythm Electrophysiol 2017;10.

724. Kim TH, Uhm JS, Kim JY, Joung B, Lee MH, Pak HN. Does additional electrogram-guided ablation after linear ablation reduce recurrence after catheter ablation for longstanding persistent atrial fibrillation? A prospective randomized study. J Am Heart Assoc 2017;6:e004811.

725. Kircher S, Arya A, Altmann D, Rolf S, Bollmann A, Sommer P, Dagres N, Richter S, Breithardt OA, Dinov B, Husser D, Eitel C, Gaspar T, Piorkowski C, Hindricks G. Individually tailored vs. standardized substrate modification during radiofrequency catheter ablation for atrial fibrillation: a randomized study. Europace 2018;20:1766-1775.

726. Ammar-Busch S, Bourier F, Reents T, Semmler V, Telishevska M, Kathan S, Hofmann M, Hessling G, Deisenhofer I. Ablation of complex fractionated electrograms with or without ADditional LINEar Lesions for Persistent Atrial Fibrillation (the ADLINE trial). J Cardiovasc Electrophysiol 2017;28:636-641.

727. Blandino A, Bianchi F, Grossi S, Biondi-Zoccai G, Conte MR, Gaido L, Gaita F, Scaglione M, Rametta F. Left atrial substrate modification targeting low-voltage areas for catheter ablation of atrial fibrillation: a systematic review and metaanalysis. Pacing Clin Electrophysiol 2017;40:199-212.

728. Yang B, Jiang C, Lin Y, Yang G, Chu H, Cai H, Lu F, Zhan X, Xu J, Wang X, Ching CK, Singh B, Kim YH, Chen M; STABLE-SR Investigators. STABLE-SR (Electrophysiological Substrate Ablation in the Left Atrium During Sinus Rhythm) for the treatment of nonparoxysmal atrial fibrillation: a prospective, multicenter randomized clinical trial. Circ Arrhythm Electrophysiol 2017;10:pii: e005405.

729. Yu HT, Shim J, Park J, Kim IS, Kim TH, Uhm JS, Joung B, Lee MH, Kim YH, Pak $\mathrm{HN}$. Pulmonary vein isolation alone versus additional linear ablation in patients with persistent atrial fibrillation converted to paroxysmal type with 
antiarrhythmic drug therapy: a multicenter, prospective, randomized study. Circ Arrhythm Electrophysiol 2017;10:pii: e004915.

730. Wang YL, Liu X, Zhang Y, jiang WF, Zhou L, Qin M, Zhang DL, Zhang XD, Wu $\mathrm{SH}, \mathrm{Xu} \mathrm{K}$. Optimal endpoint for catheter ablation of longstanding persistent atrial fibrillation: a randomized clinical trial. Pacing Clin Electrophysiol 2018;41:172-178.

731. Perez FJ, Schubert CM, Parvez B, Pathak V, Ellenbogen KA, Wood MA. Longterm outcomes after catheter ablation of cavo-tricuspid isthmus dependent atrial flutter: a meta-analysis. Circ Arrhythm Electrophysiol 2009;2:393-401.

732. Natale A, Newby KH, Pisano E, Leonelli F, Fanelli R, Potenza D, Beheiry S, Tomassoni G. Prospective randomized comparison of antiarrhythmic therapy versus first-line radiofrequency ablation in patients with atrial flutter. J Am Coll Cardiol 2000;35:1898-1904.

733. Wazni O, Marrouche NF, Martin DO, Gillinov AM, Saliba W, Saad E, Klein A, Bhargava M, Bash D, Schweikert R, Erciyes D, Abdul-Karim A, Brachman J, Gunther J, Pisano E, Potenza D, Fanelli R, Natale A. Randomized study comparing combined pulmonary vein-left atrial junction disconnection and cavotricuspid isthmus ablation versus pulmonary vein-left atrial junction disconnection alone in patients presenting with typical atrial flutter and atrial fibrillation. Circulation 2003;108:2479-2483.

734. Shah DC, Sunthorn H, Burri H, Gentil-Baron P. Evaluation of an individualized strategy of cavotricuspid isthmus ablation as an adjunct to atrial fibrillation ablation. J Cardiovasc Electrophysiol 2007;18:926-930.

735. Neumann T, Kuniss M, Conradi G, Janin S, Berkowitsch A, Wojcik M, Rixe J, Erkapic D, Zaltsberg S, Rolf A, Bachmann G, Dill T, Hamm CW, Pitschner HF. MEDAFI-Trial (Micro-embolization during ablation of atrial fibrillation): comparison of pulmonary vein isolation using cryoballoon technique vs. radiofrequency energy. Europace 2011;13:37-44.

736. Herrera Siklody C, Deneke T, Hocini M, Lehrmann H, Shin DI, Miyazaki S, Henschke S, Fluegel P, Schiebeling-Romer J, Bansmann PM, Bourdias T, Dousset $V$, Haissaguerre $M$, Arentz $T$. Incidence of asymptomatic intracranial embolic events after pulmonary vein isolation: comparison of different atrial fibrillation ablation technologies in a multicenter study. J Am Coll Cardiol 2011;58:681-688.

737. Herrera Siklody C, Arentz T, Minners J, Jesel L, Stratz C, Valina CM, Weber R, Kalusche D, Toti F, Morel O, Trenk D. Cellular damage, platelet activation, and inflammatory response after pulmonary vein isolation: a randomized study comparing radiofrequency ablation with cryoablation. Heart Rhythm 2012;9:189-196.

738. Pokushalov E, Romanov A, Artyomenko S, Baranova V, Losik D, Bairamova S, Karaskov A, Mittal S, Steinberg JS. Cryoballoon versus radiofrequency for pulmonary vein re-isolation after a failed initial ablation procedure in patients with paroxysmal atrial fibrillation. J Cardiovasc Electrophysiol 2013;24:274-279.

739. Schmidt M, Dorwarth U, Andresen D, Brachmann J, Kuck KH, Kuniss M, Lewalter T, Spitzer S, Willems S, Senges J, Junger C, Hoffmann E. Cryoballoon versus RF ablation in paroxysmal atrial fibrillation: results from the German Ablation Registry. J Cardiovasc Electrophysiol 2014;25:1-7.

740. Perez-Castellano N, Fernandez-Cavazos R, Moreno J, Canadas V, Conde A, Gonzalez-Ferrer JJ, Macaya C, Perez-Villacastin J. The COR trial: a randomized study with continuous rhythm monitoring to compare the efficacy of cryoenergy and radiofrequency for pulmonary vein isolation. Heart Rhythm 2014;11:8-14.

741. Hunter RJ, Baker V, Finlay MC, Duncan ER, Lovell MJ, Tayebjee MH, Ullah W, Siddiqui MS, Mc LA, Richmond L, Kirkby C, Ginks MR, Dhinoja M, Sporton S, Earley MJ, Schilling RJ. Point-by-point radiofrequency ablation versus the cryoballoon or a novel combined approach: a randomized trial comparing 3 methods of pulmonary vein isolation for paroxysmal atrial fibrillation (the Cryo Versus RF trial). J Cardiovasc Electrophysiol 2015;26:1307-1314.

742. Squara F, Zhao A, Marijon E, Latcu DG, Providencia R, Di Giovanni G, Jauvert G, Jourda F, Chierchia GB, De Asmundis C, Ciconte G, Alonso C, Grimard C, Boveda S, Cauchemez B, Saoudi N, Brugada P, Albenque JP, Thomas O. Comparison between radiofrequency with contact force-sensing and secondgeneration cryoballoon for paroxysmal atrial fibrillation catheter ablation: a multicentre European evaluation. Europace 2015;17:718-724

743. Straube F, Dorwarth U, Ammar-Busch S, Peter T, Noelker G, Massa T, Kuniss M, Ewertsen NC, Chun KR, Tebbenjohanns J, Tilz R, Kuck KH, Ouarrak T, Senges J, Hoffmann E; Freeze Cohort Investigators. First-line catheter ablation of paroxysmal atrial fibrillation: outcome of radiofrequency vs. cryoballoon pulmonary vein isolation. Europace 2016;18:368-375.

744. Schmidt M, Dorwarth U, Andresen D, Brachmann J, Kuck K, Kuniss M, Willems S, Deneke T, Tebbenjohanns J, Gerds-Li JH, Spitzer S, Senges J, Hochadel M, Hoffmann E. German ablation registry: cryoballoon vs. radiofrequency ablation in paroxysmal atrial fibrillation - one-year outcome data. Heart Rhythm 2016;13:836-844.

745. Boveda S, Providencia R, Defaye P, Pavin D, Cebron JP, Anselme F, Halimi F, Khoueiry Z, Combes N, Combes S, Jacob S, Albenque JP, Sousa P. Outcomes after cryoballoon or radiofrequency ablation for persistent atrial fibrillation: a multicentric propensity-score matched study. J Interv Card Electrophysiol 2016;47:133-142.

746. Kuck KH, Furnkranz A, Chun KR, Metzner A, Ouyang F, Schluter M, Elvan A Lim HW, Kueffer FJ, Arentz T, Albenque JP, Tondo C, Kuhne M, Sticherling C, Brugada J; FIRE AND ICE Investigators. Cryoballoon or radiofrequency ablation for symptomatic paroxysmal atrial fibrillation: reintervention, rehospitalization, and quality-of-life outcomes in the FIRE AND ICE trial. Eur Heart 2016;37:2858-2865.

747. Buist TJ, Adiyaman A, Smit JJJ, Ramdat Misier AR, Elvan A. Arrhythmia-free survival and pulmonary vein reconnection patterns after second-generation cryoballoon and contact-force radiofrequency pulmonary vein isolation. Clin Res Cardiol 2018;107:498-506.

748. Gunawardene MA, Hoffmann BA, Schaeffer B, Chung DU, Moser J, Akbulak RO, Jularic M, Eickholt C, Nuehrich J, Meyer C, Willems S. Influence of energy source on early atrial fibrillation recurrences: a comparison of cryoballoon vs. radiofrequency current energy ablation with the endpoint of unexcitability in pulmonary vein isolation. Europace 2018;20:43-49.

749. Mortsell D, Arbelo E, Dagres N, Brugada J, Laroche C, Trines SA, Malmborg H Hoglund N, Tavazzi L, Pokushalov E, Stabile G, Blomstrom-Lundqvist C; ESCEHRA Atrial Fibrillation Ablation Long-Term Registry Investigators. Cryoballoon vs. radiofrequency ablation for atrial fibrillation: a study of outcome and safety based on the ESC-EHRA atrial fibrillation ablation long-term registry and the Swedish catheter ablation registry. Europace 2019;21:581-589.

750. Akkaya E, Berkowitsch A, Zaltsberg S, Greiss H, Hamm CW, Sperzel J, Neumann T, Kuniss M. Ice or fire? Comparison of second-generation cryoballoon ablation and radiofrequency ablation in patients with symptomatic persistent atrial fibrillation and an enlarged left atrium. J Cardiovasc Electrophysiol 2018;29:375-384.

751. Murray MI, Arnold A, Younis M, Varghese S, Zeiher AM. Cryoballoon versus radiofrequency ablation for paroxysmal atrial fibrillation: a meta-analysis of randomized controlled trials. Clin Res Cardiol 2018;107:658-669.

752. Chen CF, Gao XF, Duan X, Chen B, Liu XH, Xu YZ. Comparison of catheter ablation for paroxysmal atrial fibrillation between cryoballoon and radiofrequency: a meta-analysis. J Interv Card Electrophysiol 2017;48:351-366.

753. Buiatti A, von Olshausen G, Barthel P, Schneider S, Luik A, Kaess B, Laugwitz KL, Hoppmann P. Cryoballoon vs. radiofrequency ablation for paroxysmal atrial fibrillation: an updated meta-analysis of randomized and observational studies. Europace 2017;19:378-384.

754. Cardoso R, Mendirichaga R, Fernandes G, Healy C, Lambrakos LK, VilesGonzalez JF, Goldberger JJ, Mitrani RD. Cryoballoon versus radiofrequency catheter ablation in atrial fibrillation: a meta-analysis. J Cardiovasc Electrophysiol 2016;27:1151-1159.

755. Kabunga P, Phan K, Ha H, Sy RW. Meta-analysis of contemporary atrial fibrillation ablation strategies: irrigated radiofrequency versus duty-cycled phased radiofrequency versus cryoballoon ablation. JACC Clin Electrophysio 2016;2:377-390.

756. Bollmann A, Ueberham L, Schuler E, Wiedemann M, Reithmann C, Sause A, Tebbenjohanns J, Schade A, Shin DI, Staudt A, Zacharzowsky U, Ulbrich M, Wetzel U, Neuser H, Bode K, Kuhlen R, Hindricks G. Cardiac tamponade in catheter ablation of atrial fibrillation: German-wide analysis of 21141 procedures in the Helios atrial fibrillation ablation registry (SAFER). Europace 2018;20:1944-1951.

757. Ueberham L, Schuler E, Hindricks G, Kuhlen R, Bollmann A. SAFER. Eur Heart 2018;39:2023-2024.

758. Hummel J, Michaud G, Hoyt R, DeLurgio D, Rasekh A, Kusumoto F, Giudici M, Dan D, Tschopp D, Calkins H, Boersma L; TTOP-AF Investigators. Phased RF ablation in persistent atrial fibrillation. Heart Rhythm 2014;11:202-209.

759. Boersma LV, van der Voort P, Debruyne P, Dekker L, Simmers T, Rossenbacker T, Balt J, Wijffels M, Degreef $Y$. Multielectrode pulmonary vein isolation versus single tip wide area catheter ablation for paroxysmal atrial fibrillation: a multinational multicenter randomized clinical trial. Circ Arrhythm Electrophysiol 2016;9:e003151.

760. Nagashima K, Okumura Y, Watanabe I, Nakahara S, Hori Y, Iso K, Watanabe R, Arai M, Wakamatsu Y, Kurokawa S, Mano H, Nakai T, Ohkubo K, Hirayama A. Hot balloon versus cryoballoon ablation for atrial fibrillation: lesion characteristics and middle-term outcomes. Circ Arrhythm Electrophysiol 2018;11:e005861.

761. Ucer E, Janeczko Y, Seegers J, Fredersdorf S, Friemel S, Poschenrieder F, Maier LS, Jungbauer CG. A RAndomized Trial to compare the acute reconnection after pulmonary vein ISolation with Laser-BalloON versus radiofrequency Ablation: RATISBONA trial. J Cardiovasc Electrophysiol 2018;29:733-739.

762. De Greef Y, Stroker E, Schwagten B, Kupics K, De Cocker J, Chierchia GB, de Asmundis C, Stockman D, Buysschaert I. Complications of pulmonary vein isolation in atrial fibrillation: predictors and comparison between four different ablation techniques: results from the Middelheim PVI-registry. Europace 2018;20:1279-1286. 
763. Steinbeck G, Sinner MF, Lutz M, Muller-Nurasyid M, Kaab S, Reinecke H. Incidence of complications related to catheter ablation of atrial fibrillation and atrial flutter: a nationwide in-hospital analysis of administrative data for Germany in 2014. Eur Heart J 2018;39:4020-4029.

764. Fink T, Metzner A, Willems S, Eckardt L, Ince H, Brachmann J, Spitzer SG, Deneke T, Schmitt C, Hochadel M, Senges J, Rillig A. Procedural success, safety and patients satisfaction after second ablation of atrial fibrillation in the elderly: results from the German ablation registry. Clin Res Cardiol 2019;108:1354-1363.

765. Szegedi N, Szeplaki G, Herczeg S, Tahin T, Sallo Z, Nagy VK, Osztheimer I, Ozcan EE, Merkely B, Geller L. Repeat procedure is a new independent predictor of complications of atrial fibrillation ablation. Europace 2019;21:732-737.

766. Cappato R, Calkins H, Chen SA, Davies W, lesaka Y, Kalman J, Kim YH, Klein G, Natale A, Packer D, Skanes A, Ambrogi F, Biganzoli E. Updated worldwide survey on the methods, efficacy, and safety of catheter ablation for human atrial fibrillation. Circ Arrhythm Electrophysiol 2010;3:32-38.

767. Lee G, Sparks PB, Morton JB, Kistler PM, Vohra JK, Medi C, Rosso R, Teh A, Halloran K, Kalman JM. Low risk of major complications associated with pulmonary vein antral isolation for atrial fibrillation: results of 500 consecutive ablation procedures in patients with low prevalence of structural heart disease from a single center. J Cardiovasc Electrophysiol 2011;22:163-168.

768. Deshmukh A, Patel NJ, Pant S, Shah N, Chothani A, Mehta K, Grover P, Singh V, Vallurupalli S, Savani GT, Badheka A, Tuliani T, Dabhadkar K, Dibu G, Reddy YM, Sewani A, Kowalski M, Mitrani R, Paydak H, Viles-Gonzalez JF. In-hospital complications associated with catheter ablation of atrial fibrillation in the United States between 2000 and 2010: analysis of 93801 procedures. Circulation 2013;128:2104-2112.

769. Tripathi B, Arora S, Kumar V, Abdelrahman M, Lahewala S, Dave M, Shah M, Tan B, Savani S, Badheka A, Gopalan R, Shantha GPS, Viles-Gonzalez J, Deshmukh A. Temporal trends of in-hospital complications associated with catheter ablation of atrial fibrillation in the United States: an update from Nationwide Inpatient Sample database (2011-2014). J Cardiovasc Electrophysiol 2018;29:715-724.

770. Voskoboinik A, Sparks PB, Morton JB, Lee G, Joseph SA, Hawson JJ, Kistler PM, Kalman JM. Low rates of major complications for radiofrequency ablation of atrial fibrillation maintained over 14 years: a single centre experience of 2750 consecutive cases. Heart Lung Circ 2018;27:976-983.

771. Berger WR, Meulendijks ER, Limpens J, van den Berg NWE, Neefs J, Driessen AHG, Krul SPJ, van Boven WJP, de Groot JR. Persistent atrial fibrillation: a systematic review and meta-analysis of invasive strategies. Int I Cardiol 2019;278:137-143.

772. Shah AN, Mittal S, Sichrovsky TC, Cotiga D, Arshad A, Maleki K, Pierce W], Steinberg JS. Long-term outcome following successful pulmonary vein isolation: pattern and prediction of very late recurrence. J Cardiovasc Electrophysiol 2008; 19:661-667

773. Sawhney N, Anousheh R, Chen WC, Narayan S, Feld GK. Five-year outcomes after segmental pulmonary vein isolation for paroxysmal atrial fibrillation. Am J Cardiol 2009;104:366-372.

774. Ouyang F, Tilz R, Chun J, Schmidt B, Wissner E, Zerm T, Neven K, Kokturk B, Konstantinidou M, Metzner A, Fuernkranz A, Kuck KH. Long-term results of catheter ablation in paroxysmal atrial fibrillation: lessons from a 5-year followup. Circulation 2010;122:2368-2377.

775. Bertaglia E, Tondo C, De Simone A, Zoppo F, Mantica M, Turco P, luliano A, Forleo G, La Rocca V, Stabile G. Does catheter ablation cure atrial fibrillation? Single-procedure outcome of drug-refractory atrial fibrillation ablation: a 6-year multicentre experience. Europace 2010;12:181-187.

776. Weerasooriya R, Khairy P, Litalien J, Macle L, Hocini M, Sacher F, Lellouche N, Knecht S, Wright M, Nault I, Miyazaki S, Scavee C, Clementy J, Haissaguerre M, Jais $P$. Catheter ablation for atrial fibrillation: are results maintained at 5 years of follow-up?] Am Coll Cardiol 2011;57:160-166.

777. Medi C, Sparks PB, Morton JB, Kistler PM, Halloran K, Rosso R, Vohra JK, Kumar S, Kalman JM. Pulmonary vein antral isolation for paroxysmal atrial fibrillation: results from long-term follow-up. I Cardiovasc Electrophysiol 2011;22:137-141.

778. Schreiber D, Rostock T, Frohlich M, Sultan A, Servatius H, Hoffmann BA, Luker J, Berner I, Schaffer B, Wegscheider K, Lezius S, Willems S, Steven D. Five-year follow-up after catheter ablation of persistent atrial fibrillation using the stepwise approach and prognostic factors for success. Circ Arrhythm Electrophysiol 2015;8:308-317

779. Scherr D, Khairy P, Miyazaki S, Aurillac-Lavignolle V, Pascale P, Wilton SB, Ramoul K, Komatsu Y, Roten L, Jadidi A, Linton N, Pedersen M, Daly M, O'Neill M, Knecht S, Weerasooriya R, Rostock T, Manninger M, Cochet H, Shah AJ, Yeim S, Denis A, Derval N, Hocini M, Sacher F, Haissaguerre M, Jais P. Five-year outcome of catheter ablation of persistent atrial fibrillation using termination of atrial fibrillation as a procedural endpoint. Circ Arrhythm Electrophysiol 2015;8:18-24.
780. Bertaglia E, Senatore G, De Michieli L, De Simone A, Amellone C, Ferretto S, La Rocca V, Giuggia M, Corrado D, Zoppo F, Stabile G. Twelve-year follow-up of catheter ablation for atrial fibrillation: a prospective, multicenter, randomized study. Heart Rhythm 2017;14:486-492.

781. Skelly A, Hashimoto R, Al-Khatib S, et alet alCatheter Ablation for Treatment of Atrial Fibrillation [Internet]. Rockville, MD: Agency for Healthcare Research and Quality (US). Available from https://www.ncbi.nlm.nih.gov/books/NBK305763/ (accessed 20 April 2015).

782. Zheng YR, Chen ZY, Ye LF, Wang LH. Long-term stroke rates after catheter ablation or antiarrhythmic drug therapy for atrial fibrillation: a meta-analysis of randomized trials. J Geriatr Cardiol 2015;12:507-514.

783. Reynolds MR, Walczak J, White SA, Cohen DJ, Wilber DJ. Improvements in symptoms and quality of life in patients with paroxysmal atrial fibrillation treated with radiofrequency catheter ablation versus antiarrhythmic drugs. Circ Cardiovasc Qual Outcomes 2010;3:615-623.

784. Walfridsson $H$, Walfridsson $U$, Nielsen JC, Johannessen A, Raatikainen $P$, Janzon M, Levin LA, Aronsson M, Hindricks G, Kongstad O, Pehrson S, Englund A, Hartikainen J, Mortensen LS, Hansen PS. Radiofrequency ablation as initial therapy in paroxysmal atrial fibrillation: results on health-related quality of life and symptom burden. The MANTRA-PAF trial. Europace 2015;17:215-221.

785. De Greef Y, Schwagten B, Chierchia GB, de Asmundis C, Stockman D, Buysschaert I. Diagnosis-to-ablation time as a predictor of success: early choice for pulmonary vein isolation and long-term outcome in atrial fibrillation: results from the Middelheim-PVI registry. Europace 2018;20:589-595.

786. Wong CX, Abed HS, Molaee P, Nelson AJ, Brooks AG, Sharma G, Leong DP, Lau DH, Middeldorp ME, Roberts-Thomson KC, Wittert GA, Abhayaratna WP, Worthley SG, Sanders P. Pericardial fat is associated with atrial fibrillation severity and ablation outcome. I Am Coll Cardiol 2011;5 7:1745-1751

787. Kang JH, Lee DI, Kim S, Kim MN, Park YM, Ban JE, Choi JI, Lim HE, Park SW, $\mathrm{Kim} \mathrm{YH}$. Prediction of long-term outcomes of catheter ablation of persistent atrial fibrillation by parameters of preablation DC cardioversion. J Cardiovasc Electrophysiol 2012;23:1165-1170.

788. Mahajan R, Lau DH, Brooks AG, Shipp NJ, Manavis J, Wood JPM, Finnie JW, Samuel CS, Royce SG, Twomey DJ, Thanigaimani S, Kalman JM, Sanders P. Electrophysiological, electroanatomical, and structural remodeling of the atria as consequences of sustained obesity. J Am Coll Cardiol 2015;66:1-11.

789. Winkle RA, Mead RH, Engel G, Kong MH, Fleming W, Salcedo J, Patrawala RA. Impact of obesity on atrial fibrillation ablation: patient characteristics, long-term outcomes, and complications. Heart Rhythm 2017;14:819-827.

790. De Maat GE, Mulder B, Berretty WL, Al-Jazairi MIH, Tan YES, Wiesfeld ACP, Mariani MA, Van Gelder IC, Rienstra M, Blaauw Y. Obesity is associated with impaired long-term success of pulmonary vein isolation: a plea for risk factor management before ablation. Open Heart 2018;5:e000771.

791. Glover BM, Hong KL, Dagres N, Arbelo E, Laroche C, Riahi S, Bertini M, Mikhaylov EN, Galvin J, Kiliszek M, Pokushalov E, Kautzner J, Calvo N, Blomstrom-Lundqvist C, Brugada J; ESC-EHRA Atrial Fibrillation Ablation LongTerm Registry Investigators. Impact of body mass index on the outcome of catheter ablation of atrial fibrillation. Heart 2019;105:244-250.

792. Chang SL, Tuan TC, Tai CT, Lin YJ, Lo LW, Hu YF, Tsao HM, Chang CJ, Tsai WC, Chen SA. Comparison of outcome in catheter ablation of atrial fibrillation in patients with versus without the metabolic syndrome. Am J Cardiol 2009;103:67-72.

793. Tang RB, Dong JZ, Liu XP, Long DY, Yu RH, Kalifa J, Ma CS. Metabolic syndrome and risk of recurrence of atrial fibrillation after catheter ablation. Circ J 2009;73:438-443.

794. Mohanty S, Mohanty P, Di Biase L, Bai R, Pump A, Santangeli P, Burkhardt D, Gallinghouse JG, Horton R, Sanchez JE, Bailey S, Zagrodzky J, Natale A. Impact of metabolic syndrome on procedural outcomes in patients with atrial fibrillation undergoing catheter ablation. J Am Coll Cardiol 2012;59:1295-1301.

795. Mulder AA, Wijffels MC, Wever EF, Kelder JC, Boersma LV. Arrhythmia detection after atrial fibrillation ablation: value of incremental monitoring time. Pacing Clin Electrophysiol 2012;35:164-169.

796. Steven D, Rostock T, Lutomsky B, Klemm H, Servatius H, Drewitz I, Friedrichs $\mathrm{K}$, Ventura R, Meinertz T, Willems $\mathrm{S}$. What is the real atrial fibrillation burden after catheter ablation of atrial fibrillation? A prospective rhythm analysis in pacemaker patients with continuous atrial monitoring. Eur Heart J 2008;29:1037-1042.

797. Kaitani K, Inoue K, Kobori A, Nakazawa Y, Ozawa T, Kurotobi T, Morishima I, Miura F, Watanabe T, Masuda M, Naito M, Fujimoto H, Nishida T, Furukawa Y, Shirayama T, Tanaka M, Okajima K, Yao T, Egami Y, Satomi K, Noda T, Miyamoto K, Haruna T, Kawaji T, Yoshizawa T, Toyota T, Yahata M, Nakai K, Sugiyama $H$, Higashi $Y$, Ito $M$, Horie M, Kusano KF, Shimizu W, Kamakura S, Morimoto T, Kimura T, Shizuta S; EAST-AF Trial Investigators. Efficacy of Antiarrhythmic Drugs Short-Term Use After Catheter Ablation for Atrial Fibrillation (EAST-AF) trial. Eur Heart J 2016;37:610-618. 
798. Willems S, Khairy P, Andrade JG, Hoffmann BA, Levesque S, Verma A, Weerasooriya R, Novak P, Arentz T, Deisenhofer I, Rostock T, Steven D, Rivard L, Guerra PG, Dyrda K, Mondesert B, Dubuc M, Thibault B, Talajic M, Roy D, Nattel S, Macle L, ADVICE Trial Investigators. Redefining the blanking period after catheter ablation for paroxysmal atrial fibrillation: insights from the ADVICE (Adenosine Following Pulmonary Vein Isolation to Target Dormant Conduction Elimination) trial. Circ Arrhythm Electrophysiol 2016;9: e003909.

799. Klemm HU, Ventura R, Rostock T, Brandstrup B, Risius T, Meinertz T, Willems S. Correlation of symptoms to ECG diagnosis following atrial fibrillation ablation. J Cardiovasc Electrophysiol 2006;17:146-150.

800. Vasamreddy CR, Dalal D, Dong J, Cheng A, Spragg D, Lamiy SZ, Meininger G, Henrikson CA, Marine JE, Berger R, Calkins H. Symptomatic and asymptomatic atrial fibrillation in patients undergoing radiofrequency catheter ablation. J Cardiovasc Electrophysiol 2006;17:134-139.

801. Oral H, Veerareddy S, Good E, Hall B, Cheung P, Tamirisa K, Han J, Fortino J, Chugh A, Bogun F, Pelosi F Jr, Morady F. Prevalence of asymptomatic recurrences of atrial fibrillation after successful radiofrequency catheter ablation. J Cardiovasc Electrophysiol 2004;15:920-924.

802. Hindricks G, Piorkowski C, Tanner H, Kobza R, Gerds-Li JH, Carbucicchio C, Kottkamp $\mathrm{H}$. Perception of atrial fibrillation before and after radiofrequency catheter ablation: relevance of asymptomatic arrhythmia recurrence. Circulation 2005; 112:307-313

803. Senatore G, Stabile G, Bertaglia E, Donnici G, De Simone A, Zoppo F, Turco P, Pascotto P, Fazzari M. Role of transtelephonic electrocardiographic monitoring in detecting short-term arrhythmia recurrences after radiofrequency ablation in patients with atrial fibrillation. J Am Coll Cardiol 2005;45:873-876.

804. Roux JF, Zado E, Callans DJ, Garcia F, Lin D, Marchlinski FE, Bala R, Dixit S, Riley M, Russo AM, Hutchinson MD, Cooper J, Verdino R, Patel V, Joy PS, Gerstenfeld EP. Antiarrhythmics After Ablation of Atrial Fibrillation (5A Study) Circulation 2009;120:1036-1040.

805. Duytschaever M, Demolder A, Phlips T, Sarkozy A, El Haddad M, Taghji P, Knecht S, Tavernier R, Vandekerckhove $\mathrm{Y}$, De Potter T. PulmOnary vein isolation With vs. without continued antiarrhythmic Drug trEatment in subjects with Recurrent Atrial Fibrillation (POWDER AF): results from a multicentre randomized trial. Eur Heart J 2018;39:1429-1437.

806. Mardigyan V, Verma A, Birnie D, Guerra P, Redfearn D, Becker G, Champagne J, Sapp J, Gula L, Parkash R, Macle L, Crystal E, O'Hara G, Khaykin Y, Sturmer M, Veenhuyzen GD, Greiss I, Sarrazin JF, Mangat I, Novak P, Skanes A, Roux JF, Chauhan V, Hadjis T, Morillo CA, Essebag V. Anticoagulation management preand post atrial fibrillation ablation: a survey of Canadian centres. Can J Cardiol 2013;29:219-223.

807. Nakahara S, Hori Y, Kobayashi S, Sakai Y, Taguchi I, Takayanagi K, Nagashima K, Sonoda K, Kogawa R, Sasaki N, Watanabe I, Okumura Y. Epicardial adipose tissue-based defragmentation approach to persistent atrial fibrillation: its impact on complex fractionated electrograms and ablation outcome. Heart Rhythm 2014;11:1343-1351.

808. Chao TF, Hung CL, Tsao HM, Lin YJ, Yun CH, Lai YH, Chang SL, Lo LW, Hu YF, Tuan TC, Chang HY, Kuo JY, Yeh HI, Wu TJ, Hsieh MH, Yu WC, Chen SA. Epicardial adipose tissue thickness and ablation outcome of atrial fibrillation. PLoS One 2013;8:e74926.

809. Masuda M, Mizuno H, Enchi Y, Minamiguchi H, Konishi S, Ohtani T, Yamaguchi O, Okuyama Y, Nanto S, Sakata Y. Abundant epicardial adipose tissue surrounding the left atrium predicts early rather than late recurrence of atrial fibrillation after catheter ablation. J Interv Card Electrophysiol 2015;44:31-37.

810. Sepehri Shamloo A, Dagres N, Dinov B, Sommer P, Husser-Bollmann D, Bollmann A, Hindricks G, Arya A. Is epicardial fat tissue associated with atrial fibrillation recurrence after ablation? A systematic review and meta-analysis. Int J Cardiol Heart Vasc 2019;22:132-138.

811. Blanche C, Tran N, Rigamonti F, Burri H, Zimmermann M. Value of P-wave signal averaging to predict atrial fibrillation recurrences after pulmonary vein isolation. Europace 2013;15:198-204.

812. Bhargava M, Di Biase L, Mohanty P, Prasad S, Martin DO, Williams-Andrews M, Wazni OM, Burkhardt JD, Cummings JE, Khaykin Y, Verma A, Hao S, Beheiry S, Hongo R, Rossillo A, Raviele A, Bonso A, Themistoclakis S, Stewart K, Saliba WI, Schweikert RA, Natale A. Impact of type of atrial fibrillation and repeat catheter ablation on long-term freedom from atrial fibrillation: results from a multicenter study. Heart Rhythm 2009;6:1403-1412.

813. Winkle RA, Mead RH, Engel G, Patrawala RA. Long-term results of atrial fibrillation ablation: the importance of all initial ablation failures undergoing a repeat ablation. Am Heart J 2011;162:193-200.

814. Mohanty S, Mohanty P, DIB L, Bai R, Trivedi C, Santangeli P, Santoro F, Hongo R, Hao S, Beheiry S, Burkhardt D, Gallinghouse JG, Horton R, Sanchez JE, Bailey S, Hranitzky PM, Zagrodzky J, Natale A. Long-term outcome of catheter ablation in atrial fibrillation patients with coexistent metabolic syndrome and obstructive sleep apnea: impact of repeat procedures versus lifestyle changes. J Cardiovasc Electrophysiol 2014;25:930-938.

815. Ejima K, Shoda M, Arai K, Suzuki A, Yagishita D, Yagishita Y, Yashiro B, Sato T, Manaka T, Ashihara K, Hagiwara N. Impact of diastolic dysfunction on the outcome of catheter ablation in patients with atrial fibrillation. Int J Cardio 2013;164:88-93.

816. Hocini M, Sanders P, Deisenhofer I, Jais P, Hsu LF, Scavee C, Weerasoriya R, Raybaud F, Macle L, Shah DC, Garrigue S, Le Metayer P, Clementy J, Haissaguerre M. Reverse remodeling of sinus node function after catheter ablation of atrial fibrillation in patients with prolonged sinus pauses. Circulation 2003;108:1172-1175.

817. Chen YW, Bai R, Lin T, Salim M, Sang CH, Long DY, Yu RH, Tang RB, Guo XY, Yan XL, Nie JG, Du X, Dong JZ, Ma CS. Pacing or ablation: which is better for paroxysmal atrial fibrillation-related tachycardia-bradycardia syndrome?Pacing Clin Electrophysiol 2014;37:403-411.

818. Inada K, Yamane T, Tokutake K, Yokoyama K, Mishima T, Hioki M, Narui R, Ito K, Tanigawa S, Yamashita S, Tokuda M, Matsuo S, Shibayama K, Miyanaga S, Date $T$, Sugimoto $K$, Yoshimura $M$. The role of successful catheter ablation in patients with paroxysmal atrial fibrillation and prolonged sinus pauses: outcome during a 5-year follow-up. Europace 2014;16:208-213.

819. Cummings JE, Schweikert R, Saliba W, Hao S, Martin DO, Marrouche NF, Burkhardt JD, Kilicaslan F, Verma A, Beheiry S, Belden W, Natale A. Left atrial flutter following pulmonary vein antrum isolation with radiofrequency energy: linear lesions or repeat isolation. J Cardiovasc Electrophysiol 2005;16:293-297.

820. Schneider R, Lauschke J, Tischer T, Schneider C, Voss W, Moehlenkamp F, Glass A, Diedrich D, Bansch D. Pulmonary vein triggers play an important role in the initiation of atrial flutter: initial results from the prospective randomized Atrial Fibrillation Ablation in Atrial Flutter (Triple A) trial. Heart Rhythm 2015;12:865-871.

821. Patel NJ, Deshmukh A, Pau D, Goyal V, Patel SV, Patel N, Agnihotri K, Asirvatham S, Noseworthy P, Di Biase L, Natale A, Viles-Gonzalez JF. Contemporary utilization and safety outcomes of catheter ablation of atrial flutter in the United States: analysis of 89,638 procedures. Heart Rhythm 2016;13:1317-1325.

822. Cox JL, Schuessler RB, Boineau JP. The development of the maze procedure for the treatment of atrial fibrillation. Semin Thorac Cardiovasc Surg 2000;12:2-14.

823. Melby SJ, Zierer A, Bailey MS, Cox JL, Lawton JS, Munfakh N, Crabtree TD, Moazami N, Huddleston CB, Moon MR, Damiano RJ Jr. A new era in the surgical treatment of atrial fibrillation: the impact of ablation technology and lesion set on procedural efficacy. Ann Surg 2006;244:583-592.

824. Badhwar V, Rankin JS, Damiano RJ, Jr., Gillinov AM, Bakaeen FG, Edgerton JR, Philpott JM, McCarthy PM, Bolling SF, Roberts HG, Thourani VH, Suri RM, Shemin RJ, Firestone S, Ad N. The Society of Thoracic Surgeons 2017 Clinical Practice Guidelines for the surgical treatment of atrial fibrillation. Ann Thorac Surg 2017;103:329-341.

825. Belley-Cote EP, Singal RK, McClure G, Devereaux K, Brady K, An K, Healey JS Connolly SJ, Whitlock RP. Perspective and practice of surgical atrial fibrillation ablation: an international survey of cardiac surgeons. Europace 2019;21:445-450.

826. Barnett SD, Ad N. Surgical ablation as treatment for the elimination of atrial fibrillation: a meta-analysis. J Thorac Cardiovasc Surg 2006;131:1029-1035.

827. McClure GR, Belley-Cote EP, Jaffer IH, Dvirnik N, An KR, Fortin G, Spence J, Healey J, Singal RK, Whitlock RP. Surgical ablation of atrial fibrillation: a systematic review and meta-analysis of randomized controlled trials. Europace 2018;20:1442-1450.

828. Osmancik P, Budera P, Talavera D, Hlavicka J, Herman D, Holy J, Cervinka P, Smid J, Hanak P, Hatala R, Widimsky P. Five-year outcomes in cardiac surgery patients with atrial fibrillation undergoing concomitant surgical ablation versus no ablation. The long-term follow-up of the PRAGUE-12 study. Heart Rhythm 201916:1334-1340.

829. Sharples L, Everett C, Singh J, Mills C, Spyt T, Abu-Omar Y, Fynn S, Thorpe B, Stoneman V, Goddard H, Fox-Rushby J, Nashef S. Amaze: a double-blind, multicentre randomised controlled trial to investigate the clinical effectiveness and cost-effectiveness of adding an ablation device-based maze procedure as an adjunct to routine cardiac surgery for patients with pre-existing atrial fibrillation. Health Technol Assess 2018;22:1-132.

830. Bagge L, Probst J, Jensen SM, Blomstrom P, Thelin S, Holmgren A, BlomstromLundqvist C. Quality of life is not improved after mitral valve surgery combined with epicardial left atrial cryoablation as compared with mitral valve surgery alone: a substudy of the double blind randomized SWEDish Multicentre Atrial Fibrillation study (SWEDMAF). Europace 2018;20:f343-f350.

831. Suwalski P, Kowalewski M, Jasinski M, Staromlynski J, Zembala M, Widenka K Brykczynski M, Skiba J, Zembala MO, Bartus K, Hirnle T, Dziembowska I, Deja M, Tobota Z, Maruszewski BJ. Surgical ablation for atrial fibrillation during isolated coronary artery bypass surgery. Eur J Cardiothorac Surg 2019. 
832. Suwalski P, Kowalewski M, Jasinski M, Staromlynski J, Zembala M, Widenka K, Brykczynski M, Skiba J, Zembala MO, Bartus K, Hirnle T, Dziembowska I, Tobota Z, Maruszewski BJ; KROK Investigators. Survival after surgical ablation for atrial fibrillation in mitral valve surgery: analysis from the Polish National Registry of Cardiac Surgery Procedures (KROK). J Thorac Cardiovasc Surg 2018; doi: 10.1016/j.jtcvs.2018.07.099.

833. Gillinov AM, Bakaeen F, McCarthy PM, Blackstone EH, Rajeswaran J, Pettersson G, Sabik JF 3rd, Najam F, Hill KM, Svensson LG, Cosgrove DM, Marrouche N, Natale A. Surgery for paroxysmal atrial fibrillation in the setting of mitral valve disease: a role for pulmonary vein isolation? Ann Thorac Surg 2006;81:19-26; discussion 27-28.

834. Basu S, Nagendran M, Maruthappu M. How effective is bipolar radiofrequency ablation for atrial fibrillation during concomitant cardiac surgery? Interact Cardiovasc Thorac Surg 2012;15:741-748.

835. Gillinov AM, Bhavani S, Blackstone EH, Rajeswaran J, Svensson LG, Navia JL, Pettersson BG, Sabik JF, 3rd, Smedira NG, Mihaljevic T, McCarthy PM, Shewchik J, Natale A. Surgery for permanent atrial fibrillation: impact of patient factors and lesion set. Ann Thorac Surg 2006;82:502-513; discussion 513-514.

836. Beukema WP, Sie HT, Misier AR, Delnoy PP, Wellens HJ, Elvan A. Predictive factors of sustained sinus rhythm and recurrent atrial fibrillation after a radiofrequency modified maze procedure. Eur J Cardiothorac Surg 2008;34:771-775.

837. Lee SH, Kim JB, Cho WC, Chung CH, Jung SH, Choo SJ, Lee JW. The influence of age on atrial fibrillation recurrence after the maze procedure in patients with giant left atrium. J Thorac Cardiovasc Surg 2011;141:1015-1019.

838. Damiano RJ Jr, Schwartz FH, Bailey MS, Maniar HS, Munfakh NA, Moon MR, Schuessler RB. The Cox maze IV procedure: predictors of late recurrence. J Thorac Cardiovasc Surg 2011;141:113-121.

839. Sunderland N, Maruthappu M, Nagendran M. What size of left atrium significantly impairs the success of maze surgery for atrial fibrillation?Interact Cardiovasc Thorac Surg 2011;13:332-338.

840. Bakker RC, Akin S, Rizopoulos D, Kik C, Takkenberg JJ, Bogers AJ. Results of clinical application of the modified maze procedure as concomitant surgery. Interact Cardiovasc Thorac Surg 2013;16:151-156.

841. Ad N, Holmes SD. Prediction of sinus rhythm in patients undergoing concomitant Cox maze procedure through a median sternotomy. J Thorac Cardiovasc Surg 2014;148:881-886.

842. Blomstrom-Lundqvist C, Johansson B, Berglin E, Nilsson L, Jensen SM, Thelin S, Holmgren A, Edvardsson N, Kallner G, Blomstrom P. A randomized doubleblind study of epicardial left atrial cryoablation for permanent atrial fibrillation in patients undergoing mitral valve surgery: the SWEDish Multicentre Atrial Fibrillation study (SWEDMAF). Eur Heart J 2007;28:2902-2908.

843. Huffman MD, Karmali KN, Berendsen MA, Andrei AC, Kruse J, McCarthy PM, Malaisrie SC. Concomitant atrial fibrillation surgery for people undergoing cardiac surgery. Cochrane Database Syst Rev 2016:CD011814.

844. Budera P, Straka Z, Osmancik P, Vanek T, Jelinek S, Hlavicka J, Fojt R, Cervinka $P$, Hulman M, Smid M, Maly M, Widimsky P. Comparison of cardiac surgery with left atrial surgical ablation vs. cardiac surgery without atrial ablation in patients with coronary and/or valvular heart disease plus atrial fibrillation: final results of the PRAGUE-12 randomized multicentre study. Eur Heart J 2012;33:2644-2652.

845. Wang JG, Xin M, Han J, Li Y, Luo TG, Wang J, Meng F, Meng X. Ablation in selective patients with long-standing persistent atrial fibrillation: medium-term results of the Dallas lesion set. Eur J Cardiothorac Surg 2014;46:213-220.

846. Badhwar V, Rankin JS, Ad N, Grau-Sepulveda M, Damiano RJ, Gillinov AM, McCarthy PM, Thourani VH, Suri RM, Jacobs JP, Cox JL. Surgical ablation of atrial fibrillation in the United States: trends and propensity matched outcomes. Ann Thorac Surg 2017;104:493-500.

847. Joshibayev S, Bolatbekov B. Early and long-term outcomes and quality of life after concomitant mitral valve surgery, left atrial size reduction, and radiofrequency surgical ablation of atrial fibrillation. Anatol J Cardiol 2016;16:797-803.

848. Driessen AHG, Berger WR, Bierhuizen MFA, Piersma FR, van den Berg NWE, Neefs J, Krul SPJ, van Boven WP, de Groot JR. Quality of life improves after thoracoscopic surgical ablation of advanced atrial fibrillation: results of the Atrial Fibrillation Ablation and Autonomic Modulation via Thoracoscopic Surgery (AFACT) study. J Thorac Cardiovasc Surg 2018;155:972-980.

849. Castella M, Kotecha D, van Laar C, Wintgens L, Castillo Y, Kelder J, Aragon D, Nunez M, Sandoval E, Casellas A, Mont L, van Boven WJ, Boersma LVA, van Putte BP. Thoracoscopic vs. catheter ablation for atrial fibrillation: long-term follow-up of the FAST randomized trial. Europace 2019;21:746-753.

850. Osmancik P, Budera P, Talavera D, Herman D, Vesela J, Prochazkova R, Rizov $\checkmark$, Kacer P. Improvement in the quality of life of patients with persistent or long-standing persistent atrial fibrillation after hybrid ablation. J Interv Card Electrophysiol 2020;57:435-442.

851. Kim HJ, Kim JS, Kim TS. Epicardial thoracoscopic ablation versus endocardial catheter ablation for management of atrial fibrillation: a systematic review and meta-analysis. Interact Cardiovasc Thorac Surg 2016;22:729-737.
852. Gammie JS, Haddad M, Milford-Beland S, Welke KF, Ferguson TB Jr, O'Brien $\mathrm{SM}$, Griffith BP, Peterson ED. Atrial fibrillation correction surgery: lessons from the Society of Thoracic Surgeons National Cardiac Database. Ann Thorac Surg 2008;85:909-914.

853. Calkins H, Kuck KH, Cappato R, Brugada J, Camm AJ, Chen SA, Crijns HJ, Damiano RJ, Jr, Davies DW, DiMarco J, Edgerton J, Ellenbogen K, Ezekowitz MD, Haines DE, Haissaguerre M, Hindricks G, lesaka $Y$, Jackman W, Jalife J, Jais P, Kalman J, Keane D, Kim YH, Kirchhof P, Klein G, Kottkamp H, Kumagai K, Lindsay BD, Mansour M, Marchlinski FE, McCarthy PM, Mont JL, Morady F, Nademanee K, Nakagawa H, Natale A, Nattel S, Packer DL, Pappone C, Prystowsky E, Raviele A, Reddy V, Ruskin JN, Shemin RJ, Tsao HM, Wilber D. $2012 \mathrm{HRS} / \mathrm{EHRA} / \mathrm{ECAS}$ expert consensus statement on catheter and surgical ablation of atrial fibrillation: recommendations for patient selection, procedural techniques, patient management and follow-up, definitions, endpoints, and research trial design. Europace 2012;14:528-606.

854. van der Heijden CAJ, Vroomen M, Luermans JG, Vos R, Crijns H, Gelsomino S, La Meir M, Pison L, Maesen B. Hybrid versus catheter ablation in patients with persistent and longstanding persistent atrial fibrillation: a systematic review and meta-analysis. Eur J Cardiothorac Surg 2019;56:433-443.

855. Velagic V, DEA C, Mugnai G, Irfan G, Hunuk B, Stroker E, Hacioglu E, Umbrain V, Beckers S, Czapla J, Wellens F, Nijs J, Brugada P, M LAM, Chierchia GB. Repeat procedures after hybrid thoracoscopic ablation in the setting of longstanding persistent atrial fibrillation: electrophysiological findings and 2-year clinical outcome. J Cardiovasc Electrophysiol 2016;27:41 - 50.

856. Osmancik P, Budera P, Zdarska J, Herman D, Petr R, Straka Z Electrophysiological findings after surgical thoracoscopic atrial fibrillation ablation. Heart Rhythm 2016;13:1246-1252.

857. Wang H, Han J, Wang Z, Yin Z, Liu Z, Jin Y, Han H. A prospective randomized trial of the cut-and-sew maze procedure in patients undergoing surgery for rheumatic mitral valve disease. J Thorac Cardiovasc Surg 2018;155: 608-617.

858. Lawrance CP, Henn MC, Miller JR, Sinn LA, Schuessler RB, Maniar HS, Damiano RJ Jr. A minimally invasive Cox maze IV procedure is as effective as sternotomy while decreasing major morbidity and hospital stay. I Thorac Cardiovasc Surg 2014;148:955-961.

859. Weimar T, Schena S, Bailey MS, Maniar HS, Schuessler RB, Cox JL, Damiano RJ Jr. The Cox-maze procedure for lone atrial fibrillation: a single-center experience over 2 decades. Circ Arrhythm Electrophysiol 2012;5:8-14.

860. Nuotio I, Hartikainen JE, Gronberg T, Biancari F, Airaksinen KE. Time to cardioversion for acute atrial fibrillation and thromboembolic complications. JAMA 2014;312:647-649.

861. Airaksinen KE, Gronberg T, Nuotio I, Nikkinen M, Ylitalo A, Biancari F, Hartikainen JE. Thromboembolic complications after cardioversion of acute atrial fibrillation: the FinCV (Finnish CardioVersion) study. J Am Coll Cardiol 2013;62:1187-1192.

862. Hansen ML, Jepsen RM, Olesen JB, Ruwald MH, Karasoy D, Gislason GH, Hansen J, Kober L, Husted S, Torp-Pedersen C. Thromboembolic risk in 16 274 atrial fibrillation patients undergoing direct current cardioversion with and without oral anticoagulant therapy. Europace 2015;17:18-23.

863. Lip GY. Cardioversion of atrial fibrillation. Postgrad Med J 1995;71:457-465.

864. Garg A, Khunger M, Seicean S, Chung MK, Tchou PJ. Incidence of thromboembolic complications within 30 days of electrical cardioversion performed within 48 hours of atrial fibrillation onset. JACC Clin Electrophysiol 2016;2:487-494.

865. Tampieri A, Cipriano V, Mucci F, Rusconi AM, Lenzi T, Cenni P. Safety of cardioversion in atrial fibrillation lasting less than $48 \mathrm{~h}$ without post-procedural anticoagulation in patients at low cardioembolic risk. Intern Emerg Med 2018;13:87-93.

866. Klein AL, Grimm RA, Murray RD, Apperson-Hansen C, Asinger RW, Black IW, Davidoff R, Erbel R, Halperin JL, Orsinelli DA, Porter TR, Stoddard MF; Assessment of Cardioversion Using Transesophageal Echocardiography Investigators. Use of transesophageal echocardiography to guide cardioversion in patients with atrial fibrillation. N Engl J Med 2001;344:1411-1420.

867. Stellbrink C, Nixdorff U, Hofmann T, Lehmacher W, Daniel WG, Hanrath P, Geller C, Mugge A, Sehnert W, Schmidt-Lucke C, Schmidt-Lucke JA, Group ACES. Safety and efficacy of enoxaparin compared with unfractionated heparin and oral anticoagulants for prevention of thromboembolic complications in cardioversion of nonvalvular atrial fibrillation: the Anticoagulation in Cardioversion using Enoxaparin (ACE) trial. Circulation 2004;109:997-1003.

868. Cappato R, Ezekowitz MD, Klein AL, Camm AJ, Ma CS, Le Heuzey JY, Talajic M, Scanavacca M, Vardas PE, Kirchhof P, Hemmrich M, Lanius V, Meng IL, Wildgoose P, van Eickels M, Hohnloser SH; X-VERT Investigators. Rivaroxaban vs. vitamin $\mathrm{K}$ antagonists for cardioversion in atrial fibrillation. Eur Heart J 2014;35:3346-3355.

869. Ezekowitz MD, Pollack CV Jr, Halperin JL, England RD, VanPelt Nguyen S, Spahr J, Sudworth M, Cater NB, Breazna A, Oldgren J, Kirchhof P. Apixaban 
compared to heparin/vitamin $\mathrm{K}$ antagonist in patients with atrial fibrillation scheduled for cardioversion: the EMANATE trial. Eur Heart J 2018;39:2959-2971.

870. Goette A, Merino JL, Ezekowitz MD, Zamoryakhin D, Melino M, Jin J, Mercuri MF, Grosso MA, Fernandez V, Al-Saady N, Pelekh N, Merkely B, Zenin S, Kushnir M, Spinar J, Batushkin V, de Groot JR, Lip GY; ENSURE-AF investigators. Edoxaban versus enoxaparin-warfarin in patients undergoing cardioversion of atrial fibrillation (ENSURE-AF): a randomised, open-label, phase $3 \mathrm{~b}$ trial. Lancet 2016;388:1995-2003.

871. Telles-Garcia N, Dahal K, Kocherla C, Lip GYH, Reddy P, Dominic P. Non-vitamin $\mathrm{K}$ antagonists oral anticoagulants are as safe and effective as warfarin for cardioversion of atrial fibrillation: a systematic review and meta-analysis. Int J Cardiol 2018;268:143-148.

872. Brunetti ND, Tarantino N, De Gennaro L, Correale M, Santoro F, Di Biase M. Direct oral anti-coagulants compared to vitamin- $K$ antagonists in cardioversion of atrial fibrillation: an updated meta-analysis. I Thromb Thrombolysis 2018;45:550-556.

873. Kotecha D, Pollack CV Jr, De Caterina R, Renda G, Kirchhof P. Direct oral anticoagulants halve thromboembolic events after cardioversion of AF compared with warfarin. J Am Coll Cardiol 2018;72:1984-1986.

874. Itainen S, Lehto M, Vasankari T, Mustonen P, Kotamaki M, Numminen A, Lahtela H, Bah A, Hartikainen J, Hekkala AM, Airaksinen JKE. Non-vitamin K antagonist oral anticoagulants in atrial fibrillation patients undergoing elective cardioversion. Europace 2018;20:565-568.

875. Lip GY, Hammerstingl C, Marin F, Cappato R, Meng IL, Kirsch B, van Eickels M, Cohen A; X-TRA study and CLOT-AF Registry Investigators. Left atrial thrombus resolution in atrial fibrillation or flutter: results of a prospective study with rivaroxaban (X-TRA) and a retrospective observational registry providing baseline data (CLOT-AF). Am Heart J 2016;178:126-134.

876. Steinberg JS, Sadaniantz A, Kron J, Krahn A, Denny DM, Daubert J, Campbell WB, Havranek E, Murray K, Olshansky B, O'Neill G, Sami M, Schmidt S, Storm R, Zabalgoitia M, Miller J, Chandler M, Nasco EM, Greene HL. Analysis of cause-specific mortality in the Atrial Fibrillation Follow-up Investigation of Rhythm Management (AFFIRM) study. Circulation 2004;109:1973-1980.

877. Cardoso R, Knijnik L, Bhonsale A, Miller J, Nasi G, Rivera M, Blumer V, Calkins $\mathrm{H}$. An updated meta-analysis of novel oral anticoagulants versus vitamin $\mathrm{K}$ antagonists for uninterrupted anticoagulation in atrial fibrillation catheter ablation. Heart Rhythm 2018;15:107-115.

878. Calkins H, Willems S, Gerstenfeld EP, Verma A, Schilling R, Hohnloser SH, Okumura K, Serota H, Nordaby M, Guiver K, Biss B, Brouwer MA, Grimaldi M; RE-CIRCUIT Investigators. Uninterrupted dabigatran versus warfarin for ablation in atrial fibrillation. N Engl J Med 2017;376:1627-1636.

879. Cappato R, Marchlinski FE, Hohnloser SH, Naccarelli GV, Xiang J, Wilber DJ, Ma CS, Hess S, Wells DS, Juang G, Vijgen J, Hugl BJ, Balasubramaniam R, De Chillou C, Davies DW, Fields LE, Natale A; VENTURE-AF Investigators. Uninterrupted rivaroxaban vs. uninterrupted vitamin $\mathrm{K}$ antagonists for catheter ablation in non-valvular atrial fibrillation. Eur Heart J 2015;36:1805-1811.

880. Kirchhof P, Haeusler KG, Blank B, De Bono J, Callans D, Elvan A, Fetsch T, Van Gelder IC, Gentlesk P, Grimaldi M, Hansen J, Hindricks G, Al-Khalidi HR, Massaro T, Mont L, Nielsen JC, Nolker G, Piccini JP, De Potter T, Scherr D, Schotten $U$, Themistoclakis S, Todd D, Vijgen J, Di Biase L. Apixaban in patients at risk of stroke undergoing atrial fibrillation ablation. Eur Heart J 2018:39:2942-2955.

881. Hohnloser SH, Camm J, Cappato R, Diener HC, Heidbuchel H, Mont L, Morillo CA, Abozguia K, Grimaldi M, Rauer H, Reimitz PE, Smolnik R, Monninghoff C, Kautzner J. Uninterrupted edoxaban vs. vitamin $\mathrm{K}$ antagonists for ablation of atrial fibrillation: the ELIMINATE-AF trial. Eur Heart J 2019;40:3013-3021.

882. European Heart Rhythm Association, European Association for CardioThoracic Surgery, Camm AJ, Kirchhof P, Lip GY, Schotten U, Savelieva I, Ernst S, Van Gelder IC, Al-Attar N, Hindricks G, Prendergast B, Heidbuchel H, Alfieri O, Angelini A, Atar D, Colonna P, De Caterina R, De Sutter J, Goette A, Gorenek B, Heldal M, Hohloser SH, Kolh P, Le Heuzey JY, Ponikowski P, Rutten FH. Guidelines for the management of atrial fibrillation: the Task Force for the management of atrial fibrillation of the European Society of Cardiology (ESC). Eur Heart J 2010;31:2369-2429.

883. Lafuente-Lafuente C, Longas-Tejero MA, Bergmann JF, Belmin J. Antiarrhythmics for maintaining sinus rhythm after cardioversion of atrial fibrillation. Cochrane Database Syst Rev 2012:CD005049.

884. Valembois L, Audureau E, Takeda A, Jarzebowski W, Belmin J, LafuenteLafuente $C$. Antiarrhythmics for maintaining sinus rhythm after cardioversion of atrial fibrillation. Cochrane Database Syst Rev 2019;9:CD005049.

885. Nabauer M, Gerth A, Limbourg T, Schneider S, Oeff M, Kirchhof P, Goette A, Lewalter T, Ravens U, Meinertz T, Breithardt G, Steinbeck G. The registry of the German Competence NETwork on Atrial Fibrillation: patient characteristics and initial management. Europace 2009;11:423-434.
886. Darkner S, Chen X, Hansen J, Pehrson S, Johannessen A, Nielsen JB, Svendsen $\mathrm{JH}$. Recurrence of arrhythmia following short-term oral AMIOdarone after CATheter ablation for atrial fibrillation: a double-blind, randomized, placebocontrolled study (AMIO-CAT trial). Eur Heart / 2014;35:3356-3364.

887. Leong-Sit P, Roux JF, Zado E, Callans DJ, Garcia F, Lin D, Marchlinski FE, Bala R, Dixit S, Riley M, Hutchinson MD, Cooper J, Russo AM, Verdino R, Gerstenfeld EP. Antiarrhythmics After Ablation of Atrial Fibrillation (5A Study): six-month follow-up study. Circ Arrhythm Electrophysiol 2011;4:11-14.

888. Abed HS, Wittert GA, Leong DP, Shirazi MG, Bahrami B, Middeldorp ME, Lorimer MF, Lau DH, Antic NA, Brooks AG, Abhayaratna WP, Kalman JM, Sanders P. Effect of weight reduction and cardiometabolic risk factor management on symptom burden and severity in patients with atrial fibrillation: a randomized clinical trial. JAMA 2013;310:2050-2060.

889. Pathak RK, Middeldorp ME, Meredith M, Mehta AB, Mahajan R, Wong CX, Twomey D, Elliott AD, Kalman JM, Abhayaratna WP, Lau DH, Sanders P. Longterm effect of goal-directed weight management in an atrial fibrillation cohort: a long-term follow-up study (LEGACY). J Am Coll Cardiol 2015;65:2159-2169.

890. Lafuente-Lafuente C, Valembois L, Bergmann JF, Belmin J. Antiarrhythmics for maintaining sinus rhythm after cardioversion of atrial fibrillation. Cochrane Database Syst Rev 2015;3:CD005049.

891. Kochiadakis GE, Igoumenidis NE, Marketou ME, Kaleboubas MD, Simantirakis EN, Vardas PE. Low dose amiodarone and sotalol in the treatment of recurrent, symptomatic atrial fibrillation: a comparative, placebo controlled study. Heart 2000;84:251-257.

892. Galperin J, Elizari MV, Chiale PA, Molina RT, Ledesma R, Scapin AO, Vazquez Blanco M; GEFCA Investigators-GEMA Group. Efficacy of amiodarone for the termination of chronic atrial fibrillation and maintenance of normal sinus rhythm: a prospective, multicenter, randomized, controlled, double blind trial. J Cardiovasc Pharmacol Ther 2001;6:341 - 350.

893. Channer KS, Birchall A, Steeds RP, Walters SJ, Yeo WW, West JN, Muthusamy R, Rhoden WE, Saeed BT, Batin P, Brooksby WP, Wilson I, Grant S. A randomized placebo-controlled trial of pre-treatment and short- or long-term maintenance therapy with amiodarone supporting DC cardioversion for persistent atrial fibrillation. Eur Heart J 2004;25:144-150.

894. Singh SN, Singh BN, Reda DJ, Fye CL, Ezekowitz MD, Fletcher RD, Sharma SC, Atwood JE, Jacobson AK, Lewis HD Jr, Antman EM, Falk RH, Lopez B, Tang $X C$. Comparison of sotalol versus amiodarone in maintaining stability of sinus rhythm in patients with atrial fibrillation (Sotalol-Amiodarone Fibrillation Efficacy Trial [Safe-T]). Am J Cardiol 2003;92:468-472.

895. Kochiadakis GE, Igoumenidis NE, Hamilos ME, Tzerakis PG, Klapsinos NC Chlouverakis GI, Vardas PE. Sotalol versus propafenone for long-term maintenance of normal sinus rhythm in patients with recurrent symptomatic atrial fibrillation. Am J Cardiol 2004;94:1563-1566.

896. Gulizia M, Mangiameli S, Orazi S, Chiaranda G, Piccione G, Di Giovanni N Colletti A, Pensabene O, Lisi F, Vasquez L, Grammatico A, Boriani G; PITAGORA Study Investigators. A randomized comparison of amiodarone and class IC antiarrhythmic drugs to treat atrial fibrillation in patients paced for sinus node disease: the Prevention Investigation and Treatment: A Group for Observation and Research on Atrial arrhythmias (PITAGORA) trial. Am Heart J 2008;155:100-107.e1.

897. Freemantle N, Lafuente-Lafuente C, Mitchell S, Eckert L, Reynolds M. Mixed treatment comparison of dronedarone, amiodarone, sotalol, flecainide, and propafenone, for the management of atrial fibrillation. Europace 2011;13:329-345

898. Cadrin-Tourigny J, Wyse DG, Roy D, Blondeau L, Levesque S, Talajic M, Andrade JG, Dubuc M, Thibault B, Guerra PG, Macle L, Rivard L, Khairy P. Efficacy of amiodarone in patients with atrial fibrillation with and without left ventricular dysfunction: a pooled analysis of AFFIRM and AF-CHF trials. Cardiovasc Electrophysiol 2014;25:1306-1313.

899. Massie BM, Fisher SG, Radford M, Deedwania PC, Singh BN, Fletcher RD, Singh $\mathrm{SN}$. Effect of amiodarone on clinical status and left ventricular function in patients with congestive heart failure. CHF-STAT Investigators. Circulation 1996;93:2128-2134.

900. Singh SN, Fletcher RD, Fisher SG, Singh BN, Lewis HD, Deedwania PC, Massie BM, Colling C, Lazzeri D. Amiodarone in patients with congestive heart failure and asymptomatic ventricular arrhythmia. Survival trial of antiarrhythmic therapy in congestive heart failure. N Engl J Med 1995;333:77-82.

901. Zimetbaum P. Antiarrhythmic drug therapy for atrial fibrillation. Circulation 2012;125:381-389.

902. Kirchhof P, Franz MR, Bardai A, Wilde AM. Giant T-U waves precede torsades de pointes in long QT syndrome: a systematic electrocardiographic analysis in patients with acquired and congenital QT prolongation. J Am Coll Cardiol 2009;54:143-149.

903. Orr CF, Ahlskog JE. Frequency, characteristics, and risk factors for amiodarone neurotoxicity. Arch Neurol 2009;66:865-869. 
904. Lai SW, Lin CL, Liao KF, Lin CY. Amiodarone use and risk of acute pancreatitis: a population-based case-control study. Heart Rhythm 2015;12:163-166.

905. Epstein AE, Olshansky B, Naccarelli GV, Kennedy JI Jr, Murphy EJ, Goldschlager $\mathrm{N}$. Practical management guide for clinicians who treat patients with amiodarone. Am J Med 2016;129:468-475.

906. Basaria S, Cooper DS. Amiodarone and the thyroid. Am J Med 2005;118:706-714.

907. Colby R, Geyer H. Amiodarone-induced pulmonary toxicity. JAAPA 2017;30:23-26

908. Van Gelder IC, Crijns HJ, Van Gilst WH, Van Wijk LM, Hamer HP, Lie KI. Efficacy and safety of flecainide acetate in the maintenance of sinus rhythm after electrical cardioversion of chronic atrial fibrillation or atrial flutter. Am J Cardiol 1989;64:1317-1321.

909. Anderson JL, Gilbert EM, Alpert BL, Henthorn RW, Waldo AL, Bhandari AK, Hawkinson RW, Pritchett EL. Prevention of symptomatic recurrences of paroxysmal atrial fibrillation in patients initially tolerating antiarrhythmic therapy. A multicenter, double-blind, crossover study of flecainide and placebo with transtelephonic monitoring. Flecainide Supraventricular Tachycardia Study Group. Circulation 1989;80:1557-1570.

910. Stroobandt R, Stiels B, Hoebrechts R. Propafenone for conversion and prophylaxis of atrial fibrillation. Propafenone Atrial Fibrillation Trial Investigators. Am J Cardiol 1997;79:418-423.

911. Echt DS, Liebson PR, Mitchell LB, Peters RW, Obias-Manno D, Barker AH, Arensberg D, Baker A, Friedman L, Greene HL, et alet alMortality and morbidity in patients receiving encainide, flecainide, or placebo. The Cardiac Arrhythmia Suppression Trial. N Engl J Med 1991;324:781-788.

912. Flaker GC, Blackshear JL, McBride R, Kronmal RA, Halperin JL, Hart RG. Antiarrhythmic drug therapy and cardiac mortality in atrial fibrillation. The Stroke Prevention in Atrial Fibrillation Investigators. I Am Coll Cardiol 1992;20:527-532.

913. Podrid PJ, Anderson JL. Safety and tolerability of long-term propafenone therapy for supraventricular tachyarrhythmias. The Propafenone Multicenter Study Group. Am J Cardiol 1996;78:430-434.

914. Brembilla-Perrot B, Laporte F, Sellal JM, Schwartz J, Olivier A, Zinzius PY, Manenti V, Beurrier D, Andronache M, Louis P, Selton O, de la Chaise AT, De Chillou C. 1: 1 atrial-flutter. Prevalence and clinical characteristics. Int J Cardiol 2013;168:3287-3290.

915. Gao X, Guha A, Buck B, Patel D, Snider MJ, Boyd M, Afzal M, Badin A, Godara H, Liu Z, Tyler J, Weiss R, Kalbfleisch S, Hummel J, Augostini R, Houmsse M, Daoud EG. Initiation and outcomes with Class Ic antiarrhythmic drug therapy. Indian Pacing Electrophysiol J 2018;18:68-72.

916. Richiardi E, Gaita F, Greco C, Gaschino G, Comba Costa G, Rosettani E, Brusca A. [Propafenone versus hydroquinidine in long-term pharmacological prophylaxis of atrial fibrillation]. Cardiologia 1992;37:123-127.

917. Chimienti M, Cullen MT, Jr., Casadei G. Safety of long-term flecainide and propafenone in the management of patients with symptomatic paroxysmal atrial fibrillation: report from theFlecainide and Propafenone Italian Study Investigators. Am J Cardiol 1996;77:60A-75A.

918. Reimold SC, Cantillon CO, Friedman PL, Antman EM. Propafenone versus sotalol for suppression of recurrent symptomatic atrial fibrillation. Am J Cardiol 1993;71:558-563.

919. Aliot E, Denjoy I. Comparison of the safety and efficacy of flecainide versus propafenone in hospital out-patients with symptomatic paroxysmal atrial fibrillation/flutter. The Flecainide AF French Study Group. Am J Cardiol 1996;77:66A-71A.

920. Bellandi F, Simonetti I, Leoncini M, Frascarelli F, Giovannini T, Maioli M, Dabizzi RP. Long-term efficacy and safety of propafenone and sotalol for the maintenance of sinus rhythm after conversion of recurrent symptomatic atrial fibrillation. Am J Cardiol 2001;88:640-645.

921. Meinertz T, Lip GY, Lombardi F, Sadowski ZP, Kalsch B, Camez A, Hewkin A, Eberle S; ERAFT Investigators. Efficacy and safety of propafenone sustained release in the prophylaxis of symptomatic paroxysmal atrial fibrillation (The European Rythmol/Rytmonorm Atrial Fibrillation Trial [ERAFT] Study). Am J Cardiol 2002;90:1300-1306.

922. Pritchett EL, Page RL, Carlson M, Undesser K, Fava G; Rythmol Atrial Fibrillation Trial (RAFT) Investigators. Efficacy and safety of sustained-release propafenone (propafenone SR) for patients with atrial fibrillation. Am J Cardiol 2003;92:941-946.

923. Hohnloser SH, Crijns HJ, van Eickels M, Gaudin C, Page RL, Torp-Pedersen C, Connolly SJ; Athena Investigators. Effect of dronedarone on cardiovascular events in atrial fibrillation. N Engl J Med 2009;360:668-678.

924. Le Heuzey JY, De Ferrari GM, Radzik D, Santini M, Zhu J, Davy JM. A shortterm, randomized, double-blind, parallel-group study to evaluate the efficacy and safety of dronedarone versus amiodarone in patients with persistent atrial fibrillation: the DIONYSOS study. I Cardiovasc Electrophysiol 2010;21:597-605
925. Singh BN, Connolly SJ, Crijns HJ, Roy D, Kowey PR, Capucci A, Radzik D, Aliot EM, Hohnloser SH, for the EURIDIS and ADONIS Investigators. Dronedarone for maintenance of sinus rhythm in atrial fibrillation or flutter. $N$ Engl J Med 2007;357:987-999.

926. Touboul P, Brugada J, Capucci A, Crijns HJ, Edvardsson N, Hohnloser SH. Dronedarone for prevention of atrial fibrillation: a dose-ranging study. Eur Heart J 2003;24:1481-1487.

927. Kober L, Torp-Pedersen C, McMurray JJ, Gotzsche O, Levy S, Crijns H, Amlie J, Carlsen J, Dronedarone Study G. Increased mortality after dronedarone therapy for severe heart failure. N Engl J Med 2008;358:2678-2687.

928. Wu C, Tcherny-Lessenot S, Dai W, Wang Y, Kechemir H, Gandhi S, Lin S, Juhaeri J. Assessing the risk for peripheral neuropathy in patients treated with dronedarone compared with that in other antiarrhythmics. Clin Ther 2018;40:450-455.e1.

929. Piccini JP, Hasselblad V, Peterson ED, Washam JB, Califf RM, Kong DF. Comparative efficacy of dronedarone and amiodarone for the maintenance of sinus rhythm in patients with atrial fibrillation. I Am Coll Cardiol 2009;54:1089-1095.

930. Gao S, Dai W, Zhang L, Juhaeri J, Wang Y, Caubel P. Risk of cardiovascular events, stroke, congestive heart failure, interstitial lung disease, and acute liver injury: dronedarone versus amiodarone and other antiarrhythmics. I Atr Fibrillation 2013;6:890

931. Pisters R, Hohnloser SH, Connolly SJ, Torp-Pedersen C, Naditch-Brule L, Page RL, Crijns HJ; ATHENA Investigators. Effect of dronedarone on clinical end points in patients with atrial fibrillation and coronary heart disease: insights from the ATHENA trial. Europace 2014;16:174-181.

932. Connolly SJ, Camm AJ, Halperin JL, Joyner C, Alings M, Amerena J, Atar D, Avezum A, Blomstrom P, Borggrefe M, Budaj A, Chen SA, Ching CK, Commerford P, Dans A, Davy JM, Delacretaz E, Di Pasquale G, Diaz R, Dorian P, Flaker G, Golitsyn S, Gonzalez-Hermosillo A, Granger CB, Heidbuchel H, Kautzner J, Kim JS, Lanas F, Lewis BS, Merino JL, Morillo C, Murin J, Narasimhan C, Paolasso E, Parkhomenko A, Peters NS, Sim KH, Stiles MK, Tanomsup S, Toivonen L, Tomcsanyi J, Torp-Pedersen C, Tse HF, Vardas P, Vinereanu D, Xavier D, Zhu J, Zhu JR, Baret-Cormel L, Weinling E, Staiger C, Yusuf S, Chrolavicius S, Afzal R, Hohnloser SH;PALLAS Investigators. Dronedarone in high-risk permanent atrial fibrillation. N Engl J Med 2011;365:2268-2276.

933. Friberg L. Safety of dronedarone in routine clinical care. J Am Coll Cardiol 2014;63:2376-2384.

934. Friberg L. Ventricular arrhythmia and death among atrial fibrillation patients using anti-arrhythmic drugs. Am Heart J 2018;205:118-127.

935. Khan MH, Rochlani Y, Aronow WS. Efficacy and safety of dronedarone in the treatment of patients with atrial fibrillation. Expert Opin Drug Saf 2017; 16:1407-1412.

936. Vamos M, Hohnloser SH. Amiodarone and dronedarone: an update. Trends Cardiovasc Med 2016;26:597-602.

937. Tschuppert Y, Buclin T, Rothuizen LE, Decosterd LA, Galleyrand J, Gaud C, Biollaz J. Effect of dronedarone on renal function in healthy subjects. $\mathrm{Br} J \mathrm{Clin}$ Pharmacol 2007;64:785-791.

938. Vijayalakshmi K, Whittaker VJ, Sutton A, Campbell P, Wright RA, Hall JA, Harcombe AA, Linker NJ, Stewart MJ, Davies A, de Belder MA. A randomized trial of prophylactic antiarrhythmic agents (amiodarone and sotalol) in patients with atrial fibrillation for whom direct current cardioversion is planned. Am Heart J 2006;151:863.e1-6.

939. Capucci A, Botto G, Molon G, Spampinato A, Favale S, Proclemer A, Porfilio A, Marotta T, Vimercati M, Boriani G; DAPHNE Study Investigators. The Drug And Pace Health cliNical Evaluation (DAPHNE) study: a randomized trial comparing sotalol versus beta-blockers to treat symptomatic atrial fibrillation in patients with brady-tachycardia syndrome implanted with an antitachycardia pacemaker. Am Heart J 2008;156:373.e1-8.

940. Juul-Moller S, Edvardsson N, Rehnqvist-Ahlberg N. Sotalol versus quinidine for the maintenance of sinus rhythm after direct current conversion of atrial fibrillation. Circulation 1990;82:1932-199.

941. MacNeil DJ, Davies RO, Deitchman D. Clinical safety profile of sotalol in the treatment of arrhythmias. Am J Cardiol 1993;72:44A-50A.

942. Roy D, Talajic M, Dorian P, Connolly S, Eisenberg MJ, Green M, Kus T, Lambert J, Dubuc M, Gagne P, Nattel S, Thibault B. Amiodarone to prevent recurrence of atrial fibrillation. Canadian Trial of Atrial Fibrillation Investigators. N Engl J Med 2000;342:913-920.

943. Andersen SS, Hansen ML, Gislason GH, Schramm TK, Folke F, Fosbol E, Abildstrom SZ, Madsen M, Kober L, Torp-Pedersen C. Antiarrhythmic therapy and risk of death in patients with atrial fibrillation: a nationwide study. Europace 2009;11:886-891.

944. Crijns HJ, Gosselink AT, Lie KI. Propafenone versus disopyramide for maintenance of sinus rhythm after electrical cardioversion of chronic atrial fibrillation: a randomized, double-blind study. PRODIS Study Group. Cardiovasc Drugs Ther 1996;10:145-152. 
945. Lloyd EA, Gersh BJ, Forman R. The efficacy of quinidine and disopyramide in the maintenance of sinus rhythm after electroconversion from atrial fibrillation. A double-blind study comparing quinidine, disopyramide and placebo. S Afr Med | 1984;65:367-369.

946. Karlson BW, Torstensson I, Abjorn C, Jansson SO, Peterson LE. Disopyramide in the maintenance of sinus rhythm after electroconversion of atrial fibrillation. A placebo-controlled one-year follow-up study. Eur Heart J 1988;9:284-290.

947. Coplen SE, Antman EM, Berlin JA, Hewitt P, Chalmers TC. Efficacy and safety of quinidine therapy for maintenance of sinus rhythm after cardioversion. A meta-analysis of randomized control trials. Circulation 1990;82:1106-1116.

948. Lip GY, Laroche C, Dan GA, Santini M, Kalarus Z, Rasmussen LH, Oliveira MM, Mairesse G, Crijns HJ, Simantirakis E, Atar D, Kirchhof P, Vardas P, Tavazzi L, Maggioni AP. A prospective survey in European Society of Cardiology member countries of atrial fibrillation management: baseline results of EURObservational Research Programme Atrial Fibrillation (EORP-AF) pilot general registry. Europace 2014;16:308-319.

949. Dagres N, Lewalter T, Lip GY, Pison L, Proclemer A, Blomstrom-Lundqvist C, Scientific Initiatives Committee EHRA. Current practice of antiarrhythmic drug therapy for prevention of atrial fibrillation in Europe: the European Heart Rhythm Association survey. Europace 2013;15:478-481.

950. Sherrid MV, Barac I, McKenna WJ, Elliott PM, Dickie S, Chojnowska L, Casey S, Maron BJ. Multicenter study of the efficacy and safety of disopyramide in obstructive hypertrophic cardiomyopathy. J Am Coll Cardiol 2005; 45:1251-1258.

951. Goette A, Staack T, Rocken C, Arndt M, Geller JC, Huth C, Ansorge S, Klein $\mathrm{HU}$, Lendeckel U. Increased expression of extracellular signal-regulated kinase and angiotensin-converting enzyme in human atria during atrial fibrillation. J Am Coll Cardiol 2000;35:1669-1677.

952. Thanigaimani S, Lau DH, Agbaedeng T, Elliott AD, Mahajan R, Sanders P. Molecular mechanisms of atrial fibrosis: implications for the clinic. Expert Rev Cardiovasc Ther 2017;15:247-256.

953. Kumagai K, Nakashima H, Urata H, Gondo N, Arakawa K, Saku K. Effects of angiotensin II type 1 receptor antagonist on electrical and structural remodeling in atrial fibrillation. J Am Coll Cardiol 2003;41:2197-2204.

954. Schneider MP, Hua TA, Bohm M, Wachtell K, Kjeldsen SE, Schmieder RE. Prevention of atrial fibrillation by renin-angiotensin system inhibition a metaanalysis. J Am Coll Cardiol 2010;55:2299-2307.

955. Healey JS, Baranchuk A, Crystal E, Morillo CA, Garfinkle M, Yusuf S, Connolly SJ. Prevention of atrial fibrillation with angiotensin-converting enzyme inhibitors and angiotensin receptor blockers: a meta-analysis. I Am Coll Cardiol 2005;45:1832-1839.

956. Ducharme A, Swedberg K, Pfeffer MA, Cohen-Solal A, Granger CB, Maggioni AP, Michelson EL, McMurray JJ, Olsson L, Rouleau JL, Young JB, Yusuf S. Prevention of atrial fibrillation in patients with symptomatic chronic heart failure by candesartan in the Candesartan in Heart failure: Assessment of Reduction in Mortality and morbidity (CHARM) program. Am Heart J 2006;152:86-92

957. McMurray JJ, Young JB, Dunlap ME, Granger CB, Hainer J, Michelson EL, Earle S, Olofsson B, Ostergren J, Yusuf S, Swedberg K, Pfeffer MA, CHARM Investigators. Relationship of dose of background angiotensin-converting enzyme inhibitor to the benefits of candesartan in the Candesartan in Heart failure: Assessment of Reduction in Mortality and morbidity (CHARM)-Added trial. Am Heart / 2006;151:985-991.

958. Wachtell K, Lehto M, Gerdts E, Olsen MH, Hornestam B, Dahlof B, Ibsen H, Julius S, Kjeldsen SE, Lindholm LH, Nieminen MS, Devereux RB. Angiotensin II receptor blockade reduces new-onset atrial fibrillation and subsequent stroke compared to atenolol: the Losartan Intervention For End Point Reduction in Hypertension (LIFE) study. J Am Coll Cardiol 2005;45:712-719.

959. Anand K, Mooss AN, Hee TT, Mohiuddin SM. Meta-analysis: inhibition of reninangiotensin system prevents new-onset atrial fibrillation. Am Heart J 2006;152:217-222

960. Okin PM, Wachtell K, Devereux RB, Harris KE, Jern S, Kjeldsen SE, Julius S, Lindholm LH, Nieminen MS, Edelman JM, Hille DA, Dahlof B. Regression of electrocardiographic left ventricular hypertrophy and decreased incidence of new-onset atrial fibrillation in patients with hypertension. JAMA 2006;296:1242-1248.

961. Olsson LG, Swedberg K, Ducharme A, Granger CB, Michelson EL, McMurray JJ, Puu M, Yusuf S, Pfeffer MA; CHARM Investigators. Atrial fibrillation and risk of clinical events in chronic heart failure with and without left ventricular systolic dysfunction: results from the Candesartan in Heart failure: Assessment of Reduction in Mortality and morbidity (CHARM) program. J Am Coll Cardiol 2006;47:1997-2004

962. Marott SC, Nielsen SF, Benn M, Nordestgaard BG. Antihypertensive treatment and risk of atrial fibrillation: a nationwide study. Eur Heart J 2014;35:1205-1214.
963. Goette A, Schon N, Kirchhof P, Breithardt G, Fetsch T, Hausler KG, Klein HU, Steinbeck G, Wegscheider K, Meinertz T. Angiotensin Il-antagonist in paroxysmal atrial fibrillation (ANTIPAF) trial. Circ Arrhythm Electrophysiol 2012;5:43-51.

964. Savelieva I, Kakouros N, Kourliouros A, Camm AJ. Upstream therapies for management of atrial fibrillation: review of clinical evidence and implications for European Society of Cardiology guidelines. Part II: secondary prevention. Europace 2011;13:610-625.

965. Reil JC, Hohl M, Selejan S, Lipp P, Drautz F, Kazakow A, Munz BM, Muller P, Steendijk P, Reil GH, Allessie MA, Bohm M, Neuberger HR. Aldosterone promotes atrial fibrillation. Eur Heart J 2012;33:2098-2108.

966. Reil JC, Tauchnitz M, Tian Q, Hohl M, Linz D, Oberhofer M, Kaestner L, Reil $\mathrm{GH}$, Thiele H, Steendijk P, Bohm M, Neuberger HR, Lipp P. Hyperaldosteronism induces left atrial systolic and diastolic dysfunction. Am J Physiol Heart Circ Physiol 2016;311:H1014-H1023.

967. Tsai CT, Chiang FT, Tseng CD, Hwang J, Kuo KT, Wu CK, Yu CC, Wang YC, Lai LP, Lin JL. Increased expression of mineralocorticoid receptor in human atrial fibrillation and a cellular model of atrial fibrillation. J Am Coll Cardiol 2010;55:758-770.

968. Pitt B, Remme W, Zannad F, Neaton J, Martinez F, Roniker B, Bittman R, Hurley S, Kleiman J, Gatlin M; Eplerenone Post-Acute Myocardial Infarction Heart Failure Efficacy Survival Study Investigators. Eplerenone, a selective aldosterone blocker, in patients with left ventricular dysfunction after myocardial infarction. N Engl J Med 2003;348:1309-1321.

969. Swedberg K, Zannad F, McMurray J], Krum H, van Veldhuisen DJ, Shi H, Vincent J, Pitt B; EMPHASIS-HF Study Investigators. Eplerenone and atrial fibrillation in mild systolic heart failure: results from the EMPHASIS-HF (Eplerenone in Mild Patients Hospitalization And Survlval Study in Heart Failure) study. J Am Coll Cardiol 2012;59:1598-1603.

970. Cikes M, Claggett B, Shah AM, Desai AS, Lewis EF, Shah SJ, Anand IS, O'Meara E, Rouleau JL, Sweitzer NK, Fang JC, Saksena S, Pitt B, Pfeffer MA, Solomon SD. Atrial fibrillation in heart failure with preserved ejection fraction: the TOPCAT trial. JACC Heart Fail 2018;6:689-697.

971. Neefs J, van den Berg NW, Limpens J, Berger WR, Boekholdt SM, Sanders P, de Groot JR. Aldosterone pathway blockade to prevent atrial fibrillation: a systematic review and meta-analysis. Int J Cardiol 2017;231:155-161.

972. Kuhlkamp V, Schirdewan A, Stangl K, Homberg M, Ploch M, Beck OA. Use of metoprolol $C R / X L$ to maintain sinus rhythm after conversion from persistent atrial fibrillation: a randomized, double-blind, placebo-controlled study. J Am Coll Cardiol 2000;36:139-146.

973. Nergardh AK, Rosenqvist M, Nordlander R, Frick M. Maintenance of sinus rhythm with metoprolol $C R$ initiated before cardioversion and repeated cardioversion of atrial fibrillation: a randomized double-blind placebo-controlled study. Eur Heart J 2007;28:1351-1357.

974. Zheng Z, Jayaram R, Jiang L, Emberson J, Zhao Y, Li Q, Du J, Guarguagli S, Hill M, Chen Z, Collins R, Casadei B. Perioperative rosuvastatin in cardiac surgery. N Engl J Med 2016;374:1744-1753.

975. Rahimi K, Emberson J, McGale P, Majoni W, Merhi A, Asselbergs FW, Krane V Macfarlane PW, PROSPER Executive. Effect of statins on atrial fibrillation: collaborative meta-analysis of published and unpublished evidence from randomised controlled trials. BMJ 2011;342:d1250.

976. Pinho-Gomes AC, Reilly S, Brandes RP, Casadei B. Targeting inflammation and oxidative stress in atrial fibrillation: role of 3-hydroxy-3-methylglutaryl-coenzyme a reductase inhibition with statins. Antioxid Redox Signal 2014;20:1268-1285.

977. Humphries KH, Lee M, Sheldon R, Ramanathan K, Dorian P, Green M, Kerr CR CARAF Investigators. Statin use and recurrence of atrial fibrillation after successful cardioversion. Am Heart / 2007;154:908-913.

978. Bianconi L, Calo L, Mennuni M, Santini L, Morosetti P, Azzolini P, Barbato G, Biscione F, Romano P, Santini M. n-3 polyunsaturated fatty acids for the prevention of arrhythmia recurrence after electrical cardioversion of chronic persistent atrial fibrillation: a randomized, double-blind, multicentre study. Europace 2011;13:174-181.

979. Mozaffarian D, Marchioli R, Macchia A, Silletta MG, Ferrazzi P, Gardner TJ, Latini R, Libby P, Lombardi F, O'Gara PT, Page RL, Tavazzi L, Tognoni G: OPERA Investigators. Fish oil and postoperative atrial fibrillation: the Omega-3 Fatty Acids for Prevention of Post-operative Atrial Fibrillation (OPERA) randomized trial. JAMA 2012;308:2001-2011.

980. Yamashita T, Inoue H, Okumura K, Kodama I, Aizawa Y, Atarashi H, Ohe T, Ohtsu H, Kato T, Kamakura S, Kumagai K, Kurachi Y, Koretsune Y, Saikawa T, Sakurai M, Sato T, Sugi K, Nakaya H, Hirai M, Hirayama A, Fukatani M, Mitamura H, Yamazaki T, Watanabe E, Ogawa S; J-RHYTHM II Investigators. Randomized trial of angiotensin II-receptor blocker vs. dihydropiridine calcium channel blocker in the treatment of paroxysmal atrial fibrillation with hypertension (J-RHYTHM II study). Europace 2011;13:473-479.

981. Macchia A, Grancelli H, Varini S, Nul D, Laffaye N, Mariani J, Ferrante D, Badra R, Figal J, Ramos S, Tognoni G, Doval HC; GESICA Investigators. Omega-3 fatty 
acids for the prevention of recurrent symptomatic atrial fibrillation: results of the FORWARD (Randomized Trial to Assess Efficacy of PUFA for the Maintenance of Sinus Rhythm in Persistent Atrial Fibrillation) trial. J Am Coll Cardiol 2013;61:463-468.

982. Kowey PR, Reiffel JA, Ellenbogen KA, Naccarelli GV, Pratt CM. Efficacy and safety of prescription omega-3 fatty acids for the prevention of recurrent symptomatic atrial fibrillation: a randomized controlled trial. JAMA 2010;304:2363-2372.

983. Kochiadakis GE, Marketou ME, Igoumenidis NE, Chrysostomakis SI, Mavrakis $\mathrm{HE}$, Kaleboubas MD, Vardas PE. Amiodarone, sotalol, or propafenone in atrial fibrillation: which is preferred to maintain normal sinus rhythm?Pacing Clin Electrophysiol 2000;23:1883-1887.

984. Boriani G, Biffi M, Capucci A, Botto GL, Broffoni T, Rubino I, Della Casa S, Sanguinetti M, Magnani B. Oral propafenone to convert recent-onset atrial fibrillation in patients with and without underlying heart disease. A randomized, controlled trial. Ann Intern Med 1997;126:621-625.

985. Ehrlich JR, Look C, Kostev K, Israel CW, Goette A. Impact of dronedarone on the risk of myocardial infarction and stroke in atrial fibrillation patients followed in general practices in Germany. Int J Cardiol 2019;278:126-132.

986. Camm AJ. Hopes and disappointments with antiarrhythmic drugs. Int J Cardiol 2017;237:71-74.

987. De Vecchis R. Long-term antiarrhythmic drug treatment after atrial fibrillation ablation: does a too obstinate rhythm control strategy bring serious risk of proarrhythmia to ablated patients?Eur Heart J Cardiovasc Pharmacother 2019;5:117-118

988. Fabritz L, Kirchhof P. Predictable and less predictable unwanted cardiac drugs effects: individual pre-disposition and transient precipitating factors. Basic Clin Pharmacol Toxicol 2010;106:263-268.

989. Reimold FR, Reynolds MR. Proarrhythmia and death with antiarrhythmic drugs for atrial fibrillation, and the unfulfilled promise of comparative effectiveness research. Am Heart J 2018;205:128-130.

990. Coughtrie AL, Behr ER, Layton D, Marshall V, Camm AJ, Shakir SAW. Drugs and life-threatening ventricular arrhythmia risk: results from the DARE study cohort. BMJ Open 2017;7:e016627.

991. Haverkamp W, Breithardt G, Camm AJ, Janse MJ, Rosen MR, Antzelevitch C, Escande D, Franz M, Malik M, Moss A, Shah R. The potential for QT prolongation and proarrhythmia by non-antiarrhythmic drugs: clinical and regulatory implications. Report on a policy conference of the European Society of Cardiology. Eur Heart J 2000;21:1216-1231.

992. Milan DJ, Saul JP, Somberg JC, Molnar J. Efficacy of intravenous and oral sotalol in pharmacologic conversion of atrial fibrillation: a systematic review and metaanalysis. Cardiology 2017;136:52-60.

993. Agusala K, Oesterle A, Kulkarni C, Caprio T, Subacius H, Passman R. Risk prediction for adverse events during initiation of sotalol and dofetilide for the treatment of atrial fibrillation. Pacing Clin Electrophysiol 2015;38:490-498.

994. Lin CY, Lin YJ, Lo LW, Chen YY, Chong E, Chang SL, Chung FP, Chao TF, Hu YF, Tuan TC, Liao JN, Chang Y, Chien KL, Chiou CW, Chen SA. Factors predisposing to ventricular proarrhythmia during antiarrhythmic drug therapy for atrial fibrillation in patients with structurally normal heart. Heart Rhythm 2015;12:1490-1500.

995. Kaab S, Hinterseer M, Nabauer M, Steinbeck G. Sotalol testing unmasks altered repolarization in patients with suspected acquired long-QT-syndrome - a case-control pilot study using i.v. sotalol. Eur Heart J 2003;24:649-657.

996. Lehtonen A, Fodstad H, Laitinen-Forsblom P, Toivonen L, Kontula K, Swan H. Further evidence of inherited long QT syndrome gene mutations in antiarrhythmic drug-associated torsades de pointes. Heart Rhythm 2007;4:603-607.

997. Patten M, Maas R, Bauer P, Luderitz B, Sonntag F, Dluzniewski M, Hatala R, Opolski G, Muller HW, Meinertz T; SOPAT Investigators. Suppression of paroxysmal atrial tachyarrhythmias - results of the SOPAT trial. Eur Heart J 2004;25:1395-1404

998. Fetsch T, Bauer P, Engberding R, Koch HP, Lukl J, Meinertz T, Oeff M, Seipel L, Trappe HJ, Treese N, Breithardt G; Prevention of Atrial Fibrillation after Cardioversion Investigators. Prevention of atrial fibrillation after cardioversion: results of the PAFAC trial. Eur Heart J 2004;25:1385-1394.

999. Vallurupalli S, Pothineni NV, Deshmukh A, Paydak H. Utility of routine exercise testing to detect rate-related QRS widening in patients without structural heart disease on class Ic antiarrhythmic agents (flecainide and propafenone). Am J Cardiol 2015;116:730-732.

1000. Parkash R, Wells GA, Sapp JL, Healey JS, Tardif J-C, Greiss I, Rivard L, Roux JF, Gula L, Nault I. Effect of aggressive blood pressure control on the recurrence of atrial fibrillation after catheter ablation: a randomized, open-label clinical trial (SMAC-AF [Substrate Modification With Aggressive Blood Pressure Control]). Circulation 2017;135:1788-1798.

1001. Huxley RR, Misialek JR, Agarwal SK, Loehr LR, Soliman EZ, Chen LY, Alonso A. Physical activity, obesity, weight change, and risk of atrial fibrillation: the
Atherosclerosis Risk in Communities study. Circ Arrhythm Electrophysiol 2014;7:620-625.

1002. Murphy NF, Maclntyre K, Stewart S, Hart CL, Hole D, McMurray JJ. Longterm cardiovascular consequences of obesity: 20-year follow-up of more than 15000 middle-aged men and women (the Renfrew-Paisley study). Eur Heart J 2006;27:96-106.

1003. Wanahita N, Messerli FH, Bangalore S, Gami AS, Somers VK, Steinberg JS. Atrial fibrillation and obesity - results of a meta-analysis. Am Heart J 2008;155:310-315.

1004. Wang TJ, Parise H, Levy D, D'Agostino RB Sr, Wolf PA, Vasan RS, Benjamin EJ. Obesity and the risk of new-onset atrial fibrillation. JAMA 2004;292:2471-2477.

1005. Baek YS, Yang PS, Kim TH, Uhm JS, Park J, Pak HN, Lee MH, Joung B. Associations of abdominal obesity and new-onset atrial fibrillation in the general population. J Am Heart Assoc 2017;6.

1006. Proietti M, Guiducci E, Cheli P, Lip GY. Is there an obesity paradox for outcomes in atrial fibrillation? A systematic review and meta-analysis of nonvitamin K antagonist oral anticoagulant trials. Stroke 2017;48:857-866.

1007. Piepoli MF, Hoes AW, Agewall S, Albus C, Brotons C, Catapano AL, Cooney MT, Corra U, Cosyns B, Deaton C, Graham I, Hall MS, Hobbs FDR, Lochen ML, Lollgen H, Marques-Vidal P, Perk J, Prescott E, Redon J, Richter DJ, Sattar N, Smulders Y, Tiberi M, van der Worp HB, van Dis I, Verschuren WMM, Binno S, ESC Scientific Document Group. 2016 European Guidelines on cardiovascular disease prevention in clinical practice: the Sixth Joint Task Force of the European Society of Cardiology and other societies on cardiovascular disease prevention in clinical practice (constituted by representatives of 10 societies and by invited experts). Developed with the special contribution of the European Association for Cardiovascular Prevention \& Rehabilitation (EACPR). Eur Heart J 2016;37:2315-2381.

1008. Cha YM, Friedman PA, Asirvatham SJ, Shen WK, Munger TM, Rea RF, Brady PA, Jahangir A, Monahan KH, Hodge DO, Meverden RA, Gersh BJ, Hammill SC, Packer DL. Catheter ablation for atrial fibrillation in patients with obesity. Circulation 2008;117:2583-2590.

1009. Ector J, Dragusin O, Adriaenssens B, Huybrechts W, Willems R, Ector H, Heidbuchel $\mathrm{H}$. Obesity is a major determinant of radiation dose in patients undergoing pulmonary vein isolation for atrial fibrillation. J Am Coll Cardiol 2007;50:234-242

1010. Shoemaker MB, Muhammad R, Farrell M, Parvez B, White BW, Streur M, Stubblefield T, Rytlewski J, Parvathaneni S, Nagarakanti R, Roden DM, Saavedra P, Ellis C, Whalen SP, Darbar D. Relation of morbid obesity and female gender to risk of procedural complications in patients undergoing atrial fibrillation ablation. Am J Cardiol 2013;111:368-373.

1011. Ettinger PO, Wu CF, De La Cruz C Jr, Weisse AB, Ahmed SS, Regan TJ. Arrhythmias and the 'holiday heart': alcohol-associated cardiac rhythm disorders. Am Heart J 1978;95:555-562.

1012. Conen D, Albert CM. Alcohol consumption and risk of atrial fibrillation: how much is too much?] Am Coll Cardiol 2014;64:290-292.

1013. Liang Y, Mente A, Yusuf S, Gao P, Sleight P, Zhu J, Fagard R, Lonn E, Teo KK; ONTARGET and TRANSCEND Investigators. Alcohol consumption and the risk of incident atrial fibrillation among people with cardiovascular disease. CMAJ 2012;184:E857-866.

1014. Larsson SC, Drca N, Wolk A. Alcohol consumption and risk of atrial fibrillation: a prospective study and dose-response meta-analysis. J Am Coll Cardiol 2014;64:281-289.

1015. Overvad TF, Rasmussen LH, Skjoth F, Overvad K, Albertsen IE, Lane DA, Lip GY, Larsen TB. Alcohol intake and prognosis of atrial fibrillation. Heart 2013;99:1093-1099.

1016. Voskoboinik A, Kalman JM, De Silva A, Nicholls T, Costello B, Nanayakkara S, Prabhu S, Stub D, Azzopardi S, Vizi D, Wong G, Nalliah C, Sugumar H, Wong M, Kotschet E, Kaye D, Taylor AJ, Kistler PM. Alcohol abstinence in drinkers with atrial fibrillation. N Engl J Med 2020;382:20-28.

1017. Lavie CJ, Thomas RJ, Squires RW, Allison TG, Milani RV. Exercise training and cardiac rehabilitation in primary and secondary prevention of coronary heart disease. Mayo Clin Proc 2009;84:373-383.

1018. Mont L. Arrhythmias and sport practice. Heart 2010;96:398-405.

1019. Menezes AR, Lavie CJ, De Schutter A, Milani RV, O'Keefe J, DiNicolantonio JJ, Morin DP, Abi-Samra FM. Lifestyle modification in the prevention and treatment of atrial fibrillation. Prog Cardiovasc Dis 2015;58:117-125.

1020. Karjalainen J, Kujala UM, Kaprio J, Sarna S, Viitasalo M. Lone atrial fibrillation in vigorously exercising middle aged men: case-control study. BMJ 1998;316:1784-1785.

1021. Baldesberger S, Bauersfeld U, Candinas R, Seifert B, Zuber M, Ritter M, Jenni R, Oechslin E, Luthi P, Scharf C, Marti B, Attenhofer Jost CH. Sinus node disease and arrhythmias in the long-term follow-up of former professional cyclists. Eur Heart J 2008;29:71-78.

1022. Molina L, Mont L, Marrugat J, Berruezo A, Brugada J, Bruguera J, Rebato C Elosua R. Long-term endurance sport practice increases the incidence of lone atrial fibrillation in men: a follow-up study. Europace 2008;10:618-623. 
1023. Nielsen JR, Wachtell K, Abdulla J. The relationship between physical activity and risk of atrial fibrillation - a systematic review and meta-analysis. J Atr Fibrillation 2013;5:789.

1024. Risom SS, Zwisler AD, Johansen PP, Sibilitz KL, Lindschou J, Gluud C, Taylor RS, Svendsen JH, Berg SK. Exercise-based cardiac rehabilitation for adults with atrial fibrillation. Cochrane Database Syst Rev 2017;2:CD011197.

1025. Dzeshka MS, Shantsila A, Shantsila E, Lip GYH. Atrial fibrillation and hypertension. Hypertension 2017;70:854-861.

1026. Williams B, Mancia G, Spiering W, Agabiti Rosei E, Azizi M, Burnier M, Clement DL, Coca A, de Simone G, Dominiczak A, Kahan T, Mahfoud F, Redon J, Ruilope L, Zanchetti A, Kerins M, Kjeldsen SE, Kreutz R, Laurent S, Lip GYH, McManus R, Narkiewicz K, Ruschitzka F, Schmieder RE, Shlyakhto E, Tsioufis C, Aboyans V, Desormais I, ESC Scientific Document Group. 2018 ESC/ESH Guidelines for the management of arterial hypertension. Eur Heart J 2018;39:3021-3104.

1027. Kim TH, Yang PS, Yu HT, Jang E, Shin H, Kim HY, Uhm JS, Kim JY, Sung JH, Pak HN, Lee MH, Joung B, Lip GYH. Effect of hypertension duration and blood pressure level on ischaemic stroke risk in atrial fibrillation: nationwide data covering the entire Korean population. Eur Heart J 2019;40:809-819.

1028. Lip GY, Frison L, Grind M. Effect of hypertension on anticoagulated patients with atrial fibrillation. Eur Heart J 2007;28:752-759.

1029. Steinberg JS, Shabanov V, Ponomarev D, Losik D, Ivanickiy E, Kropotkin E, Polyakov K, Ptaszynski P, Keweloh B, Yao CJ, Pokushalov EA, Romanov AB. Effect of renal denervation and catheter ablation vs catheter ablation alone on atrial fibrillation recurrence among patients with paroxysmal atrial fibrillation and hypertension: the ERADICATE-AF randomized clinical trial. JAMA 2020;323:248-255.

1030. Du X, Ninomiya T, de Galan B, Abadir E, Chalmers J, Pillai A, Woodward M, Cooper M, Harrap S, Hamet P, Poulter N, Lip GY, Patel A, Group AC. Risks of cardiovascular events and effects of routine blood pressure lowering among patients with type 2 diabetes and atrial fibrillation: results of the ADVANCE study. Eur Heart J 2009;30:1128-1135.

1031. Pallisgaard JL, Schjerning AM, Lindhardt TB, Procida K, Hansen ML, TorpPedersen C, Gislason GH. Risk of atrial fibrillation in diabetes mellitus: a nationwide cohort study. Eur J Prev Cardiol 2016;23:621-627.

1032. Rizzo MR, Sasso FC, Marfella R, Siniscalchi M, Paolisso P, Carbonara O, Capoluongo MC, Lascar N, Pace C, Sardu C, Passavanti B, Barbieri M, Mauro C, Paolisso G. Autonomic dysfunction is associated with brief episodes of atrial fibrillation in type 2 diabetes. J Diabetes Complications 2015;29:88-92.

1033. Movahed MR, Hashemzadeh M, Jamal MM. Diabetes mellitus is a strong, independent risk for atrial fibrillation and flutter in addition to other cardiovascular disease. Int / Cardiol 2005;105:315-318.

1034. Lee SR, Choi EK, Rhee TM, Lee HJ, Lim WH, Kang SH, Han KD, Cha MJ, Cho Y, Oh IY, Oh S. Evaluation of the association between diabetic retinopathy and the incidence of atrial fibrillation: a nationwide population-based study. Int J Cardiol 2016;223:953-957.

1035. Mozaffarian D, Benjamin EJ, Go AS, Arnett DK, Blaha MJ, Cushman M, de Ferranti S, Despres JP, Fullerton HJ, Howard VJ, Huffman MD, Judd SE, Kissela BM, Lackland DT, Lichtman JH, Lisabeth LD, Liu S, Mackey RH, Matchar DB, McGuire DK, Mohler ER, 3rd, Moy CS, Muntner P, Mussolino ME, Nasir K, Neumar RW, Nichol G, Palaniappan L, Pandey DK, Reeves MJ, Rodriguez C], Sorlie PD, Stein J, Towfighi A, Turan TN, Virani SS, Willey JZ, Woo D, Yeh RW, Turner MB, American Heart Association Statistics Committee and Stroke Statistics Subcommittee. Heart disease and stroke statistics - 2015 update: a report from the American Heart Association. Circulation 2015;131:e29-322.

1036. Fatemi O, Yuriditsky E, Tsioufis C, Tsachris D, Morgan T, Basile J, Bigger T, Cushman W, Goff D, Soliman EZ, Thomas A, Papademetriou V. Impact of intensive glycemic control on the incidence of atrial fibrillation and associated cardiovascular outcomes in patients with type 2 diabetes mellitus (from the Action to Control Cardiovascular Risk in Diabetes study). Am J Cardiol 2014;114:1217-1222.

1037. Chang SH, Wu LS, Chiou MJ, Liu JR, Yu KH, Kuo CF, Wen MS, Chen WJ, Yeh $\mathrm{YH}$, See LC. Association of metformin with lower atrial fibrillation risk among patients with type 2 diabetes mellitus: a population-based dynamic cohort and in vitro studies. Cardiovasc Diabetol 2014;13:123.

1038. Zhang Z, Zhang X, Korantzopoulos P, Letsas KP, Tse G, Gong M, Meng L, Li G, Liu T. Thiazolidinedione use and atrial fibrillation in diabetic patients: a meta-analysis. BMC Cardiovasc Disord 2017;17:96.

1039. Bell DSH, Goncalves E. Atrial fibrillation and type 2 diabetes: prevalence, etiology, pathophysiology and effect of anti-diabetic therapies. Diabetes Obes Metab 2019;21:210-217.

1040. Patti G, Di Gioia G, Cavallari I, Nenna A. Safety and efficacy of nonvitamin K antagonist oral anticoagulants versus warfarin in diabetic patients with atrial fibrillation: a study-level meta-analysis of phase III randomized trials. Diabetes Metab Res Rev 2017;33.
1041. Hylek EM, Held C, Alexander JH, Lopes RD, De Caterina R, Wojdyla DM, Huber K, Jansky P, Steg PG, Hanna M, Thomas L, Wallentin L, Granger CB. Major bleeding in patients with atrial fibrillation receiving apixaban or warfarin: the ARISTOTLE trial (Apixaban for Reduction in Stroke and Other Thromboembolic Events in Atrial Fibrillation): predictors, characteristics, and clinical outcomes. J Am Coll Cardiol 2014;63:2141-2147.

1042. Donnellan E, Aagaard P, Kanj M, Jaber W, Elshazly M, Hoosien M, Baranowski B, Hussein A, Saliba W, Wazni O. Association between pre-ablation glycemic control and outcomes among patients with diabetes undergoing atrial fibrillation ablation. JACC Clin Electrophysiol 2019;5:897-903.

1043. Linz D, Baumert M, Catcheside P, Floras J, Sanders P, Levy P, Cowie MR, Doug McEvoy R. Assessment and interpretation of sleep disordered breathing severity in cardiology: clinical implications and perspectives. Int J Cardiol 2018;271:281-288.

1044. Gami AS, Pressman G, Caples SM, Kanagala R, Gard JJ, Davison DE, Malouf JF, Ammash NM, Friedman PA, Somers VK. Association of atrial fibrillation and obstructive sleep apnea. Circulation 2004;110:364-367.

1045. Goudis CA, Ketikoglou DG. Obstructive sleep and atrial fibrillation: pathophysiological mechanisms and therapeutic implications. Int J Cardiol 2017;230:293-300.

1046. Epstein LJ, Kristo D, Strollo PJ Jr, Friedman N, Malhotra A, Patil SP, Ramar K, Rogers R, Schwab RJ, Weaver EM, Weinstein MD, Adult Obstructive Sleep Apnea Task Force of the American Academy of Sleep Medicine. Clinical guideline for the evaluation, management and long-term care of obstructive sleep apnea in adults. J Clin Sleep Med 2009;5:263-276.

1047. Linz D, McEvoy RD, Cowie MR, Somers VK, Nattel S, Levy P, Kalman JM, Sanders P. Associations of obstructive sleep apnea with atrial fibrillation and continuous positive airway pressure treatment: a review. JAMA Cardiol 2018;3:532-540.

1048. Fein AS, Shvilkin A, Shah D, Haffajee Cl, Das S, Kumar K, Kramer DB Zimetbaum PJ, Buxton AE, Josephson ME, Anter E. Treatment of obstructive sleep apnea reduces the risk of atrial fibrillation recurrence after catheter ablation. J Am Coll Cardiol 2013;62:300-305.

1049. Neilan TG, Farhad H, Dodson JA, Shah RV, Abbasi SA, Bakker JP, Michaud GF, van der Geest R, Blankstein R, Steigner M, John RM, Jerosch-Herold M, Malhotra A, Kwong RY. Effect of sleep apnea and continuous positive airway pressure on cardiac structure and recurrence of atrial fibrillation. J Am Heart Assoc 2013;2:e000421.

1050. Qureshi WT, Nasir UB, Alqalyoobi S, O'Neal WT, Mawri S, Sabbagh S, Soliman EZ, Al-Mallah MH. Meta-analysis of continuous positive airway pressure as a therapy of atrial fibrillation in obstructive sleep apnea. Am J Cardio 2015;116:1767-1773.

1051. Shukla A, Aizer A, Holmes D, Fowler S, Park DS, Bernstein S, Bernstein N Chinitz L. Effect of obstructive sleep apnea treatment on atrial fibrillation recurrence: a meta-analysis. JACC Clin Electrophysiol 2015;1:41-51.

1052. Pathak RK, Elliott A, Middeldorp ME, Meredith M, Mehta AB, Mahajan R Hendriks JM, Twomey D, Kalman JM, Abhayaratna WP, Lau DH, Sanders P. Impact of CARDIOrespiratory FITness on Arrhythmia Recurrence in Obese Individuals With Atrial Fibrillation: the CARDIO-FIT study. J Am Coll Cardiol 2015;66:985-996.

1053. Bonfanti L, Annovi A, Sanchis-Gomar F, Saccenti C, Meschi T, Ticinesi A Cervellin G. Effectiveness and safety of electrical cardioversion for acute-onset atrial fibrillation in the emergency department: a real-world 10-year single center experience. Clin Exp Emerg Med 2019;6:64-69.

1054. Scheuermeyer FX, Grafstein E, Stenstrom R, Innes G, Heslop C, MacPhee J, Pourvali R, Heilbron B, McGrath L, Christenson J. Thirty-day and 1-year outcomes of emergency department patients with atrial fibrillation and no acute underlying medical cause. Ann Emerg Med 2012;60:755-765.e2.

1055. Boriani G, Proietti M, Laroche C, Diemberger I, Popescu MI, Riahi S, Shantsila A, Dan GA, Tavazzi L, Maggioni AP, Lip GYH; EORP-A Pilot General Registry Investigators. Changes to oral anticoagulant therapy and risk of death over a 3-year follow-up of a contemporary cohort of European patients with atrial fibrillation final report of the EURObservational Research Programme on Atrial Fibrillation (EORP-AF) pilot general registry. Int J Cardiol 2018;271:68-74.

1056. Boriani G, Laroche C, Diemberger I, Fantecchi E, Popescu MI, Rasmussen LH, Dan GA, Kalarus Z, Tavazzi L, Maggioni AP, Lip GY. 'Real-world' management and outcomes of patients with paroxysmal vs. non-paroxysmal atrial fibrillation in Europe: the EURObservational Research Programme-Atrial Fibrillation (EORP-AF) general pilot registry. Europace 2016;18:648-657.

1057. Gulizia MM, Cemin R, Colivicchi F, De Luca L, Di Lenarda A, Boriani G, Di Pasquale G, Nardi F, Scherillo M, Lucci D, Fabbri G, Maggioni AP; BLITZ-AF Investigators. Management of atrial fibrillation in the emergency room and in the cardiology ward: the BLITZ AF study. Europace 2019;21:230-238.

1058. Gonzalez-Pacheco H, Marquez MF, Arias-Mendoza A, Alvarez-Sangabriel A Eid-Lidt G, Gonzalez-Hermosillo A, Azar-Manzur F, Altamirano-Castillo A, Briseno-Cruz JL, Garcia-Martinez A, Mendoza-Garcia S, Martinez-Sanchez C. 
Clinical features and in-hospital mortality associated with different types of atrial fibrillation in patients with acute coronary syndrome with and without ST elevation. J Cardiol 2015;66:148-154.

1059. Krijthe BP, Leening MJ, Heeringa J, Kors JA, Hofman A, Franco OH, Witteman JC, Stricker BH. Unrecognized myocardial infarction and risk of atrial fibrillation: the Rotterdam Study. Int J Cardiol 2013;168:1453-1457.

1060. Chao TF, Huang YC, Liu CJ, Chen SJ, Wang KL, Lin YJ, Chang SL, Lo LW, Hu YF, Tuan TC, Chen TJ, Hsieh MH, Lip GY, Chen SA. Acute myocardial infarction in patients with atrial fibrillation with a CHA2DS2-VASc score of 0 or 1: a nationwide cohort study. Heart Rhythm 2014;11:1941-1947.

1061. Sanclemente C, Yeste M, Suarez C, Coll R, Aguilar E, Sahuquillo JC, Lerma R, Monreal M; FRENA Investigators. Predictors of outcome in stable outpatients with peripheral artery disease. Intern Emerg Med 2014;9:69-77.

1062. Soliman EZ, Lopez F, O'Neal WT, Chen LY, Bengtson L, Zhang ZM, Loehr L, Cushman M, Alonso A. Atrial fibrillation and risk of ST-segment-elevation versus non-ST-segment-elevation myocardial infarction: the Atherosclerosis Risk in Communities (ARIC) study. Circulation 2015;131:1843-1850.

1063. Soliman EZ, Safford MM, Muntner P, Khodneva Y, Dawood FZ, Zakai NA, Thacker EL, Judd S, Howard VJ, Howard G, Herrington DM, Cushman M. Atrial fibrillation and the risk of myocardial infarction. JAMA Intern Med 2014; 174:107-114

1064. Kralev S, Schneider K, Lang S, Suselbeck T, Borggrefe M. Incidence and severity of coronary artery disease in patients with atrial fibrillation undergoing first-time coronary angiography. PLoS One 2011;6:e24964.

1065. Guimaraes PO, Zakroysky P, Goyal A, Lopes RD, Kaltenbach LA, Wang TY. Usefulness of antithrombotic therapy in patients with atrial fibrillation and acute myocardial infarction. Am J Cardiol 2019;123:12-18.

1066. Erez A, Goldenberg I, Sabbag A, Nof E, Zahger D, Atar S, Pollak A, DobreckyMerye I, Beigel R, Matetzky S, Glikson M, Beinart R. Temporal trends and outcomes associated with atrial fibrillation observed during acute coronary syndrome: real-world data from the Acute Coronary Syndrome Israeli Survey (ACSIS), 2000-2013. Clin Cardiol 2017;40:275-280.

1067. Neumann FJ, Sousa-Uva M, Ahlsson A, Alfonso F, Banning AP, Benedetto U, Byrne RA, Collet JP, Falk V, Head SJ, Juni P, Kastrati A, Koller A, Kristensen SD, Niebauer J, Richter DJ, Seferovic PM, Sibbing D, Stefanini GG, Windecker S, Yadav R, Zembala MO, ESC Scientific Document Group. 2018 ESC/EACTS Guidelines on myocardial revascularization. Eur Heart J 2019;40:87-165.

1068. Knuuti J, Wijns W, Saraste A, Capodanno D, Barbato E, Funck-Brentano C, Prescott E, Storey RF, Deaton C, Cuisset T, Agewall S, Dickstein K, Edvardsen T, Escaned J, Gersh B], Svitil P, Gilard M, Hasdai D, Hatala R, Mahfoud F, Masip J, Muneretto C, Valgimigli M, Achenbach S, Bax JJ, ESC Scientific Document Group. 2019 ESC Guidelines for the diagnosis and management of chronic coronary syndromes. Eur Heart J 2020;41:407-477.

1069. Lip GYH, Collet JP, Haude M, Byrne R, Chung EH, Fauchier L, Halvorsen S, Lau D, Lopez-Cabanillas N, Lettino M, Marin F, Obel I, Rubboli A, Storey RF, Valgimigli M, Huber K, ESC Scientific Document Group. 2018 Joint European consensus document on the management of antithrombotic therapy in atrial fibrillation patients presenting with acute coronary syndrome and/or undergoing percutaneous cardiovascular interventions: a joint consensus document of the European Heart Rhythm Association (EHRA), European Society of Cardiology Working Group on Thrombosis, European Association of Percutaneous Cardiovascular Interventions (EAPCI), and European Association of Acute Cardiac Care (ACCA) endorsed by the Heart Rhythm Society (HRS), Asia-Pacific Heart Rhythm Society (APHRS), Latin America Heart Rhythm Society (LAHRS), and Cardiac Arrhythmia Society of Southern Africa (CASSA). Europace 2019;21:192-193.

1070. Gargiulo G, Goette A, Tijssen J, Eckardt L, Lewalter T, Vranckx P, Valgimigli $M$. Safety and efficacy outcomes of double vs. triple antithrombotic therapy in patients with atrial fibrillation following percutaneous coronary intervention: a systematic review and meta-analysis of non-vitamin $\mathrm{K}$ antagonist oral anticoagulant-based randomized clinical trials. Eur Heart J 2019;40:3757-3767.

1071. Potpara TS, Mujovic N, Proietti M, Dagres N, Hindricks G, Collet JP, Valgimigli $M$, Heidbuchel H, Lip GYH. Revisiting the effects of omitting aspirin in combined antithrombotic therapies for atrial fibrillation and acute coronary syndromes or percutaneous coronary interventions: meta-analysis of pooled data from the PIONEER AF-PCI, RE-DUAL PCI, and AUGUSTUS trials. Europace 2020;22:33-46.

1072. Yasuda S, Kaikita K, Akao M, Ako J, Matoba T, Nakamura M, Miyauchi K, Hagiwara N, Kimura K, Hirayama A, Matsui K, Ogawa H; AFIRE Investigators. Antithrombotic therapy for atrial fibrillation with stable coronary disease. $\mathrm{N}$ Engl J Med 2019;381:1103-1113.

1073. Karjalainen PP, Vikman S, Niemela M, Porela P, Ylitalo A, Vaittinen MA, Puurunen M, Airaksinen TJ, Nyman K, Vahlberg T, Airaksinen KE. Safety of percutaneous coronary intervention during uninterrupted oral anticoagulant treatment. Eur Heart J 2008;29:1001-1010.
1074. Vranckx P, Leebeek FW, Tijssen JG, Koolen J, Stammen F, Herman JP, de Winter RJ, van THAW, Backx B, Lindeboom W, Kim SY, Kirsch B, van Eickels M, Misselwitz F, Verheugt FW. Peri-procedural use of rivaroxaban in elective percutaneous coronary intervention to treat stable coronary artery disease. The X-PLORER trial. Thromb Haemost 2015;114:258-267.

1075. Vranckx P, Verheugt FW, de Maat MP, Ulmans VA, Regar E, Smits P, ten Berg JM, Lindeboom W, Jones RL, Friedman J, Reilly P, Leebeek FW. A randomised study of dabigatran in elective percutaneous coronary intervention in stable coronary artery disease patients. Eurolntervention 2013;8:1052-1060.

1076. Fiedler KA, Maeng M, Mehilli J, Schulz-Schupke S, Byrne RA, Sibbing D, Hoppmann P, Schneider S, Fusaro M, Ott I, Kristensen SD, Ibrahim T, Massberg S, Schunkert H, Laugwitz KL, Kastrati A, Sarafoff N. Duration of triple therapy in patients requiring oral anticoagulation after drug-eluting stent implantation: the ISAR-TRIPLE trial. J Am Coll Cardiol 2015;65:1619-1629.

1077. Lamberts M, Gislason GH, Olesen JB, Kristensen SL, Schjerning Olsen AM, Mikkelsen A, Christensen CB, Lip GY, Kober L, Torp-Pedersen C, Hansen ML. Oral anticoagulation and antiplatelets in atrial fibrillation patients after myocardial infarction and coronary intervention. J Am Coll Cardiol 2013;62:981-989.

1078. Dewilde WJ, Oirbans T, Verheugt FW, Kelder JC, De Smet BJ, Herrman JP, Adriaenssens T, Vrolix M, Heestermans AA, Vis MM, Tijsen JG, van 't Hof AW, ten Berg JM, for the WOEST Study Investigators. Use of clopidogrel with or without aspirin in patients taking oral anticoagulant therapy and undergoing percutaneous coronary intervention: an open-label, randomised, controlled trial. Lancet 2013;381:1107-1115.

1079. Cannon CP, Bhatt DL, Oldgren J, Lip GYH, Ellis SG, Kimura T, Maeng M, Merkely B, Zeymer U, Gropper S, Nordaby M, Kleine E, Harper R, Manassie J, Januzzi JL, Ten Berg JM, Steg PG, Hohnloser SH; RE-DUAL PCl Steering Committee Investigators. Dual antithrombotic therapy with dabigatran after $\mathrm{PCl}$ in atrial fibrillation. N Engl J Med 2017;377:1513-1524.

1080. Gibson CM, Mehran R, Bode C, Halperin J, Verheugt FW, Wildgoose P, Birmingham M, lanus J, Burton P, van Eickels M, Korjian S, Daaboul Y, Lip GY, Cohen M, Husted S, Peterson ED, Fox KA. Prevention of bleeding in patients with atrial fibrillation undergoing $\mathrm{PCl} . \mathrm{N}$ Engl J Med 2016;375:2423-2434.

1081. Lopes RD, Heizer G, Aronson R, Vora AN, Massaro T, Mehran R, Goodman SG, Windecker S, Darius H, Li J, Averkov O, Bahit MC, Berwanger O, Budaj A, Hijazi Z, Parkhomenko A, Sinnaeve P, Storey RF, Thiele H, Vinereanu D, Granger CB, Alexander JH; AUGUSTUS Investigators. Antithrombotic therapy after acute coronary syndrome or $\mathrm{PCl}$ in atrial fibrillation. $N$ Engl J Med 2019;380:1509-1524.

1082. Vranckx P, Valgimigli M, Eckardt L, Tijssen J, Lewalter T, Gargiulo G, Batushkin V, Campo G, Lysak Z, Vakaliuk I, Milewski K, Laeis P, Reimitz PE, Smolnik R, Zierhut W, Goette A. Edoxaban-based versus vitamin $K$ antagonist-based antithrombotic regimen after successful coronary stenting in patients with atrial fibrillation (ENTRUST-AF PCI): a randomised, open-label, phase $3 \mathrm{~b}$ trial. Lancet 2019;394:1335-1343.

1083. Golwala HB, Cannon CP, Steg PG, Doros G, Qamar A, Ellis SG, Oldgren J, Ten Berg JM, Kimura T, Hohnloser SH, Lip GYH, Bhatt DL. Safety and efficacy of dual vs. triple antithrombotic therapy in patients with atrial fibrillation following percutaneous coronary intervention: a systematic review and metaanalysis of randomized clinical trials. Eur Heart J 2018;39:1726-1735a.

1084. Lopes RD, Hong H, Harskamp RE, Bhatt DL, Mehran R, Cannon CP, Granger CB, Verheugt FWA, Li J, Ten Berg JM, Sarafoff N, Gibson CM, Alexander JH. Safety and efficacy of antithrombotic strategies in patients with atrial fibrillation undergoing percutaneous coronary intervention: a network meta-analysis of randomized controlled trials. JAMA Cardiol 2019:pii:2735647.

1085. Andreou I, Briasoulis A, Pappas C, Ikonomidis I, Alexopoulos D. Ticagrelor versus clopidogrel as part of dual or triple antithrombotic therapy: a systematic review and meta-analysis. Cardiovasc Drugs Ther 2018;32:287-294.

1086. Fu A, Singh K, Abunassar J, Malhotra N, Le May M, Labinaz M, Glover C, Marquis JF, Froeschl M, Dick A, Hibbert B, Chong AY, So DY; CAPITAL Investigators. Ticagrelor in triple antithrombotic therapy: predictors of ischemic and bleeding complications. Clin Cardiol 2016;39:19-23.

1087. Jackson LR, 2nd, Ju C, Zettler M, Messenger JC, Cohen DJ, Stone GW, Baker BA, Effron M, Peterson ED, Wang TY. Outcomes of patients with acute myocardial infarction undergoing percutaneous coronary intervention receiving an oral anticoagulant and dual antiplatelet therapy: a comparison of clopidogrel versus prasugrel from the TRANSLATE-ACS study. JACC Cardiovasc Interv 2015;8:1880-1889.

1088. Sarafoff N, Martischnig A, Wealer J, Mayer K, Mehilli J, Sibbing D, Kastrati A. Triple therapy with aspirin, prasugrel, and vitamin $\mathrm{K}$ antagonists in patients with drug-eluting stent implantation and an indication for oral anticoagulation. J Am Coll Cardiol 2013;61:2060-2066.

1089. Verlinden NJ, Coons JC, lasella CJ, Kane-Gill SL. Triple antithrombotic therapy with aspirin, P2Y12 inhibitor, and warfarin after percutaneous coronary 
intervention: an evaluation of prasugrel or ticagrelor versus clopidogrel. J Cardiovasc Pharmacol Ther 2017;22:546-551.

1090. Steffel J, Verhamme P, Potpara TS, Albaladejo P, Antz M, Desteghe L, Haeusler KG, Oldgren J, Reinecke H, Roldan-Schilling V, Rowell N, Sinnaeve P, Collins R, Camm AJ, Heidbuchel H, ESC Scientific Document Group. The 2018 European Heart Rhythm Association Practical Guide on the use of nonvitamin $\mathrm{K}$ antagonist oral anticoagulants in patients with atrial fibrillation. Eur Heart J 2018;39:1330-1393.

1091. Pikija S, Sztriha LK, Sebastian Mutzenbach J, Golaszewski SM, Sellner J. Idarucizumab in dabigatran-treated patients with acute ischemic stroke receiving alteplase: a systematic review of the available evidence. CNS Drugs 2017;31:747-757.

1092. Saxena R, Lewis S, Berge E, Sandercock PA, Koudstaal PJ. Risk of early death and recurrent stroke and effect of heparin in 3169 patients with acute ischemic stroke and atrial fibrillation in the International Stroke Trial. Stroke 2001;32:2333-2337.

1093. Hart RG, Coull BM, Hart D. Early recurrent embolism associated with nonvalvular atrial fibrillation: a retrospective study. Stroke 1983;14:688-693.

1094. Berge E, Abdelnoor M, Nakstad PH, Sandset PM. Low molecular-weight heparin versus aspirin in patients with acute ischaemic stroke and atrial fibrillation: a double-blind randomised study. HAEST Study Group. Heparin in Acute Embolic Stroke Trial. Lancet 2000;355:1205-1210.

1095. Paciaroni M, Agnelli G, Micheli S, Caso V. Efficacy and safety of anticoagulant treatment in acute cardioembolic stroke: a meta-analysis of randomized controlled trials. Stroke 2007;38:423-430.

1096. von Kummer R, Broderick JP, Campbell BC, Demchuk A, Goyal M, Hill MD, Treurniet KM, Majoie CB, Marquering HA, Mazya MV, San Roman L, Saver JL, Strbian D, Whiteley W, Hacke W. The Heidelberg Bleeding Classification: classification of bleeding events after ischemicstroke and reperfusion therapy. Stroke 2015;46:2981-2986.

1097. Paciaroni M, Agnelli G, Corea F, Ageno W, Alberti A, Lanari A, Caso V, Micheli S, Bertolani L, Venti M, Palmerini F, Biagini S, Comi G, Previdi P, Silvestrelli $G$. Early hemorrhagic transformation of brain infarction: rate, predictive factors, and influence on clinical outcome: results of a prospective multicenter study. Stroke 2008;39:2249-2256.

1098. Kablau M, Kreisel SH, Sauer T, Binder J, Szabo K, Hennerici MG, Kern R. Predictors and early outcome of hemorrhagic transformation after acute ischemic stroke. Cerebrovasc Dis 2011;32:334-341.

1099. Seiffge DJ, Werring DJ, Paciaroni M, Dawson J, Warach S, Milling TJ, Engelter ST, Fischer $U$, Norrving B. Timing of anticoagulation after recent ischaemic stroke in patients with atrial fibrillation. Lancet Neurol 2019;18:117-126.

1100. Paciaroni M, Agnelli G, Falocci N, Tsivgoulis G, Vadikolias K, Liantinioti C, Chondrogianni M, Bovi P, Carletti M, Cappellari M, Zedde M, Ntaios G, Karagkiozi E, Athanasakis G, Makaritsis K, Silvestrelli G, Lanari A, Ciccone A, Putaala J, Tomppo L, Tatlisumak T, Abdul-Rahim AH, Lees KR, Alberti A, Venti M, Acciarresi M, D’Amore C, Becattini C, Mosconi MG, Cimini LA, Soloperto R, Masotti L, Vannucchi V, Lorenzini G, Tassi R, Guideri F, Acampa M, Martini G, Sohn SI, Marcheselli S, Mumoli N, De Lodovici ML, Bono G, Furie KL, Tadi P, Yaghi S, Toni D, Letteri F, Tassinari T, Kargiotis O, Lotti EM, Flomin Y, Mancuso M, Maccarrone M, Giannini N, Bandini F, Pezzini A, Poli L, Padovani A, Scoditti U, Denti L, Consoli D, Galati F, Sacco S, Carolei A, Tiseo C, Gourbali V, Orlandi G, Giuntini M, Chiti A, Giorli E, Gialdini G, Corea F, Ageno W, Bellesini M, Colombo G, Monaco S, Maimone Baronello M, Karapanayiotides T, Caso V. Early Recurrence and Major Bleeding in Patients With Acute Ischemic Stroke and Atrial Fibrillation Treated With NonVitamin-K Oral Anticoagulants (RAF-NOACs) study. J Am Heart Assoc 2017;6.

1101. Seiffge DJ, Traenka C, Polymeris A, Hert L, Peters N, Lyrer P, Engelter ST, Bonati LH, De Marchis GM. Early start of DOAC after ischemic stroke: risk of intracranial hemorrhage and recurrent events. Neurology 2016;87:1856-1862.

1102. Arihiro S, Todo K, Koga M, Furui E, Kinoshita N, Kimura K, Yamagami H, Terasaki T, Yoshimura S, Shiokawa Y, Kamiyama K, Takizawa S, Okuda S, Okada Y, Nagakane Y, Kameda T, Hasegawa Y, Shibuya S, Ito Y, Nakashima T, Takamatsu K, Nishiyama K, Matsuki T, Homma K, Takasugi J, Tokunaga K, Sato S, Kario K, Kitazono T, Toyoda K; SAMURAI Study Investigators. Three-month risk-benefit profile of anticoagulation after stroke with atrial fibrillation: the SAMURAI-Nonvalvular Atrial Fibrillation (NVAF) study. Int J Stroke 2016;11:565-574.

1103. Hong KS, Kwon SU, Lee SH, Lee JS, Kim YJ, Song TJ, Kim YD, Park MS, Kim EG, Cha JK, Sung SM, Yoon BW, Bang OY, Seo WK, Hwang YH, Ahn SH, Kang DW, Kang HG, Yu KH, Phase 2 exploratory clinical study to assess the effects of xarelto versus warfarin on ischemia $B$, hospital stay in acute cerebral infarction patients with non-valvular atrial fibrillation study $G$. Rivaroxaban vs warfarin sodium in the ultra-early period after atrial fibrillation-related mild ischemic stroke: a randomized clinical trial. JAMA Neurol 2017;74:1206-1215.

1104. Ntaios G, Papavasileiou V, Diener HC, Makaritsis K, Michel P. Nonvitamin-Kantagonist oral anticoagulants versus warfarin in patients with atrial fibrillation and previous stroke or transient ischemic attack: an updated systematic review and meta-analysis of randomized controlled trials. Int J Stroke 2017;12:589-596.

1105. Gonzalez ME, Klein FR, Riccio PM, Cassara FP, Munoz Giacomelli F, Racosta JM, Roberts ES, Sposato, LA. Atrial fibrillation detected after acute ischemic stroke: evidence supporting the neurogenic hypothesis. J Stroke Cerebrovasc Dis 2013;22:e486-491.

1106. Sposato LA, Cerasuolo JO, Cipriano LE, Fang J, Fridman S, Paquet M, Saposnik G. Atrial fibrillation detected after stroke is related to a low risk of ischemic stroke recurrence. Neurology 2018;90:e924-e931.

1107. Steinberg BA, Shrader P, Pieper K, Thomas L, Allen LA, Ansell J, Chan PS, Ezekowitz MD, Fonarow GC, Freeman JV, Gersh BJ, Kowey PR, Mahaffey KW, Naccarelli GV, Reiffel JA, Singer, DE, Peterson ED, Piccini, JP; Outcomes Registry for Better Informed Treatment of Atrial Fibrillation (ORBIT-AF) II Investigators. Frequency and outcomes of reduced dose non-vitamin $\mathrm{K}$ antagonist anticoagulants: results from ORBIT-AF II (the Outcomes Registry for Better Informed Treatment of Atrial Fibrillation II). J Am Heart Assoc 2018;7:e007633.

1108. Hart RG, Sharma M, Mundl H, Kasner SE, Bangdiwala SI, Berkowitz SD, Swaminathan B, Lavados P, Wang Y, Wang Y, Davalos A, Shamalov N, Mikulik R, Cunha L, Lindgren A, Arauz A, Lang W, Czlonkowska A, Eckstein J, Gagliardi RJ, Amarenco P, Ameriso SF, Tatlisumak T, Veltkamp R, Hankey GJ, Toni D, Bereczki D, Uchiyama S, Ntaios G, Yoon BW, Brouns R, Endres M, Muir KW, Bornstein N, Ozturk S, O’Donnell MJ, De Vries Basson MM, Pare G, Pater C, Kirsch B, Sheridan P, Peters G, Weitz Jl, Peacock WF, Shoamanesh A, Benavente OR, Joyner C, Themeles E, Connolly SJ; NAVIGATE ESUS Investigators. Rivaroxaban for Stroke Prevention after Embolic Stroke of Undetermined Source. N Engl J Med 2018;378:2191-2201.

1109. Diener HC, Sacco RL, Easton JD, Granger CB, Bernstein RA, Uchiyama S, Kreuzer J, Cronin L, Cotton D, Grauer C, Brueckmann M, Chernyatina M, Donnan G, Ferro JM, Grond M, Kallmunzer B, Krupinski J, Lee BC, Lemmens R, Masjuan J, Odinak M, Saver JL, Schellinger PD, Toni D, Toyoda K; RESPECT ESUS Steering Committee Investigators. Dabigatran for prevention of stroke after embolic stroke of undetermined source. N Engl J Med 2019;380:1906-1917.

1110. Healey JS, Gladstone DJ, Swaminathan B, Eckstein J, Mundl H, Epstein AE, Haeusler KG, Mikulik R, Kasner SE, Toni D, Arauz A, Ntaios G, Hankey GJ, Perera K, Pagola J, Shuaib A, Lutsep H, Yang X, Uchiyama S, Endres M, Coutts SB, Karlinski M, Czlonkowska A, Molina CA, Santo G, Berkowitz SD, Hart RG, Connolly SJ. Recurrent stroke with rivaroxaban compared with aspirin according to predictors of atrial fibrillation: secondary analysis of the NAVIGATE ESUS randomized clinical trial. JAMA Neurol 2019.

1111. Geisler T, Poli S, Meisner C, Schreieck J, Zuern CS, Nagele T, Brachmann J, Jung W, Gahn G, Schmid E, Baezner H, Keller T, Petzold GC, Schrickel JW, Liman J, Wachter R, Schon F, Schabet M, Lindner A, Ludolph AC, Kimmig H, Jander S, Schlegel U, Gawaz M, Ziemann U. Apixaban for treatment of embolic stroke of undetermined source (ATTICUS randomized trial): rationale and study design. Int J Stroke 2017;12:985-990.

1112. Li Y-G, Bisson A, Bodin A, Herbert J, Grammatico-Guillon L, Joung B, Wang Y-T, Lip GYH, Fauchier L. C2HEST score and prediction of incident atrial fibrillation in poststroke patients: a French nationwide study. J Am Heart Assoc 2019;8:e012546.

1113. Gladstone DJ, Spring M, Dorian P, Panzov V, Thorpe KE, Hall J, Vaid $H$ O'Donnell M, Laupacis A, Cote R, Sharma M, Blakely JA, Shuaib A, Hachinski V, Coutts SB, Sahlas DJ, Teal P, Yip S, Spence JD, Buck B, Verreault S, Casaubon LK, Penn A, Selchen D, Jin A, Howse D, Mehdiratta M, Boyle K, Aviv R, Kapral MK, Mamdani M; EMBRACE Investigators and Coordinators. Atrial fibrillation in patients with cryptogenic stroke. N Engl J Med 2014;370:2467-2477.

1114. Sanna T, Diener HC, Passman RS, Di Lazzaro V, Bernstein RA, Morillo CA, Rymer MM, Thijs V, Rogers T, Beckers F, Lindborg K, Brachmann J; CRYSTAL AF Investigators. Cryptogenic stroke and underlying atrial fibrillation. N Engl J Med 2014;370:2478-2486.

1115. Wachter R, Groschel K, Gelbrich G, Hamann GF, Kermer P, Liman J, Seegers J, Wasser K, Schulte A, Jurries F, Messerschmid A, Behnke N, Groschel S, Uphaus T, Grings A, Ibis T, Klimpe S, Wagner-Heck M, Arnold M, Protsenko E, Heuschmann PU, Conen D, Weber-Kruger M; Find-AF(randomised) Investigators and Coordinators. Holter-electrocardiogram-monitoring in patients with acute ischaemic stroke (Find-AFRANDOMISED): an open-label randomised controlled trial. Lancet Neurol 2017;16:282-290.

1116. Grond M, Jauss M, Hamann G, Stark E, Veltkamp R, Nabavi D, Horn M, Weimar C, Kohrmann M, Wachter R, Rosin L, Kirchhof P. Improved detection of silent atrial fibrillation using 72-hour Holter ECG in patients with ischemic stroke: a prospective multicenter cohort study. Stroke 2013;44:3357-3364.

1117. Kishore A, Vail A, Majid A, Dawson J, Lees KR, Tyrrell PJ, Smith CJ. Detection of atrial fibrillation after ischemic stroke or transient ischemic attack: a systematic review and meta-analysis. Stroke 2014;45:520-526. 
1118. Sposato LA, Cipriano LE, Saposnik G, Ruiz Vargas E, Riccio PM, Hachinski V. Diagnosis of atrial fibrillation after stroke and transient ischaemic attack: a systematic review and meta-analysis. Lancet Neurol 2015;14:377-387.

1119. Thijs VN, Brachmann J, Morillo CA, Passman RS, Sanna T, Bernstein RA, Diener HC, Di Lazzaro V, Rymer MM, Hogge L, Rogers TB, Ziegler PD, Assar MD. Predictors for atrial fibrillation detection after cryptogenic stroke: results from CRYSTAL AF. Neurology 2016;86:261-269.

1120. Levin LA, Husberg M, Sobocinski PD, Kull VF, Friberg L, Rosenqvist M, Davidson T. A cost-effectiveness analysis of screening for silent atrial fibrillation after ischaemic stroke. Europace 2015;17:207-214.

1121. Yong JH, Thavorn K, Hoch JS, Mamdani M, Thorpe KE, Dorian P, Sharma M, Laupacis A, Gladstone DJ, on behalf of the EMBRACE Steering Committee. Potential cost-effectiveness of ambulatory cardiac rhythm monitoring after cryptogenic stroke. Stroke 2016;47:2380-2385.

1122. Shen AY, Yao JF, Brar SS, Jorgensen MB, Chen W. Racial/ethnic differences in the risk of intracranial hemorrhage among patients with atrial fibrillation. J Am Coll Cardiol 2007;50:309-315.

1123. Wilson D, Ambler G, Lee KJ, Lim JS, Shiozawa M, Koga M, Li L, Lovelock C, Chabriat H, Hennerici M, Wong YK, Mak HKF, Prats-Sanchez L, MartinezDomeno A, Inamura S, Yoshifuji K, Arsava EM, Horstmann S, Purrucker J, Lam BYK, Wong A, Kim YD, Song TJ, Schrooten M, Lemmens R, Eppinger S, Gattringer T, Uysal E, Tanriverdi Z, Bornstein NM, Assayag EB, Hallevi $H$, Tanaka J, Hara H, Coutts SB, Hert L, Polymeris A, Seiffge DJ, Lyrer P, Algra A, Kappelle J, Al-Shahi Salman R, Jager HR, Lip GYH, Mattle HP, Panos LD, Mas JL, Legrand L, Karayiannis C, Phan T, Gunkel S, Christ N, Abrigo J, Leung T, Chu W, Chappell F, Makin S, Hayden D, Williams DJ, Kooi ME, van DamNolen DHK, Barbato C, Browning S, Wiegertjes K, Tuladhar AM, Maaijwee N, Guevarra C, Yatawara C, Mendyk AM, Delmaire C, Kohler S, van Oostenbrugge R, Zhou Y, Xu C, Hilal S, Gyanwali B, Chen C, Lou M, Staals J, Bordet R, Kandiah N, de Leeuw FE, Simister R, van der Lugt A, Kelly PJ, Wardlaw JM, Soo Y, Fluri F, Srikanth V, Calvet D, Jung S, Kwa VIH, Engelter ST, Peters N, Smith EE, Yakushiji Y, Orken DN, Fazekas F, Thijs V, Heo JH, Mok V, Veltkamp R, Ay H, Imaizumi T, Gomez-Anson B, Lau KK, Jouvent E, Rothwell PM, Toyoda K, Bae HJ, Marti-Fabregas J, Werring DJ. Cerebral microbleeds and stroke risk after ischaemic stroke or transient ischaemic attack: a pooled analysis of individual patient data from cohort studies. Lancet Neurol 2019;18:653-665.

1124. Murthy SB, Gupta A, Merkler AE, Navi BB, Mandava P, ladecola C, Sheth KN, Hanley DF, Ziai WC, Kamel H. Restarting anticoagulant therapy after intracranial hemorrhage: a systematic review and meta-analysis. Stroke 2017;48:1594-1600.

1125. Diener HC, Connolly SJ, Ezekowitz MD, Wallentin L, Reilly PA, Yang S, Xavier D, Di Pasquale G, Yusuf S. Dabigatran compared with warfarin in patients with atrial fibrillation and previous transient ischaemic attack or stroke: a subgroup analysis of the RE-LY trial. Lancet Neurol 2010;9:1157-1163.

1126. Easton JD, Lopes RD, Bahit MC, Wojdyla DM, Granger CB, Wallentin L, Alings M, Goto S, Lewis BS, Rosenqvist M, Hanna M, Mohan P, Alexander JH, Diener HC; ARISTOTLE Committees and Investigators. Apixaban compared with warfarin in patients with atrial fibrillation and previous stroke or transient ischaemic attack: a subgroup analysis of the ARISTOTLE trial. Lancet Neurol 2012;11:503-511.

1127. Hankey GJ, Patel MR, Stevens SR, Becker RC, Breithardt G, Carolei A, Diener HC, Donnan GA, Halperin JL, Mahaffey KW, Mas JL, Massaro A, Norrving B, Nessel CC, Paolini JF, Roine RO, Singer DE, Wong L, Califf RM, Fox KA, Hacke W; ROCKET AF Steering Committee Investigators. Rivaroxaban compared with warfarin in patients with atrial fibrillation and previous stroke or transient ischaemic attack: a subgroup analysis of ROCKET AF. Lancet Neurol 2012;11:315-322.

1128. Rost NS, Giugliano RP, Ruff CT, Murphy SA, Crompton AE, Norden AD, Silverman S, Singhal AB, Nicolau JC, SomaRaju B, Mercuri MF, Antman EM, Braunwald $E$. Outcomes with edoxaban versus warfarin in patients with previous cerebrovascular events: findings from ENGAGE AF-TIMI 48 (Effective Anticoagulation With Factor $\mathrm{Xa}$ Next Generation in Atrial FibrillationThrombolysis in Myocardial Infarction 48). Stroke 2016;47:2075-2082.

1129. Diener HC, Eikelboom J, Connolly SJ, Joyner CD, Hart RG, Lip GY, O'Donnell M, Hohnloser SH, Hankey GJ, Shestakovska O, Yusuf S. Apixaban versus aspirin in patients with atrial fibrillation and previous stroke or transient ischaemic attack: a predefined subgroup analysis from AVERROES, a randomised trial. Lancet Neurol 2012;11:225-231.

1130. Secondary prevention in non-rheumatic atrial fibrillation after transient ischaemic attack or minor stroke. EAFT (European Atrial Fibrillation Trial) Study Group. Lancet 1993;342:1255-1262.

1131. Cuker A. Laboratory measurement of the non-vitamin $\mathrm{K}$ antagonist oral anticoagulants: selecting the optimal assay based on drug, assay availability, and clinical indication. J Thromb Thrombolysis 2016;41:241-247.
1132. Salmonson T, Dogne JM, Janssen H, Garcia Burgos J, Blake P. Non-vitamin-K oral anticoagulants and laboratory testing: now and in the future: views from a workshop at the European Medicines Agency (EMA). Eur Heart J Cardiovasc Pharmacother 2017;3:42-47.

1133. Chai-Adisaksopha C, Hillis C, Lim W, Boonyawat K, Moffat K, Crowther M. Hemodialysis for the treatment of dabigatran-associated bleeding: a case report and systematic review. J Thromb Haemost 2015;13:1790-1798.

1134. Anderson I, Cifu AS. Management of bleeding in patients taking oral anticoagulants. JAMA 2018;319:2032-2033.

1135. Milling TJ Jr, Refaai MA, Sarode R, Lewis B, Mangione A, Durn BL, Harman A, Lee ML, Goldstein JN. Safety of a four-factor prothrombin complex concentrate versus plasma for vitamin $\mathrm{K}$ antagonist reversal: an integrated analysis of two phase Illb clinical trials. Acad Emerg Med 2016;23:466-475.

1136. Pollack CV Jr, Reilly PA, van Ryn J, Eikelboom JW, Glund S, Bernstein RA, Dubiel R, Huisman MV, Hylek EM, Kam CW, Kamphuisen PW, Kreuzer J, Levy JH, Royle G, Sellke FW, Stangier J, Steiner T, Verhamme P, Wang B, Young L, Weitz Jl. Idarucizumab for dabigatran reversal - full cohort analysis. N Engl J Med 2017;377:431-441.

1137. Connolly SJ, Crowther M, Eikelboom JW, Gibson CM, Curnutte JT, Lawrence $\mathrm{JH}$, Yue P, Bronson MD, Lu G, Conley PB, Verhamme P, Schmidt J, Middeldorp S, Cohen AT, Beyer-Westendorf J, Albaladejo P, Lopez-Sendon J, Demchuk AM, Pallin DJ, Concha M, Goodman S, Leeds J, Souza S, Siegal DM, Zotova E, Meeks B, Ahmad S, Nakamya J, Milling TJ Jr; ANNEXA Investigators. Full study report of andexanet alfa for bleeding associated with factor $\mathrm{Xa}$ inhibitors. N Engl J Med 2019;380:1326-1335.

1138. Levi M, Moore KT, Castillejos CF, Kubitza D, Berkowitz SD, Goldhaber SZ, Raghoebar M, Patel MR, Weitz Jl, Levy JH. Comparison of three-factor and four-factor prothrombin complex concentrates regarding reversal of the anticoagulant effects of rivaroxaban in healthy volunteers. J Thromb Haemost 2014;12:1428-1436

1139. Nagalla S, Thomson L, Oppong Y, Bachman B, Chervoneva I, Kraft WK. Reversibility of apixaban anticoagulation with a four-factor prothrombin complex concentrate in healthy volunteers. Clin Transl Sci 2016;9:176-180.

1140. Zahir H, Brown KS, Vandell AG, Desai M, Maa JF, Dishy V, Lomeli B, Feussner A, Feng W, He L, Grosso MA, Lanz HJ, Antman EM. Edoxaban effects on bleeding following punch biopsy and reversal by a 4-factor prothrombin complex concentrate. Circulation 2015;131:82-90.

1141. Isnard R, Bauer F, Cohen-Solal A, Damy T, Donal E, Galinier M, Hagege A, Jourdain P, Leclercq C, Sabatier R, Trochu JN, Cohen A. Non-vitamin K antagonist oral anticoagulants and heart failure. Arch Cardiovasc Dis 2016;109:641-650.

1142. Xiong Q, Lau YC, Senoo K, Lane DA, Hong K, Lip GY. Non-vitamin K antagonist oral anticoagulants (NOACs) in patients with concomitant atrial fibrillation and heart failure: a systemic review and meta-analysis of randomized trials. Eur J Heart Fail 2015;17:1192-1200.

1143. Ezekowitz JA, O'Meara E, McDonald MA, Abrams H, Chan M, Ducharme A, Giannetti N, Grzeslo A, Hamilton PG, Heckman GA, Howlett JG, Koshman SL, Lepage S, McKelvie RS, Moe GW, Rajda M, Swiggum E, Virani SA, Zieroth S, Al-Hesayen A, Cohen-Solal A, D'Astous M, De S, Estrella-Holder E, Fremes S, Green L, Haddad H, Harkness K, Hernandez AF, Kouz S, LeBlanc MH, Masoudi FA, Ross HJ, Roussin A, Sussex B. 2017 comprehensive update of the Canadian Cardiovascular Society Guidelines for the management of heart failure. Can J Cardiol 2017;33:1342-1433.

1144. Atherton J], Sindone A, De Pasquale CG, Driscoll A, MacDonald PS, Hopper I, Kistler PM, Briffa T, Wong J, Abhayaratna W, Thomas L, Audehm R, Newton P, O'Loughlin J, Branagan M, Connell C. National Heart Foundation of Australia and Cardiac Society of Australia and New Zealand: Guidelines for the prevention, detection, and management of heart failure in Australia 2018. Heart Lung Circ 2018;27:1123-1208.

1145. Ponikowski P, Voors AA, Anker SD, Bueno H, Cleland JGF, Coats AJS, Falk V, Gonzalez-Juanatey JR, Harjola VP, Jankowska EA, Jessup M, Linde C, Nihoyannopoulos P, Parissis JT, Pieske B, Riley JP, Rosano GMC, Ruilope LM, Ruschitzka F, Rutten FH, van der Meer P; ESC Scientific Document Group. 2016 ESC Guidelines for the diagnosis and treatment of acute and chronic heart failure: the Task Force for the diagnosis and treatment of acute and chronic heart failure of the European Society of Cardiology (ESC). Developed with the special contribution of the Heart Failure Association (HFA) of the ESC. Eur Heart J 2016;37:2129-2200.

1146. Kelly JP, DeVore AD, Wu J, Hammill BG, Sharma A, Cooper LB, Felker GM, Piccini JP, Allen LA, Heidenreich PA, Peterson ED, Yancy CW, Fonarow GC, Hernandez AF. Rhythm control versus rate control in patients with atrial fibrillation and heart failure with preserved ejection fraction: insights from Get With The Guidelines-Heart Failure. J Am Heart Assoc 2019;8:e011560.

1147. Filippatos G, Farmakis D. How to use beta-blockers in heart failure with reduced ejection fraction and atrial fibrillation. I Am Coll Cardiol 2017;69:2897-2900. 
1148. Nielsen PB, Larsen TB, Gorst-Rasmussen A, Skjoth F, Lip GY. beta-Blockers in atrial fibrillation patients with or without heart failure: association with mortality in a nationwide cohort study. Circ Heart Fail 2016;9:e002597.

1149. Nkomo VT, Gardin JM, Skelton TN, Gottdiener JS, Scott CG, Enriquez-Sarano M. Burden of valvular heart diseases: a population-based study. Lancet 2006;368:1005-1011.

1150. Barbash IM, Minha S, Ben-Dor I, Dvir D, Torguson R, Aly M, Bond E, Satler LF, Pichard AD, Waksman R. Predictors and clinical implications of atrial fibrillation in patients with severe aortic stenosis undergoing transcatheter aortic valve implantation. Catheter Cardiovasc Interv 2015;85:468-477.

1151. Eguchi K, Ohtaki E, Matsumura T, Tanaka K, Tohbaru T, Iguchi N, Misu K, Asano R, Nagayama M, Sumiyoshi T, Kasegawa H, Hosoda S. Pre-operative atrial fibrillation as the key determinant of outcome of mitral valve repair for degenerative mitral regurgitation. Eur Heart J 2005;26:1866-1872.

1152. Maan A, Heist EK, Passeri J, Inglessis I, Baker J, Ptaszek L, Vlahakes G, Ruskin JN, Palacios I, Sundt T, Mansour M. Impact of atrial fibrillation on outcomes in patients who underwent transcatheter aortic valve replacement. Am J Cardiol 2015;115:220-226.

1153. Ngaage DL, Schaff HV, Barnes SA, Sundt TM 3rd, Mullany CJ, Dearani JA, Daly RC, Orszulak TA. Prognostic implications of preoperative atrial fibrillation in patients undergoing aortic valve replacement: is there an argument for concomitant arrhythmia surgery?Ann Thorac Surg 2006;82:1392-1399.

1154. Ngaage DL, Schaff HV, Mullany CJ, Barnes S, Dearani JA, Daly RC, Orszulak TA, Sundt TM 3rd. Influence of preoperative atrial fibrillation on late results of mitral repair: is concomitant ablation justified?Ann Thorac Surg 2007;84:434-442.

1155. Lim E, Barlow CW, Hosseinpour AR, Wisbey C, Wilson K, Pidgeon W, Charman S, Barlow JB, Wells FC. Influence of atrial fibrillation on outcome following mitral valve repair. Circulation 2001;104:159-63.

1156. Philippart R, Brunet-Bernard A, Clementy N, Bourguignon T, Mirza A, Babuty D, Angoulvant D, Lip GY, Fauchier L. Prognostic value of CHA2DS2-VASc score in patients with 'non-valvular atrial fibrillation' and valvular heart disease: the Loire Valley Atrial Fibrillation Project. Eur Heart J 2015;36:1822-1830.

11547. Lip GYH, Jensen M, Melgaard L, Skjoth F, Nielsen PB, Larsen TB. Stroke and bleeding risk scores in patients with atrial fibrillation and valvular heart disease: evaluating 'valvular heart disease' in a nationwide cohort study. Europace 2019;21:33-40.

1158. Szekely P. Systemic embolism and anticoagulant prophylaxis in rheumatic heart disease. Br Med J 1964;1:1209-1212.

1159. Philippart R, Brunet-Bernard A, Clementy N, Bourguignon T, Mirza A, Angoulvant D, Babuty D, Lip GY, Fauchier L. Oral anticoagulation, stroke and thromboembolism in patients with atrial fibrillation and valve bioprosthesis. The Loire Valley Atrial Fibrillation Project. Thromb Haemost 2016;115:1056-1063.

1160. Siontis KC, Yao X, Gersh BJ, Noseworthy PA. Direct oral anticoagulants in patients with atrial fibrillation and valvular heart disease other than significant mitral stenosis and mechanical valves: a meta-analysis. Circulation 2017;135:714-716.

1161. Kim JY, Kim SH, Myong JP, Kim YR, Kim TS, Kim JH, Jang SW, Oh YS, Lee MY, Rho TH. Outcomes of direct oral anticoagulants in patients with mitral stenosis. J Am Coll Cardiol 2019;73:1123-1131.

1162. Bisson A, Bodin A, Clementy N, Bernard A, Babuty D, Lip GYH, Fauchier L. Stroke, thromboembolism and bleeding in patients with atrial fibrillation according to the EHRA valvular heart disease classification. Int J Cardiol 2018;260:93-98.

1163. Seeger J, Gonska B, Rodewald C, Rottbauer W, Wohrle J. Apixaban in patients with atrial fibrillation after transfemoral aortic valve replacement. JACC Cardiovasc Interv 2017;10:66-74.

1164. Dangas GD, Tijssen JGP, Wohrle J, Sondergaard L, Gilard M, Mollmann H, Makkar RR, Herrmann HC, Giustino G, Baldus S, De Backer O, Guimaraes AHC, Gullestad L, Kini A, von Lewinski D, Mack M, Moreno R, Schafer U, Seeger J, Tchetche D, Thomitzek K, Valgimigli M, Vranckx P, Welsh RC, Wildgoose P, Volkl AA, Zazula A, van Amsterdam RGM, Mehran R, Windecker $S$, for the GALILEO Investigators. A controlled trial of rivaroxaban after transcatheter aortic-valve replacement. $N$ Engl J Med 2020;382:120-129.

1165. Eikelboom JW, Connolly SJ, Brueckmann M, Granger CB, Kappetein AP, Mack MJ, Blatchford J, Devenny K, Friedman J, Guiver K, Harper R, Khder Y, Lobmeyer MT, Maas H, Voigt JU, Simoon ML, Van de Werf, F; RE-ALIGN Investigators. Dabigatran versus warfarin in patients with mechanical heart valves. N Engl I Med 2013;369:1206-1214.

1166. Potpara TS, Ferro C], Lip GYH. Use of oral anticoagulants in patients with atrial fibrillation and renal dysfunction. Nat Rev Nephrol 2018;14:337-351.

1167. Albertsen IE, Rasmussen LH, Overvad TF, Graungaard T, Larsen TB, Lip GY. Risk of stroke or systemic embolism in atrial fibrillation patients treated with warfarin: a systematic review and meta-analysis. Stroke 2013;44:1329-1336.
1168. Olesen JB, Lip GY, Kamper AL, Hommel K, Kober L, Lane DA, Lindhardsen J, Gislason GH, Torp-Pedersen C. Stroke and bleeding in atrial fibrillation with chronic kidney disease. N Engl J Med 2012;367:625-635.

1169. Hart RG, Eikelboom JW, Brimble KS, McMurtry MS, Ingram AJ. Stroke prevention in atrial fibrillation patients with chronic kidney disease. Can J Cardio 2013;29:S71-78.

1170. Zimmerman D, Sood MM, Rigatto C, Holden RM, Hiremath S, Clase CM. Systematic review and meta-analysis of incidence, prevalence and outcomes of atrial fibrillation in patients on dialysis. Nephrol Dial Transplant 2012;27:3816-3822.

1171. Banerjee A, Fauchier L, Vourc'h P, Andres CR, Taillandier S, Halimi JM, LiP $\mathrm{GYH}$. A prospective study of estimated glomerular filtration rate and outcomes in patients with atrial fibrillation: the Loire Valley Atrial Fibrillation Project. Chest 2014;145:1370-1382.

1172. Fauchier L, Bisson A, Clementy N, Vourc'h P, Angoulvant D, Babuty D, Halimi JM, Lip GYH. Changes in glomerular filtration rate and outcomes in patients with atrial fibrillation. Am Heart J 2018;198:39-45.

1173. Bohula EA, Giugliano RP, Ruff CT, Kuder JF, Murphy SA, Antman EM, Braunwald E. Impact of renal function on outcomes with edoxaban in the ENGAGE AF-TIMI 48 trial. Circulation 2016;134:24-36.

1174. Hijazi Z, Hohnloser SH, Oldgren J, Andersson U, Connolly SJ, Eikelboom JW Ezekowitz MD, Reilly PA, Siegbahn A, Yusuf S, Wallentin L. Efficacy and safety of dabigatran compared with warfarin in relation to baseline renal function in patients with atrial fibrillation: a RE-LY (Randomized Evaluation of Long-term Anticoagulation Therapy) trial analysis. Circulation 2014;129:961-970.

1175. Fox KA, Piccini JP, Wojdyla D, Becker RC, Halperin JL, Nessel CC, Paolini JF, Hankey G], Mahaffey KW, Patel MR, Singer DE, Califf RM. Prevention of stroke and systemic embolism with rivaroxaban compared with warfarin in patients with non-valvular atrial fibrillation and moderate renal impairment. Eur Heart J 2011;32:2387-2394.

1176. Hohnloser SH, Hijazi Z, Thomas L, Alexander JH, Amerena J, Hanna M, Keltai M, Lanas F, Lopes RD, Lopez-Sendon J, Granger CB, Wallentin L. Efficacy of apixaban when compared with warfarin in relation to renal function in patients with atrial fibrillation: insights from the ARISTOTLE trial. Eur Heart 2012;33:2821-2830.

1177. Coleman Cl, Kreutz R, Sood NA, Bunz TJ, Eriksson D, Meinecke AK, Baker WL. Rivaroxaban versus warfarin in patients with nonvalvular atrial fibrillation and severe kidney disease or undergoing hemodialysis. Am J Med 2019;132:1078-1083.

1178. Ha JT, Neuen BL, Cheng LP, Jun M, Toyama T, Gallagher MP, Jardine MJ, Sood MM, Garg AX, Palmer SC, Mark PB, Wheeler DC, Jha V, Freedman B, Johnson DW, Perkovic V, Badve SV. Benefits and harms of oral anticoagulant therapy in chronic kidney disease: a systematic review and meta-analysis. Ann Intern Med 2019;171:181-189.

1179. Pokorney SD. RENal hemodialysis patients ALlocated apixaban versus warfarin in Atrial Fibrillation (RENAL-AF). Presentation at the American Heart Association Annual Scientific Sessions (AHA 2019), Philadelphia, PA, 16 November 2019

1180. Violi F, Davi G, Hiatt W, Lip GY, Corazza GR, Perticone F, Proietti M, Pignatelli P, Vestri AR, Basili S; ARAPACIS Study Investigators. Prevalence of peripheral artery disease by abnormal ankle-brachial index in atrial fibrillation: implications for risk and therapy. J Am Coll Cardiol 2013;62:2255-2256.

1181. Bruere H, Fauchier L, Bernard Brunet A, Pierre B, Simeon E, Babuty D, Clementy N. History of thyroid disorders in relation to clinical outcomes in atrial fibrillation. Am J Med 2015;128:30-37.

1182. Nakazawa HK, Sakurai K, Hamada N, Momotani N, Ito K. Management of atrial fibrillation in the post-thyrotoxic state. Am J Med 1982;72:903-906.

1183. Kristensen SL, Lindhardsen J, Ahlehoff $O$, Erichsen R, Lamberts $M$, Khalid $U$, Torp-Pedersen C, Nielsen OH, Gislason GH, Hansen PR. Increased risk of atrial fibrillation and stroke during active stages of inflammatory bowel disease: a nationwide study. Europace 2014;16:477-484.

1184. Holster IL, Valkhoff VE, Kuipers EJ, Tjwa ETTL. New oral anticoagulants increase risk for gastrointestinal bleeding: a systematic review and meta-analysis. Gastroenterology 2013;145:105-112.e15.

1185. Caldeira D, Barra M, Ferreira A, Rocha A, Augusto A, Pinto FJ, Costa J, Ferreira JJ. Systematic review with meta-analysis: the risk of major gastrointestinal bleeding with non-vitamin $\mathrm{K}$ antagonist oral anticoagulants. Aliment Pharmacol Ther 2015;42:1239-1249.

1186. Kolb JM, Flack KF, Chatterjee-Murphy P, Desai J, Wallentin LC, Ezekowitz M, Connolly S, Reilly P, Brueckmann M, Ilgenfritz J, Aisenberg J. Locations and mucosal lesions responsible for major gastrointestinal bleeding in patients on warfarin or dabigatran. Dig Dis Sci 2018;63:1878-1889.

1187. Chai-Adisaksopha C, Hillis C, Monreal M, Witt DM, Crowther M. Thromboembolic events, recurrent bleeding and mortality after resuming anticoagulant following gastrointestinal bleeding. A meta-analysis. Thromb Haemost 2015;114:819-825. 
1188. O'Dea D, Whetteckey J, Ting N. A prospective, randomized, open-label study to evaluate two management strategies for gastrointestinal symptoms in patients newly on treatment with dabigatran. Cardiol Ther 2016;5:187-201.

1189. Lai HC, Chien WC, Chung CH, Lee WL, Wu TJ, Wang KY, Liu CN, Liu TJ. Atrial fibrillation, liver disease, antithrombotics and risk of cerebrovascular events: a population-based cohort study. Int J Cardiol 2016;223:829-837.

1190. Lip GY, Frison L, Halperin JL, Lane DA. Comparative validation of a novel risk score for predicting bleeding risk in anticoagulated patients with atrial fibrillation: the HAS-BLED (Hypertension, Abnormal Renal/Liver Function, Stroke, Bleeding History or Predisposition, Labile INR, Elderly, Drugs/Alcohol Concomitantly) score. J Am Coll Cardiol 2011;57:173-180.

1191. Potpara TS, Lip GY. Drug-induced liver injury with oral anticoagulants: a threat or not? Heart 2017;103:809-811.

1192. Hoolwerf EW, Kraaijpoel N, Buller HR, van Es N. Direct oral anticoagulants in patients with liver cirrhosis: a systematic review. Thromb Res 2018:170:102-108.

1193. Kubitza D, Roth A, Becka M, Alatrach A, Halabi A, Hinrichsen H, Mueck W. Effect of hepatic impairment on the pharmacokinetics and pharmacodynamics of a single dose of rivaroxaban, an oral, direct factor $\mathrm{Xa}$ inhibitor. $\mathrm{Br} J \mathrm{Clin}$ Pharmacol 2013;76:89-98.

1194. Bonde AN, Blanche P, Staerk L, Gerds TA, Gundlund A, Gislason G, TorpPedersen C, Lip GYH, Hlatky MA, Olesen JB. Oral anticoagulation among atrial fibrillation patients with anaemia: an observational cohort study. Eur Heart / 2019.

1195. Arun M, Brauneis D, Doros G, Shelton AC, Sloan JM, Quillen K, Ruberg FL, Sanchorawala $V$, Varga $C$. The incidence of atrial fibrillation among patients with $\mathrm{AL}$ amyloidosis undergoing high-dose melphalan and stem cell transplantation: experience at a single institution. Bone Marrow Transplant 2017;52:1349-1351.

1196. Yuan M, Tse G, Zhang Z, Han X, Wu WKK, Li G, Xia Y, Liu T. The incidence of atrial fibrillation with trastuzumab treatment: a systematic review and metaanalysis. Cardiovasc Ther 2018;36:e12475.

1197. Ganatra S, Sharma A, Shah S, Chaudhry GM, Martin DT, Neilan TG, Mahmood SS, Barac A, Groarke JD, Hayek SS, Dani S, Venesy D, Patten R, Nohria A. Ibrutinib-associated atrial fibrillation. JACC Clin Electrophysiol 2018:4:1491-1500

1198. Boriani G, Corradini P, Cuneo A, Falanga A, Foa R, Gaidano G, Ghia PP, Martelli M, Marasca R, Massaia M, Mauro FR, Minotti G, Molica S, Montillo M, Pinto A, Tedeschi A, Vitolo U, Zinzani PL. Practical management of ibrutinib in the real life: focus on atrial fibrillation and bleeding. Hematol Oncol 2018;36:624-632.

1199. Yun S, Vincelette ND, Acharya U, Abraham I. Risk of atrial fibrillation and bleeding diathesis associated with ibrutinib treatment: a systematic review and pooled analysis of four randomized controlled trials. Clin Lymphoma Myeloma Leuk 2017; 17:31-37.e13.

1200. Feinberg WM, Blackshear JL, Laupacis A, Kronmal R, Hart RG. Prevalence, age distribution, and gender of patients with atrial fibrillation. Analysis and implications. Arch Intern Med 1995;155:469-473.

1201. Lane DA, Skjoth F, Lip GYH, Larsen TB, Kotecha D. Temporal trends in incidence, prevalence, and mortality of atrial fibrillation in primary care. J Am Heart Assoc 2017;6:e005155.

1202. Miyasaka Y, Barnes ME, Gersh BJ, Cha SS, Bailey KR, Abhayaratna WP, Seward JB, Tsang TS. Secular trends in incidence of atrial fibrillation in Olmsted County, Minnesota, 1980 to 2000, and implications on the projections for future prevalence. Circulation 2006;114:119-125.

1203. Ohlmeier C, Mikolajczyk R, Haverkamp W, Garbe E. Incidence, prevalence, and antithrombotic management of atrial fibrillation in elderly Germans. Europace 2013;15:1436-1444.

1204. Go AS, Hylek EM, Phillips KA, Chang Y, Henault LE, Selby JV, Singer DE. Prevalence of diagnosed atrial fibrillation in adults: national implications for rhythm management and stroke prevention: the AnTicoagulation and Risk Factors in Atrial Fibrillation (ATRIA) Study. JAMA 2001;285:2370-2375.

1205. Wilke T, Groth A, Mueller S, Pfannkuche M, Verheyen F, Linder R, Maywald $\mathrm{U}$, Bauersachs R, Breithardt G. Incidence and prevalence of atrial fibrillation: an analysis based on 8.3 million patients. Europace 2013;15:486-493.

1206. Zoni-Berisso M, Lercari F, Carazza T, Domenicucci S. Epidemiology of atrial fibrillation: European perspective. Clin Epidemiol 2014;6:213-220.

1207. Marinigh R, Lip GY, Fiotti N, Giansante C, Lane DA. Age as a risk factor for stroke in atrial fibrillation patients: implications for thromboprophylaxis. J Am Coll Cardiol 2010;56:827-837.

1208. Stroke Risk in Atrial Fibrillation Working Group. Independent predictors of stroke in patients with atrial fibrillation: a systematic review. Neurology 2007;69:546-554.

1209. Graham DJ, Reichman ME, Wernecke M, Zhang R, Southworth MR, Levenson M, Sheu TC, Mott K, Goulding MR, Houstoun M, MaCurdy TE, Worrall C, Kelman JA. Cardiovascular, bleeding, and mortality risks in elderly Medicare patients treated with dabigatran or warfarin for nonvalvular atrial fibrillation. Circulation 2015;131:157-164.

1210. Biteker M, Basaran O, Dogan V, Altun I, Ozpamuk Karadeniz F, Tekkesin Al, Cakilli Y, Turkkan C, Hamidi M, Demir V, Gursoy MO, Tek Ozturk M, Aksan G, Seyis S, Balli M, Alici MH, Bozyel S. Real-world clinical characteristics and treatment patterns of individuals aged 80 and older with nonvalvular atrial fibrillation: results from the ReAl-life Multicenter Survey Evaluating Stroke Study. J Am Geriatr Soc 2017;65:1684-1690.

1211. Gage BF, Boechler M, Doggette AL, Fortune G, Flaker GC, Rich MW, Radford MJ. Adverse outcomes and predictors of underuse of antithrombotic therapy in Medicare beneficiaries with chronic atrial fibrillation. Stroke 2000;31:822-827.

1212. Ghaswalla PK, Harpe SE, Slattum PW. Warfarin use in nursing home residents: results from the 2004 national nursing home survey. Am J Geriatr Pharmacother 2012;10:25-36.e2

1213. Kotecha D, Chudasama R, Lane DA, Kirchhof P, Lip GY. Atrial fibrillation and heart failure due to reduced versus preserved ejection fraction: a systematic review and meta-analysis of death and adverse outcomes. Int I Cardiol 2016;203:660-666.

1214. Oqab Z, Pournazari P, Sheldon RS. What is the impact of frailty on prescription of anticoagulation in elderly patients with atrial fibrillation? A systematic review and meta-analysis. J Atr Fibrillation 2018;10:1870.

1215. Proietti M, Laroche C, Opolski G, Maggioni AP, Boriani G, Lip GYH, on behalf of the AF Gen Pilot Investigators. 'Real-world' atrial fibrillation management in Europe: observations from the 2-year follow-up of the EURObservational Research Programme-Atrial Fibrillation General Registry Pilot Phase. Europace 2017:19:722-733.

1216. Singh P, Arrevad PS, Peterson GM, Bereznicki LR. Evaluation of antithrombotic usage for atrial fibrillation in aged care facilities. J Clin Pharm Ther 2011;36:166-171

1217. Annoni G. Mazzola P. Real-world characteristics of hospitalized frail elderly patients with atrial fibrillation: can we improve the current prescription of anticoagulants?] Geriatr Cardiol 2016;13:226-232.

1218. Deandrea S, Bravi F, Turati F, Lucenteforte E, La Vecchia C, Negri E. Risk factors for falls in older people in nursing homes and hospitals. A systematic review and meta-analysis. Arch Gerontol Geriatr 2013;56:407-415.

1219. Phelan EA, Mahoney JE, Voit JC, Stevens JA. Assessment and management of fall risk in primary care settings. Med Clin North Am 2015;99:281-293.

1220. Donze J, Clair C, Hug B, Rodondi N, Waeber G, Cornuz J, Aujesky D. Risk of falls and major bleeds in patients on oral anticoagulation therapy. Am J Med 2012;125:773-778.

1221. Garwood CL, Corbett TL. Use of anticoagulation in elderly patients with atrial fibrillation who are at risk for falls. Ann Pharmacother 2008;42 $523-532$.

1222. Hart RG, Pearce LA, Aguilar MI. Adjusted-dose warfarin versus aspirin for preventing stroke in patients with atrial fibrillation. Ann Intern Med 2007: 147:590-592

1223. Jacobs LG, Billett HH, Freeman K, Dinglas C, Jumaquio L. Anticoagulation for stroke prevention in elderly patients with atrial fibrillation, including those with falls and/or early-stage dementia: a single-center, retrospective, observational study. Am J Geriatr Pharmacother 2009;7:159-166.

1224. Rash A, Downes T, Portner R, Yeo WW, Morgan N, Channer KS. A randomised controlled trial of warfarin versus aspirin for stroke prevention in octogenarians with atrial fibrillation (WASPO). Age Ageing 2007;36:151-156.

1225. Sardar P, Chatterjee S, Chaudhari S, Lip GY. New oral anticoagulants in elderly adults: evidence from a meta-analysis of randomized trials. J Am Geriatr Soc 2014;62:857-864

1226. Siu CW, Tse HF. Net clinical benefit of warfarin therapy in elderly Chinese patients with atrial fibrillation. Circ Arrhythm Electrophysiol 2014;7:300-306.

1227. Alnsasra H, Haim M, Senderey AB, Reges $O$, Leventer-Roberts $M$, Arnson $Y$, Leibowitz M, Hoshen M, Avgil-Tsadok M. Net clinical benefit of anticoagulant treatments in elderly patients with nonvalvular atrial fibrillation: experience from the real world. Heart Rhythm 2019;16:31-37.

1228. Eikelboom JW, Wallentin L, Connolly SJ, Ezekowitz M, Healey JS, Oldgren J, Yang S, Alings M, Kaatz S, Hohnloser SH, Diener HC, Franzosi MG, Huber K, Reilly P, Varrone J, Yusuf S. Risk of bleeding with 2 doses of dabigatran compared with warfarin in older and younger patients with atrial fibrillation: an analysis of the randomized evaluation of long-term anticoagulant therapy (RELY) trial. Circulation 2011;123:2363-2372.

1229. Halperin JL, Hankey G], Wojdyla DM, Piccini JP, Lokhnygina Y, Patel MR, Breithardt G, Singer DE, Becker RC, Hacke W, Paolini JF, Nessel CC, Mahaffey KW, Califf RM, Fox KA; ROCKET AF Steering Committee Investigators. Efficacy and safety of rivaroxaban compared with warfarin among elderly patients with nonvalvular atrial fibrillation in the Rivaroxaban Once Daily, Oral, Direct Factor Xa Inhibition Compared With Vitamin K 
Antagonism for Prevention of Stroke and Embolism Trial in Atrial Fibrillation (ROCKET AF). Circulation 2014;130:138-146.

1230. Halvorsen S, Atar D, Yang H, De Caterina R, Erol C, Garcia D, Granger CB, Hanna M, Held C, Husted S, Hylek EM, Jansky P, Lopes RD, Ruzyllo W, Thomas L, Wallentin L. Efficacy and safety of apixaban compared with warfarin according to age for stroke prevention in atrial fibrillation: observations from the ARISTOTLE trial. Eur Heart J 2014;35:1864-1872.

1231. Hori M, Matsumoto M, Tanahashi N, Momomura S, Uchiyama S, Goto S, Izumi T, Koretsune Y, Kajikawa M, Kato M, Ueda H, lekushi K, Yamanaka S, Tajiri M; J-ROCKET AF Study Investigators. Rivaroxaban vs. warfarin in Japanese patients with non-valvular atrial fibrillation in relation to age. Circ J 2014;78:1349-1356.

1232. Kato ET, Giugliano RP, Ruff CT, Koretsune Y, Yamashita T, Kiss RG, Nordio F, Murphy SA, Kimura T, Jin J, Lanz H, Mercuri M, Braunwald E, Antman, EM. Efficacy and safety of edoxaban in elderly patients with atrial fibrillation in the ENGAGE AF-TIMI 48 trial. J Am Heart Assoc 2016;5:e003432.

1233. Kim IS, Kim HJ, Kim TH, Uhm JS, Joung B, Lee MH, Pak HN. Non-vitamin K antagonist oral anticoagulants have better efficacy and equivalent safety compared to warfarin in elderly patients with atrial fibrillation: a systematic review and meta-analysis. J Cardiol 2018;72:105-112.

1234. Ng KH, Shestakovska O, Connolly SJ, Eikelboom JW, Avezum A, Diaz R, Lanas F, Yusuf S, Hart RG. Efficacy and safety of apixaban compared with aspirin in the elderly: a subgroup analysis from the AVERROES trial. Age Ageing 2016;45:77-83.

1235. Ruiz Ortiz M, Muniz J, Rana Miguez P, Roldan I, Marin F, Asuncion EstevePastor M Cequier A, Martinez-Selles M, Bertomeu V, Anguita M; FANTASIIA Study Investigators. Inappropriate doses of direct oral anticoagulants in realworld clinical practice: prevalence and associated factors. A subanalysis of the FANTASIIA Registry. Europace 2018;20:1577-1583.

1236. Deitelzweig S, Keshishian A, Li X, Kang A, Dhamane AD, Luo X, Balachander N, Rosenblatt L, Mardekian J, Pan X, Nadkarni A, Di Fusco M, Garcia Reeves $A B$, Yuce H, Lip GYH. Comparisons between oral anticoagulants among older nonvalvular atrial fibrillation patients. J Am Geriatr Soc 2019;67:1662-1671.

1237. Dillinger JG, Aleil B, Cheggour S, Benhamou Y, Bejot Y, Marechaux S, Delluc $A$, Bertoletti L, Lellouche N. Dosing issues with non-vitamin $K$ antagonist oral anticoagulants for the treatment of non-valvular atrial fibrillation: why we should not underdose our patients. Arch Cardiovasc Dis 2018;111:85-94.

1238. Nieuwlaat R, Olsson SB, Lip GY, Camm AJ, Breithardt G, Capucci A, Meeder JG, Prins MH, Levy S, Crijns HJ; Euro Heart Survey Investigators. Guidelineadherent antithrombotic treatment is associated with improved outcomes compared with undertreatment in high-risk patients with atrial fibrillation. The Euro Heart Survey on Atrial Fibrillation. Am Heart J 2007;153:1006-1012.

1239. Fumagalli S, Said SAM, Laroche C, Gabbai D, Marchionni N, Boriani G, Maggioni AP, Popescu MI, Rasmussen LH, Crijns H, Lip GYH; EORP-AF Investigators. Age-related differences in presentation, treatment, and outcome of patients with atrial fibrillation in europe: the EORP-AF General Pilot Registry (EURObservational Research Programme-Atrial Fibrillation). JACC Clin Electrophysiol 2015;1:326-334.

1240. Paciullo F, Proietti M, Bianconi V, Nobili A, Pirro M, Mannucci PM, Lip GYH, Lupattelli G; REPOSI Investigators. Choice and outcomes of rate control versus rhythm control in elderly patients with atrial fibrillation: a report from the REPOSI Study. Drugs Aging 2018;35:365 - 373.

1241. Shariff N, Desai RV, Patel K, Ahmed MI, Fonarow GC, Rich MW, Aban IB, Banach M, Love TE, White M, Aronow WS, Epstein AE, Ahmed A. Rate-control versus rhythm-control strategies and outcomes in septuagenarians with atrial fibrillation. Am J Med 2013;126:887-893.

1242. Purmah Y, Proietti M, Laroche C, Mazurek M, Tahmatzidis D, Boriani G, Novo S, Lip GYH; EORP-AF General Pilot Registry Investigators. Rate vs. rhythm control and adverse outcomes among European patients with atrial fibrillation. Europace 2018;20:243-252.

1243. Abdin A, Yalin K, Lyan E, Sawan N, Liosis S, Meyer-Saraei R, Elsner C, Lange SA, Heeger CH, Eitel C, Eitel I, Tilz RR. Safety and efficacy of cryoballoon ablation for the treatment of atrial fibrillation in elderly patients. Clin Res Cardiol 2019;108:167-174.

1244. Bhargava M, Marrouche NF, Martin DO, Schweikert RA, Saliba W, Saad EB, Bash D, Williams-Andrews M, Rossillo A, Erciyes D, Khaykin Y, Burkhardt JD, Joseph G, Tchou PJ, Natale A. Impact of age on the outcome of pulmonary vein isolation for atrial fibrillation using circular mapping technique and cooled-tip ablation catheter. J Cardiovasc Electrophysiol 2004;15:8-13.

1245. Bulava A, Hanis J, Dusek L. Clinical outcomes of radiofrequency catheter ablation of atrial fibrillation in octogenarians-10-year experience of a one highvolume center. J Geriatr Cardiol 2017;14:575-581.

1246. Bunch TJ, Weiss JP, Crandall BG, May HT, Bair TL, Osborn JS, Anderson JL, Lappe DL, Muhlestein JB, Nelson J, Day JD. Long-term clinical efficacy and risk of catheter ablation for atrial fibrillation in octogenarians. Pacing Clin Electrophysiol 2010;33:146-152.
1247. Heeger CH, Bellmann B, Fink T, Bohnen JE, Wissner E, Wohlmuth P, Rottner L, Sohns C, Tilz RR, Mathew S, Reissmann B, Lemes C, Maurer T, Luker J, Sultan A, Plenge T, Goldmann B, Ouyang F, Kuck KH, Metzner I, Metzner A, Steven D, Rillig A. Efficacy and safety of cryoballoon ablation in the elderly: a multicenter study. Int J Cardiol 2019;278:108-113.

1248. Kis Z, Noten AM, Martirosyan M, Hendriks AA, Bhagwandien R, Szili-Torok T. Comparison of long-term outcome between patients aged $<65$ years vs. $>/$ $=65$ years after atrial fibrillation ablation. J Geriatr Cardiol 2017;14:569-574.

1249. Lim T, Day D, Weiss P, Crandall BG, May HAT, Bair TL, Osborn JS, Anderson JL, Muhlestein JB, Lappe DL, Mahapatra S, Bunch TJ. More aggressive left atrial ablation in elderly patients does not increase procedural complications. J Innov Card Rhythm Manag 2011;2:206-211.

1250. Lin T, Du X, Bai R, Chen YW, Yu RH, Long DY, Tang RB, Sang CH, Li SN, Ma CS, Dong JZ. Long-term results of single-procedure catheter ablation for atrial fibrillationin pre- and post-menopausal women. J Geriatr Cardiol 2014; 11:120-125.

1251. Lioni L, Letsas KP, Efremidis M, Vlachos K, Giannopoulos G, Kareliotis V Deftereos S, Sideris A. Catheter ablation of atrial fibrillation in the elderly. J Geriatr Cardiol 2014;11:291-295.

1252. Metzner I, Wissner E, Tilz RR, Rillig A, Mathew S, Schmidt B, Chun J, Wohlmuth P, Deiss S, Lemes C, Maurer T, Fink T, Heeger C, Ouyang F, Kuck $\mathrm{KH}$, Metzner A. Ablation of atrial fibrillation in patients $>/=75$ years: longterm clinical outcome and safety. Europace 2016;18:543-549.

1253. Santangeli P, Di Biase L, Mohanty P, Burkhardt JD, Horton R, Bai R, Mohanty S, Pump A, Gibson D, Couts L, Hongo R, Beheiry S, Natale A. Catheter ablation of atrial fibrillation in octogenarians: safety and outcomes. I Cardiovasc Electrophysiol 2012;23:687-693.

1254. Tan HW, Wang XH, Shi HF, Yang GS, Zhou L, Gu JN, Jiang WF, Liu X. Efficacy, safety and outcome of catheter ablation for atrial fibrillation in octogenarians. Int J Cardiol 2010;145:147-148.

1255. Zado E, Callans DJ, Riley M, Hutchinson M, Garcia F, Bala R, Lin D, Cooper J, Verdino R, Russo AM, Dixit S, Gerstenfeld E, Marchlinski FE. Long-term clinical efficacy and risk of catheter ablation for atrial fibrillation in the elderly. J Cardiovasc Electrophysiol 2008;19:621-626.

1256. Abugattas JP, lacopino S, Moran D, De Regibus V, Takarada K, Mugnai G, Stroker E, Coutino-Moreno HE, Choudhury R, Storti C, De Greef Y, Paparella G, Brugada P, de Asmundis C, Chierchia GB. Efficacy and safety of the second generation cryoballoon ablation for the treatment of paroxysmal atrial fibrillation in patients over 75 years: a comparison with a younger cohort. Europace 2017; 19:1798-1803.

1257. Bunch TJ, May HT, Bair TL, Jacobs V, Crandall BG, Cutler M, Weiss JP, Mallender C, Osborn JS, Anderson JL, Day JD. The impact of age on 5-year outcomes after atrial fibrillation catheter ablation. J Cardiovasc Electrophysiol 2016;27:141-146.

1258. Guiot A, Jongnarangsin K, Chugh A, Suwanagool A, Latchamsetty R, Myles JD, Jiang Q, Crawford T, Good E, Pelosi F Jr, Bogun F, Morady F, Oral H. Anticoagulant therapy and risk of cerebrovascular events after catheter ablation of atrial fibrillation in the elderly. I Cardiovasc Electrophysiol 2012;23:36-43.

1259. Kusumoto F, Prussak K, Wiesinger M, Pullen T, Lynady C. Radiofrequency catheter ablation of atrial fibrillation in older patients: outcomes and complications. J Interv Card Electrophysiol 2009;25:31-35.

1260. Liu Y, Huang H, Huang C, Zhang S, Ma C, Liu X, Yang Y, Cao K, Wu S, Wang F; National Atrial Fibrillation Working Group of Chinese Society of Pacing and Electrophysiology. Catheter ablation of atrial fibrillation in Chinese elderly patients. Int J Cardiol 2011;152:266-267.

1261. Shah RU, Freeman JV, Shilane D, Wang PJ, Go AS, Hlatky MA. Procedural complications, rehospitalizations, and repeat procedures after catheter ablation for atrial fibrillation. J Am Coll Cardiol 2012;59:143-149.

1262. Spragg DD, Dalal D, Cheema A, Scherr D, Chilukuri K, Cheng A, Henrikson CA, Marine JE, Berger RD, Dong J, Calkins H. Complications of catheter ablation for atrial fibrillation: incidence and predictors. I Cardiovasc Electrophysiol 2008; 19:627-631.

1263. Srivatsa UN, Danielsen B, Anderson I, Amsterdam E, Pezeshkian N, Yang $Y$, White $\mathrm{RH}$. Risk predictors of stroke and mortality after ablation for atrial fibrillation: the California experience 2005-2009. Heart Rhythm 2014;11:1898-1903.

1264. Friberg L, Rosenqvist M. Less dementia with oral anticoagulation in atrial fibrillation. Eur Heart J 2018:39:453-460.

1265. Jacobs V, Woller SC, Stevens S, May HT, Bair TL, Anderson JL, Crandall BG, Day JD, Johanning K, Long Y, Mallender C, Olson JL, Osborn JS, Weiss JP, Bunch TJ. Time outside of therapeutic range in atrial fibrillation patients is associated with long-term risk of dementia. Heart Rhythm 2014;11:2206-2213.

1266. Jacobs V, Woller SC, Stevens SM, May HT, Bair TL, Crandall BG, Cutler M, Day JD, Weiss JP, Osborn JS, Mallender C, Anderson JL, Bunch TJ. Percent 
time with a supratherapeutic INR in atrial fibrillation patients also using an antiplatelet agent is associated with long-term risk of dementia. J Cardiovasc Electrophysiol 2015;26:1180-1186.

1267. Jacobs V, May HT, Bair TL, Crandall BG, Cutler MJ, Day JD, Mallender C, Osborn JS, Stevens SM, Weiss JP, Woller SC, Bunch TJ. Long-term population-based cerebral ischemic event and cognitive outcomes of direct oral anticoagulants compared with warfarin among long-term anticoagulated patients for atrial fibrillation. Am J Cardiol 2016;118:210-214.

1268. Zhang C, Gu ZC, Shen L, Pan MM, Yan YD, Pu J, Liu XY, Lin HW. Non-vitamin $\mathrm{K}$ antagonist oral anticoagulants and cognitive impairment in atrial fibrillation: insights from the meta-analysis of over 90,000 patients of randomized controlled trials and real-world studies. Front Aging Neurosci 2018;10:258.

1269. Sogaard M, Skjoth F, Jensen M, Kjaeldgaard JN, Lip GYH, Larsen TB, Nielsen PB. Nonvitamin $\mathrm{K}$ antagonist oral anticoagulants versus warfarin in atrial fibrillation patients and risk of dementia: a nationwide propensity-weighted cohort study. J Am Heart Assoc 2019;8:e011358.

1270. Friberg L, Andersson T, Rosenqvist M. Less dementia and stroke in low-risk patients with atrial fibrillation taking oral anticoagulation. Eur Heart J 2019;40:2327-2335.

121. Okuno J, Yanagi $\mathrm{H}$, Tomura S. Is cognitive impairment a risk factor for poor compliance among Japanese elderly in the community?Eur J Clin Pharmacol 2001:57:589-594.

1272. Salas M, In't Veld BA, van der Linden PD, Hofman A, Breteler M, Stricker $\mathrm{BH}$. Impaired cognitive function and compliance with antihypertensive drugs in elderly: the Rotterdam Study. Clin Pharmacol Ther 2001;70:561-566.

1273. Jensen AS, Idorn L, Norager B, Vejlstrup N, Sondergaard L. Anticoagulation in adults with congenital heart disease: the who, the when and the how? Heart 2015;101:424-429.

1274. Renda G, Ricci F, Giugliano RP, De Caterina R. Non-vitamin K antagonist oral anticoagulants in patients with atrial fibrillation and valvular heart disease. J Am Coll Cardiol 2017;69:1363-1371.

1275. Caldeira D, David C, Costa J, Ferreira JJ, Pinto FJ. Non-vitamin K antagonist oral anticoagulants in patients with atrial fibrillation and valvular heart disease: systematic review and meta-analysis. Eur Heart J Cardiovasc Pharmacother 2018:4:111-118

1276. Ammash NM, Phillips SD, Hodge DO, Connolly HM, Grogan MA, Friedman PA, Warnes CA, Asirvatham SJ. Outcome of direct current cardioversion for atrial arrhythmias in adults with congenital heart disease. Int J Cardiol 2012;154:270-274

1277. Feltes TF, Friedman RA. Transesophageal echocardiographic detection of atrial thrombi in patients with nonfibrillation atrial tachyarrhythmias and congenital heart disease. J Am Coll Cardiol 1994;24:1365-1370.

1278. Roos-Hesselink J, Meijboom F, Spitaels S, van Domburg R, van Rijen E, Utens E, Bogers A, Simoons M, Simoons ML. Excellent survival and low incidence of arrhythmias, stroke and heart failure long-term after surgical ASD closure at young age. A prospective follow-up study of 21-33 years. Eur Heart J 2003;24:190-197.

1279. Mas JL, Derumeaux G, Guillon B, Massardier E, Hosseini H, Mechtouff L, Arquizan C, Bejot Y, Vuillier F, Detante O, Guidoux C, Canaple S, Vaduva C, Dequatre-Ponchelle N, Sibon I, Garnier P, Ferrier A, Timsit S, RobinetBorgomano E, Sablot D, Lacour JC, Zuber M, Favrole P, Pinel JF, Apoil M, Reiner P, Lefebvre C, Guerin P, Piot C, Rossi R, Dubois-Rande JL, Eicher JC, Meneveau N, Lusson JR, Bertrand B, Schleich JM, Godart F, Thambo JB, Leborgne L, Michel P, Pierard L, Turc G, Barthelet M, Charles-Nelson A, Weimar C, Moulin T, Juliard JM, Chatellier G; CLOSE Investigators. Patent foramen ovale closure or anticoagulation vs. antiplatelets after stroke. $N$ Engl J Med 2017;377:1011-1021.

1280. Gutierrez SD, Earing MG, Singh AK, Tweddell JS, Bartz PJ. Atrial tachyarrhythmias and the Cox-maze procedure in congenital heart disease. Congenit Heart Dis 2013;8:434-439.

1281. Kobayashi J, Yamamoto F, Nakano K, Sasako Y, Kitamura S, Kosakai Y. Maze procedure for atrial fibrillation associated with atrial septal defect. Circulation 1998;98:11399-402.

1282. Shim H, Yang JH, Park PW, Jeong DS, Jun TG. Efficacy of the maze procedure for atrial fibrillation associated with atrial septal defect. Korean J Thorac Cardiovasc Surg 2013;46:98-103.

1283. Sherwin ED, Triedman JK, Walsh EP. Update on interventional electrophysiology in congenital heart disease: evolving solutions for complex hearts. Circ Arrhythm Electrophysiol 2013;6:1032-1040.

1284. Johnson JN, Tester DJ, Perry J, Salisbury BA, Reed CR, Ackerman MJ. Prevalence of early-onset atrial fibrillation in congenital long QT syndrome. Heart Rhythm 2008;5:704-709.

1285. Zellerhoff S, Pistulli R, Monnig G, Hinterseer M, Beckmann BM, Kobe J, Steinbeck G, Kaab S, Haverkamp W, Fabritz L, Gradaus R, Breithardt G, Schulze-Bahr E, Bocker D, Kirchhof P. Atrial arrhythmias in long-QT syndrome under daily life conditions: a nested case control study. J Cardiovasc Electrophysiol 2009;20:401-407.

1286. Gaita F, Giustetto C, Bianchi F, Wolpert C, Schimpf R, Riccardi R, Grossi S, Richiardi E, Borggrefe M. Short QT syndrome: a familial cause of sudden death. Circulation 2003;108:965-970.

1287. Borggrefe M, Wolpert C, Antzelevitch C, Veltmann C, Giustetto C, Gaita F, Schimpf R. Short QT syndrome. Genotype-phenotype correlations. J Electrocardiol 2005;38:75-80.

1288. Giustetto C, Di Monte F, Wolpert C, Borggrefe M, Schimpf R, Sbragia P, Leone G, Maury P, Anttonen O, Haissaguerre M, Gaita F. Short QT syndrome: clinical findings and diagnostic-therapeutic implications. Eur Heart 2006;27:2440-2447

1289. Giustetto C, Schimpf R, Mazzanti A, Scrocco C, Maury P, Anttonen O, Probst V, Blanc JJ, Sbragia P, Dalmasso P, Borggrefe M, Gaita F. Long-term follow-up of patients with short QT syndrome. J Am Coll Cardiol 2011;58:587-595.

1290. Gollob MH, Redpath CJ, Roberts JD. The short QT syndrome: proposed diagnostic criteria. J Am Coll Cardiol 2011;57:802-812.

1291. Bordachar P, Reuter S, Garrigue S, Cai X, Hocini M, Jais P, Haissaguerre M, Clementy J. Incidence, clinical implications and prognosis of atrial arrhythmias in Brugada syndrome. Eur Heart J 2004;25:879-884.

1292. Kusano KF, Taniyama M, Nakamura K, Miura D, Banba K, Nagase S, Morita H, Nishii N, Watanabe A, Tada T, Murakami M, Miyaji K, Hiramatsu S, Nakagawa K, Tanaka M, Miura A, Kimura H, Fuke S, Sumita W, Sakuragi S, Urakawa S, Iwasaki J, Ohe T. Atrial fibrillation in patients with Brugada syndrome relationships of gene mutation, electrophysiology, and clinical backgrounds. J Am Coll Cardiol 2008;51:1169-1175.

1293. Francis J, Antzelevitch C. Atrial fibrillation and Brugada syndrome. J Am Coll Cardiol 2008;51:1149-1153.

1294. Rodriguez-Manero M, Namdar M, Sarkozy A, Casado-Arroyo R, Ricciardi D, de Asmundis C, Chierchia GB, Wauters K, Rao JY, Bayrak F, Van Malderen S, Brugada P. Prevalence, clinical characteristics and management of atrial fibrillation in patients with Brugada syndrome. Am J Cardiol 2013;111:362-367.

1295. Sacher F, Probst V, Maury P, Babuty D, Mansourati J, Komatsu Y, Marquie C, Rosa A, Diallo A, Cassagneau R, Loizeau C, Martins R, Field ME, Derval N, Miyazaki S, Denis A, Nogami A, Ritter P, Gourraud JB, Ploux S, Rollin A, Zemmoura A, Lamaison D, Bordachar P, Pierre B, Jais P, Pasquie JL, Hocini M, Legal F, Defaye P, Boveda S, lesaka Y, Mabo P, Haissaguerre M. Outcome after implantation of a cardioverter-defibrillator in patients with Brugada syndrome: a multicenter study - part 2. Circulation 2013;128:1739-1747.

1296. Hernandez-Ojeda J, Arbelo E, Borras R, Berne P, Tolosana JM, GomezJuanatey A, Berruezo A, Campuzano O, Sarquella-Brugada G, Mont L, Brugada $\mathrm{R}$, Brugada J. Patients with Brugada syndrome and implanted cardioverterdefibrillators: long-term follow-up. J Am Coll Cardiol 2017;70:1991-2002.

1297. Sumitomo N, Sakurada H, Taniguchi K, Matsumura M, Abe O, Miyashita M, Kanamaru H, Karasawa K, Ayusawa M, Fukamizu S, Nagaoka I, Horie M, Harada K, Hiraoka M. Association of atrial arrhythmia and sinus node dysfunction in patients with catecholaminergic polymorphic ventricular tachycardia. Circ 2007;71:1606-1609.

1298. Sy RW, Gollob MH, Klein G], Yee R, Skanes AC, Gula LJ, Leong-Sit P, Gow RM, Green MS, Birnie DH, Krahn AD. Arrhythmia characterization and longterm outcomes in catecholaminergic polymorphic ventricular tachycardia. Heart Rhythm 2011;8:864-871.

1299. Siontis KC, Geske JB, Ong K, Nishimura RA, Ommen SR, Gersh BJ. Atrial fibrillation in hypertrophic cardiomyopathy: prevalence, clinical correlations, and mortality in a large high-risk population. J Am Heart Assoc 2014;3:e001002.

1300. Guttmann OP, Rahman MS, O'Mahony C, Anastasakis A, Elliott PM. Atrial fibrillation and thromboembolism in patients with hypertrophic cardiomyopathy: systematic review. Heart 2014;100:465-472.

1301. Rowin EJ, Hausvater A, Link MS, Abt P, Gionfriddo W, Wang W, Rastegar H, Estes NAM, Maron MS, Maron BJ. Clinical profile and consequences of atrial fibrillation in hypertrophic cardiomyopathy. Circulation 2017;136:2420-2436.

1302. Rowin EJ, Orfanos A, Estes NAM, Wang W, Link MS, Maron MS, Maron BJ. Occurrence and natural history of clinically silent episodes of atrial fibrillation in hypertrophic cardiomyopathy. Am J Cardiol 2017;119:1862-1865.

1303. van Velzen HG, Theuns DA, Yap SC, Michels M, Schinkel AF. Incidence of device-detected atrial fibrillation and long-term outcomes in patients with hypertrophic cardiomyopathy. Am J Cardiol 2017;119:100-105.

1304. Klopotowski M, Kwapiszewska A, Kukula K, Jamiolkowski J, Dabrowski M, Derejko P, Oreziak A, Baranowski R, Spiewak M, Marczak M, Klisiewicz A, Szepietowska B, Chmielak Z, Witkowski A. Clinical and echocardiographic parameters as risk factors for atrial fibrillation in patients with hypertrophic cardiomyopathy. Clin Cardiol 2018;41:1336-1340.

1305. Choi YJ, Choi EK, Han KD, Jung JH, Park J, Lee E, Choe W, Lee SR, Cha MJ, Lim WH, Oh S. Temporal trends of the prevalence and incidence of atrial fibrillation and stroke among Asian patients with hypertrophic cardiomyopathy: a nationwide population-based study. Int J Cardiol 2018;273:130-135. 
1306. Chu AF, Zado E, Marchlinski FE. Atrial arrhythmias in patients with arrhythmogenic right ventricular cardiomyopathy/dysplasia and ventricular tachycardia. Am J Cardiol 2010;106:720-722.

1307. Camm CF, James CA, Tichnell C, Murray B, Bhonsale A, te Riele AS, Judge DP, Tandri H, Calkins H. Prevalence of atrial arrhythmias in arrhythmogenic right ventricular dysplasia/cardiomyopathy. Heart Rhythm 2013;10:1661-1668.

1308. Saguner AM, Ganahl S, Kraus A, Baldinger SH, Medeiros-Domingo A, Saguner AR, Mueller-Burri SA, Wolber T, Haegeli LM, Krasniqi N, Tanner FC, Steffel J, Brunckhorst C, Duru F. Clinical role of atrial arrhythmias in patients with arrhythmogenic right ventricular dysplasia. Circ J 2014;78:2854-2861.

1309. Bourfiss M, Te Riele AS, Mast TP, Cramer MJ, Van Der Heijden JF, Van Veen TA, Loh P, Dooijes D, Hauer RN, Velthuis BK. Influence of genotype on structural atrial abnormalities and atrial fibrillation or flutter in arrhythmogenic right ventricular dysplasia/cardiomyopathy. I Cardiovasc Electrophysiol 2016;27:1420-1428.

1310. Mussigbrodt A, Knopp H, Efimova E, Weber A, Bertagnolli L, Hilbert S, Kosiuk J. Dinov B, Bode K, Kircher S, Dagres N, Richter S, Sommer P, Husser D, Bollmann A, Hindricks G, Arya A. Supraventricular arrhythmias in patients with arrhythmogenic right ventricular dysplasia/cardiomyopathy associate with long-term outcome after catheter ablation of ventricular tachycardias. Europace 2018;20:1182-1187.

1311. Tonet JL, Castro-Miranda R, Iwa T, Poulain F, Frank R, Fontaine GH. Frequency of supraventricular tachyarrhythmias in arrhythmogenic right ventricular dysplasia. Am / Cardiol 1991;67:1153.

1312. Hasselberg NE, Haland TF, Saberniak J, Brekke PH, Berge KE, Leren TP, Edvardsen T, Haugaa $\mathrm{KH}$. Lamin A/C cardiomyopathy: young onset, high penetrance, and frequent need for heart transplantation. Eur Heart J 2018;39:853-860.

1313. van Rijsingen IA, Nannenberg EA, Arbustini E, Elliott PM, Mogensen J, Hermans-van Ast JF, van der Kooi AJ, van Tintelen JP, van den Berg MP, Grasso M, Serio A, Jenkins S, Rowland C, Richard P, Wilde AA, Perrot A, Pankuweit S, Zwinderman AH, Charron P, Christiaans I, Pinto YM. Genderspecific differences in major cardiac events and mortality in lamin $A / C$ mutation carriers. Eur J Heart Fail 2013;15:376-384.

1314. Kumar S, Baldinger SH, Gandjbakhch E, Maury P, Sellal JM, Androulakis AF, Waintraub X, Charron P, Rollin A, Richard P, Stevenson WG, Macintyre CJ, Ho CY, Thompson T, Vohra JK, Kalman JM, Zeppenfeld K, Sacher F, Tedrow UB, Lakdawala NK. Long-term arrhythmic and nonarrhythmic outcomes of lamin A/C mutation carriers. J Am Coll Cardiol 2016;68:2299-2307.

1315. Pasotti M, Klersy C, Pilotto A, Marziliano N, Rapezzi C, Serio A, Mannarino S, Gambarin F, Favalli V, Grasso M, Agozzino M, Campana C, Gavazzi A, Febo O, Marini M, Landolina M, Mortara A, Piccolo G, Vigano M, Tavazzi L, Arbustini E. Long-term outcome and risk stratification in dilated cardiolaminopathies. J Am Coll Cardiol 2008;52:1250-1260.

1316. Stollberger C, Blazek G, Winkler-Dworak M, Finsterer J. Atrial fibrillation in left ventricular noncompaction with and without neuromuscular disorders is associated with a poor prognosis. Int J Cardiol 2009;133:41-45.

1317. Aras D, Tufekcioglu O, Ergun K, Ozeke O, Yildiz A, Topaloglu S, Deveci B, Sahin O, Kisacik HL, Korkmaz S. Clinical features of isolated ventricular noncompaction in adults long-term clinical course, echocardiographic properties, and predictors of left ventricular failure. I Card Fail 2006;12:726-733.

1318. Li S, Zhang C, Liu N, Bai H, Hou C, Wang J, Song L, Pu J. Genotype-positive status is associated with poor prognoses in patients with left ventricular noncompaction cardiomyopathy. J Am Heart Assoc 2018;7:e009910.

1319. Pappone C, Radinovic A, Manguso F, Vicedomini G, Sala S, Sacco FM, Ciconte G, Saviano M, Ferrari M, Sommariva E, Sacchi S, Ciaccio C, Kallergis EM, Santinelli $\mathrm{V}$. New-onset atrial fibrillation as first clinical manifestation of latent Brugada syndrome: prevalence and clinical significance. Eur Heart J 2009;30:2985-2992.

1320. Hong K, Bjerregaard P, Gussak I, Brugada R. Short QT syndrome and atrial fibrillation caused by mutation in $\mathrm{KCNH} 2$. I Cardiovasc Electrophysiol 2005; 16:394-396.

1321. Peters S. Atrial arrhythmias in arrhythmogenic cardiomyopathy: at the beginning or at the end of the disease story?Circ / 2015;79:446.

1322. Fatkin D, MacRae C, Sasaki T, Wolff MR, Porcu M, Frenneaux M, Atherton J, Vidaillet HJ, Jr., Spudich S, De Girolami U, Seidman JG, Seidman C, Muntoni F, Muehle G, Johnson W, McDonough B. Missense mutations in the rod domain of the lamin A/C gene as causes of dilated cardiomyopathy and conductionsystem disease. N Engl J Med 1999;341:1715-1724.

1323. Olesen MS, Yuan L, Liang B, Holst AG, Nielsen N, Nielsen JB, Hedley PL, Christiansen M, Olesen SP, Haunso S, Schmitt N, Jespersen T, Svendsen JH. High prevalence of long QT syndrome-associated SCN5A variants in patients with early-onset lone atrial fibrillation. Circ Cardiovasc Genet 2012;5:450-459.

1324. Giustetto C, Cerrato N, Gribaudo E, Scrocco C, Castagno D, Richiardi E, Giachino D, Bianchi F, Barbonaglia L, Ferraro A. Atrial fibrillation in a large population with Brugada electrocardiographic pattern: prevalence, management, and correlation with prognosis. Heart Rhythm 2014;11:259-265

1325. Conte G, Dewals W, Sieira J, de Asmundis C, Ciconte G, Chierchia GB, D Giovanni G, Baltogiannis G, Saitoh Y, Levinstein M, La Meir M, Wellens F, Pappaert G, Brugada P. Drug-induced Brugada syndrome in children: clinical features, device-based management, and long-term follow-up. J Am Coll Cardiol 2014;63:2272-2279.

1326. Lee SE, Park JK, Uhm JS, Kim JY, Pak HN, Lee MH, Joung B. Impact of atrial fibrillation on the clinical course of apical hypertrophic cardiomyopathy. Heart 2017;103:1496-1501.

1327. Olivotto I, Cecchi F, Casey SA, Dolara A, Traverse JH, Maron BJ. Impact of atrial fibrillation on the clinical course of hypertrophic cardiomyopathy. Circulation 2001;104:2517-2524.

1328. Maron BJ, Olivotto I, Spirito P, Casey SA, Bellone P, Gohman TE, Graham KJ, Burton DA, Cecchi F. Epidemiology of hypertrophic cardiomyopathy-related death: revisited in a large non-referral-based patient population. Circulation 2000; 102:858-864.

1329. Mazzanti A, Ng K, Faragli A, Maragna R, Chiodaroli E, Orphanou N Monteforte N, Memmi M, Gambelli P, Novelli V, Bloise R, Catalano O, Moro G, Tibollo V, Morini M, Bellazzi R, Napolitano C, Bagnardi V, Priori SG. Arrhythmogenic right ventricular cardiomyopathy: clinical course and predictors of arrhythmic risk. J Am Coll Cardiol 2016;68:2540-2550.

1330. Pizzale S, Gollob MH, Gow R, Birnie DH. Sudden death in a young man with catecholaminergic polymorphic ventricular tachycardia and paroxysmal atrial fibrillation. J Cardiovasc Electrophysiol 2008;19:1319-1321.

1331. Roses-Noguer F, Jarman JW, Clague JR, Till J. Outcomes of defibrillator therapy in catecholaminergic polymorphic ventricular tachycardia. Heart Rhythm 2014;11:58-66.

1332. van der Werf C, Lieve KV, Bos JM, Lane CM, Denjoy I, Roses-Noguer F, Aiba T, Wada Y, Ingles J, Leren IS, Rudic B, Schwartz PJ, Maltret A, Sacher F, Skinner JR, Krahn AD, Roston TM, Tfelt-Hansen J, Swan H, Robyns T, Ohno S, Roberts JD, van den Berg MP, Kammeraad JA, Probst V, Kannankeril PJ, Blom NA, Behr ER, Borggrefe M, Haugaa KH, Semsarian C, Horie M, Shimizu W, Till JA, Leenhardt A, Ackerman MJ, Wilde AA. Implantable cardioverterdefibrillators in previously undiagnosed patients with catecholaminergic polymorphic ventricular tachycardia resuscitated from sudden cardiac arrest. Eur Heart J 2019;40:2953-2961.

1333. Sugiyasu A, Oginosawa Y, Nogami A, Hata Y. A case with catecholaminergic polymorphic ventricular tachycardia unmasked after successful ablation of atrial tachycardias from pulmonary veins. Pacing Clin Electrophysiol 2009;32:e21-24.

1334. Veltmann C, Kuschyk J, Schimpf R, Streitner F, Schoene N, Borggrefe M, Wolpert $C$. Prevention of inappropriate ICD shocks in patients with Brugada syndrome. Clin Res Cardiol 2010;99:37-44.

1335. Klein GJ, Bashore TM, Sellers TD, Pritchett EL, Smith WM, Gallagher JJ. Ventricular fibrillation in the Wolff-Parkinson-White syndrome. N Engl J Med 1979;301:1080-1085.

1336. Brugada J, Katritsis DG, Arbelo E, Arribas F, Bax J], Blomstrom-Lundqvist C Calkins H, Corrado D, Deftereos SG, Diller GP, Gomez-Doblas J], Gorenek B, Grace A, Ho SY, Kaski JC, Kuck KH, Lambiase PD, Sacher F, Sarquella-Brugada G, Suwalski P, Zaza A; ESC Scientific Document Group. 2019 ESC Guidelines for the management of patients with supraventricular tachycardia. The Task Force for the management of patients with supraventricular tachycardia of the European Society of Cardiology (ESC). Eur Heart J 2020;41:655-720.

1337. Morady F, DiCarlo LA, Jr., Baerman JM, De Buitleir M. Effect of propranolol on ventricular rate during atrial fibrillation in the Wolff-Parkinson-White syndrome. Pacing Clin Electrophysiol 1987;10:492-496.

1338. Sellers TD Jr, Bashore TM, Gallagher JJ. Digitalis in the pre-excitation syndrome. Analysis during atrial fibrillation. Circulation 1977;56:260-267.

1339. Glatter KA, Dorostkar PC, Yang Y, Lee RJ, Van Hare GF, Keung E, Modin G, Scheinman MM. Electrophysiological effects of ibutilide in patients with accessory pathways. Circulation 2001;104:1933-1939.

1340. Ludmer PL, McGowan NE, Antman EM, Friedman PL. Efficacy of propafenone in Wolff-Parkinson-White syndrome: electrophysiologic findings and longterm follow-up. J Am Coll Cardiol 1987;9:1357-1363.

1341. Boahene KA, Klein GJ, Yee R, Sharma AD, Fujimura O. Termination of acute atrial fibrillation in the Wolff-Parkinson-White syndrome by procainamide and propafenone: importance of atrial fibrillatory cycle length. J Am Coll Cardiol 1990;16:1408-1414.

1342. Crijns HJ, den Heijer P, van Wijk LM, Lie KI. Successful use of flecainide in atrial fibrillation with rapid ventricular rate in the Wolff-Parkinson-White syndrome. Am Heart J 1988;115:1317-1321.

1343. Simonian SM, Lotfipour S, Wall C, Langdorf MI. Challenging the superiority of amiodarone for rate control in Wolff-Parkinson-White and atrial fibrillation. Intern Emerg Med 2010;5:421 - 426. 
1344. Vaidya VR, Arora S, Patel N, Badheka AO, Patel N, Agnihotri K, Billimoria Z, Turakhia MP, Friedman PA, Madhavan M, Kapa S, Noseworthy PA, Cha YM, Gersh B, Asirvatham SJ, Deshmukh AJ. Burden of arrhythmia in pregnancy. Circulation 2017;135:619-621.

1345. Drenthen W, Pieper PG, Roos-Hesselink JW, van Lottum WA, Voors AA, Mulder BJ, van Dijk AP, Vliegen HW, Yap SC, Moons P, Ebels T, van Veldhuisen DJ; Zahara Investigators. Outcome of pregnancy in women with congenital heart disease: a literature review. J Am Coll Cardiol 2007;49:2303-2311.

1346. Opotowsky AR, Siddiqi OK, D'Souza B, Webb GD, Fernandes SM, Landzberg MJ. Maternal cardiovascular events during childbirth among women with congenital heart disease. Heart 2012;98:145-151.

1347. Lee MS, Chen W, Zhang Z, Duan L, Ng A, Spencer HT, Kwan DM, Shen AY. Atrial fibrillation and atrial flutter in pregnant women - a population-based study. J Am Heart Assoc 2016;5:e003182.

1348. Li JM, Nguyen C, Joglar JA, Hamdan MH, Page RL. Frequency and outcome of arrhythmias complicating admission during pregnancy: experience from a high-volume and ethnically-diverse obstetric service. Clin Cardiol 2008;31:538-541.

1349. Regitz-Zagrosek V, Roos-Hesselink JW, Bauersachs J, Blomstrom-Lundqvist C, Cifkova R, De Bonis M, lung B, Johnson MR, Kintscher U, Kranke P, Lang IM, Morais J, Pieper PG, Presbitero P, Price S, Rosano GMC, Seeland U, Simoncini T, Swan L, Warnes CA; ESC Scientific Document Group. 2018 ESC Guidelines for the management of cardiovascular diseases during pregnancy. Eur Heart J 2018;39:3165-3241.

1350. Wang YC, Chen CH, Su HY, Yu MH. The impact of maternal cardioversion on fetal haemodynamics. Eur J Obstet Gynecol Reprod Biol 2006;126:268-269.

1351. Moore JS, Teefey P, Rao K, Berlowitz MS, Chae SH, Yankowitz J. Maternal arrhythmia: a case report and review of the literature. Obstet Gynecol Surv 2012;67:298-312.

1352. Page RL. Treatment of arrhythmias during pregnancy. Am Heart J 1995;130:871-876

1353. Barnes EJ, Eben F, Patterson D. Direct current cardioversion during pregnancy should be performed with facilities available for fetal monitoring and emergency caesarean section. BJOG 2002;109:1406-1407.

1354. Katritsis DG, Boriani G, Cosio FG, Hindricks G, Jais P, Josephson ME, Keegan R, Kim YH, Knight BP, Kuck KH, Lane DA, Lip GYH, Malmborg H, Oral H, Pappone C, Themistoclakis S, Wood KA, Blomstrom-Lundqvist C. European Heart Rhythm Association (EHRA) consensus document on the management of supraventricular arrhythmias, endorsed by Heart Rhythm Society (HRS), Asia-Pacific Heart Rhythm Society (APHRS), and Sociedad Latinoamericana de Estimulacion Cardiaca y Electrofisiologia (SOLAECE). Eur Heart J 2018;39:1442-1445.

1355. Kockova R, Kocka V, Kiernan T, Fahy G]. Ibutilide-induced cardioversion of atrial fibrillation during pregnancy. J Cardiovasc Electrophysiol 2007;18:545-547.

1356. Lip GY, Beevers M, Churchill D, Shaffer LM, Beevers DG. Effect of atenolol on birth weight. Am J Cardiol 1997;79:1436-1438.

1357. Heidbuchel H, Anne W, Willems R, Adriaenssens B, Van de Werf F, Ector H. Endurance sports is a risk factor for atrial fibrillation after ablation for atrial flutter. Int J Cardiol 2006;107:67-72.

1358. Calvo N, Ramos P, Montserrat S, Guasch E, Coll-Vinent B, Domenech M, Bisbal F, Hevia S, Vidorreta S, Borras R, Falces C, Embid C, Montserrat JM, Berruezo A, Coca A, Sitges M, Brugada J, Mont L. Emerging risk factors and the dose-response relationship between physical activity and lone atrial fibrillation: a prospective case-control study. Europace 2016;18:57-63.

1359. Crump C, Sundquist J, Winkleby MA, Sundquist K. Height, weight, and aerobic fitness level in relation to the risk of atrial fibrillation. Am J Epidemiol 2018; 187:417-426

1360. Mont L, Sambola A, Brugada J, Vacca M, Marrugat J, Elosua R, Pare C, Azqueta M, Sanz G. Long-lasting sport practice and lone atrial fibrillation. Eur Heart J 2002;23:477-482.

1361. Mont L, Tamborero D, Elosua R, Molina I, Coll-Vinent B, Sitges M, Vidal B, Scalise A, Tejeira A, Berruezo A, Brugada J; GIRAFA Investigators. Physical activity, height, and left atrial size are independent risk factors for lone atrial fibrillation in middle-aged healthy individuals. Europace 2008;10:15-20.

1362. Guasch E, Mont L. Diagnosis, pathophysiology, and management of exerciseinduced arrhythmias. Nat Rev Cardiol 2017;14:88-101.

1363. Stein R, Medeiros CM, Rosito GA, Zimerman LI, Ribeiro JP. Intrinsic sinus and atrioventricular node electrophysiologic adaptations in endurance athletes. J Am Coll Cardiol 2002;39:1033-1038.

1364. Calvo N, Mont L, Tamborero D, Berruezo A, Viola G, Guasch E, Nadal M, Andreu D, Vidal B, Sitges M, Brugada J. Efficacy of circumferential pulmonary vein ablation of atrial fibrillation in endurance athletes. Europace 2010;12:30-36.

1365. Koopman P, Nuyens D, Garweg C, La Gerche A, De Buck S, Van Casteren L, Alzand B, Willems R, Heidbuchel H. Efficacy of radiofrequency catheter ablation in athletes with atrial fibrillation. Europace 2011;13:1386-1393.
1366. Aizer A, Gaziano JM, Cook NR, Manson JE, Buring JE, Albert CM. Relation of vigorous exercise to risk of atrial fibrillation. $A m 」$ Cardiol 2009;103:1572-1577.

1367. Mozaffarian D, Furberg CD, Psaty BM, Siscovick D. Physical activity and incidence of atrial fibrillation in older adults: the cardiovascular health study. Circulation 2008;118:800-807.

1368. Thelle DS, Selmer R, Gjesdal K, Sakshaug S, Jugessur A, Graff-Iversen S, Tverdal A, Nystad W. Resting heart rate and physical activity as risk factors for lone atrial fibrillation: a prospective study of 309,540 men and women. Heart 2013;99:1755-1760.

1369. O'Brien B, Burrage PS, Ngai JY, Prutkin JM, Huang CC, Xu X, Chae SH, Bollen BA, Piccini JP, Schwann NM, Mahajan A, Ruel M, Body SC, Sellke FW, Mathew J, Muehlschlegel JD. Society of Cardiovascular Anesthesiologists/European Association of Cardiothoracic Anaesthetists practice advisory for the management of perioperative atrial fibrillation in patients undergoing cardiac surgery. J Cardiothorac Vasc Anesth 2019;33:12-26.

1370. Lubitz SA, Yin X, Rienstra M, Schnabel RB, Walkey AJ, Magnani JW, Rahman F, McManus DD, Tadros TM, Levy D, Vasan RS, Larson MG, Ellinor PT, Benjamin EJ. Long-term outcomes of secondary atrial fibrillation in the community: the Framingham Heart Study. Circulation 2015;131:1648-1655.

1371. Dobrev D, Aguilar M, Heijman J, Guichard JB, Nattel S. Postoperative atrial fibrillation: mechanisms, manifestations and management. Nat Rev Cardiol 2019;16:417-436.

1372. Echahidi N, Pibarot P, O'Hara G, Mathieu P. Mechanisms, prevention, and treatment of atrial fibrillation after cardiac surgery. I Am Coll Cardiol 2008;51:793-801.

1373. Gillinov AM, Bagiella E, Moskowitz AJ, Raiten JM, Groh MA, Bowdish ME, Ailawadi G, Kirkwood KA, Perrault LP, Parides MK, Smith RL 2nd, Kern JA, Dussault G, Hackmann AE, Jeffries NO, Miller MA, Taddei-Peters WC, Rose EA, Weisel RD, Williams DL, Mangusan RF, Argenziano M, Moquete EG, O'Sullivan KL, Pellerin M, Shah KJ, Gammie JS, Mayer ML, Voisine P, Gelijns AC, O'Gara PT, Mack MJ, CTSN. Rate control versus rhythm control for atrial fibrillation after cardiac surgery. N Engl J Med 2016;374:1911-1921.

1374. Amar D. Postthoracotomy atrial fibrillation. Curr Opin Anaesthesiol 2007;20:43-47.

1375. Philip I, Berroeta C, Leblanc I. Perioperative challenges of atrial fibrillation. Curr Opin Anaesthesiol 2014;27:344-352.

1376. Lowres N, Mulcahy G, Jin K, Gallagher R, Neubeck L, Freedman B. Incidence of postoperative atrial fibrillation recurrence in patients discharged in sinus rhythm after cardiac surgery: a systematic review and meta-analysis. Interact Cardiovasc Thorac Surg 2018;26:504-511.

1377. Lee SH, Kang DR, Uhm JS, Shim J, Sung JH, Kim JY, Pak HN, Lee MH, Joung B. New-onset atrial fibrillation predicts long-term newly developed atrial fibrillation after coronary artery bypass graft. Am Heart J 2014;167:593600.e1.

1378. Konstantino Y, Zelnik Yovel D, Friger MD, Sahar G, Knyazer B, Amit G. Postoperative atrial fibrillation following coronary artery bypass graft surgery predicts long-term atrial fibrillation and stroke. Isr Med Assoc J 2016;18:744-748.

1379. Lin MH, Kamel H, Singer DE, Wu YL, Lee M, Ovbiagele B. Perioperative/postoperative atrial fibrillation and risk of subsequent stroke and/or mortality. Stroke 2019;50:1364-1371.

1380. AlTurki A, Marafi M, Proietti R, Cardinale D, Blackwell R, Dorian P, Bessissow A, Vieira L, Greiss I, Essebag V, Healey JS, Huynh T. Major adverse cardiovascular events associated with postoperative atrial fibrillation after noncardiac surgery: a systematic review and meta-analysis. Circ Arrhythm Electrophysiol 2020;13:e007437.

1381. Mathew JP, Fontes ML, Tudor IC, Ramsay J, Duke P, Mazer CD, Barash PG, Hsu PH, Mangano DT; Investigators of the Ischemia Research and Education Foundation, Multicenter Study of Perioperative Ischemia Research Group. A multicenter risk index for atrial fibrillation after cardiac surgery. JAMA 2004;291:1720-1729.

1382. Villareal RP, Hariharan R, Liu BC, Kar B, Lee VV, Elayda M, Lopez JA, Rasekh A, Wilson JM, Massumi A. Postoperative atrial fibrillation and mortality after coronary artery bypass surgery. J Am Coll Cardiol 2004;43:742-748.

1383. Cardinale D, Sandri MT, Colombo A, Salvatici M, Tedeschi I, Bacchiani G, Beggiato M, Meroni CA, Civelli M, Lamantia G, Colombo N, Veglia F, Casiraghi M, Spaggiari L, Venturino M, Cipolla CM. Prevention of Atrial Fibrillation in High-risk Patients Undergoing Lung Cancer Surgery: the PRESAGE Trial. Ann Surg 2016;264:244-251.

1384. Ojima T, Nakamori M, Nakamura M, Katsuda M, Hayata K, Kato T, Kitadani J, Tabata H, Takeuchi A, Yamaue H. Randomized clinical trial of landiolol hydrochloride for the prevention of atrial fibrillation and postoperative complications after oesophagectomy for cancer. Br J Surg 2017;104:1003-1009.

1385. Arsenault KA, Yusuf AM, Crystal E, Healey JS, Morillo CA, Nair GM, Whitlock RP. Interventions for preventing post-operative atrial fibrillation in patients undergoing heart surgery. Cochrane Database Syst Rev 2013:CD003611. 
1386. Ozaydin M, Icli A, Yucel H, Akcay S, Peker O, Erdogan D, Varol E, Dogan A, Okutan $\mathrm{H}$. Metoprolol vs. carvedilol or carvedilol plus $\mathrm{N}$-acetyl cysteine on post-operative atrial fibrillation: a randomized, double-blind, placebo-controlled study. Eur Heart J 2013;34:597-604.

1387. O'Neal JB, Billings FTt, Liu X, Shotwell MS, Liang Y, Shah AS, Ehrenfeld JM, Wanderer JP, Shaw AD. Effect of preoperative beta-blocker use on outcomes following cardiac surgery. Am J Cardiol 2017;120:1293-1297.

1388. Devereaux PJ, Yang H, Yusuf S, Guyatt G, Leslie K, Villar JC, Xavier D, Chrolavicius S, Greenspan L, Pogue J, Pais P, Liu L, Xu S, Malaga G, Avezum A, Chan M, Montori VM, Jacka M, Choi P. Effects of extended-release metoprolol succinate in patients undergoing non-cardiac surgery (POISE trial): a randomised controlled trial. Lancet 2008;371:1839-1847.

1389. Zhu J, Wang C, Gao D, Zhang C, Zhang Y, Lu Y, Gao Y. Meta-analysis of amiodarone versus beta-blocker as a prophylactic therapy against atrial fibrillation following cardiac surgery. Intern Med J 2012;42:1078-1087.

1390. Auer J, Weber T, Berent R, Puschmann R, Hartl P, Ng CK, Schwarz C, Lehner E, Strasser U, Lassnig E, Lamm G, Eber B; Study of Prevention of Postoperative Atrial Fibrillation. A comparison between oral antiarrhythmic drugs in the prevention of atrial fibrillation after cardiac surgery: the pilot study of prevention of postoperative atrial fibrillation (SPPAF), a randomized, placebo-controlled trial. Am Heart J 2004;147:636-643.

1391. Buckley MS, Nolan PE Jr, Slack MK, Tisdale JE, Hilleman DE, Copeland JG. Amiodarone prophylaxis for atrial fibrillation after cardiac surgery: metaanalysis of dose response and timing of initiation. Pharmacotherapy 2007; 27:360-368.

1392. Riber LP, Christensen TD, Jensen HK, Hoejsgaard A, Pilegaard HK. Amiodarone significantly decreases atrial fibrillation in patients undergoing surgery for lung cancer. Ann Thorac Surg 2012;94:339 - 344; discussion 345 - 346.

1393. Tisdale JE, Wroblewski HA, Wall DS, Rieger KM, Hammoud ZT, Young JV, Kesler KA. A randomized trial evaluating amiodarone for prevention of atrial fibrillation after pulmonary resection. Ann Thorac Surg 2009;88:886-893; discussion 894-895.

1394. Yuan X, Du J, Liu Q, Zhang L. Defining the role of perioperative statin treatment in patients after cardiac surgery: a meta-analysis and systematic review of 20 randomized controlled trials. Int J Cardiol 2017;228:958-966.

1395. Fairley JL, Zhang L, Glassford NJ, Bellomo R. Magnesium status and magnesium therapy in cardiac surgery: a systematic review and meta-analysis focusing on arrhythmia prevention. J Crit Care 2017;42:69-77.

1396. Tabbalat RA, Hamad NM, Alhaddad IA, Hammoudeh A, Akasheh BF, Khader Y. Effect of ColchiciNe on the InciDence of atrial fibrillation in open heart surgery patients: END-AF trial. Am Heart J 2016;178:102-107.

1397. Ali-Hasan-Al-Saegh S, Mirhosseini SJ, Liakopoulos O, Sabashnikov A, Dehghan HR, Sedaghat-Hamedani F, Kayvanpour E, Ghaffari N, Vahabzadeh V, Aghabagheri M, Mozayan MR, Popov AF. Posterior pericardiotomy in cardiac surgery: systematic review and meta-analysis. Asian Cardiovasc Thorac Ann 2015;23:354-362.

1398. Hu XL, Chen Y, Zhou ZD, Ying J, Hu YH, Xu GH. Posterior pericardiotomy for the prevention of atrial fibrillation after coronary artery bypass grafting: a meta-analysis of randomized controlled trials. Int J Cardiol 2016;215:252-256.

1399. Wang W, Mei YQ, Yuan XH, Feng XD. Clinical efficacy of epicardial application of drug-releasing hydrogels to prevent postoperative atrial fibrillation. J Thorac Cardiovasc Surg 2016;151:80-85.

1400. Dieleman JM, Nierich AP, Rosseel PM, van der Maaten JM, Hofland J, Diephuis JC, Schepp RM, Boer C, Moons KG, van Herwerden LA, Tijssen JG, Numan SC, Kalkman CJ, van Dijk D; Dexamethasone for Cardiac Surgery Study Group. Intraoperative high-dose dexamethasone for cardiac surgery: a randomized controlled trial. JAMA 2012;308:1761-1767.

1401. Whitlock RP, Devereaux PJ, Teoh KH, Lamy A, Vincent J, Pogue J, Paparella D, Sessler DI, Karthikeyan G, Villar JC, Zuo Y, Avezum A, Quantz M, Tagarakis GI, Shah PJ, Abbasi SH, Zheng H, Pettit S, Chrolavicius S, Yusuf S; SIRS Investigators. Methylprednisolone in patients undergoing cardiopulmonary bypass (SIRS): a randomised, double-blind, placebo-controlled trial. Lancet 2015;386:1243-1253.

1402. Saxena A, Dinh DT, Smith JA, Shardey GC, Reid CM, Newcomb AE. Usefulness of postoperative atrial fibrillation as an independent predictor for worse early and late outcomes after isolated coronary artery bypass grafting (multicenter Australian study of 19,497 patients). Am J Cardiol 2012;109:219-225.

1403. Almassi GH, Schowalter T, Nicolosi AC, Aggarwal A, Moritz TE, Henderson WG, Tarazi R, Shroyer AL, Sethi GK, Grover FL, Hammermeister KE. Atrial fibrillation after cardiac surgery: a major morbid event?Ann Surg 1997;226:501-511; discussion 511-513.

1404. Gialdini G, Nearing K, Bhave PD, Bonuccelli U, ladecola C, Healey JS, Kamel $\mathrm{H}$. Perioperative atrial fibrillation and the long-term risk of ischemic stroke. JAMA 2014;312:616-622.
1405. Horwich P, Buth KJ, Legare JF. New onset postoperative atrial fibrillation is associated with a long-term risk for stroke and death following cardiac surgery. J Card Surg 2013;28:8-13.

1406. Ahlsson A, Fengsrud E, Bodin L, Englund A. Postoperative atrial fibrillation in patients undergoing aortocoronary bypass surgery carries an eightfold risk of future atrial fibrillation and a doubled cardiovascular mortality. Eur J Cardiothorac Surg 2010;37:1353-1359.

1407. Rubin DA, Nieminski KE, Reed GE, Herman MV. Predictors, prevention, and long-term prognosis of atrial fibrillation after coronary artery bypass graft operations. J Thorac Cardiovasc Surg 1987;94:331-335.

1408. Butt JH, Xian Y, Peterson ED, Olsen PS, Rorth R, Gundlund A, Olesen JB, Gislason GH, Torp-Pedersen C, Kober L, Fosbol EL. Long-term thromboembolic risk in patients with postoperative atrial fibrillation after coronary artery bypass graft surgery and patients with nonvalvular atrial fibrillation. JAMA Cardiol 2018;3:417-424.

1409. Butt JH, Olesen JB, Havers-Borgersen E, Gundlund A, Andersson C, Gislason $\mathrm{GH}$, Torp-Pedersen C, Kober L, Fosbol EL. Risk of thromboembolism associated with atrial fibrillation following noncardiac surgery. J Am Coll Cardiol 2018;72:2027-2036.

1410. POISE Study group., Devereaux PJ, Yang H, Yusuf S, Guyatt G, Leslie K, Villar JC, Xavier D, Chrolavicius S, Greenspan L, Pogue J, Pais P, Liu L, Xu S, Malaga G, Avezum A, Chan M, Montori VM, Jacka M, Choi P. Effects of extendedrelease metoprolol succinate in patients undergoing non-cardiac surgery (POISE trial): a randomised controlled trial. Lancet 2008;371:1839-1847.

1411. Leonardi M, Bissett J. Prevention of atrial fibrillation. Curr Opin Cardiol 2005;20:417-423.

1412. Roberts JD, Dewland TA, Glidden DV, Hoffmann TJ, Arking DE, Chen LY, Psaty BM, Olgin JE, Alonso A, Heckbert SR, Marcus GM. Impact of genetic variants on the upstream efficacy of renin-angiotensin system inhibitors for the prevention of atrial fibrillation. Am Heart J 2016;175:9-17.

1413. Pena JM, MacFadyen J, Glynn RJ, Ridker PM. High-sensitivity C-reactive protein, statin therapy, and risks of atrial fibrillation: an exploratory analysis of the JUPITER trial. Eur Heart J 2012;33:531 - 537.

1414. Maggioni AP, Fabbri G, Lucci D, Marchioli R, Franzosi MG, Latini R, Nicolosi GL, Porcu M, Cosmi F, Stefanelli S, Tognoni G; GISSI-HF Investigators. Effects of rosuvastatin on atrial fibrillation occurrence: ancillary results of the GISSIHF trial. Eur Heart J 2009;30:2327-2336.

1415. Zhou X, Du JL, Yuan J, Chen YQ. Statin therapy is beneficial for the prevention of atrial fibrillation in patients with coronary artery disease: a meta-analysis. Eur J Pharmacol 2013;707:104-111.

1416. Fang WT, Li HJ, Zhang H, Jiang S. The role of statin therapy in the prevention of atrial fibrillation: a meta-analysis of randomized controlled trials. $\mathrm{Br} J \mathrm{Clin}$ Pharmacol 2012;74:744-756.

1417. Macfarlane PW, Murray H, Sattar N, Stott DJ, Ford I, Buckley B, Jukema JW Westendorp RG, Shepherd J. The incidence and risk factors for new onset atrial fibrillation in the PROSPER study. Europace 2011;13:634-639.

1418. Haywood LJ, Ford CE, Crow RS, Davis BR, Massie BM, Einhorn PT, Williard A; ALLHAT Collaborative Research Group. Atrial fibrillation at baseline and during follow-up in ALLHAT (Antihypertensive and Lipid-Lowering Treatment to Prevent Heart Attack Trial). J Am Coll Cardiol 2009;54: 2023-2031.

1419. Schwartz GG, Chaitman BR, Goldberger JJ, Messig M. High-dose atorvastatin and risk of atrial fibrillation in patients with prior stroke or transient ischemic attack: analysis of the Stroke Prevention by Aggressive Reduction in Cholesterol Levels (SPARCL) trial. Am Heart J 2011;161:993-999.

1420. Fauchier L, Clementy N, Babuty D. Statin therapy and atrial fibrillation: systematic review and updated meta-analysis of published randomized controlled trials. Curr Opin Cardiol 2013;28:7-18.

1421. Yang Q, Qi X, Li Y. The preventive effect of atorvastatin on atrial fibrillation: a meta-analysis of randomized controlled trials. BMC Cardiovasc Disord 2014;14:99.

1422. Martino A, Pezzi L, Magnano R, Salustri E, Penco M, Calo L. Omega 3 and atrial fibrillation: where are we?World J Cardiol 2016;8:114-119.

1423. Linde C, Bongiorni MG, Birgersdotter-Green U, Curtis AB, Deisenhofer I, Furokawa T, Gillis AM, Haugaa KH, Lip GYH, Van Gelder I, Malik M, Poole J, Potpara T, Savelieva I, Sarkozy A; ESC Scientific Document Group. Sex differences in cardiac arrhythmia: a consensus document of the European Heart Rhythm Association, endorsed by the Heart Rhythm Society and Asia Pacific Heart Rhythm Society. Europace 2018;20:1565-1565ao.

1424. Potpara TS, Marinkovic JM, Polovina MM, Stankovic GR, Seferovic PM, Ostojic MC, Lip GY. Gender-related differences in presentation, treatment and longterm outcome in patients with first-diagnosed atrial fibrillation and structurally normal heart: the Belgrade atrial fibrillation study. Int J Cardiol 2012;161:39-44.

1425. Potpara TS, Blomstrom-Lundqvist C. Sex-related differences in atrial fibrillation: can we discern true disparities from biases? Heart 2017;103:979-981. 
1426. Piccini JP, Simon DN, Steinberg BA, Thomas L, Allen LA, Fonarow GC, Gersh B, Hylek E, Kowey PR, Reiffel JA, Naccarelli GV, Chan PS, Spertus JA, Peterson ED; Outcomes Registry for Better Informed Treatment of Atrial Fibrillation Investigators and Patients. Differences in clinical and functional outcomes of atrial fibrillation in women and men: two-year results from the ORBIT-AF registry. JAMA Cardiol 2016;1:282-291.

1427. Lang C, Seyfang L, Ferrari J, Gattringer T, Greisenegger S, Willeit K, Toell T, Krebs S, Brainin M, Kiechl S, Willeit J, Lang W, Knoflach M; Austrian Stroke Registry Collaborators. Do women with atrial fibrillation experience more severe strokes? Results from the Austrian Stroke Unit Registry. Stroke 2017;48:778-780.

1428. Sullivan RM, Zhang J, Zamba G, Lip GY, Olshansky B. Relation of genderspecific risk of ischemic stroke in patients with atrial fibrillation to differences in warfarin anticoagulation control (from AFFIRM). Am J Cardiol 2012;110:1799-1802.

1429. Rienstra M, Van Veldhuisen DJ, Hagens VE, Ranchor AV, Veeger NJ, Crijns HJ, Van Gelder IC; RACE Investigators. Gender-related differences in rhythm control treatment in persistent atrial fibrillation: data of the Rate Control Versus Electrical Cardioversion (RACE) study. J Am Coll Cardiol 2005;46:1298-1306.

1430. Lehmann MH, Timothy KW, Frankovich D, Fromm BS, Keating M, Locati EH, Taggart RT, Towbin JA, Moss AJ, Schwartz PJ, Vincent GM. Age-gender influence on the rate-corrected QT interval and the QT-heart rate relation in families with genotypically characterized long QT syndrome. J Am Coll Cardiol 1997;29:93-99.

1431. Zylla MM, Brachmann J, Lewalter T, Hoffmann E, Kuck KH, Andresen D, Willems S, Eckardt L, Tebbenjohanns J, Spitzer SG, Schumacher B, Hochadel M, Senges J, Katus HA, Thomas D. Sex-related outcome of atrial fibrillation ablation: insights from the German Ablation Registry. Heart Rhythm 2016;13:1837-1844.

1432. Patel N, Deshmukh A, Thakkar B, Coffey JO, Agnihotri K, Patel A, Ainani N, Nalluri N, Patel N, Patel N, Patel N, Badheka AO, Kowalski M, Hendel R, Viles-Gonzalez J, Noseworthy PA, Asirvatham S, Lo K, Myerburg RJ, Mitrani $\mathrm{RD}$. Gender, race, and health insurance status in patients undergoing catheter ablation for atrial fibrillation. Am J Cardiol 2016;117:1117-1126.

1433. Emdin CA, Wong CX, Hsiao AJ, Altman DG, Peters SA, Woodward M, Odutayo AA. Atrialfibrillation as risk factor for cardiovascular disease and death in women compared with men: systematic review and meta-analysis of cohort studies. BMJ 2016;532:h7013.

1434. Lip GY, Laroche C, Popescu MI, Rasmussen LH, Vitali-Serdoz L, Dan GA, Kalarus Z, Crijns HJ, Oliveira MM, Tavazzi L, Maggioni AP, Boriani G. Improved outcomes with European Society of Cardiology guideline-adherent antithrombotic treatment in high-risk patients with atrial fibrillation: a report from the EORP-AF General Pilot Registry. Europace 2015;17:1777-1786.

1435. Proietti M, Nobili A, Raparelli V, Napoleone L, Mannucci PM, Lip GY; REPOSI Investigators. Adherence to antithrombotic therapy guidelines improves mortality among elderly patients with atrial fibrillation: insights from the REPOSI study. Clin Res Cardiol 2016;105:912-920.

1436. Kirchhof P, Ammentorp B, Darius H, De Caterina R, Le Heuzey JY, Schilling RJ, Schmitt J, Zamorano JL. Management of atrial fibrillation in seven European countries after the publication of the 2010 ESC Guidelines on atrial fibrillation: primary results of the PREvention of thromboemolic events - European Registry in Atrial Fibrillation (PREFER in AF). Europace 2014;16:6-14.

1437. Lip GY, Laroche C, loachim PM, Rasmussen LH, Vitali-Serdoz L, Petrescu L, Darabantiu D, Crijns HJ, Kirchhof P, Vardas P, Tavazzi L, Maggioni AP, Boriani G. Prognosis and treatment of atrial fibrillation patients by European cardiologists: one year follow-up of the EURObservational Research Programme Atrial Fibrillation General Registry Pilot Phase (EORP-AF Pilot registry). Eur Heart J 2014;35:3365-3376.

1438. Jortveit J, Pripp AH, Langorgen J, Halvorsen S. Poor adherence to guideline recommendations among patients with atrial fibrillation and acute myocardial infarction. Eur J Prev Cardiol 2019:2047487319841940.

1439. Potpara TS, Dan GA, Trendafilova E, Goda A, Kusljugic Z, Manola S, Music L, Musetescu R, Badila E, Mitic G, Paparisto V, Dimitrova ES, Polovina MM, Petranov SL, Djergo H, Loncar D, Bijedic A, Brusich S, Lip GY; Balkan-AF Investigators. Stroke prevention in atrial fibrillation and 'real world' adherence to guidelines in the Balkan region: the BALKAN-AFSurvey. Sci Rep 2016;6:20432

1440. Kim H, Kim TH, Cha MJ, Lee JM, Park J, Park JK, Kang KW, Shim J, Uhm JS, Kim J, Park HW, Choi EK, Kim JB, Kim C, Lee YS, Joung B. A prospective survey of atrial fibrillation management for real-world guideline adherence: COmparison study of Drugs for symptom control and complication prEvention of Atrial Fibrillation (CODE-AF) Registry. Korean Circ J 2017; 47:877-887.

1441. Miyazawa K, Li YG, Rashed WA, Al Mahmeed W, Shehab A, Zubaid M, Lip $\mathrm{GYH}$. Secondary stroke prevention and guideline adherent antithrombotic treatment in patients with atrial fibrillation: insights from the Gulf Survey of Atrial Fibrillation events (Gulf SAFE). Int J Cardiol 2019;274:126-131.

1442. Camm AJ, Accetta G, Ambrosio G, Atar D, Bassand JP, Berge E, Cools F, Fitzmaurice DA, Goldhaber SZ, Goto S, Haas S, Kayani G, Koretsune Y, Mantovani LG, Misselwitz F, Oh S, Turpie AG, Verheugt FW, Kakkar AK; GARFIELD-AF Investigators. Evolving antithrombotic treatment patterns for patients with newly diagnosed atrial fibrillation. Heart 2017;103:307-314.

1443. Steinberg BA, Gao H, Shrader P, Pieper K, Thomas L, Camm AJ, Ezekowitz MD, Fonarow GC, Gersh BJ, Goldhaber S, Haas S, Hacke W, Kowey PR, Ansell J, Mahaffey KW, Naccarelli G, Reiffel JA, Turpie A, Verheugt F, Piccini JP, Kakkar A, Peterson ED, Fox KAA, Garfield AF; ORBIT-AF Investigators. International trends in clinical characteristics and oral anticoagulation treatment for patients with atrial fibrillation: results from the GARFIELD-AF, ORBIT-AF I, and ORBIT-AF II registries. Am Heart J 2017;194:132-140.

1444. Leef GC, Perino AC, Askari M, Fan J, Ho PM, Olivier CB, Longo L, Mahaffey $\mathrm{KW}$, Turakhia MP. Appropriateness of direct oral anticoagulant dosing in patients with atrial fibrillation: insights from the Veterans Health Administration. J Pharm Pract 2019:897190019828270.

1445. Dupree L, DeLosSantos M, Smotherman C. Evaluation of adherence to guideline-directed antithrombotic therapy for atrial fibrillation at hospital discharge. J Cardiovasc Pharmacol Ther 2018;23:502-508.

1446. Heidbuchel H, Dagres N, Antz M, Kuck KH, Lazure P, Murray S, Carrera C, Hindricks G, Vahanian A. Major knowledge gaps and system barriers to guideline implementation among European physicians treating patients with atrial fibrillation: a European Society of Cardiology international educational needs assessment. Europace 2018;20:1919-1928.

1447. Desteghe L, Germeys J, Vijgen J, Koopman P, Dilling-Boer D, Schurmans J, Delesie M, Dendale P, Heidbuchel $H$. Effectiveness and usability of an online tailored education platform for atrial fibrillation patients undergoing a direct current cardioversion or pulmonary vein isolation. Int $J$ Cardiol 2018;272:123-129.

1448. Marquez-Contreras E, Martell-Claros N, Marquez-Rivero S, Hermida-Campa E, Gracia-Diez C, Sanchez-Lopez E, Gil-Guillen V; Compliance and Inertia Working Group, Spanish Society of Hypertension (SEH-LELHA). Strategies for improving dabigatran adherence for stroke prevention in patients with non-valvular atrial fibrillation: education and drug intake reminders (FACILITA study). Curr Med Res Opin 2018;34:1301-1308.

1449. Piccini JP, Xu H, Cox M, Matsouaka RA, Fonarow GC, Butler J, Curtis AB, Desai N, Fang M, McCabe PJ, Page li RL, Turakhia M, Russo AM, Knight BP, Sidhu M, Hurwitz JL, Ellenbogen KA, Lewis WR; Get With The GuidelinesAFIB Clinical Working Group and Hospitals. Adherence to guideline-directed stroke prevention therapy for atrial fibrillation is achievable. Circulation 2019;139:1497-1506.

1450. Richardson KM, Singh J, Munoz D, Damp JB, Mendes LA. Improving practice guideline adherence through peer feedback: impact of an ambulatory cardiology curriculum. Teach Learn Med 2018;30:328-336.

1451. Ferguson C, Hickman LD, Phillips J, Newton PJ, Inglis SC, Lam L, Bajorek BV. An mHealth intervention to improve nurses' atrial fibrillation and anticoagulation knowledge and practice: the EVICOAG study. Eur J Cardiovasc Nurs 2019;18:7-15.

1452. Siebenhofer A, Ulrich LR, Mergenthal K, Berghold A, Pregartner G, Kemperdick B, Schulz-Rothe S, Rauck S, Harder S, Gerlach FM, Petersen JJ. Primary care management for patients receiving long-term antithrombotic treatment: a cluster-randomized controlled trial. PLoS One 2019;14:e0209366.

1453. Heidenreich PA, Solis P, Estes NAM 3rd, Fonarow GC, Jurgens CY, Marine JE, McManus DD, McNamara RL. 2016 ACC/AHA clinical performance and quality measures for adults with atrial fibrillation or atrial flutter: a report of the American College of Cardiology/American Heart Association Task Force on performance measures. J Am Coll Cardiol 2016;68:525-568.

1454. Lewis WR, Piccini JP, Turakhia MP, Curtis AB, Fang M, Suter RE, Page RL, 2nd, Fonarow GC. Get With The Guidelines AFIB: novel quality improvement registry for hospitalized patients with atrial fibrillation. Circ Cardiovasc Qual Outcomes 2014;7:770-777.

1455. Friedman DJ, Al-Khatib SM. Measuring quality in electrophysiology. J Interv Card Electrophysiol 2016;47:5-10.

1456. Schulman S, Kearon C; Subcommittee on Control of Anticoagulation of the Scientific and Standardization Committee of the International Society on Thrombosis and Haemostasis. Definition of major bleeding in clinical investigations of antihemostatic medicinal products in non-surgical patients. J Thromb Haemost 2005;3:692-694.

1457. Schulman S, Angeras U, Bergqvist D, Eriksson B, Lassen MR, Fisher W; Subcommittee on Control of Anticoagulation of the Scientific and Standardization Committee of the International Society on Thrombosis and Haemostasis. Definition of major bleeding in clinical investigations of antihemostatic medicinal products in surgical patients. J Thromb Haemost 2010;8:202-204. 
1458. Diederichsen SZ, Haugan KJ, Brandes A, Lanng MB, Graff C, Krieger D, Kronborg C, Holst AG, Kober L, Hojberg S, Svendsen JH. Natural history of subclinical atrial fibrillation detected by implanted loop recorders. I Am Coll Cardiol 2019;74:2771-2781.

1459. Swiryn S, Orlov MV, Benditt DG, DiMarco JP, Lloyd-Jones DM, Karst E, Qu F, Slawsky MT, Turkel M, Waldo AL; RATE Registry Investigators. Clinical implications of brief device-detected atrial tachyarrhythmias in a cardiac rhythm management device population: results from the Registry of Atrial Tachycardia and Atrial Fibrillation Episodes. Circulation 2016;134:1130-1140.

1460. Pastori D, Miyazawa K, Li Y, Szekely O, Shahid F, Farcomeni A, Lip GYH. Atrial high-rate episodes and risk of major adverse cardiovascular events in patients with cardiac implantable electronic devices. Clin Res Cardiol 2020;109:96-102.

1461. Gonzalez M, Keating RJ, Markowitz SM, Liu CF, Thomas G, Ip JE, Lerman BB, Cheung JW. Newly detected atrial high rate episodes predict long-term mortality outcomes in patients with permanent pacemakers. Heart Rhythm 2014;11:2214-2221.

1462. Gorenek BC, Bax J, Boriani G, Chen SA, Dagres N, Glotzer TV, Healey JS, Israel CW, Kudaiberdieva G, Levin LA, Lip GYH, Martin D, Okumura K, Svendsen JH, Tse HF, Botto GLC-C; ESC Scientific Document Group. Devicedetected subclinical atrial tachyarrhythmias: definition, implications and management - an European Heart Rhythm Association (EHRA) consensus document, endorsed by Heart Rhythm Society (HRS), Asia Pacific Heart Rhythm Society (APHRS) and Sociedad Latinoamericana de Estimulacion Cardiaca y Electrofisiologia (SOLEACE). Europace 2017;19:1556-1578.

1463. Nasir JM, Pomeroy W, Marler A, Hann M, Baykaner T, Jones R, Stoll R, Hursey K, Meadows A, Walker J, Kindsvater S. Predicting determinants of atrial fibrillation or flutter for therapy elucidation in Patients at Risk for Thromboembolic Events (PREDATE AF) study. Heart Rhythm 2017;14:955-961.

1464. Reiffel JA, Verma A, Kowey PR, Halperin JL, Gersh BJ, Wachter R, Pouliot E, Ziegler PD; REVEAL AF Investigators. Incidence of previously undiagnosed atrial fibrillation using insertable cardiac monitors in a high-risk population: the REVEAL AF study. JAMA Cardiol 2017;2:1120-1127.

1465. Kaplan RM, Koehler J, Ziegler PD, Sarkar S, Zweibel S, Passman RS. Stroke risk as a function of atrial fibrillation duration and CHA2DS2-VASc score. Circulation 2019;140:1639-1646.

1466. Celikyurt U, Knecht S, Kuehne M, Reichlin T, Muehl A, Spies F, Osswald S, Sticherling $C$. Incidence of new-onset atrial fibrillation after cavotricuspid isthmus ablation for atrial flutter. Europace 2017;19:1776-1780.

1467. Bertaglia E, Bonso A, Zoppo F, Proclemer A, Verlato R, Coro L, Mantovan R, Themistoclakis S, Raviele A, Pascotto P; North-Eastern Italian Study on Atrial Flutter Ablation Investigators. Different clinical courses and predictors of atrial fibrillation occurrence after transisthmic ablation in patients with preablation lone atrial flutter, coexistent atrial fibrillation, and drug induced atrial flutter. Pacing Clin Electrophysiol 2004;27:1507-1512.

1468. Nabar A, Rodriguez LM, Timmermans C, van Mechelen R, Wellens HJ. Class IC antiarrhythmic drug induced atrial flutter: electrocardiographic and electrophysiological findings and their importance for long term outcome after right atrial isthmus ablation. Heart 2001;85:424-429.

1469. Enriquez A, Sarrias A, Villuendas R, Ali FS, Conde D, Hopman WM, Redfearn DP, Michael K, Simpson C, De Luna AB, Bayes-Genis A, Baranchuk A. Newonset atrial fibrillation after cavotricuspid isthmus ablation: identification of advanced interatrial block is key. Europace 2015;17:1289-1293.

1470. Maskoun W, Pino MI, Ayoub K, Llanos OL, Almomani A, Nairooz R, Hakeem A, Miller J. Incidence of atrial fibrillation after atrial flutter ablation. JACC Clin Electrophysiol 2016;2:682-690.

1471. Reithmann C, Hoffmann E, Spitzlberger G, Dorwarth U, Gerth A, Remp T, Steinbeck G. Catheter ablation of atrial flutter due to amiodarone therapy for paroxysmal atrial fibrillation. Eur Heart J 2000;21:565-572.

1472. Vadmann H, Nielsen PB, Hjortshoj SP, Riahi S, Rasmussen LH, Lip GY, Larsen TB. Atrial flutter and thromboembolic risk: a systematic review. Heart 2015;101:1446-1455

1473. Van Gelder IC, Crijns HJ, Van Gilst WH, Verwer R, Lie KI. Prediction of uneventful cardioversion and maintenance of sinus rhythm from direct-current electrical cardioversion of chronic atrial fibrillation and flutter. Am J Cardiol 1991;68:41-46.

1474. Gillis AM, Unterberg-Buchwald C, Schmidinger H, Massimo S, Wolfe K, Kavaney DJ, Otterness MF, Hohnloser SH; GEM III AT Worldwide Investigators. Safety and efficacy of advanced atrial pacing therapies for atrial tachyarrhythmias in patients with a new implantable dual chamber cardioverter-defibrillator. J Am Coll Cardiol 2002;40:1653-1659.
1475. Crijns HJ, Van Gelder IC, Kingma JH, Dunselman PH, Gosselink AT, Lie KI. Atrial flutter can be terminated by a class III antiarrhythmic drug but not by a class IC drug. Eur Heart J 1994;15:1403-1408.

1476. Ellenbogen KA, Stambler BS, Wood MA, Sager PT, Wesley RC, Jr., Meissner MC, Zoble RG, Wakefield LK, Perry KT, Vanderlugt JT. Efficacy of intravenous ibutilide for rapid termination of atrial fibrillation and atrial flutter: a doseresponse study. J Am Coll Cardiol 1996;28:130-136.

1477. Falk RH, Pollak A, Singh SN, Friedrich T. Intravenous dofetilide, a class III antiarrhythmic agent, for the termination of sustained atrial fibrillation or flutter. Intravenous Dofetilide Investigators. I Am Coll Cardiol 1997;29:385-390.

1478. Singh S, Zoble RG, Yellen L, Brodsky MA, Feld GK, Berk M, Billing CB, Jr. Efficacy and safety of oral dofetilide in converting to and maintaining sinus rhythm in patients with chronic atrial fibrillation or atrial flutter: the symptomatic atrial fibrillation investigative research on dofetilide (SAFIRE-D) study. Circulation 2000;102:2385-2390.

1479. Crozier IG, Ikram H, Kenealy M, Levy L. Flecainide acetate for conversion of acute supraventricular tachycardia to sinus rhythm. Am J Cardiol 1987;59:607-609.

1480. Murdock C], Kyles AE, Yeung-Lai-Wah JA, Qi A, Vorderbrugge S, Kerr CR Atrial flutter in patients treated for atrial fibrillation with propafenone. Am J Cardiol 1990;66:755-757.

1481. Da Costa A, Thevenin J, Roche F, Romeyer-Bouchard C, Abdellaoui L, Messier M, Denis L, Faure E, Gonthier R, Kruszynski G, Pages JM, Bonijoly S, Lamaison D, Defaye P, Barthelemy JC, Gouttard T, Isaaz K; Loire-ArdècheDrôme-Isère-Puy-de-Dôme Trial of Atrial Flutter Investigators. Results from the Loire-Ardèche-Drôme-Isère-Puy-de-Dome (LADIP) trial on atrial flutter, a multicentric prospective randomized study comparing amiodarone and radiofrequency ablation after the first episode of symptomatic atrial flutter. Circulation 2006;114:1676-1681.

1482. Schwartzman D, Callans DJ, Gottlieb CD, Dillon SM, Movsowitz C Marchlinski FE. Conduction block in the inferior vena caval-tricuspid valve isthmus: association with outcome of radiofrequency ablation of type I atrial flutter. J Am Coll Cardiol 1996;28:1519-1531.

1483. Wasmer K, Monnig G, Bittner A, Dechering D, Zellerhoff S, Milberg P, Kobe J, Eckardt L. Incidence, characteristics, and outcome of left atrial tachycardias after circumferential antral ablation of atrial fibrillation. Heart Rhythm 2012;9:1660-1666.

1484. Satomi K, Bansch D, Tilz R, Chun J, Ernst S, Antz M, Greten H, Kuck KH, Ouyang F. Left atrial and pulmonary vein macroreentrant tachycardia associated with double conduction gaps: a novel type of man-made tachycardia after circumferential pulmonary vein isolation. Heart Rhythm 2008;5:43-51.

1485. Chugh A, Oral H, Lemola K, Hall B, Cheung P, Good E, Tamirisa K, Han J, Bogun F, Pelosi F Jr, Morady F. Prevalence, mechanisms, and clinical significance of macroreentrant atrial tachycardia during and following left atrial ablation for atrial fibrillation. Heart Rhythm 2005;2:464-471.

1486. Goette A, Auricchio A, Boriani G, Braunschweig F, Terradellas JB, Burri $H$, Camm AJ, Crijns H, Dagres N, Deharo JC, Dobrev D, Hatala R, Hindricks G, Hohnloser SH, Leclercq C, Lewalter T, Lip GYH, Merino JL, Mont L, Prinzen F, Proclemer A, Purerfellner H, Savelieva I, Schilling R, Steffel J, van Gelder IC, Zeppenfeld K, Zupan I, Heidbuchel H. EHRA White Paper: knowledge gaps in arrhythmia management - status 2019. Europace 2019; 21:993-994.

1487. De With RR, Marcos EG, Dudink E, Spronk HM, Crijns H, Rienstra M, Van Gelder IC. Atrial fibrillation progression risk factors and associated cardiovascular outcome in well-phenotyped patients: data from the AF-RISK study. Europace 2020;22:352-360.

1488. Andrade JG, Deyell MW, Lee AY, Macle L. Sex differences in atrial fibrillation. Canadian J Cardiol 2018;34:429-436.

1489. Potpara TS, Ferro C, Lip GYH, Dan GA, Lenarczyk R, Mallamaci F, Ortiz A Sarafidis P, Ekart R, Dagres N. Management of atrial fibrillation in patients with chronic kidney disease in clinical practice: a joint European Heart Rhythm Association (EHRA) and European Renal Association/European Dialysis and Transplantation Association (ERA/EDTA) physician-based survey. Europace 2020;22:496-505.

1490. Al-Khatib SM, Benjamin EJ, Buxton AE, Calkins $H$, Chung MK, Curtis $A B$, Desvigne-Nickens P, Jais P, Packer DL, Piccini JP, Rosenberg Y, Russo AM, Wang PJ, Cooper LS, Go AS, Workshop C. Research needs and priorities for catheter ablation of atrial fibrillation: a report from a National Heart, Lung, and Blood Institute Virtual Workshop. Circulation 2020;141:482-492.

1491. Nielsen JC, Lin YJ, de Oliveira Figueiredo MJ, Sepehri Shamloo A, Alfie A, Boveda S, Dagres N, Di Toro D, Eckhardt LL, Ellenbogen K, Hardy C, Ikeda T, 
Jaswal A, Kaufman E, Krahn A, Kusano K, Kutyifa V, Lim HS, Lip GYH, NavaTownsend S, Pak HN, RodrÚguez Diez G, Sauer W, Saxena A, Svendsen JH, Vanegas D, Vaseghi M, Wilde A, Bunch T]; ESC Scientific Document Group, Buxton AE, Calvimontes G, Chao TF, Eckardt L, Estner H, Gillis AM, Isa R, Kautzner J, Maury P, Moss JD, Nam GB, Olshansky B, Pava Molano LF, Pimentel M, Prabhu M, Tzou WS, Sommer P, Swampillai J, Vidal A, Deneke T, Hindricks G, Leclercq C. European Heart Rhythm Association (EHRA)/Heart Rhythm Society (HRS)/Asia Pacific Heart Rhythm Society (APHRS)/Latin
American Heart Rhythm Society (LAHRS) expert consensus on risk assessment in cardiac arrhythmias: use the right tool for the right outcome, in the right population. Europace 2020;22:1147-1148.

1492. Blessberger $H$, Lewis SR, Pritchard MW, Fawcett LJ, Domanovits $H$, Schlager O, Wildner B, Kammler J, Steinwender C. Perioperative beta-blockers for preventing surgery-related mortality and morbidity in adults undergoing cardiac surgery. Cochrane Database Syst Rev 2019 23;9:CD013435. 